\title{
Pentanidium- and Bisguanidinium-Catalyzed Enantioselective Alkylations Using Silylamide as Brønsted Probase
}

Bo Teng*,${ }^{\dagger}$ Wenchao Chen, ${ }^{\dagger}$ Shen Dong, ${ }^{\dagger}$ Choon Wee Kee, ${ }^{\dagger}$ Dhika Aditya Gandamana, ${ }^{\dagger}$ Lili Zong, ${ }^{\dagger}$ Choon-Hong Tan ${ }^{*}, \dagger$

†Division of Chemistry \& Biological Chemistry, School of Physical \& Mathematical Sciences, Nanyang Technological University, Singapore 637371, Singapore

*choonhong@ntu.edu.sg

*bteng001@,ntu.edu.sg

\section{Supporting Information}

\section{Contents}

I: General Experimental

II: Table S1, S2, S3

S3-4

III: General Procedure for Synthesis of Dihydrocoumarins and Cyclic Ketones

IV: General Procedure for Catalytic Enantioselective Alkylation

V: Preparation of Silyl Ketene Acetal and Silyl Enol Ether

VI: Determination of Absolute Configuration

VII: Characterization of Starting Materials and Products

VIII: Calculation Result

IX: ${ }^{1} \mathrm{H}$ NMR, ${ }^{13} \mathrm{C}$ NMR Spectra of Starting Materials and Products

X: Characterization of Starting Materials and Products

S179-237 


\section{I: General Experimental}

Commercially available materials purchased from Alfa Aesar or Aldrich was used as received. HPLC grade solvents (toluene and methanol) were used without further purification. Proton nuclear magnetic resonance $\left({ }^{1} \mathrm{H}\right.$ NMR) spectra were recorded on a Bruker AV400 (400 MHz) spectrometer. Chemical shifts were recorded in parts per million $(\mathrm{ppm}, \delta)$ relative to tetramethylsilane $(\delta 0.00) .{ }^{1} \mathrm{H}$ NMR splitting patterns are designated as singlet (s), doublet (d), triplet (t), quartet (q), dd (doublet of doublets); m (multiplets), and etc. All first-order splitting patterns were assigned on the basis of the appearance of the multiplet. Splitting patterns that could not be easily interpreted are designated as multiplet (m) or broad (br). Carbon nuclear magnetic resonance $\left({ }^{13} \mathrm{C} N M R\right)$ spectra were recorded on a Bruker AV400 (100 MHz) spectrometer. High resolution mass spectral analysis (HRMS) was performed on Waters Q-TOF Premier mass spectrometer. IR spectra were recorded on a Shimadzu IRPrestige-21 FT-IR spectrometer as neat thinfilms between $\mathrm{NaCl}$ plates. Optical rotations were measured using a $1 \mathrm{~mL}$ cell with a $1 \mathrm{~cm}$ path length on a Jasco P1030 digital polarimeter and are reported as follows: $[\alpha]^{t} \mathrm{D}$. Flash chromatography was performed using Merck silica gel 60 with distilled solvents. Analytical thin-layer chromatography (TLC) was carried out on Merck 60 F254 pre-coated silica gel plate (0.2 mm thickness). Visualization was performed using a UV lamp. 


\section{II: Table S1, S2, S3}

Table S1. Alkylation of Dihydrocoumarin 1a Using Different Base ${ }^{a}$
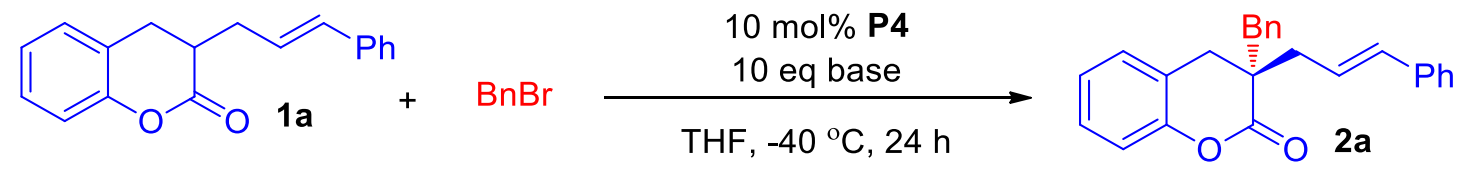

\begin{tabular}{ccc}
\hline entry & base & result ${ }^{b, c}$ \\
\hline 1 & $\mathrm{Na}_{2} \mathrm{CO}_{3}$ & $\mathrm{NR}$ \\
2 & $\mathrm{~K}_{2} \mathrm{CO}_{3}$ & $\mathrm{NR}$ \\
3 & $\mathrm{Cs}_{2} \mathrm{CO}_{3}$ & $\mathrm{NR}$ \\
4 & $\mathrm{~K}_{3} \mathrm{PO}_{4}$ & SM decompose \\
5 & $\mathrm{KOAc}$ & $\mathrm{NR}$ \\
6 & $\mathrm{PhCO}_{2} \mathrm{~K}$ & $\mathrm{NR}$ \\
7 & $\mathrm{LiOH} \cdot \mathrm{H}_{2} \mathrm{O}$ & SM decompose \\
8 & $\mathrm{NaOH}$ & SM decompose \\
9 & $\mathrm{KOH}$ & SM decompose \\
10 & $\mathrm{CsOH} \cdot \mathrm{H}_{2} \mathrm{O}$ & SM decompose \\
$11^{d}$ & $\mathrm{NaOMe}$ & SM decompose \\
$12^{d}$ & $t$ BuOK & Low yield, $0 \%$ ee \\
13 & Triethylamine & NR \\
$14^{e}$ & $\mathrm{LiHMDS}$ & $65 \%$ yield, $0 \%$ ee
\end{tabular}

${ }^{a}$ Reaction was conducted with of $1 \mathrm{a}(0.1 \mathrm{mmol}, 1.0$ equiv), $\mathrm{BnBr}(0.2 \mathrm{mmol}, 2.0$ equiv) in $0.4 \mathrm{~mL}$ THF in the presence of $10 \mathrm{~mol} \% \mathbf{P 4}$ for $24 \mathrm{~h}$ at $-40{ }^{\circ} \mathrm{C}$. ${ }^{b}$ Isolated yields. ${ }^{c}$ Determined by HPLC analysis on a chiral stationary phase. ${ }^{d} 3$ eq base was used in reaction. ${ }^{e} 1.5$ eq base was used in reaction.

The controlling experiments proved our Brønsted probase strategy could generate a strong base with weak nucleophilicity. Probase pb4 has strong basicity than carbonate salts, phosphrate salts, acetate salts. In comparision with hydroxide salts, probase pb4 has similar basicity but weaker nucleophilicity. For strong base $t \mathrm{BuOK}$ and LiHMDS, the in situ generation of base involved PTC catalyst can avoid background reaction, so excellent enantioselectivity was obtained. 
Table S2. Direct Alkylation of Linear Ketone

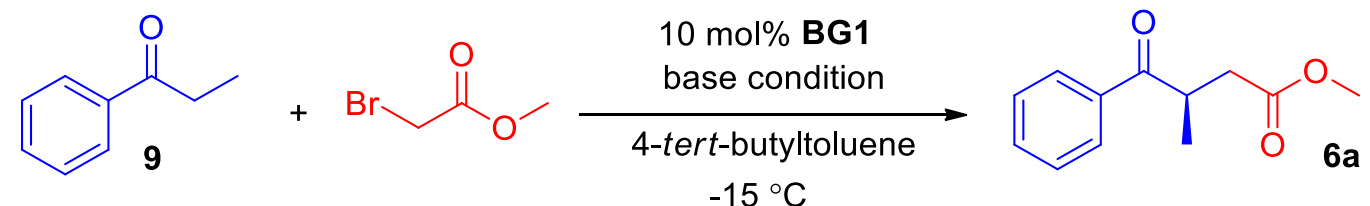

\begin{tabular}{cccc}
\hline entry & base condition & yield $^{c}$ & ee $^{d}$ \\
\hline $1^{a}$ & $5 \mathrm{eq} \mathrm{LiOH} \cdot \mathrm{H}_{2} \mathrm{O}$ & trace & -- \\
$2^{a}$ & $5 \mathrm{eq} \mathrm{NaOH}$ & trace & -- \\
$3^{a}$ & $5 \mathrm{eq} \mathrm{KOH}$ & trace & -- \\
$4^{a}$ & $5 \mathrm{eq} \mathrm{CsOH} \cdot \mathrm{H}_{2} \mathrm{O}$ & trace & -- \\
$5^{b}$ & $2 \mathrm{eq} \mathrm{pb4}$ and $5 \mathrm{eq} \mathrm{CsF}$ & $35^{e}$ & 56 \\
$6^{b}$ & $2 \mathrm{eq} \mathrm{pb5}$ and $5 \mathrm{eq} \mathrm{CsF}$ & trace & -- \\
\hline
\end{tabular}

${ }^{a}$ Reaction was carried out with $9(0.10 \mathrm{mmol})$, methyl bromoacetate $(0.20 \mathrm{mmol})$, base $(0.50 \mathrm{mmol})$ and catalyst $(10 \mathrm{~mol} \%)$ in $0.4 \mathrm{~mL}$ 4-tert-butyltoluene for $24 \mathrm{~h}$ at $-15{ }^{\circ} \mathrm{C}$. ${ }^{b}$ Reaction was carried out with $9(0.10$ $\mathrm{mmol})$, methyl bromoacetate $(0.20 \mathrm{mmol})$, probase $(0.20 \mathrm{mmol}), \mathrm{CsF}(0.50 \mathrm{mmol})$ and catalyst $(10 \mathrm{~mol} \%)$ in $0.4 \mathrm{~mL}$ 4-tert-butyltoluene for $24 \mathrm{~h}$ at $-15{ }^{\circ} \mathrm{C}$. Isolated yield. ${ }^{d}$ Determined by HPLC analysis on a chiral stationary phase. ${ }^{e}$ Silyl enol ether byproduct was formed.

Table S3. Alkylation of Cyclic Ketone Using Inorganic Bases ${ }^{a, b, c}$

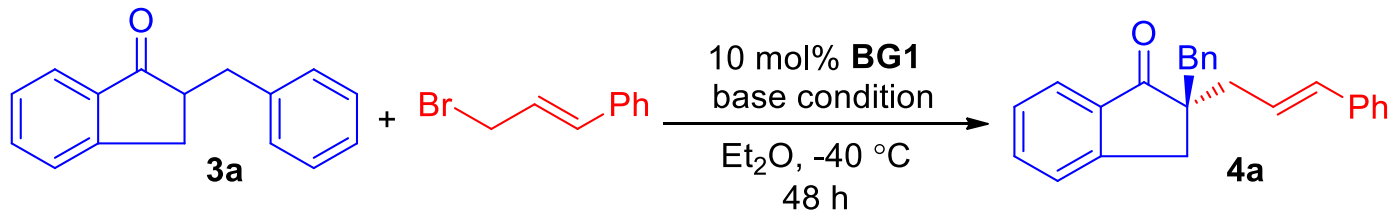

\begin{tabular}{cccc}
\hline entry & base condition & yield & ee \\
\hline 1 & $5 \mathrm{eq} \mathrm{LiOH} \cdot \mathrm{H}_{2} \mathrm{O}$ & trace & - \\
2 & $5 \mathrm{eq} \mathrm{NaOH}$ & 56 & 34 \\
3 & $5 \mathrm{eq} \mathrm{KOH}$ & 75 & 58 \\
4 & $5 \mathrm{eq} \mathrm{CsOH} \cdot \mathrm{H}_{2} \mathrm{O}$ & 93 & 37 \\
\hline
\end{tabular}

${ }^{a}$ Reaction was carried out with 3a $(0.10 \mathrm{mmol})$, cinnamyl bromide $(0.20 \mathrm{mmol})$, base $(0.50 \mathrm{mmol})$ and catalyst BG1 (10 mol\%) in $0.4 \mathrm{~mL} \mathrm{Et}_{2} \mathrm{O}$ for $48 \mathrm{~h}$ at $-40{ }^{\circ} \mathrm{C} .{ }^{b}$ Isolated yield. ${ }^{c}$ Determined by HPLC analysis on a chiral stationary phase. 


\section{III: General Procedure for Synthesis of Dihydrocoumarins and Cyclic Ketones}

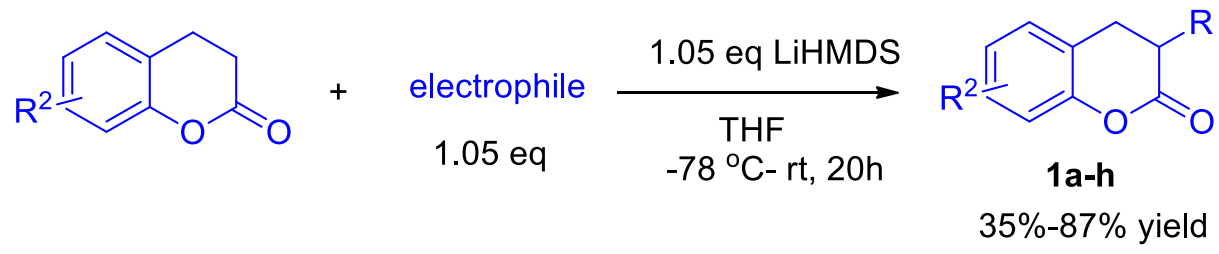

To a dry RBF equipped with a magnetic stir bar was added dihydrocoumarin and dry THF. The solution then was cooled to $-78^{\circ} \mathrm{C}$ and 1.05 eq LiHMDS was slowly added. The reaction was stirred at $-78{ }^{\circ} \mathrm{C}$ for $45 \mathrm{~min}$. 1.05 eq electrophile was then slowly added to the yellow solution. Keeping the reaction slowly increase temperature from $-78{ }^{\circ} \mathrm{C}$ to room temperature overnight. $\mathrm{NH}_{4} \mathrm{Cl}$ solution was added to quench the reaction. The organic phase was extracted with EA, dried with $\mathrm{Na}_{2} \mathrm{SO}_{4}$, after concentration, the residue was purified by flash chromatography (EA/Hexane: 1:50-1:20) to get the desired product in $35 \%$ to $87 \%$ yield.

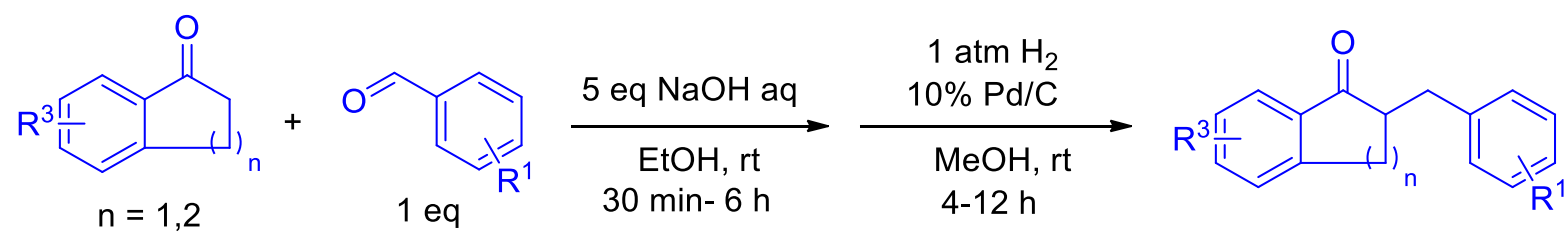

To a RBF equipped with a magnetic stir bar was added ketone substrate, aldehyde and EtOH. $\mathrm{NaOH}$ aqueous solution was slowly added to the RBF, precipitate was formed soon. The mixture was stirred at $\mathrm{rt}$ for $30 \mathrm{~min}$ to $6 \mathrm{hrs}$ and then put at $0^{\circ} \mathrm{C}$ for $1 \mathrm{~h}$. After filtration and washing with cooled $\mathrm{MeOH}$, the solid product was dissolved in $\mathrm{MeOH} .10 \% \mathrm{Pd} / \mathrm{C}$ was added to the solution and the reaction was stirred at 1 atm $\mathrm{H}_{2}$ for 4-12 to provide the desired product in $45-90 \%$ yield. 


\section{IV: General Procedure for Catalytic Enantioselective Alkylation}

General procedure for scheme 2 in article:

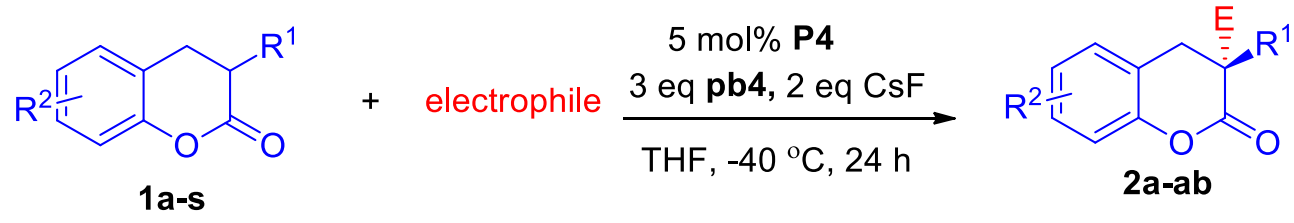

To a dry Schlenk tube equipped with a magnetic stir bar, was added $5 \mathrm{~mol} \%$ catalyst $\mathbf{P 4}, 3$ eq pb4, 2 eq CsF and 2 eq electrophile. The tube was closed after addition of $0.4 \mathrm{~mL}$ THF. The resulting mixture was stirred at $-40{ }^{\circ} \mathrm{C}$ for $24 \mathrm{~h}$. The reaction mixture was then directly applied to silica gel chromatography (EA/Hexane: 1:200-1:30) to obtain product.

General procedure for scheme 3 in article:

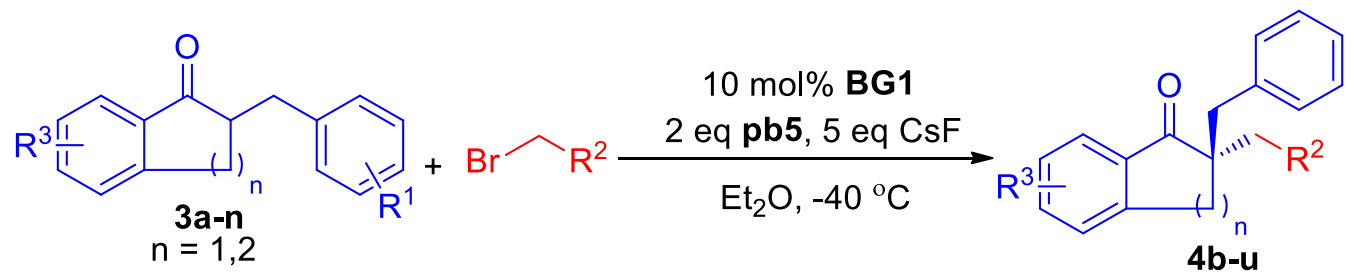

To a dry Schlenk tube equipped with a magnetic stir bar, was added $10 \mathrm{~mol} \%$ catalyst BG1, 2 eq pb5, 5 eq $\mathrm{CsF}$ and 2 eq electrophile. The tube was closed after addition of $0.4 \mathrm{~mL} \mathrm{Et}_{2} \mathrm{O}$. The resulting mixture was stirred at $-40{ }^{\circ} \mathrm{C}$ for $24-96 \mathrm{~h}$. The reaction mixture was then directly applied to silica gel chromatography (EA/Hexane: 1:200-1:30) to obtain product.

General procedure for scheme 4 in article:

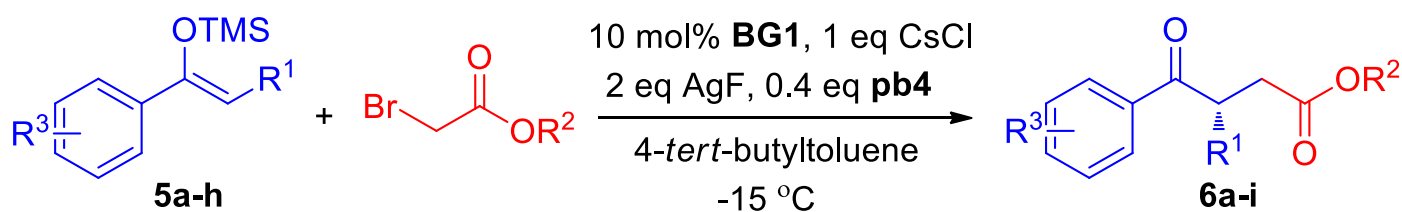

To a dry Schlenk tube equipped with a magnetic stir bar, was added $10 \mathrm{~mol} \%$ catalyst BG1, 0.4 eq pb4, 2 eq $\mathrm{AgF}, 1$ eq $\mathrm{CsCl}$ and 2 eq electrophile. The tube was closed after addition of $0.4 \mathrm{~mL}$ 4-tert-butyltoluene. The resulting mixture was stirred at $-15^{\circ} \mathrm{C}$ for $12-24 \mathrm{~h}$ under avoiding light. The reaction mixture was then directly applied to silica gel chromatography (EA/Hexane: 1:10) to obtain product. 
Gram scale experiment:<smiles>O=C1Oc2ccccc2CC1C/C=C/c1ccccc1</smiles>

$1.2 \mathrm{~g}$
$10 \mathrm{~mol} \% \mathbf{P 4}$

$\underset{\mathrm{THF},-40^{\circ} \mathrm{C}, 54 \mathrm{~h}}{\stackrel{4 \text { eq pb4, } 6 \text { eq } \mathrm{CsF}}{\longrightarrow}}$<smiles>O=C1Oc2ccccc2CC1(Br)C/C=C/c1ccccc1</smiles>

$92 \%$ yield, $1.48 \mathrm{~g}$ $93 \%$ ee

To a dry Schlenk tube equipped with a magnetic stir bar, was added substrate $1 \mathrm{a}(1.2 \mathrm{~g} 4.5$ mmol), 10 mol\% catalyst $\mathbf{P 4}(800 \mathrm{mg}), 4$ eq pb4 (4.2 mL, $18.0 \mathrm{mmol}), 6$ eq CsF (4.1 g, 27.0 $\mathrm{mmol})$ and 4 eq $\operatorname{BnBr}(2.2 \mathrm{~mL}, 18.0 \mathrm{mmol})$. The tube was closed after addition of $20 \mathrm{~mL}$ THF. The resulting mixture was stirred at $-40{ }^{\circ} \mathrm{C}$ for 54 h. $20 \mathrm{~mL} \mathrm{NaCl}$ solution was added to the reaction mixture and the aqueous phase was extracted with DCM (10 mL*3). The combination organic phase was dried with $\mathrm{Na}_{2} \mathrm{SO}_{4}$. After filtration, concentration, the residue was applied to silica gel chromatography (Hexane to EA/Hexane: 1:30 to EA to EA/MeOH: $15: 1)$ to obtain product $2 \mathrm{a}$ as white solid (1.48g, 92\% yield, 93\% ee) and recovered $384 \mathrm{mg}$ catalyst $\mathbf{P 4}$. 


\section{V: Preparation of Silyl Ketene Acetal and Silyl Enol Ether}

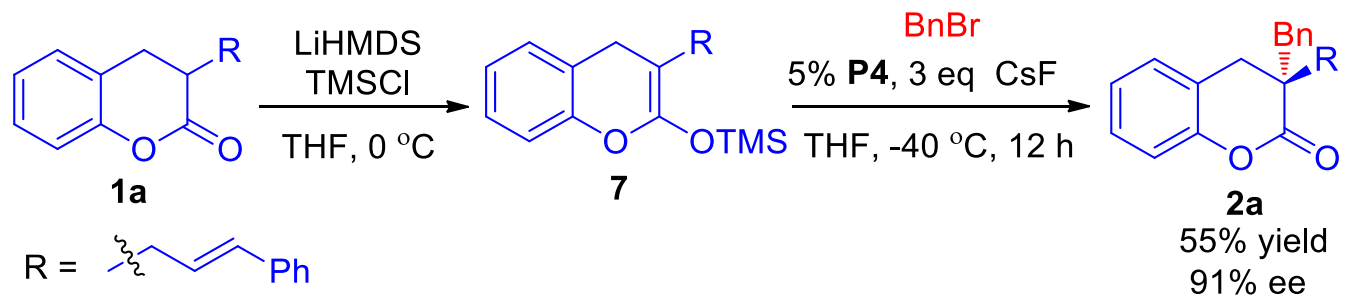

To a dry Schlenk tube equipped with a magnetic stir bar, was added 1a. The solid was dissolved in dry THF and the solution was cooled to $0^{\circ} \mathrm{C} .1 .1$ eq LiHMDS was slowly added to the solution. After stirring at $0^{\circ}$ for $30 \mathrm{~min}, 1.4$ eq $\mathrm{TMSCl}$ was slowly added to the reaction. After stirring at $\mathrm{rt}$ for $3 \mathrm{~h}$, the solvent was removed by rotavapor and the residue was washed with hexane. After concentration, the product was added in a dry Schlenk tube with 2eq bromide, 5\% mol P4 and 3 eq CsF. The resulting mixture was stirred at $-40{ }^{\circ} \mathrm{C}$ for $20 \mathrm{~h}$. The reaction mixture was then directly applied to silica gel chromatography (EA/Hexane: $1: 200-1: 30)$ to obtain product.

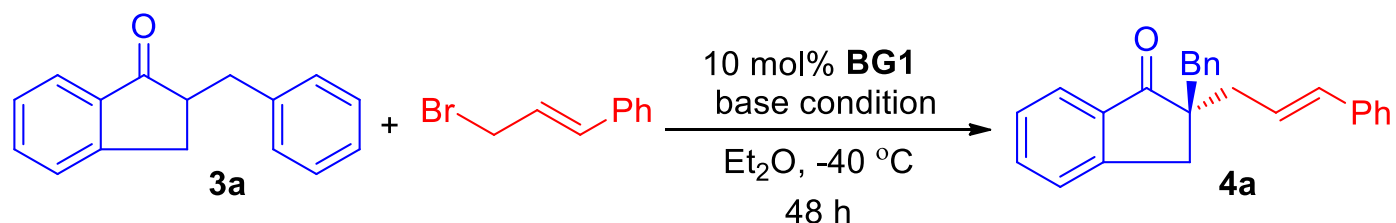

To a Schlenk tube equipped with a magnetic stir bar, was added 3a, 10 mol\% catalyst BG1, 2 eq bromide and 5 eq inorganic base $(\mathrm{LiOH} / \mathrm{NaOH} / \mathrm{KOH} / \mathrm{CsOH})$. The tube was closed after addition of $\mathrm{Et}_{2} \mathrm{O}$. The resulting mixture was stirred at $-40{ }^{\circ} \mathrm{C}$ for $48 \mathrm{~h}$. The reaction mixture was then directly applied to silica gel chromatography (EA/Hexane: 1:200-1:30) to obtain product. 


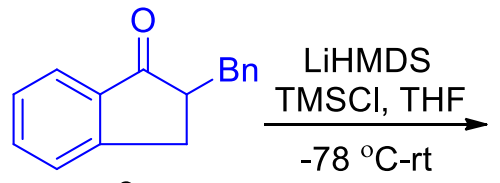

$3 \mathbf{a}$

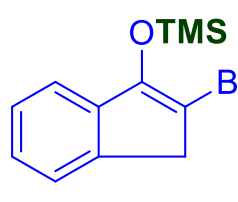

$8 \mathbf{a}$

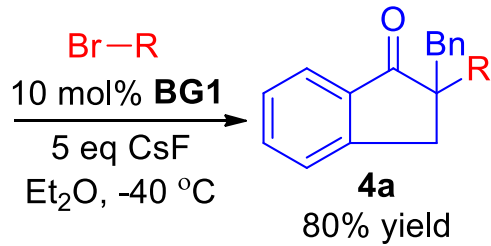

$80 \%$ ee<smiles>O=C1c2ccccc2CC1P</smiles>

3a

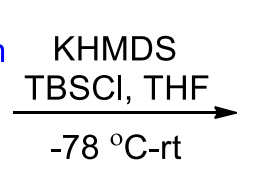
$-78^{\circ} \mathrm{C}-\mathrm{rt}$ $\mathrm{R}=\overbrace{2} / \mathrm{Ph}$<smiles>O=[Sb]1CC(Br)=C([O-])c2ccccc21</smiles>

$8 b$

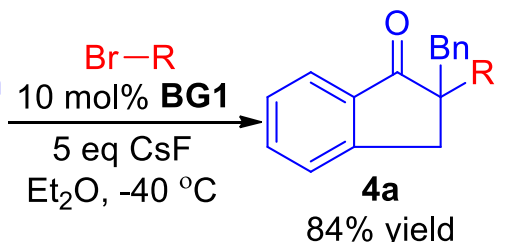

$94 \%$ ee

To a dry Schlenk tube equipped with a magnetic stir bar, was added 3a. The solid was dissolved in dry THF and the solution was cooled to $-78^{\circ} \mathrm{C} .1 .1 \mathrm{eq}$ LiHMDS/KHMDS was slowly added to the solution. After stirring at $-78{ }^{\circ} \mathrm{C}$ for $30 \mathrm{~min}, 1.4 \mathrm{eq} \mathrm{TMSCl} / \mathrm{TBSCl}$ was slowly added to the reaction. After stirring at $\mathrm{rt}$ for $12 \mathrm{~h}$, The solvent was removed by rotavapor and the residue was purified by flash chromatography (EA:Hex = 1:40). The product 8a/8b was added in a dry Schlenk tube with 2eq bromide, $10 \mathrm{~mol} \%$ BG1 and 5 eq $\mathrm{CsF}$. The resulting mixture was stirred at $-40{ }^{\circ} \mathrm{C}$ for $24 \mathrm{~h}$. The reaction mixture was then directly applied to silica gel chromatography (EA/Hexane: 1:200- 1:30) to obtain product. 


\section{VI: Determination of Absolute Configuration}
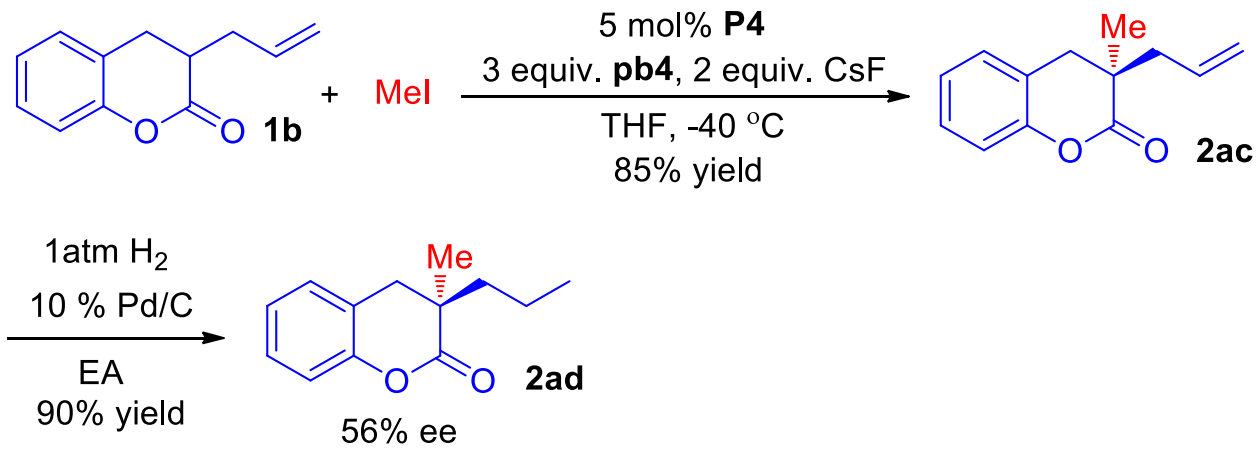

To a dry Schlenk tube equipped with a magnetic stir bar, was added $5 \mathrm{~mol} \%$ catalyst $\mathbf{P 4}, 3$ eq pb4, 2 eq CsF and 2 eq electrophile. The tube was closed after addition of $0.4 \mathrm{~mL}$ THF. The resulting mixture was stirred at $-40{ }^{\circ} \mathrm{C}$ for $24 \mathrm{~h}$. The reaction mixture was then directly applied to silica gel chromatography (EA/Hexane: 1:200- 1:30) to obtain product 2ac. Then 2ac was stirred with $10 \% \mathrm{Pd} / \mathrm{C}$ in EA under 1 atm $\mathrm{H}_{2}$ over $12 \mathrm{~h}$ to give $\mathbf{2 a d}$ in $90 \%$ yield. Absolute configuration of $\mathbf{2 a d}$ was determined by comparing with HPLC result of compound 2 in reference 1.
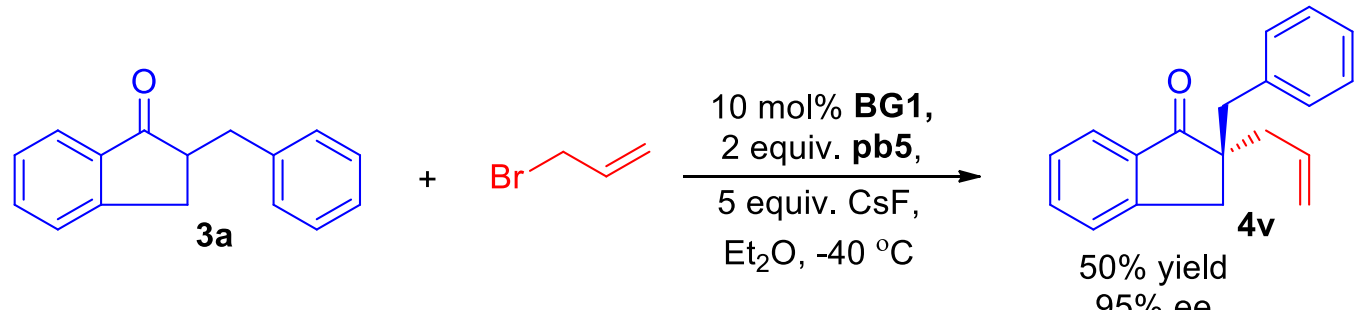

To a dry Schlenk tube equipped with a magnetic stir bar, was added $10 \mathrm{~mol} \%$ catalyst BG1, 2 eq pb5, 5 eq $\mathrm{CsF}$ and 2 eq electrophile. The tube was closed after addition of $0.4 \mathrm{~mL} \mathrm{Et}_{2} \mathrm{O}$. The resulting mixture was stirred at $-40{ }^{\circ} \mathrm{C}$ for $48 \mathrm{~h}$. The reaction mixture was then directly applied to silica gel chromatography (EA/Hexane: 1:200- 1:30) to obtain product $\mathbf{4 v}$. Absolute configuration of $\mathbf{4} \mathbf{v}$ was determined by comparing with HPLC result of table 17 entry 5 in reference 2.<smiles>COC(=O)C[C@H](C)C(=O)c1ccccc1</smiles>

Absolute configuration of $\mathbf{6 a}[\alpha]^{26}{ }_{\mathrm{D}}(c$ 1.09, THF):-6.6 was determined by comparing with optical rotation of $5 \mathrm{a}\left([\alpha]_{\mathrm{D}}(c 1.00, \mathrm{THF}):-1.6\right)$ in reference 3. 


\section{References}

(1) Murakata, M.; Jono, T.; Mizuno, Y.; Hoshino, O. J. Am. Chem. Soc. 1997, 119, 1171311714.

(2) Behenna, D.; Mohr, J.; Sherden, N.; Marinescu, S.; Harned, A.; Tani, K.; Seto, M.; Ma, S.; Novak, Z.; Krout, M.; McFadden, R.; Roizen, J.; Enquist, J.; White, D.; Levine, S.; Petrova, K.; Iwashita, A.; Virgil, S.; Stoltz, B. M. Chem. Eur. J. 2011, 17, 14199-14223.

(3) Blanco, L.; Rousseau, J-P.; Barnier, E. Tetrahedron: Asymmetry 1993, 5, 783-792. 


\section{VII: Characterization of Starting Materials and Products}

\section{3-cinnamylchroman-2-one (1a)}<smiles>O=C1Oc2ccccc2CC1C/C=C/c1ccccc1</smiles>

mp: $105.0-106.2^{\circ} \mathrm{C}$

${ }^{1}$ H NMR: $\left(400 \mathrm{MHz}, \mathrm{CDCl}_{3}\right) \delta 7.40$ - $7.24(\mathrm{~m}, 6 \mathrm{H}), 7.20$ - $7.18(\mathrm{~m}, 1 \mathrm{H}), 7.13-7.00(\mathrm{~m}$, 2H), $6.52(\mathrm{~d}, J=16.0 \mathrm{~Hz}, 1 \mathrm{H}), 6.30-6.22(\mathrm{~m}, 1 \mathrm{H}), 3.06-3.00(\mathrm{~m}, 1 \mathrm{H}), 2.93-2.83(\mathrm{~m}$, $3 \mathrm{H}), 2.55-2.52(\mathrm{~m}, 1 \mathrm{H})$;

${ }^{13}$ C NMR: $\left(100 \mathrm{MHz}, \mathrm{CDCl}_{3}\right) \delta 170.4,151.6,136.9,133.4,128.6,128.2,128.1,127.4$, $126.2,125.8,124.4,122.6,116.6,39.2,33.2,28.7$;

HRMS: (ESI) $[\mathrm{M}+\mathrm{H}]^{+}$calcd. for $\mathrm{C}_{18} \mathrm{H}_{17} \mathrm{O}_{2}, 265.1229$ found, 265.1232;

IR (film): $v_{\max } 1764,1489,1458,1232,1134,966,756,694 \mathrm{~cm}^{-1}$

\section{3-allylchroman-2-one (1b)}<smiles>C=CCC1Cc2ccccc2OC1=O</smiles>

${ }^{1}$ H NMR: $\left(400 \mathrm{MHz}, \mathrm{CDCl}_{3}\right) \delta 7.29-7.22(\mathrm{~m}, 1 \mathrm{H}), 7.20-7.15(\mathrm{~m}, 1 \mathrm{H}), 7.13-7.01$ (m, 2H), $5.92-5.80(\mathrm{~m}, 1 \mathrm{H}), 5.20-5.12(\mathrm{~m}, 2 \mathrm{H}), 3.06(\mathrm{dd}, J=16.0,4.0 \mathrm{~Hz}, 1 \mathrm{H}), 2.88-2.63$ (m, 3H), $2.41-2.32(\mathrm{~m}, 1 \mathrm{H})$;

${ }^{13}$ C NMR: $\left(100 \mathrm{MHz}, \mathrm{CDCl}_{3}\right) \delta 170.4,151.6,134.3,128.2,128.1,124.3,118.2,116.6,38.7$, $33.9,28.6$;

HRMS: (ESI) $[\mathrm{M}+\mathrm{H}]^{+}$calcd. for $\mathrm{C}_{12} \mathrm{H}_{13} \mathrm{O}_{2}, 189.0916$ found, 189.0914;

IR (film): $v_{\max } 1770,1489,1458,1232,1145,918,756 \mathrm{~cm}^{-1}$

\section{3-(3-methylbut-2-en-1-yl)chroman-2-one (1c)}<smiles>CC(C)=CCC1Cc2ccccc2OC1=O</smiles>

${ }^{1}$ H NMR: $\left(400 \mathrm{MHz}, \mathrm{CDCl}_{3}\right) \delta 7.29$ - $7.22(\mathrm{~m}, 1 \mathrm{H}), 7.20-7.15(\mathrm{~m}, 1 \mathrm{H}), 7.13-7.01(\mathrm{~m}$, 2H), $5.22-5.16(\mathrm{~m}, 1 \mathrm{H}), 5.20-5.12(\mathrm{~m}, 2 \mathrm{H}), 3.00(\mathrm{dd}, J=16.0,8.0 \mathrm{~Hz}, 1 \mathrm{H}), 2.81(\mathrm{dd}, J=$ 16.0, 8.0 Hz, 1H), $2.75-2.67(\mathrm{~m}, 1 \mathrm{H}), 2.64-2.55(\mathrm{~m}, 1 \mathrm{H}), 1.75(\mathrm{~s}, 3 \mathrm{H}), 1.61(\mathrm{~s}, 3 \mathrm{H})$;

${ }^{13}$ C NMR: $\left(100 \mathrm{MHz}, \mathrm{CDCl}_{3}\right) \delta 170.8,151.7,135.1,128.2,128.1,124.2,122.7,119.9,116.5$, 
$39.5,28.6,28.1,25.8,17.9$;

HRMS: (ESI) $[\mathrm{M}+\mathrm{H}]^{+}$calcd. for $\mathrm{C}_{14} \mathrm{H}_{17} \mathrm{O}_{2}, 217.1229$ found, 217.1225;

IR (film): $v_{\max } 1768,1489,1458,1234,918,756 \mathrm{~cm}^{-1}$

\section{3-(prop-2-yn-1-yl)chroman-2-one (1d)}<smiles>C#CCC1Cc2ccccc2OC1=O</smiles>

${ }^{1}$ H NMR: $\left(400 \mathrm{MHz}, \mathrm{CDCl}_{3}\right) \delta 7.30-7.22(\mathrm{~m}, 2 \mathrm{H}), 7.15-7.10(\mathrm{~m}, 1 \mathrm{H}), 7.08-7.03(\mathrm{~m}$, $1 \mathrm{H}), 3.20(\mathrm{dd}, J=16.0,4.0 \mathrm{~Hz}, 1 \mathrm{H}), 3.05-2.95(\mathrm{~m}, 1 \mathrm{H}), 2.93-2.81(\mathrm{~m}, 2 \mathrm{H}), 2.66-2.55$ $(\mathrm{m}, 1 \mathrm{H}), 2.12-2.09(\mathrm{~m}, 1 \mathrm{H})$;

${ }^{13}$ C NMR: $\left(100 \mathrm{MHz}, \mathrm{CDCl}_{3}\right) \delta 169.2,151.5,128.4,128.1,124.5,122.4,116.6,80.4,71.0$, $38.2,28.5,19.5$;

HRMS: (ESI) $[\mathrm{M}+\mathrm{H}]^{+}$calcd. for $\mathrm{C}_{12} \mathrm{H}_{11} \mathrm{O}_{2}, 187.0759$ found, 187.0760;

IR (film): $v_{\max } 3290,1766,1489,1458,1232,1141,756 \mathrm{~cm}^{-1}$

\section{3-(3-phenylprop-2-yn-1-yl)chroman-2-one (1e)}<smiles>O=C1Oc2ccccc2CC1CC#Cc1ccccc1</smiles>

mp: $54.5-56.5^{\circ} \mathrm{C}$

${ }^{1}$ H NMR: $\left(400 \mathrm{MHz}, \mathrm{CDCl}_{3}\right) \delta 7.48-7.40(\mathrm{~m}, 2 \mathrm{H}), 7.36-7.23(\mathrm{~m}, 5 \mathrm{H}), 7.17-7.06(\mathrm{~m}$, 2H), $3.28(\mathrm{dd}, J=16.0,8.0 \mathrm{~Hz}, 1 \mathrm{H}), 3.14(\mathrm{dd}, J=16.0,8.0 \mathrm{~Hz}, 1 \mathrm{H}), 3.12-3.03(\mathrm{~m}, 1 \mathrm{H})$, $3.02-2.91(\mathrm{~m}, 1 \mathrm{H}), 2.84(\mathrm{dd}, J=16.0,8.0 \mathrm{~Hz}, 1 \mathrm{H})$;

${ }^{13}$ C NMR: $\left(100 \mathrm{MHz}, \mathrm{CDCl}_{3}\right) \delta 169.5,151.6,131.7,128.4,128.3,128.4,128.1,124.5$, $123.1,122.4,116.7,85.8,83.0,38.6,28.8,20.5$;

HRMS: (ESI) $[\mathrm{M}+\mathrm{H}]^{+}$calcd. for $\mathrm{C}_{18} \mathrm{H}_{15} \mathrm{O}_{2}, 263.1072$ found, 263.1067;

IR (film): $v_{\max } 1768,1489,1458,1232,1138,756 \mathrm{~cm}^{-1}$

\section{3-(3-(trimethylsilyl)prop-2-yn-1-yl)chroman-2-one (1f)}

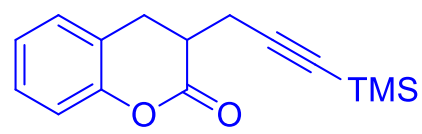

mp: $67.5-68.7^{\circ} \mathrm{C}$

${ }^{1}$ H NMR: $\left(400 \mathrm{MHz}, \mathrm{CDCl}_{3}\right) \delta 7.31-7.21(\mathrm{~m}, 2 \mathrm{H}), 7.15-7.09(\mathrm{~m}, 1 \mathrm{H}), 7.07-7.02(\mathrm{~m}$, 
1H), $3.21(\mathrm{dd}, J=16.0,4.0 \mathrm{~Hz}, 1 \mathrm{H}), 3.04-2.83(\mathrm{~m}, 2 \mathrm{H}), 2.61(\mathrm{dd}, J=16.0,8.0 \mathrm{~Hz}, 1 \mathrm{H})$, $0.19(\mathrm{~s}, 9 \mathrm{H})$;

${ }^{13}$ C NMR: $\left(100 \mathrm{MHz}, \mathrm{CDCl}_{3}\right) \delta 169.4,151.6,128.4,128.2,124.5,122.5,116.7,102.6,87.6$, $38.3,28.6,20.9,0.1$;

HRMS: (ESI) $[\mathrm{M}+\mathrm{H}]^{+}$calcd. for $\mathrm{C}_{15} \mathrm{H}_{19} \mathrm{O}_{2} \mathrm{Si}, 259.1154$ found, 259.1157;

IR (film): $v_{\max } 2177,1768,1489,1460,1249,1232,1138,842,758 \mathrm{~cm}^{-1}$

(Z)-3-(3,7-dimethylocta-2,6-dien-1-yl)chroman-2-one (1g)<smiles>CC(C)=CCC/C(C)=C\CC1Cc2ccccc2OC1=O</smiles>

${ }^{1}$ H NMR: $\left(400 \mathrm{MHz}, \mathrm{CDCl}_{3}\right) \delta 7.30-7.22(\mathrm{~m}, 1 \mathrm{H}), 7.20-7.15(\mathrm{~m}, 1 \mathrm{H}), 7.12-7.02(\mathrm{~m}$, 2H), $5.22-5.15(\mathrm{~m}, 1 \mathrm{H}), 5.13-5.06(\mathrm{~m}, 1 \mathrm{H}), 3.00(\mathrm{dd}, J=16.0,8.0 \mathrm{~Hz}, 1 \mathrm{H}), 2.81(\mathrm{dd}, J=$ 16.0, $8.0 \mathrm{~Hz}, 1 \mathrm{H}), 2.77-2.67(\mathrm{~m}, 1 \mathrm{H}), 2.66-2.56(\mathrm{~m}, 1 \mathrm{H}), 2.38-2.28(\mathrm{~m}, 1 \mathrm{H}), 2.15-2.01$ $(\mathrm{m}, 4 \mathrm{H}), 1.70(\mathrm{~s}, 3 \mathrm{H}), 1.63(\mathrm{~s}, 3 \mathrm{H}), 1.60(\mathrm{~s}, 3 \mathrm{H})$;

${ }^{13}$ C NMR: (100 MHz, $\left.\mathrm{CDCl}_{3}\right) \delta 170.8,151.7,138.7,131.6,128.2,128.1,124.2,124.1$, $122.7,119.9,116.5,39.7,39.5,28.5,28.0,26.5,25.7,17.7,16.1$;

HRMS: (ESI) $[\mathrm{M}+\mathrm{H}]^{+}$calcd. for $\mathrm{C}_{19} \mathrm{H}_{25} \mathrm{O}_{2}, 285.1855$ found, 285.1855;

IR (film): $v_{\max } 1768,1489,1458,1232,1130,756 \mathrm{~cm}^{-1}$

ethyl 2-(2-oxochroman-3-yl)acetate (1h)<smiles>CCOC(=O)CC1Cc2ccccc2OC1=O</smiles>

mp: $75.9-77.2^{\circ} \mathrm{C}$

${ }^{1}$ H NMR: $\left(400 \mathrm{MHz}, \mathrm{CDCl}_{3}\right) \delta 7.24-7.11(\mathrm{~m}, 1 \mathrm{H}), 7.16-7.11(\mathrm{~m}, 1 \mathrm{H}), 7.08-7.02(\mathrm{~m}$, 1H), $7.00-6.95(\mathrm{~m}, 1 \mathrm{H}), 4.17(\mathrm{~d}, J=8.0 \mathrm{~Hz}, 1 \mathrm{H}), 4.13(\mathrm{~d}, J=8.0 \mathrm{~Hz}, 1 \mathrm{H}), 3.18-3.07$ (m, $1 \mathrm{H}), 3.03-2.85(\mathrm{~m}, 3 \mathrm{H}), 2.55(\mathrm{dd}, J=16.0,8.0 \mathrm{~Hz}, 1 \mathrm{H}), 1.24(\mathrm{t}, J=8.0 \mathrm{~Hz}, 3 \mathrm{H})$;

${ }^{13}$ C NMR: $\left(100 \mathrm{MHz}, \mathrm{CDCl}_{3}\right) \delta 171.1,169.9,151.5,128.3,128.0,124.4,122.6,116.6,60.8$, 36.0, 34.5, 29.3, 14.1;

HRMS: (ESI) $[\mathrm{M}+\mathrm{H}]^{+}$calcd. for $\mathrm{C}_{13} \mathrm{H}_{15} \mathrm{O}_{4}, 235.0969$ found, 235.0970;

IR (film): $v_{\max } 1766,1732,1489,1458,1230,1138,767 \mathrm{~cm}^{-1}$ 
3-benzylchroman-2-one (1i)<smiles>O=C1Oc2ccccc2CC1Cc1ccccc1</smiles>

mp: $104.5-105.5^{\circ} \mathrm{C}$

${ }^{1}$ H NMR: $\left(400 \mathrm{MHz}, \mathrm{CDCl}_{3}\right) \delta 7.39$ - $7.19(\mathrm{~m}, 6 \mathrm{H}), 7.13$ - $7.05(\mathrm{~m}, 3 \mathrm{H}), 3.40(\mathrm{dd}, J=16.0$, $8.0 \mathrm{~Hz}, 1 \mathrm{H}), 3.02-2.93(\mathrm{~m}, 1 \mathrm{H}), 2.87(\mathrm{dd}, J=16.0,8.0 \mathrm{~Hz}, 1 \mathrm{H}), 2.80-2.70(\mathrm{~m}, 2 \mathrm{H})$;

${ }^{13}$ C NMR: $\left(100 \mathrm{MHz}, \mathrm{CDCl}_{3}\right) \delta 170.6,151.6,138.0,129.2,128.7,128.3,128.2,126.8$, $124.4,122.3,116.6,40.8,35.6,28.2$;

HRMS: (ESI) $[\mathrm{M}+\mathrm{H}]^{+}$calcd. for $\mathrm{C}_{16} \mathrm{H}_{15} \mathrm{O}_{2}, 239.1072$ found, 239.1071;

IR (film): $v_{\max } 1762,1489,1458,1230,1128,700 \mathrm{~cm}^{-1}$

\section{6,8-dibromo-3-(3-methylbut-2-en-1-yl)chroman-2-one (1j)}<smiles>CC(C)=CCC1Cc2cc(Br)cc(Br)c2OC1=O</smiles>

mp: $88.0-89.1^{\circ} \mathrm{C}$

${ }^{1}$ H NMR: $\left(400 \mathrm{MHz}, \mathrm{CDCl}_{3}\right) \delta 7.50(\mathrm{~d}, J=4.0 \mathrm{~Hz}, 1 \mathrm{H}), 7.20(\mathrm{~d}, J=4.0 \mathrm{~Hz}, 1 \mathrm{H}), 5.18-5.10$ (m, 1H), $3.01-2.81(\mathrm{~m}, 3 \mathrm{H}), 2.46-2.25(\mathrm{~m}, 2 \mathrm{H}), 1.73(\mathrm{~s}, 3 \mathrm{H}), 1.62(\mathrm{~s}, 3 \mathrm{H})$;

${ }^{13}$ C NMR: $\left(100 \mathrm{MHz}, \mathrm{CDCl}_{3}\right) \delta 181.2,149.8,134.8,133.2,132.4,128.8,120.1,112.3,111.2$, $45.2,32.2,30.6,25.8,17.9$;

HRMS: (ESI) $[\mathrm{M}+\mathrm{H}]^{+}$calcd. for $\mathrm{C}_{14} \mathrm{H}_{15} \mathrm{Br}_{2} \mathrm{O}_{2}, 372.9439$ found, 372.9427 ;

IR (film): $v_{\max } 1705,1460,1215,756 \mathrm{~cm}^{-1}$

\section{8-methyl-3-(3-methylbut-2-en-1-yl)chroman-2-one (1k)}<smiles>CC(C)=CCC1Cc2cccc(C)c2OC1=O</smiles>

${ }^{1}$ H NMR: $\left(400 \mathrm{MHz}, \mathrm{CDCl}_{3}\right) \delta 7.12-7.08(\mathrm{~m}, 1 \mathrm{H}), 7.03-6.95(\mathrm{~m}, 2 \mathrm{H}), 5.24-5.16(\mathrm{~m}$, $1 \mathrm{H}), 3.00(\mathrm{dd}, J=16.0,4.0 \mathrm{~Hz}, 1 \mathrm{H}), 2.78(\mathrm{dd}, J=16.0,8.0 \mathrm{~Hz}, 1 \mathrm{H}), 2.74-2.54(\mathrm{~m}, 2 \mathrm{H})$, $2.36-2.31(\mathrm{~m}, 1 \mathrm{H}), 2.31(\mathrm{~s}, 3 \mathrm{H}), 1.76(\mathrm{~s}, 3 \mathrm{H}), 1.62(\mathrm{~s}, 3 \mathrm{H})$;

${ }^{13}$ C NMR: $\left(100 \mathrm{MHz}, \mathrm{CDCl}_{3}\right) \delta 171.0,149.9,135.0,129.7,125.8,125.7,123.8,122.5$, 
$120.1,39.6,28.8,28.1,25.8,17.9,15.7$;

HRMS: (ESI) $[\mathrm{M}+\mathrm{H}]^{+}$calcd. for $\mathrm{C}_{15} \mathrm{H}_{19} \mathrm{O}_{2}, 231.1385$ found, 231.1385;

IR (film): $v_{\max } 1766,1469,1269,1132,767 \mathrm{~cm}^{-1}$

\section{3-cinnamyl-6-methylchroman-2-one (11)}<smiles>Cc1ccc2c(c1)CC(C/C=C/c1ccccc1)C(=O)O2</smiles>

mp: $78.5-79.7^{\circ} \mathrm{C}$

${ }^{1}$ H NMR: $\left(400 \mathrm{MHz}, \mathrm{CDCl}_{3}\right) \delta 7.42-7.21(\mathrm{~m}, 5 \mathrm{H}), 7.20-7.18(\mathrm{~m}, 1 \mathrm{H}), 7.08-7.04(\mathrm{~m}$, 1H), $7.00-6.94(\mathrm{~m}, 2 \mathrm{H}), 6.50(\mathrm{~d}, J=16.0 \mathrm{~Hz}, 1 \mathrm{H}), 6.30-6.21(\mathrm{~m}, 1 \mathrm{H}), 3.04-2.93(\mathrm{~m}$, $1 \mathrm{H}), 2.91-2.80(\mathrm{~m}, 3 \mathrm{H}), 2.60-2.49(\mathrm{~m}, 1 \mathrm{H}), 2.33(\mathrm{~s}, 3 \mathrm{H})$;

${ }^{13}$ C NMR: $\left(100 \mathrm{MHz}, \mathrm{CDCl}_{3}\right) \delta 170.6,149.5,137.0,133.9,133.3,128.7,128.6,127.5$, $126.2,125.9,122.2,116.3,39.3,33.2,28.7,20.7$;

HRMS: (ESI) $[\mathrm{M}+\mathrm{H}]^{+}$calcd. for $\mathrm{C}_{19} \mathrm{H}_{19} \mathrm{O}_{2}, 279.1385$ found, 279.1388;

IR (film): $v_{\max } 1764,1494,1257,1213,1136,742 \mathrm{~cm}^{-1}$

\section{6-methoxy-3-(3-methylbut-2-en-1-yl)chroman-2-one (1m)}<smiles>COc1ccc2c(c1)CC(CC=C(C)C)C(=O)O2</smiles>

${ }^{1}$ H NMR: $\left(400 \mathrm{MHz}, \mathrm{CDCl}_{3}\right) \delta 6.94(\mathrm{~d}, J=8.0 \mathrm{~Hz}, 1 \mathrm{H}), 6.75(\mathrm{dd}, J=8.0,4.0 \mathrm{~Hz}, 1 \mathrm{H}), 6.69$ $(\mathrm{d}, J=4.0 \mathrm{~Hz}, 1 \mathrm{H}), 5.20-5.12(\mathrm{~m}, 1 \mathrm{H}), 3.77(\mathrm{~s}, 3 \mathrm{H}), 2.93(\mathrm{dd}, J=16.0,4.0 \mathrm{~Hz}, 1 \mathrm{H}), 2.75$ $(\mathrm{dd}, J=16.0,8.0 \mathrm{~Hz}, 1 \mathrm{H}), 2.70-2.51(\mathrm{~m}, 2 \mathrm{H}), 2.34-2.21(\mathrm{~m}, 1 \mathrm{H}), 1.73(\mathrm{~s}, 3 \mathrm{H}), 1.60(\mathrm{~s}$, $3 \mathrm{H})$;

${ }^{13}$ C NMR: $\left(100 \mathrm{MHz}, \mathrm{CDCl}_{3}\right) \delta 171.0,156.0,145.5,125.1,123.6,120.0,117.2,113.3,113.2$, $55.6,39.4,28.8,28.1,25.8,17.9$;

HRMS: (ESI) $[\mathrm{M}+\mathrm{H}]^{+}$calcd. for $\mathrm{C}_{15} \mathrm{H}_{19} \mathrm{O}_{3}, 247.1334$ found, 247.1336;

IR (film): $v_{\max } 1759,1494,1433,1205,1157,1037,813 \mathrm{~cm}^{-1}$

\section{3-cinnamyl-7-methoxychroman-2-one (1n)}<smiles>COc1ccc2c(c1)OC(=O)C(C/C=C/c1ccccc1)C2</smiles>

mp: $56.0-57.0^{\circ} \mathrm{C}$ 
${ }^{1}$ H NMR: $\left(400 \mathrm{MHz}, \mathrm{CDCl}_{3}\right) \delta 7.43-7.19(\mathrm{~m}, 4 \mathrm{H}), 7.07(\mathrm{~d}, J=8.0 \mathrm{~Hz}, 1 \mathrm{H}), 6.71-6.58(\mathrm{~m}$, 2H), $6.50(\mathrm{~d}, J=16.0 \mathrm{~Hz}, 1 \mathrm{H}), 6.30-6.20(\mathrm{~m}, 1 \mathrm{H}), 3.81(\mathrm{~s}, 3 \mathrm{H}), 3.04-2.72(\mathrm{~m}, 3 \mathrm{H}), 2.60-$ $2.49(\mathrm{~m}, 1 \mathrm{H})$;

${ }^{13}$ C NMR: $\left(100 \mathrm{MHz}, \mathrm{CDCl}_{3}\right) \delta 170.4,159.7,152.3,137.0,133.3,128.6,127.5,126.2$, $125.9,114.3,110.4,102.3,55.5,39.4,33.3,28.1$;

HRMS: (ESI) $[\mathrm{M}+\mathrm{H}]^{+}$calcd. for $\mathrm{C}_{19} \mathrm{H}_{19} \mathrm{O}_{3}, 295.1334$ found, 295.1329;

IR (film): $v_{\max } 1762,1625,1589,1508,1155,1130,694 \mathrm{~cm}^{-1}$

\section{2-cinnamyl-1H-benzo[f]chromen-3(2H)-one (10)}<smiles>O=C1Oc2ccc3ccccc3c2CC1C/C=C/c1ccccc1</smiles>

mp: $105.8-107.4^{\circ} \mathrm{C}$

${ }^{1}$ H NMR: (400 MHz, $\left.\mathrm{CDCl}_{3}\right) \delta 7.93-7.75(\mathrm{~m}, 3 \mathrm{H}), 7.63-7.53(\mathrm{~m}, 1 \mathrm{H}), 7.52-7.44(\mathrm{~m}$, 1H), $7.43-7.30(\mathrm{~m}, 4 \mathrm{H}), 7.29-7.22(\mathrm{~m}, 2 \mathrm{H}), 6.53(\mathrm{~d}, J=16.0 \mathrm{~Hz}, 1 \mathrm{H}), 6.38-6.26(\mathrm{~m}$, 1H), $3.53(\mathrm{dd}, J=24.0,8.0 \mathrm{~Hz}, 1 \mathrm{H}), 3.13$ (dd, $J=24.0,8.0 \mathrm{~Hz}, 1 \mathrm{H}), 3.05-2.89(\mathrm{~m}, 2 \mathrm{H})$, $2.71-2.61(\mathrm{~m}, 1 \mathrm{H})$;

${ }^{13}$ C NMR: $\left(100 \mathrm{MHz}, \mathrm{CDCl}_{3}\right) \delta 170.3,149.2,136.9,133.6,131.2,130.8,128.9,128.8$, $128.6,127.5,127.1,126.2,125.7,125.1,122.9,117.1,115.4,38.6,33.5,24.9$;

HRMS: (ESI) $[\mathrm{M}+\mathrm{H}]^{+}$calcd. for $\mathrm{C}_{22} \mathrm{H}_{19} \mathrm{O}_{2}, 315.1385$ found, 315.1382;

IR (film): $v_{\max } 1762,1224,1138,810,746 \mathrm{~cm}^{-1}$

\section{3-(3-methylbut-2-en-1-yl)-3,4-dihydro-2H-benzo[ $h]$ chromen-2-one (1p)}<smiles>CC(C)=CCC1Cc2ccc3ccccc3c2OC1=O</smiles>

${ }^{1}$ H NMR: $\left(400 \mathrm{MHz}, \mathrm{CDCl}_{3}\right) \delta 8.28-8.20(\mathrm{~m}, 1 \mathrm{H}), 7.86-7.79(\mathrm{~m}, 1 \mathrm{H}), 7.62-7.48(\mathrm{~m}$, $3 \mathrm{H}), 7.30-7.23(\mathrm{~m}, 1 \mathrm{H}), 5.28-5.18(\mathrm{~m}, 1 \mathrm{H}), 3.13(\mathrm{dd}, J=24.0,8.0 \mathrm{~Hz}, 1 \mathrm{H}), 2.93(\mathrm{dd}, J=$ 24.0, 8.0 Hz, 1H), $2.88-2.77(\mathrm{~m}, 1 \mathrm{H}), 2.71-2.61(\mathrm{~m}, 1 \mathrm{H}), 2.43-2.33(\mathrm{~m}, 1 \mathrm{H}), 1.77(\mathrm{~s}$, $3 \mathrm{H}), 1.63(\mathrm{~s}, 3 \mathrm{H})$;

${ }^{13}$ C NMR: $\left(100 \mathrm{MHz}, \mathrm{CDCl}_{3}\right) \delta 170.8,146.4,135.2,133.4,127.5,126.5,126.4,125.6$, 
$123.8,123.5,121.0,120.3,117.1,39.5,28.8,28.2,25.9,17.9$;

HRMS: (ESI) $[\mathrm{M}+\mathrm{H}]^{+}$calcd. for $\mathrm{C}_{18} \mathrm{H}_{19} \mathrm{O}_{2}, 267.1385$ found, 267.1386;

IR (film): $v_{\max } 1762,1379,1126,808 \mathrm{~cm}^{-1}$

\section{8-bromo-2-cinnamyl-1H-benzo[f]chromen-3(2H)-one (1q)}<smiles>O=C1Oc2ccc3cc(Br)ccc3c2CC1C/C=C/c1ccccc1</smiles>

${ }^{1}$ H NMR: $\left(400 \mathrm{MHz}, \mathrm{CDCl}_{3}\right) \delta 8.02-7.96(\mathrm{~m}, 1 \mathrm{H}), 7.77$ - $7.56(\mathrm{~m}, 3 \mathrm{H}), 7.42-7.30(\mathrm{~m}$, 4H), $7.29-7.22(\mathrm{~m}, 2 \mathrm{H}), 6.52(\mathrm{~d}, J=16.0 \mathrm{~Hz}, 1 \mathrm{H}), 6.35-6.25(\mathrm{~m}, 1 \mathrm{H}), 3.46(\mathrm{dd}, J=16.0$, $8.0 \mathrm{~Hz}, 1 \mathrm{H}), 3.12-3.03(\mathrm{~m}, 1 \mathrm{H}), 3.02-2.88(\mathrm{~m}, 2 \mathrm{H}), 2.71-2.58(\mathrm{~m}, 1 \mathrm{H})$;

${ }^{13}$ C NMR: $\left(100 \mathrm{MHz}, \mathrm{CDCl}_{3}\right) \delta 169.8,149.4,136.8,133.7,131.9,130.6,130.3,129.7$, $128.6,128.0,127.5,126.2,125.5,124.6,119.0,118.3,115.7,38.4,33.4,24.9$;

HRMS: (ESI) $[\mathrm{M}+\mathrm{H}]^{+}$calcd. for $\mathrm{C}_{22} \mathrm{H}_{18} \mathrm{O}_{2} \mathrm{~B}_{\mathrm{r}}, 393.0492$ found, 393.0490;

IR (film): $v_{\max } 1766,1224,1134,810,694 \mathrm{~cm}^{-1}$

\section{9-bromo-2-cinnamyl-1H-benzo[f]chromen-3(2H)-one (1r)}<smiles>O=C1Oc2ccc3ccc(Br)cc3c2CC1C/C=C/c1ccccc1</smiles>

${ }^{1}$ H NMR: $\left(400 \mathrm{MHz}, \mathrm{CDCl}_{3}\right) \delta 8.06-8.00(\mathrm{~m}, 1 \mathrm{H}), 7.78-7.67(\mathrm{~m}, 2 \mathrm{H}), 7.57-7.51(\mathrm{~m}$, 1H), $7.42-7.30(\mathrm{~m}, 4 \mathrm{H}), 7.29-7.21(\mathrm{~m}, 2 \mathrm{H}), 6.53(\mathrm{~d}, J=16.0 \mathrm{~Hz}, 1 \mathrm{H}), 6.36-6.26(\mathrm{~m}$, $1 \mathrm{H}), 3.41(\mathrm{dd}, J=16.0,8.0 \mathrm{~Hz}, 1 \mathrm{H}), 3.11-2.85$ (m, 3H), $2.72-2.60$ (m, 1H);

${ }^{13}$ C NMR: $\left(100 \mathrm{MHz}, \mathrm{CDCl}_{3}\right) \delta 169.7,149.9,136.9,133.7,132.5,130.3,129.1,128.9$, $128.6,128.5,127.5,126.2,125.4,125.3,121.7,117.6,114.7,38.4,33.4,24.9$;

HRMS: (ESI) $[\mathrm{M}+\mathrm{H}]^{+}$calcd. for $\mathrm{C}_{22} \mathrm{H}_{18} \mathrm{O}_{2} \mathrm{Br}, 393.0490$ found, 393.0490;

IR (film): $v_{\max } 1766,1620,1141,830,756 \mathrm{~cm}^{-1}$ 
<smiles>COc1ccc2ccc3c(c2c1)CC(/C=C/c1ccccc1)C(=O)O3</smiles>

${ }^{1}$ H NMR: (400 MHz, $\left.\mathrm{CDCl}_{3}\right) \delta 7.78-7.66(\mathrm{~m}, 2 \mathrm{H}), 7.42-7.29(\mathrm{~m}, 4 \mathrm{H}), 7.29-7.23(\mathrm{~m}$, 1H), $7.18-7.07$ (m, 3H), 6.50 (d, $J=16.0 \mathrm{~Hz}, 1 \mathrm{H}), 6.37-6.27$ (m, 1H), 3.91 (s, 3H), 3.37 $(\mathrm{dd}, J=16.0,8.0 \mathrm{~Hz}, 1 \mathrm{H}), 3.10-2.84(\mathrm{~m}, 3 \mathrm{H}), 2.70-2.59(\mathrm{~m}, 1 \mathrm{H})$;

${ }^{13}$ C NMR: $\left(100 \mathrm{MHz}, \mathrm{CDCl}_{3}\right) \delta 170.2,158.8,149.8,137.0,133.5,132.6,130.3,128.6$, $127.5,126.2,126.1,125.8,117.2,114.5,114.1,101.9,55.3,38.6,33.4,25.0$;

HRMS: (ESI) $[\mathrm{M}+\mathrm{H}]^{+}$calcd. for $\mathrm{C}_{23} \mathrm{H}_{21} \mathrm{O}_{3}, 345.1496$ found, 345.1491;

IR (film): $v_{\max } 1766,1627,1225,1134,833,746 \mathrm{~cm}^{-1}$

(S)-3-benzyl-3-cinnamylchroman-2-one (2a)<smiles>O=C1Oc2ccccc2CC1(Br)C/C=C/c1ccccc1</smiles>

mp: $114.6-116.4^{\circ} \mathrm{C}$

Opt. Rot.: $[\alpha]^{24}{ }_{\mathrm{D}}\left(c 1.30, \mathrm{CHCl}_{3}\right):-2.16$

${ }^{1}$ H NMR: $\left(400 \mathrm{MHz}, \mathrm{CDCl}_{3}\right) \delta 7.38-7.21(\mathrm{~m}, 9 \mathrm{H}), 7.21-7.10(\mathrm{~m}, 4 \mathrm{H}), 7.09-7.03(\mathrm{~m}$, $1 \mathrm{H}), 6.42$ (d, $J=16.0 \mathrm{~Hz}, 1 \mathrm{H}), 6.23$ (ddd, $J=4.0,8.0,16.0 \mathrm{~Hz}, 1 \mathrm{H}), 3.10$ (d, $J=16.0 \mathrm{~Hz}$, 1H), $2.98(\mathrm{~d}, J=16.0 \mathrm{~Hz}, 1 \mathrm{H}), 2.90(\mathrm{~d}, J=16.0 \mathrm{~Hz}, 1 \mathrm{H}), 2.83-2.72(\mathrm{~m}, 2 \mathrm{H}), 2.39(\mathrm{ddd}, J=$ 8.0, 16.0, $24.0 \mathrm{~Hz}, 1 \mathrm{H})$;

${ }^{13}$ C NMR: $\left(100 \mathrm{MHz}, \mathrm{CDCl}_{3}\right) \delta 171.2,151.1,136.9,135.6,134.8,130.6,128.6,128.5$, 128.3, 128.2, 127.5, 127.1, 126.4, 124.6, 124.1, 121.8, 116.2, 45.9, 40.9, 38.8, 32.6;

HRMS: (ESI) $[\mathrm{M}+\mathrm{H}]^{+}$calcd. for $\mathrm{C}_{25} \mathrm{H}_{23} \mathrm{O}_{2}, 355.1698$ found, 355.1698;

IR (film): $v_{\max } 1759,1489,1456,1232,1143,754,700 \mathrm{~cm}^{-1}$

HPLC: $96 \%$ ee determined by HPLC (OD-H, 90/10 hexanes/i-PrOH, $1.0 \mathrm{ml} / \mathrm{min}$ ) $t r=6.5$ $\min , t r=8.9 \mathrm{~min}$.

(S)-3-allyl-3-benzylchroman-2-one (2b)<smiles>C=CC[C@]1(Br)Cc2ccccc2OC1=O</smiles>

Opt. Rot.: $[\alpha]^{24}{ }_{\mathrm{D}}\left(\mathrm{c} 1.40, \mathrm{CHCl}_{3}\right)$ : -27.6

${ }^{1}$ H NMR: $\left(400 \mathrm{MHz} \mathrm{CDCl}_{3}\right) \delta 7.35-7.22(\mathrm{~m}, 4 \mathrm{H}), 7.18-7.08(\mathrm{~m}, 4 \mathrm{H}), 7.07-7.02(\mathrm{~m}$, 
1H), $5.92-5.80(\mathrm{~m}, 1 \mathrm{H}), 5.19(\mathrm{~d}, J=8.0 \mathrm{~Hz}, 1 \mathrm{H}), 5.10(\mathrm{~d}, J=8.0 \mathrm{~Hz}, 1 \mathrm{H}), 3.06(\mathrm{~d}, J=16.0$ $\mathrm{Hz}, 1 \mathrm{H}), 2.96-2.70(\mathrm{~m}, 3 \mathrm{H}), 2.56(\mathrm{dd}, J=8.0,16.0 \mathrm{~Hz}, 1 \mathrm{H}), 2.24(\mathrm{dd}, J=8.0,16.0 \mathrm{~Hz}, 1 \mathrm{H})$; ${ }^{13}$ C NMR: $\left(100 \mathrm{MHz}, \mathrm{CDCl}_{3}\right) \delta 171.1,151.0,135.7,132.6,130.6,128.6,128.2,128.1$, 127.0, 124.5, 121.7, 119.9, 116.1, 45.4, 40.6, 39.3, 32.5;

HRMS: (ESI) $[\mathrm{M}+\mathrm{H}]^{+}$calcd. for $\mathrm{C}_{19} \mathrm{H}_{19} \mathrm{O}_{2}, 279.1385$ found, 279.1391;

IR (film): $v_{\max } 1759,1489,1456,1232,1155,1134,756,702 \mathrm{~cm}^{-1}$

HPLC: $85 \%$ ee termined by HPLC (OJ-H, 95/5 hexanes/i-PrOH, $1.0 \mathrm{ml} / \mathrm{min}) \operatorname{tr}(R)=9.6$ $\min , \operatorname{tr}(S)=13.9 \mathrm{~min}$.

\section{(S)-3-benzyl-3-(3-methylbut-2-en-1-yl)chroman-2-one (2c)}<smiles>CC(C)=CC[C@]1(Br)Cc2ccccc2OC1=O</smiles>

Opt. Rot.: $[\alpha]^{24}\left(c 2.07, \mathrm{CHCl}_{3}\right):+4.3$

${ }^{1}$ H NMR: (400 MHz, $\left.\mathrm{CDCl}_{3}\right) \delta 7.42-7.02(\mathrm{~m}, 9 \mathrm{H}), 5.25-5.20(\mathrm{~m}, 1 \mathrm{H}), 3.11(\mathrm{~d}, J=16.0$ $\mathrm{Hz}, 1 \mathrm{H}), 2.85(\mathrm{~d}, J=16.0 \mathrm{~Hz}, 1 \mathrm{H}), 2.80(\mathrm{~d}, J=16.0 \mathrm{~Hz}, 1 \mathrm{H}), 2.76(\mathrm{~d}, J=16.0 \mathrm{~Hz}, 1 \mathrm{H}), 2.75$ $-2.70(\mathrm{~m}, 1 \mathrm{H}), 2.34(\mathrm{dd}, J=8.0,16.0 \mathrm{~Hz}, 1 \mathrm{H}), 2.32(\mathrm{dd}, J=8.0,16.0 \mathrm{~Hz}, 1 \mathrm{H}), 1.75(\mathrm{~s}, 3 \mathrm{H})$, $1.52(\mathrm{~s}, 3 \mathrm{H})$;

${ }^{13}$ C NMR: $\left(100 \mathrm{MHz}, \mathrm{CDCl}_{3}\right) \delta 171.6,151.1,136.2,136.0,130.6,128.6,128.2,128.0$, $126.9,124.4,121.9,118.0,116.0,45.9,40.7,33.5,32.6,26.0,18.0$;

HRMS: (ESI) $[\mathrm{M}+\mathrm{H}]^{+}$calcd. for $\mathrm{C}_{21} \mathrm{H}_{23} \mathrm{O}_{2}, 307.1698$ found, 307.1693;

IR (film): $v_{\max } 1759,1489,1456,1232,1141,756,649 \mathrm{~cm}^{-1}$

HPLC: $93 \%$ ee determined by HPLC (AD-H, 99/1 hexanes/i-PrOH, $1.0 \mathrm{ml} / \mathrm{min}) t r=12.0$ $\min , t r=15.9 \min$.

\section{(S)-3-benzyl-3-(prop-2-yn-1-yl)chroman-2-one (2d)}<smiles>C#CC[C@]1(Br)Cc2ccccc2OC1=O</smiles>

mp: $94.2-95.4^{\circ} \mathrm{C}$

Opt. Rot.: $[\alpha]^{24}\left(c 1.60, \mathrm{CHCl}_{3}\right):+2.2$

${ }^{1}$ H NMR: (400 MHz, $\left.\mathrm{CDCl}_{3}\right) \delta 7.45-7.08(\mathrm{~m}, 9 \mathrm{H}), 3.21(\mathrm{~d}, J=16.0 \mathrm{~Hz}, 1 \mathrm{H}), 3.05(\mathrm{~d}, J=$ $16.0 \mathrm{~Hz}, 1 \mathrm{H}), 3.12-2.94(\mathrm{~m}, 2 \mathrm{H}), 2.61-2.56(\mathrm{~s}, 2 \mathrm{H}), 2.25-2.20(\mathrm{~m}, 1 \mathrm{H})$;

${ }^{13}$ C NMR: $\left(100 \mathrm{MHz}, \mathrm{CDCl}_{3}\right) \delta 170.2,151.1,134.9,130.2,128.8,128.5,128.4,127.3$, 
124.7, 121.2, 116.3, 79.7, 72.3, 45.1, 39.2, 33.4, 24.9;

HRMS: (ESI) $[\mathrm{M}+\mathrm{H}]^{+}$calcd. for $\mathrm{C}_{19} \mathrm{H}_{17} \mathrm{O}_{2}, 277.1229$ found, 277.1228;

IR (film): $v_{\max } 3296,1707,1456,1232,756,702 \mathrm{~cm}^{-1}$

HPLC: $94 \%$ ee determined by HPLC (OD-H, 99/1 hexanes/i-PrOH, $1.0 \mathrm{ml} / \mathrm{min}) t r=14.4$ $\min , t r=16.3 \mathrm{~min}$.

(R)-3-benzyl-3-(3-phenylprop-2-yn-1-yl)chroman-2-one (2e)<smiles>O=C1Oc2ccccc2CC1(Br)CC#Cc1ccccc1</smiles>

mp: $145.0-147.0^{\circ} \mathrm{C}$

Opt. Rot.: $[\alpha]^{24}{ }_{\mathrm{D}}\left(\mathrm{c} 1.23, \mathrm{CHCl}_{3}\right):-1.36$

${ }^{1}$ H NMR: $\left(400 \mathrm{MHz}, \mathrm{CDCl}_{3}\right) \delta 7.52-7.42(\mathrm{~m}, 2 \mathrm{H}), 7.38-7.05(\mathrm{~m}, 12 \mathrm{H}), 3.24(\mathrm{~d}, J=16.0$ $\mathrm{Hz}, 1 \mathrm{H}), 3.08(\mathrm{~d}, J=16.0 \mathrm{~Hz}, 1 \mathrm{H}), 3.01(\mathrm{~d}, J=16.0 \mathrm{~Hz}, 1 \mathrm{H}), 3.12-2.96(\mathrm{~m}, 1 \mathrm{H}), 2.81-$ $2.72(\mathrm{~s}, 2 \mathrm{H})$;

${ }^{13} \mathrm{C}$ NMR: $\left(100 \mathrm{MHz}, \mathrm{CDCl}_{3}\right) \delta 170.4,151.1,135.2,131.7,130.3,128.2,128.4,128.3$, $128.2,127.3,124.7,123.2,121.3,116.3,85.0,84.4,45.6,39.6,33.7,25.9$;

HRMS: (ESI) $[\mathrm{M}+\mathrm{H}]^{+}$calcd. for $\mathrm{C}_{25} \mathrm{H}_{21} \mathrm{O}_{2}, 353.1542$ found, 353.1549;

IR (film): $v_{\max } 1761,1489,1234,1141,754 \mathrm{~cm}^{-1}$

HPLC: $90 \%$ ee determined by HPLC (OJ-H, 85/15 hexanes/i-PrOH, $1.0 \mathrm{ml} / \mathrm{min}) t r=15.2$ $\min , t r=21.8 \mathrm{~min}$.

(S)-3-benzyl-3-(3-(trimethylsilyl)prop-2-yn-1-yl)chroman-2-one (2f)<smiles>CC#CCC1(Cc2ccccc2)Cc2ccccc2OC1=O</smiles>

mp: $107.7-109.1^{\circ} \mathrm{C}$

Opt. Rot.: $[\alpha]^{24} \mathrm{D}\left(c 1.00, \mathrm{CHCl}_{3}\right):+1.37$

${ }^{1}$ H NMR: $\left(400 \mathrm{MHz}, \mathrm{CDCl}_{3}\right) \delta 7.34-7.08(\mathrm{~m}, 9 \mathrm{H}), 3.26(\mathrm{~d}, J=16.0 \mathrm{~Hz}, 1 \mathrm{H}), 3.23-3.02$ $(\mathrm{m}, 3 \mathrm{H}), 2.54-2.40(\mathrm{~m}, 2 \mathrm{H}), 0.31(\mathrm{~s}, 9 \mathrm{H})$;

${ }^{13}$ C NMR: $\left(100 \mathrm{MHz}, \mathrm{CDCl}_{3}\right) \delta 170.2,151.1,135.2,130.2,128.8,128.4,128.3,127.2$, $124.6,121.3,116.2,101.9,89.2,45.2,39.3,33.5,26.4,0.1$;

${ }^{19}$ F NMR: $\left(376 \mathrm{MHz}, \mathrm{CDCl}_{3}\right)-114.6--114.68(\mathrm{~m}, 1 \mathrm{~F})$;

HRMS: (ESI) $[\mathrm{M}+\mathrm{H}]^{+}$calcd. for $\mathrm{C}_{22} \mathrm{H}_{25} \mathrm{O}_{2} \mathrm{Si}, 349.1624$ found, 349.1624; 
IR (film): $v_{\max } 1732,1489,1456,1249,1232,842,756,702 \mathrm{~cm}^{-1}$

HPLC: $94 \%$ ee determined by HPLC (OD-H, 99/1 hexanes/i-PrOH, $1.0 \mathrm{ml} / \mathrm{min}) t r=14.4$ $\min , t r=16.3 \mathrm{~min}$.

(S,Z)-3-benzyl-3-(3,7-dimethylocta-2,6-dien-1-yl)chroman-2-one (2g)<smiles>CC(C)=CCC/C(C)=C\C[C@]1(Cc2ccccc2)Cc2ccccc2OC1=O</smiles>

mp: $82.5-83.0^{\circ} \mathrm{C}$

Opt. Rot.: $[\alpha]^{24}\left(c 0.86, \mathrm{CHCl}_{3}\right):-9.8$

${ }^{1}$ H NMR: $\left(400 \mathrm{MHz}, \mathrm{CDCl}_{3}\right) \delta 7.35$ - $6.98(\mathrm{~m}, 9 \mathrm{H}), 5.36$ - $5.29(\mathrm{~m}, 1 \mathrm{H}), 5.15-5.08(\mathrm{~m}$, 1H), $3.09(\mathrm{~d}, J=16.0 \mathrm{~Hz}, 1 \mathrm{H}), 2.93-2.72(\mathrm{~m}, 3 \mathrm{H}), 2.44-2.33(\mathrm{~m}, 2 \mathrm{H}), 2.32-2.23(\mathrm{~m}$, $2 \mathrm{H}), 2.15-1.97(\mathrm{~m}, 4 \mathrm{H}), 1.67(\mathrm{~s}, 3 \mathrm{H}), 1.61(\mathrm{~s}, 3 \mathrm{H}), 1.51(\mathrm{~s}, 3 \mathrm{H})$;

${ }^{13}$ C NMR: $\left(100 \mathrm{MHz}, \mathrm{CDCl}_{3}\right) \delta 171.6,151.1,139.7,136.1,131.6,130.6,128.6,128.2$, $128.0,126.9,124.4,124.1,122.0,118.1,116.1,46.0,40.1,39.9,33.4,32.6,26.5,25.7,17.7$, 16.3;

HRMS: (ESI) $[\mathrm{M}+\mathrm{H}]^{+}$calcd. for $\mathrm{C}_{26} \mathrm{H}_{31} \mathrm{O}_{2}, 375.2324$ found, 375.2320;

IR (film): $v_{\max } 1761,1489,1458,1234,1136,756,702 \mathrm{~cm}^{-1}$

HPLC: 93\% ee determined by HPLC (AD-H, 99/1 hexanes/i-PrOH, $1.0 \mathrm{ml} / \mathrm{min}$ ) $t r=7.3$ $\min , t r=11.0 \mathrm{~min}$.

(S)-ethyl 2-(3-benzyl-2-oxochroman-3-yl)acetate (2h)<smiles>CCOC(=O)CC1(Br)Cc2ccccc2OC1=O</smiles>

mp: $72.6-73.1^{\circ} \mathrm{C}$

Opt. Rot.: $[\alpha]^{24}{ }_{\mathrm{D}}\left(c 1.00, \mathrm{CHCl}_{3}\right):-21.7$

${ }^{1}$ H NMR: (400 MHz, $\left.\mathrm{CDCl}_{3}\right) \delta 7.34-7.24(\mathrm{~m}, 4 \mathrm{H}), 7.22-7.13(\mathrm{~m}, 2 \mathrm{H}), 7.12-7.06(\mathrm{~m}$, $3 \mathrm{H}), 4.18(\mathrm{~d}, J=8.0 \mathrm{~Hz}, 1 \mathrm{H}), 4.14(\mathrm{~d}, J=8.0 \mathrm{~Hz}, 1 \mathrm{H}), 3.48(\mathrm{~d}, J=16.0 \mathrm{~Hz}, 1 \mathrm{H}), 3.02(\mathrm{~d}, J=$ $16.0 \mathrm{~Hz}, 1 \mathrm{H}), 2.94(\mathrm{~d}, J=16.0 \mathrm{~Hz}, 1 \mathrm{H}), 2.89$ (d, $J=16.0 \mathrm{~Hz}, 1 \mathrm{H}), 2.80$ (d, $J=16.0 \mathrm{~Hz}, 1 \mathrm{H})$, $2.45(\mathrm{~d}, J=16.0 \mathrm{~Hz}, 1 \mathrm{H}), 1.28(\mathrm{t}, J=8.0 \mathrm{~Hz}, 1 \mathrm{H})$;

${ }^{13}$ C NMR: $\left(100 \mathrm{MHz}, \mathrm{CDCl}_{3}\right) \delta 171.2,170.7,151.2,134.6,130.5,128.6,128.4,128.3$, 
$127.3,124.7,121.3,116.4,60.8,44.0,40.4,39.1,32.4,14.1$

HRMS: (ESI) $[\mathrm{M}+\mathrm{H}]^{+}$calcd. for $\mathrm{C}_{20} \mathrm{H}_{21} \mathrm{O}_{4}, 325.1444$ found, 325.1440;

IR (film): $v_{\max } 1759,1728,1489,1458,1022,763 \mathrm{~cm}^{-1}$

HPLC: $86 \%$ ee determined by HPLC (OJ-H, 90/10 hexanes/i-PrOH, $1.0 \mathrm{ml} / \mathrm{min}) \mathrm{tr}=15.1$ $\min , \operatorname{tr}=19.3 \mathrm{~min}$.

\section{(S)-3-benzyl-3-(4-chlorobenzyl)chroman-2-one (2i)}

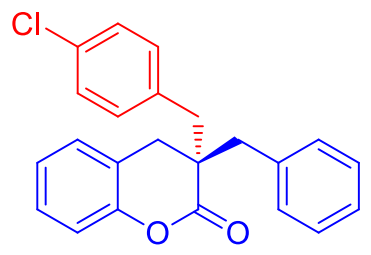

mp: $97.5-98.4^{\circ} \mathrm{C}$

Opt. Rot.: $[\alpha]^{24}{ }_{\mathrm{D}}\left(c \mathrm{1} 1.30, \mathrm{CHCl}_{3}\right):+11.0$

${ }^{1}$ H NMR: $\left(400 \mathrm{MHz}, \mathrm{CDCl}_{3}\right) \delta 7.38-7.01(\mathrm{~m}, 13 \mathrm{H}), 3.24(\mathrm{~d}, J=16.0 \mathrm{~Hz}, 1 \mathrm{H}), 2.20(\mathrm{~d}, J=$ $16.0 \mathrm{~Hz}, 1 \mathrm{H}), 2.79-2.70(\mathrm{~m}, 4 \mathrm{H})$;

${ }^{13}$ C NMR: $\left(100 \mathrm{MHz}, \mathrm{CDCl}_{3}\right) \delta 170.9,150.8,135.4,134.4,133.1,132.1,130.8,128.6$, $128.4,128.3,128.2,127.2,124.7,121.7,116.2,47.0,41.5,40.7,31.7$;

HRMS: (ESI) $[\mathrm{M}+\mathrm{H}]^{+}$calcd. for $\mathrm{C}_{23} \mathrm{H}_{20} \mathrm{ClO}_{2}, 363.1152$ found, 363.1157;

IR (film): $v_{\max } 1759,1489,1457,1016,756 \mathrm{~cm}^{-1}$

HPLC: $93 \%$ ee determined by HPLC (IC, 99/1 hexanes/i-PrOH, $0.5 \mathrm{ml} / \mathrm{min}$ ) $t r=29.6 \mathrm{~min}, t r$ $=32.7 \mathrm{~min}$.

(R)-3-benzyl-3-(2-methylallyl)chroman-2-one (2j)

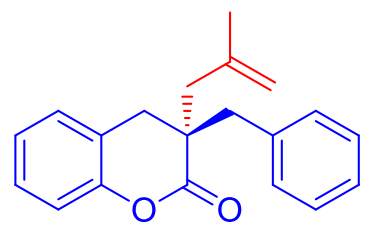

Opt. Rot.: $[\alpha]^{24}{ }_{\mathrm{D}}\left(c 1.40, \mathrm{CHCl}_{3}\right):-11.4$

${ }^{1}$ H NMR: $\left(400 \mathrm{MHz}, \mathrm{CDCl}_{3}\right) \delta 7.32-7.05(\mathrm{~m}, 9 \mathrm{H}), 4.96-4.93(\mathrm{~m}, 1 \mathrm{H}), 4.76-4.73(\mathrm{~m}$, 1H), $3.21(\mathrm{~d}, J=16.0 \mathrm{~Hz}, 1 \mathrm{H}), 2.89(\mathrm{~d}, J=24.0 \mathrm{~Hz}, 1 \mathrm{H}), 2.83-2.71(\mathrm{~m}, 2 \mathrm{H}), 2.66(\mathrm{~d}, J=$ $16.0 \mathrm{~Hz}, 1 \mathrm{H}), 1.78(\mathrm{~s}, 3 \mathrm{H})$;

${ }^{13}$ C NMR: $\left(100 \mathrm{MHz}, \mathrm{CDCl}_{3}\right) \delta 171.6,151.0,140.9,135.8,130.8,128.6,128.2,128.1$, $127.0,124.5,122.0,116.5,116.1,45.4,43.1,42.0,32.4,24.3$;

HRMS: (ESI) $[\mathrm{M}+\mathrm{H}]^{+}$calcd. for $\mathrm{C}_{20} \mathrm{H}_{21} \mathrm{O}_{2}, 293.1542$ found, 293.1537; 
IR (film): $v_{\max } 1759,1489,1456,1234,1139,754,702 \mathrm{~cm}^{-1}$

HPLC: $90 \%$ ee determined by HPLC (OD-H, 95/5 hexanes/i-PrOH, $0.5 \mathrm{ml} / \mathrm{min}$ ) $t r=9.6$ $\min , t r=10.4 \mathrm{~min}$.

(S)-3-cinnamyl-3-(2-methylallyl)chroman-2-one (2k)<smiles>C=C(C)C[C@]1(/C=C/c2ccccc2)Cc2ccccc2OC1=O</smiles>

mp: $84.5-85.8^{\circ} \mathrm{C}$

Opt. Rot.: $[\alpha]^{25} \mathrm{D}\left(c 1.19, \mathrm{CHCl}_{3}\right):-0.6$

${ }^{1}$ H NMR: (400 MHz, $\left.\mathrm{CDCl}_{3}\right) \delta 7.35-7.03(\mathrm{~m}, 9 \mathrm{H}), 6.43(\mathrm{~d}, J=16.0 \mathrm{~Hz}, 1 \mathrm{H}), 6.25-6.14$ $(\mathrm{m}, 1 \mathrm{H}), 4.97-4.94(\mathrm{~m}, 1 \mathrm{H}), 4.77-4.74(\mathrm{~m}, 1 \mathrm{H}), 3.00(\mathrm{~d}, J=16.0 \mathrm{~Hz}, 1 \mathrm{H}), 2.89$ (d, $J=$ $16.0 \mathrm{~Hz}, 1 \mathrm{H}), 2.79-2.71(\mathrm{~m}, 1 \mathrm{H}), 2.55(\mathrm{~d}, J=16.0 \mathrm{~Hz}, 1 \mathrm{H}), 2.51-2.44(\mathrm{~m}, 1 \mathrm{H}), 2.32(\mathrm{~d}, J$ $=16.0 \mathrm{~Hz}, 1 \mathrm{H}), 1.78(\mathrm{~s}, 3 \mathrm{H})$;

${ }^{13}$ C NMR: $\left(100 \mathrm{MHz}, \mathrm{CDCl}_{3}\right) \delta 172.6,151.1,140.7,137.0,134.7,128.6,128.5,128.1$, $127.5,126.2,124.5,124.2,121.9,116.5,116.1,44.6,43.0,39.6,33.4,24.1$;

HRMS: (ESI) $[\mathrm{M}+\mathrm{H}]^{+}$calcd. for $\mathrm{C}_{22} \mathrm{H}_{23} \mathrm{O}_{2}, 319.1698$ found, 319.1708;

IR (film): $v_{\max } 1759,1489,1458,1232,1141,756,694 \mathrm{~cm}^{-1}$

HPLC: $89 \%$ ee determined by HPLC (OD-H, 90/10 hexanes/i-PrOH, $0.5 \mathrm{ml} / \mathrm{min}$ ) $t r=10.4$ $\min , t r=11.8 \mathrm{~min}$.

(S)-3-cinnamyl-3-((E)-2-methyl-3-phenylallyl)chroman-2-one (2l)<smiles>C/C(=C\c1ccccc1)C[C@]1(/C=C/c2ccccc2)Cc2ccccc2OC1=O</smiles>

mp: $123.0-125.0^{\circ} \mathrm{C}$

Opt. Rot.: $[\alpha]^{25}{ }_{\mathrm{D}}\left(c 0.65, \mathrm{CHCl}_{3}\right):-25.2$

${ }^{1}$ H NMR: $\left(400 \mathrm{MHz}, \mathrm{CDCl}_{3}\right) \delta 7.39-7.03(\mathrm{~m}, 14 \mathrm{H}), 6.48(\mathrm{~d}, J=16.0 \mathrm{~Hz}, 1 \mathrm{H}), 6.33-6.19$ $(\mathrm{m}, 2 \mathrm{H}), 3.12(\mathrm{~d}, J=16.0 \mathrm{~Hz}, 1 \mathrm{H}), 2.95-2.75(\mathrm{~m}, 2 \mathrm{H}), 2.67(\mathrm{~d}, J=16.0 \mathrm{~Hz}, 1 \mathrm{H}), 2.57-$ $2.44(\mathrm{~m}, 2 \mathrm{H}), 1.89$ (s, 3H);

${ }^{13}$ C NMR: $\left(100 \mathrm{MHz}, \mathrm{CDCl}_{3}\right) \delta 171.7,151.1,137.6,137.0,134.8,133.6,130.9,129.0$, $128.7,128.6,128.2$, 128.1 127.5, 126.5, 126.3, 124.6, 124.3, 121.9, 116.2, 46.7, 45.2, 40.0, 
33.6, 19.9;

HRMS: (ESI) $[\mathrm{M}+\mathrm{H}]^{+}$calcd. for $\mathrm{C}_{28} \mathrm{H}_{27} \mathrm{O}_{2}, 395.2011$ found, 395.2013;

IR (film): $v_{\max } 1755,1489,1458,1234,1141,754 \mathrm{~cm}^{-1}$

HPLC: $91 \%$ ee determined by HPLC (OD-H, 95/5 hexanes/i-PrOH, $0.5 \mathrm{ml} / \mathrm{min}) \mathrm{tr}=17.6$ $\min , \operatorname{tr}=26.4 \mathrm{~min}$.

(S)-3-cinnamyl-3-(naphthalen-2-ylmethyl)chroman-2-one (2m)<smiles>O=C1Oc2ccccc2C[C@H]1C/C=C/Pc1ccccc1</smiles>

mp: $148.5-151.4^{\circ} \mathrm{C}$

Opt. Rot.: $[\alpha]^{24}{ }_{\mathrm{D}}\left(c \mathrm{0} .40, \mathrm{CHCl}_{3}\right):-6.6$

${ }^{1}$ H NMR: $\left(400 \mathrm{MHz}, \mathrm{CDCl}_{3}\right) \delta 8.02-7.03(\mathrm{~m}, 16 \mathrm{H}), 6.42(\mathrm{~d}, J=16.0 \mathrm{~Hz}, 1 \mathrm{H}), 6.33-6.20$ (m, 1H), $3.30(\mathrm{~d}, J=16.0 \mathrm{~Hz}, 1 \mathrm{H}), 3.07(\mathrm{~d}, J=16.0 \mathrm{~Hz}, 1 \mathrm{H}), 3.01(\mathrm{~d}, J=16.0 \mathrm{~Hz}, 1 \mathrm{H}), 2.85$ $-2.75(\mathrm{~m}, 2 \mathrm{H}), 2.44(\mathrm{dd}, J=8.0,16.0 \mathrm{~Hz}, 1 \mathrm{H})$;

${ }^{13}$ C NMR: $\left(100 \mathrm{MHz}, \mathrm{CDCl}_{3}\right) \delta 171.3,151.1,136.9,134.9,133.2,133.1,132.5,129.5$, $128.7,128.6,128.5,128.2,127.8,127.7,127.6,127.5,126.3,126.2,125.8,124.6,124.1$, $121.8,116.2,46.0,41.1,38.9,32.6$;

HRMS: (ESI) $[\mathrm{M}+\mathrm{H}]^{+}$calcd. for $\mathrm{C}_{29} \mathrm{H}_{25} \mathrm{O}_{2}, 405.1855$ found, 405.1842;

IR (film): $v_{\max } 1755,1489,1458,1234,1143,754 \mathrm{~cm}^{-1}$

HPLC: $91 \%$ ee determined by HPLC (OD-H, 95/5 hexanes/i-PrOH, $1.0 \mathrm{ml} / \mathrm{min}$ ) tr $=11.9$ $\min , t r=15.1 \mathrm{~min}$.

\section{(S)-3-cinnamyl-3-(3-methoxybenzyl)chroman-2-one (2n)}<smiles>COc1cccc(C[C@@H]2Cc3ccccc3OC2=O)c1</smiles>

Opt. Rot.: $[\alpha]^{25}\left(c 1.40, \mathrm{CHCl}_{3}\right):-1.6$

${ }^{1}$ H NMR: $\left(400 \mathrm{MHz}, \mathrm{CDCl}_{3}\right) \delta 7.42-7.02(\mathrm{~m}, 10 \mathrm{H}), 6.82(\mathrm{dd}, J=4.0,8.0 \mathrm{~Hz}, 1 \mathrm{H}), 6.78-$ $6.70(\mathrm{~m}, 2 \mathrm{H}), 6.42(\mathrm{~d}, J=16.0 \mathrm{~Hz}, 1 \mathrm{H}), 6.27-6.16(\mathrm{~m}, 1 \mathrm{H}), 3.81(\mathrm{~s}, 3 \mathrm{H}), 3.09(\mathrm{~d}, J=16.0$ $\mathrm{Hz}, 1 \mathrm{H}), 2.97(\mathrm{~d}, J=16.0 \mathrm{~Hz}, 1 \mathrm{H}), 2.88-2.72(\mathrm{~m}, 3 \mathrm{H}), 2.44-2.36(\mathrm{~m}, 1 \mathrm{H})$; 
${ }^{13}$ C NMR: $\left(100 \mathrm{MHz}, \mathrm{CDCl}_{3}\right) \delta 171.3,159.5,151.1,137.1,136.9,134.8,129.2,128.6$, $128.5,128.2$, 127.5, 126.2, 124.6, 124.1, 123.9, 121.8, 116.4, 116.2, 112.4, 55.2, 45.8, 40.9, $38.9,32.6$;

HRMS: (ESI) $[\mathrm{M}+\mathrm{H}]^{+}$calcd. for $\mathrm{C}_{26} \mathrm{H}_{25} \mathrm{O}_{3}, 385.1084$ found, 385.1083;

IR (film): $v_{\max } 1759,1583,1489,1456,1263,1232,1141,756,694 \mathrm{~cm}^{-1}$

HPLC: $95 \%$ ee determined by HPLC (OD-H, 90/10 hexanes/i-PrOH, $1.0 \mathrm{ml} / \mathrm{min}$ ) tr $=8.2$ $\min , t r=10.5 \min$.

(R)-3-(2-bromobenzyl)-3-cinnamylchroman-2-one (2o)<smiles>O=C1Oc2ccccc2C[C@]1(C/C=C/c1ccccc1)Cc1ccccc1</smiles>

mp: $107.0-109.0^{\circ} \mathrm{C}$

Opt. Rot.: $[\alpha]^{25}\left(c 1.85, \mathrm{CHCl}_{3}\right):-1.2$

${ }^{1}$ H NMR: $\left(400 \mathrm{MHz}, \mathrm{CDCl}_{3}\right) \delta 7.55-7.51(\mathrm{~m}, 1 \mathrm{H}), 7.40-6.95(\mathrm{~m}, 12 \mathrm{H}), 6.44(\mathrm{~d}, J=16.0$ $\mathrm{Hz}, 1 \mathrm{H}), 6.27-6.18(\mathrm{~m}, 1 \mathrm{H}), 3.41$ (d, $J=16.0 \mathrm{~Hz}, 1 \mathrm{H}), 3.30$ (d, $J=16.0 \mathrm{~Hz}, 1 \mathrm{H}), 3.13$ (d, $J$ $=16.0 \mathrm{~Hz}, 1 \mathrm{H}), 2.87(\mathrm{~d}, J=16.0 \mathrm{~Hz}, 1 \mathrm{H}), 2.76(\mathrm{ddd}, J=4.0,8.0,16.0 \mathrm{~Hz}, 1 \mathrm{H}), 2.51(\mathrm{ddd}, J$ $=4.0,8.0,16.0 \mathrm{~Hz}, 1 \mathrm{H})$;

${ }^{13} \mathrm{C}$ NMR: $\left(100 \mathrm{MHz}, \mathrm{CDCl}_{3}\right) \delta 171.2,150.8,136.9,136.2,134.9,133.0,132.0,128.7$, $128.6,128.5,128.1,127.6,127.5,126.3,124.5,123.7,121.7,116.0,47.0,40.0,39.8,32.3$;

HRMS: (ESI) $[\mathrm{M}+\mathrm{H}]^{+}$calcd. for $\mathrm{C}_{25} \mathrm{H}_{22} \mathrm{BrO}_{2}, 433.0803$ found, 433.0801;

IR (film): $v_{\max } 1759,1489,1458,1234,1143,754 \mathrm{~cm}^{-1}$

HPLC: $89 \%$ ee determined by HPLC (OD-H, 95/5 hexanes/i-PrOH, $0.5 \mathrm{ml} / \mathrm{min}) t r=14.9$ $\min , t r=16.7 \mathrm{~min}$.

(S)-3-cinnamyl-3-(4-methylbenzyl)chroman-2-one (2p)<smiles>Cc1ccc(C/C=C/Pc2ccccc2)cc1</smiles>

mp: $114.8-116.8^{\circ} \mathrm{C}$ 
Opt. Rot.: $[\alpha]^{26}{ }_{\mathrm{D}}\left(c 0.82, \mathrm{CHCl}_{3}\right):-1.7$

${ }^{1}$ H NMR: $\left(400 \mathrm{MHz}, \mathrm{CDCl}_{3}\right) \delta 7.37-7.03(\mathrm{~m}, 13 \mathrm{H}), 6.43(\mathrm{~d}, J=16.0 \mathrm{~Hz}, 1 \mathrm{H}), 6.28-6.18$ (m, 1H), $3.04(\mathrm{~d}, J=16.0 \mathrm{~Hz}, 1 \mathrm{H}), 2.98(\mathrm{~d}, J=16.0 \mathrm{~Hz}, 1 \mathrm{H}), 2.87(\mathrm{~d}, J=16.0 \mathrm{~Hz}, 1 \mathrm{H}), 2.83$ $-2.73(\mathrm{~m}, 2 \mathrm{H}), 2.41-2.29$ (m, 1H), 2.38 (s, 3H);

${ }^{13}$ C NMR: $\left(100 \mathrm{MHz}, \mathrm{CDCl}_{3}\right) \delta 171.4,151.1,137.0,136.7,134.7,132.4,130.5,129.0$, $128.6,128.5,128.1,127.5,126.2,124.6,14.3,121.9,116.2,45.9,40.5,38.7,32.5,21.1$;

HRMS: (ESI) $[\mathrm{M}+\mathrm{H}]^{+}$calcd. for $\mathrm{C}_{26} \mathrm{H}_{25} \mathrm{O}_{2}, 369.1855$ found, 369.1854;

IR (film): $v_{\max } 1759,1489,1458,1232,1141,756,694 \mathrm{~cm}^{-1}$

HPLC: $91 \%$ ee determined by HPLC (OD-H, 9O/10 hexanes/i-PrOH, $1.0 \mathrm{ml} / \mathrm{min}) \mathrm{tr}=6.0$ $\min , \operatorname{tr}(R)=7.2 \mathrm{~min}$.

\section{(S)-3-cinnamyl-3-(4-fluorobenzyl)chroman-2-one (2q)}<smiles>O=C1Oc2ccccc2C[C@H]1C/C=C/Pc1ccccc1</smiles>

mp: $106.5-118.0^{\circ} \mathrm{C}$

Opt. Rot:: $[\alpha]^{25} \mathrm{D}\left(c 0.52, \mathrm{CHCl}_{3}\right):-4.5$

${ }^{1}$ H NMR: $\left(400 \mathrm{MHz}, \mathrm{CDCl}_{3}\right) \delta 7.37-6.95(\mathrm{~m}, 13 \mathrm{H}), 6.43(\mathrm{~d}, J=16.0 \mathrm{~Hz}, 1 \mathrm{H}), 6.26-6.15$ (m, 1H), $3.09(\mathrm{~d}, J=16.0 \mathrm{~Hz}, 1 \mathrm{H}), 2.98(\mathrm{~d}, J=16.0 \mathrm{~Hz}, 1 \mathrm{H}), 2.86(\mathrm{~d}, J=16.0 \mathrm{~Hz}, 1 \mathrm{H}), 2.78$ $(\mathrm{d}, J=16.0 \mathrm{~Hz}, 1 \mathrm{H}), 2.43-2.35(\mathrm{~m}, 1 \mathrm{H})$;

${ }^{13}$ C NMR: $\left(100 \mathrm{MHz}, \mathrm{CDCl}_{3}\right) \delta 171.1,151.0,136.8,134.9,132.0,131.4,131.3,128.6$, 128.3, 127.6, 126.2 124.7, 123.8, 121.6, 116.2, 115.3, 115.1, 45.9, 40.3, 38.9, 32.6;

${ }^{19}$ F NMR: $\left(376 \mathrm{MHz}, \mathrm{CDCl}_{3}\right) \delta$-115.6;

HRMS: (ESI) $[\mathrm{M}+\mathrm{H}]^{+}$calcd. for $\mathrm{C}_{25} \mathrm{H}_{22} \mathrm{FO}_{2}, 373.1604$ found, 373.1611;

IR (film): $v_{\max } 1759,1508,1489,1458,1232,1143,756 \mathrm{~cm}^{-1}$

HPLC: $93 \%$ ee determined by HPLC (OD-H, 95/5 hexanes/i-PrOH, $1.0 \mathrm{ml} / \mathrm{min}$ ) $t r=9.1$ $\min , t r=16.6 \mathrm{~min}$.

(S)-3-cinnamyl-3-(4-(trifluoromethyl)benzyl)chroman-2-one (2r) 


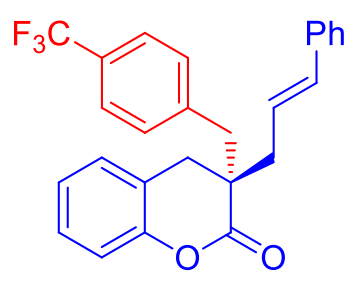

mp: $99.2-101.0^{\circ} \mathrm{C}$

Opt. Rot.: $[\alpha]^{25}{ }_{\mathrm{D}}\left(c 0.60, \mathrm{CHCl}_{3}\right):-5.6$

${ }^{1}$ H NMR: (400 MHz, $\left.\mathrm{CDCl}_{3}\right) \delta 7.59$ - $7.52(\mathrm{~m}, 2 \mathrm{H}), 7.38$ - $7.10(\mathrm{~m}, 10 \mathrm{H}), 7.07-7.02(\mathrm{~m}$, 1H), $6.43(\mathrm{~d}, J=16.0 \mathrm{~Hz}, 1 \mathrm{H}), 6.25-6.15(\mathrm{~m}, 1 \mathrm{H}), 3.21(\mathrm{~d}, J=16.0 \mathrm{~Hz}, 1 \mathrm{H}), 2.99(\mathrm{~d}, J=$ $16.0 \mathrm{~Hz}, 1 \mathrm{H}), 2.93(\mathrm{~d}, J=16.0 \mathrm{~Hz}, 1 \mathrm{H}), 2.79(\mathrm{~d}, J=16.0 \mathrm{~Hz}, 1 \mathrm{H}), 2.71$ (ddd, $J=4.0,8.0$, $16.0 \mathrm{~Hz}, 1 \mathrm{H}), 2.42$ (ddd, $J=4.0,8.0,16.0 \mathrm{~Hz}, 1 \mathrm{H})$;

${ }^{13}$ C NMR: $\left(100 \mathrm{MHz}, \mathrm{CDCl}_{3}\right) \delta 170.8,150.9,139.9,136.7,135.1,130.9,128.6,128.5$, $128.4,127.7,126.3,125.2,125.1,124.7,123.4,121.4,116.2,45.9,40.9,39.0,32.8$;

${ }^{19}$ F NMR: $\left(376 \mathrm{MHz}, \mathrm{CDCl}_{3}\right) \delta-62.5$;

HRMS: (ESI) $[\mathrm{M}+\mathrm{H}]^{+}$calcd. for $\mathrm{C}_{26} \mathrm{H}_{22} \mathrm{~F}_{3} \mathrm{O}_{2}, 423.1572$ found, 423.1588 ;

IR (film): $v_{\max } 1759,1489,1458,1325,1234,1122,1068,756 \mathrm{~cm}^{-1}$

HPLC: 91\% ee determined by HPLC (OD-H, 90/10 hexanes/i-PrOH, $1.0 \mathrm{ml} / \mathrm{min}) \mathrm{tr}=7.9$ $\min , \operatorname{tr}=11.0 \mathrm{~min}$.

(S)-3-benzyl-6,8-dibromo-3-(3-methylbut-2-en-1-yl)chroman-2-one (2s)<smiles>CC(C)=CC[C@]1(Br)Cc2cc(Br)cc(Br)c2OC1=O</smiles>

Opt. Rot.: $[\alpha]^{26}{ }_{\mathrm{D}}\left(\mathrm{c} 1.80, \mathrm{CHCl}_{3}\right):-63.7$

${ }^{1}$ H NMR: $\left(400 \mathrm{MHz}, \mathrm{CDCl}_{3}\right) \delta 7.62(\mathrm{~d}, J=4.0 \mathrm{~Hz}, 1 \mathrm{H}), 7.33-7.12(\mathrm{~m}, 6 \mathrm{H}), 5.23-5.15(\mathrm{~m}$, $1 \mathrm{H}), 3.13(\mathrm{~d}, J=16.0 \mathrm{~Hz}, 1 \mathrm{H}), 2.85(\mathrm{~d}, J=16.0 \mathrm{~Hz}, 1 \mathrm{H}), 2.84-2.80(\mathrm{~m}, 2 \mathrm{H}), 2.36(\mathrm{dd}, J=$ 8.0, 16.0 Hz, 1H), 2.27 (dd, $J=8.0,16.0 \mathrm{~Hz}, 1 \mathrm{H}), 1.74$ (s, 3H), 1.54 (s, 3H);

${ }^{13}$ C NMR: $\left(100 \mathrm{MHz}, \mathrm{CDCl}_{3}\right) \delta 169.7,147.3,137.0,135.5,134.0,130.5,130.4,128.3$, $127.2,125.5,117.4,116.7,110.7,45.8,41.1,33.9,33.2,26.1,18.0$;

HRMS: (ESI) $[\mathrm{M}+\mathrm{H}]^{+}$calcd. for $\mathrm{C}_{21} \mathrm{H}_{21} \mathrm{Br}_{2} \mathrm{O}_{2}, 462.9908$ found, 462.9906;

IR (film): $v_{\max } 1772,1489,1458,1134,756,702 \mathrm{~cm}^{-1}$

HPLC: $90 \%$ ee determined by HPLC (IC, 99/1 hexanes/i-PrOH, $0.5 \mathrm{ml} / \mathrm{min}$ ) $t r=19.1 \mathrm{~min}, t r$ $=21.9 \mathrm{~min}$. 
(S)-3-benzyl-8-methyl-3-(3-methylbut-2-en-1-yl)chroman-2-one (2t)<smiles>CC(C)=CC[C@@]1(Br)Cc2cccc(C)c2OC1=O</smiles>

Opt. Rot.: $[\alpha]^{26} \mathrm{D}\left(c 1.51, \mathrm{CHCl}_{3}\right):+25.5$

${ }^{1}$ H NMR: $\left(400 \mathrm{MHz}, \mathrm{CDCl}_{3}\right) \delta 7.32-7.20(\mathrm{~m}, 3 \mathrm{H}), 7.18-7.12(\mathrm{~m}, 2 \mathrm{H}), 7.11-7.06(\mathrm{~m}$, 1H), $7.01-6.93(\mathrm{~m}, 2 \mathrm{H}), 5.27-5.20(\mathrm{~m}, 1 \mathrm{H}), 3.09$ (d, $J=16.0 \mathrm{~Hz}, 1 \mathrm{H}), 2.87$ (d, $J=16.0$ $\mathrm{Hz}, 1 \mathrm{H}), 2.82(\mathrm{~d}, J=16.0 \mathrm{~Hz}, 1 \mathrm{H}), 2.76(\mathrm{~d}, J=16.0 \mathrm{~Hz}, 1 \mathrm{H}), 2.31(\mathrm{~s}, 3 \mathrm{H}), 2.39-2.22(\mathrm{~m}$, $2 \mathrm{H}), 1.74(\mathrm{~s}, 3 \mathrm{H}), 1.52(\mathrm{~s}, 3 \mathrm{H})$;

${ }^{13}$ C NMR: $\left(100 \mathrm{MHz}, \mathrm{CDCl}_{3}\right) \delta 171.7,149.3,136.2,137.1,130.6,129.5,128.1,126.8$, $126.1,125.3,123.9,121.7,118.2,45.9,40.7,33.4,32.9,26.0,18.0,15.7$;

HRMS: (ESI) $[\mathrm{M}+\mathrm{H}]^{+}$calcd. for $\mathrm{C}_{22} \mathrm{H}_{25} \mathrm{O}_{2}, 321.1855$ found, 321.1857;

IR (film): $v_{\max } 1757,1489,1458,1193,1139,756,702 \mathrm{~cm}^{-1}$

HPLC: 95\% ee determined by HPLC (OJ-H, 99/1 hexanes/i-PrOH, $0.5 \mathrm{ml} / \mathrm{min}) t r=12.6$ $\min , \operatorname{tr}=15.4 \mathrm{~min}$.

(S)-3-benzyl-3-cinnamyl-7-methylchroman-2-one (2u)<smiles>Cc1ccc2c(c1)OC(=O)C(Br)(C/C=C\c1ccccc1)C2</smiles>

mp: $154.1-155.3^{\circ} \mathrm{C}$

Opt. Rot.: $[\alpha]^{26}{ }_{\mathrm{D}}\left(c \mathrm{1} 1.03, \mathrm{CHCl}_{3}\right):-3.2$

${ }^{1}$ H NMR: (400 MHz, $\left.\mathrm{CDCl}_{3}\right) \delta 7.37-7.20(\mathrm{~m}, 8 \mathrm{H}), 7.20-7.15(\mathrm{~m}, 2 \mathrm{H}), 7.08-7.03(\mathrm{~m}$, $1 \mathrm{H}), 6.98-6.92(\mathrm{~m}, 2 \mathrm{H}), 6.42(\mathrm{~d}, J=16.0 \mathrm{~Hz}, 1 \mathrm{H}), 6.28-6.16(\mathrm{~m}, 1 \mathrm{H}), 3.08(\mathrm{~d}, J=16.0$ $\mathrm{Hz}, 1 \mathrm{H}), 2.93(\mathrm{~d}, J=16.0 \mathrm{~Hz}, 1 \mathrm{H}), 2.89$ (d, $J=16.0 \mathrm{~Hz}, 1 \mathrm{H}), 2.40-2.33(\mathrm{~m}, 1 \mathrm{H}), 2.34$ (s, $1 \mathrm{H})$;

${ }^{13}$ C NMR: (100 MHz, $\left.\mathrm{CDCl}_{3}\right) \delta 171.4,149.0,137.0,135.7,134.7,134.1,130.6,129.0$, $128.6,128.5,128.3,127.5,127.1,126.2,124.2$, 121.4, 115.9, 45.8, 40.9, 38.7, 32.7, 20.8;

HRMS: (ESI) $[\mathrm{M}+\mathrm{H}]^{+}$calcd. for $\mathrm{C}_{26} \mathrm{H}_{25} \mathrm{O}_{2}, 369.1855$ found, 369.1855; 
IR (film): $v_{\max } 1751,1489,1458,1215,754,669 \mathrm{~cm}^{-1}$

HPLC: $90 \%$ ee determined by HPLC (OD-H, 90/10 hexanes/i-PrOH, $0.5 \mathrm{ml} / \mathrm{min}$ ) $t r=11.7$ $\min , t r=16.1 \mathrm{~min}$.

(S)-3-benzyl-6-methoxy-3-(3-methylbut-2-en-1-yl)chroman-2-one (2v)<smiles>COc1ccc2c(c1)C[C@@](CC=C(C)C)(Cc1ccccc1)C(=O)O2</smiles>

mp: $103.2-104.7^{\circ} \mathrm{C}$

Opt. Rot.: $[\alpha]^{26}\left(c 1.20, \mathrm{CHCl}_{3}\right):-1.0$

${ }^{1}$ H NMR: $\left(400 \mathrm{MHz}, \mathrm{CDCl}_{3}\right) \delta 7.32-7.22(\mathrm{~m}, 3 \mathrm{H}), 7.19-7.13(\mathrm{~m}, 2 \mathrm{H}), 7.10-7.05(\mathrm{~m}$, 1H), $6.98-6.93(\mathrm{~m}, 1 \mathrm{H}), 6.81-6.75(\mathrm{~m}, 1 \mathrm{H}), 6.11-6.65(\mathrm{~m}, 1 \mathrm{H}), 5.36-6.31(\mathrm{~m}, 1 \mathrm{H}), 3.80$ (s, 3H), 3.13 (d, $J=16.0 \mathrm{~Hz}, 1 \mathrm{H}), 2.87$ (d, $J=16.0 \mathrm{~Hz}, 1 \mathrm{H}), 2.79$ (d, $J=16.0 \mathrm{~Hz}, 1 \mathrm{H}), 2.73$ $(\mathrm{d}, J=16.0 \mathrm{~Hz}, 1 \mathrm{H}), 2.36(\mathrm{dd}, J=8.0,16.0 \mathrm{~Hz}, 1 \mathrm{H}), 2.25(\mathrm{dd}, J=8.0,16.0 \mathrm{~Hz}, 1 \mathrm{H}), 1.75$ (s, $3 \mathrm{H}), 1.52(\mathrm{~s}, 3 \mathrm{H})$;

${ }^{13}$ C NMR: $\left(100 \mathrm{MHz}, \mathrm{CDCl}_{3}\right) \delta 170.6,149.5,137.0,138.0,133.3,128.7,128.6,127.5$, $126.2,125.9,122.2,116.3,39.3,33.2,28.7,20.7$;

HRMS: (ESI) $[\mathrm{M}+\mathrm{H}]^{+}$calcd. for $\mathrm{C}_{22} \mathrm{H}_{25} \mathrm{O}_{3}, 337.1804$ found, 337.1809;

IR (film): $v_{\max } 1749,1489,1458,1215,754,702 \mathrm{~cm}^{-1}$

HPLC: $96 \%$ ee determined by HPLC (AD-H, 95/5 hexanes/i-PrOH, $0.5 \mathrm{ml} / \mathrm{min}) t r=22.6$ $\min , t r=26.0 \mathrm{~min}$.

(S)-3-benzyl-3-cinnamyl-7-methoxychroman-2-one (2w)<smiles>COc1ccc2c(c1)OC(=O)C(CBr)(C/C=C/c1ccccc1)C2</smiles>

mp: $74.5-76.0^{\circ} \mathrm{C}$

Opt. Rot.: $[\alpha]^{26}\left(c 1.63, \mathrm{CHCl}_{3}\right):-6.9$

${ }^{1}$ H NMR: $\left(400 \mathrm{MHz}, \mathrm{CDCl}_{3}\right) \delta 7.37-7.20(\mathrm{~m}, 8 \mathrm{H}), 7.19-7.15(\mathrm{~m}, 2 \mathrm{H}), 7.05(\mathrm{~d}, J=8.0 \mathrm{~Hz}$, 1H), $6.69(\mathrm{dd}, J=4.0,8.0 \mathrm{~Hz}, 1 \mathrm{H}), 6.63(\mathrm{~d}, J=4.0 \mathrm{~Hz}, 1 \mathrm{H}), 6.42(\mathrm{~d}, J=16.0 \mathrm{~Hz}, 1 \mathrm{H}), 6.26-$ $6.16(\mathrm{~m}, 1 \mathrm{H}), 3.81(\mathrm{~s}, 3 \mathrm{H}), 3.08(\mathrm{~d}, J=16.0 \mathrm{~Hz}, 1 \mathrm{H}), 2.90(\mathrm{~d}, J=16.0 \mathrm{~Hz}, 1 \mathrm{H}), 2.78-2.69$ $(\mathrm{m}, 2 \mathrm{H}), 2.38(\mathrm{ddd}, J=8.0,16.0,24.0 \mathrm{~Hz}, 1 \mathrm{H})$; 
${ }^{13}$ C NMR: $\left(100 \mathrm{MHz}, \mathrm{CDCl}_{3}\right) \delta 171.3,159.6,151.7,137.0,135.7,134.6,130.6,129.1$, $128.5,128.3,127.5,127.1,126.2,124.2,113.5,110.6,101.8,55.5,46.0,41.0,38.8,31.9$;

HRMS: (ESI) $[\mathrm{M}+\mathrm{H}]^{+}$calcd. for $\mathrm{C}_{26} \mathrm{H}_{25} \mathrm{O}_{3}, 385.1804$ found, 385.1804;

IR (film): $v_{\max } 1759,1627,1589,1508,1442,1141,750,700 \mathrm{~cm}^{-1}$

HPLC: 94\% ee determined by HPLC (OD-H, 90/10 hexanes/i-PrOH, $1.0 \mathrm{ml} / \mathrm{min}$ ) $t r=8.0$ $\min , t r=10.0 \mathrm{~min}$.

(S)-2-benzyl-2-cinnamyl-1H-benzo[f $]$ chromen-3(2H)-one $(2 x)$<smiles>O=C1Oc2ccc3ccccc3c2C[C@]1(C/C=C/c1ccccc1)Cc1ccccc1</smiles>

Opt. Rot.: $[\alpha]^{25} \mathrm{D}\left(c 1.41, \mathrm{CHCl}_{3}\right):-0.7$

${ }^{1}$ H NMR: (400 MHz, $\left.\mathrm{CDCl}_{3}\right) \delta 7.90-7.75(\mathrm{~m}, 3 \mathrm{H}), 7.61-7.46(\mathrm{~m}, 2 \mathrm{H}), 7.36-7.15(\mathrm{~m}$, $10 \mathrm{H}), 7.14-7.07(\mathrm{~m}, 2 \mathrm{H}), 6.43(\mathrm{~d}, J=16.0 \mathrm{~Hz}, 1 \mathrm{H}), 6.34-6.24(\mathrm{~m}, 1 \mathrm{H}), 3.27(\mathrm{~d}, J=16.0$ Hz, 1H), 3.17 (d, $J=16.0 \mathrm{~Hz}, 1 \mathrm{H}), 3.16(\mathrm{~d}, J=16.0 \mathrm{~Hz}, 1 \mathrm{H}), 2.96(\mathrm{~d}, J=16.0 \mathrm{~Hz}, 1 \mathrm{H}), 2.89$ (ddd, $J=4.0,8.0,16.0 \mathrm{~Hz}, 1 \mathrm{H}), 2.46(\mathrm{ddd}, J=4.0,8.0,16.0 \mathrm{~Hz}, 1 \mathrm{H})$;

${ }^{13}$ C NMR: $\left(100 \mathrm{MHz}, \mathrm{CDCl}_{3}\right) \delta 171.4,148.6,136.9,135.4,134.9,131.4,131.0,128.9$, $128.8,128.5,128.3,127.5,127.2,127.1,126.3,125.1,124.1,122.8,116.8,114.6,45.6,41.5$, 39.1, 28.4;

HRMS: (ESI) $[\mathrm{M}+\mathrm{H}]^{+}$calcd. for $\mathrm{C}_{29} \mathrm{H}_{25} \mathrm{O}_{2}, 405.1855$ found, 405.1854;

IR (film): $v_{\max } 1757,1489,1458,1217,756,702 \mathrm{~cm}^{-1}$

HPLC: 90\% ee determined by HPLC (OD-H, 90/10 hexanes/i-PrOH, $1.0 \mathrm{ml} / \mathrm{min}$ ) $t r=9.0$ $\min , t r=11.6 \min$.

(S)-3-benzyl-3-(3-methylbut-2-en-1-yl)-3,4-dihydro-2H-benzo[ $h]$ chromen-2-one (2y)<smiles>CC(C)=CC[C@]1(Br)Cc2ccc3ccccc3c2OC1=O</smiles>

Opt. Rot.: $[\alpha]^{24}\left(c 1.00, \mathrm{CHCl}_{3}\right):+2.3$

${ }^{1}$ H NMR: $\left(400 \mathrm{MHz}, \mathrm{CDCl}_{3}\right) \delta 8.27(\mathrm{~d}, J=8.0 \mathrm{~Hz}, 1 \mathrm{H}), 7.84(\mathrm{~d}, J=8.0 \mathrm{~Hz}, 1 \mathrm{H}), 7.63-7.48$ $(\mathrm{m}, 3 \mathrm{H}), 7.33-7.15(\mathrm{~m}, 6 \mathrm{H}), 5.31-5.23(\mathrm{~m}, 1 \mathrm{H}), 3.15(\mathrm{~d}, J=16.0 \mathrm{~Hz}, 1 \mathrm{H}), 3.05-2.88(\mathrm{~m}$, 
3H), $2.45(\mathrm{dd}, J=8.0,16.0 \mathrm{~Hz}, 1 \mathrm{H}), 2.34(\mathrm{dd}, J=8.0,16.0 \mathrm{~Hz}, 1 \mathrm{H}), 1.73(\mathrm{~s}, 3 \mathrm{H}), 1.52$ (s, $3 \mathrm{H})$;

${ }^{13}$ C NMR: $\left(100 \mathrm{MHz}, \mathrm{CDCl}_{3}\right) \delta 171.5,145.7,136.2,136.0,133.4,130.6,128.2,127.6$, $126.9,126.5,126.3,125.9,124.0,123.2,121.0,118.1,116.4,46.0,40.8,33.5,32.9,26.1$, 18.1 ;

HRMS: (ESI) $[\mathrm{M}+\mathrm{H}]^{+}$calcd. for $\mathrm{C}_{25} \mathrm{H}_{25} \mathrm{O}_{2}, 357.1855$ found, 357.1854;

IR (film): $v_{\max } 1761,1489,1458,1377,1132,756 \mathrm{~cm}^{-1}$

HPLC: $93 \%$ ee determined by HPLC (AD-H, 95/5 hexanes/i-PrOH, $0.5 \mathrm{ml} / \mathrm{min}$ ) $t r=12.4$ $\min , t r=15.5 \min$.

(S)-2-benzyl-8-bromo-2-cinnamyl-1H-benzo[f]chromen-3(2H)-one (2z)<smiles>O=C1Oc2ccc3cc(Br)ccc3c2C[C@@]1(CBr)C/C=C/c1ccccc1</smiles>

Opt. Rot.: $[\alpha]^{25}{ }_{\mathrm{D}}\left(c 0.61, \mathrm{CHCl}_{3}\right):-1.3$.

${ }^{1}$ H NMR: (400 MHz, $\left.\mathrm{CDCl}_{3}\right) \delta 8.05-8.00(\mathrm{~m}, 1 \mathrm{H}), 7.73-7.56(\mathrm{~m}, 3 \mathrm{H}), 7.34-7.18(\mathrm{~m}$, 9H), $7.12-7.03(\mathrm{~m}, 2 \mathrm{H}), 6.42(\mathrm{~d}, J=16.0 \mathrm{~Hz}, 1 \mathrm{H}), 6.32-6.21(\mathrm{~m}, 1 \mathrm{H}), 3.23(\mathrm{~d}, J=16.0$ Hz, 1H), $3.15(\mathrm{~d}, J=16.0 \mathrm{~Hz}, 1 \mathrm{H}), 3.11(\mathrm{~d}, J=16.0 \mathrm{~Hz}, 1 \mathrm{H}), 2.98-2.84(\mathrm{~m}, 2 \mathrm{H}), 2.47-$ $2.40(\mathrm{~m}, 1 \mathrm{H})$;

${ }^{13}$ C NMR: $\left(100 \mathrm{MHz}, \mathrm{CDCl}_{3}\right) \delta 171.0,148.8,136.7,135.1,135.0,132.1,130.7,130.6$, $130.3,129.9,128.5,128.3,127.9,127.5,127.2,126.2,124.5,123.9,119.0,117.9,115.0$, 45.4, 41.6, 39.2, 28.3;

HRMS: (ESI) $[\mathrm{M}+\mathrm{H}]^{+}$calcd. for $\mathrm{C}_{29} \mathrm{H}_{24} \mathrm{O}_{2} \mathrm{~B}_{\mathrm{r}}, 483.0958$ found, 483.0960;

IR (film): $v_{\max } 1759,1489,1357,1149,810,649 \mathrm{~cm}^{-1}$

HPLC: $95 \%$ ee determined by HPLC (OD-H, 90/10 hexanes/i-PrOH, $1.0 \mathrm{ml} / \mathrm{min}$ ) $t r=8.2$ $\min , t r=13.2 \mathrm{~min}$.

\section{(S)-2-benzyl-9-bromo-2-cinnamyl-1H-benzo[f]chromen-3(2H)-one (2aa)}<smiles>O=C1Oc2ccc3ccc(Br)cc3c2CC1(Br)C/C=C/c1ccccc1</smiles> 
Opt. Rot.: $[\alpha]^{25}{ }_{\mathrm{D}}\left(c 0.81, \mathrm{CHCl}_{3}\right): 26.0$.

${ }^{1}$ H NMR: (400 MHz, MeOD) $\delta 8.05-8.02(\mathrm{~m}, 1 \mathrm{H}), 7.79-7.73(\mathrm{~m}, 2 \mathrm{H}), 7.56-7.51(\mathrm{~m}$, 1H), $7.29-7.11(\mathrm{~m}, 11 \mathrm{H}), 6.38(\mathrm{~d}, J=16.0 \mathrm{~Hz}, 1 \mathrm{H}), 6.30-6.19(\mathrm{~m}, 1 \mathrm{H}), 3.23$ (d, $J=16.0$ $\mathrm{Hz}, 1 \mathrm{H}), 3.1 \mathrm{I} 8-3.10(\mathrm{~m}, 2 \mathrm{H}), 2.92(\mathrm{~d}, J=16.0 \mathrm{~Hz}, 1 \mathrm{H}), 2.75(\mathrm{ddd}, J=4.0,8.0,16.0 \mathrm{~Hz}$, $1 \mathrm{H}), 2.49$ (ddd, $J=4.0,8.0,16.0 \mathrm{~Hz}, 1 \mathrm{H})$;

${ }^{13}$ C NMR: (100 MHz, MeOD) $\delta$ 171.1, 149.0, 136.9, 135.7, 134.7, 132.6, 130.2, 130.1, $129.4,128.6,128.1,128.0,127.8,127.0,126.7,125.8,125.0,123.5,121.2,116.7,114.3$, 45.5, 41.4, 39.3, 28.4;

HRMS: (ESI) $[\mathrm{M}+\mathrm{H}]^{+}$calcd. for $\mathrm{C}_{29} \mathrm{H}_{24} \mathrm{O}_{2} \mathrm{Br}$, 483.0951 found, 483.0960;

IR (film): $v_{\max } 1759,1496,1357,1149,833,640 \mathrm{~cm}^{-1}$

HPLC: 94\% ee determined by HPLC (OD-H, 95/5 hexanes/i-PrOH, $1.0 \mathrm{ml} / \mathrm{min}$ ) $t r=10.2$ $\min , \operatorname{tr}=12.7 \mathrm{~min}$.

(S)-2-benzyl-2-cinnamyl-9-methoxy-1H-benzo[f]chromen-3(2H)-one (2ab)<smiles>COc1ccc2ccc3c(c2c1)C[C@](Br)(C/C=C/c1ccccc1)C(=O)O3</smiles>

Opt. Rot.: $[\alpha]^{25} \mathrm{D}\left(c\right.$ 1.90, $\left.\mathrm{CHCl}_{3}\right)$ : -53.9.

${ }^{1}$ H NMR: $\left(400 \mathrm{MHz}, \mathrm{CDCl}_{3}\right) \delta 7.81-7.67(\mathrm{~m}, 2 \mathrm{H}), 7.35-7.20(\mathrm{~m}, 8 \mathrm{H}), 7.18-7.07$ (m, 4H), $7.06-7.02(\mathrm{~m}, 1 \mathrm{H}), 6.43(\mathrm{~d}, J=16.0 \mathrm{~Hz}, 1 \mathrm{H}), 6.35-6.25(\mathrm{~m}, 1 \mathrm{H}), 3.90(\mathrm{~s}, 3 \mathrm{H}), 3.22-$ $2.88(\mathrm{~m}, 5 \mathrm{H}), 2.48-2.39(\mathrm{~m}, 1 \mathrm{H})$;

${ }^{13}$ C NMR: $\left(100 \mathrm{MHz}, \mathrm{CDCl}_{3}\right) \delta 171.4,158.8,149.3,136.9,135.3,134.9,132.7,130.8$, $130.3,128.6,128.5,128.2$, 127.5, 127.1, 126.3, 126.2, 124.2, 117.3, 114.2, 113.4, 101.7, 55.3, 45.6, 41.5, 39.0, 28.1;

HRMS: (ESI) $[\mathrm{M}+\mathrm{H}]^{+}$calcd. for $\mathrm{C}_{30} \mathrm{H}_{27} \mathrm{O}_{3}, 435.1965$ found, 435.1960;

IR (film): $v_{\max } 1759,1627,1149,833,694 \mathrm{~cm}^{-1}$

HPLC: $96 \%$ ee determined by HPLC (OD-H, 90/10 hexanes/i-PrOH, $1.0 \mathrm{ml} / \mathrm{min}$ ) $t r=8.9$ $\min , t r=12.7 \mathrm{~min}$.

(S,E)-4-((7-bromo-3-cinnamyl-2-oxo-1,2,3,4-tetrahydrophenanthren-3yl)methyl)benzonitrile (2ac) 


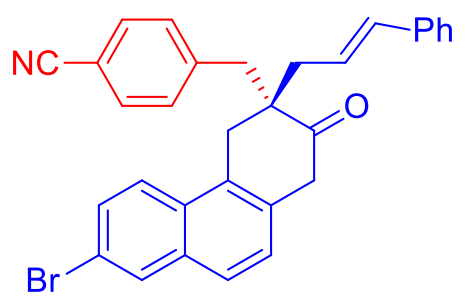

Opt. Rot.: $[\alpha]^{25}{ }_{\mathrm{D}}\left(\mathrm{c} 1.21, \mathrm{CHCl}_{3}\right):-4.0$.

${ }^{1}$ H NMR: $\left(400 \mathrm{MHz}, \mathrm{CDCl}_{3}\right) \delta 8.06-8.03(\mathrm{~m}, 1 \mathrm{H}), 7.74-7.62(\mathrm{~m}, 3 \mathrm{H}), 7.60-7.52(\mathrm{~m}$, 2H), $7.38-7.21(\mathrm{~m}, 8 \mathrm{H}), 6.42(\mathrm{~d}, J=16.0 \mathrm{~Hz}, 1 \mathrm{H}), 6.28-6.17(\mathrm{~m}, 1 \mathrm{H}), 3.34-3.25(\mathrm{~m}$, 2H), $3.08(\mathrm{~d}, J=16.0 \mathrm{~Hz}, 1 \mathrm{H}), 2.97(\mathrm{~d}, J=16.0 \mathrm{~Hz}, 1 \mathrm{H}), 2.82(\mathrm{ddd}, J=4.0,8.0,16.0 \mathrm{~Hz}$, 1H), 2.49 (ddd, $J=4.0,8.0,16.0 \mathrm{~Hz}, 1 \mathrm{H}$ );

${ }^{13}$ C NMR: $\left(100 \mathrm{MHz}, \mathrm{CDCl}_{3}\right) \delta 170.3,148.5,135.6,132.1,132.0,131.2,130.9,130.6$, $128.6,128.2,127.8,126.2,124.2,122.8,117.8,114.4,45.6,41.8,39.6,28.7$;

HRMS: (ESI) $[\mathrm{M}+\mathrm{H}]^{+}$calcd. for $\mathrm{C}_{30} \mathrm{H}_{23} \mathrm{NO}_{2} \mathrm{~B}_{\mathrm{r}}, 508.0893$ found, 508.0912;

IR (film): $v_{\max } 2230,1651,1435,1263,1149,725,667 \mathrm{~cm}^{-1}$

HPLC: $90 \%$ ee determined by HPLC (OD-H, 70/30 hexanes/i-PrOH, $1.0 \mathrm{ml} / \mathrm{min}$ ) $t r=16.8$ $\min , t r=22.7 \mathrm{~min}$.

\section{2-benzyl-2,3-dihydro-1H-inden-1-one (3a)}<smiles>O=C1c2ccccc2CC1Cc1ccccc1</smiles>

${ }^{1}$ H NMR: $\left(400 \mathrm{MHz}, \mathrm{CDCl}_{3}\right) \delta 7.80(\mathrm{~d}, J=8.0 \mathrm{~Hz}, 1 \mathrm{H}), 7.60-7.55(\mathrm{~m}, 1 \mathrm{H}), 7.42-7.20(\mathrm{~m}$, $7 \mathrm{H}), 3.42(\mathrm{dd}, J=4.0,16.0 \mathrm{~Hz}, 1 \mathrm{H}), 3.15(\mathrm{dd}, J=8.0,16.0 \mathrm{~Hz}, 1 \mathrm{H}), 3.03-2.94(\mathrm{~m}, 1 \mathrm{H})$, $2.85(\mathrm{dd}, J=8.0,16.0 \mathrm{~Hz}, 1 \mathrm{H}), 2.68(\mathrm{dd}, J=4.0,16.0 \mathrm{~Hz}, 1 \mathrm{H})$;

${ }^{13}$ C NMR: $\left(100 \mathrm{MHz}, \mathrm{CDCl}_{3}\right) \delta 207.6,153.6,139.6,136.6,134.8,128.9,128.5,127.4$, $126.6,126.4,123.9,48.9,36.9,32.2$;

HRMS: (ESI) $[\mathrm{M}+\mathrm{H}]^{+}$calcd. for $\mathrm{C}_{16} \mathrm{H}_{15} \mathrm{O}, 223.1125$ found, 223.1123;

IR (film): $v_{\max } 1759,1627,1589,1508,1442,1141,750,700 \mathrm{~cm}^{-1}$

2-(2-(trifluoromethyl)benzyl)-2,3-dihydro-1H-inden-1-one (3b)<smiles>O=C1c2ccccc2CC1Cc1ccccc1C(F)(F)F</smiles>

${ }^{1}$ H NMR: $\left(400 \mathrm{MHz}, \mathrm{CDCl}_{3}\right) \delta 7.84-7.30(\mathrm{~m}, 7 \mathrm{H}), 3.61(\mathrm{dd}, J=4.0,16.0 \mathrm{~Hz}, 1 \mathrm{H}), 3.22$ 
$(\mathrm{dd}, J=8.0,16.0 \mathrm{~Hz}, 1 \mathrm{H}), 3.14-3.04(\mathrm{~m}, 1 \mathrm{H}), 2.94(\mathrm{dd}, J=8.0,16.0 \mathrm{~Hz}, 1 \mathrm{H}), 2.84(\mathrm{dd}, J=$ 4.0, $16.0 \mathrm{~Hz}, 1 \mathrm{H})$;

${ }^{13}$ C NMR: $\left(100 \mathrm{MHz}, \mathrm{CDCl}_{3}\right) \delta 207.1,153.2,138.6,138.5,136.3,134.9,131.2,130.8$, $129.4,129.1,128.8,128.6,128.5,127.5,126.5,126.4,126.3,126.2,126.1,126.1,125.9$, $124.0,123.2,120.4,48.2,32.9,32.8,32.3$;

${ }^{19}$ F NMR: $\left(376 \mathrm{MHz}, \mathrm{CDCl}_{3}\right) \delta-62.4$;

HRMS: (ESI) $[\mathrm{M}+\mathrm{H}]^{+}$calcd. for $\mathrm{C}_{19} \mathrm{H}_{20} \mathrm{OF}_{3}, 321.1465$ found, 321.1466;

IR (film): $v_{\max } 1712,1604,1465,1311,1118,694 \mathrm{~cm}^{-1}$

\section{2-(3-(trifluoromethyl)benzyl)-2,3-dihydro-1H-inden-1-one (3c)}<smiles>O=C1c2ccccc2CC1Cc1cccc(C(F)(F)F)c1</smiles>

${ }^{1}$ H NMR: $\left(400 \mathrm{MHz}, \mathrm{CDCl}_{3}\right) \delta 7.78(\mathrm{~d}, J=8.0 \mathrm{~Hz}, 1 \mathrm{H}), 7.61-7.32(\mathrm{~m}, 6 \mathrm{H}), 3.43(\mathrm{dd}, J=$ $4.0,16.0 \mathrm{~Hz}, 1 \mathrm{H}), 3.19(\mathrm{dd}, J=8.0,16.0 \mathrm{~Hz}, 1 \mathrm{H}), 3.04-2.95(\mathrm{~m}, 1 \mathrm{H}), 2.83(\mathrm{dd}, J=4.0,16.0$ $\mathrm{Hz}, 1 \mathrm{H}), 2.75(\mathrm{dd}, J=8.0,16.0 \mathrm{~Hz}, 1 \mathrm{H})$;

${ }^{13}$ C NMR: $\left(100 \mathrm{MHz}, \mathrm{CDCl}_{3}\right) \delta$ 207.0, 153.2, 140.6, 136.4, 134.9, 132.3, 131.2, 130.9, $130.6,130.3,129.0,128.2,127.5,126.6,125.6,125.6,125.5,125.4,124.0,123.3,123.2$, $123.2,123.2,122.8,120.1,48.5,36.7,32.1$;

${ }^{19}$ F NMR: $\left(376 \mathrm{MHz}, \mathrm{CDCl}_{3}\right) \delta-62.5$;

HRMS: (ESI) $[\mathrm{M}+\mathrm{H}]^{+}$calcd. for $\mathrm{C}_{19} \mathrm{H}_{20} \mathrm{OF}_{3}, 321.1465$ found, 321.1466;

IR (film): $v_{\max } 1712,1612,1465,1327,1126,702 \mathrm{~cm}^{-1}$

\section{2-(4-(trifluoromethyl)benzyl)-2,3-dihydro-1H-inden-1-one (3d)}<smiles>O=C1c2ccccc2CC1Cc1ccc(C(F)(F)F)cc1</smiles>

${ }^{1}$ H NMR: $\left(400 \mathrm{MHz}, \mathrm{CDCl}_{3}\right) \delta 7.80(\mathrm{~d}, J=8.0 \mathrm{~Hz}, 1 \mathrm{H}), 7.63-7.54(\mathrm{~m}, 3 \mathrm{H}), 7.45-7.34(\mathrm{~m}$, 4H), $3.45(\mathrm{dd}, J=4.0,16.0 \mathrm{~Hz}, 1 \mathrm{H}), 3.21$ (dd, $J=8.0,16.0 \mathrm{~Hz}, 1 \mathrm{H}), 3.06-2.97(\mathrm{~m}, 1 \mathrm{H})$, 2.89-2.74 (m, 2H);

${ }^{13}$ C NMR: $\left(100 \mathrm{MHz}, \mathrm{CDCl}_{3}\right) \delta 207.1,153.3,143.9,143.8,136.3,135.0,129.2,128.9$, $128.6,127.6,126.6,125.6,125.5,125.4,125.4,125.3,124.0,122.9,48.5,36.6,32.0$;

${ }^{19}$ F NMR: $\left(376 \mathrm{MHz}, \mathrm{CDCl}_{3}\right) \delta$-58.99;

HRMS: (ESI) $[\mathrm{M}+\mathrm{H}]^{+}$calcd. for $\mathrm{C}_{19} \mathrm{H}_{20} \mathrm{OF}_{3}, 321.1473$ found, 321.1466; 
IR (film): $v_{\max } 1705,1612,1327,1118,694 \mathrm{~cm}^{-1}$

\section{2-(3-bromobenzyl)-2,3-dihydro-1H-inden-1-one (3e)}<smiles>O=C1c2ccccc2CC1Cc1cccc(Br)c1</smiles>

${ }^{1}$ H NMR: $\left(400 \mathrm{MHz}, \mathrm{CDCl}_{3}\right) \delta 7.84-7.14(\mathrm{~m}, 8 \mathrm{H}), 3.38(\mathrm{dd}, J=4.0,16.0 \mathrm{~Hz}, 1 \mathrm{H}), 3.20$ $(\mathrm{dd}, J=8.0,16.0 \mathrm{~Hz}, 1 \mathrm{H}), 3.05-2.94(\mathrm{~m}, 1 \mathrm{H}), 2.83(\mathrm{dd}, J=4.0,16.0 \mathrm{~Hz}, 1 \mathrm{H}), 2.63(\mathrm{dd}, J=$ 8.0, $16.0 \mathrm{~Hz}, 1 \mathrm{H})$;

${ }^{13}$ C NMR: $\left(100 \mathrm{MHz}, \mathrm{CDCl}_{3}\right) \delta 207.1,153.3,142.0,136.4,134.9,131.9,130.1,129.5$, $127.5,126.6,124.0,122.6,48.6,36.5,32.1$;

HRMS: (ESI) $[\mathrm{M}+\mathrm{H}]^{+}$calcd. for $\mathrm{C}_{19} \mathrm{H}_{22} \mathrm{OB}_{\mathrm{r},} 345.0853$ found, 345.0854;

IR (film): $v_{\max } 1705,1604,1465,1288,694 \mathrm{~cm}^{-1}$

\section{2-(thiophen-3-ylmethyl)-2,3-dihydro-1H-inden-1-one (3f)}<smiles>O=C1c2ccccc2CC1Cc1ccsc1</smiles>

${ }^{1}$ H NMR: $\left(400 \mathrm{MHz}, \mathrm{CDCl}_{3}\right) \delta 7.78(\mathrm{~d}, J=8.0 \mathrm{~Hz}, 1 \mathrm{H}), 7.60-7.53(\mathrm{~m}, 1 \mathrm{H}), 7.45-7.31(\mathrm{~m}$, 2H), $7.26-7.21(\mathrm{~m}, 1 \mathrm{H}), 7.04-6.93(\mathrm{~m}, 2 \mathrm{H}), 6.26(\mathrm{dd}, J=2.0,2.8 \mathrm{~Hz}, 1 \mathrm{H}), 6.07-6.03(\mathrm{~m}$, 1H), $3.33(\mathrm{dd}, J=4.0,16.0 \mathrm{~Hz}, 1 \mathrm{H}), 3.23(\mathrm{dd}, J=8.0,16.0 \mathrm{~Hz}, 1 \mathrm{H}), 3.02-2.93(\mathrm{~m}, 1 \mathrm{H})$, $2.91-2.77(\mathrm{~m}, 2 \mathrm{H})$;

${ }^{13}$ C NMR: $\left(100 \mathrm{MHz}, \mathrm{CDCl}_{3}\right) \delta 207.6,153.6,139.7,136.5,134.8,128.3,127.4,126.6$, $125.7,123.9,121.4,48.2,32.3,31.4$;

HRMS: (ESI) $[\mathrm{M}+\mathrm{H}]^{+}$calcd. for $\mathrm{C}_{14} \mathrm{H}_{13} \mathrm{OS}, 229.1071$ found, 229.1078;

IR (film): $v_{\max } 1705,1604,1465,694 \mathrm{~cm}^{-1}$

\section{2-(furan-2-ylmethyl)-2,3-dihydro-1H-inden-1-one (3g)}<smiles>O=C1c2ccccc2CC1Cc1ccco1</smiles>

${ }^{1}$ H NMR: $\left(400 \mathrm{MHz}, \mathrm{CDCl}_{3}\right) \delta 7.75(\mathrm{~d}, J=8.0 \mathrm{~Hz}, 1 \mathrm{H}), 7.59-7.51(\mathrm{~m}, 1 \mathrm{H}), 7.41(\mathrm{~d}, J=8.0$ $\mathrm{Hz}, 1 \mathrm{H}), 7.37-7.25(\mathrm{~m}, 2 \mathrm{H}), 6.26(\mathrm{dd}, J=2.0,2.8 \mathrm{~Hz}, 1 \mathrm{H}), 6.07-6.03(\mathrm{~m}, 1 \mathrm{H}), 3.35-3.21$ (m, 2H), $3.03-2.86(\mathrm{~m}, 2 \mathrm{H}), 2.78(\mathrm{dd}, J=8.0,16.0 \mathrm{~Hz}, 1 \mathrm{H})$; 
${ }^{13}$ C NMR: $\left(100 \mathrm{MHz}, \mathrm{CDCl}_{3}\right) \delta 207.1,153.6,153.5,141.4,136.4,134.8,127.4,126.6$, $123.9,110.2,106.3,46.4,32.4,29.3$;

HRMS: (ESI) $[\mathrm{M}+\mathrm{H}]^{+}$calcd. for $\mathrm{C}_{14} \mathrm{H}_{13} \mathrm{O}_{2}, 213.0911$ found, 213.0916;

IR (film): $v_{\max } 1712,1612,1149,1010,694 \mathrm{~cm}^{-1}$

6-methyl-2-(2-(trifluoromethyl)benzyl)-2,3-dihydro-1H-inden-1-one (3h)<smiles>Cc1ccc2c(c1)C(=O)C(Cc1ccccc1C(F)(F)F)C2</smiles>

${ }^{1}$ H NMR: $\left(400 \mathrm{MHz}, \mathrm{CDCl}_{3}\right) \delta 7.71-7.58(\mathrm{~m}, 2 \mathrm{H}), 7.53$ - $7.408(\mathrm{~m}, 3 \mathrm{H}), 7.38$ - 7.27 (m, 2H), $3.59(\mathrm{dd}, J=4.0,16.0 \mathrm{~Hz}, 1 \mathrm{H}), 3.22-3.05(\mathrm{~m}, 1 \mathrm{H}), 2.94(\mathrm{dd}, J=8.0,16.0 \mathrm{~Hz}, 1 \mathrm{H})$, $2.79(\mathrm{dd}, J=4.0,16.0 \mathrm{~Hz}, 1 \mathrm{H}), 2.43(\mathrm{~s}, 3 \mathrm{H})$;

${ }^{13}$ C NMR: $\left(100 \mathrm{MHz}, \mathrm{CDCl}_{3}\right) \delta$ 207.2, 138.6, 137.4, 136.4, 136.1, 131.8, 130.8, 129.1, $128.8,126.4,126.2,126.1,125.8,123.9,32.8,31.9,21.0$;

${ }^{19}$ F NMR: $\left(376 \mathrm{MHz}, \mathrm{CDCl}_{3}\right) \delta-59.0$;

HRMS: (ESI) $[\mathrm{M}+\mathrm{H}]^{+}$calcd. for $\mathrm{C}_{18} \mathrm{H}_{16} \mathrm{OF}_{3}, 305.1158$ found, 305.1153;

IR (film): $v_{\max } 1705,1311,1118,700 \mathrm{~cm}^{-1}$

\section{2-benzyl-5-chloro-2,3-dihydro-1H-inden-1-one (3i)}<smiles>O=C1c2ccc(Cl)cc2CC1Cc1ccccc1</smiles>

${ }^{1}$ H NMR: $\left(400 \mathrm{MHz}, \mathrm{CDCl}_{3}\right) \delta 7.74-7.67(\mathrm{~m}, 1 \mathrm{H}), 7.42-7.20(\mathrm{~m}, 7 \mathrm{H}), 3.39(\mathrm{dd}, J=4.0$, $16.0 \mathrm{~Hz}, 1 \mathrm{H}), 3.16(\mathrm{dd}, J=8.0,16.0 \mathrm{~Hz}, 1 \mathrm{H}), 3.08-2.99(\mathrm{~m}, 1 \mathrm{H}), 2.86(\mathrm{dd}, J=8.0,16.0 \mathrm{~Hz}$, $1 \mathrm{H}), 2.71(\mathrm{dd}, J=4.0,16.0 \mathrm{~Hz}, 1 \mathrm{H})$;

${ }^{13}$ C NMR: $\left(100 \mathrm{MHz}, \mathrm{CDCl}_{3}\right) \delta 206.2,155.0,141.3,139.2,135.0,128.9,128.6,128.2$, $126.7,126.4,125.1,49.0,36.9,31.8$;

HRMS: (ESI) $[\mathrm{M}+\mathrm{H}]^{+}$calcd. for $\mathrm{C}_{16} \mathrm{H}_{14} \mathrm{OCl}, 257.0730$ found, 257.0733;

IR (film): $v_{\max } 1705,1612,1147,694 \mathrm{~cm}^{-1}$

2-(4-(trifluoromethyl)benzyl)-3,4-dihydronaphthalen-1(2H)-one (3j)<smiles>O=C1c2ccccc2CCC1Cc1ccc(C(F)(F)F)cc1</smiles> 
${ }^{1}$ H NMR: $\left(400 \mathrm{MHz}, \mathrm{CDCl}_{3}\right) \delta 8.12-8.06(\mathrm{~m}, 1 \mathrm{H}), 7.63-7.54(\mathrm{~m}, 2 \mathrm{H}), 7.52-7.46(\mathrm{~m}$, 1H), $7.42-7.30(\mathrm{~m}, 3 \mathrm{H}), 7.28-7.22(\mathrm{~m}, 1 \mathrm{H}), 3.61-3.49(\mathrm{~m}, 1 \mathrm{H}), 2.98(\mathrm{dd}, J=4.0,8.0 \mathrm{~Hz}$, $1 \mathrm{H}), 2.85-2.72(\mathrm{~m}, 2 \mathrm{H}), 2.18-2.07(\mathrm{~m}, 1 \mathrm{H}), 1.91-1.75(\mathrm{~m}, 1 \mathrm{H})$;

${ }^{13}$ C NMR: $\left(100 \mathrm{MHz}, \mathrm{CDCl}_{3}\right) \delta 198.7,144.3,143.8,133.4,132.3,129.6,128.8,128.7$, $128.3,127.5,126.7,125.6,125.4,125.3,125.3,125.2,122.9,49.2,35.6,28.7,27.9$;

${ }^{19}$ F NMR: $\left(376 \mathrm{MHz}, \mathrm{CDCl}_{3}\right) \delta-62.3$;

HRMS: (ESI) $[\mathrm{M}+\mathrm{H}]^{+}$calcd. for $\mathrm{C}_{21} \mathrm{H}_{24} \mathrm{OF}_{3}, 349.1722$ found, 349.1779;

IR (film): $v_{\max }$ 1674, 1465, 1327, 1118, $694 \mathrm{~cm}^{-1}$

\section{2-(3-(trifluoromethyl)benzyl)-3,4-dihydronaphthalen-1(2H)-one (3k)}<smiles>O=C1c2ccccc2CCC1Cc1cccc(C(F)(F)F)c1</smiles>

${ }^{1}$ H NMR: $\left(400 \mathrm{MHz}, \mathrm{CDCl}_{3}\right) \delta 8.13-8.06(\mathrm{~m}, 1 \mathrm{H}), 7.56$ - $7.39(\mathrm{~m}, 5 \mathrm{H}), 7.52-7.46(\mathrm{~m}$, $1 \mathrm{H}), 7.36-7.28(\mathrm{~m}, 1 \mathrm{H}), 7.27-7.21(\mathrm{~m}, 1 \mathrm{H}), 3.62-3.50(\mathrm{~m}, 1 \mathrm{H}), 2.97(\mathrm{dd}, J=4.0,8.0 \mathrm{~Hz}$, $1 \mathrm{H}), 2.84-2.72(\mathrm{~m}, 2 \mathrm{H}), 2.17-2.08(\mathrm{~m}, 1 \mathrm{H}), 1.90-1.74(\mathrm{~m}, 1 \mathrm{H})$;

${ }^{13}$ C NMR: $\left(100 \mathrm{MHz}, \mathrm{CDCl}_{3}\right) \delta 198.7,144.9,141.1,133.4,132.7,132.3,130.8,130.5$, $128.8,128.7,127.5,126.6,125.9,125.9,125.6,123.1,123.0,122.9,49.2,35.6,28.7,27.9$;

${ }^{19}$ F NMR: $\left(376 \mathrm{MHz}, \mathrm{CDCl}_{3}\right) \delta-62.4$;

HRMS: (ESI) $[\mathrm{M}+\mathrm{H}]^{+}$calcd. for $\mathrm{C}_{21} \mathrm{H}_{24} \mathrm{OF}_{3}, 349.1722$ found, 349.1777;

IR (film): $v_{\max } 1705,1643,1465,1327,1126,694 \mathrm{~cm}^{-1}$

\section{2-(4-fluorobenzyl)-3,4-dihydronaphthalen-1(2H)-one (3I)}<smiles>O=C1c2ccccc2CCC1Cc1ccc(F)cc1</smiles>

${ }^{1}$ H NMR: $\left(400 \mathrm{MHz}, \mathrm{CDCl}_{3}\right) \delta 8.12-8.06(\mathrm{~m}, 1 \mathrm{H}), 7.56-7.39(\mathrm{~m}, 5 \mathrm{H}), 7.52-7.44(\mathrm{~m}$, 1H), $7.37-7.30(\mathrm{~m}, 1 \mathrm{H}), 7.27-7.16(\mathrm{~m}, 3 \mathrm{H}), 7.06-7.94(\mathrm{~m}, 2 \mathrm{H}), 3.51-3.37(\mathrm{~m}, 1 \mathrm{H})$, $3.04-2.88(\mathrm{~m}, 2 \mathrm{H}), 2.79-2.65(\mathrm{~m}, 2 \mathrm{H}), 2.18-2.06(\mathrm{~m}, 1 \mathrm{H}), 1.88-1.72(\mathrm{~m}, 1 \mathrm{H})$;

${ }^{13}$ C NMR: $\left(100 \mathrm{MHz}, \mathrm{CDCl}_{3}\right) \delta 198.1,162.7,160.2,143.9,135.6,135.5,133.3,132.4$, $130.6,130.5,128.7,127.5,126.6,115.2,115.0,49.4,34.8,28.6,27.7$;

${ }^{19}$ F NMR: $\left(376 \mathrm{MHz}, \mathrm{CDCl}_{3}\right) \delta-117.2$;

HRMS: (ESI) $[\mathrm{M}+\mathrm{H}]^{+}$calcd. for $\mathrm{C}_{20} \mathrm{H}_{24} \mathrm{OF} 299.1815$ found, 299.1811; 
IR (film): $v_{\max } 1681,1512,1296,694 \mathrm{~cm}^{-1}$

2-(4-bromobenzyl)-3,4-dihydronaphthalen-1(2H)-one (3m)<smiles>O=C1c2ccccc2CCC1Cc1ccc(Br)cc1</smiles>

${ }^{1}$ H NMR: $\left(400 \mathrm{MHz}, \mathrm{CDCl}_{3}\right) \delta 8.11-8.05(\mathrm{~m}, 1 \mathrm{H}), 7.53-7.40(\mathrm{~m}, 3 \mathrm{H}), 7.37-7.30(\mathrm{~m}$, 1H), $7.28-7.21(\mathrm{~m}, 1 \mathrm{H}), 7.17-7.09(\mathrm{~m}, 2 \mathrm{H}), 3.43(\mathrm{dd}, J=4.0,16.0 \mathrm{~Hz}, 1 \mathrm{H}), 3.03-2.89$ (m, 2H), $2.79-2.65(\mathrm{~m}, 2 \mathrm{H}), 2.17-2.07(\mathrm{~m}, 1 \mathrm{H}), 1.87-1.73(\mathrm{~m}, 1 \mathrm{H})$;

${ }^{13}$ C NMR: $\left(100 \mathrm{MHz}, \mathrm{CDCl}_{3}\right) \delta 198.9,143.9,139.0,133.3,132.4,131.4,131.0,128.7$, $127.5,126.6,119.9,49.2,35.1,28.7,27.8$;

HRMS: (ESI) $[\mathrm{M}+\mathrm{H}]^{+}$calcd. for $\mathrm{C}_{19} \mathrm{H}_{22} \mathrm{OB}_{\mathrm{r}}, 345.0851$ found, 345.0854;

IR (film): $v_{\max } 1759,1627,1589,1508,1442,1141,750,700 \mathrm{~cm}^{-1}$

\section{2-(thiophen-3-ylmethyl)-3,4-dihydronaphthalen-1(2H)-one (3n)}<smiles>O=C1c2ccccc2CCC1Cc1ccsc1</smiles>

${ }^{1}$ H NMR: $\left(400 \mathrm{MHz}, \mathrm{CDCl}_{3}\right) \delta 8.12-8.06(\mathrm{~m}, 1 \mathrm{H}), 7.52-7.44(\mathrm{~m}, 1 \mathrm{H}), 7.37-7.21(\mathrm{~m}$, 3H), $7.06-6.96(\mathrm{~m}, 2 \mathrm{H}), 3.41(\mathrm{dd}, J=4.0,16.0 \mathrm{~Hz}, 1 \mathrm{H}), 3.03-2.94(\mathrm{~m}, 2 \mathrm{H}), 2.87((\mathrm{dd}, J=$ 4.0, $16.0 \mathrm{~Hz}, 1 \mathrm{H}), 2.81-2.72(\mathrm{~m}, 1 \mathrm{H}), 2.23-2.12(\mathrm{~m}, 1 \mathrm{H}), 1.89-1.75(\mathrm{~m}, 1 \mathrm{H})$;

${ }^{13}$ C NMR: $\left(100 \mathrm{MHz}, \mathrm{CDCl}_{3}\right) \delta 199.2,144.0,140.0,133.3,132.5,128.7,128.6,127.5$, 126.6, 125.4, 121.7, 48.7, 30.1, 27.7, 27.9;

HRMS: (ESI) $[\mathrm{M}+\mathrm{H}]^{+}$calcd. for $\mathrm{C}_{17} \mathrm{H}_{21} \mathrm{OS}, 273.1315$ found, 273.1313;

IR (film): $v_{\max } 1681,1465,1288,1010,694 \mathrm{~cm}^{-1}$

(S)-2-benzyl-2-cinnamyl-2,3-dihydro-1H-inden-1-one (4a)<smiles>O=C1c2ccccc2CC1(C/C=C/c1ccccc1)Cc1ccccc1</smiles>

Opt. Rot.: $[\alpha]^{26}{ }_{\mathrm{D}}\left(c \mathrm{1.43}, \mathrm{CHCl}_{3}\right): 2.5$;

${ }^{1}$ H NMR: (400 MHz, MeOD) $\delta 7.68(\mathrm{~d}, J=8.0 \mathrm{~Hz}, 1 \mathrm{H}), 7.67$ - $7.61(\mathrm{~m}, 1 \mathrm{H}), 7.38$ - 7.05 (m, 
$12 \mathrm{H}), 6.45(\mathrm{~d}, J=16.0 \mathrm{~Hz}, 1 \mathrm{H}), 6.06-5.95(\mathrm{~m}, 1 \mathrm{H}),, 3.27-3.16(\mathrm{~m}, 2 \mathrm{H}), 3.11(\mathrm{~d}, J=16.0$ $\mathrm{Hz}, 1 \mathrm{H}), 2.67$ (ddd, $J=4.0,8.0,16.0 \mathrm{~Hz}, 1 \mathrm{H}), 2.52$ (ddd, $J=4.0,8.0,16.0 \mathrm{~Hz}, 1 \mathrm{H})$;

${ }^{13}$ C NMR: (100 MHz, MeOD) $\delta$ 211.5, 153.6, 137.1, 134.9, 133.5, 129.7, 128.0, 127.6, 127.0, 126.8, 126.2, 126.1, 125.6, 124.4, 122.9, 54.3, 42.3, 41.7, 35.1;

HRMS: (ESI) $[\mathrm{M}+\mathrm{H}]^{+}$calcd. for $\mathrm{C}_{25} \mathrm{H}_{23} \mathrm{O}, 339.1747$ found, 339.1749;

IR (film): $v_{\max } 1705,1604,1465,1296,1010,694 \mathrm{~cm}^{-1}$

HPLC: $94 \%$ ee determined by HPLC (OJ-H, 70/30 hexanes/i-PrOH, $1.0 \mathrm{ml} / \mathrm{min}) t r=8.8$ $\min , \operatorname{tr}=15.3 \mathrm{~min}$.

(R)-2-cinnamyl-2-(2-(trifluoromethyl)benzyl)-2,3-dihydro-1H-inden-1-one (4b)<smiles>O=C1c2ccccc2CC1(C/C=C/c1ccccc1)Cc1ccccc1C(F)(F)F</smiles>

Opt. Rot.: $[\alpha]^{26}{ }_{\mathrm{D}}\left(c\right.$ 1.57, $\left.\mathrm{CHCl}_{3}\right): 1.57$;

${ }^{1}$ H NMR: (400 MHz, MeOD) $\delta 7.74(\mathrm{~d}, J=8.0 \mathrm{~Hz}, 1 \mathrm{H}), 7.67-7.61(\mathrm{~m}, 1 \mathrm{H}), 7.58-7.51(\mathrm{~m}$, 1H), $7.41-7.11(\mathrm{~m}, 10 \mathrm{H}), 6.45(\mathrm{~d}, J=16.0 \mathrm{~Hz}, 1 \mathrm{H}), 6.03-5.93(\mathrm{~m}, 1 \mathrm{H}), 3.51(\mathrm{~d}, J=16.0$ $\mathrm{Hz}, 1 \mathrm{H}), 3.30-3.23(\mathrm{~m}, 1 \mathrm{H}), 3.11(\mathrm{~d}, J=16.0 \mathrm{~Hz}, 1 \mathrm{H}), 2.80(\mathrm{~d}, J=16.0 \mathrm{~Hz}, 1 \mathrm{H}), 2.63-$ $2.48(\mathrm{~m}, 2 \mathrm{H})$;

${ }^{13}$ C NMR: (100 MHz, MeOD) $\delta$ 211.2, 153.6, 137.1, 136.3, 135.2, 133.8, 131.6, 130.8, $128.0,127.2,126.9,126.6,126.2,125.7,125.6,124.0,123.2,54.4,42.9,36.4,35.0$;

${ }^{19}$ F NMR: $\left(376 \mathrm{MHz}, \mathrm{CDCl}_{3}\right) \delta-59.3$;

HRMS: (ESI) $[\mathrm{M}+\mathrm{H}]^{+}$calcd. for $\mathrm{C}_{26} \mathrm{H}_{22} \mathrm{OF}_{3}, 407.1627$ found, 407.1623;

IR (film): $v_{\max } 1705,1604,1458,1311,1118,694 \mathrm{~cm}^{-1}$

HPLC: 95\% ee determined by HPLC (OJ-H, 95/5 hexanes/i-PrOH, $1.0 \mathrm{ml} / \mathrm{min}) t r=8.1 \mathrm{~min}$, $\operatorname{tr}=10.0 \mathrm{~min}$.

(S)-2-cinnamyl-2-(3-(trifluoromethyl)benzyl)-2,3-dihydro-1H-inden-1-one (4c)

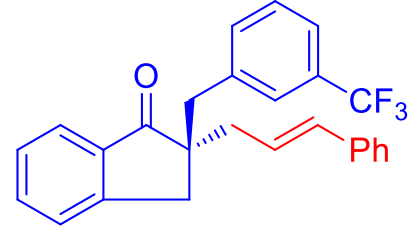

Opt. Rot.: $[\alpha]^{26}\left(c 1.85, \mathrm{CHCl}_{3}\right): 0.7$;

${ }^{1}$ H NMR: (400 MHz, MeOD) $\delta 7.62(\mathrm{~d}, J=8.0 \mathrm{~Hz}, 1 \mathrm{H}), 7.54-7.47(\mathrm{~m}, 1 \mathrm{H}), 7.43-7.11(\mathrm{~m}$, 
$11 \mathrm{H}), 6.46(\mathrm{~d}, J=16.0 \mathrm{~Hz}, 1 \mathrm{H}), 6.07-5.97(\mathrm{~m}, 1 \mathrm{H}), 3.25(\mathrm{~d}, J=16.0 \mathrm{~Hz}, 1 \mathrm{H}), 3.15(\mathrm{~m}, 1 \mathrm{H})$, $2.99(\mathrm{~d}, J=16.0 \mathrm{~Hz}, 1 \mathrm{H}), 2.67(\mathrm{ddd}, J=4.0,8.0,16.0 \mathrm{~Hz}, 1 \mathrm{H}), 2.53(\mathrm{ddd}, J=4.0,8.0,16.0$ $\mathrm{Hz}, 1 \mathrm{H})$;

${ }^{13}$ C NMR: (100 MHz, MeOD) $\delta$ 210.8, 153.3, 138.4, 137.1, 136.6, 135.1, 133.7, 133.5, $130.0,128.3,128.0,127.1,126.9,126.3,126.2,126.1,125.7,124.2,123.0,122.9,122.8$, 54.1, 42.0, 41.5, 35.1;

${ }^{19}$ F NMR: $\left(376 \mathrm{MHz}, \mathrm{CDCl}_{3}\right) \delta-64.1$;

HRMS: (ESI) $[\mathrm{M}+\mathrm{H}]^{+}$calcd. for $\mathrm{C}_{26} \mathrm{H}_{22} \mathrm{OF}_{3}, 407.1622$ found, 407.1623;

IR (film): $v_{\max } 1705,1604,1450,1327,1126,702 \mathrm{~cm}^{-1}$

HPLC: $93 \%$ ee determined by HPLC (OJ-H, 99/1 hexanes/i-PrOH, $1.0 \mathrm{ml} / \mathrm{min}) t r=8.1 \mathrm{~min}$, $\operatorname{tr}=14.8 \mathrm{~min}$.

(S)-2-cinnamyl-2-(4-(trifluoromethyl)benzyl)-2,3-dihydro-1H-inden-1-one (4d)

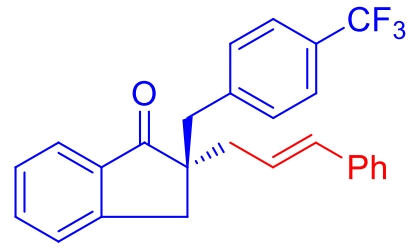

Opt. Rot.: $[\alpha]^{26}{ }_{\mathrm{D}}\left(c \mathrm{1} 1.66, \mathrm{CHCl}_{3}\right): 2.6$;

${ }^{1}$ H NMR: (400 MHz, MeOD) $\delta 7.64(\mathrm{~d}, J=8.0 \mathrm{~Hz}, 1 \mathrm{H}), 7.56-7.49(\mathrm{~m}, 1 \mathrm{H}), 7.46$ - 7.41 (m, 2H), $7.38-7.28(\mathrm{~m}, 4 \mathrm{H}), 7.23-7.11(\mathrm{~m}, 5 \mathrm{H}), 6.45(\mathrm{~d}, J=16.0 \mathrm{~Hz}, 1 \mathrm{H}), 6.05-5.95(\mathrm{~m}, 1 \mathrm{H})$, $3.25(\mathrm{~d}, J=16.0 \mathrm{~Hz}, 1 \mathrm{H}), 3.16(\mathrm{dd}, J=4.0,16.0 \mathrm{~Hz}, 2 \mathrm{H}), 2.98$ (d, $J=16.0 \mathrm{~Hz}, 1 \mathrm{H}), 2.66$ (ddd, $J=4.0,8.0,16.0 \mathrm{~Hz}, 1 \mathrm{H}), 2.52(\mathrm{ddd}, J=4.0,8.0,16.0 \mathrm{~Hz}, 1 \mathrm{H})$;

${ }^{13}$ C NMR: $\left(100 \mathrm{MHz}, \mathrm{CDCl}_{3}\right) \delta 209.5,152.6,141.5,137.0,136.4,135.0,133.9,130.4$, $128.4,127.5,127.3,126.3,126.1,125.0,124.9,124.7,123.9,54.2,42.2,41.8,35.6$;

${ }^{19}$ F NMR: $\left(376 \mathrm{MHz}, \mathrm{CDCl}_{3}\right) \delta-62.4$;

HRMS: (ESI) $[\mathrm{M}+\mathrm{H}]^{+}$calcd. for $\mathrm{C}_{26} \mathrm{H}_{22} \mathrm{OF}_{3}, 407.1622$ found, 407.1623;

IR (film): $v_{\max } 1705,1612,1465,1327,1118,694 \mathrm{~cm}^{-1}$

HPLC: $95 \%$ ee determined by HPLC (OD-H, 99/1 hexanes/i-PrOH, $1.0 \mathrm{ml} / \mathrm{min}) t r=34.9$ $\min , t r=41.1 \mathrm{~min}$.

(S)-2-(3-bromobenzyl)-2-cinnamyl-2,3-dihydro-1H-inden-1-one (4e) 


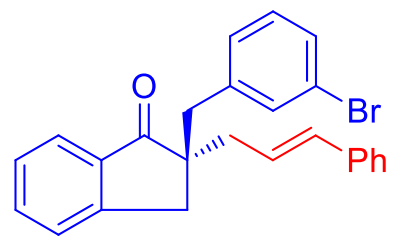

Opt. Rot.: $[\alpha]^{26}{ }_{\mathrm{D}}\left(c\right.$ 1.46, $\left.\mathrm{CHCl}_{3}\right)$ : 11.8;

${ }^{1}$ H NMR: (400 MHz, MeOD) $\delta 7.65(\mathrm{~d}, J=8.0 \mathrm{~Hz}, 1 \mathrm{H}), 7.57-7.49(\mathrm{~m}, 1 \mathrm{H}), 7.39-7.27$ (m, 3H), $7.26-7.00(\mathrm{~m}, 8 \mathrm{H}), 6.43(\mathrm{~d}, J=16.0 \mathrm{~Hz}, 1 \mathrm{H}), 6.05-5.93(\mathrm{~m}, 1 \mathrm{H}), 3.21-3.08(\mathrm{~m}$, $3 \mathrm{H}), 2.87(\mathrm{~d}, J=16.0 \mathrm{~Hz}, 1 \mathrm{H}), 2.64(\mathrm{ddd}, J=4.0,8.0,16.0 \mathrm{~Hz}, 1 \mathrm{H}), 2.50(\mathrm{ddd}, J=4.0,8.0$, $16.0 \mathrm{~Hz}, 1 \mathrm{H})$;

${ }^{13}$ C NMR: (100 MHz, MeOD) $\delta$ 210.9, 153.4, 139.8, 137.1, 136.6, 135.1, 133.6, 132.7, $129.3,129.2$, 128.6, 128.0, 127.1, 126.8, 126.1, 125.6, 124.2, 123.0, 121.5, 54.1, 41.8, 41.6, 35.1 ;

HRMS: (ESI) $[\mathrm{M}+\mathrm{H}]^{+}$calcd. for $\mathrm{C}_{25} \mathrm{H}_{22} \mathrm{OB}_{\mathrm{r},} 417.0844$ found, 417.0854;

IR (film): $v_{\max } 1705,1604,1465,1296,694 \mathrm{~cm}^{-1}$

HPLC: $99 \%$ ee determined by HPLC (IA, 98/2 hexanes/i-PrOH, $1.0 \mathrm{ml} / \mathrm{min}$ ) $t r=11.6 \mathrm{~min}, t r$ $=18.2 \mathrm{~min}$.

(R)-2-cinnamyl-2-(thiophen-3-ylmethyl)-2,3-dihydro-1H-inden-1-one (4f)

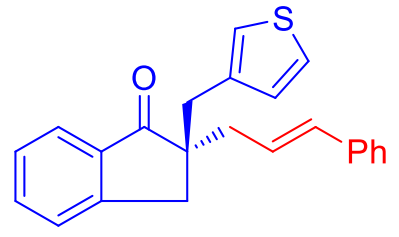

Opt. Rot.: $[\alpha]^{26}{ }_{\mathrm{D}}\left(c\right.$ 2.31, $\left.\mathrm{CHCl}_{3}\right): 5.17$;

${ }^{1}$ H NMR: (400 MHz, MeOD) $\delta 7.65(\mathrm{~d}, J=8.0 \mathrm{~Hz}, 1 \mathrm{H}), 7.58-7.51(\mathrm{~m}, 1 \mathrm{H}), 7.41-7.28(\mathrm{~m}$, 2H), $7.23-7.10(\mathrm{~m}, 6 \mathrm{H}), 7.04-6.98(\mathrm{~m}, 1 \mathrm{H}), 6.85-6.79(\mathrm{~m}, 1 \mathrm{H}), 6.43(\mathrm{~d}, J=16.0 \mathrm{~Hz}$, $1 \mathrm{H}), 6.04-5.93(\mathrm{~m}, 1 \mathrm{H}), 3.24-3.07(\mathrm{~m}, 3 \mathrm{H}), 2.97(\mathrm{~d}, J=16.0 \mathrm{~Hz}, 1 \mathrm{H}), 2.62(\mathrm{ddd}, J=4.0$, 8.0, 16.0 Hz, 1H), 2.50 (ddd, $J=4.0,8.0,16.0 \mathrm{~Hz}, 1 \mathrm{H}$ );

${ }^{13}$ C NMR: (100 MHz, MeOD) $\delta 211.5,153.7,137.3,137.1,136.6,135.0,133.4,128.8$, 128.0, 127.0, 126.8, 126.2, 125.6, 124.6, 124.4, 123.0, 122.5, 54.0, 41.5, 36.6, 35.6;

HRMS: (ESI) $[\mathrm{M}+\mathrm{H}]^{+}$calcd. for $\mathrm{C}_{23} \mathrm{H}_{21} \mathrm{OS}, 345.1313$ found, 345.1313;

IR (film): $v_{\max } 1705,1604,1465,964,694 \mathrm{~cm}^{-1}$

HPLC: $89 \%$ ee determined by HPLC (OJ-H, 80/20 hexanes/i-PrOH, $1.0 \mathrm{ml} / \mathrm{min})$ tr $=11.8$ $\min , t r=22.3 \mathrm{~min}$. 
(R)-2-cinnamyl-2-(furan-2-ylmethyl)-2,3-dihydro-1H-inden-1-one (4g)

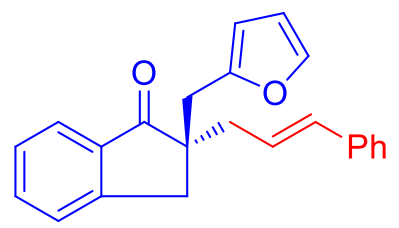

Opt. Rot.: $[\alpha]^{26}{ }_{\mathrm{D}}\left(c\right.$ 1.23, $\left.\mathrm{CHCl}_{3}\right): 5.18$;

${ }^{1}$ H NMR: (400 MHz, MeOD) $\delta 7.67(\mathrm{~d}, J=8.0 \mathrm{~Hz}, 1 \mathrm{H}), 7.62-7.55(\mathrm{~m}, 1 \mathrm{H}), 7.45-7.32$ (m, 2H), $7.23-7.11(\mathrm{~m}, 6 \mathrm{H}), 6.43(\mathrm{~d}, J=16.0 \mathrm{~Hz}, 1 \mathrm{H}), 6.20-6.17(\mathrm{~m}, 1 \mathrm{H}), 6.06-5.92(\mathrm{~m}, 2 \mathrm{H})$, $3.24(\mathrm{~d}, J=16.0 \mathrm{~Hz}, 1 \mathrm{H}), 3.15(\mathrm{~d}, J=16.0 \mathrm{~Hz}, 1 \mathrm{H}), 3.08$ (d, $J=16.0 \mathrm{~Hz}, 1 \mathrm{H}), 3.00(\mathrm{~d}, J=$ $16.0 \mathrm{~Hz}, 1 \mathrm{H}), 2.61$ (ddd, $J=4.0,8.0,16.0 \mathrm{~Hz}, 1 \mathrm{H}), 2.49$ (ddd, $J=4.0,8.0,16.0 \mathrm{~Hz}, 1 \mathrm{H})$;

${ }^{13}$ C NMR: (100 MHz, MeOD) $\delta 211.5,153.4,151.9,141.2,137.1,136.2,135.0,133.6$, $128.0,127.1,126.8,126.2,125.6,124.2,123.1,109.6,107.4,53.1,41.0,36.0,34.5$;

HRMS: (ESI) $[\mathrm{M}+\mathrm{H}]^{+}$calcd. for $\mathrm{C}_{23} \mathrm{H}_{21} \mathrm{O}_{2}, 329.1547$ found, 329.1542;

IR (film): $v_{\max } 1705,1604,1465,1296,1010,694 \mathrm{~cm}^{-1}$

HPLC: $85 \%$ ee determined by HPLC (OD-H, 98/2 hexanes/i-PrOH, $1.0 \mathrm{ml} / \mathrm{min}) t r=10.5$ $\min , \operatorname{tr}=13.0 \mathrm{~min}$.

(S)-2-(3-bromobenzyl)-2-(3-methylbut-2-en-1-yl)-2,3-dihydro-1H-inden-1-one (4h)

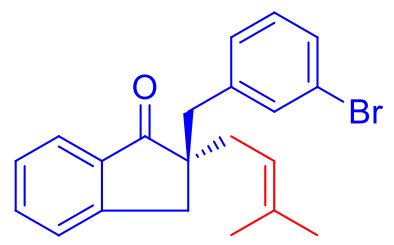

Opt. Rot.: $[\alpha]^{26}{ }_{\mathrm{D}}\left(c\right.$ 2.39, $\left.\mathrm{CHCl}_{3}\right): 1.58$;

${ }^{1}$ H NMR: $\left(400 \mathrm{MHz}, \mathrm{CDCl}_{3}\right) \delta 7.74-7.66(\mathrm{~m}, 1 \mathrm{H}), 7.54-7.46(\mathrm{~m}, 1 \mathrm{H}), 7.34-7.21(\mathrm{~m}$, 4H), $7.05-6.96(\mathrm{~m}, 2 \mathrm{H}), 5.06-4.99(\mathrm{~m}, 1 \mathrm{H}), 3.10(\mathrm{~d}, J=16.0 \mathrm{~Hz}, 1 \mathrm{H}), 3.04(\mathrm{~d}, J=16.0$ $\mathrm{Hz}, 1 \mathrm{H}), 2.94(\mathrm{~d}, J=16.0 \mathrm{~Hz}, 1 \mathrm{H}), 2.80(\mathrm{~d}, J=16.0 \mathrm{~Hz}, 1 \mathrm{H}), 2.44-2.29(\mathrm{~m}, 2 \mathrm{H}), 1.63$ (s, $3 \mathrm{H}), 1.61(\mathrm{~s}, 3 \mathrm{H})$;

${ }^{13}$ C NMR: $\left(100 \mathrm{MHz}, \mathrm{CDCl}_{3}\right) \delta 210.1,152.9,139.9,136.7,135.2,134.7,133.0,129.5$, $129.4,128.7,127.2,126.2,123.8,122.0,118.7,54.3,41.8,36.3,35.6,25.9,18.0$;

HRMS: (ESI) $[\mathrm{M}+\mathrm{H}]^{+}$calcd. for $\mathrm{C}_{21} \mathrm{H}_{22} \mathrm{OB}$, 369.0856 found, 369.0854;

IR (film): $v_{\max } 1705,1604,1465,1296,1010,694 \mathrm{~cm}^{-1}$

HPLC: $91 \%$ ee determined by HPLC (OJ-H, 99/1 hexanes/i-PrOH, $1.0 \mathrm{ml} / \mathrm{min}) t r=7.9 \mathrm{~min}$, $\operatorname{tr}=8.9 \mathrm{~min}$. 
(R)-2-(but-2-yn-1-yl)-2-(thiophen-3-ylmethyl)-2,3-dihydro-1H-inden-1-one (4i)

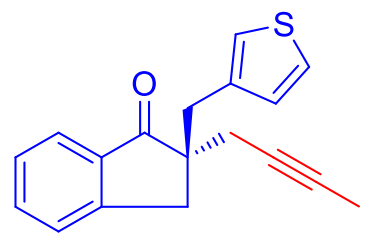

Opt. Rot.: $[\alpha]^{26}{ }_{\mathrm{D}}\left(c \mathrm{1} 1.07, \mathrm{CHCl}_{3}\right):-16.9$;

${ }^{1}$ H NMR: (400 MHz, MeOD) $\delta 7.68$ - $7.54(\mathrm{~m}, 2 \mathrm{H}), 7.45-7.38(\mathrm{~m}, 1 \mathrm{H}), 7.37-7.31$ (m, 1H), $7.17-7.12(\mathrm{~m}, 1 \mathrm{H}), 7.02-6.97(\mathrm{~m}, 1 \mathrm{H}), 6.82-6.76(\mathrm{~m}, 1 \mathrm{H}), 3.05(\mathrm{~d}, J=16.0 \mathrm{~Hz}$, $1 \mathrm{H}), 2.98(\mathrm{~d}, J=16.0 \mathrm{~Hz}, 1 \mathrm{H}), 2.54-2.36(\mathrm{~m}, 2 \mathrm{H}), 1.58(\mathrm{t}, J=2.4 \mathrm{~Hz}, 3 \mathrm{H})$;

${ }^{13}$ C NMR: (100 MHz, MeOD) $\delta$ 210.5, 153.8, 137.0, 136.4, 134.9, 128.7, 126.9, 126.1, 124.6, 123.1, 122.6, 77.3, 74.3, 53.0, 36.2, 27.0, 1.4;

HRMS: (ESI) $[\mathrm{M}+\mathrm{H}]^{+}$calcd. for $\mathrm{C}_{18} \mathrm{H}_{17} \mathrm{OS}, 281.0998$ found, 281.1000;

IR (film): $v_{\max } 1705,1604,1465,1296,1010,694 \mathrm{~cm}^{-1}$

HPLC: 95\% ee determined by HPLC (OD-H, 99/1 hexanes/i-PrOH, $1.0 \mathrm{ml} / \mathrm{min}$ ) $t r=10.7$ $\min , \operatorname{tr}=11.7 \mathrm{~min}$.

(R)-2-(but-2-yn-1-yl)-2-(furan-2-ylmethyl)-2,3-dihydro-1H-inden-1-one (4j)

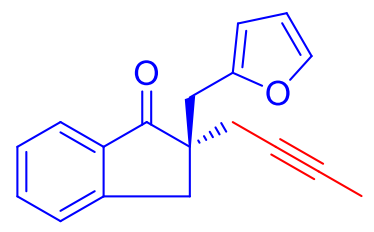

Opt. Rot.: $[\alpha]^{26}{ }_{\mathrm{D}}\left(c 1.29, \mathrm{CHCl}_{3}\right):-2.9$;

${ }^{1}$ H NMR: (400 MHz, MeOD) $\delta 7.69$ - $7.56(\mathrm{~m}, 2 \mathrm{H}), 7.48$ - $7.43(\mathrm{~m}, 1 \mathrm{H}), 7.40-7.34(\mathrm{~m}$, 1H), $7.19-7.17(\mathrm{~m}, 1 \mathrm{H}), 6.17-6.15(\mathrm{~m}, 1 \mathrm{H}), 6.01-5.99(\mathrm{~m}, 1 \mathrm{H}), 3.23(\mathrm{~m}, 1 \mathrm{H}), 2.99$ (m, $1 \mathrm{H}), 2.52-2.35(\mathrm{~m}, 2 \mathrm{H}), 1.56(\mathrm{t}, J=2.4 \mathrm{~Hz}, 3 \mathrm{H})$;

${ }^{13}$ C NMR: (100 MHz, MeOD) $\delta$ 209.6, 153.7, 151.6, 141.3, 135.9, 135.0, 127.0, 126.1, 123.2, 109.6, 107.5, 77.3, 74.1, 36.4, 34.2, 26.6, 1.4;

HRMS: (ESI) $[\mathrm{M}+\mathrm{H}]^{+}$calcd. for $\mathrm{C}_{18} \mathrm{H}_{17} \mathrm{O}_{2}, 265.1226$ found, 265.1229;

IR (film): $v_{\max } 1705,1604,1465,1296,1010,694 \mathrm{~cm}^{-1}$

HPLC: $96 \%$ ee determined by HPLC (OD-H, 99/1 hexanes/i-PrOH, $1.0 \mathrm{ml} / \mathrm{min}) t r=9.1$ $\min , t r=9.9 \mathrm{~min}$.

(S)-methyl 2-(1-oxo-2-(3-(trifluoromethyl)benzyl)-2,3-dihydro-1H-inden-2-yl)acetate $(4 k)$ 


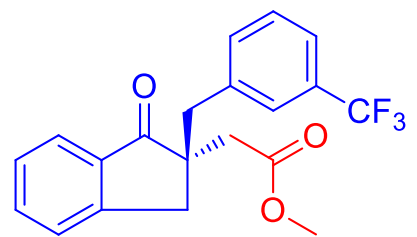

Opt. Rot:: $[\alpha]^{26}{ }_{\mathrm{D}}\left(c 1.31, \mathrm{CHCl}_{3}\right): 0.4$;

${ }^{1}$ H NMR: $\left(400 \mathrm{MHz}, \mathrm{CDCl}_{3}\right) \delta 7.73(\mathrm{~d}, J=8.0 \mathrm{~Hz}, 1 \mathrm{H}), 7.53-7.47(\mathrm{~m}, 1 \mathrm{H}), 7.41-7.25(\mathrm{~m}$, $6 \mathrm{H}), 3.59(\mathrm{~s}, 3 \mathrm{H}), 3.25-3.12(\mathrm{~m}, 2 \mathrm{H}), 3.06(\mathrm{~d}, J=16.0 \mathrm{~Hz}, 1 \mathrm{H}), 2.93(\mathrm{~d}, J=16.0 \mathrm{~Hz}, 1 \mathrm{H})$, $2.92(\mathrm{~d}, J=16.0 \mathrm{~Hz}, 1 \mathrm{H}), 2.71(\mathrm{~d}, J=16.0 \mathrm{~Hz}, 1 \mathrm{H})$;

${ }^{13}$ C NMR: $\left(100 \mathrm{MHz}, \mathrm{CDCl}_{3}\right) \delta 208.2,171.4,152.1,137.1,136.2,134.9,133.5,128.4$, $127.5,126.7,126.1,124.0,123.6,123.5,51.6,51.1,42.9,40.6,36.6$;

${ }^{19}$ F NMR: $\left(376 \mathrm{MHz}, \mathrm{CDCl}_{3}\right) \delta-62.7$;

HRMS: (ESI) $[\mathrm{M}+\mathrm{H}]^{+}$calcd. for $\mathrm{C}_{20} \mathrm{H}_{18} \mathrm{O}_{3} \mathrm{~F}_{3}, 363.1207$ found, 363.1208;

IR (film): $v_{\max } 1712,1705,1604,1465,1311,1118,1010,694 \mathrm{~cm}^{-1}$

HPLC: $90 \%$ ee determined by HPLC (OD-H, 90/10 hexanes/i-PrOH, $1.0 \mathrm{ml} / \mathrm{min}$ ) $t r=6.7$ $\min , t r=8.0 \mathrm{~min}$.

(S)-methyl 2-(1-oxo-2-(4-(trifluoromethyl)benzyl)-2,3-dihydro-1H-inden-2-yl)acetate (4I)<smiles>COC(=O)C[C@]1(Cc2ccc(C(F)(F)F)cc2)Cc2ccccc2C1=O</smiles>

Opt. Rot.: $[\alpha]^{26}\left(c 1.48, \mathrm{CHCl}_{3}\right): 1.2$;

${ }^{1}$ H NMR: $\left(400 \mathrm{MHz}, \mathrm{CDCl}_{3}\right) \delta 7.77-7.72(\mathrm{~m}, 1 \mathrm{H}), 7.56-7.50(\mathrm{~m}, 1 \mathrm{H}), 7.47-7.39(\mathrm{~m}$, 2H), $7.37-7.31(\mathrm{~m}, 2 \mathrm{H}), 7.25-7.18(\mathrm{~m}, 2 \mathrm{H}), 3.58(\mathrm{~s}, 3 \mathrm{H}), 3.19(\mathrm{~m}, 2 \mathrm{H}), 3.05(\mathrm{~d}, J=16.0$ $\mathrm{Hz}, 1 \mathrm{H}), 2.93$ (d, $J=16.0 \mathrm{~Hz}, 1 \mathrm{H}), 2.91$ (d, $J=16.0 \mathrm{~Hz}, 1 \mathrm{H}), 2.69$ (d, $J=16.0 \mathrm{~Hz}, 1 \mathrm{H})$;

${ }^{13}$ C NMR: $\left(100 \mathrm{MHz}, \mathrm{CDCl}_{3}\right) \delta 208.1,171.3,152.1,140.4,136.0,134.9,130.5,127.5$, $126.2,125.0,124.9,124.0,51.6,51.1,42.7,40.6,36.6$;

${ }^{19}$ F NMR: $\left(376 \mathrm{MHz}, \mathrm{CDCl}_{3}\right) \delta-62.6$;

HRMS: (ESI) $[\mathrm{M}+\mathrm{H}]^{+}$calcd. for $\mathrm{C}_{20} \mathrm{H}_{18} \mathrm{O}_{3} \mathrm{~F}_{3}, 363.1205$ found, 363.1208;

IR (film): $v_{\max } 1712,1705,1604,1465,1327,1296,1118,1010,694 \mathrm{~cm}^{-1}$

HPLC: $87 \%$ ee determined by HPLC (OD-H, 90/10 hexanes/i-PrOH, $1.0 \mathrm{ml} / \mathrm{min}$ ) $t r=6.9$ $\min , \operatorname{tr}=8.4 \mathrm{~min}$. 
(4m)

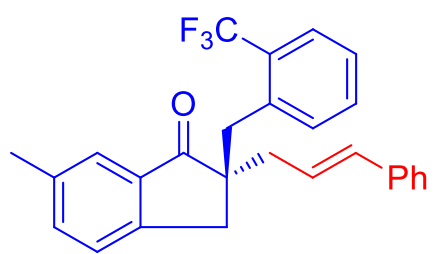

Opt. Rot.: $[\alpha]^{26}{ }_{\mathrm{D}}\left(c \mathrm{2} 2.28, \mathrm{CHCl}_{3}\right):-59.4$;

${ }^{1}$ H NMR: (400 MHz, MeOD) $\delta 7.66-7.60(\mathrm{~m}, 1 \mathrm{H}), 7.56-7.51(\mathrm{~m}, 1 \mathrm{H}), 7.41-7.36(\mathrm{~m}$, 1H), $7.31-7.10(\mathrm{~m}, 9 \mathrm{H}), 6.44(\mathrm{~d}, J=16.0 \mathrm{~Hz}, 1 \mathrm{H}), 6.04-5.92(\mathrm{~m}, 1 \mathrm{H}), 3.49$ (d, $J=16.0$ $\mathrm{Hz}, 1 \mathrm{H}), 3.29-3.21(\mathrm{~m}, 1 \mathrm{H}), 3.05(\mathrm{~d}, J=16.0 \mathrm{~Hz}, 1 \mathrm{H}), 2.73(\mathrm{~d}, J=16.0 \mathrm{~Hz}, 1 \mathrm{H}), 2.58$ (ddd, $J=4.0,8.0,16.0 \mathrm{~Hz}, 1 \mathrm{H}), 2.50(\mathrm{ddd}, J=4.0,8.0,16.0 \mathrm{~Hz}, 1 \mathrm{H}), 2.37$ (s, 3H);

${ }^{13}$ C NMR: (100 MHz, MeOD) $\delta$ 211.3, 151.0, 137.5, 137.1, 137.0, 136.5, 137.4, 133.7, $131.5,130.7,128.7,128.0,126.8,126.5,125.9,125.7,125.6,124.1,123.0,54.7,43.0,36.5$, 34.6, 19.6;

${ }^{19}$ F NMR: (376 MHz, MeOD) $\delta$-59.3;

HRMS: (ESI) $[\mathrm{M}+\mathrm{H}]^{+}$calcd. for $\mathrm{C}_{27} \mathrm{H}_{24} \mathrm{OF}_{3}, 421.1774$ found, 421.1779;

IR (film): $v_{\max } 1705,1604,1465,1311,1118,694 \mathrm{~cm}^{-1}$

HPLC: 98\% ee determined by HPLC (OJ-H, 99/1 hexanes/i-PrOH, $1.0 \mathrm{ml} / \mathrm{min}) \mathrm{tr}=11.6$ $\min , t r=13.6 \mathrm{~min}$.

(S)-2-benzyl-5-chloro-2-cinnamyl-2,3-dihydro-1H-inden-1-one (4n)

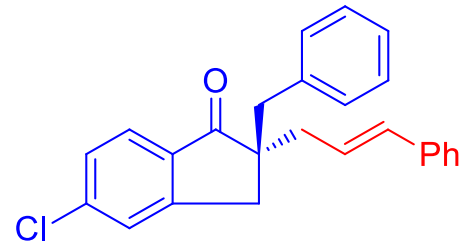

Opt. Rot.: $[\alpha]^{26}{ }_{\mathrm{D}}\left(c \mathrm{1} 1.92, \mathrm{CHCl}_{3}\right):-12.4$;

${ }^{1}$ H NMR: (400 MHz, MeOD) $\delta 7.60-7.55(\mathrm{~m}, 1 \mathrm{H}), 7.37-7.33(\mathrm{~m}, 1 \mathrm{H}), 7.31-7.26(\mathrm{~m}$, 1H), $7.23-7.06(\mathrm{~m}, 10 \mathrm{H}), 6.44(\mathrm{~d}, J=16.0 \mathrm{~Hz}, 1 \mathrm{H}), 6.05-5.93(\mathrm{~m}, 1 \mathrm{H}), 3.23-3.14(\mathrm{~m}$, 2H), $3.09(\mathrm{~d}, J=16.0 \mathrm{~Hz}, 1 \mathrm{H}), 2.87(\mathrm{~d}, J=16.0 \mathrm{~Hz}, 1 \mathrm{H}), 2.65(\mathrm{ddd}, J=4.0,8.0,16.0 \mathrm{~Hz}$, $1 \mathrm{H}), 2.51$ (ddd, $J=4.0,8.0,16.0 \mathrm{~Hz}, 1 \mathrm{H})$;

${ }^{13}$ C NMR: (100 MHz, MeOD) $\delta$ 209.8, 155.1, 141.2, 137.1, 136.9, 135.3, 133.7, 129.7, $128.0,127.8,127.7,126.9,126.3,126.2,125.6,124.3,124.2,54.6,42.4,41.6,34.9$;

${ }^{19}$ F NMR: (376 MHz, MeOD) $\delta$-59.3;

HRMS: (ESI) $[\mathrm{M}+\mathrm{H}]^{+}$calcd. for $\mathrm{C}_{25} \mathrm{H}_{22} \mathrm{OCl}, 373.1353$ found, 373.1359; 
IR (film): $v_{\max } 1705,1597,1496,694 \mathrm{~cm}^{-1}$

HPLC: $92 \%$ ee determined by HPLC (OJ-H, 90/10 hexanes/i-PrOH, $1.0 \mathrm{ml} / \mathrm{min})$ tr $=8.8$ $\min , \operatorname{tr}=12.3 \mathrm{~min}$.

(S)-2-cinnamyl-2-(4-(trifluoromethyl)benzyl)-3,4-dihydronaphthalen-1(2H)-one (40)<smiles>O=C1c2ccccc2CC[C@]1(C/C=C/c1ccccc1)Cc1ccc(C(F)(F)F)cc1</smiles>

Opt. Rot.: $[\alpha]^{26}{ }_{\mathrm{D}}\left(c 1.85, \mathrm{CHCl}_{3}\right): 26.6$;

${ }^{1}$ H NMR: (400 MHz, MeOD) $\delta 8.05-7.99(\mathrm{~m}, 1 \mathrm{H}), 7.57-7.47(\mathrm{~m}, 3 \mathrm{H}), 7.38-7.23(\mathrm{~m}$, $8 \mathrm{H}), 7.22-7.16(\mathrm{~m}, 1 \mathrm{H}), 6.46(\mathrm{~d}, J=16.0 \mathrm{~Hz}, 1 \mathrm{H}), 6.28-6.16(\mathrm{~m}, 1 \mathrm{H}), 3.38(\mathrm{~d}, J=16.0$ $\mathrm{Hz}, 1 \mathrm{H}), 3.19-2.97(\mathrm{~m}, 2 \mathrm{H}), 2.93(\mathrm{~d}, J=16.0 \mathrm{~Hz}, 1 \mathrm{H}), 2.66$ (ddd, $J=4.0,8.0,16.0 \mathrm{~Hz}, 1 \mathrm{H})$, $2.41(\mathrm{ddd}, J=4.0,8.0,16.0 \mathrm{~Hz}, 1 \mathrm{H}), 2.17-2.07(\mathrm{~m}, 1 \mathrm{H}), 2.01-1.92(\mathrm{~m}, 1 \mathrm{H})$;

${ }^{13}$ C NMR: (100 MHz, MeOD) $\delta$ 200.9, 143.4, 142.4, 137.2, 133.7, 133.3, 131.7, 130.9, $128.6,128.2$, 128.1, 127.3, 126.8, 126.4, 126.1, 125.7, 124.6, 124.4, 124.3, 49.7, 40.2, 38.6, 30.4, 24.6;

${ }^{19}$ F NMR: $\left(376 \mathrm{MHz}, \mathrm{CDCl}_{3}\right) \delta-63.8$;

HRMS: (ESI) $[\mathrm{M}+\mathrm{H}]^{+}$calcd. for $\mathrm{C}_{27} \mathrm{H}_{24} \mathrm{OF}_{3}, 421.1780$ found, 421.1779;

IR (film): $v_{\max } 1681,1604,1450,1327,1118,694 \mathrm{~cm}^{-1}$

HPLC: $90 \%$ ee determined by HPLC (OD-H, 98/2 hexanes/i-PrOH, $1.0 \mathrm{ml} / \mathrm{min}$ ) $t r=14.2$ $\min , \operatorname{tr}=15.8 \mathrm{~min}$.

(S)-2-cinnamyl-2-(3-(trifluoromethyl)benzyl)-3,4-dihydronaphthalen-1(2H)-one (4p)<smiles>O=C1c2ccccc2CC[C@]1(C/C=C/c1ccccc1)Cc1ccccc1</smiles>

Opt. Rot.: $[\alpha]^{26}{ }_{\mathrm{D}}\left(c\right.$ 1.82, $\left.\mathrm{CHCl}_{3}\right): 57.3$;

${ }^{1}$ H NMR: (400 MHz, MeOD) $\delta 8.03-7.97(\mathrm{~m}, 1 \mathrm{H}), 7.54-7.14(\mathrm{~m}, 12 \mathrm{H}), 6.44(\mathrm{~d}, J=16.0$ $\mathrm{Hz}, 1 \mathrm{H}), 6.28-6.17(\mathrm{~m}, 1 \mathrm{H}), 3.35(\mathrm{~d}, J=16.0 \mathrm{~Hz}, 1 \mathrm{H}), 3.17-2.94(\mathrm{~m}, 2 \mathrm{H}), 2.91$ (d, $J=$ $16.0 \mathrm{~Hz}, 1 \mathrm{H}), 2.62(\mathrm{ddd}, J=4.0,8.0,16.0 \mathrm{~Hz}, 1 \mathrm{H}), 2.40(\mathrm{ddd}, J=4.0,8.0,16.0 \mathrm{~Hz}, 1 \mathrm{H}), 2.14$ $-2.03(\mathrm{~m}, 1 \mathrm{H}), 2.00-1.89(\mathrm{~m}, 1 \mathrm{H})$;

${ }^{13}$ C NMR: (100 MHz, MeOD) $\delta$ 200.9,143.4, 139.0, 137.2, 134.1, 133.7, 133.3, 131.7, 
$130.0,129.7,128.6,128.3,128.1,127.2,126.9,126.4,125.7,124.6,122.8,122.7,49.6,40.2$, $38.5,30.4,24.5$;

${ }^{19}$ F NMR: $\left(376 \mathrm{MHz}, \mathrm{CDCl}_{3}\right) \delta-64.0$;

HRMS: (ESI) $[\mathrm{M}+\mathrm{H}]^{+}$calcd. for $\mathrm{C}_{27} \mathrm{H}_{24} \mathrm{OF}_{3}, 421.1780$ found, 421.1779;

IR (film): $v_{\max } 1681,1604,1450,1327,1126,694 \mathrm{~cm}^{-1}$

HPLC: $91 \%$ ee determined by HPLC (OJ-H, 90/10 hexanes/i-PrOH, $1.0 \mathrm{ml} / \mathrm{min}) t r=7.9$ $\min , t r=9.9 \min$.

(S)-2-cinnamyl-2-(4-fluorobenzyl)-3,4-dihydronaphthalen-1(2H)-one (4q)<smiles>O=C1c2ccccc2CC[C@]1(C/C=C/c1ccccc1)Cc1ccc(F)cc1</smiles>

Opt. Rot.: $[\alpha]^{26}{ }_{\mathrm{D}}\left(c\right.$ 1.82, $\left.\mathrm{CHCl}_{3}\right): 3.9$;

${ }^{1}$ H NMR: (400 MHz, MeOD) $\delta 8.03-7.97(\mathrm{~m}, 1 \mathrm{H}), 7.53-7.47(\mathrm{~m}, 1 \mathrm{H}), 7.36-7.10(\mathrm{~m}$, $9 \mathrm{H}), 6.98-6.90(\mathrm{~m}, 2 \mathrm{H}), 6.42(\mathrm{~d}, J=16.0 \mathrm{~Hz}, 1 \mathrm{H}), 6.26-6.16(\mathrm{~m}, 1 \mathrm{H}), 3.25(\mathrm{~d}, J=16.0$ $\mathrm{Hz}, 1 \mathrm{H}), 3.14-2.91(\mathrm{~m}, 2 \mathrm{H}), 2.81$ (d, $J=16.0 \mathrm{~Hz}, 1 \mathrm{H}), 2.64(\mathrm{ddd}, J=4.0,8.0,16.0 \mathrm{~Hz}, 1 \mathrm{H})$, $2.35(\mathrm{ddd}, J=4.0,8.0,16.0 \mathrm{~Hz}, 1 \mathrm{H}), 2.13-2.02(\mathrm{~m}, 1 \mathrm{H}), 2.00-1.91(\mathrm{~m}, 1 \mathrm{H})$;

${ }^{13}$ C NMR: (100 MHz, MeOD) $\delta$ 201.3, 143.5, 137.3, 133.5, 133.4, 133.3, 132.0, 131.9, $131.8,128.6,128.1,127.2,126.8,126.4,125.7,124.8,114.2,114.0,49.6,39.7,38.7,30.3$, 24.6;

${ }^{19}$ F NMR: $\left(376 \mathrm{MHz}, \mathrm{CDCl}_{3}\right) \delta-118.9$;

HRMS: (ESI) $[\mathrm{M}+\mathrm{H}]^{+}$calcd. for $\mathrm{C}_{26} \mathrm{H}_{24} \mathrm{OF}, 371.1809$ found, 371.1811;

IR (film): $v_{\max } 1674,1604,1450,1226,694 \mathrm{~cm}^{-1}$

HPLC: $90 \%$ ee determined by HPLC (IA, 98/2 hexanes/i-PrOH, $1.0 \mathrm{ml} / \mathrm{min}$ ) $t r=10.8 \mathrm{~min}$, $\operatorname{tr}=12.5 \mathrm{~min}$.

(S)-2-(3-methylbut-2-en-1-yl)-2-(4-(trifluoromethyl)benzyl)-3,4-dihydronaphthalen1(2H)-one (4r) 


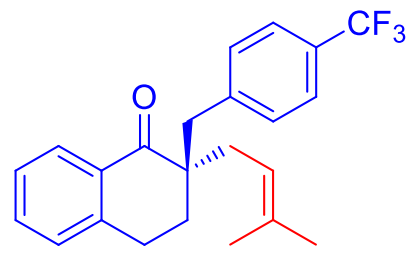

Opt. Rot.: $[\alpha]^{26}\left(c 1.89, \mathrm{CHCl}_{3}\right): 1.8$;

${ }^{1}$ H NMR: (400 MHz, MeOD) $\delta 8.02$ - $7.94(\mathrm{~m}, 1 \mathrm{H}), 7.56$ - $7.46(\mathrm{~m}, 3 \mathrm{H}), 7.37$ - 7.22 (m, 4H), $5.25-5.18(\mathrm{~m}, 1 \mathrm{H}), 3.32(\mathrm{~d}, J=16.0 \mathrm{~Hz}, 1 \mathrm{H}), 3.15-2.92(\mathrm{~m}, 2 \mathrm{H}), 2.85(\mathrm{~d}, J=16.0$ $\mathrm{Hz}, 1 \mathrm{H}), 2.37$ (ddd, $J=4.0,8.0,16.0 \mathrm{~Hz}, 1 \mathrm{H}), 2.28$ (ddd, $J=4.0,8.0,16.0 \mathrm{~Hz}, 1 \mathrm{H}), 2.11-$ $2.01(\mathrm{~m}, 1 \mathrm{H}), 1.95-1.86(\mathrm{~m}, 1 \mathrm{H}), 1.73(\mathrm{~s}, 3 \mathrm{H}), 1.57$ (s, 3H);

${ }^{13}$ C NMR: (100 MHz, MeOD) $\delta$ 201.2, 143.3, 142.6, 134.6, 133.2, 131.8, 130.9, 128.5, $127.2,126.3,124.3,124.2,118.6,49.8,39.9,33.2,30.2,24.7,24.6,16.7$;

${ }^{19}$ F NMR: $\left(376 \mathrm{MHz}, \mathrm{CDCl}_{3}\right) \delta-63.8$;

HRMS: (ESI) $[\mathrm{M}+\mathrm{H}]^{+}$calcd. for $\mathrm{C}_{23} \mathrm{H}_{24} \mathrm{OF}_{3}, 373.1777$ found, 373.1779;

IR (film): $v_{\max } 1681,1604,1450,1327,1165,1118,694 \mathrm{~cm}^{-1}$

HPLC: $92 \%$ ee determined by HPLC (OJ-H, 85/15 hexanes/i-PrOH, $1.0 \mathrm{ml} / \mathrm{min})$ tr $=11.5$ $\min , \operatorname{tr}=22.3 \mathrm{~min}$.

(R)-2-(4-bromobenzyl)-2-(but-2-yn-1-yl)-3,4-dihydronaphthalen-1(2H)-one (4s)<smiles>CC#CC[C@]1(Cc2ccc(Br)cc2)CCc2ccccc2C1=O</smiles>

Opt. Rot.: $[\alpha]^{26}{ }_{\mathrm{D}}\left(c 1.21, \mathrm{CHCl}_{3}\right):-6.1$;

${ }^{1}$ H NMR: (400 MHz, MeOD) $\delta 7.97$ - $7.92(\mathrm{~m}, 1 \mathrm{H}), 7.56$ - $7.49(\mathrm{~m}, 1 \mathrm{H}), 7.40-7.29$ (m, 4H), $7.04-6.98(\mathrm{~m}, 2 \mathrm{H}), 3.19-2.98(\mathrm{~m}, 3 \mathrm{H}), 2.95$ (d, J = 16.0 Hz, 1H), $2.43-2.28$ (m, 2H), $2.26-2.09(\mathrm{~m}, 2 \mathrm{H}), 1.79(\mathrm{t}, J=2.4 \mathrm{~Hz}, 3 \mathrm{H})$;

${ }^{13}$ C NMR: (100 MHz, MeOD) $\delta$ 200.4, 143.3, 136.3, 133.3, 131.9, 131.6, 130.6, 128.7, $127.1,126.4,120.0,78.6,74.5,48.8,38.5,30.8,24.7,24.5,1.6$;

HRMS: (ESI) $[\mathrm{M}+\mathrm{H}]^{+}$calcd. for $\mathrm{C}_{21} \mathrm{H}_{20} \mathrm{OB}_{\mathrm{r}}, 367.0699$ found, 367.0698;

IR (film): $v_{\max } 1681,1604,1450,1288,1010,694 \mathrm{~cm}^{-1}$

HPLC: $87 \%$ ee determined by HPLC (OJ-H, 98/2 hexanes/i-PrOH, $1.0 \mathrm{ml} / \mathrm{min}) t r=9.2 \mathrm{~min}$, $\operatorname{tr}=12.4 \mathrm{~min}$.

(S)-2-(but-2-yn-1-yl)-2-(thiophen-3-ylmethyl)-3,4-dihydronaphthalen-1(2H)-one (4t) 


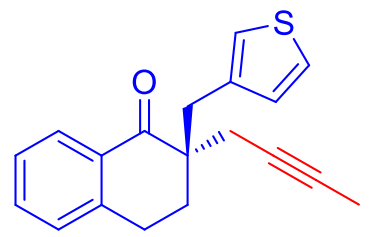

Opt. Rot.: $[\alpha]^{26}\left(c 1.37, \mathrm{CHCl}_{3}\right): 5.2$;

${ }^{1}$ H NMR: (400 MHz, MeOD) $\delta 7.67-7.61(\mathrm{~m}, 1 \mathrm{H}), 7.60-7.54(\mathrm{~m}, 1 \mathrm{H}), 7.44-7.39(\mathrm{~m}$, 1H), $7.37-7.31(\mathrm{~m}, 1 \mathrm{H}), 7.17-7.12(\mathrm{~m}, 2 \mathrm{H}), 7.02-6.96(\mathrm{~m}, 1 \mathrm{H}), 6.81-6.76(\mathrm{~m}, 1 \mathrm{H}), 3.20$ $(\mathrm{m}, 2 \mathrm{H}), 3.05(\mathrm{~d}, J=16.0 \mathrm{~Hz}, 1 \mathrm{H}), 2.98(\mathrm{~d}, J=16.0 \mathrm{~Hz}, 1 \mathrm{H}), 2.53-2.37(\mathrm{~m}, 2 \mathrm{H}), 1.58(\mathrm{t}, J=$ $2.4 \mathrm{~Hz}, 3 \mathrm{H})$;

${ }^{13}$ C NMR: (100 MHz, MeOD) $\delta$ 210.5, 153.8, 137.0, 136.4, 134.9, 128.7, 126.9, 126.1, 124.6, 123.1, 122.6, 77.3, 74.3, 53.0, 36.2, 27.0, 1.4;

HRMS: (ESI) $[\mathrm{M}+\mathrm{H}]^{+}$calcd. for $\mathrm{C}_{19} \mathrm{H}_{19} \mathrm{OS}, 295.1157$ found, 295.1157;

IR (film): $v_{\max } 1674,1604,1450,1288,1010,694 \mathrm{~cm}^{-1}$

HPLC: $92 \%$ ee determined by HPLC (OJ-H, 99/1 hexanes/i-PrOH, $1.0 \mathrm{ml} / \mathrm{min}) t r=14.5$ $\min , t r=28.3 \mathrm{~min}$.

(R)-methyl 2-(1-oxo-2-(4-(trifluoromethyl)benzyl)-1,2,3,4-tetrahydronaphthalen-2yl)acetate $(4 u)$<smiles>COC(=O)C[C@]1(Cc2ccc(C(F)(F)F)cc2)CCc2ccccc2C1=O</smiles>

Opt. Rot:: $[\alpha]^{26}{ }_{\mathrm{D}}\left(\mathrm{c} 1.72, \mathrm{CHCl}_{3}\right): 18.7$;

${ }^{1}$ H NMR: (400 MHz, MeOD) $\delta 8.04-7.97(\mathrm{~m}, 1 \mathrm{H}), 7.61-7.53(\mathrm{~m}, 3 \mathrm{H}), 7.42-7.31(\mathrm{~m}$, 4H), $3.62(\mathrm{~s}, 3 \mathrm{H}), 3.32-3.23(\mathrm{~m}, 1 \mathrm{H}), 3.20(\mathrm{~d}, J=16.0 \mathrm{~Hz}, 1 \mathrm{H}), 3.10-2.99(\mathrm{~m}, 2 \mathrm{H}), 2.88$ $(\mathrm{d}, J=16.0 \mathrm{~Hz}, 1 \mathrm{H}), 2.51-2.43(\mathrm{~m}, 1 \mathrm{H}), 2.40(\mathrm{~d}, J=16.0 \mathrm{~Hz}, 1 \mathrm{H}), 2.07-1.98(\mathrm{~m}, 1 \mathrm{H})$;

${ }^{13}$ C NMR: (100 MHz, MeOD) $\delta$ 200.1, 171.9, 143.1, 133.4, 131.4, 130.9, 128.7, 127.3, $126.5,124.5,124.4,50.5,39.2,39.1,30.3,24.5$;

${ }^{19}$ F NMR: $\left(376 \mathrm{MHz}, \mathrm{CDCl}_{3}\right) \delta-62.4$;

HRMS: (ESI) $[\mathrm{M}+\mathrm{H}]^{+}$calcd. for $\mathrm{C}_{21} \mathrm{H}_{20} \mathrm{O}_{3} \mathrm{~F}_{3}, 376.1412$ found, 376.1418;

IR (film): $v_{\max } 1705,1604,1465,1296,1010,694 \mathrm{~cm}^{-1}$

HPLC: 77\% ee determined by HPLC (OD-H, 90/10 hexanes/i-PrOH, $1.0 \mathrm{ml} / \mathrm{min}$ ) $t r=11.3$ $\min , t r=13.7 \mathrm{~min}$. 
(S)-methyl 3-methyl-4-oxo-4-phenylbutanoate ${ }^{1}$ (6a)<smiles>COC(=O)C[C@H](C)C(=O)c1ccccc1</smiles>

Opt. Rot.: $[\alpha]^{26}{ }_{\mathrm{D}}(c$ 1.09, THF):-6.6;

HPLC: $85 \%$ ee determined by HPLC (OD-H, 90/10 hexanes/i-PrOH, $1.0 \mathrm{ml} / \mathrm{min}$ ) tr $=6.1$ $\min , t r=8.0 \mathrm{~min}$.

(S)-methyl 3-benzoylheptanoate (6b)<smiles>CCCC[C@H](CC(=O)OC)C(=O)c1ccccc1</smiles>

Opt. Rot.: $[\alpha]^{26}{ }_{\mathrm{D}}\left(c 0.64, \mathrm{CHCl}_{3}\right): 3.6$;

${ }^{1}$ H NMR: $\left(300 \mathrm{MHz}, \mathrm{CDCl}_{3}\right) \delta 8.04-7.98(\mathrm{~m}, 2 \mathrm{H}), 7.62-7.55(\mathrm{~m}, 1 \mathrm{H}), 7.53-7.45(\mathrm{~m}$, 2H), $4.00-3.85(\mathrm{~m}, 1 \mathrm{H}), 3.84(\mathrm{~s}, 3 \mathrm{H}), 2.97(\mathrm{dd}, J=9.0,15.0 \mathrm{~Hz}, 1 \mathrm{H}), 2.53(\mathrm{dd}, J=9.0,15.0$ $\mathrm{Hz}, 1 \mathrm{H}), 1.78-1.59(\mathrm{~m}, 2 \mathrm{H}), 1.55-1.40(\mathrm{~m}, 1 \mathrm{H}), 1.39-1.25(\mathrm{~m}, 3 \mathrm{H}), 0.89(\mathrm{t}, J=6.0 \mathrm{~Hz}$, $3 \mathrm{H})$;

${ }^{13}$ C NMR: $\left(75 \mathrm{MHz}, \mathrm{CDCl}_{3}\right) \delta 202.9,173.0,136.7,128.6,128.3,51.7,42.0,35.5,34.6$, 20.2, 14.0;

HRMS: (ESI) $[\mathrm{M}+\mathrm{H}]^{+}$calcd. for $\mathrm{C}_{15} \mathrm{H}_{21} \mathrm{O}_{3}, 249.1492$ found, 249.1491;

IR (film): $v_{\max } 1643,1604,694 \mathrm{~cm}^{-1}$

HPLC: $89 \%$ ee determined by HPLC (OD-H, 90/10 hexanes/i-PrOH, $1.0 \mathrm{ml} / \mathrm{min}) t r=5.1$ $\min , t r=7.6 \mathrm{~min}$.

(S)-methyl 3-benzoylnonanoate (6c)<smiles>CCCCCC[C@H](CC(=O)OC)C(=O)c1ccccc1</smiles>

Opt. Rot:: $[\alpha]^{26}{ }_{\mathrm{D}}\left(c 0.66, \mathrm{CHCl}_{3}\right): 3.1$;

${ }^{1}$ H NMR: (400 MHz, $\left.\mathrm{CDCl}_{3}\right) \delta 8.03-7.97(\mathrm{~m}, 2 \mathrm{H}), 7.61-7.56(\mathrm{~m}, 1 \mathrm{H}), 7.53-7.46(\mathrm{~m}$, 2H), $3.96-3.88(\mathrm{~m}, 1 \mathrm{H}), 3.64(\mathrm{~s}, 3 \mathrm{H}), 2.97(\mathrm{dd}, J=8.0,16.0 \mathrm{~Hz}, 1 \mathrm{H}), 2.53(\mathrm{dd}, J=8.0,16.0$ $\mathrm{Hz}, 1 \mathrm{H}), 1.78-1.66(\mathrm{~m}, 1 \mathrm{H}), 1.55-1.43(\mathrm{~m}, 1 \mathrm{H}), 1.35-1.16(\mathrm{~m}, 8 \mathrm{H}), 0.86(\mathrm{t}, J=8.0 \mathrm{~Hz}$, 
$3 \mathrm{H})$;

${ }^{13}$ C NMR: $\left(100 \mathrm{MHz}, \mathrm{CDCl}_{3}\right) \delta 202.9,173.0,136.7,132.9,128.6,128.3,51.6,42.2,35.5$, $32.4,31.5,29.9,26.9,22.4,13.9$;

HRMS: (ESI) $[\mathrm{M}+\mathrm{H}]^{+}$calcd. for $\mathrm{C}_{17} \mathrm{H}_{25} \mathrm{O}_{3}, 277.1805$ found, 277.1804;

IR (film): $v_{\max } 1705,1604,694 \mathrm{~cm}^{-1}$

HPLC: $89 \%$ ee determined by HPLC (OD-H, 90/10 hexanes/i-PrOH, $1.0 \mathrm{ml} / \mathrm{min}$ ) $t r=4.6$ $\min , t r=5.4 \mathrm{~min}$.

(S)-methyl 3-benzoyltridecanoate (6d)<smiles>CCCCCCCCC[C@H](CC(=O)OC)C(=O)c1ccccc1</smiles>

Opt. Rot.: $[\alpha]^{26}{ }_{\mathrm{D}}\left(c 3.13, \mathrm{CHCl}_{3}\right): 1.2$;

${ }^{1}$ H NMR: $\left(300 \mathrm{MHz}, \mathrm{CDCl}_{3}\right) \delta 8.04-7.96(\mathrm{~m}, 2 \mathrm{H}), 7.61-7.56(\mathrm{~m}, 1 \mathrm{H}), 7.63-7.43(\mathrm{~m}$, 3H), $3.98-3.85(\mathrm{~m}, 1 \mathrm{H}), 3.64(\mathrm{~s}, 3 \mathrm{H}), 2.97(\mathrm{dd}, J=9.0,15.0 \mathrm{~Hz}, 1 \mathrm{H}), 2.53(\mathrm{dd}, J=9.0,15.0$ $\mathrm{Hz}, 1 \mathrm{H}), 1.80-1.57(\mathrm{~m}, 1 \mathrm{H}), 1.55-1.41(\mathrm{~m}, 1 \mathrm{H}), 1.37-1.10(\mathrm{~m}, 16 \mathrm{H}), 0.89(\mathrm{t}, J=6.0 \mathrm{~Hz}$, $3 \mathrm{H})$;

${ }^{13}$ C NMR: (75 MHz, $\left.\mathrm{CDCl}_{3}\right) \delta$ 202.9, 173.0, 136.7, 132.9, 128.6, 128.3, 51.6, 42.2, 35.5, $32.4,31.8,29.5,29.4,29.3,29.2,26.9,22.6,14.0$;

HRMS: (ESI) $[\mathrm{M}+\mathrm{H}]^{+}$calcd. for $\mathrm{C}_{21} \mathrm{H}_{33} \mathrm{O}_{3}, 333.2448$ found, 333.2450;

IR (film): $v_{\max } 1643,1604,1211,694 \mathrm{~cm}^{-1}$

HPLC: $91 \%$ ee determined by HPLC (IB, 98/2 hexanes/1-PrOH, $1.0 \mathrm{ml} / \mathrm{min}$ ) $t r=4.2 \mathrm{~min}$, $t r$ $=4.8 \mathrm{~min}$.

(S)-ethyl 3-methyl-4-oxo-4-phenylbutanoate ${ }^{2}(6 e)$<smiles>CCOC(=O)C[C@H](C)C(=O)c1ccccc1</smiles>

Opt. Rot.: $[\alpha]^{26}{ }_{\mathrm{D}}\left(c\right.$ $\left.0.70, \mathrm{CHCl}_{3}\right): 3.8$;

HPLC: $85 \%$ ee determined by HPLC (OD-H, 90/10 hexanes/i-PrOH, $1.0 \mathrm{ml} / \mathrm{min}$ ) $t r=5.2$ $\min , t r=6.8 \mathrm{~min}$.

(S)-methyl 4-(4-bromophenyl)-3-methyl-4-oxobutanoate (6f) 
$\overbrace{O=}^{O M M e}$

Opt. Rot.: $[\alpha]^{26}\left(c 0.78, \mathrm{CHCl}_{3}\right): 6.1$;

${ }^{1}$ H NMR: $\left(400 \mathrm{MHz}, \mathrm{CDCl}_{3}\right) \delta 7.89-7.83(\mathrm{~m}, 2 \mathrm{H}), 7.66-7.61(\mathrm{~m}, 2 \mathrm{H}), 3.95-3.82(\mathrm{~m}$, $1 \mathrm{H}), 3.66(\mathrm{~s}, 3 \mathrm{H}), 2.97$ (dd, $J=8.0,16.0 \mathrm{~Hz}, 1 \mathrm{H}), 2.47$ (dd, $J=8.0,16.0 \mathrm{~Hz}, 1 \mathrm{H}), 1.22(\mathrm{~d}, J=$ $8.0 \mathrm{~Hz}, 3 \mathrm{H})$;

${ }^{13}$ C NMR: $\left(100 \mathrm{MHz}, \mathrm{CDCl}_{3}\right) \delta 201.7,172.6,134.6,132.0,129.9,128.2,51.7,37.1,17.7$;

HRMS: (ESI) $[\mathrm{M}+\mathrm{H}]^{+}$calcd. for $\mathrm{C}_{26} \mathrm{H}_{25} \mathrm{O}_{3}, 385.1804$ found, 385.1804;

IR (film): $v_{\max } 1759,1627,1589,1508,1442,1141,750,700 \mathrm{~cm}^{-1}$

HPLC: $85 \%$ ee determined by HPLC (OD-H, 90/10 hexanes/i-PrOH, $1.0 \mathrm{ml} / \mathrm{min}$ ) $t r=6.1$ $\min , \operatorname{tr}=12.2 \mathrm{~min}$.

(S)-methyl 4-(4-chlorophenyl)-3-methyl-4-oxobutanoate (6g)<smiles>COC(=O)C[C@H](C)C(=O)c1ccc(Cl)cc1</smiles>

Opt. Rot.: $[\alpha]^{26}\left(c 0.73, \mathrm{CHCl}_{3}\right): 8.1$;

${ }^{1}$ H NMR: (300 MHz, $\left.\mathrm{CDCl}_{3}\right) \delta 7.98-7.90(\mathrm{~m}, 2 \mathrm{H}), 7.50$ - $7.42(\mathrm{~m}, 2 \mathrm{H}), 3.98-3.81(\mathrm{~m}$, 1H), 3.66 (s, 3H), 2.98 (dd, $J=6.0,15.0 \mathrm{~Hz}, 1 \mathrm{H}), 2.47$ (dd, $J=6.0,15.0 \mathrm{~Hz}, 1 \mathrm{H}), 1.22$ (d, $J=$ $6.0 \mathrm{~Hz}, 3 \mathrm{H})$;

${ }^{13}$ C NMR: $\left(75 \mathrm{MHz}, \mathrm{CDCl}_{3}\right) \delta 201.5,172.7,139.5,134.2,129.8,129.0,51.7,37.2,17.7$;

HRMS: (ESI) $[\mathrm{M}+\mathrm{H}]^{+}$calcd. for $\mathrm{C}_{26} \mathrm{H}_{25} \mathrm{O}_{3}, 385.1804$ found, 385.1804;

IR (film): $v_{\max } 1759,1627,1589,1508,1442,1141,750,700 \mathrm{~cm}^{-1}$

HPLC: 86\% ee determined by HPLC (OD-H, 90/10 hexanes/i-PrOH, $1.0 \mathrm{ml} / \mathrm{min}$ ) $t r=5.2$ $\min , t r=8.1 \mathrm{~min}$.

(S)-methyl 3-methyl-4-oxo-4-(p-tolyl)butanoate (6h)<smiles>COC(=O)C[C@H](C)C(=O)c1ccc(C)cc1</smiles>

Opt. Rot.: $[\alpha]^{26}\left(c 0.75, \mathrm{CHCl}_{3}\right): 1.1$;

${ }^{1}$ H NMR: (300 MHz, $\left.\mathrm{CDCl}_{3}\right) \delta 7.97-7.84(\mathrm{~m}, 2 \mathrm{H}), 7.34-7.24(\mathrm{~m}, 2 \mathrm{H}), 4.02-3.86(\mathrm{~m}$, 1H), $3.66(\mathrm{~s}, 3 \mathrm{H}), 2.96(\mathrm{dd}, J=6.0,15.0 \mathrm{~Hz}, 1 \mathrm{H}), 2.46(\mathrm{dd}, J=6.0,15.0 \mathrm{~Hz}, 1 \mathrm{H}), 2.43$ (s, 
$3 \mathrm{H}), 1.23(\mathrm{~d}, J=6.0 \mathrm{~Hz}, 3 \mathrm{H})$;

${ }^{13}$ C NMR: $\left(75 \mathrm{MHz}, \mathrm{CDCl}_{3}\right) \delta 201.5,172.7,139.5,134.2,129.8,129.0,51.7,37.2,17.7$;

HRMS: (ESI) $[\mathrm{M}+\mathrm{H}]^{+}$calcd. for $\mathrm{C}_{26} \mathrm{H}_{25} \mathrm{O}_{3}, 385.1804$ found, 385.1804;

IR (film): $v_{\max } 1759,1627,1589,1508,1442,1141,750,700 \mathrm{~cm}^{-1}$

HPLC: 90\% ee determined by HPLC (OD-H, 90/10 hexanes/i-PrOH, $1.0 \mathrm{ml} / \mathrm{min}) t r=5.8$ $\min , t r=8.7 \mathrm{~min}$.

(S)-methyl 4-(4-methoxyphenyl)-3-methyl-4-oxobutanoate (6i)<smiles>COC(=O)C[C@H](C)C(=O)c1ccc(OC)cc1</smiles>

Opt. Rot.: $[\alpha]^{26}{ }_{\mathrm{D}}\left(c 0.95, \mathrm{CHCl}_{3}\right): 17.8$;

${ }^{1}$ H NMR: $\left(400 \mathrm{MHz}, \mathrm{CDCl}_{3}\right) \delta 7.99-7.92(\mathrm{~m}, 2 \mathrm{H}), 6.96-6.90(\mathrm{~m}, 2 \mathrm{H}), 3.94-3.81(\mathrm{~m}$, $1 \mathrm{H}), 3.84(\mathrm{~s}, 3 \mathrm{H}), 3.62(\mathrm{~s}, 3 \mathrm{H}), 2.92(\mathrm{dd}, J=8.0,16.0 \mathrm{~Hz}, 1 \mathrm{H}), 2.42(\mathrm{dd}, J=8.0,16.0 \mathrm{~Hz}$, $1 \mathrm{H}), 1.20(\mathrm{~d}, J=8.0 \mathrm{~Hz}, 3 \mathrm{H})$;

${ }^{13}$ C NMR: $\left(100 \mathrm{MHz}, \mathrm{CDCl}_{3}\right) \delta 201.1,172.8,163.5,130.6,128.7,113.8,55.4,51.5,37.2$, $36.8,18.0$;

HRMS: (ESI) $[\mathrm{M}+\mathrm{H}]^{+}$calcd. for $\mathrm{C}_{26} \mathrm{H}_{25} \mathrm{O}_{3}, 385.1804$ found, 385.1804;

IR (film): $v_{\max } 1759,1627,1589,1508,1442,1141,750,700 \mathrm{~cm}^{-1}$

HPLC: $83 \%$ ee determined by HPLC (OD-H, 90/10 hexanes/i-PrOH, $1.0 \mathrm{ml} / \mathrm{min}$ ) $t r=9.2$ $\min , t r=15.3 \mathrm{~min}$.

\section{((2-benzyl-1H-inden-3-yl)oxy)trimethylsilane (8a)}<smiles>COC1=C(Br)Cc2ccccc21</smiles>

${ }^{1}$ H NMR: (400 MHz, $\left.\mathrm{CDCl}_{3}\right) \delta 7.45$ - $7.20(\mathrm{~m}, 9 \mathrm{H}), 3.85$ (s, 2H), 3.19 (s, 3H), 0.42 (s, 9H); ${ }^{13}$ C NMR: $\left(100 \mathrm{MHz}, \mathrm{CDCl}_{3}\right) \delta 148.2,142.4,141.3,140.8,128.7,128.4,126.1,126.0$, $124.5,123.6,123.0,117.8,36.1,33.1,0.96$;

\section{((2-benzyl-1H-inden-3-yl)oxy)(tert-butyl)dimethylsilane (8b)}<smiles>OCC1=C(Br)Cc2ccccc21</smiles> 
${ }^{1}$ H NMR: (400 MHz, $\left.\mathrm{CDCl}_{3}\right) \delta 7.42-7.15(\mathrm{~m}, 9 \mathrm{H}), 3.85(\mathrm{~s}, 2 \mathrm{H}), 3.16(\mathrm{~s}, 2 \mathrm{H}), 1.18(\mathrm{~s}, 9 \mathrm{H})$, $0.32(\mathrm{~s}, 6 \mathrm{H})$;

${ }^{13}$ C NMR: $\left(100 \mathrm{MHz}, \mathrm{CDCl}_{3}\right) \delta 148.1,142.4,141.4,140.8,128.7,128.4,126.0,124.4$, $123.6,122.7,118.0,36.1,33.2,25.9,18.4,-3.5$;

\section{References}

(1) Blanco, L.; Rousseau, J-P.; Barnier, E. Tetrahedron: Asymmetry 1993, 5, 783-792.

(2) Miura, K.; Fujisawa, N.; Saito, H.; Wang, D.; Hosomi, A. Org. Lett. 2001, 3, 2591-2594. 


\section{VIII: Calculation Result}

\section{Computational Method}

Calculations are performed with Gaussian 09 Revision D.01 suite of program. ${ }^{1}$ Geometry optimization was performed at M06-2X/def2-TZVP. A tight convergence criterion (via keyword opt=tight) and larger than default integration grid (via keyword: int=ultrafine) was applied for geometry optimization. Single point calculation was performed on the optimized geometries at SMD-THF-M06-2X/def2-TZVPD. The def2-TZVPD basis sets are obtained from the basis set exchange website. ${ }^{2}$ Solvent effect of THF was included in the single point calculation with the SMD implicit solvation model. Frequency calculations were performed to characterize the nature of the optimized geometries. Molecular graphic is generated from POVRAY 3.7 from scripts that are generated by CYLview. ${ }^{3}$

\section{Computational Results}

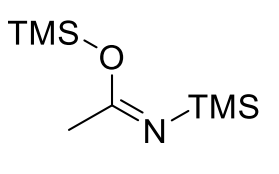

BSA-O-trans- $\mathbf{N}-$ cis
TMS

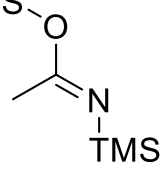

BSA-O-trans-N-trans

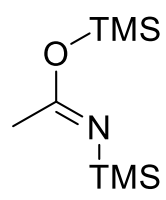

BSA-O-cis-N-trans

Figure S1. Conformations of BSA

The $\boldsymbol{O}$-cis- $\boldsymbol{N}$-cis conformation of the probase in Figure $\boldsymbol{S} \mathbf{1}$ is the most stable conformation at our current level of theory. Therefore, only this conformation is considered for the subsequent calculations unless otherwise noted. 
Table S4. Results for conformation of BSA as shown in Figure S1

\begin{tabular}{|c|c|c|c|}
\hline Relative energy $^{\mathrm{a}}$ & BSA-O-trans- $\boldsymbol{N}$-cis & BSA-O-trans- $\boldsymbol{N}$-trans & BSA-O-cis- $\boldsymbol{N}$-trans \\
\hline$\Delta \mathrm{E}$ & 2.46 & 0.03 & 0.00 \\
\hline$\Delta \mathrm{E}+\mathrm{ZPE}$ & 2.56 & 0.33 & 0.00 \\
\hline$\Delta \mathrm{U}$ & 2.38 & 0.16 & 0.00 \\
\hline$\Delta \mathrm{H}$ & 2.38 & 0.16 & 0.00 \\
\hline$\Delta \mathrm{G}$ & 3.30 & 0.75 & \\
\hline
\end{tabular}

Calculations are done at SMD ${ }_{\mathrm{THF}}-\mathrm{def} 2-\mathrm{TZVPD} / \mathrm{M} 06-2 \mathrm{X} / \mathrm{def} 2 \mathrm{TZVP} / \mathrm{M} 06-2 \mathrm{X} .{ }^{\mathrm{a}}$ in $\mathrm{kcal} / \mathrm{mol}$.

\section{Cleavage of TMS from probase}

We studied the cleavage of TMS from the probase by $\mathbf{C s F}$.

The formation of a complex between CsF and the BSA is exothermic. BSA-OFCs is more exothermic than BSA-NFCs by $7.65 \mathrm{kcal} / \mathrm{mol}$. The formation of both complexes are endogenic, BSA-OFCs is thermodynamically more stable than BSA-NFCs by $6.44 \mathrm{kcal} / \mathrm{mol}$

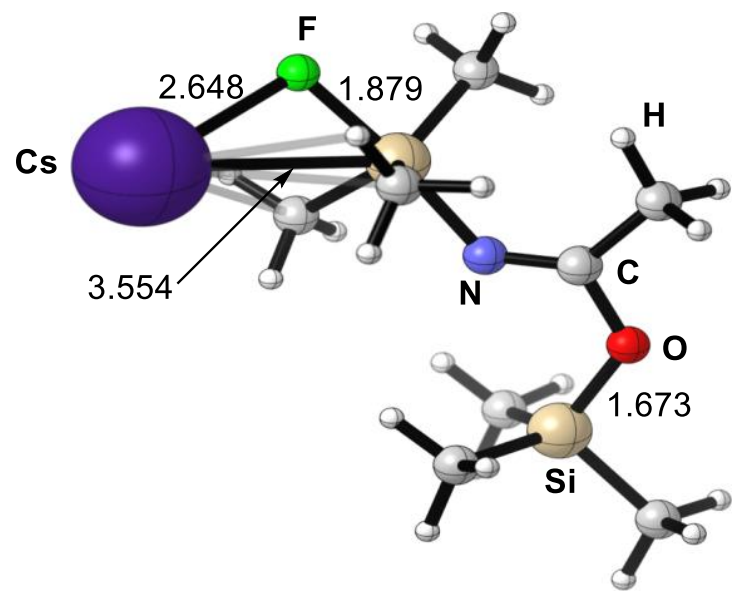

BSA-N-FCs

$\Delta \mathrm{H}=-1.82 \mathrm{kcal} / \mathrm{mol}$ $\Delta \mathrm{G}=+10.8 \mathrm{kcal} / \mathrm{mol}$

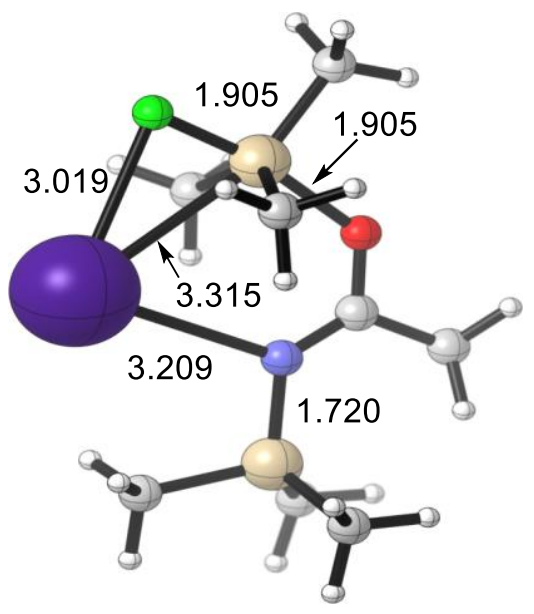

BSA-O-FCs

$\Delta \mathrm{H}=-9.47 \mathrm{kcal} / \mathrm{mol}$ $\Delta \mathrm{G}=+4.36 \mathrm{kcal} / \mathrm{mol}$

Figure S2. The CsF - BSA complexes and their relative energies.

An additional Cs-N interaction can be observed in BSA-OFCs, but because of the conformation restriction imposed by using BSA-O-cis- $\boldsymbol{N}$-trans, an analogous C-O interaction cannot be formed for BSA-NFCs. We attempted to locate a conformation for BSA-NFCs that is similar to BSA-OFCs in Figure $\boldsymbol{S}$ 2, however, a geometry optimization leads to the decomposition of BSA-NFCs to TMSO, CH3CN and TMSF. All three of these 
molecules are bonded to Cs (Figure S3).

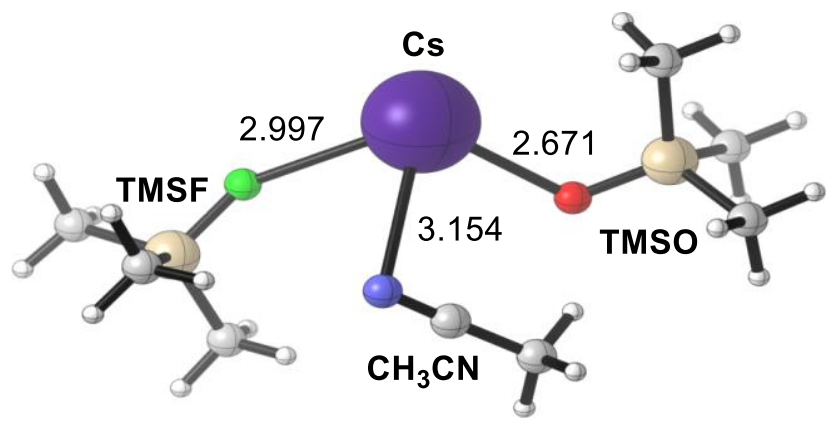

Figure S3. The intermediate which is obtained as a results of swapping the $\mathrm{O}$ and $\mathrm{N}$ in BSAO-FCs followed by a geometry optimization.

The cleavage of O-TMS from BSA-OFCs has a barrier of $11.9 \mathrm{kcal} / \mathrm{mol}$ (Figure S4). The intermediate of O-TMS cleavage is postTS-O-TMS-cleave. It is a thermodynamically stable species with a $\Delta \mathrm{G}$ of $-3.33 \mathrm{kcal} / \mathrm{mol}$. Rotation about the $\mathrm{Cs}-\mathrm{F}$ bond in postTS-O-TMScleave leads to a more stable intermediate I-O-TMS-cleave $(\Delta \mathrm{G}=-4.96 \mathrm{kcal} / \mathrm{mol})$. The dissociation of TMS-F from I-O-TMS-cleave results in highly stable intermediate which can function as a base to deprotonate the substrate.

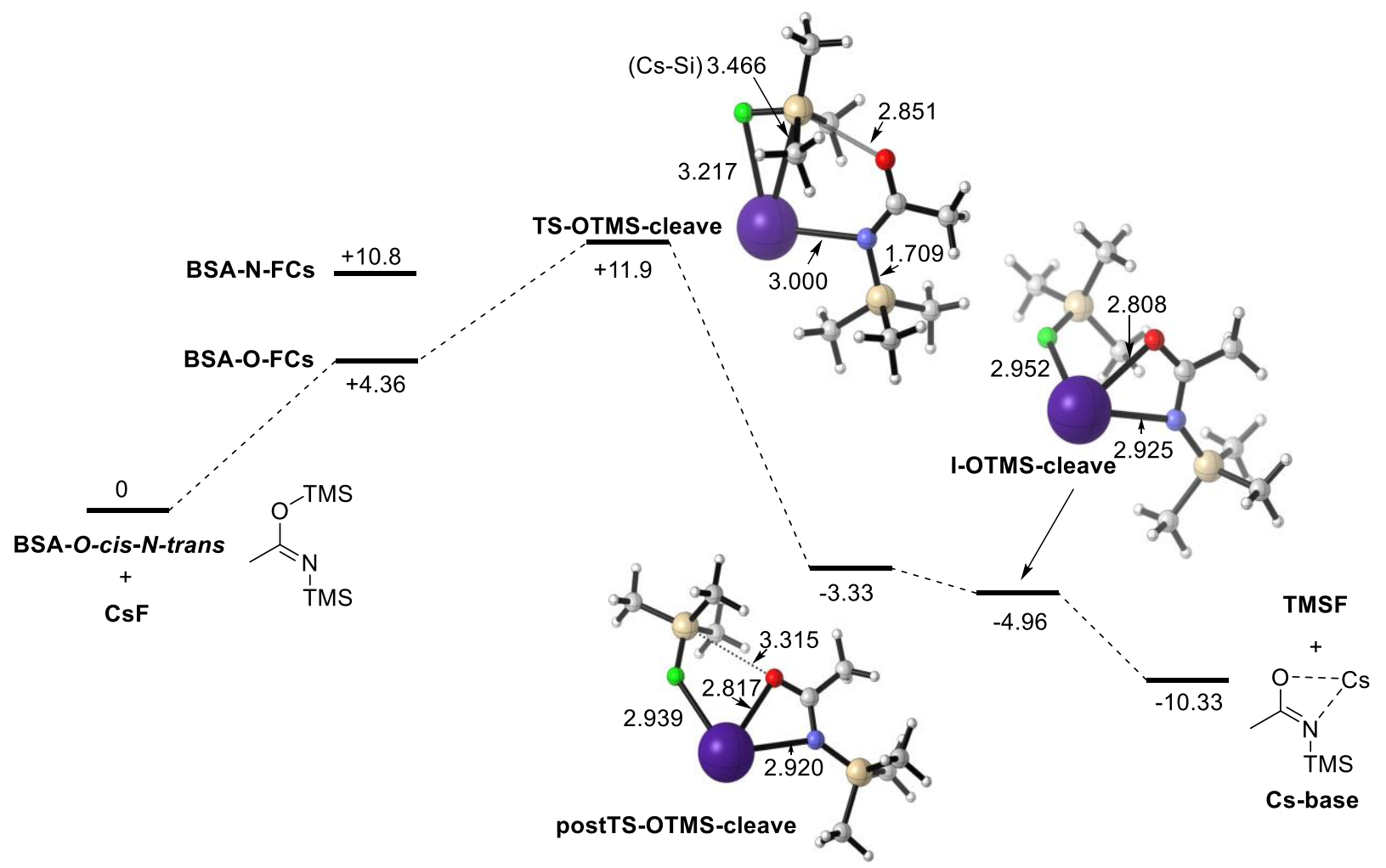

Figure S4. Calculated reaction pathway for the cleavage of the probase at the O-TMS site. Energies (in $\mathrm{kcal} / \mathrm{mol}$ ) are relative Gibbs free energy at $298.15 \mathrm{~K}$ with respect to BSA-O-cis- 


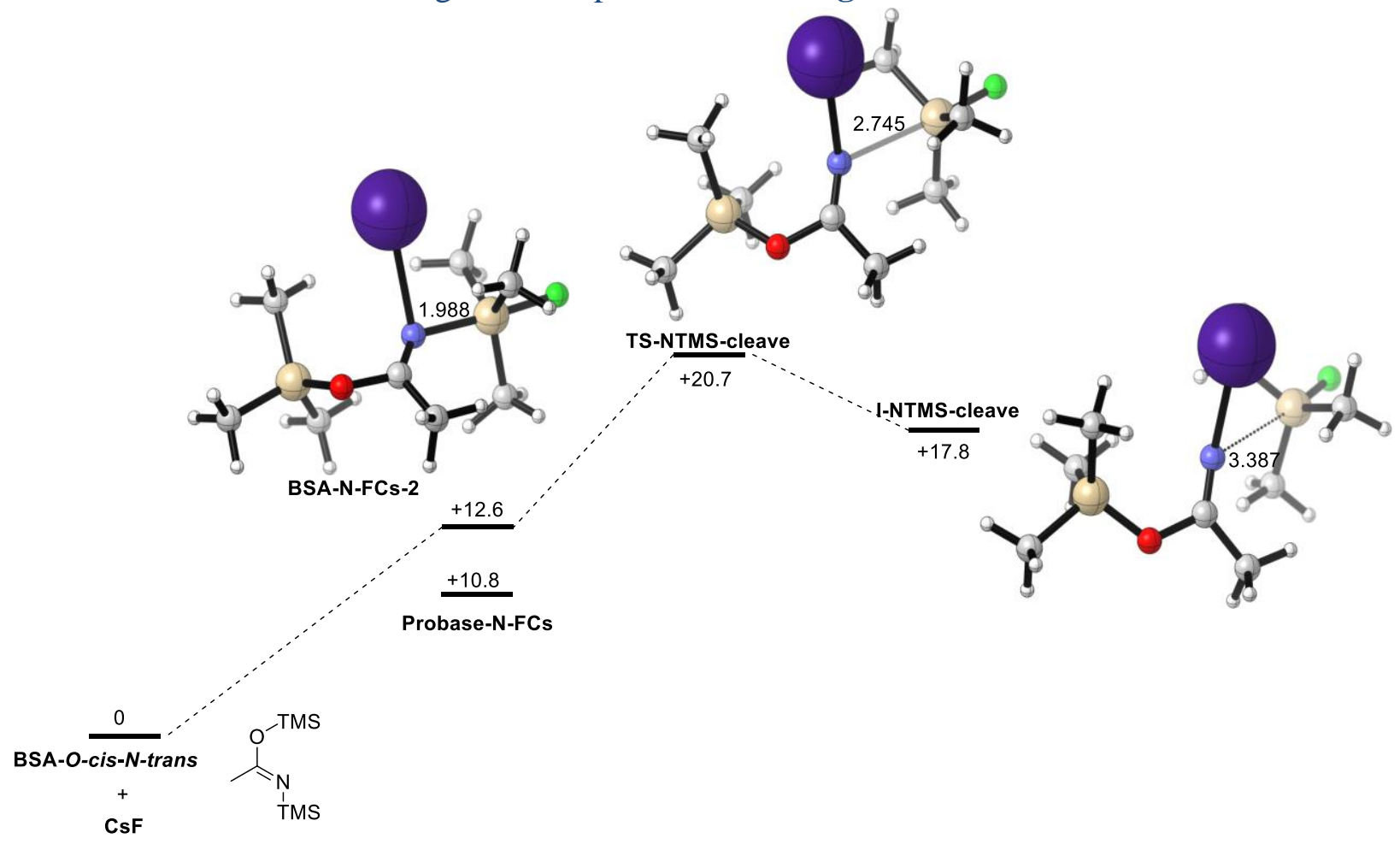

Figure S5 Calculated reaction pathway for the cleavage of the probase at the N-TMS site. Energies (in $\mathrm{kcal} / \mathrm{mol}$ ) are relative Gibbs free energy at $298.15 \mathrm{~K}$ with respect to BSA-O-cis$\mathrm{N}$-trans and CsF. For labelling of atoms please refer to Figure $\mathbf{S 2}$.

The cleavage of TMS from N of BSA takes place via a different intermediate - BSA-N-FCsC2 (Figure S5). The barrier for N-TMS cleavage from BSA is $20.7 \mathrm{kcal} / \mathrm{mol}$ which is substantially higher than that for O-TMS cleavage shown in Figure S4. Therefore, it is likely that the attack of TMS by fluoride occurs at the O site.

\section{References}

(1) Frisch, M. J.; Trucks, G. W.; Schlegel, H. B.; Scuseria, G. E.; Robb, M. A.; Cheeseman, J. R.; Scalmani, G.; Barone, V.; Mennucci, B.; Petersson, G. A.; Nakatsuji, H.; Caricato, M.; Li, X.; Hratchian, H. P.; Izmaylov, A. F.; Bloino, J.; Zheng, G.; Sonnenberg, J. L.; Hada, M.; Ehara, M.; Toyota, K.; Fukuda, R.; Hasegawa, J.; Ishida, M.; Nakajima, T.; Honda, Y.; Kitao, O.; Nakai, H.; Vreven, T.; Montgomery, J. A.; Peralta, J. E.; Ogliaro, F.; Bearpark, M.; Heyd, J. J.; Brothers, E.; Kudin, K. N.; Staroverov, V. N.; Kobayashi, R.; Normand, J.; Raghavachari, K.; Rendell, A.; Burant, J. C.; Iyengar, S. S.; Tomasi, J.; Cossi, M.; Rega, N.; Millam, J. M.; Klene, M.; Knox, J. E.; Cross, J. B.; Bakken, V.; Adamo, C.; Jaramillo, J.; 
Gomperts, R.; Stratmann, R. E.; Yazyev, O.; Austin, A. J.; Cammi, R.; Pomelli, C.; Ochterski, J. W.; Martin, R. L.; Morokuma, K.; Zakrzewski, V. G.; Voth, G. A.; Salvador, P.; Dannenberg, J. J.; Dapprich, S.; Daniels, A. D.; Farkas; Foresman, J. B.; Ortiz, J. V.; Cioslowski, J.; Fox, D. J. In Gaussian 09, Revision D.01 Wallingford CT, 2009.

(2) Schuchardt, K. L.; Didier, B. T.; Elsethagen, T.; Sun, L.; Gurumoorthi, V.; Chase, J.; Li, J.; Windus, T. L. Journal of Chemical Information and Modeling 2007, 47, 1045-1052.

(3) Legault, C. Y.; 1.0.562 BETA ed.; Université de Sherbrooke: 2012.

\section{Coordinates and thermochemical information}

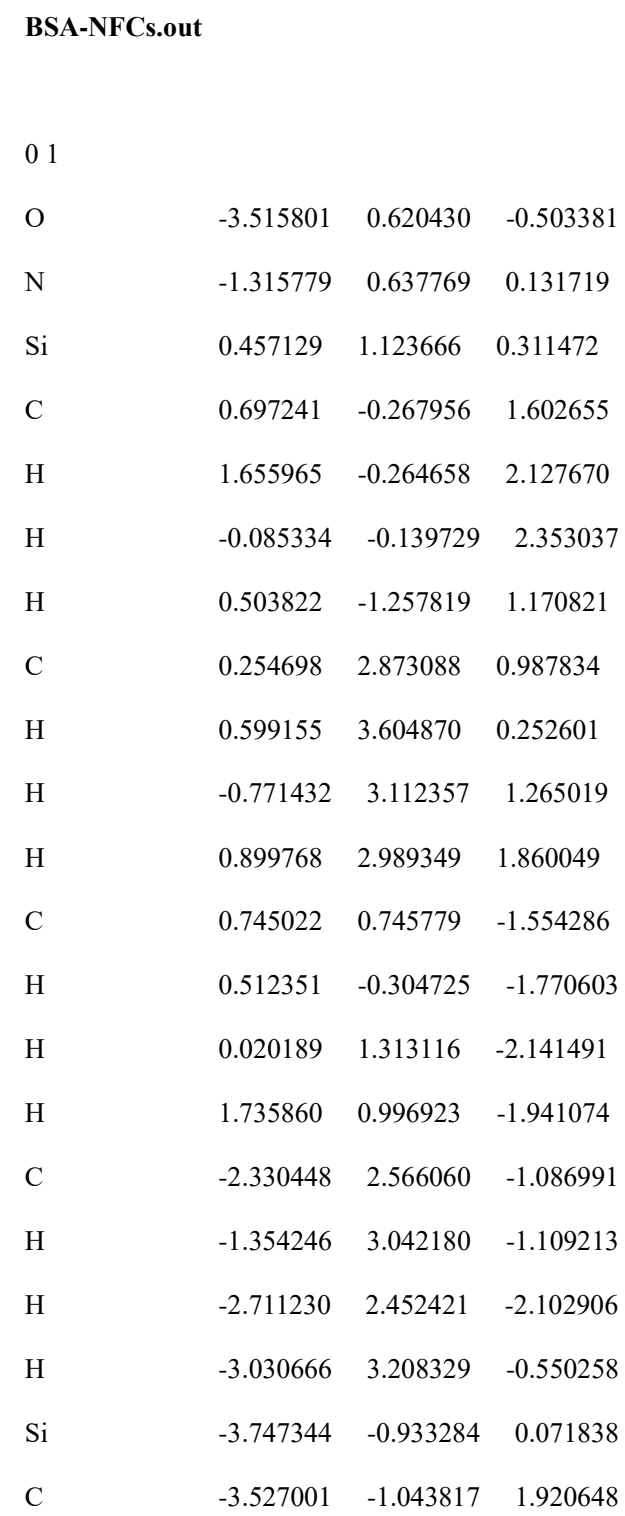




$\begin{array}{llll}\mathrm{H} & -3.886233 & -2.006973 & 2.291288 \\ \mathrm{H} & -2.476253 & -0.931453 & 2.185083 \\ \mathrm{H} & -4.094321 & -0.258661 & 2.424421 \\ \mathrm{C} & -2.652185 & -2.166481 & -0.809586 \\ \mathrm{H} & -1.625650 & -2.076144 & -0.455699 \\ \mathrm{H} & -3.003859 & -3.186210 & -0.635338 \\ \mathrm{H} & -2.661887 & -1.986396 & -1.886831 \\ \mathrm{C} & -5.532239 & -1.278141 & -0.363022 \\ \mathrm{H} & -5.828385 & -2.276827 & -0.034847 \\ \mathrm{H} & -6.195384 & -0.555098 & 0.115198 \\ \mathrm{H} & -5.690181 & -1.215332 & -1.441188 \\ \mathrm{~F} & 2.308499 & 1.429777 & 0.411716 \\ \mathrm{Cs} & 3.300416 & -0.923449 & -0.287294\end{array}$

\begin{tabular}{llll} 
Zero-point correction & \multicolumn{2}{l}{0.27981} & \\
Thermal correction to Energy & & 0.302867 & \\
Thermal correction to Enthalpy $=$ & 0.303811 & \\
Thermal correction to Gibbs Free Energy & $=$ & 0.226007 \\
Sum of electronic and zero-point Energies & $=$ & -1146.285465 \\
Sum of electronic and thermal Energies = & -1146.262408 \\
Sum of electronic and thermal Enthalpies & $=$ & -1146.261464 \\
Sum of electronic and thermal Free Energies & $=$ & -1146.339268 \\
Number of imaginary frequencies/frequency & $=$ & 0
\end{tabular}

\section{BSA-N_FCs-2.out}

$\begin{array}{llll}0 ~ 1 & & & \\ \mathrm{O} & -3.248205 & 0.181375 & -0.889710 \\ \mathrm{~N} & -1.046599 & 0.358697 & -0.218745 \\ \mathrm{Si} & 0.678114 & 1.252973 & 0.200537 \\ \mathrm{C} & 1.310854 & -0.369439 & 0.998962 \\ \mathrm{H} & 2.187588 & -0.763276 & 0.470247 \\ \mathrm{H} & 1.691379 & -0.095263 & 1.985157 \\ \mathrm{H} & 0.568233 & -1.158245 & 1.140732 \\ \mathrm{C} & -0.151028 & 2.517974 & 1.337163 \\ \mathrm{H} & -0.042981 & 3.517331 & 0.906639 \\ \mathrm{H} & -1.204832 & 2.339937 & 1.546922 \\ \mathrm{H} & 0.402570 & 2.536393 & 2.278199\end{array}$




$\begin{array}{llll}\mathrm{C} & 0.995726 & 1.557796 & -1.676892 \\ \mathrm{H} & 0.193643 & 1.308366 & -2.376860 \\ \mathrm{H} & 1.208142 & 2.623300 & -1.793885 \\ \mathrm{H} & 1.924810 & 1.062522 & -1.983173 \\ \mathrm{C} & -2.283033 & 2.326868 & -1.118188 \\ \mathrm{H} & -1.352278 & 2.886947 & -1.117281 \\ \mathrm{H} & -2.730429 & 2.347305 & -2.112691 \\ \mathrm{H} & -2.988068 & 2.805923 & -0.435999 \\ \mathrm{Si} & -3.704824 & -1.021467 & 0.166193 \\ \mathrm{C} & -3.541804 & -0.440601 & 1.926928 \\ \mathrm{H} & -3.936272 & -1.186932 & 2.619860 \\ \mathrm{H} & -2.495396 & -0.255621 & 2.173167 \\ \mathrm{H} & -4.097654 & 0.486493 & 2.081118 \\ \mathrm{C} & -2.719443 & -2.608072 & -0.056660 \\ \mathrm{H} & -1.731152 & -2.503605 & 0.395137 \\ \mathrm{H} & -3.226433 & -3.439361 & 0.438601 \\ \mathrm{H} & -2.626889 & -2.876289 & -1.113175 \\ \mathrm{H} & -5.480011 & -1.376444 & -0.279425 \\ \mathrm{C} & -5.894235 & -2.162033 & 0.355771 \\ \mathrm{H} & -6.094580 & -0.483100 & -0.158972 \\ \mathrm{H} & -5.564966 & -1.702186 & -1.317899 \\ \mathrm{H} & 2.210067 & 1.956377 & 0.491160 \\ \mathrm{H} & -235544 & -1.666977 & -1.784678\end{array}$

$\begin{array}{llll}\text { Zero-point correction } & 0.280189 & & \\ \text { Thermal correction to Energy } & & 0.302892 & \\ \text { Thermal correction to Enthalpy } & & 0.303836 & \\ \text { Thermal correction to Gibbs Free Energy } & = & 0.228163 \\ \text { Sum of electronic and zero-point Energies } & = & -1146.285137 \\ \text { Sum of electronic and thermal Energies = } & -1146.262435 \\ \text { Sum of electronic and thermal Enthalpies } & = & -1146.26149 \\ \text { Sum of electronic and thermal Free Energies } & = & -1146.337164 \\ \text { Number of imaginary frequencies/frequency } & = & 0\end{array}$

\section{BSA-O-cis-N-trans.out}

01

$\begin{array}{llll}\mathrm{O} & -1.405270 & 1.157809 & -0.000013\end{array}$ 


\begin{tabular}{|c|c|c|c|}
\hline $\mathrm{N}$ & 0.568362 & 0.034541 & -0.000101 \\
\hline $\mathrm{Si}$ & 2.267933 & -0.313053 & -0.000219 \\
\hline $\mathrm{C}$ & 2.423326 & -2.172610 & 0.000028 \\
\hline $\mathrm{H}$ & 3.468291 & -2.488605 & -0.000033 \\
\hline $\mathrm{H}$ & 1.939910 & -2.597409 & 0.881989 \\
\hline $\mathrm{H}$ & 1.939732 & -2.597657 & -0.881716 \\
\hline $\mathrm{C}$ & 3.090282 & 0.371258 & 1.538499 \\
\hline $\mathrm{H}$ & 3.065945 & 1.461666 & 1.573352 \\
\hline $\mathrm{H}$ & 2.592132 & -0.003637 & 2.434729 \\
\hline $\mathrm{H}$ & 4.136339 & 0.060047 & 1.582596 \\
\hline $\mathrm{C}$ & 3.089973 & 0.370824 & -1.539295 \\
\hline $\mathrm{H}$ & 4.136035 & 0.059644 & -1.583489 \\
\hline $\mathrm{H}$ & 2.591675 & -0.004363 & -2.435320 \\
\hline $\mathrm{H}$ & 3.065582 & 1.461220 & -1.574473 \\
\hline $\mathrm{C}$ & 0.497437 & 2.524249 & -0.000345 \\
\hline $\mathrm{H}$ & 1.583634 & 2.525552 & -0.000553 \\
\hline $\mathrm{H}$ & 0.130011 & 3.056183 & -0.878426 \\
\hline $\mathrm{H}$ & 0.130353 & 3.056287 & 0.877817 \\
\hline $\mathrm{Si}$ & -2.355904 & -0.242664 & 0.000232 \\
\hline $\mathrm{C}$ & -2.054434 & -1.238241 & 1.544173 \\
\hline $\mathrm{H}$ & -2.803817 & -2.027974 & 1.634101 \\
\hline $\mathrm{H}$ & -1.065952 & -1.695626 & 1.523789 \\
\hline $\mathrm{H}$ & -2.126333 & -0.608312 & 2.432928 \\
\hline $\mathrm{C}$ & -2.054797 & -1.238523 & -1.543598 \\
\hline $\mathrm{H}$ & -1.066287 & -1.695856 & -1.523387 \\
\hline $\mathrm{H}$ & -2.804163 & -2.028312 & -1.633174 \\
\hline $\mathrm{H}$ & -2.126964 & -0.608771 & -2.432456 \\
\hline $\mathrm{C}$ & -4.085626 & 0.449834 & 0.000373 \\
\hline $\mathrm{H}$ & -4.824196 & -0.354270 & 0.000529 \\
\hline $\mathrm{H}$ & -4.258973 & 1.067461 & 0.883155 \\
\hline $\mathrm{H}$ & -4.259180 & 1.067310 & -0.882473 \\
\hline
\end{tabular}

$\begin{array}{llll}\text { Zero-point correction }=0.276947 & & \\ \text { Thermal correction to Energy } & = & 0.297249 & \\ \text { Thermal correction to Enthalpy } & = & 0.298193 & \\ \text { Thermal correction to Gibbs Free Energy } & = & -1026.276537 \\ \text { Sum of electronic and zero-point Energies } & = & -1026.256235\end{array}$




$\begin{array}{lll}\text { Sum of electronic and thermal Enthalpies } & = & -1026.255291 \\ \text { Sum of electronic and thermal Free Energies } & = & -1026.325669 \\ \text { Number of imaginary frequencies/frequency } & = & 0\end{array}$

\section{BSA-O-trans-N-cis.out}

01

$\begin{array}{llll}\text { O } & -0.634759 & 0.199568 & -0.463562\end{array}$

$\begin{array}{llll}\mathrm{N} & 1.315176 & 1.289898 & 0.066223\end{array}$

$\begin{array}{llll}\mathrm{Si} & 2.299110 & -0.156130 & -0.006476\end{array}$

$\begin{array}{llll}\text { C } & 4.036502 & 0.361672 & 0.435851\end{array}$

$\begin{array}{llll}\mathrm{H} & 4.723035 & -0.486869 & 0.411476\end{array}$

$\begin{array}{llll}\mathrm{H} & 4.400983 & 1.115793 & -0.263869\end{array}$

$\begin{array}{llll}\mathrm{H} & 4.065527 & 0.795407 & 1.437003\end{array}$

$\begin{array}{llll}\text { C } & 2.268668 & -0.881261 & -1.730598\end{array}$

$\mathrm{H} \quad 1.259593 \quad-1.202130 \quad-1.993367$

$\begin{array}{llll}\mathrm{H} & 2.595244 & -0.145029 & -2.467301\end{array}$

$\begin{array}{llll}\mathrm{H} & 2.934458 & -1.744182 & -1.800458\end{array}$

$\begin{array}{llll}\text { C } & 1.689869 & -1.419130 & 1.238069\end{array}$

$\begin{array}{llll}\mathrm{H} & 2.383577 & -2.259585 & 1.309903\end{array}$

$\begin{array}{llll}\mathrm{H} & 1.599689 & -0.973704 & 2.231003\end{array}$

$\begin{array}{llll}\mathrm{H} & 0.713127 & -1.809775 & 0.947951\end{array}$

$\begin{array}{llll}\text { C } & -0.747969 & 2.570045 & -0.035865\end{array}$

$\begin{array}{llll}\mathrm{H} & -0.099462 & 3.420549 & 0.147531\end{array}$

$\mathrm{H} \quad-1.307371 \quad 2.713981 \quad-0.961631$

$\begin{array}{llll}\mathrm{H} & -1.473820 & 2.489128 & 0.777028\end{array}$

$\begin{array}{llll}\text { Si } & -2.131136 & -0.372737 & 0.006674\end{array}$

$\begin{array}{llll}\text { C } & -3.508195 & 0.662844 & -0.711212\end{array}$

$\mathrm{H} \quad-4.465072 \quad 0.158734 \quad-0.556845$

$\begin{array}{llll}\mathrm{H} & -3.575941 & 1.649541 & -0.252027\end{array}$

$\begin{array}{llll}\mathrm{H} & -3.372958 & 0.795528 & -1.786470\end{array}$

$\begin{array}{llll}\text { C } & -2.208991 & -0.382269 & 1.869560\end{array}$

$\begin{array}{llll}\mathrm{H} & -2.122382 & 0.623039 & 2.286338\end{array}$

H $\quad-3.157768 \quad-0.801087 \quad 2.211675$

$\begin{array}{llll}\mathrm{H} & -1.402113 & -0.988345 & 2.285840\end{array}$

$\begin{array}{llll}\text { C } & -2.196331 & -2.095238 & -0.688848\end{array}$

$\mathrm{H} \quad-3.141573 \quad-2.581901 \quad-0.441153$

$\begin{array}{llll}\mathrm{H} & -2.101591 & -2.074241 & -1.775976\end{array}$ 


$\begin{array}{llll}\text { Zero-point correction }= & 0.277105 & & \\ \text { Thermal correction to Energy } & = & 0.297127 & \\ \text { Thermal correction to Enthalpy }= & 0.298071 & \\ \text { Thermal correction to Gibbs Free Energy } & = & 0.229153 \\ \text { Sum of electronic and zero-point Energies } & = & -1026.270615 \\ \text { Sum of electronic and thermal Energies = } & -1026.250592 \\ \text { Sum of electronic and thermal Enthalpies } & = & -1026.249648 \\ \text { Sum of electronic and thermal Free Energies } & = & -1026.318566 \\ \text { Number of imaginary frequencies/frequency } & = & 0\end{array}$

\section{BSA-O-trans-N-trans.out}

01 $\begin{array}{lll}-1.552257 & 1.244200 & -0.344397\end{array}$ $\begin{array}{lll}0.498077 & 0.312358 & -0.065194\end{array}$

$\mathrm{Si}$

$\begin{array}{lll}2.165051 & -0.127638 & -0.023203\end{array}$

C

$\begin{array}{lll}2.297712 & -1.794707 & -0.856893\end{array}$

$\mathrm{H}$

$\begin{array}{lll}3.324081 & -2.166425 & -0.829973\end{array}$

$\mathrm{H}$

$\begin{array}{lll}1.658835 & -2.526583 & -0.359746\end{array}$

$1.988491 \quad-1.734307 \quad-1.902148$

C

$\begin{array}{lll}2.634261 & -0.266520 & 1.780252\end{array}$

$\mathrm{H}$

$\begin{array}{lll}2.518679 & 0.692458 & 2.288980\end{array}$

$\mathrm{H}$

$\begin{array}{lll}2.000145 & -0.994683 & 2.288809\end{array}$

$\mathrm{H}$

$\begin{array}{lll}3.672917 & -0.584445 & 1.891869\end{array}$

C

$\begin{array}{lll}3.360434 & 1.054662 & -0.860770\end{array}$

$\mathrm{H}$

$\begin{array}{lll}4.375402 & 0.662234 & -0.758983\end{array}$

$\mathrm{H}$

$3.156632 \quad 1.161185-1.927992$

$\mathrm{H}$

$3.346593 \quad 2.047323 \quad-0.406570$

C

$0.208928 \quad 2.464580 \quad-1.286133$

$\mathrm{H}$

$\begin{array}{lll}-0.107497 & 3.355011 & -0.742005\end{array}$

$\mathrm{H}$

$\begin{array}{lll}1.281871 & 2.489210 & -1.441679\end{array}$

$\mathrm{H}$

$\begin{array}{lll}-0.305715 & 2.475856 & -2.247404\end{array}$

$\begin{array}{llll}\text { Si } & -2.375697 & 0.018125 & 0.479733\end{array}$

$\begin{array}{llll}\text { C } & -4.147599 & 0.585385 & 0.381397\end{array}$

$\begin{array}{llll}\mathrm{H} & -4.809562 & -0.125854 & 0.879476\end{array}$

$\mathrm{H}$

$\begin{array}{lll}-4.274103 & 1.557163 & 0.861404\end{array}$ 


$\begin{array}{llll}\mathrm{H} & -4.470968 & 0.677253 & -0.656763 \\ \mathrm{C} & -1.819116 & -0.062317 & 2.254702 \\ \mathrm{H} & -1.869229 & 0.923821 & 2.720541 \\ \mathrm{H} & -2.468408 & -0.732227 & 2.822980 \\ \mathrm{H} & -0.794397 & -0.424988 & 2.326418 \\ \mathrm{C} & -2.168277 & -1.610443 & -0.398643 \\ \mathrm{H} & -2.827973 & -2.361836 & 0.041282 \\ \mathrm{H} & -2.428855 & -1.516316 & -1.454666 \\ \mathrm{H} & -1.140326 & -1.964053 & -0.327752\end{array}$

$\begin{array}{llll}\text { Zero-point correction }= & 0.277437 & \\ \text { Thermal correction to Energy } & = & 0.297459 & \\ \text { Thermal correction to Enthalpy }= & 0.298403 & \\ \text { Thermal correction to Gibbs Free Energy } & = & 0.22897 \\ \text { Sum of electronic and zero-point Energies } & = & -1026.27627 \\ \text { Sum of electronic and thermal Energies }= & -1026.256248 \\ \text { Sum of electronic and thermal Enthalpies } & = & -1026.255304 \\ \text { Sum of electronic and thermal Free Energies } & = & -1026.324738 \\ \text { Number of imaginary frequencies/frequency } & = & 0\end{array}$

\section{BSA-OFCs.out}

$\begin{array}{llll}0 ~ 1 & & & \\ \mathrm{O} & -0.367822 & 2.282906 & 0.036851 \\ \mathrm{~N} & 1.174768 & 0.588872 & 0.040795 \\ \mathrm{Si} & 2.772842 & -0.048328 & 0.024838 \\ \mathrm{C} & 2.627979 & -1.913596 & -0.230158 \\ \mathrm{H} & 3.624287 & -2.354810 & -0.302709 \\ \mathrm{H} & 2.130056 & -2.406719 & 0.609761 \\ \mathrm{H} & 2.103760 & -2.161204 & -1.157760 \\ \mathrm{C} & 3.669311 & 0.180726 & 1.658047 \\ \mathrm{H} & 3.880139 & 1.228486 & 1.876311 \\ \mathrm{H} & 3.067375 & -0.211748 & 2.480545 \\ \mathrm{H} & 4.621577 & -0.354855 & 1.652284 \\ \mathrm{C} & 3.842380 & 0.586431 & -1.381401 \\ \mathrm{H} & 4.763066 & 0.002574 & -1.453051 \\ \mathrm{H} & 3.315322 & 0.499714 & -2.334242 \\ \mathrm{H} & 4.122197 & 1.632199 & -1.250218 \\ & & & \end{array}$




\begin{tabular}{|c|c|c|c|}
\hline C & 2.978241 & \multicolumn{2}{|l|}{0.001609} \\
\hline $\mathrm{H}$ & 2.714736 & \multicolumn{2}{|l|}{0.486247} \\
\hline $\mathrm{H}$ & 3.217305 & \multicolumn{2}{|l|}{-1.043193} \\
\hline $\mathrm{H}$ & 3.862881 & \multicolumn{2}{|l|}{0.471741} \\
\hline $\mathrm{Si}$ & 1.240428 & \multicolumn{2}{|l|}{-0.007212} \\
\hline $\mathrm{C}$ & 0.685050 & \multicolumn{2}{|l|}{1.772935} \\
\hline $\mathrm{H}$ & -0.072702 & \multicolumn{2}{|l|}{2.174021} \\
\hline $\mathrm{H}$ & 0.383631 & \multicolumn{2}{|l|}{1.914999} \\
\hline $\mathrm{H}$ & 1.582986 & \multicolumn{2}{|l|}{2.383391} \\
\hline $\mathrm{C}$ & 0.741348 & \multicolumn{2}{|l|}{-1.787155} \\
\hline $\mathrm{H}$ & 1.657835 & \multicolumn{2}{|l|}{-2.373479} \\
\hline $\mathrm{H}$ & 0.443901 & \multicolumn{2}{|l|}{-1.902320} \\
\hline $\mathrm{H}$ & -0.003900 & \multicolumn{2}{|l|}{-2.235758} \\
\hline $\mathrm{C}$ & 2.728003 & \multicolumn{2}{|l|}{-0.005489} \\
\hline $\mathrm{H}$ & 2.711605 & \multicolumn{2}{|l|}{-0.893239} \\
\hline $\mathrm{H}$ & 2.689617 & \multicolumn{2}{|l|}{0.861956} \\
\hline $\mathrm{H}$ & 3.666656 & 0.014722 & \\
\hline $\mathrm{F}$ & 0.019308 & -0.049867 & \\
\hline Cs & -1.887849 & -0.036200 & \\
\hline Zero & 0.2 & 280902 & \\
\hline Ther & $=$ & 0.3 & \\
\hline Ther & py & 0.3 & \\
\hline Ther & Free Energy & $=$ & 0.228614 \\
\hline Sum & oint Energies & $=$ & -1146.306418 \\
\hline Sum & 1 Energies= & & 33696 \\
\hline Sum & ll Enthalpies & $=$ & -1146.282752 \\
\hline Sum & l Free Energi & gies & -1146.358706 \\
\hline Nun & ncies/frequen & ncy & 0 \\
\hline
\end{tabular}

\section{Cs-base.out}

01

$\begin{array}{llll}\text { O } & 0.182229 & 1.265883 & 2.293996\end{array}$

$\begin{array}{llll}\mathrm{N} & 1.681714 & 0.168371 & 0.990449\end{array}$

$\begin{array}{llll}\mathrm{Si} & 2.998058 & -0.049606 & -0.069504\end{array}$

$\begin{array}{lllll}\text { C } & 2.922262 & -1.839592 & -0.647094\end{array}$

$\begin{array}{llll}\mathrm{H} & 3.747360 & -2.085357 & -1.318938\end{array}$ 


$\begin{array}{llll}\mathrm{H} & 2.974739 & -2.522264 & 0.205235 \\ \mathrm{H} & 1.988686 & -2.034058 & -1.181191 \\ \mathrm{C} & 4.667231 & 0.193176 & 0.766023 \\ \mathrm{H} & 4.800884 & 1.219609 & 1.113304 \\ \mathrm{H} & 4.762104 & -0.463938 & 1.633083 \\ \mathrm{H} & 5.485947 & -0.034289 & 0.078997 \\ \mathrm{C} & 2.964973 & 1.031775 & -1.611482 \\ \mathrm{H} & 3.714850 & 0.694389 & -2.331345 \\ \mathrm{H} & 1.988182 & 0.985354 & -2.098336 \\ \mathrm{H} & 3.173853 & 2.077843 & -1.381200 \\ \mathrm{C} & 1.735557 & 2.640879 & 1.158409 \\ \mathrm{H} & 2.824315 & 2.626407 & 1.100174 \\ \mathrm{H} & 1.354287 & 2.935794 & 0.178377 \\ \mathrm{H} & 1.411626 & 3.374274 & 1.892872 \\ \mathrm{Cs} & -0.342678 & -1.440788 & 2.248342\end{array}$

\begin{tabular}{llll} 
Zero-point correction & \multicolumn{1}{l}{0.163788} & & \\
Thermal correction to Energy & & 0.177901 & \\
Thermal correction to Enthalpy & 0.178845 & \\
Thermal correction to Gibbs Free Energy & $=$ & 0.120793 \\
Sum of electronic and zero-point Energies & $=$ & -637.258278 \\
Sum of electronic and thermal Energies = & -637.244166 \\
Sum of electronic and thermal Enthalpies & $=$ & -637.243222 \\
Sum of electronic and thermal Free Energies & $=$ & -637.301273 \\
Number of imaginary frequencies/frequency & $=$ & 0
\end{tabular}

\section{Cs-TMSF-CH3CN-TMSO.out}

$\begin{array}{llll}01 & & & \\ \mathrm{Si} & 3.850023 & -0.458794 & 0.400663 \\ \mathrm{C} & 3.741590 & -2.162725 & -0.442133 \\ \mathrm{H} & 4.719201 & -2.644640 & -0.519335 \\ \mathrm{H} & 3.095815 & -2.844429 & 0.121995 \\ \mathrm{H} & 3.343842 & -2.079020 & -1.458997 \\ \mathrm{C} & 4.645572 & -0.765718 & 2.081883 \\ \mathrm{H} & 4.789051 & 0.176827 & 2.615308 \\ \mathrm{H} & 4.009169 & -1.400475 & 2.703273 \\ \mathrm{H} & 5.619891 & -1.252254 & 1.990435\end{array}$




\begin{tabular}{|c|c|c|c|}
\hline$C$ & 5.092889 & 0.542306 & -0.611929 \\
\hline $\mathrm{H}$ & 6.059602 & 0.038905 & -0.690294 \\
\hline $\mathrm{H}$ & 4.721246 & 0.712481 & -1.626035 \\
\hline & 5.266371 & 1.519734 & -0.153455 \\
\hline & 2.056030 & 2.996523 & -0.533386 \\
\hline & 2.512389 & 2.216234 & 0.091316 \\
\hline & 2.702627 & 3.228451 & -1.378075 \\
\hline & 1.851050 & 3.903046 & 0.033402 \\
\hline & -3.513520 & 1.413640 & 0.049700 \\
\hline 0 & -2.750562 & 2.881419 & 0.886728 \\
\hline & -2.589743 & 2.689747 & 1.948947 \\
\hline & -1.792525 & 3.125706 & 0.427289 \\
\hline $\mathrm{H}$ & -3.405020 & 3.751123 & 0.797837 \\
\hline . & -3.678943 & 1.635158 & -1.783157 \\
\hline $\mathrm{H}$ & -4.345821 & 2.470373 & -2.007341 \\
\hline & -2.704040 & 1.845227 & -2.224701 \\
\hline & -4.094267 & 0.741769 & -2.252987 \\
\hline${ }^{C}$ & -5.126405 & 0.933357 & 0.840741 \\
\hline & -5.536798 & 0.031313 & 0.383885 \\
\hline $\mathrm{H}$ & -5.001267 & 0.747562 & 1.908744 \\
\hline $\mathrm{H}$ & -5.862237 & 1.731658 & 0.724004 \\
\hline $\mathrm{F}$ & -2.513716 & 0.149033 & 0.288519 \\
\hline $\mathrm{Cs}$ & 0.186584 & -1.007754 & -0.306882 \\
\hline $\mathrm{O}$ & 2.418912 & 0.231003 & 0.476848 \\
\hline & -0.169821 & 1.941731 & -1.366456 \\
\hline
\end{tabular}

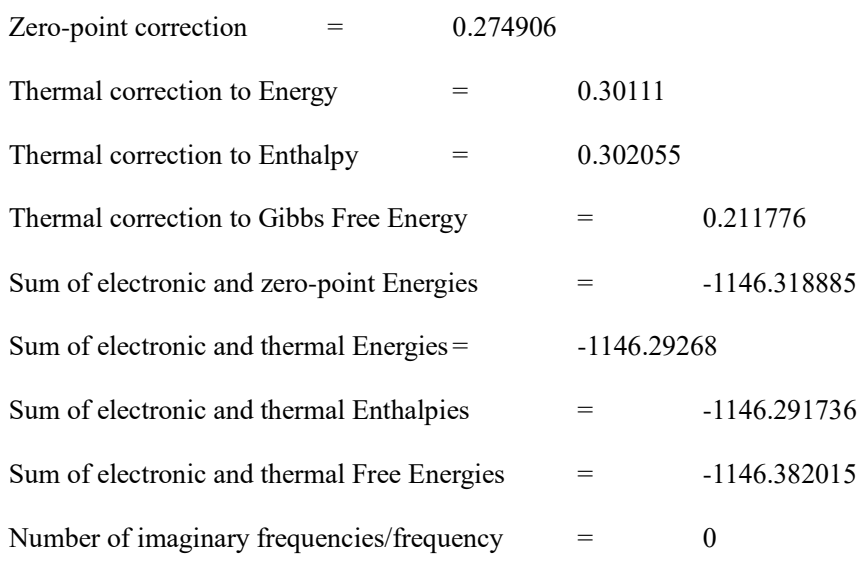

\section{CsF.out}


01

F

$\begin{array}{lll}0.000000 & 0.000000 & -2.058564\end{array}$

$\begin{array}{lll}\text { Zero-point correction }=0.000821 & \\ \text { Thermal correction to Energy } & = & 0.003531 \\ \text { Thermal correction to Enthalpy }= & 0.004475 & -0.023139 \\ \text { Thermal correction to Gibbs Free Energy } & = & -119.994006 \\ \text { Sum of electronic and zero-point Energies } & = & -119.991295 \\ \text { Sum of electronic and thermal Energies = } & = & -119.990351 \\ \text { Sum of electronic and thermal Enthalpies } & = & -120.017965 \\ \text { Sum of electronic and thermal Free Energies } & = & 0\end{array}$

\section{I-NTMS-cleave.out}

01

$\begin{array}{lll}1.592564 & 1.368535 & 0.400812\end{array}$

$\begin{array}{llll}\text { C } & 1.123623 & -0.397598 & 0.775778\end{array}$

$\begin{array}{llll}\mathrm{H} & 1.566977 & -1.102911 & 0.066598\end{array}$

$\begin{array}{llll}\mathrm{H} & 1.493621 & -0.671343 & 1.765803\end{array}$

$\begin{array}{llll}\mathrm{H} & 0.035812 & -0.489452 & 0.782431\end{array}$

$\begin{array}{llll}\text { C } & 0.754842 & 2.569277 & 1.530171\end{array}$

$\begin{array}{llll}\mathrm{H} & 1.084870 & 3.590925 & 1.334951\end{array}$

H $\quad-0.321560 \quad 2.503725 \quad 1.362651$

$\begin{array}{llll}\mathrm{H} & 0.966534 & 2.337515 & 2.575297\end{array}$

$\begin{array}{llll}\text { C } & 1.379568 & 1.825442 & -1.398458\end{array}$

$\begin{array}{llll}\mathrm{H} & 0.319378 & 1.964410 & -1.615924\end{array}$

$\begin{array}{llll}\mathrm{H} & 1.892820 & 2.767888 & -1.599158\end{array}$

$\begin{array}{llll}\mathrm{H} & 1.815869 & 1.078289 & -2.067586\end{array}$

$\begin{array}{llll}\text { C } & -2.927560 & 2.450813 & -1.445592\end{array}$

$\begin{array}{llll}\mathrm{H} & -2.846688 & 3.221541 & -0.677563\end{array}$

$\begin{array}{llll}\mathrm{H} & -2.157340 & 2.671019 & -2.190717\end{array}$

$\begin{array}{llll}\mathrm{H} & -3.909077 & 2.487131 & -1.909039\end{array}$

Si $\quad-3.792701 \quad-1.127060 \quad 0.157989$

$\begin{array}{llll}\text { C } & -3.718046 & -0.511503 & 1.926071\end{array}$

$\begin{array}{llll}\mathrm{H} & -3.886897 & -1.317865 & 2.642994\end{array}$

$\begin{array}{llll}\mathrm{H} & -2.740824 & -0.066868 & 2.125030\end{array}$

$\begin{array}{llll}\mathrm{H} & -4.476153 & 0.255885 & 2.093846\end{array}$ 


$\begin{array}{llll}\mathrm{C} & -2.569717 & -2.588015 & 0.068717 \\ \mathrm{H} & -1.553130 & -2.314017 & 0.367210 \\ \mathrm{H} & -2.902502 & -3.350125 & 0.777956 \\ \mathrm{H} & -2.546578 & -3.077445 & -0.910685 \\ \mathrm{C} & -5.469078 & -1.882940 & -0.192428 \\ \mathrm{H} & -5.696677 & -2.704073 & 0.490610 \\ \mathrm{H} & -6.249113 & -1.126335 & -0.092868 \\ \mathrm{H} & -5.514028 & -2.266818 & -1.214064 \\ \mathrm{~F} & 3.184937 & 1.452776 & 0.662831 \\ \mathrm{Cs} & -0.808707 & -0.913399 & -2.198579 \\ \mathrm{~N} & -3.556399 & 0.153516 & -0.955444 \\ \mathrm{O} & -1.526549 & 0.970078 & -0.241285\end{array}$

\section{Zero-point correction $\quad=\quad 0.27875$}

Thermal correction to Energy $\quad=\quad 0.302839$

Thermal correction to Enthalpy $\quad=\quad 0.303783$

Thermal correction to Gibbs Free Energy $\quad=\quad 0.221727$

Sum of electronic and zero-point Energies $\quad=\quad-1146.30492$

Sum of electronic and thermal Energies $=\quad-1146.280831$

Sum of electronic and thermal Enthalpies $\quad=\quad-1146.279887$

Sum of electronic and thermal Free Energies $\quad=\quad-1146.361943$

Number of imaginary frequencies/frequency $\quad=\quad 0$

\section{I-OTMS-cleave.out}

01

$\begin{array}{llll}\text { O } & 0.097297 & 1.280809 & 2.167194\end{array}$

$\begin{array}{llll}\mathrm{N} & 1.678372 & 0.175779 & 0.974255\end{array}$

Si $\quad 3.009568 \quad-0.050639 \quad-0.068039$

$\begin{array}{llll}\text { C } & 2.930131 & -1.840749 & -0.639572\end{array}$

$\begin{array}{llll}\mathrm{H} & 3.753251 & -2.092741 & -1.311414\end{array}$

$\begin{array}{llll}\mathrm{H} & 2.978301 & -2.521206 & 0.214670\end{array}$

$\mathrm{H} \quad 1.993990 \quad-2.030275 \quad-1.171124$

$\begin{array}{llll}\text { C } & 4.671786 & 0.194767 & 0.779255\end{array}$

$\begin{array}{llll}\mathrm{H} & 4.807715 & 1.223457 & 1.118684\end{array}$

$\begin{array}{llll}\mathrm{H} & 4.756413 & -0.455492 & 1.652554\end{array}$

$\begin{array}{llll}\mathrm{H} & 5.494853 & -0.043194 & 0.101099\end{array}$

C $\quad 2.974126 \quad 1.028164-1.611592$ 


\begin{tabular}{|c|c|c|c|}
\hline & 3.739550 & 0.707175 & -2.322496 \\
\hline $\mathrm{H}$ & 2.003931 & 0.957639 & -2.109513 \\
\hline & 3.155445 & 2.079415 & -1.381097 \\
\hline $\mathrm{C}$ & 1.741226 & 2.647422 & 1.164752 \\
\hline & 2.825847 & 2.618287 & 1.063467 \\
\hline $\mathrm{H}$ & 1.324644 & 2.996406 & 0.215614 \\
\hline & 1.456991 & 3.353120 & 1.942250 \\
\hline & -2.672368 & 1.076813 & -0.047682 \\
\hline 0 & -2.712415 & 2.503855 & 1.127684 \\
\hline & -3.575262 & 2.437933 & 1.793317 \\
\hline $\mathrm{H}$ & -1.797081 & 2.515092 & 1.722796 \\
\hline L & -2.788083 & 3.443280 & 0.575173 \\
\hline & -1.179906 & 1.045789 & -1.149473 \\
\hline $\mathrm{H}$ & -1.045751 & 2.011576 & -1.641804 \\
\hline & -0.275206 & 0.831123 & -0.575038 \\
\hline $\mathrm{H}$ & -1.281149 & 0.286725 & -1.928044 \\
\hline $\mathrm{C}$ & -4.247130 & 0.950755 & -1.033804 \\
\hline $\mathrm{H}$ & -4.249303 & 0.061294 & -1.666091 \\
\hline $\mathrm{H}$ & -5.119677 & 0.907422 & -0.379775 \\
\hline $\mathrm{H}$ & -4.359563 & 1.822290 & -1.682336 \\
\hline $\mathrm{F}$ & -2.614284 & -0.299155 & 0.835481 \\
\hline 0 & -0.335712 & -1.490636 & 2.285485 \\
\hline
\end{tabular}

$\begin{array}{llll}\text { Zero-point correction }= & 0.278171 & \\ \text { Thermal correction to Energy } & = & 0.302708 & \\ \text { Thermal correction to Enthalpy }= & 0.303652 & \\ \text { Thermal correction to Gibbs Free Energy } & = & 0.219396 \\ \text { Sum of electronic and zero-point Energies } & = & -1146.323486 \\ \text { Sum of electronic and thermal Energies }= & -1146.298948 \\ \text { Sum of electronic and thermal Enthalpies } & = & -1146.298004 \\ \text { Sum of electronic and thermal Free Energies } & = & -1146.382261 \\ \text { Number of imaginary frequencies/frequency } & = & 0\end{array}$

\section{TMSF.out}

01

$\begin{array}{llll}\text { C } & 0.006489 & 1.777564 & -0.517765\end{array}$

$\begin{array}{llll}\mathrm{H} & -0.874440 & 2.304317 & -0.147394\end{array}$ 


$\begin{array}{llll}\mathrm{H} & 0.891956 & 2.296801 & -0.147598 \\ \mathrm{H} & 0.006658 & 1.846549 & -1.607487 \\ \mathrm{C} & -1.541761 & -0.878170 & -0.525568 \\ \mathrm{H} & -1.598550 & -0.907914 & -1.615587 \\ \mathrm{H} & -1.558522 & -1.905989 & -0.159522 \\ \mathrm{H} & -2.435234 & -0.372551 & -0.155792 \\ \mathrm{C} & 1.532224 & -0.891111 & -0.525880 \\ \mathrm{H} & 2.429988 & -0.392903 & -0.156435 \\ \mathrm{H} & 1.540516 & -1.918980 & -0.159688 \\ \mathrm{H} & 1.588449 & -0.921476 & -1.615911 \\ \mathrm{~F} & -0.000837 & -0.003617 & 1.638932\end{array}$

\begin{tabular}{|c|c|c|c|}
\hline Zero-point correction & 0.113 & & \\
\hline Thermal correction to Energy & $=$ & \multicolumn{2}{|c|}{0.122071} \\
\hline Thermal correction to Enthalpy & $=$ & \multicolumn{2}{|c|}{0.123015} \\
\hline \multicolumn{2}{|c|}{ Thermal correction to Gibbs Free Energy } & $=$ & 0.081872 \\
\hline \multicolumn{2}{|c|}{ Sum of electronic and zero-point Energies } & $=$ & -509.047879 \\
\hline \multicolumn{2}{|c|}{ Sum of electronic and thermal Energies $=$} & \multicolumn{2}{|c|}{-509.039492} \\
\hline \multicolumn{2}{|c|}{ Sum of electronic and thermal Enthalpies } & $=$ & -509.038547 \\
\hline \multicolumn{2}{|c|}{ Sum of electronic and thermal Free Energies } & $=$ & -509.07969 \\
\hline Number of imaginary frequencie & uency & $=$ & 0 \\
\hline
\end{tabular}

TS-NTMS-cleave.out

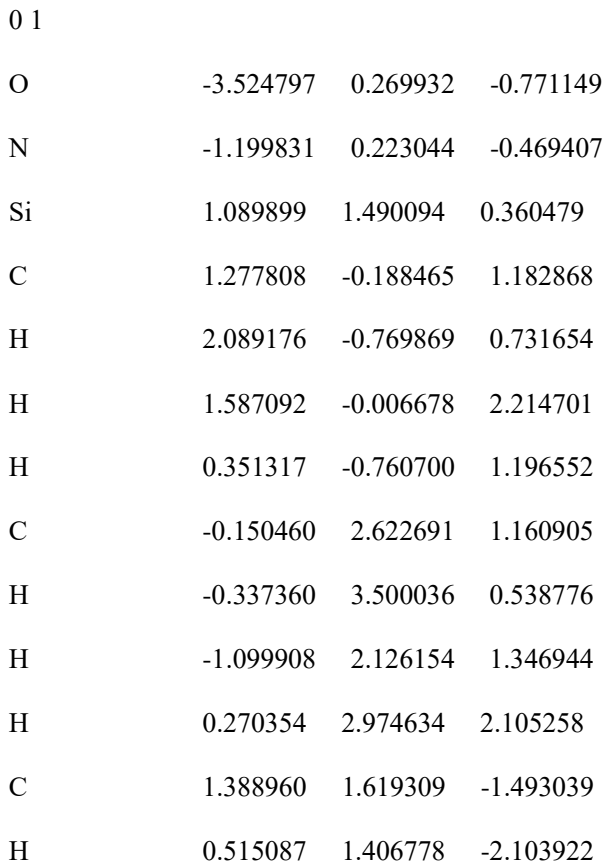




\begin{tabular}{|c|c|c|c|}
\hline 4 & 1.718971 & 2.639616 & -1.703390 \\
\hline $\mathrm{H}$ & 2.225295 & 0.975212 & -1.787195 \\
\hline $\mathrm{C}$ & -2.362467 & 2.236745 & -1.366714 \\
\hline & -1.385249 & 2.680230 & -1.544309 \\
\hline & -2.943334 & 2.233813 & -2.290225 \\
\hline & -2.907204 & 2.851319 & -0.646944 \\
\hline & -3.803508 & -1.042067 & 0.197092 \\
\hline & -3.340413 & -0.705686 & 1.973239 \\
\hline & -3.600856 & -1.553363 & 2.611496 \\
\hline & -2.269288 & -0.519075 & 2.056968 \\
\hline & -3.871469 & 0.171204 & 2.349115 \\
\hline & -2.931197 & -2.609898 & -0.388968 \\
\hline $\mathrm{H}$ & -1.958925 & -2.702241 & 0.098743 \\
\hline & -3.515505 & -3.500856 & -0.14820 \\
\hline & -2.797635 & -2.588761 & -1.47428 \\
\hline $\mathrm{C}$ & -5.643249 & -1.338258 & 0.058108 \\
\hline & -5.955740 & -2.174555 & 0.687369 \\
\hline $\mathrm{H}$ & -6.201448 & -0.453211 & 0.367507 \\
\hline $\mathrm{H}$ & -5.924856 & -1.566397 & -0.97175 \\
\hline & 2.509384 & 2.161388 & 0.843815 \\
\hline & 0.298625 & -1.673588 & -1.701439 \\
\hline
\end{tabular}

\begin{tabular}{llll} 
Zero-point correction & \multicolumn{1}{l}{0.278861} & & \\
Thermal correction to Energy & $=$ & 0.301737 & \\
Thermal correction to Enthalpy & & 0.302681 & \\
Thermal correction to Gibbs Free Energy & $=$ & 0.225124 \\
Sum of electronic and zero-point Energies & $=$ & -1146.277156 \\
Sum of electronic and thermal Energies $=$ & -1146.25428 \\
Sum of electronic and thermal Enthalpies & $=$ & -1146.253336 \\
Sum of electronic and thermal Free Energies & $=$ & -1146.330893 \\
Number of imaginary frequencies/frequency & $=$ & 1
\end{tabular}

\section{TS-OTMS-cleave.out}

01

$\begin{array}{llll}\text { O } & -0.033325 & 2.401308 & 0.060313\end{array}$

$\mathrm{N} \quad \begin{array}{llll}1.360356 & 0.585276 & -0.007445\end{array}$

$\begin{array}{llll}\mathrm{Si} & 2.922017 & -0.108735 & 0.024221\end{array}$ 


\begin{tabular}{|c|c|c|c|}
\hline $\mathrm{C}$ & 2.760575 & -1.980479 & -0.261856 \\
\hline $\mathrm{H}$ & 3.764188 & -2.410197 & -0.295359 \\
\hline $\mathrm{H}$ & 2.238257 & -2.497918 & 0.548849 \\
\hline $\mathrm{H}$ & 2.286320 & -2.229030 & -1.216365 \\
\hline $\mathrm{C}$ & 3.794474 & 0.027482 & 1.687353 \\
\hline $\mathrm{H}$ & 4.020540 & 1.060619 & 1.954980 \\
\hline $\mathrm{H}$ & 3.168494 & -0.386178 & 2.481334 \\
\hline $\mathrm{H}$ & 4.736768 & -0.526118 & 1.678015 \\
\hline $\mathrm{C}$ & 4.117215 & 0.464172 & -1.314673 \\
\hline $\mathrm{H}$ & 4.965691 & -0.221056 & -1.384278 \\
\hline $\mathrm{H}$ & 3.625489 & 0.490655 & -2.289864 \\
\hline $\mathrm{H}$ & 4.512991 & 1.460395 & -1.115112 \\
\hline $\mathrm{C}$ & 2.254434 & 2.910303 & -0.051657 \\
\hline $\mathrm{H}$ & 3.100519 & 2.627248 & 0.575060 \\
\hline $\mathrm{H}$ & 2.609948 & 2.970253 & -1.082423 \\
\hline $\mathrm{H}$ & 1.891073 & 3.889828 & 0.248154 \\
\hline $\mathrm{Si}$ & -2.581930 & 1.126012 & -0.017934 \\
\hline $\mathrm{C}$ & -1.743199 & 0.903156 & 1.639711 \\
\hline $\mathrm{H}$ & -2.340190 & 0.234754 & 2.267618 \\
\hline $\mathrm{H}$ & -0.706141 & 0.572565 & 1.586540 \\
\hline $\mathrm{H}$ & -1.698844 & 1.880507 & 2.121465 \\
\hline $\mathrm{C}$ & -1.726178 & 1.091757 & -1.682787 \\
\hline $\mathrm{H}$ & -1.683001 & 2.116520 & -2.053183 \\
\hline $\mathrm{H}$ & -0.688509 & 0.760295 & -1.658373 \\
\hline $\mathrm{H}$ & -2.313944 & 0.494536 & -2.386571 \\
\hline $\mathrm{C}$ & -3.767041 & 2.550864 & 0.054564 \\
\hline $\mathrm{H}$ & -4.398155 & 2.591339 & -0.834376 \\
\hline $\mathrm{H}$ & -4.407781 & 2.492256 & 0.935583 \\
\hline $\mathrm{H}$ & -3.193701 & 3.478976 & 0.109766 \\
\hline $\mathrm{F}$ & -3.459915 & -0.270063 & -0.103300 \\
\hline Cs & -0.578050 & -1.697960 & -0.177932 \\
\hline
\end{tabular}

$\begin{array}{llll}\text { Zero-point correction }= & 0.280308 & & \\ \text { Thermal correction to Energy } & = & 0.302637 & \\ \text { Thermal correction to Enthalpy } & = & 0.303581 & \\ \text { Thermal correction to Gibbs Free Energy } & = & -1146.294811 \\ \text { Sum of electronic and zero-point Energies } & = & -1146.272482\end{array}$


Sum of electronic and thermal Free Energies

$=\quad-1146.271538$

Number of imaginary frequencies/frequency

$-1146.347488$

1 
IX: ${ }^{1} \mathrm{H}$ NMR, ${ }^{13} \mathrm{C}$ NMR Spectra of Starting Materials and Products
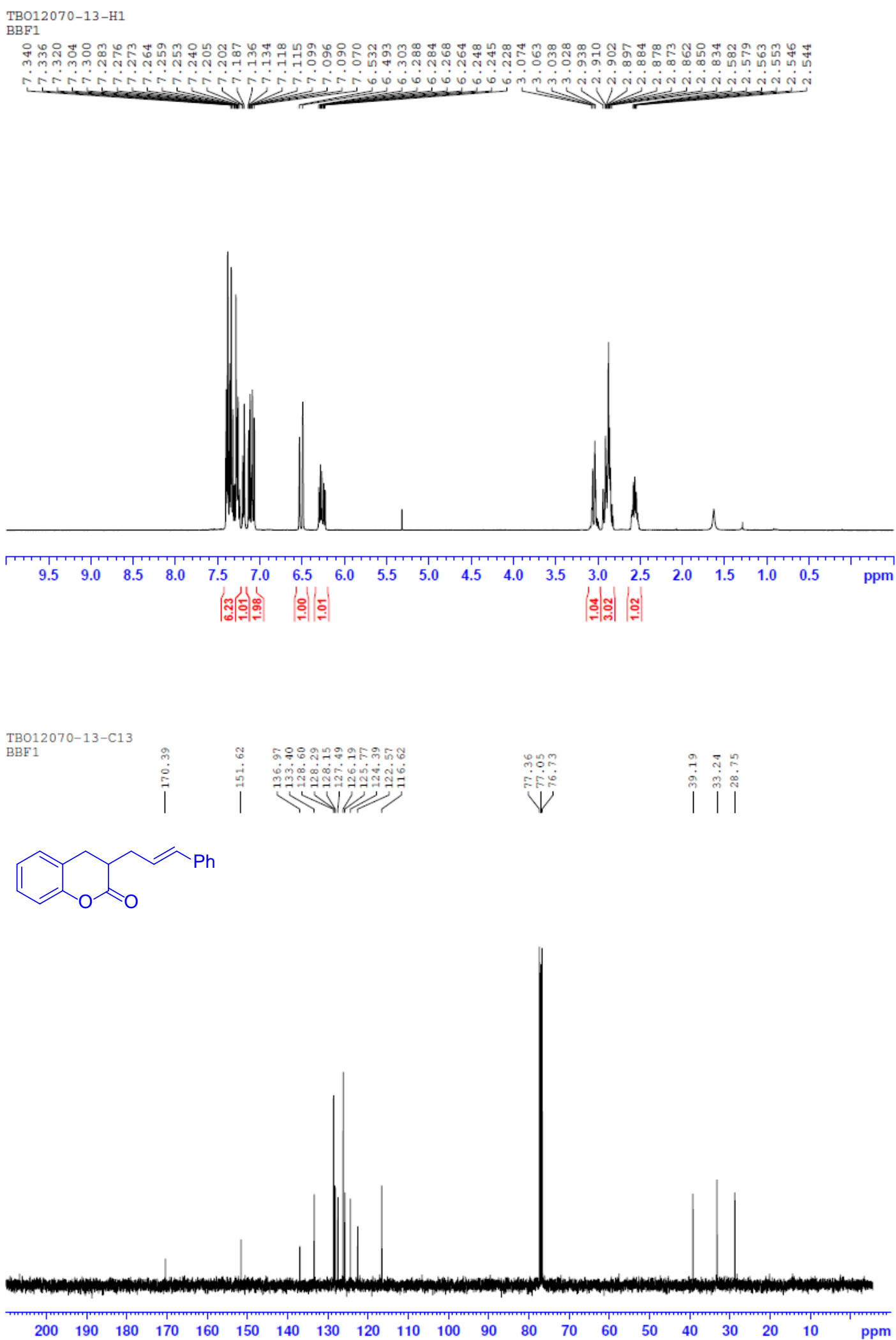

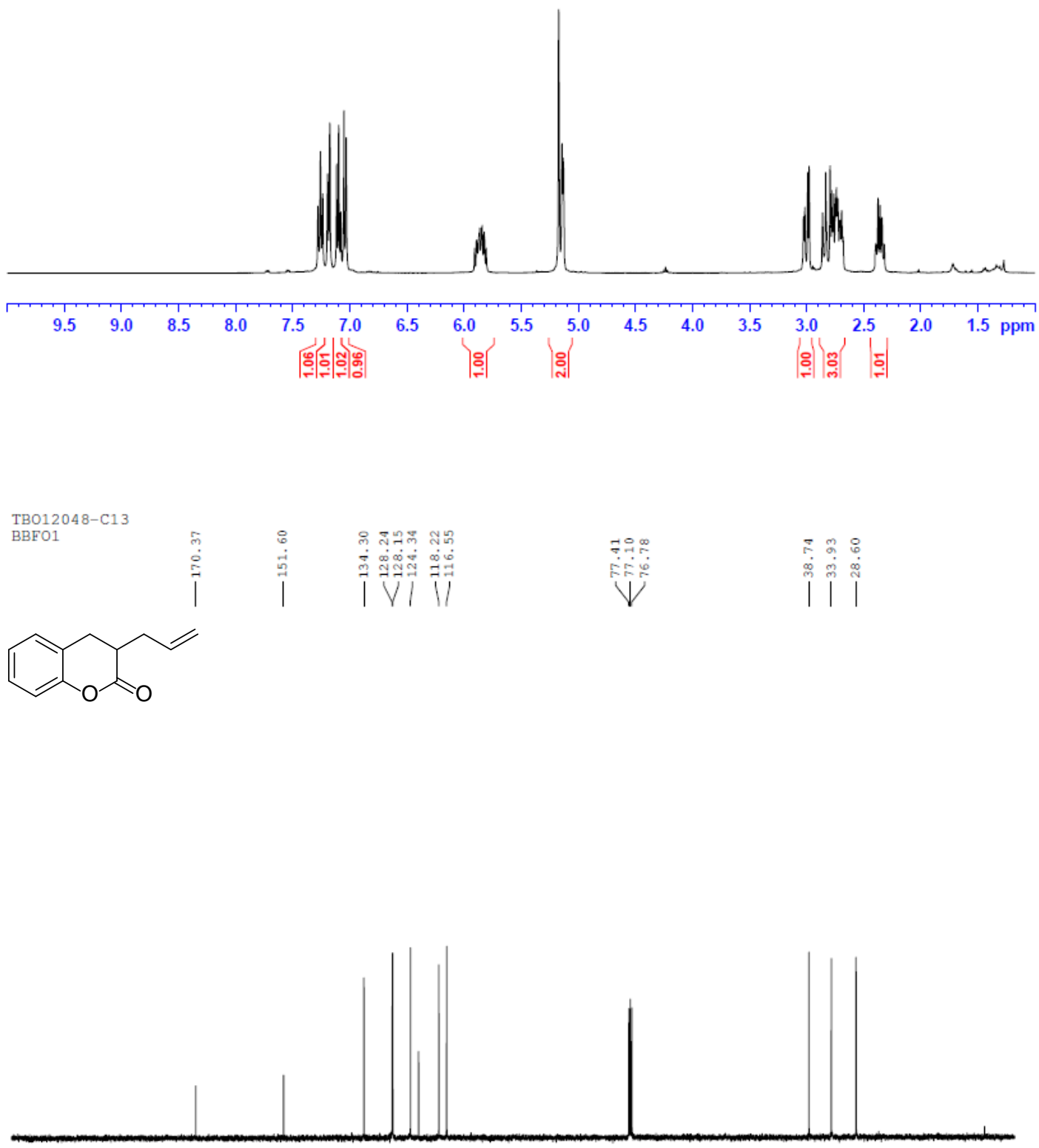

$\begin{array}{lllllllllllllllllllll}200 & 190 & 180 & 170 & 160 & 150 & 140 & 130 & 120 & 110 & 100 & 90 & 80 & 70 & 60 & 50 & 40 & 30 & 20 & 10 & \mathrm{ppm}\end{array}$ 

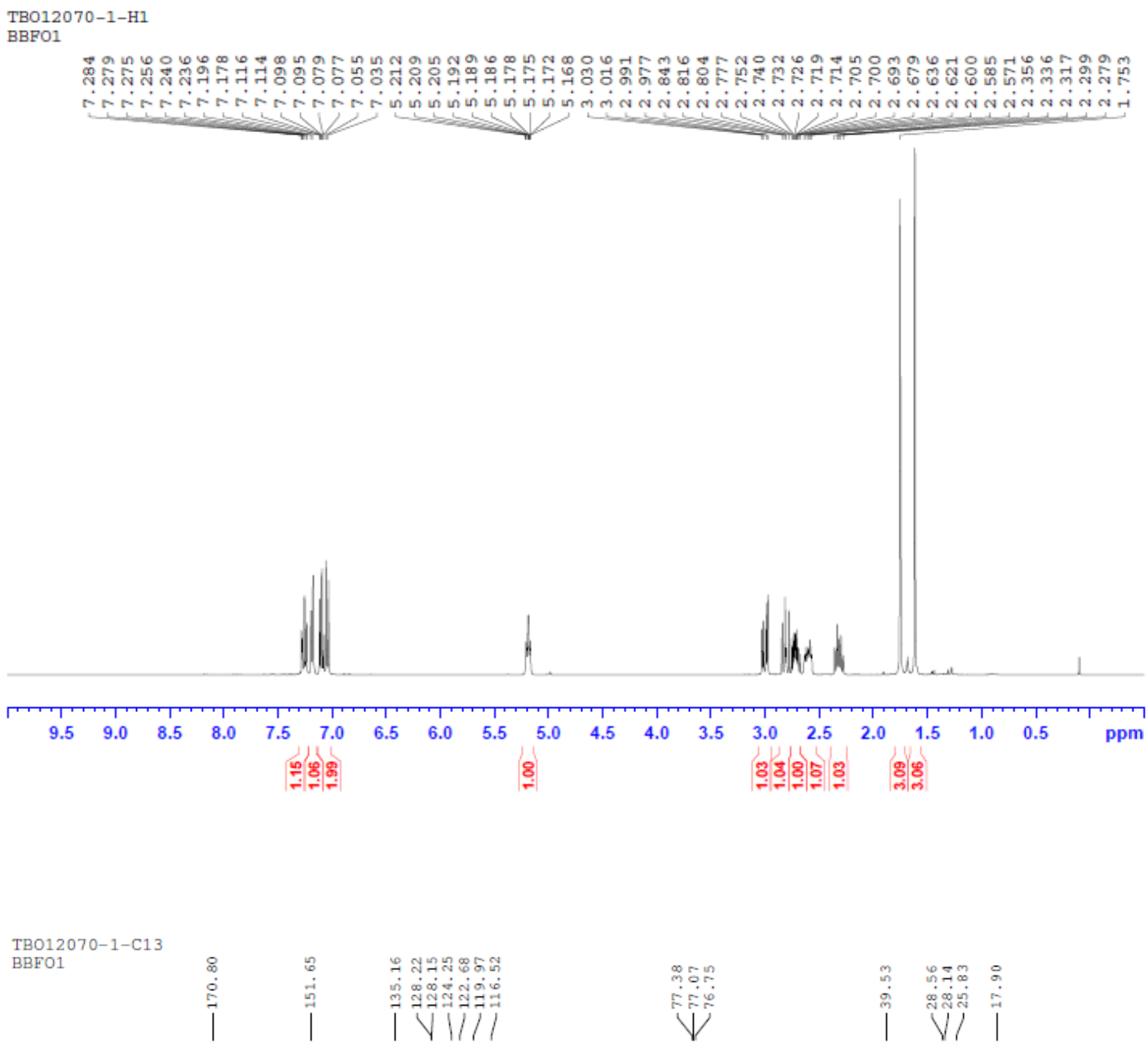<smiles>CC(C)=CCC1Cc2ccccc2OC1=O</smiles>

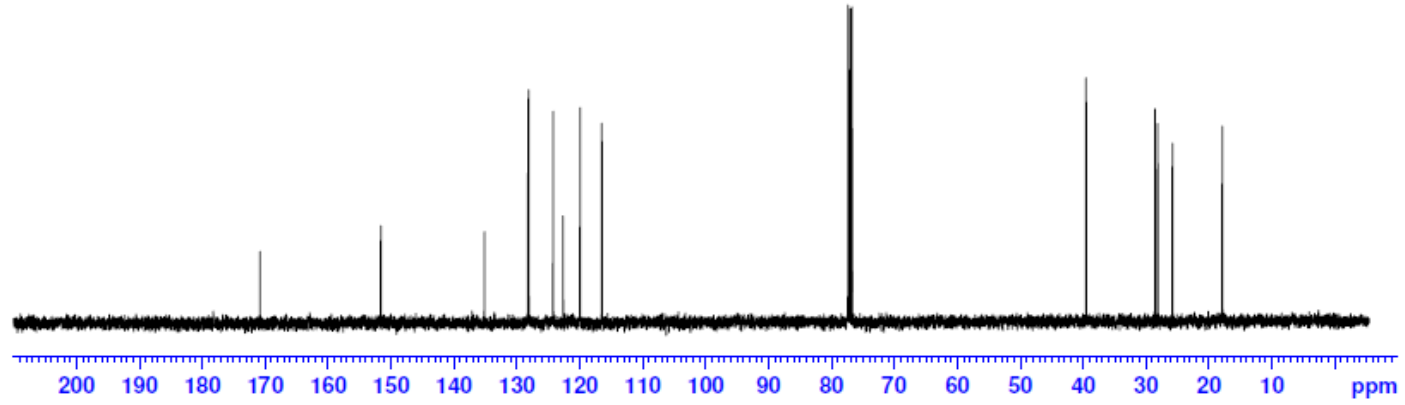




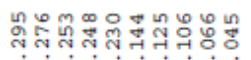

$\operatorname{rin} \cos 6$

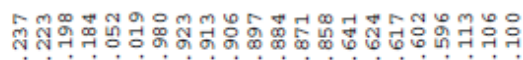

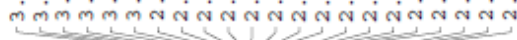

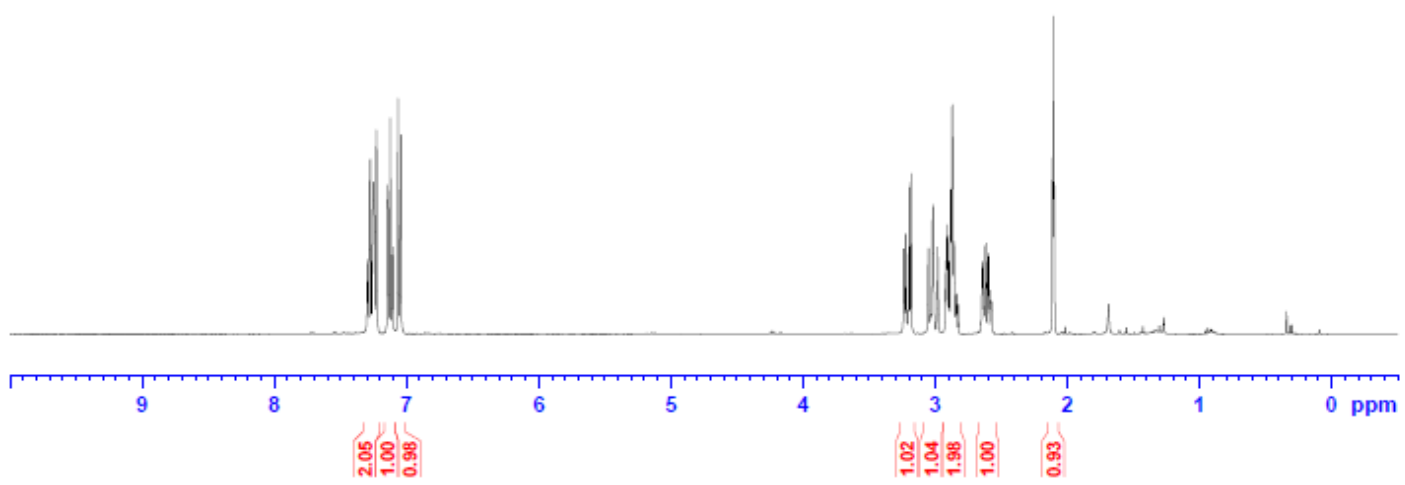

TBO12071-C13<smiles>C#CCC1Cc2ccccc2OC1=O</smiles>

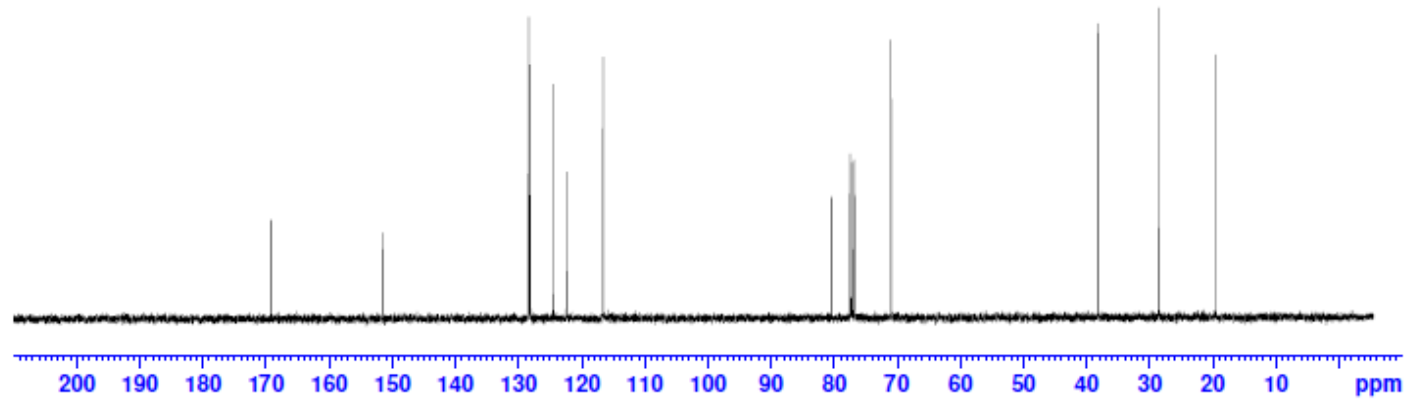




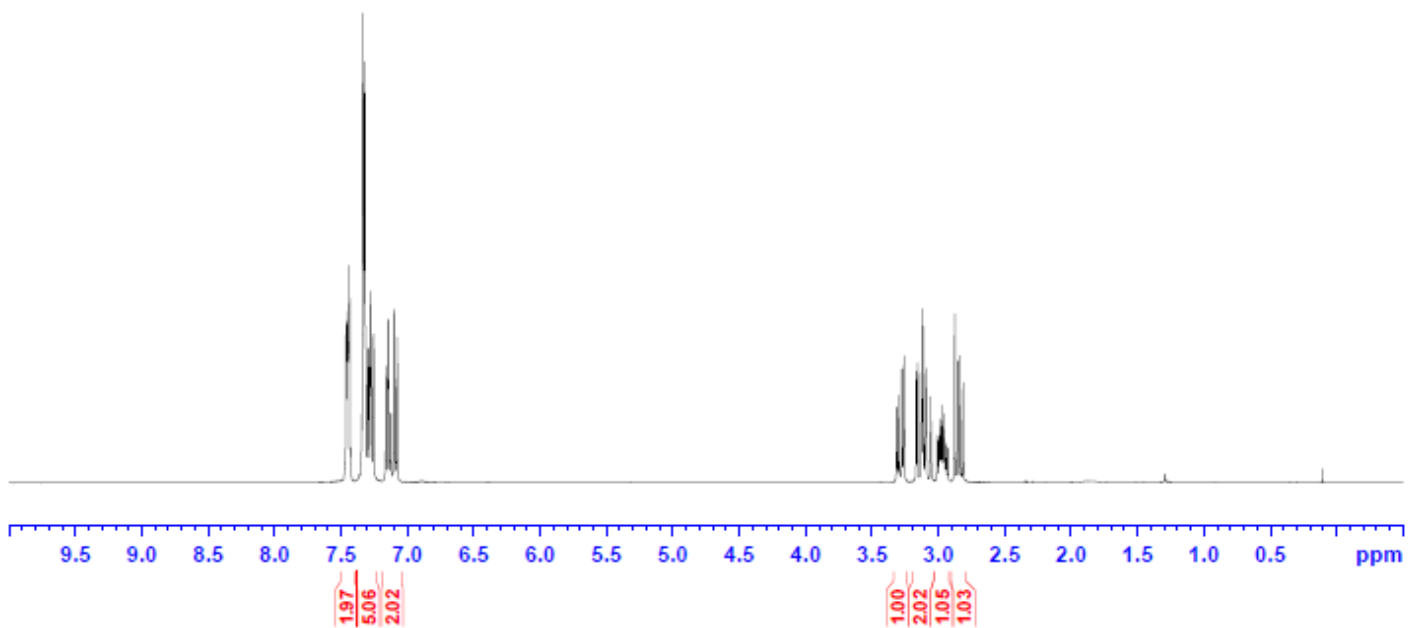<smiles>O=C1Oc2ccccc2CC1CC#Cc1ccccc1</smiles>

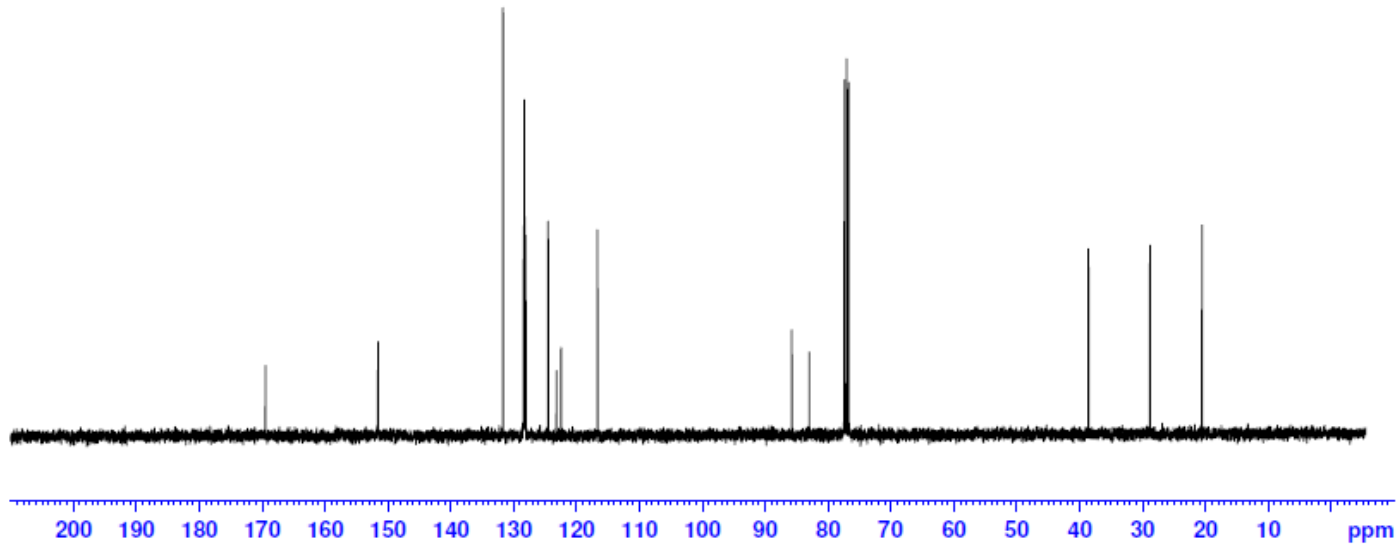


with the

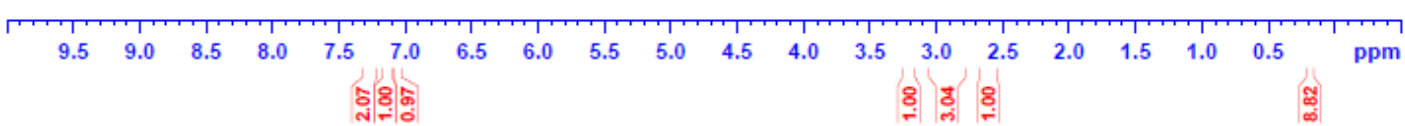

TB012070-4-C13
BBF01

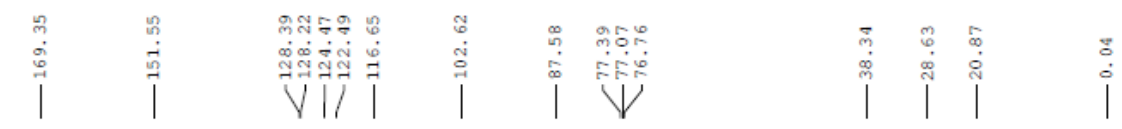

$\mathrm{N}_{\mathrm{T}}$

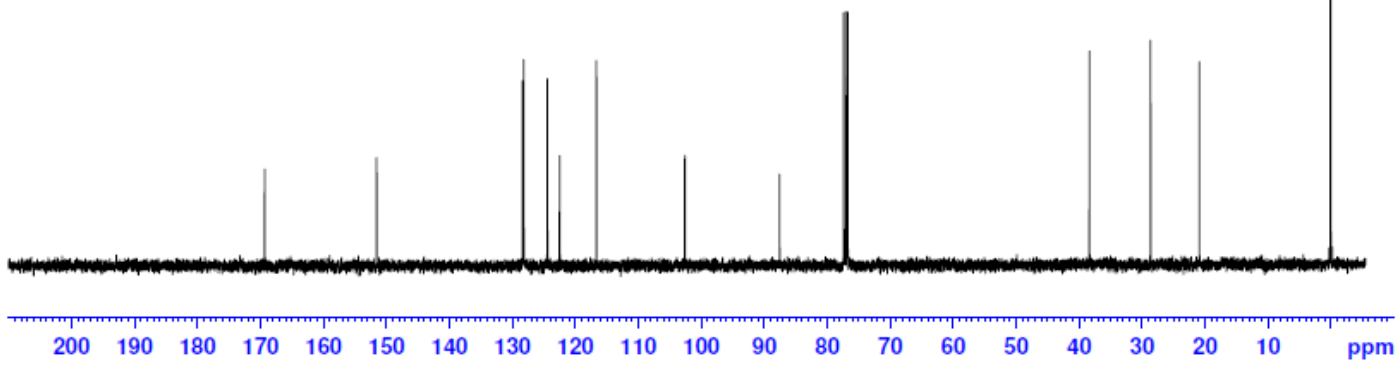




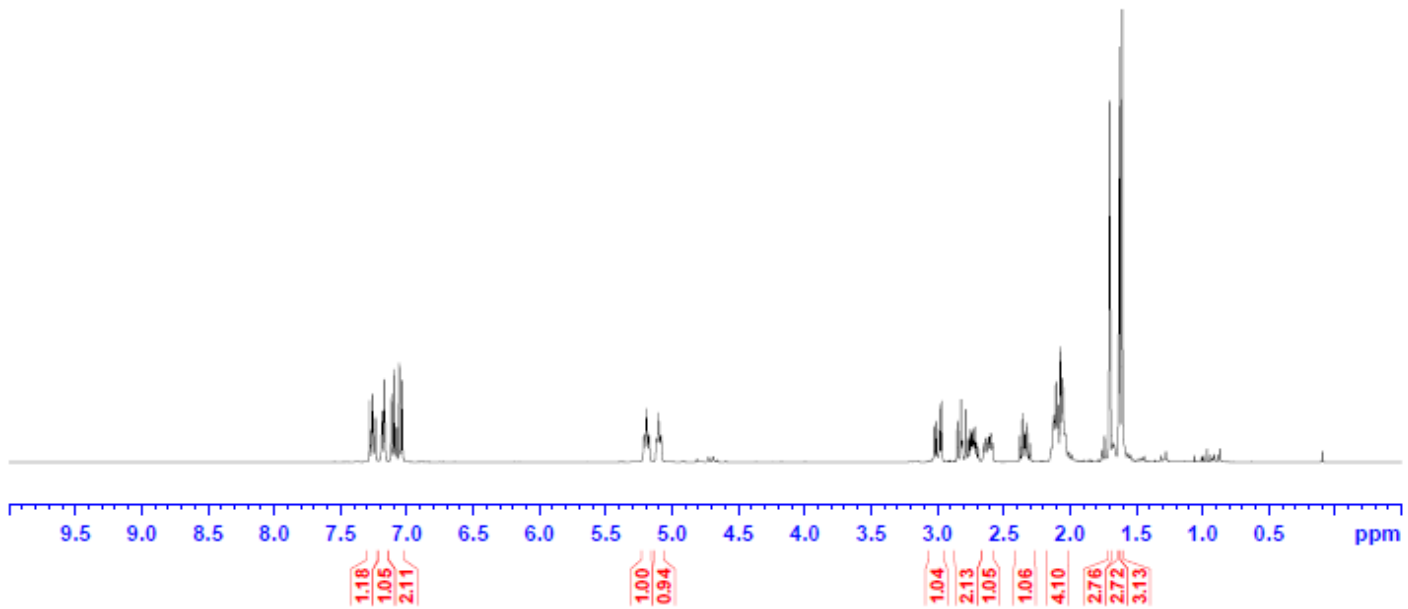<smiles>CC(C)=CCC/C(C)=C\CC1Cc2ccccc2OC1=O</smiles>

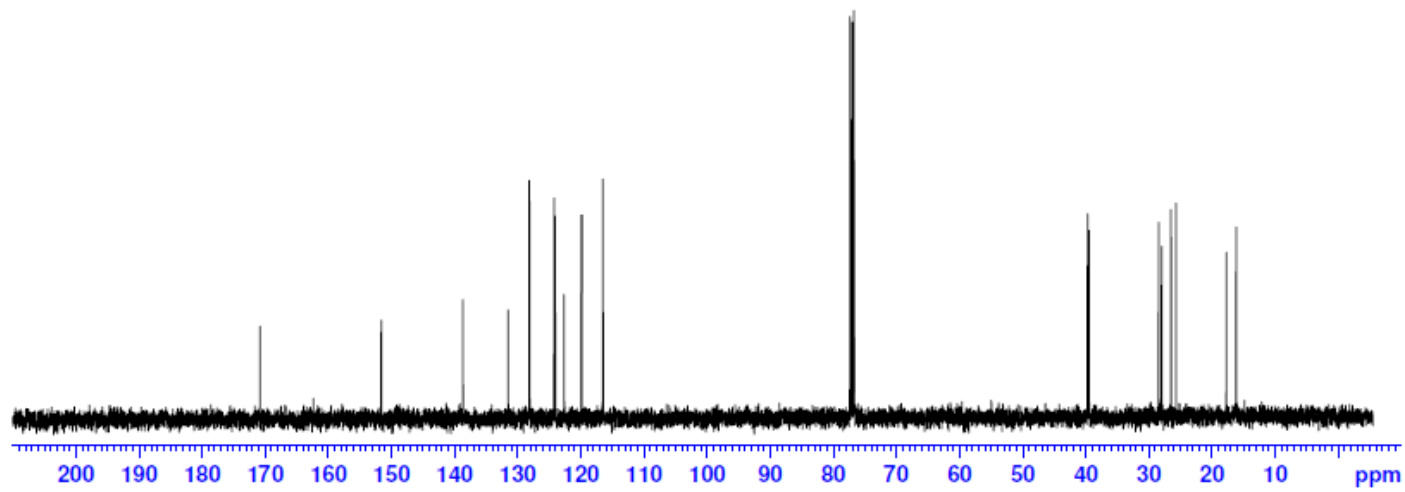




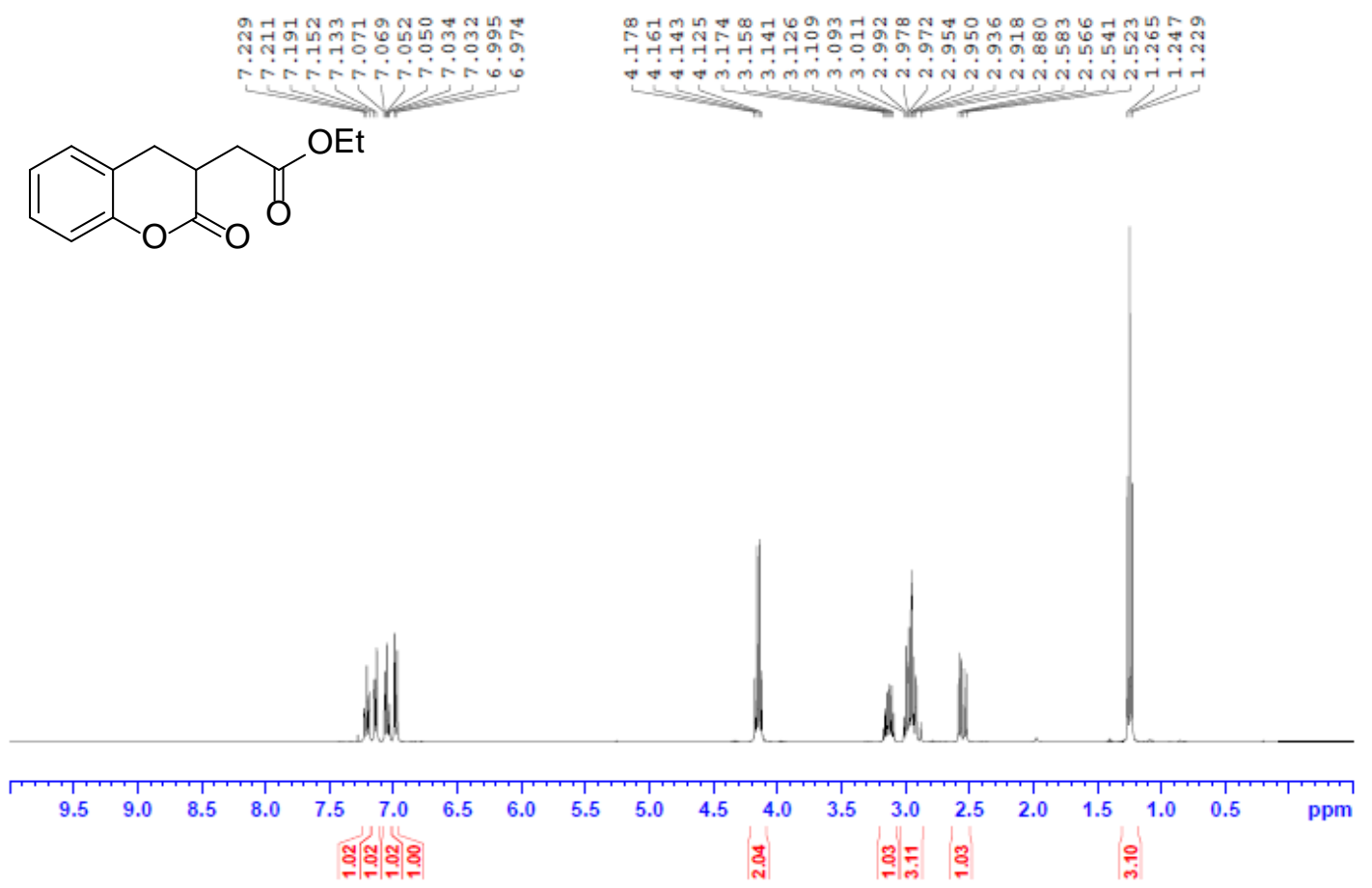

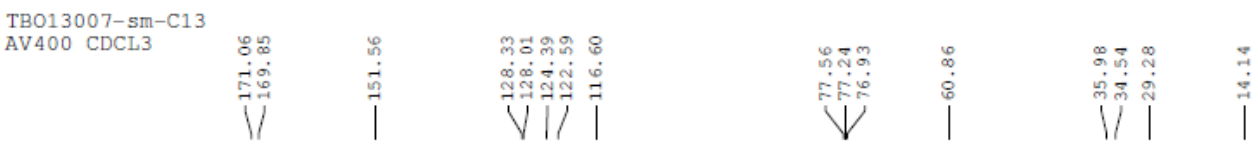<smiles>CCOC(=O)CC1Cc2ccccc2OC1=O</smiles>

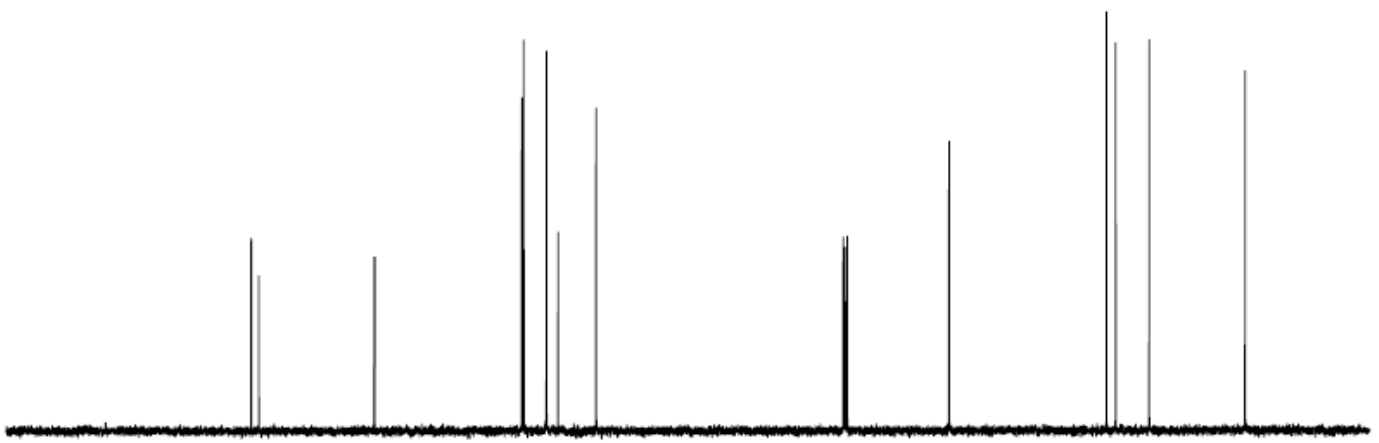

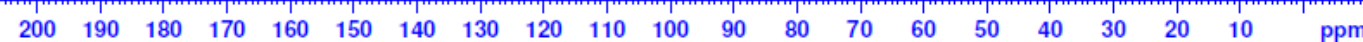




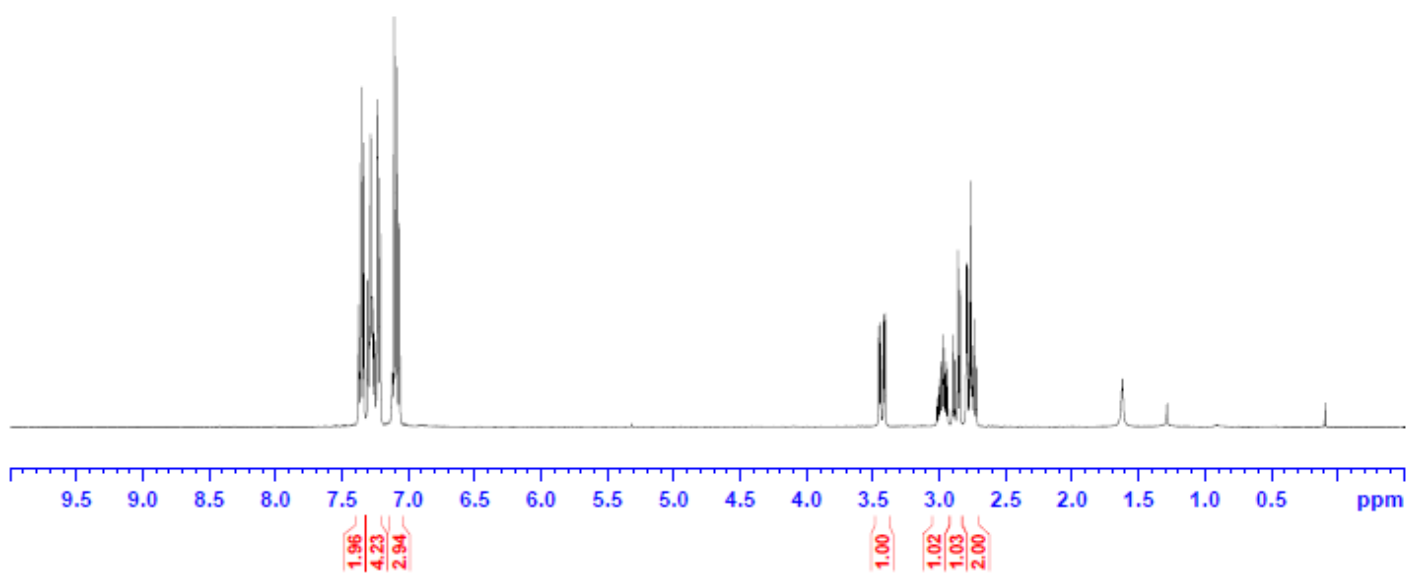<smiles>O=C1Oc2ccccc2CC1Cc1ccccc1</smiles>

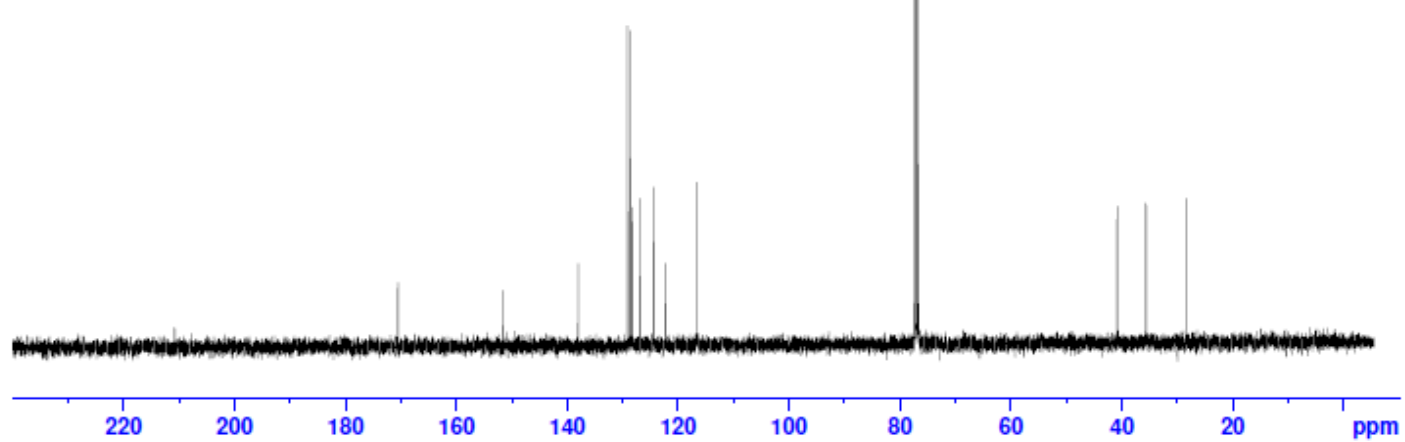


TBO12070-7-HI

BBFO1

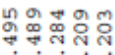

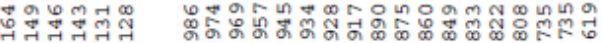

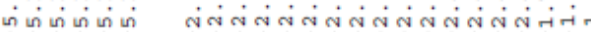

11

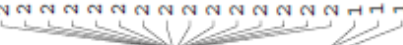

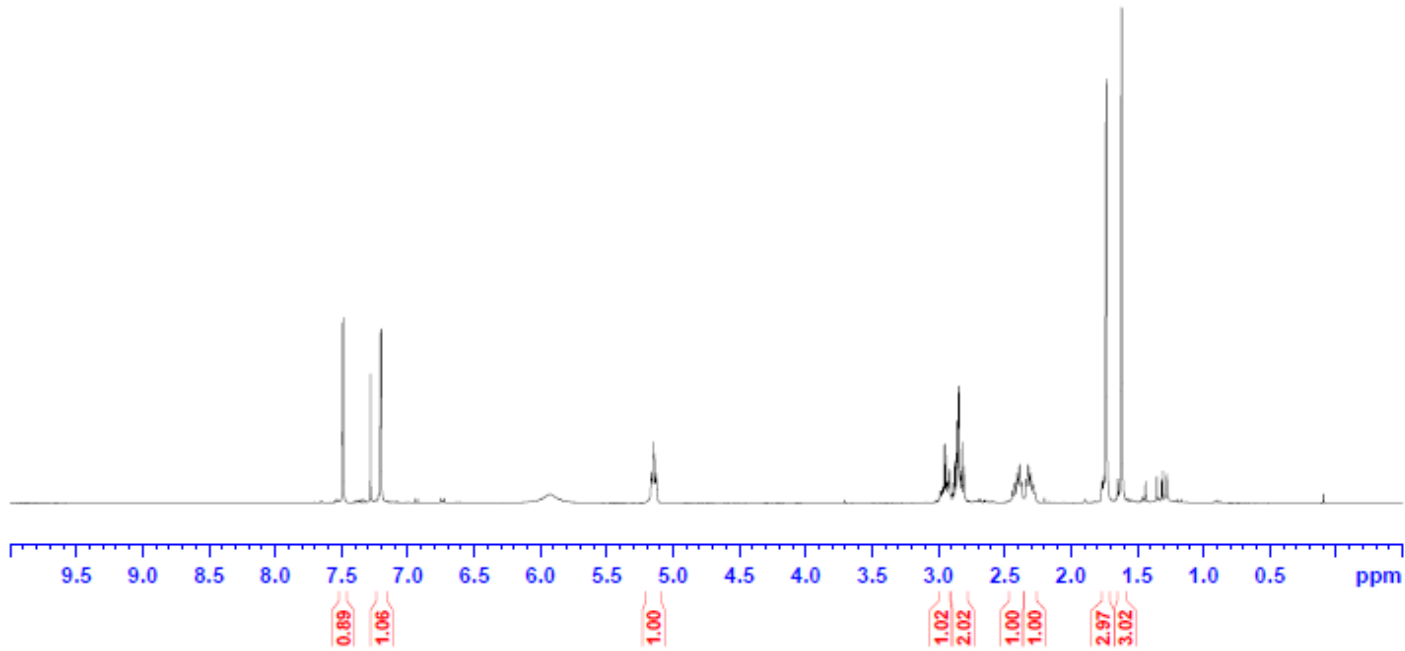

TB012070-7-C13

BBFO1<smiles>CC(C)=CCC1Cc2cc(Br)cc(Br)c2OC1=O</smiles>

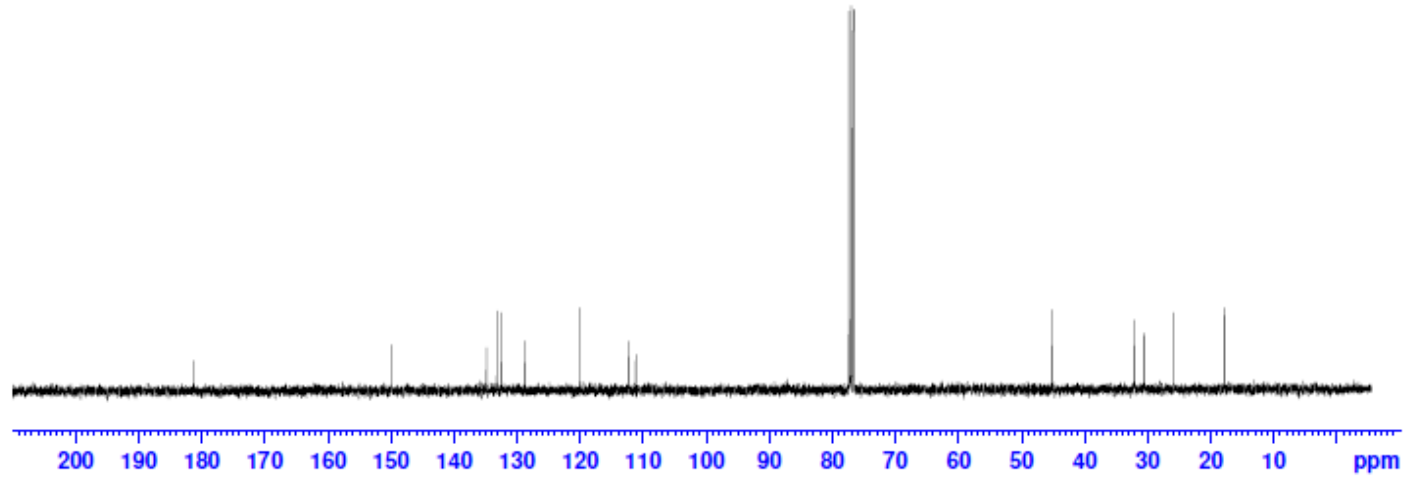




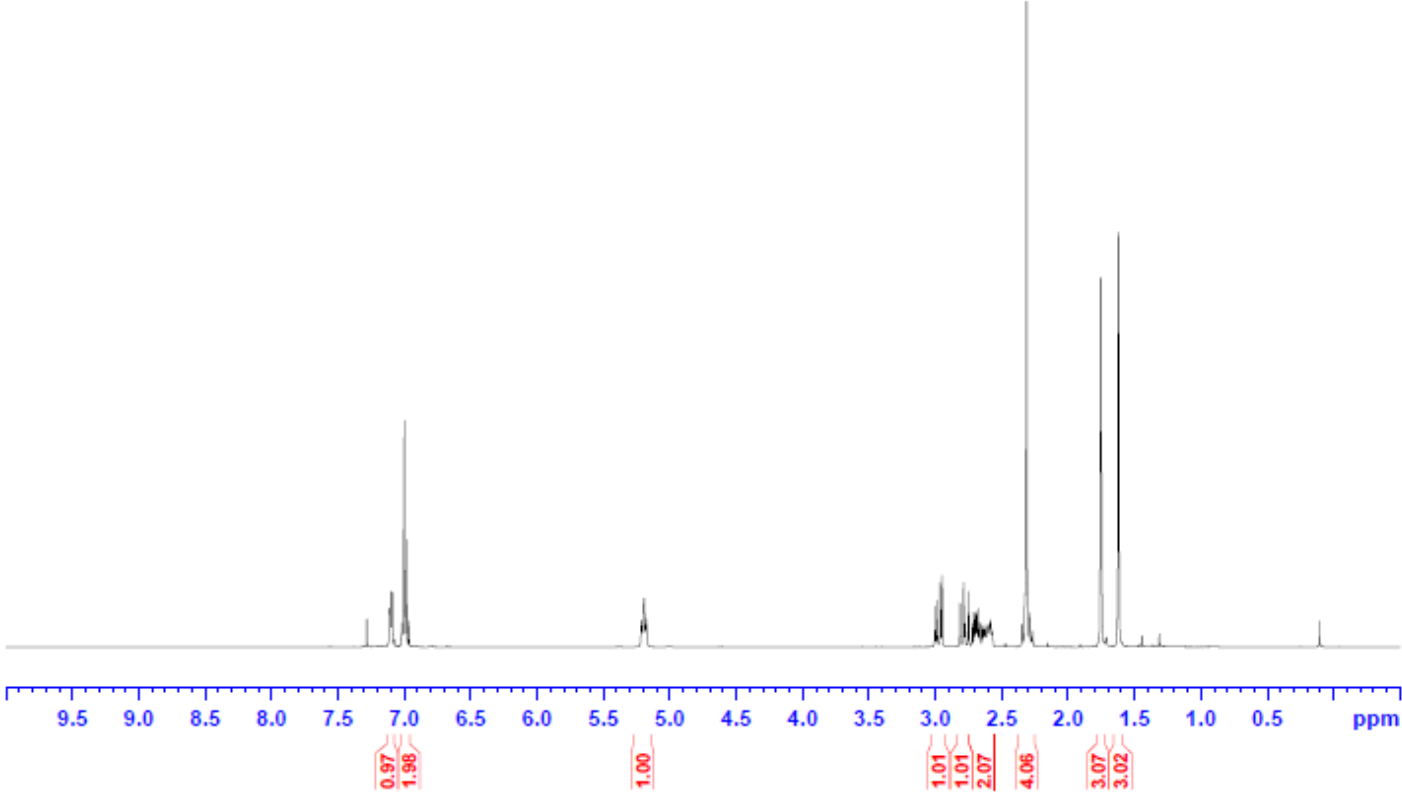<smiles>CC(C)=CCC1Cc2cccc(C)c2OC1=O</smiles>

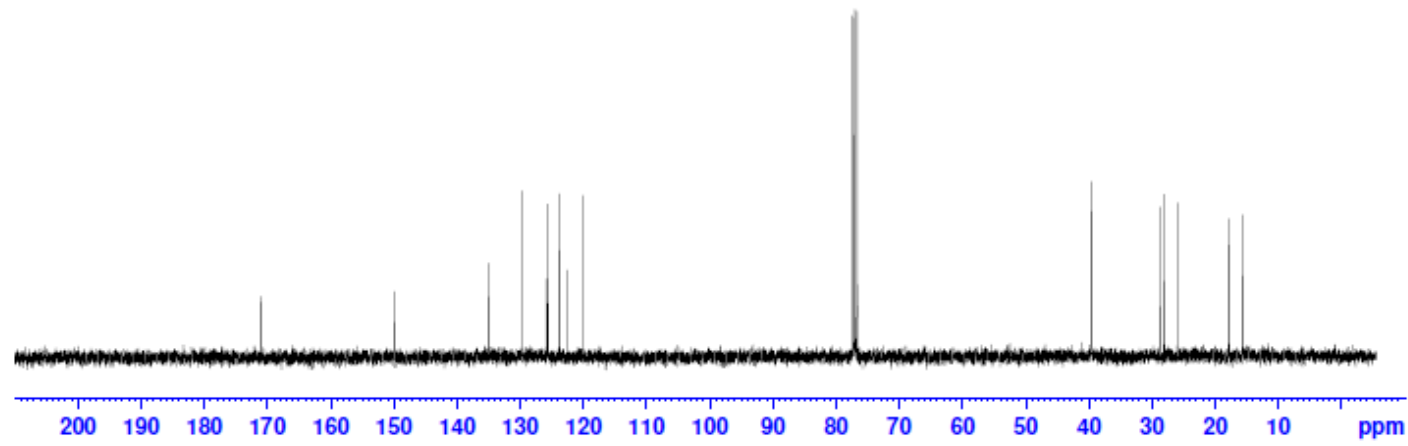




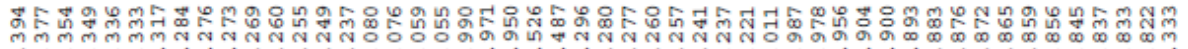

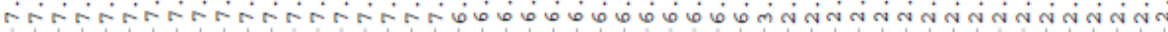

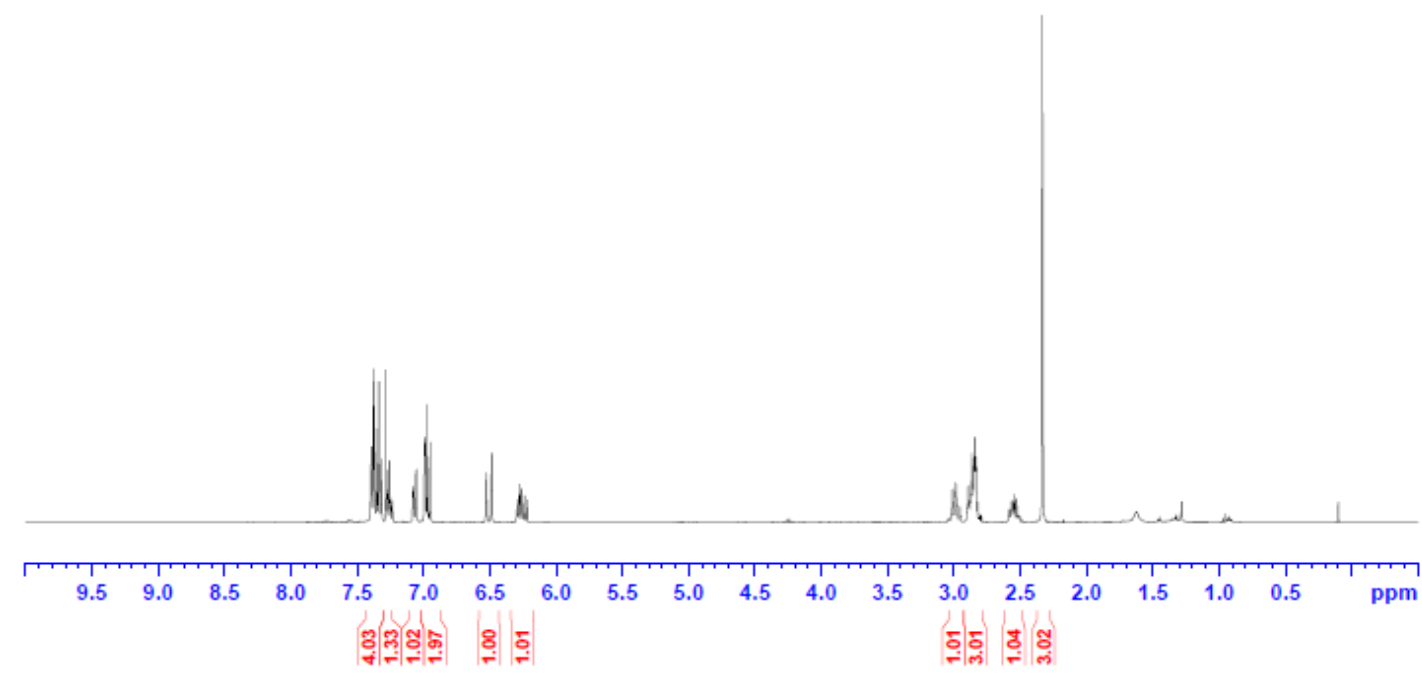

TB012070-8-C13

BBFO1

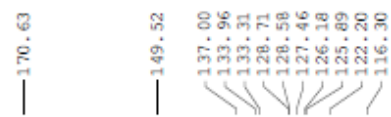

$\sqrt{n}$

|
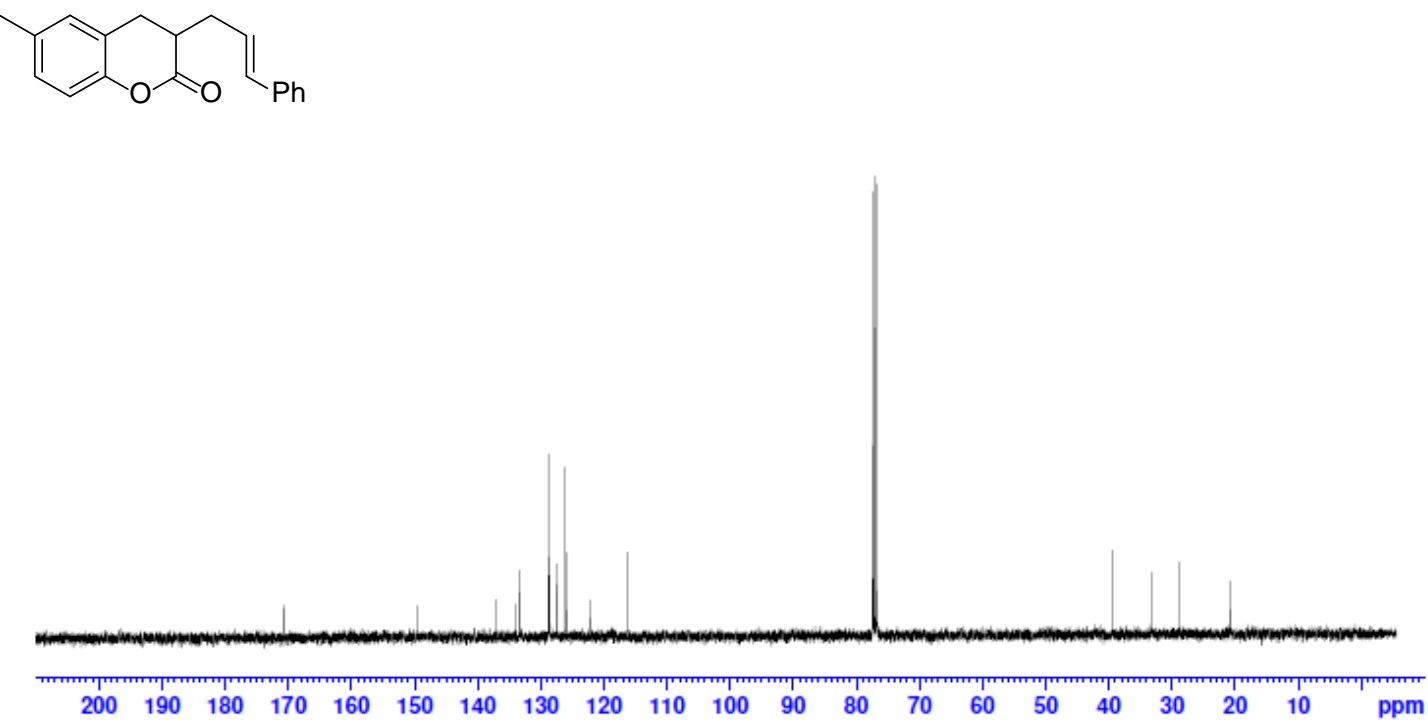


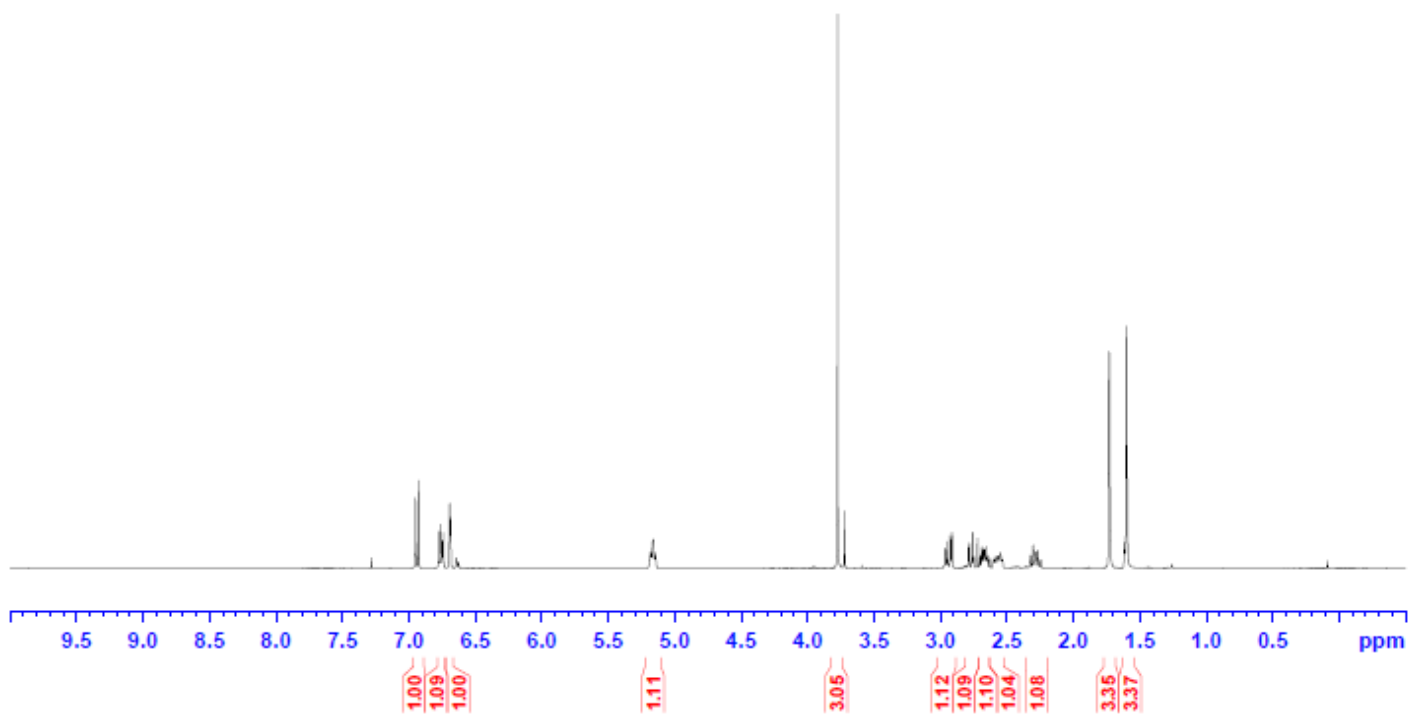<smiles>COc1ccc2c(c1)CC(CC=C(C)C)C(=O)O2</smiles>

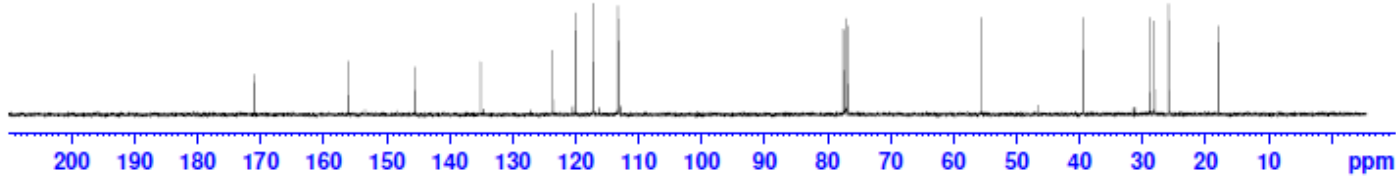




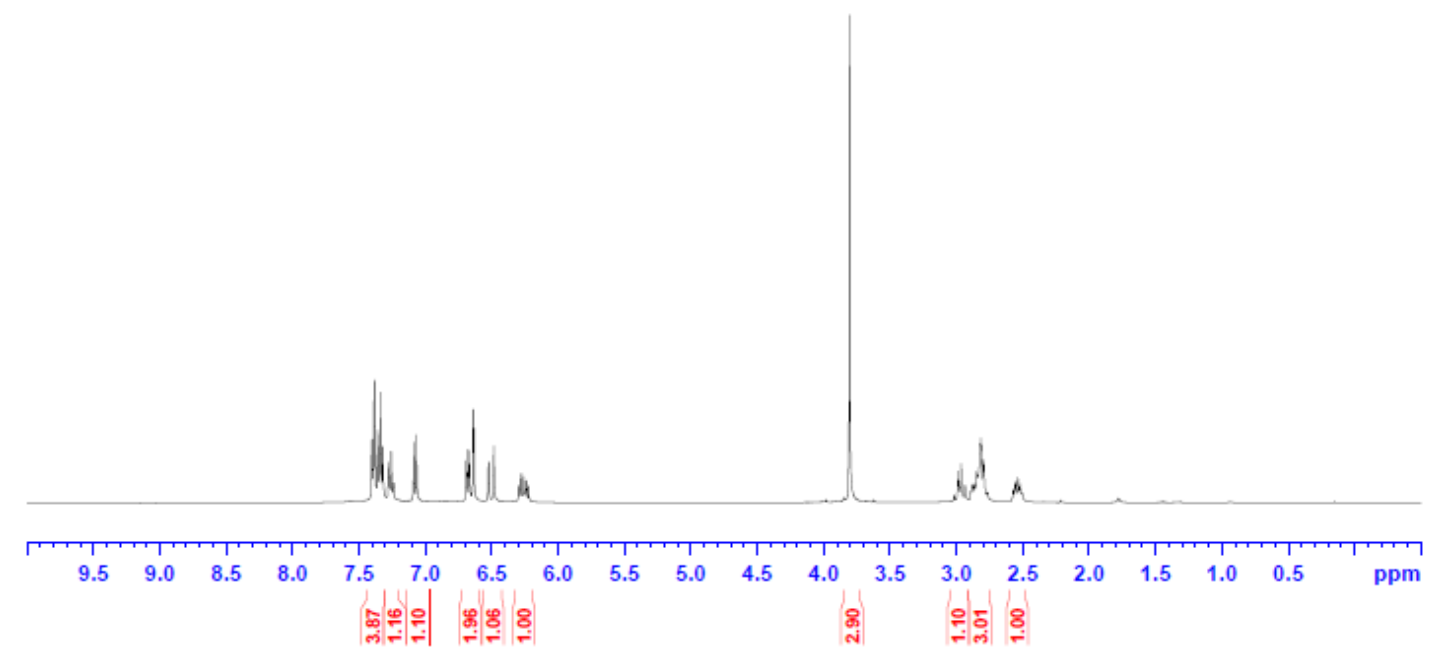

TBO12076-C13<smiles>COc1ccc2c(c1)OC(=O)C(CCC=Cc1ccccc1)C2</smiles>

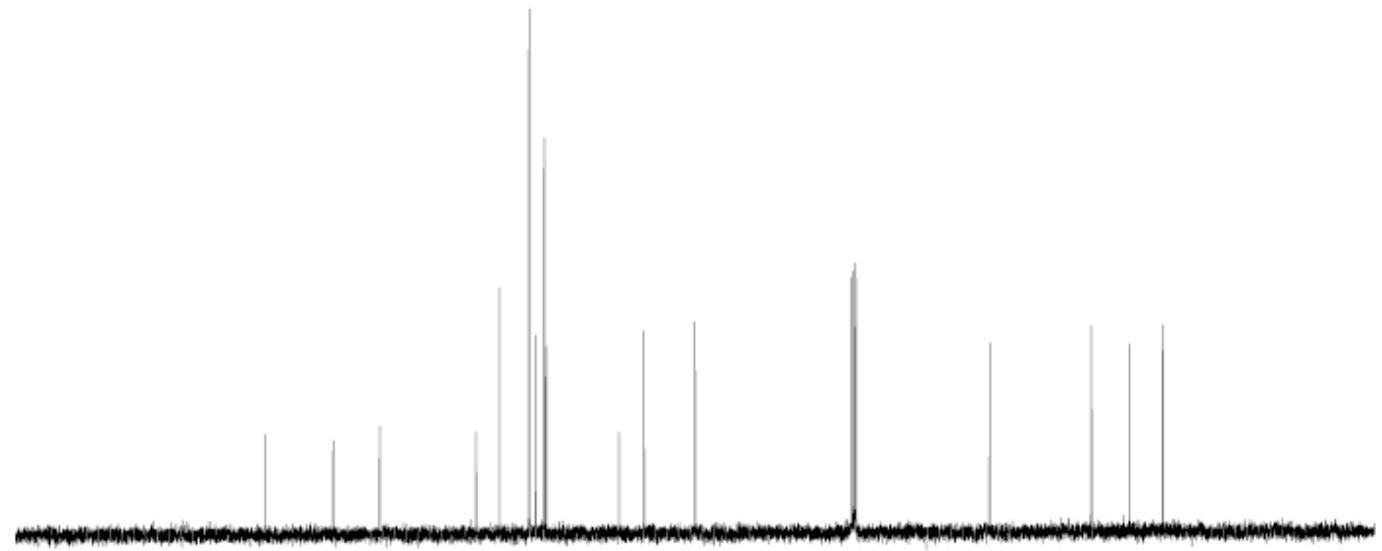



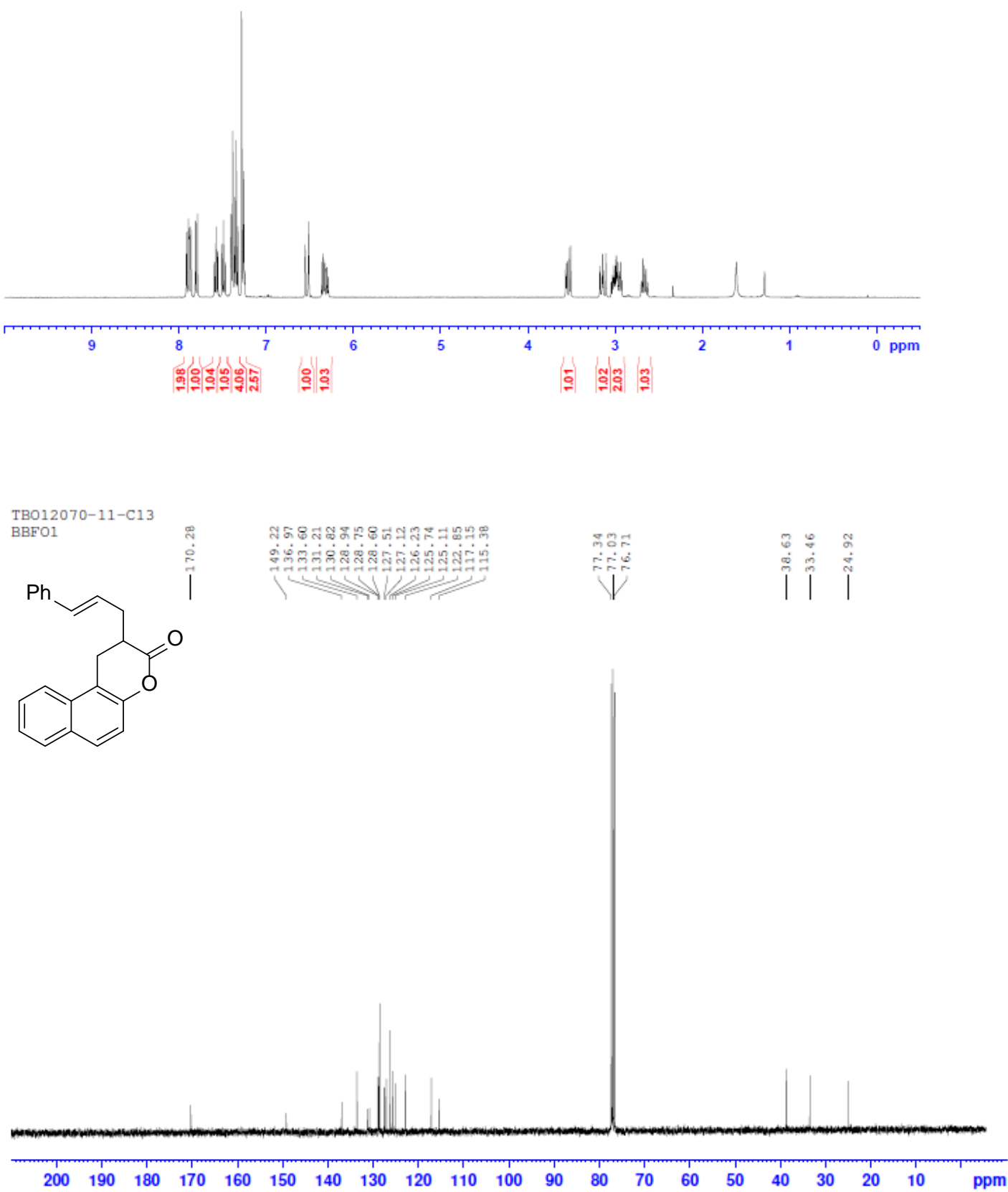


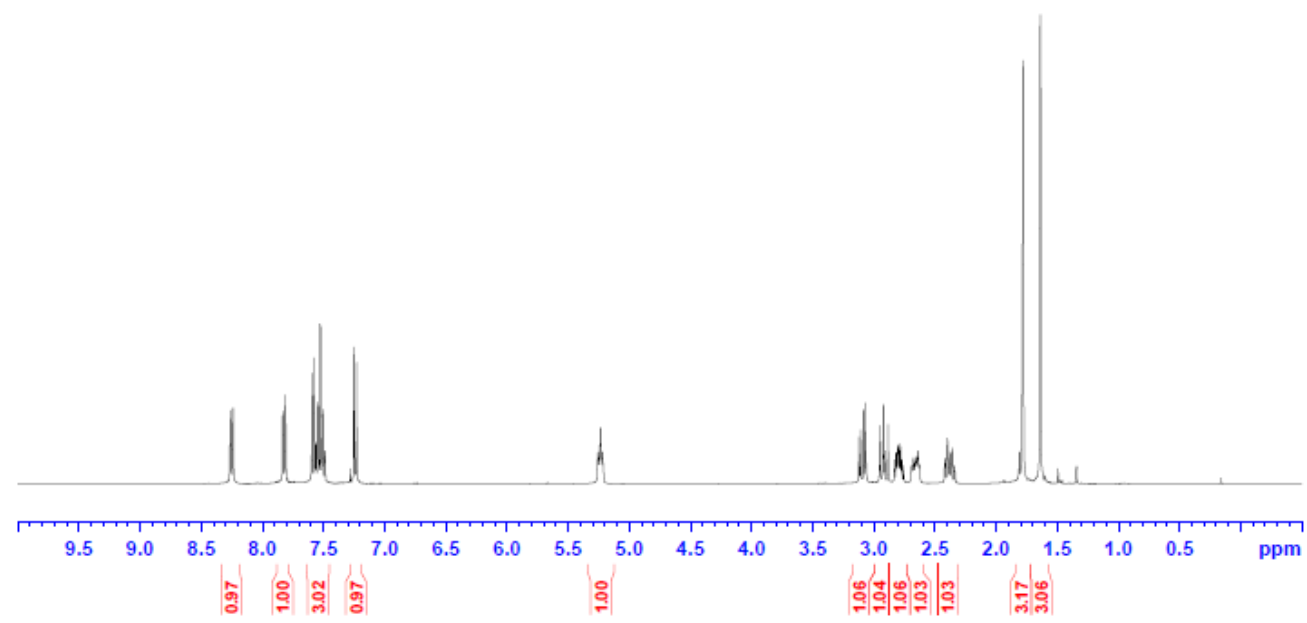

TB012070-12-C13
BBF01<smiles>CC(C)=CCC1Cc2ccc3ccccc3c2OC1=O</smiles>

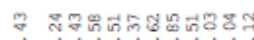

至
证

|

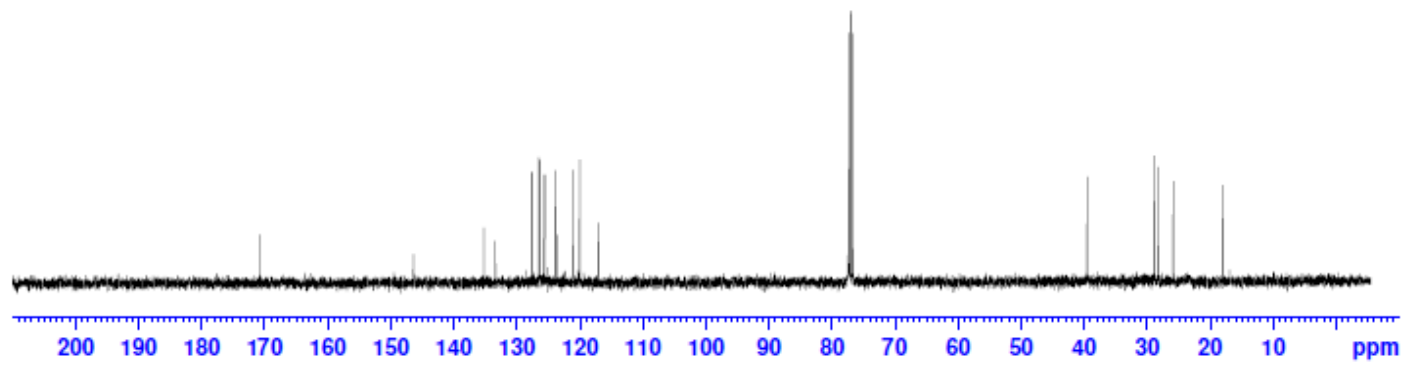



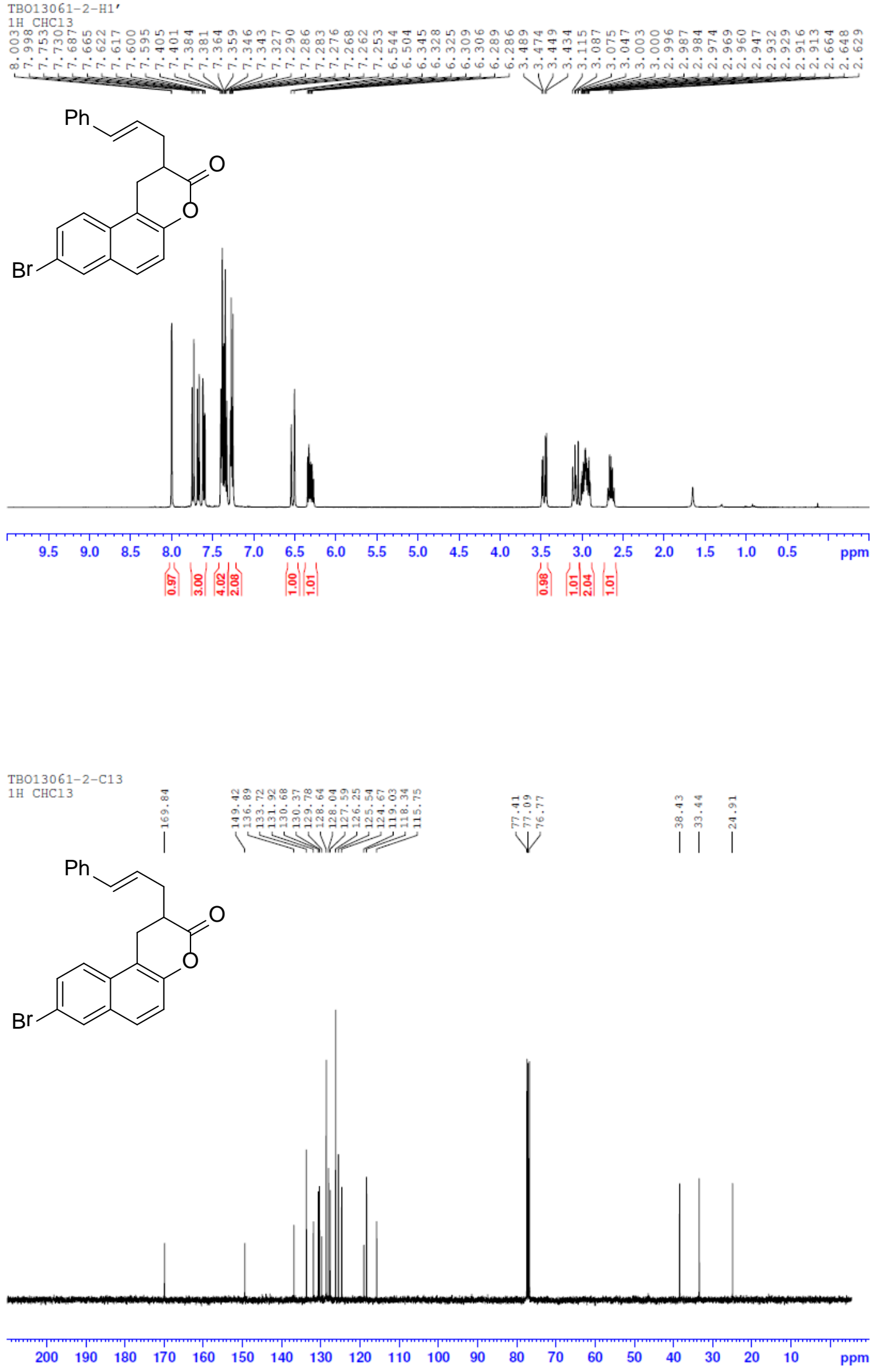


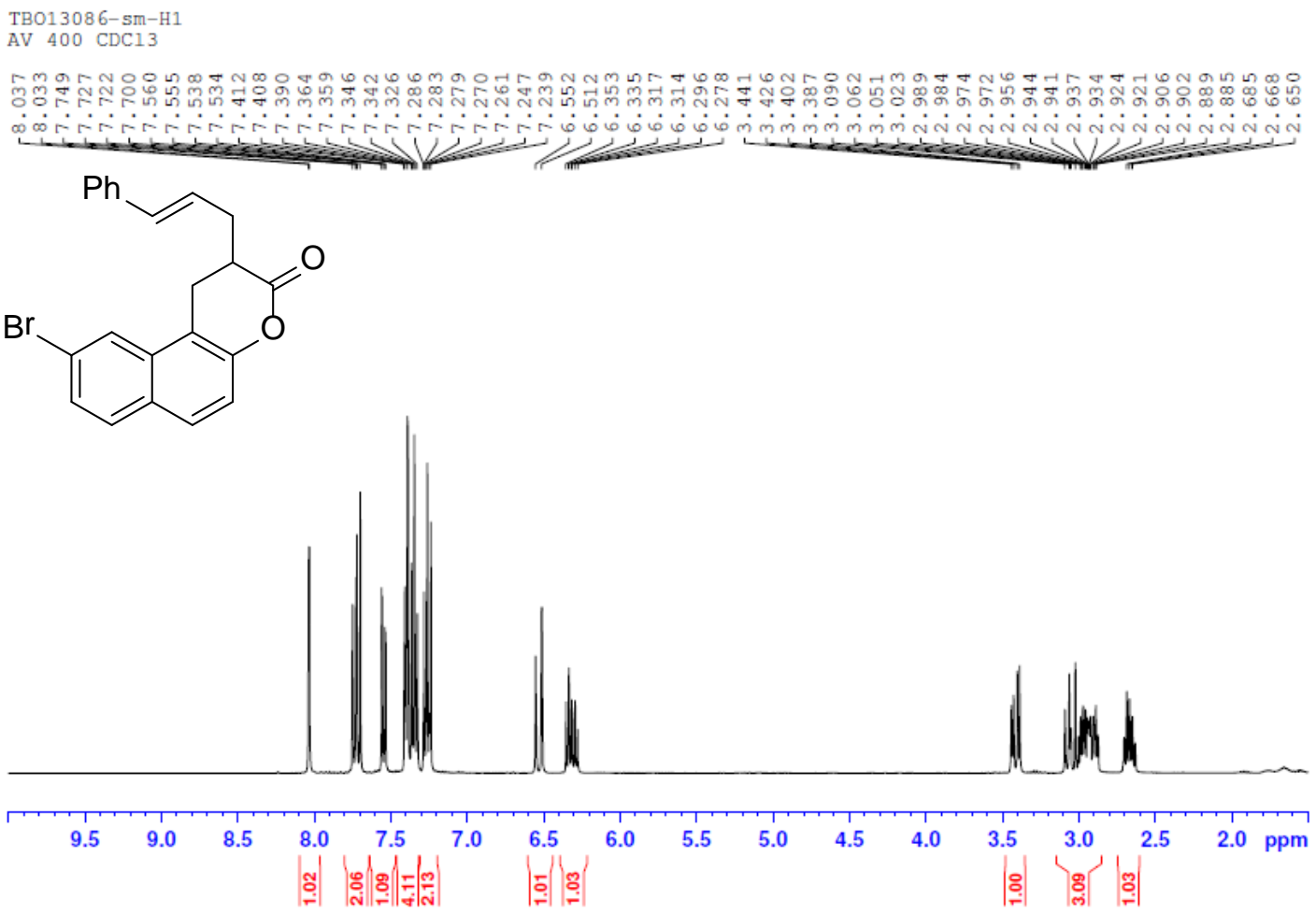

TB013086-sm-C13

AV $400 \mathrm{CDC} 13$
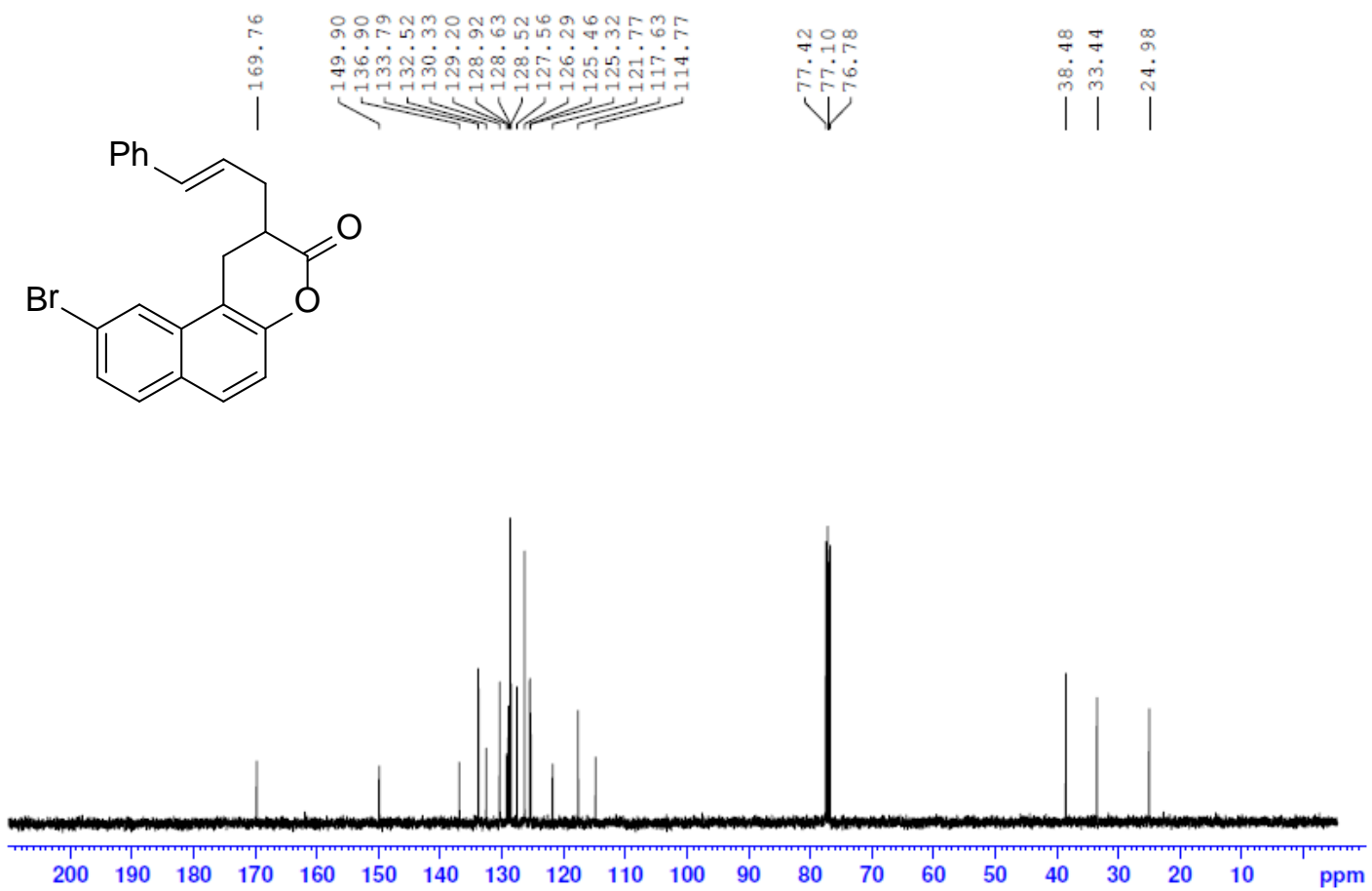


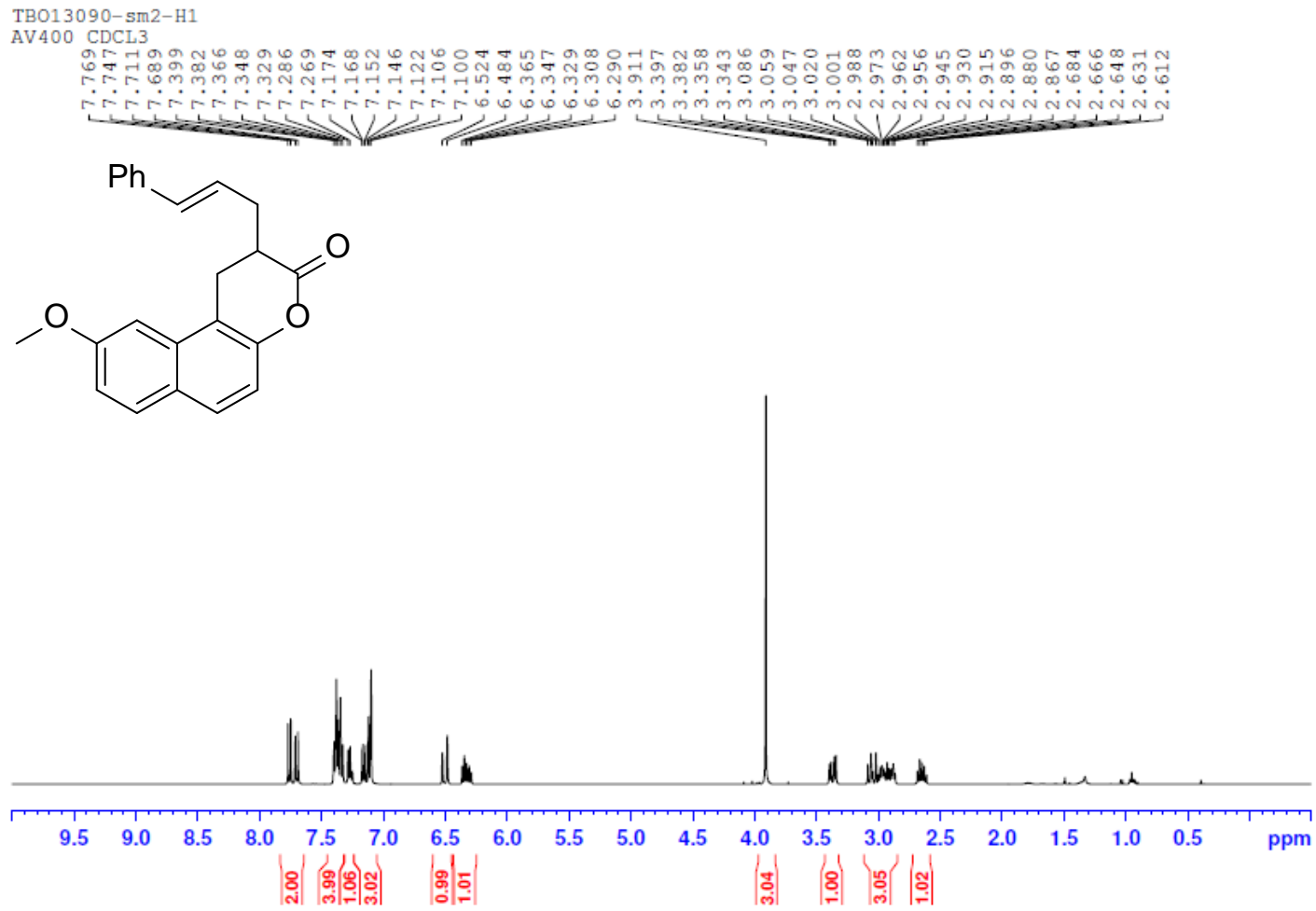

TB013090-sm-2-C13

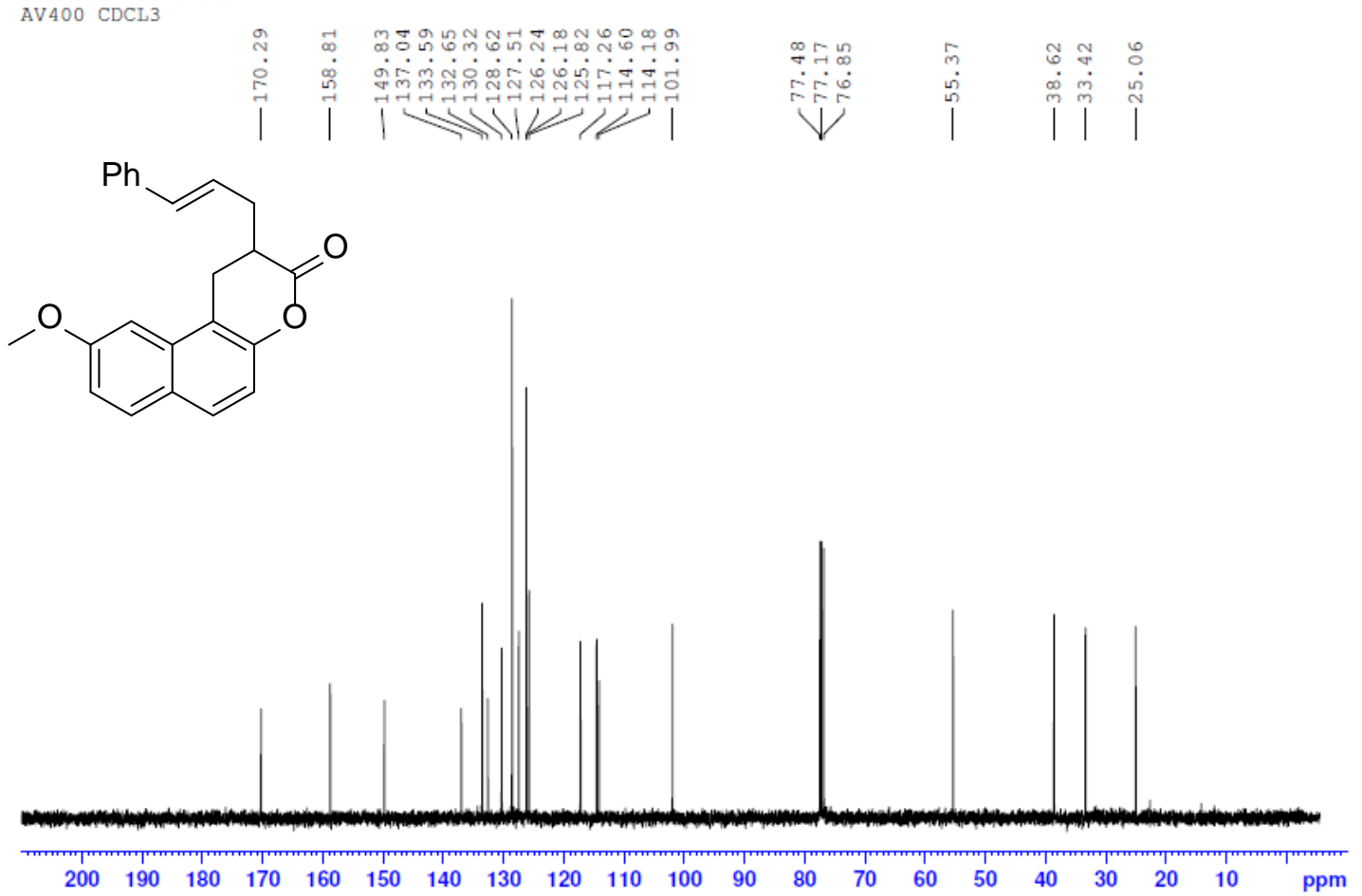



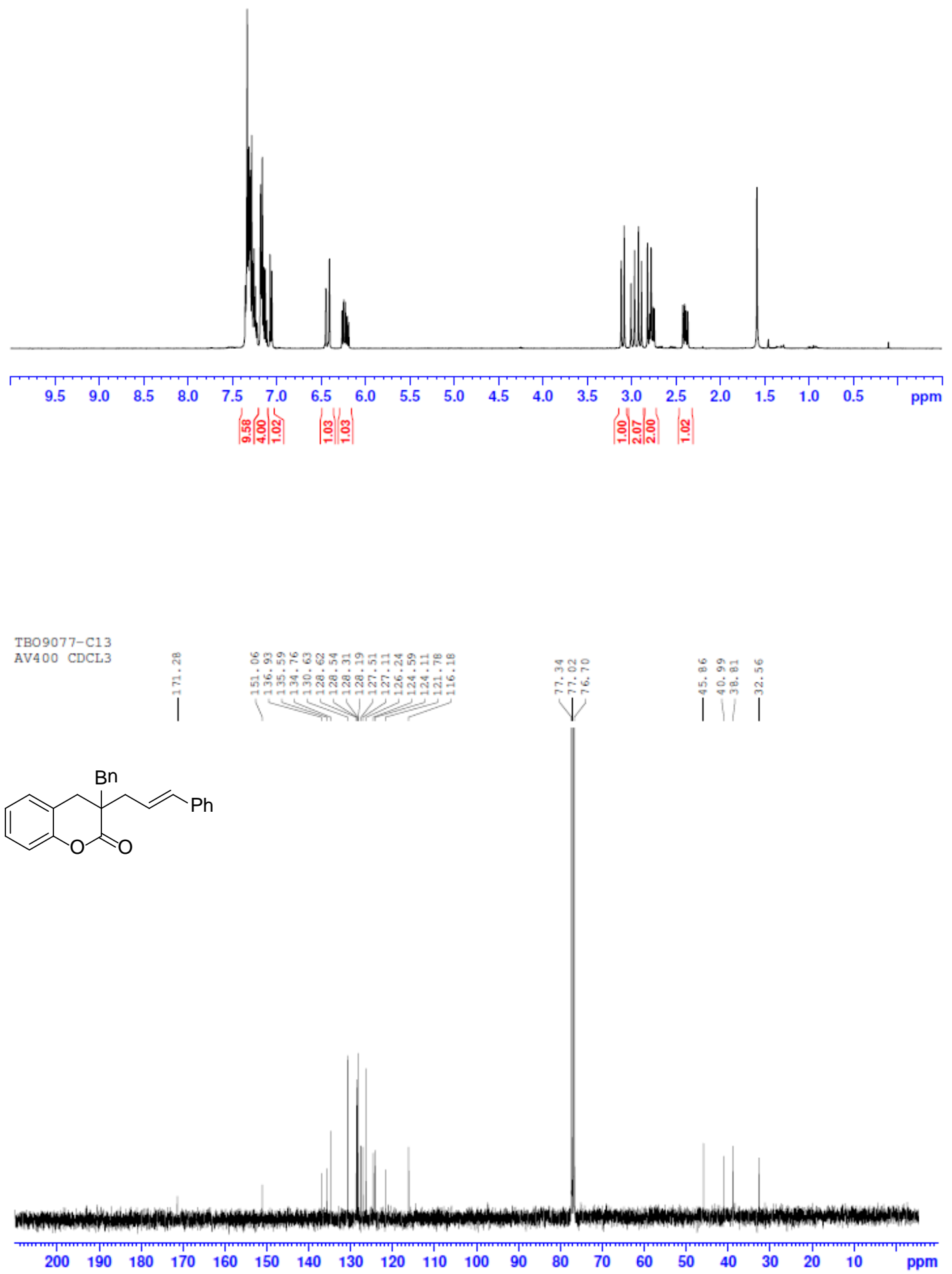
TB012117-1-H1

BBF1 CDC13

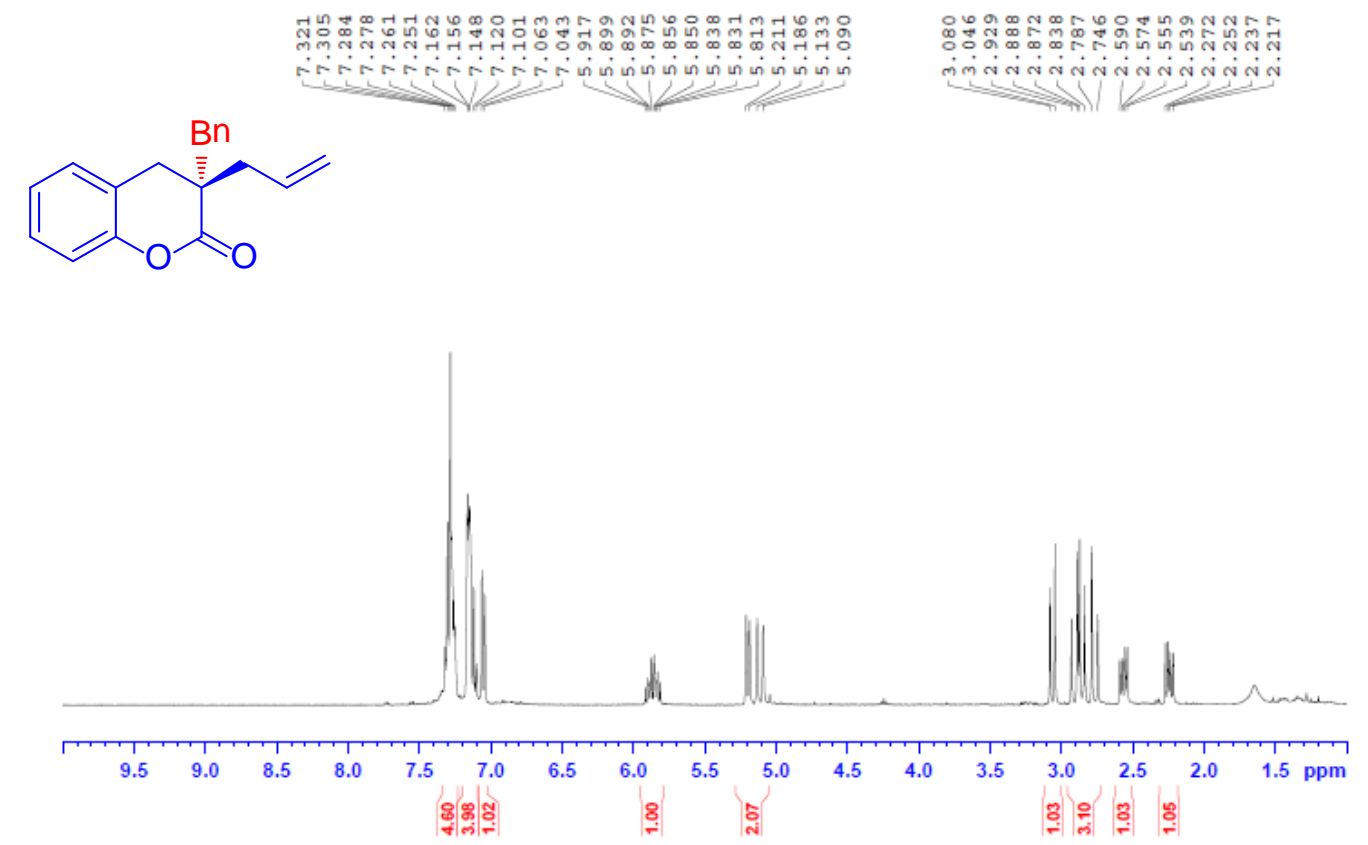

TB012117-1-C13
BBF1 CDC13

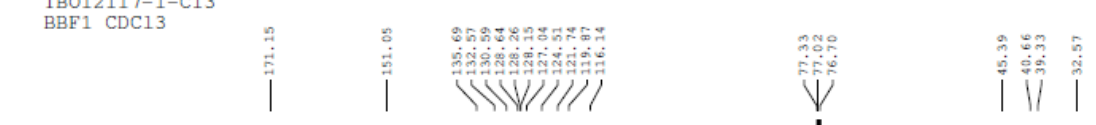<smiles>C=CC[C@]1(Br)Cc2ccccc2OC1=O</smiles>

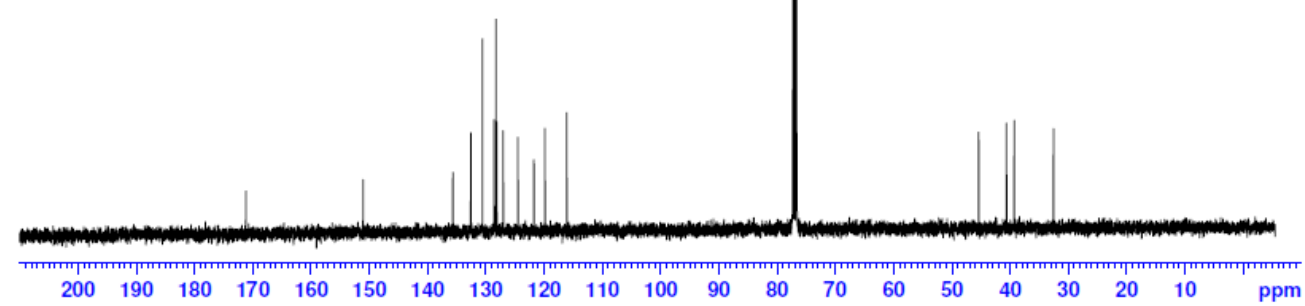


<smiles>CC(C)=CC[C@]1(Cc2ccccc2)Cc2ccccc2OC1=O</smiles>
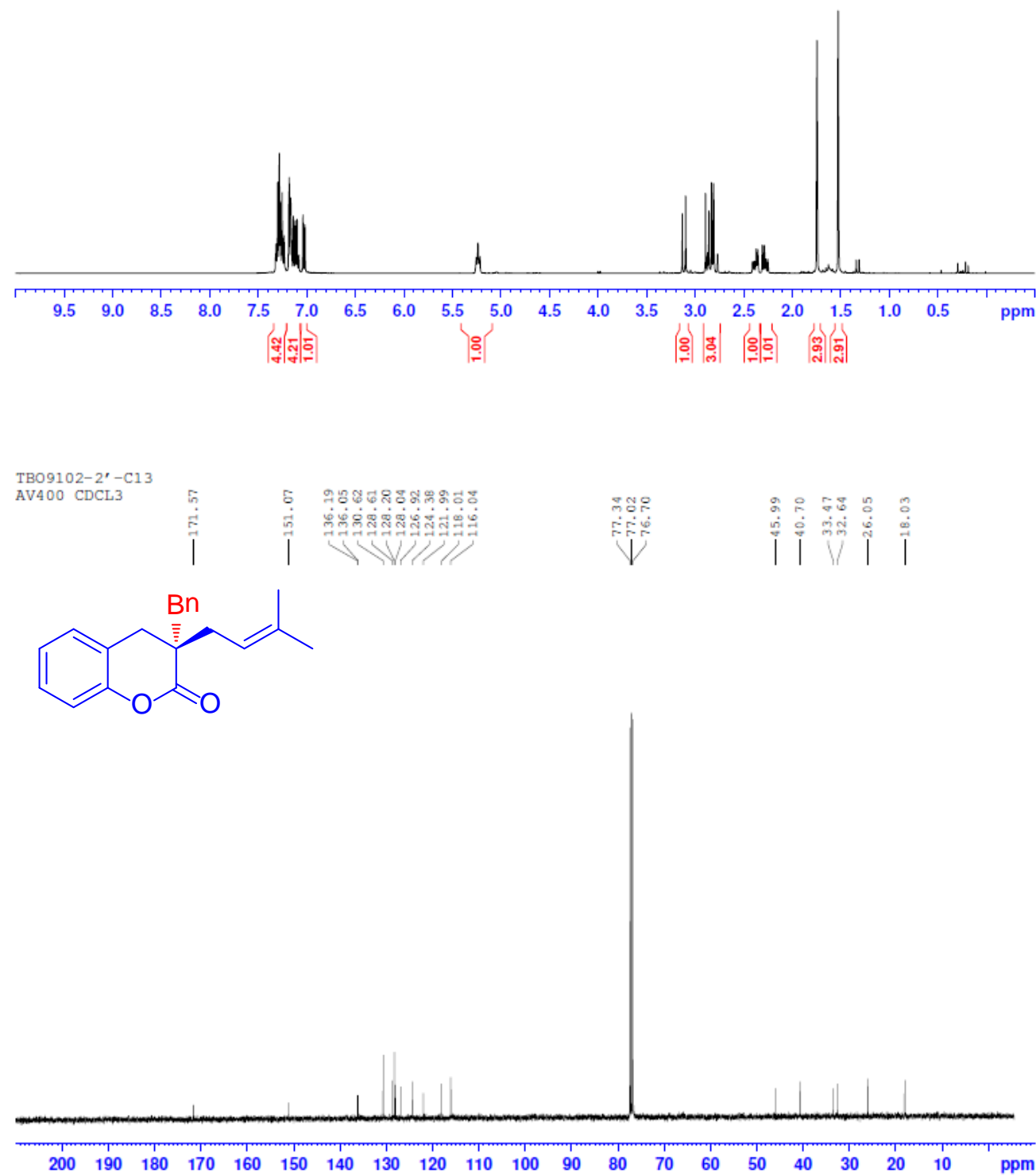
TB09117-2-H1

$\overbrace{0}^{1}$

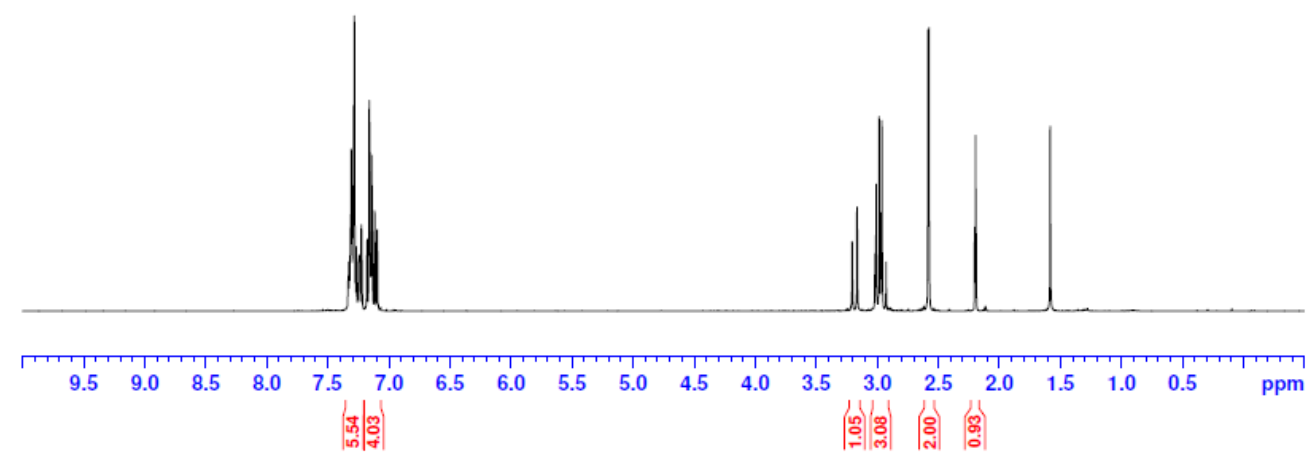

${ }_{\text {AV }}^{\text {TBO } 9117-2-C 13}$ CDCL3 3

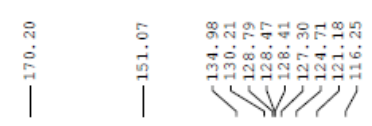

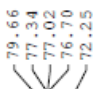

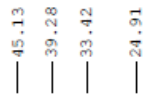

$\mathrm{Bn}$<smiles>C#CC[C@H]1Cc2ccccc2OC1=O</smiles>

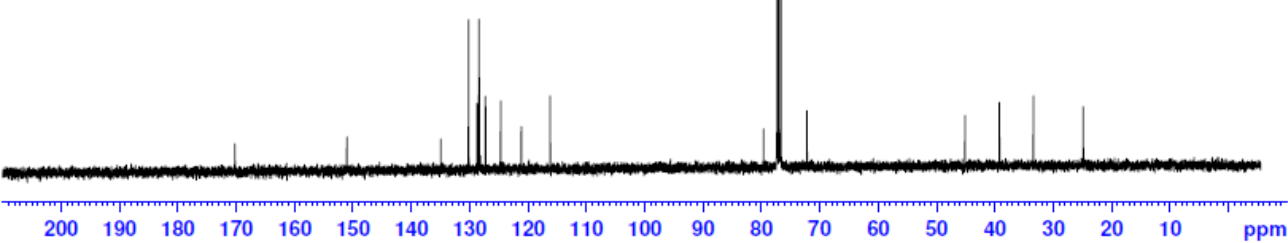


TB013073-19-H1

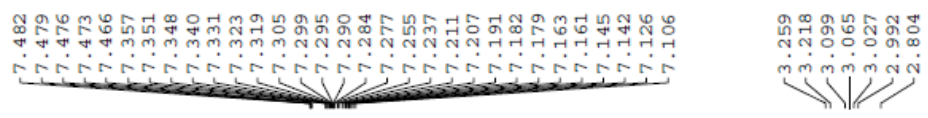

$\mathrm{Bn}$<smiles>O=C1Oc2ccccc2C[C@@H]1CC#Cc1ccccc1</smiles>

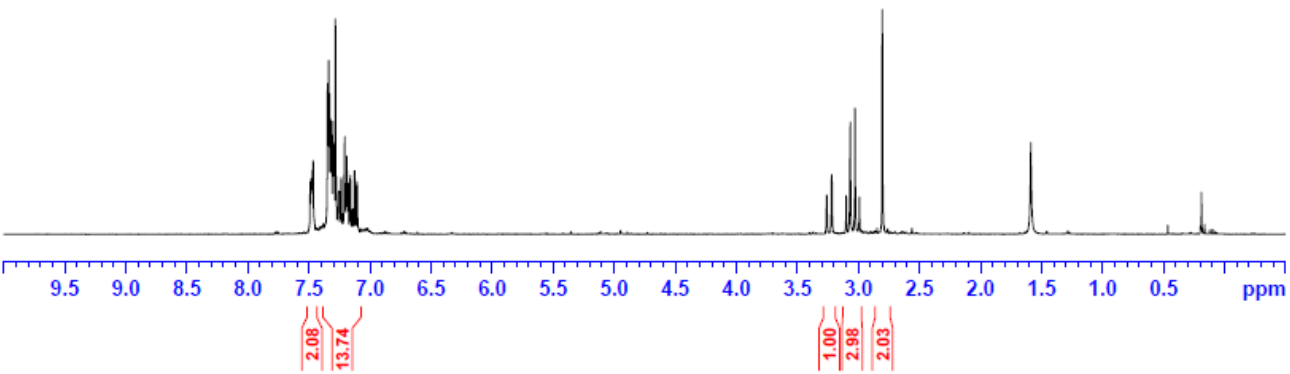

TB09107-2-C13

AV400 CDCL 3

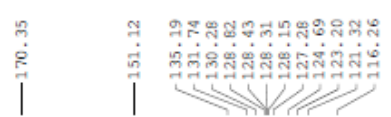

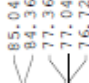

|

$\mathrm{Bn}$<smiles>O=C1Oc2ccccc2C[C@@H]1CC#Cc1ccccc1</smiles>

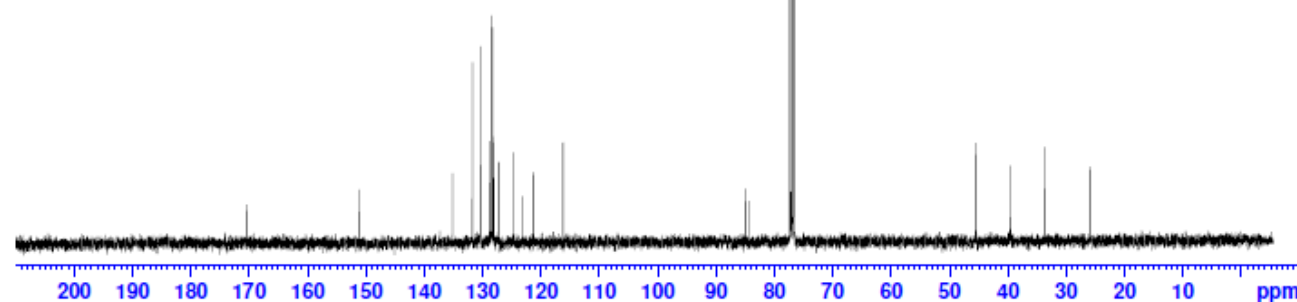




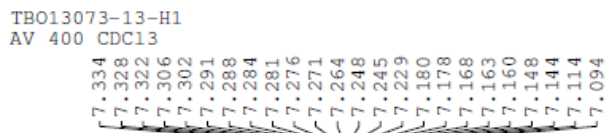

$\mathrm{Bn}$<smiles>CC#CC[C@H]1Cc2ccccc2OC1=O</smiles>

M L I

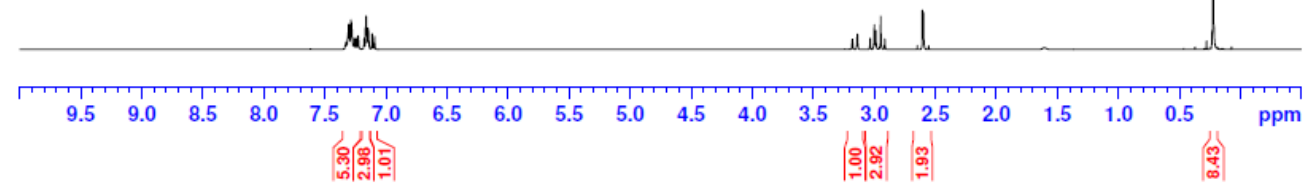
TBO9102-4-C13
AV400 CDCL 3<smiles>CC(C)(C)C#CC[C@]1(Cc2ccccc2)Cc2ccccc2OC1=O</smiles> 
TBO13073-20-H1
AV 400 CDC13

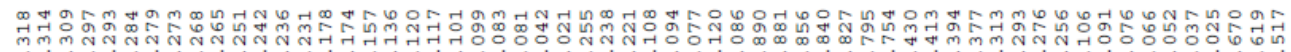

r.r.

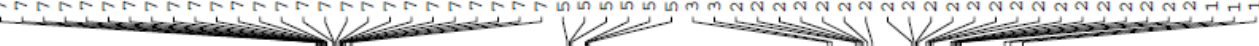<smiles>CC(C)=CCC/C(C)=C\C[C@]1(Cc2ccccc2)Cc2ccccc2OC1=O</smiles>

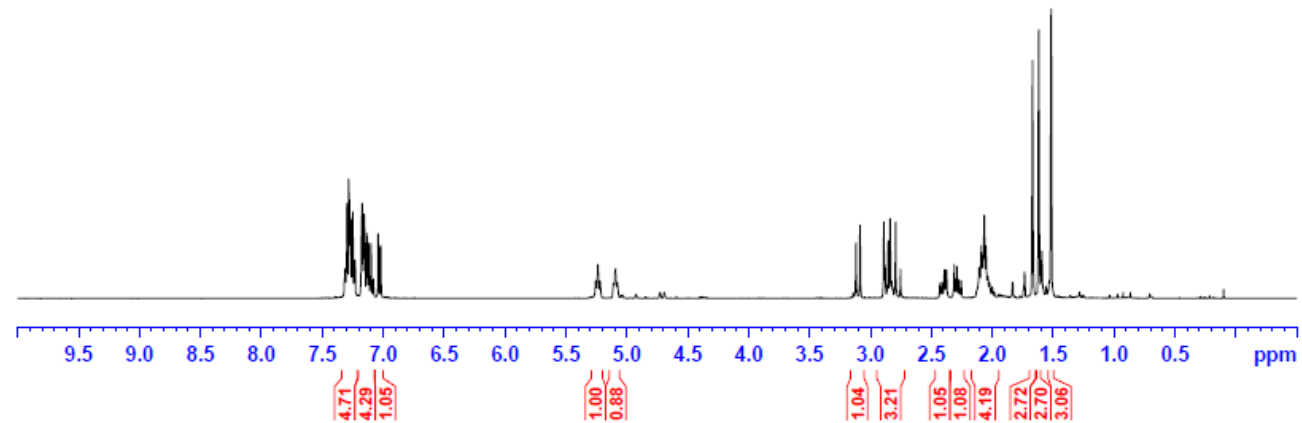<smiles>CC(C)=CCC/C(C)=C\C[C@]1(Cc2ccccc2)Cc2ccccc2OC1=O</smiles>

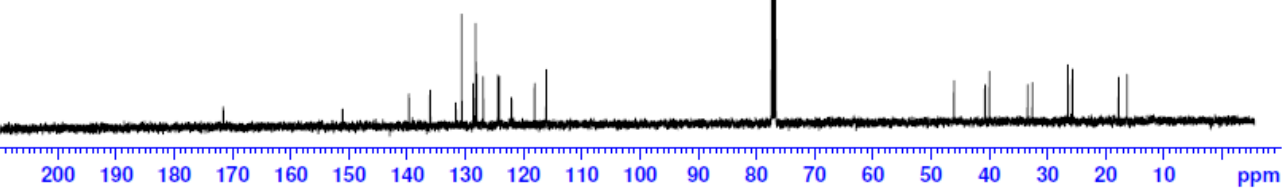


TBO13007-6-H

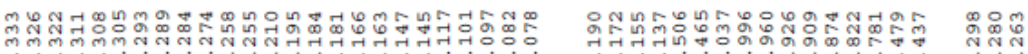

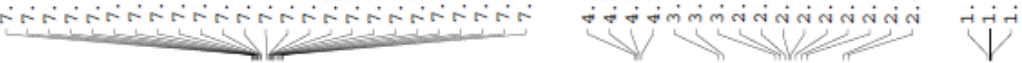

$\overbrace{0}^{\mathrm{O}}$

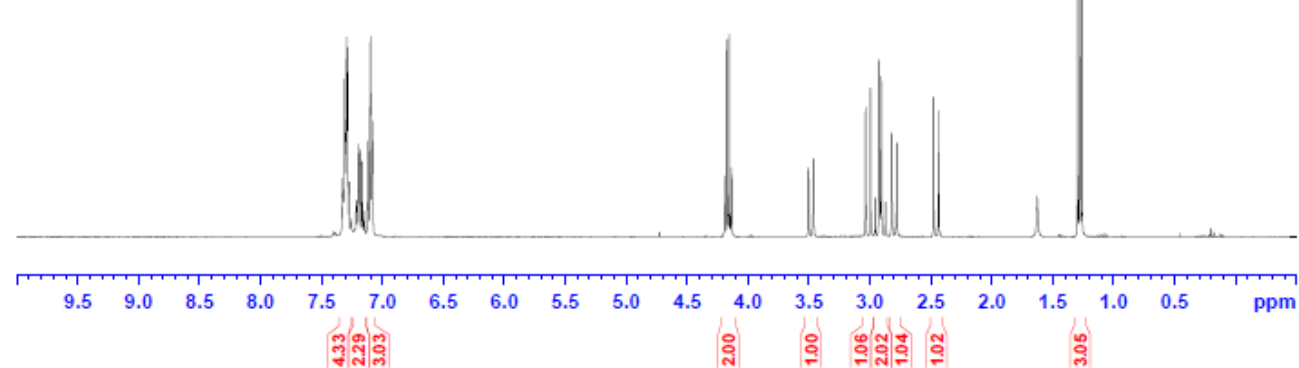

TB013007-6-C13

AV4 400 CDCL 3

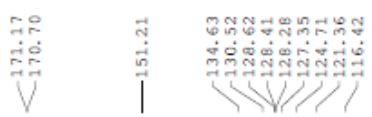

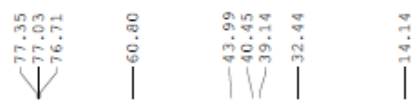

$\mathrm{Bn}$<smiles>CCOC(=O)CC1(Br)Cc2ccccc2OC1=O</smiles>

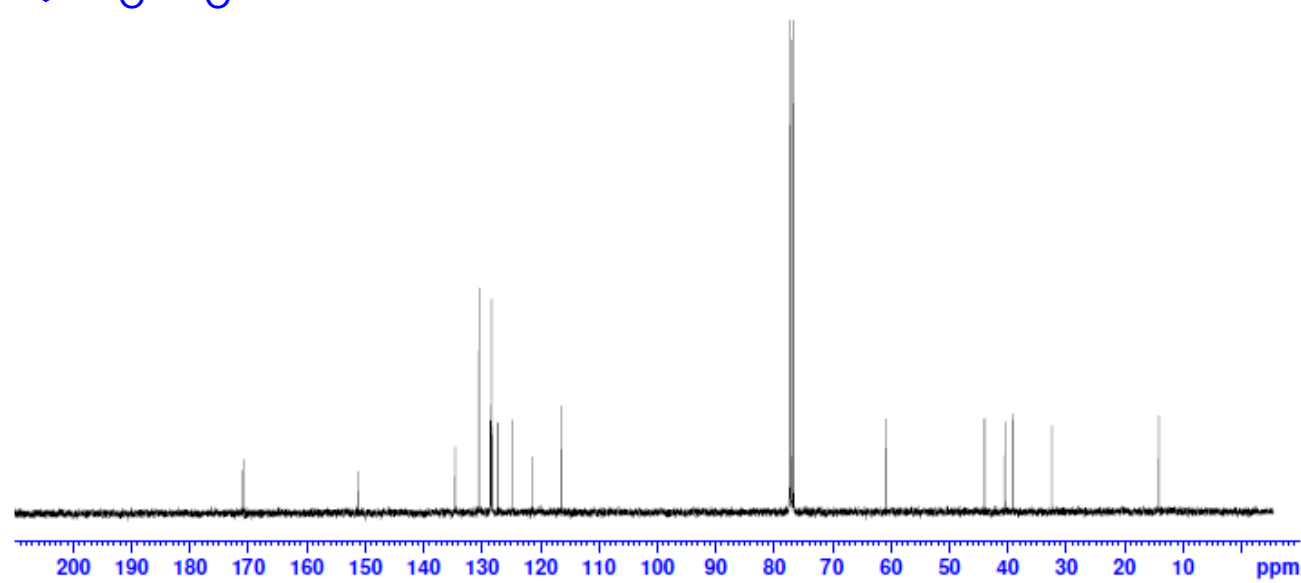



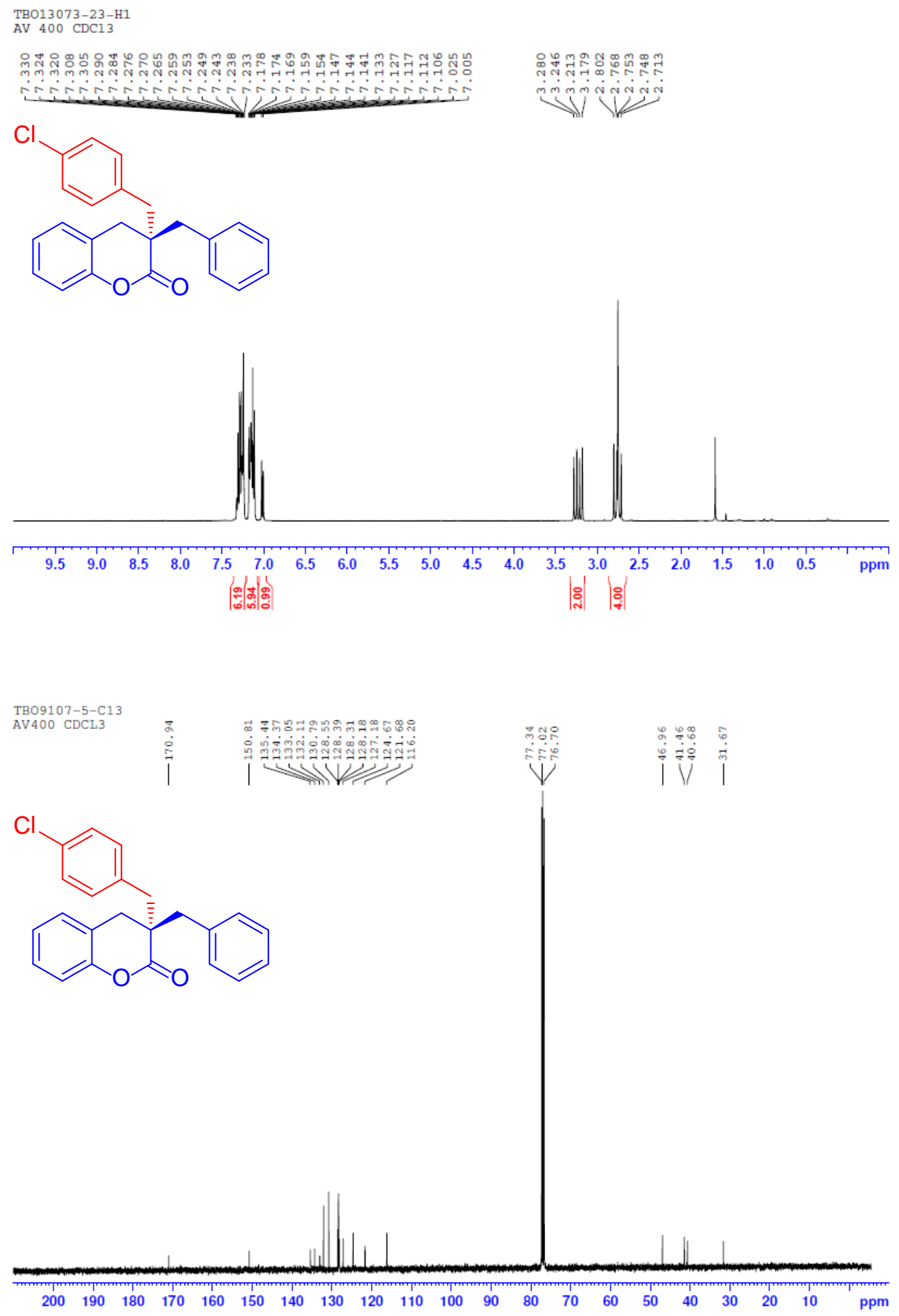

S104 
TBO12089-5-H1
BBF1

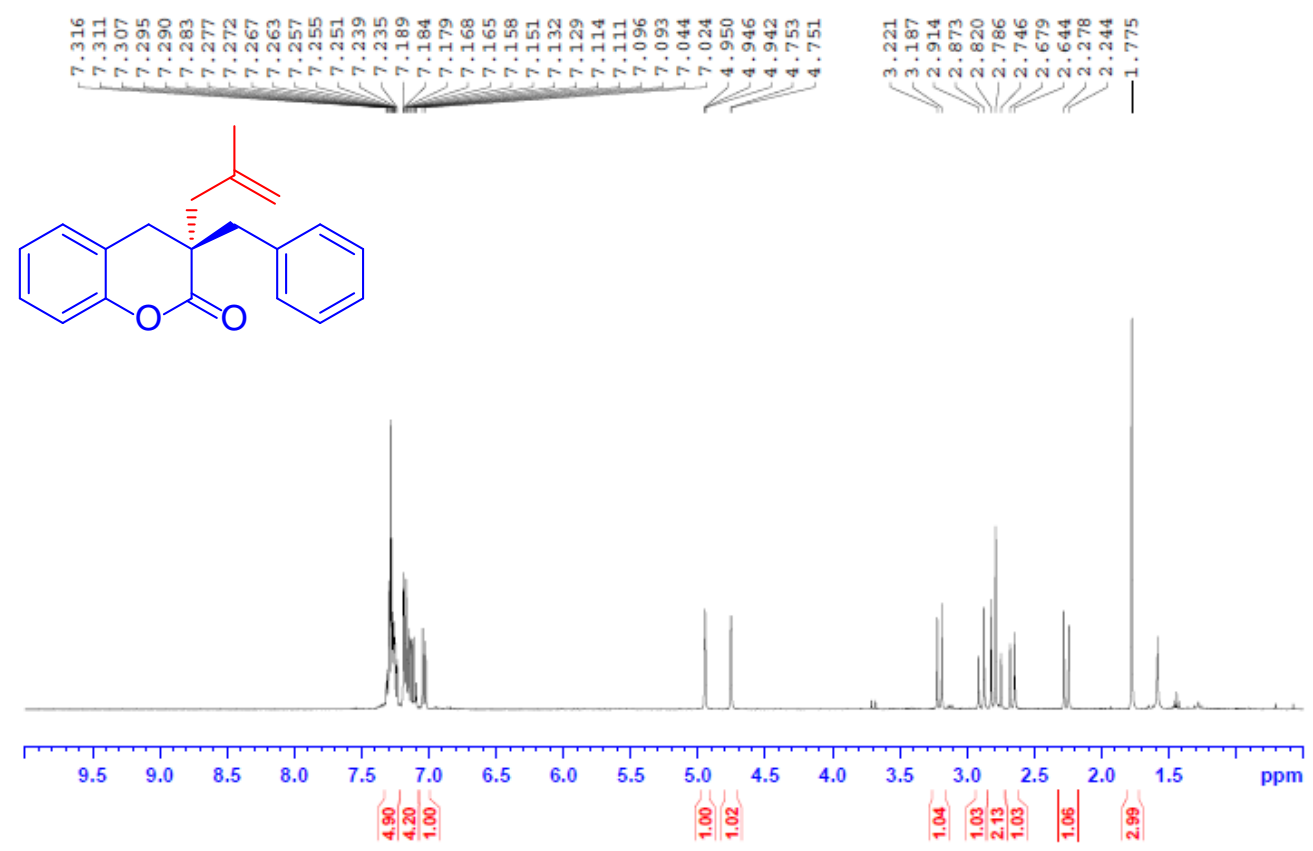<smiles>C=C(C)C[C@@]1(Cc2ccccc2)Cc2ccccc2OC1=O</smiles>

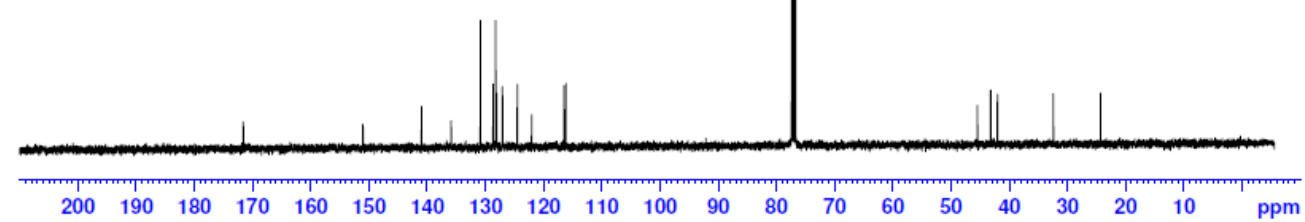


<smiles>C=C(C)C[C@]1(C/C=C/c2ccccc2)Cc2ccccc2OC1=O</smiles>

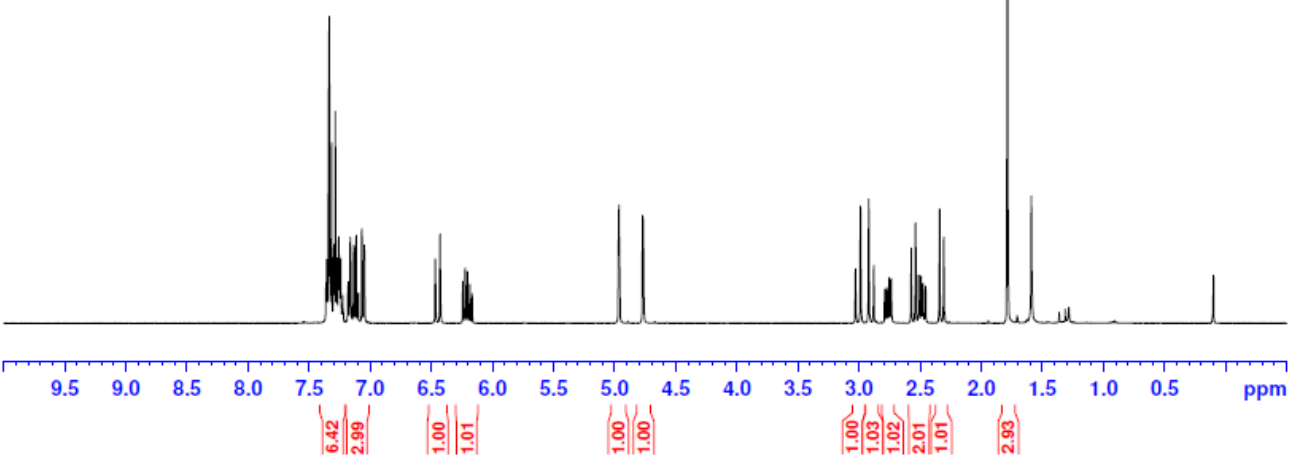<smiles>C=C[C@]1(C/C=C/c2ccccc2)Cc2ccccc2OC1=O</smiles>

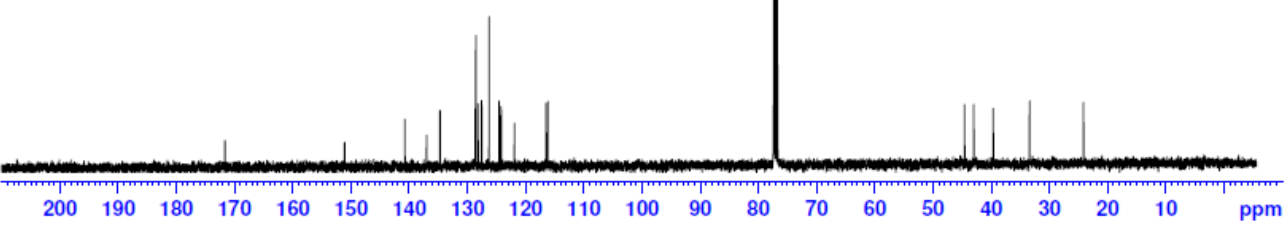



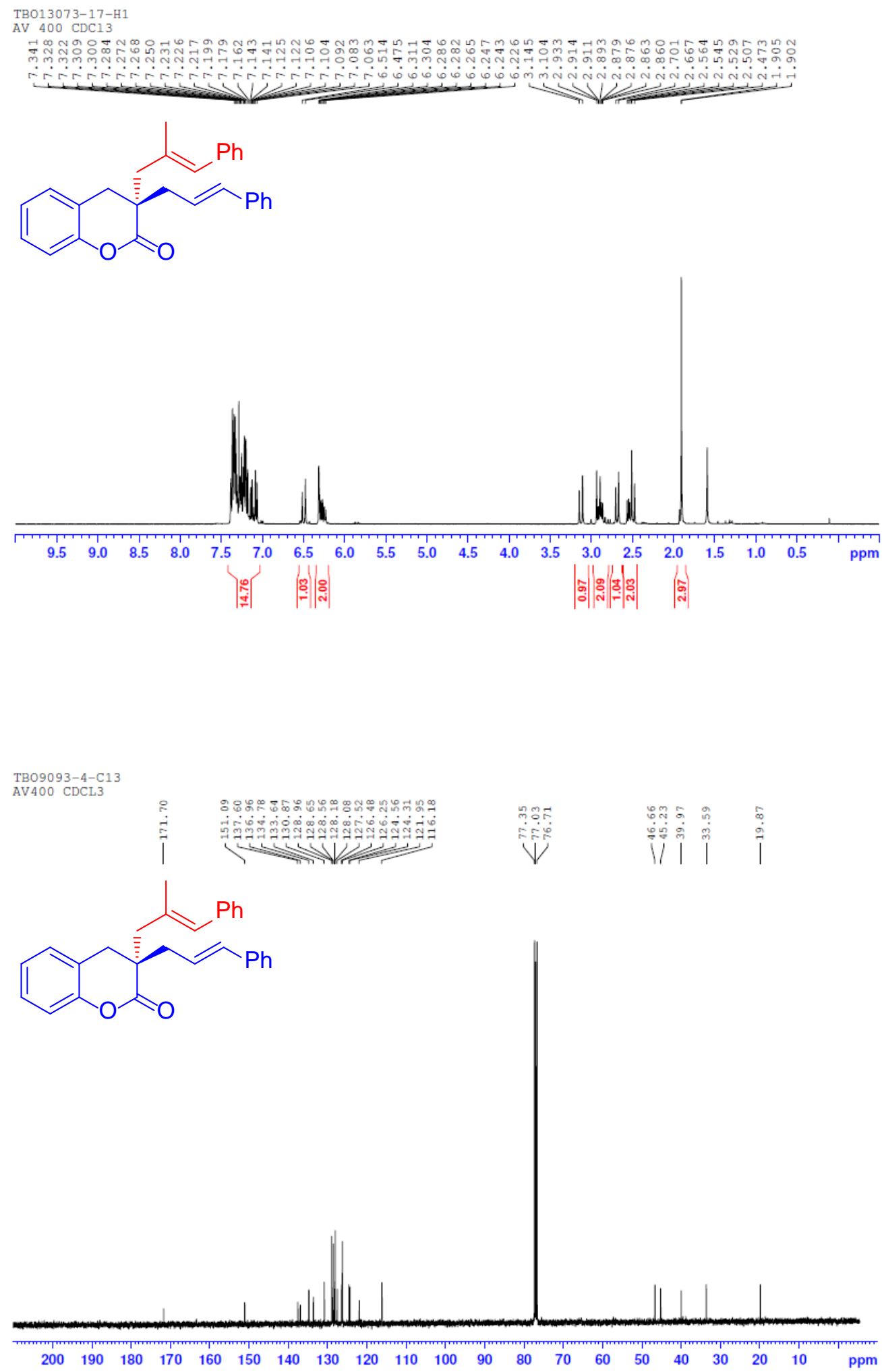

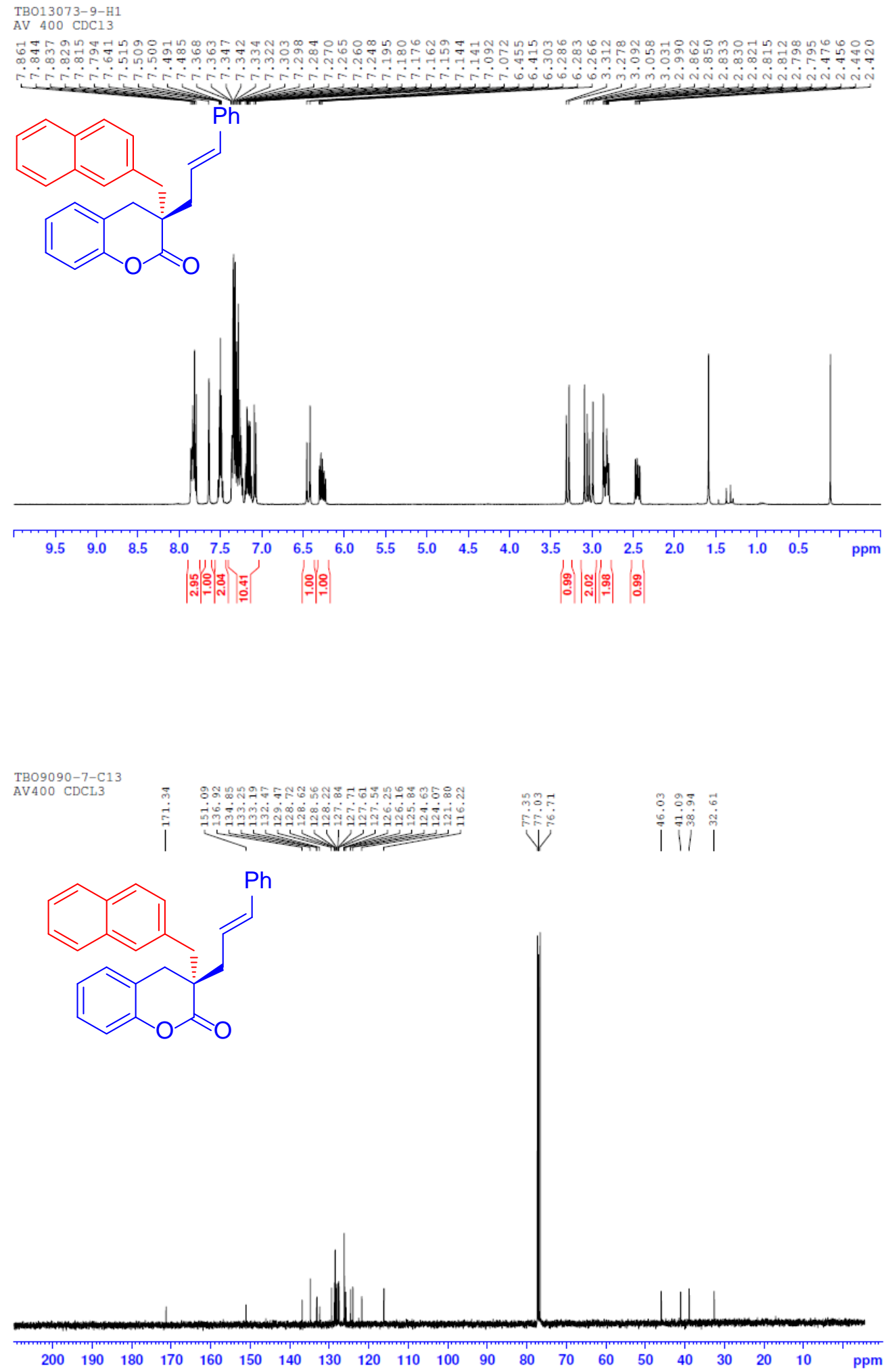


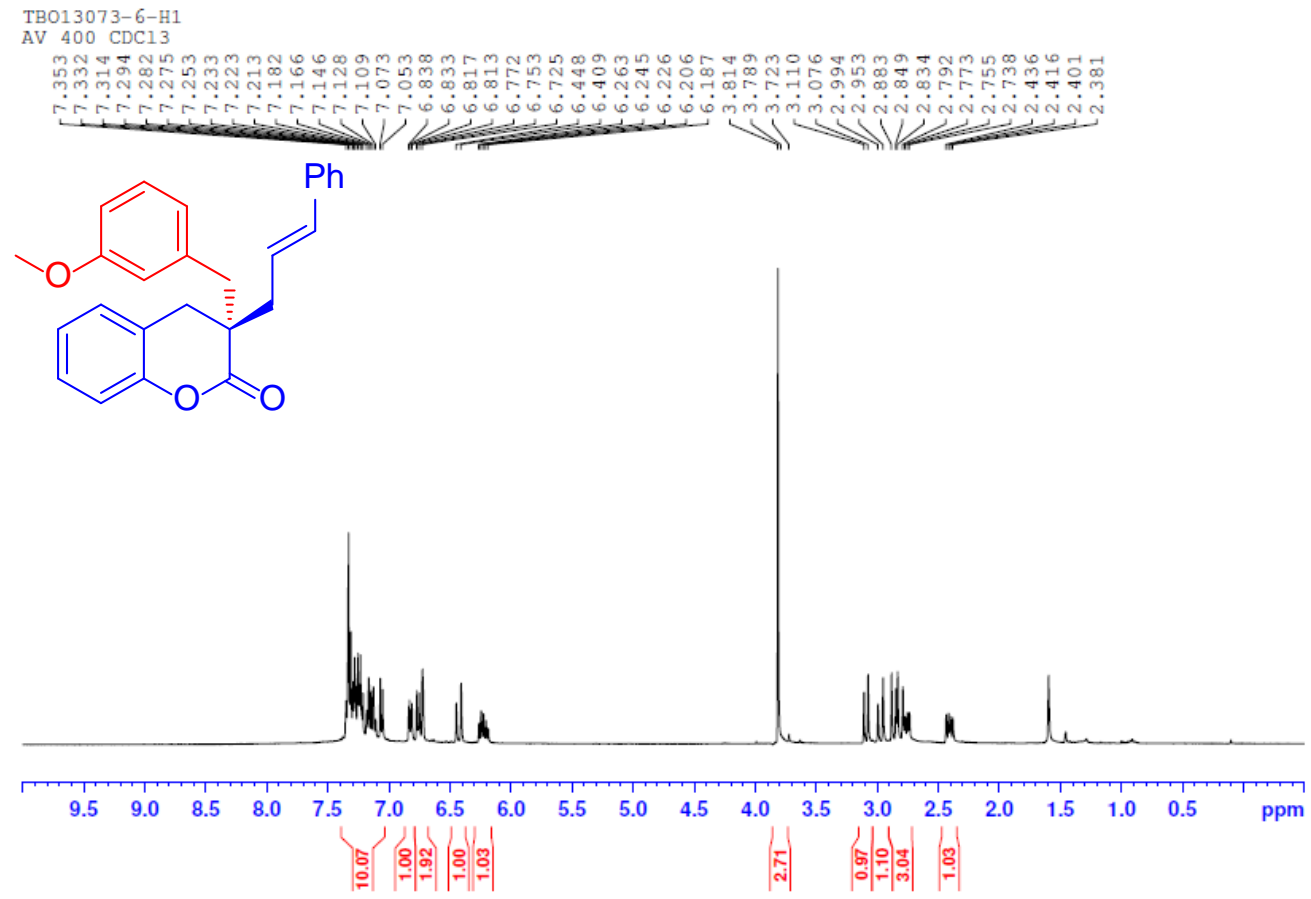

TB09090-4-C13
AV400 CDCL3<smiles>COc1cccc(C/C=C/C[C@H]2Cc3ccccc3OC(=O)[C@H]2Cc2ccccc2)c1</smiles>

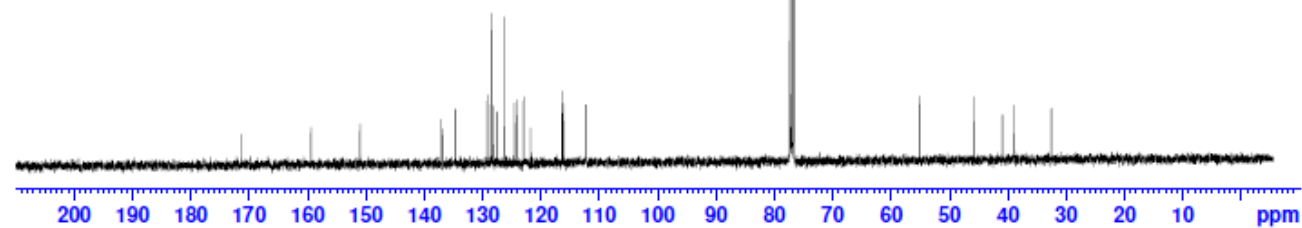


TB013073-24-H1

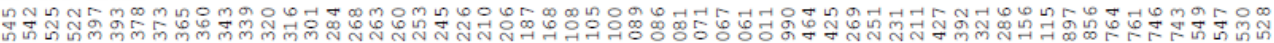

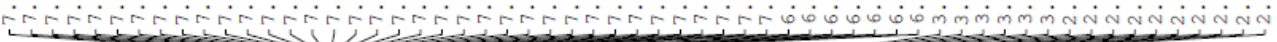<smiles>O=C1Oc2ccccc2C[C@]1(C/C=C/Pc1ccccc1)Cc1ccccc1Br</smiles>
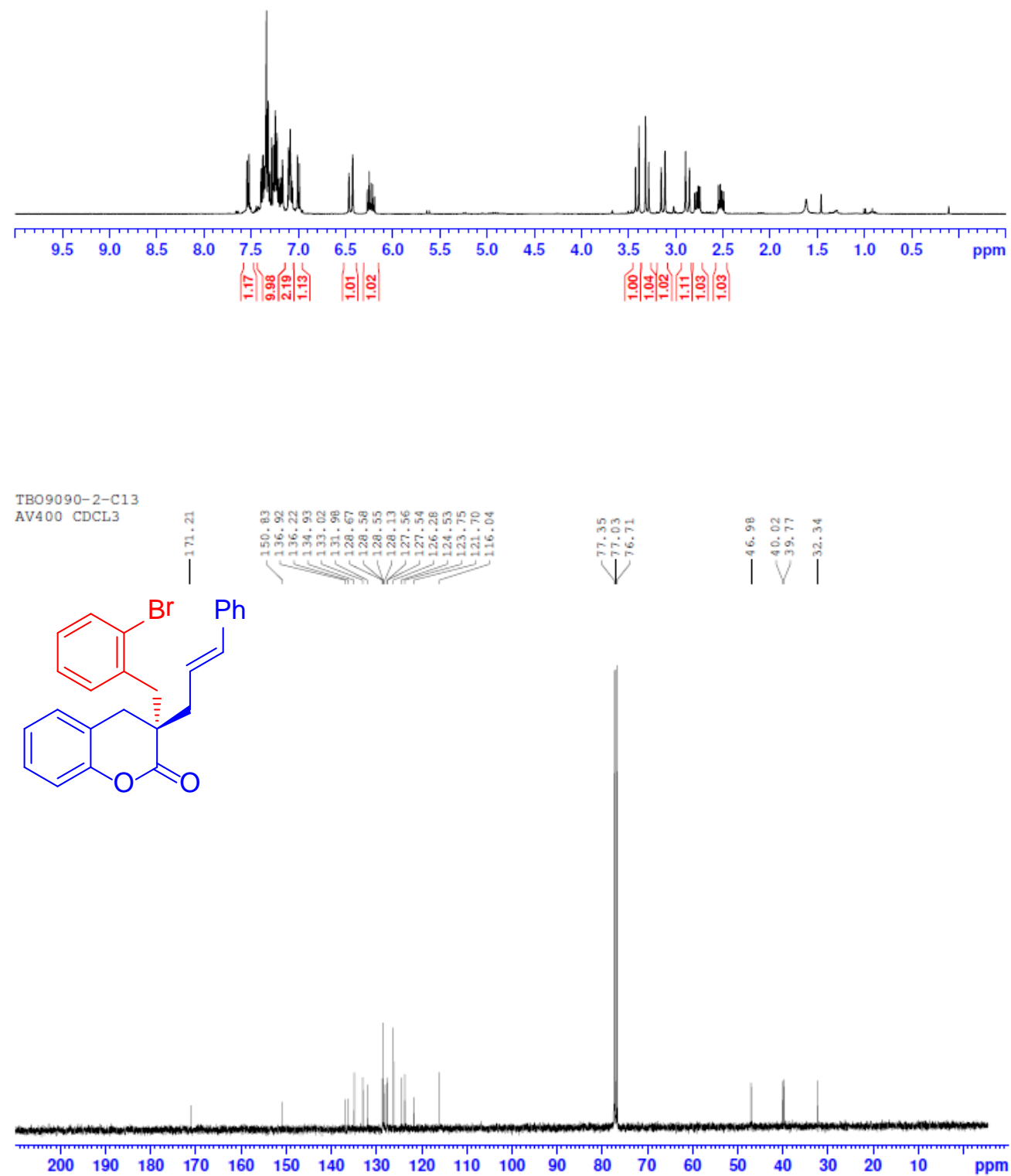

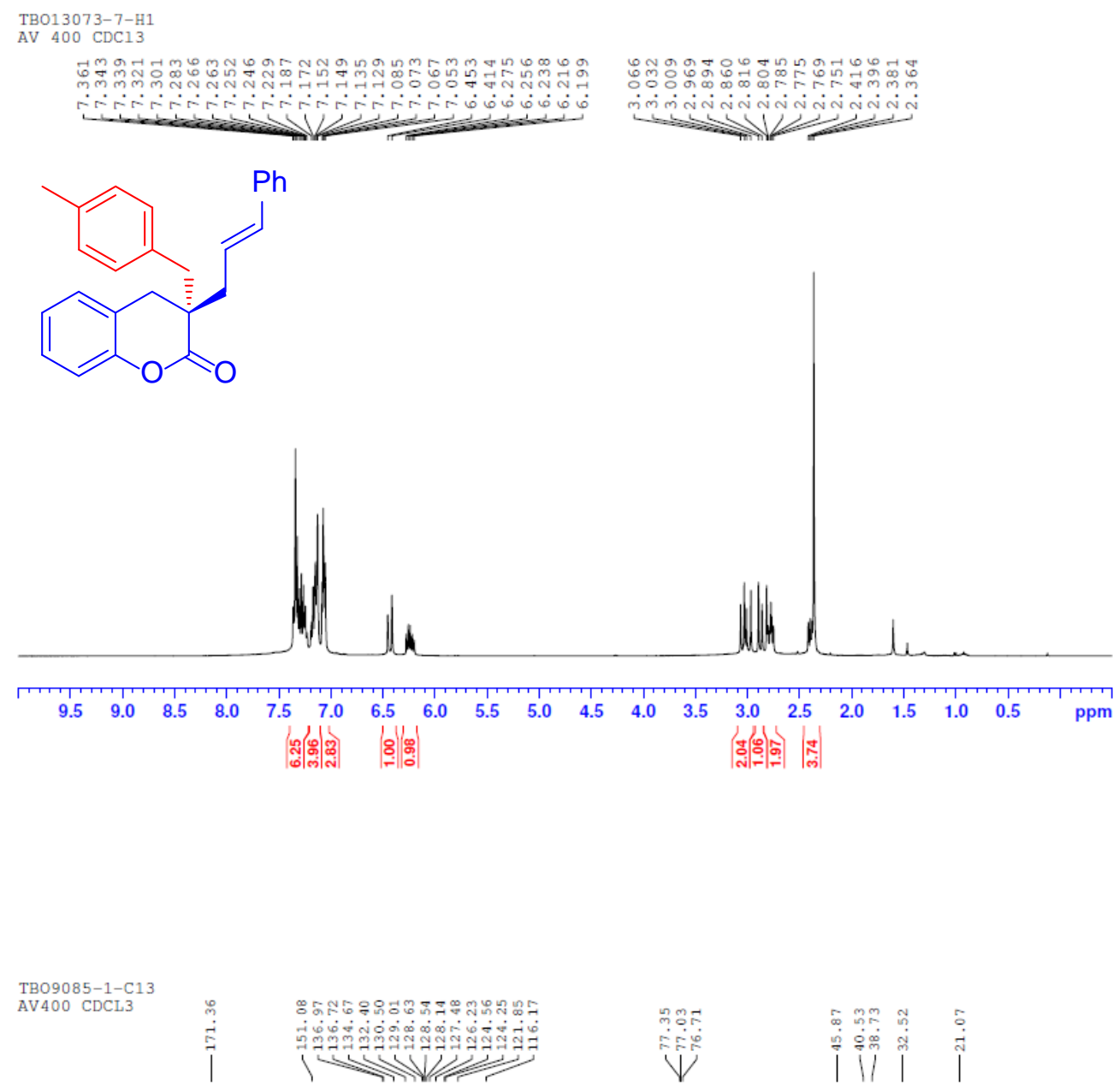<smiles>Cc1ccc(C/C=C/c2ccccc2)cc1C[C@@]1(C/C=C/c2ccccc2)Cc2ccccc2OC1=O</smiles>

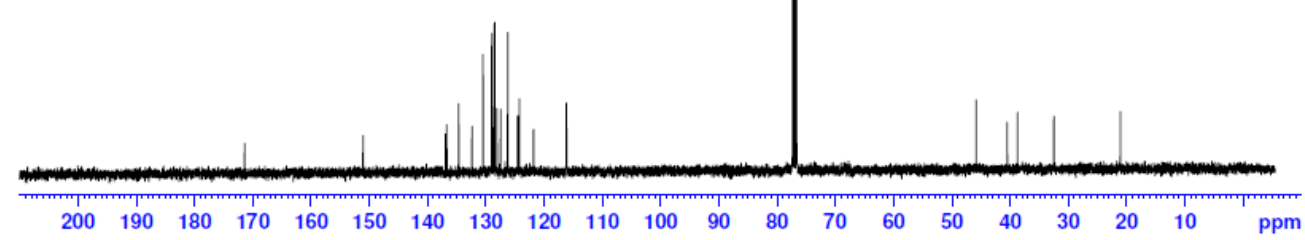


TB013073-8-H1

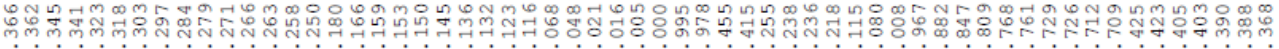

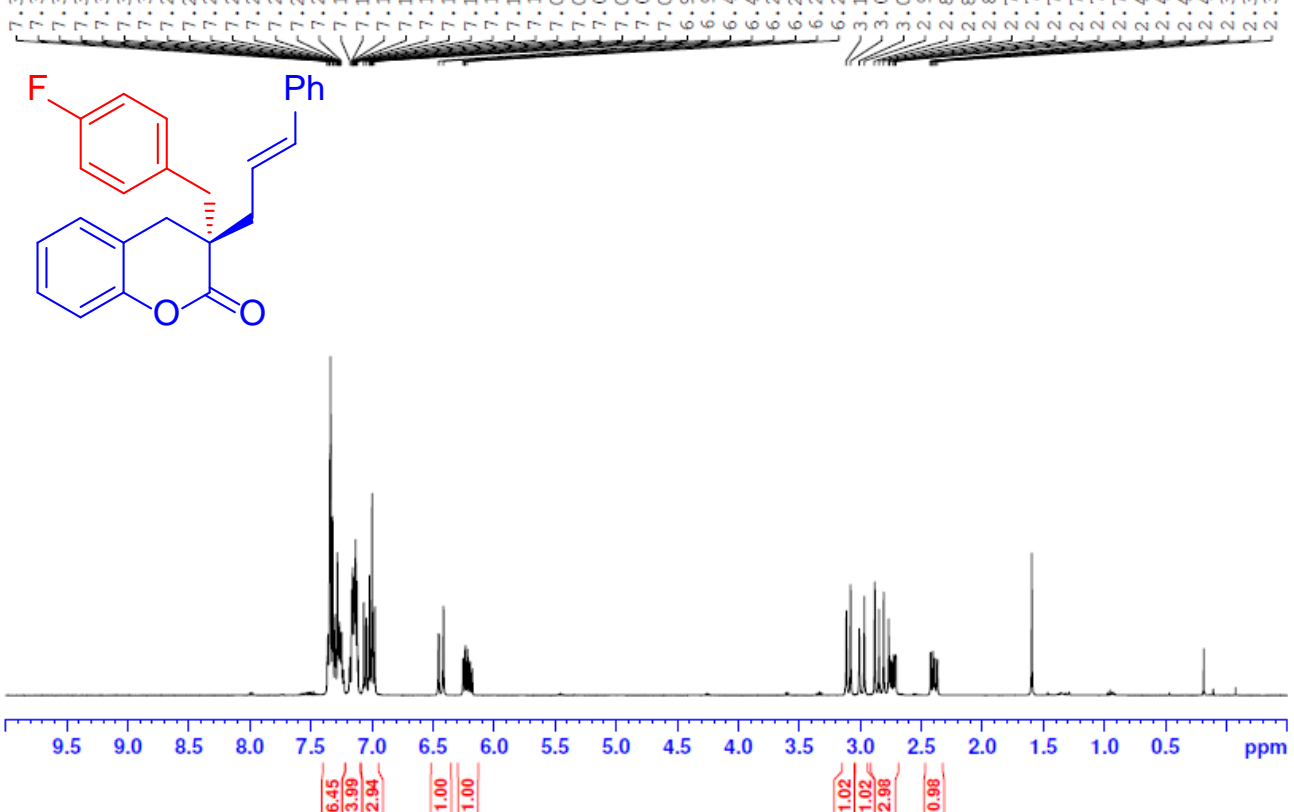

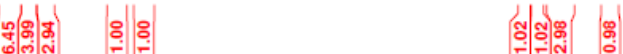

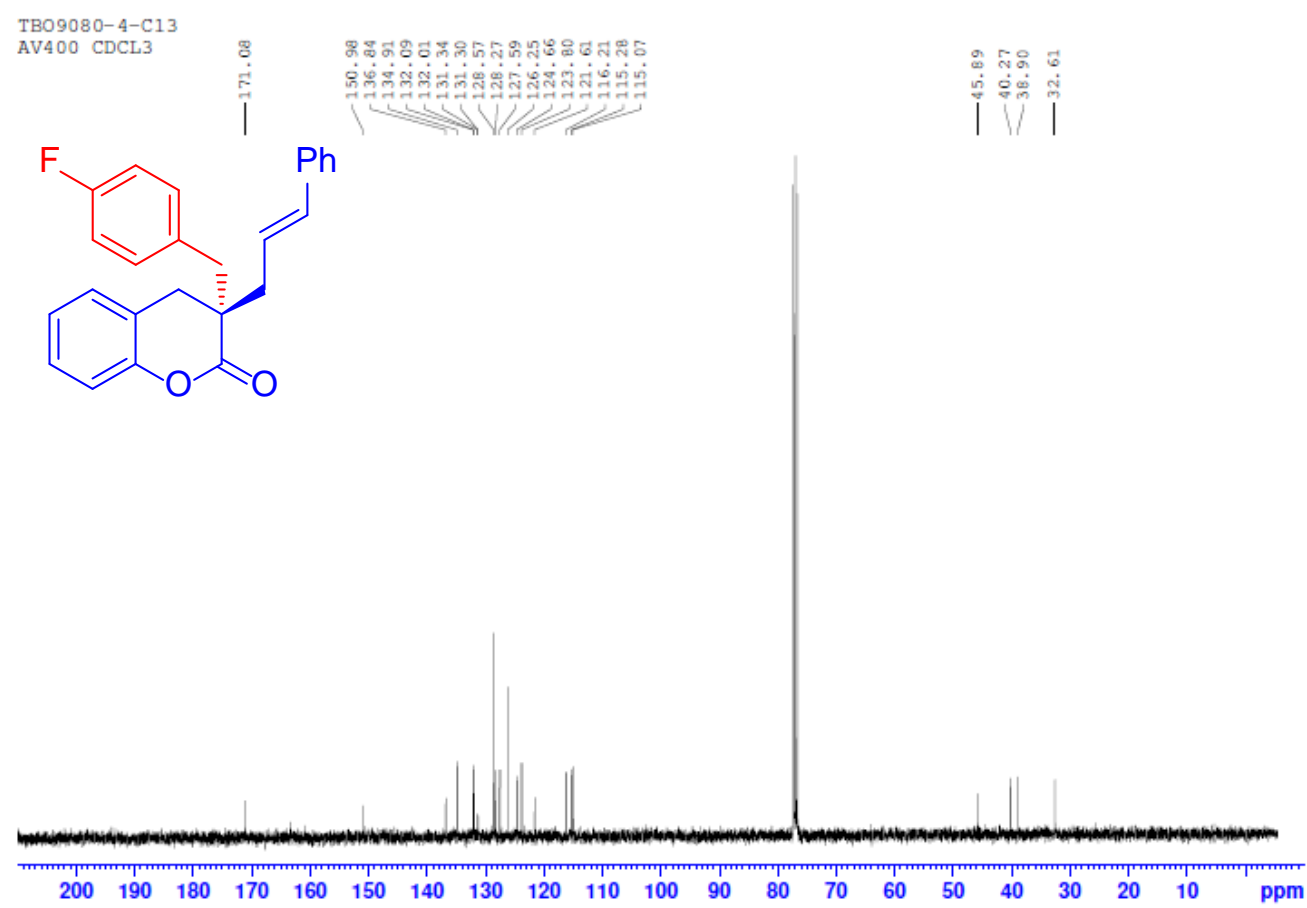




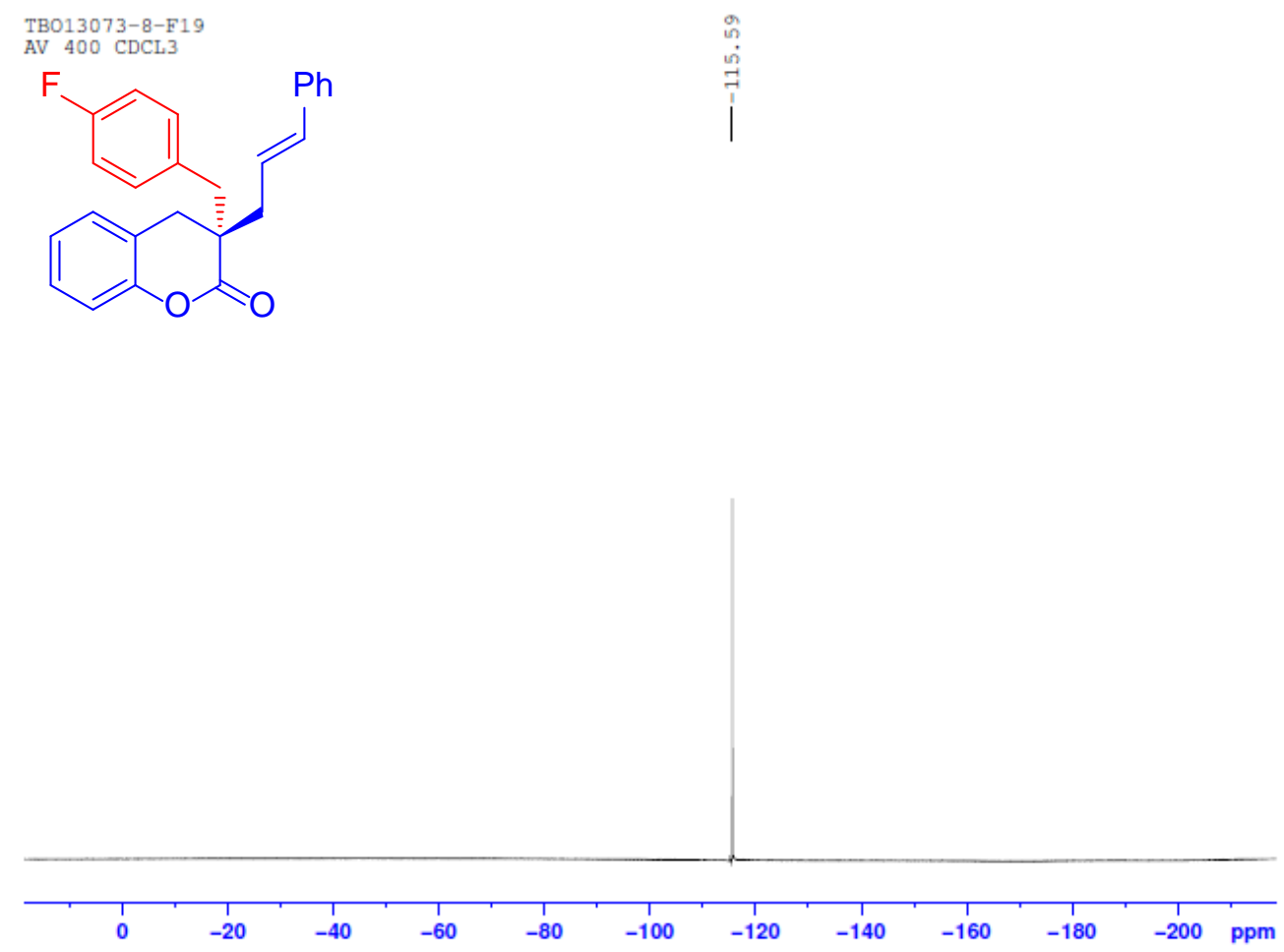

TB013073-14-H1
AV 400 CDC13

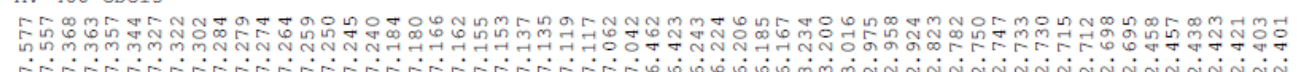

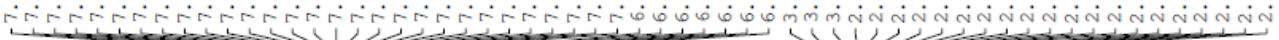

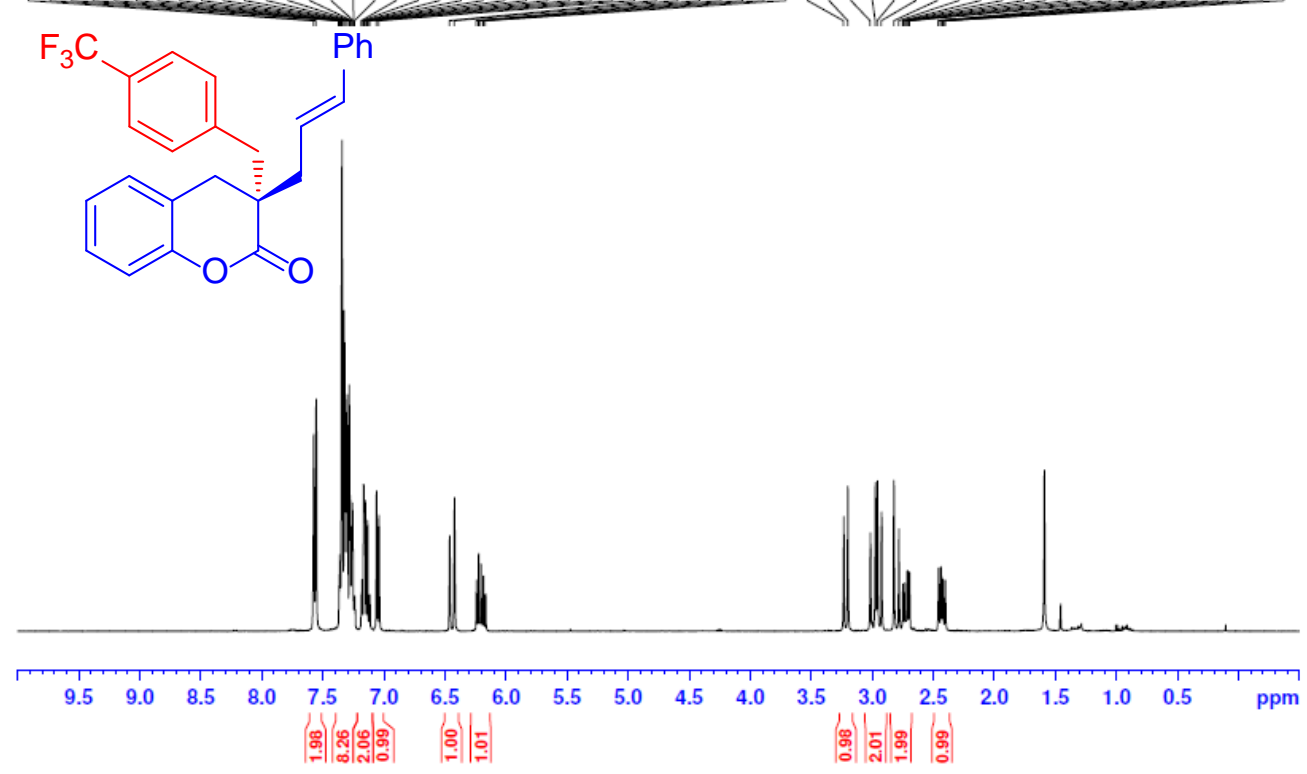



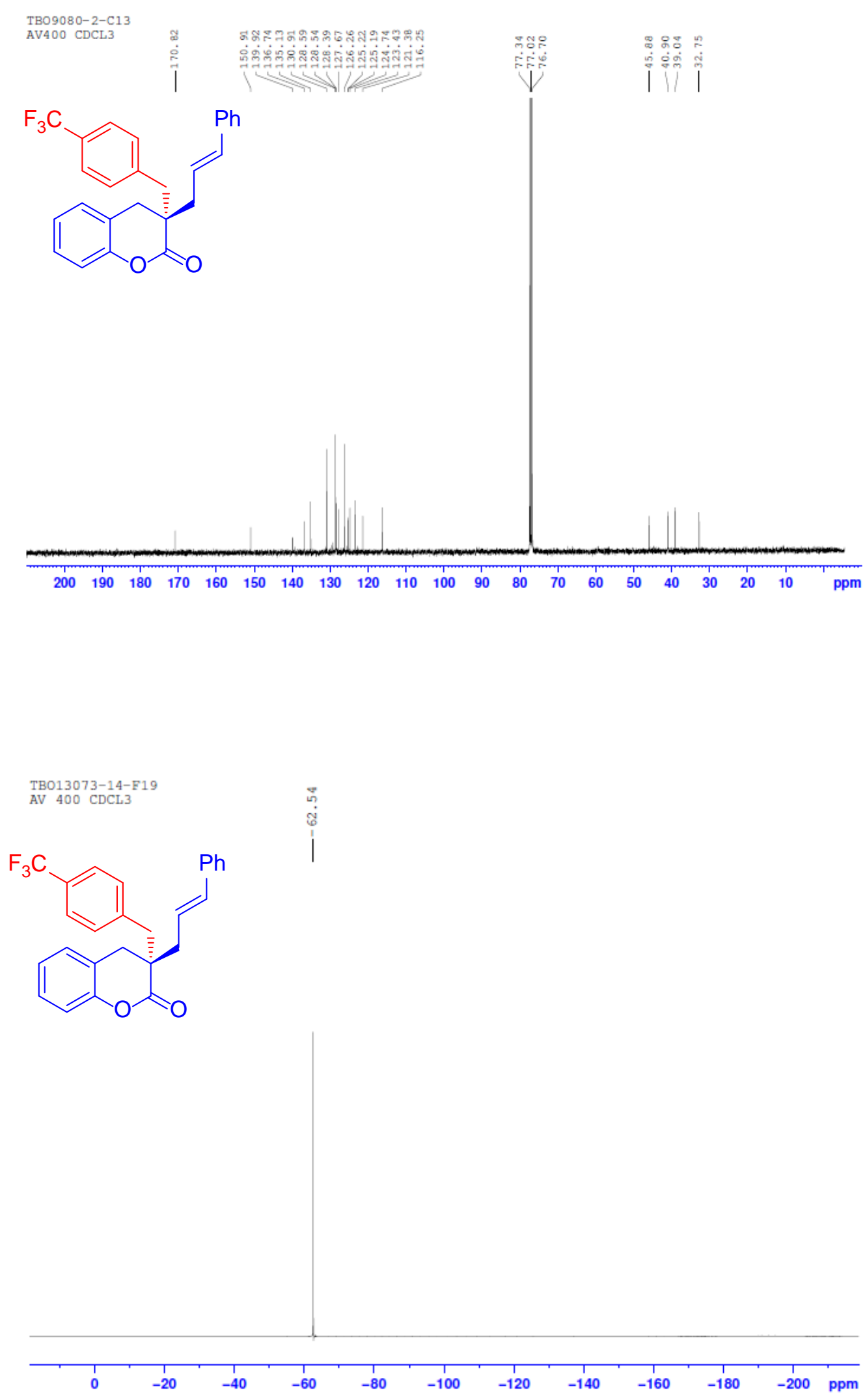
TB010003-1-H1

(c)
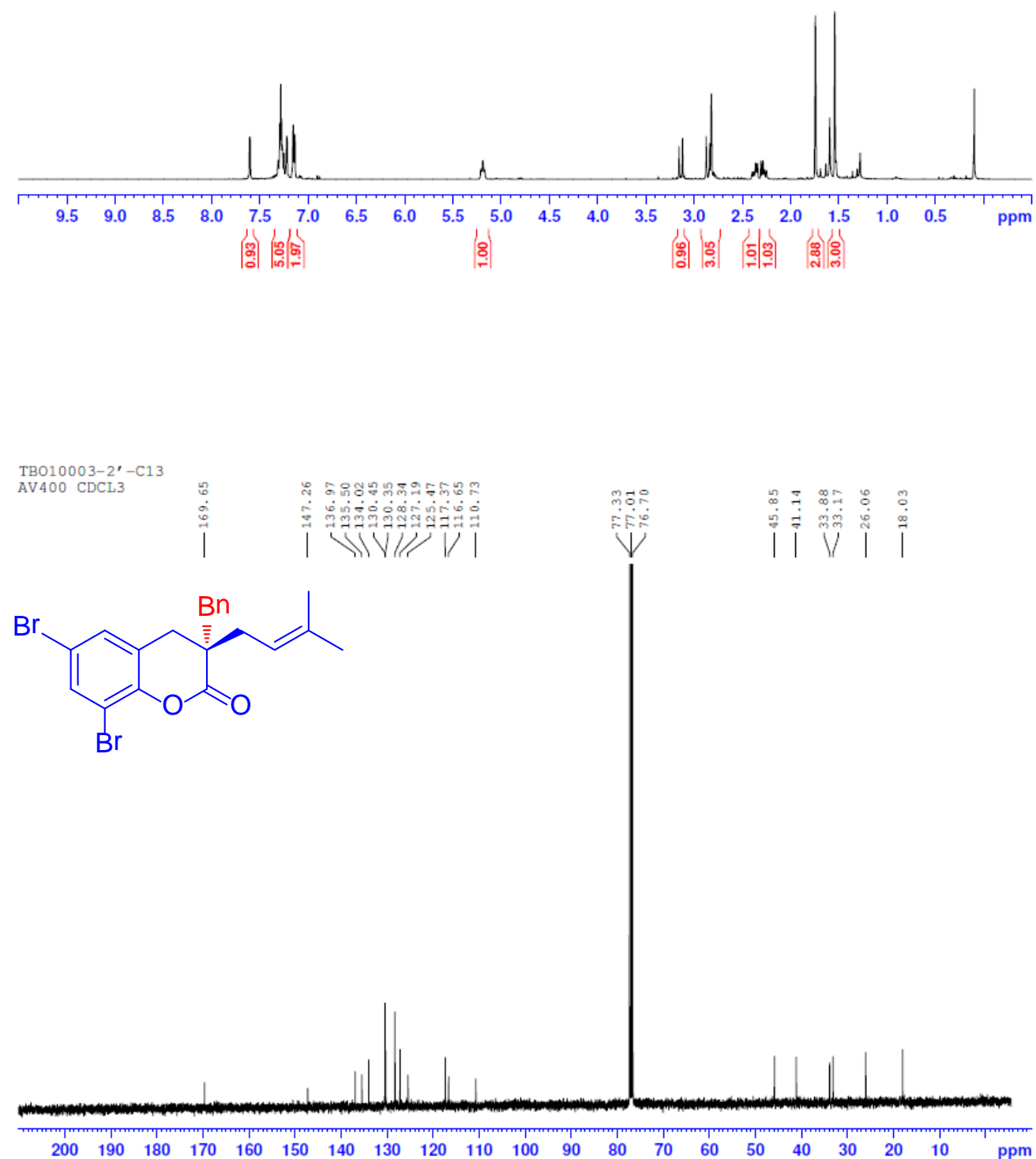
TB012089-6-H1'

BBF1

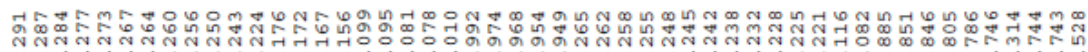

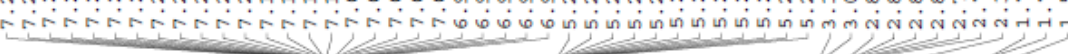<smiles>CC(C)=CC[C@]1(Br)Cc2cccc(C)c2OC1=O</smiles>

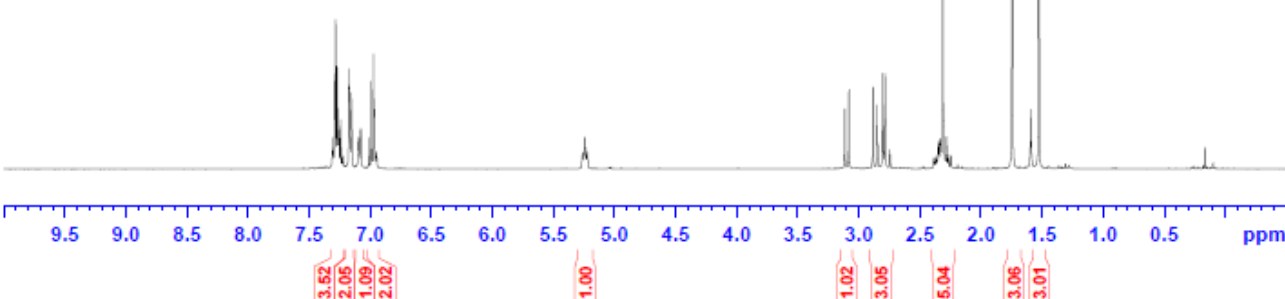

TBO10003-6-C13
AV400 CDCL3

|

ข้

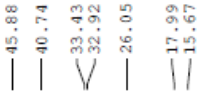<smiles>CC(C)=CC[C@]1(Br)Cc2cccc(C)c2OC1=O</smiles>

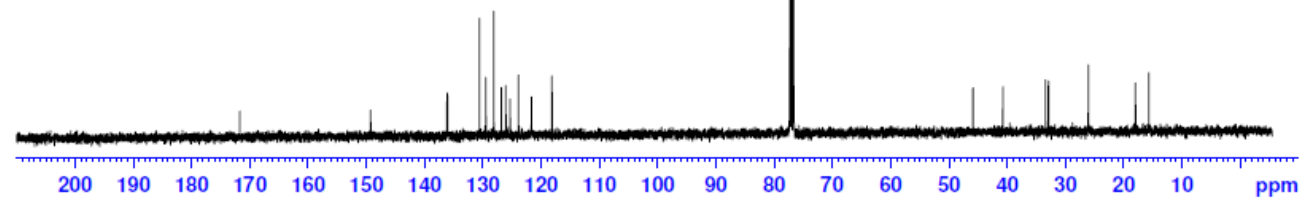


TBO12089-3-H1

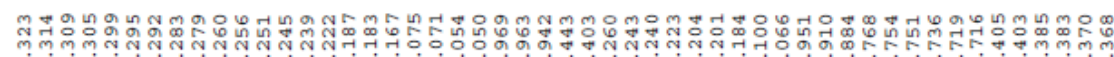

กง

$\mathrm{Bn}$<smiles>Cc1ccc2c(c1)OC(=O)C(C/C=C\c1ccccc1)(Cc1ccccc1)C2</smiles>

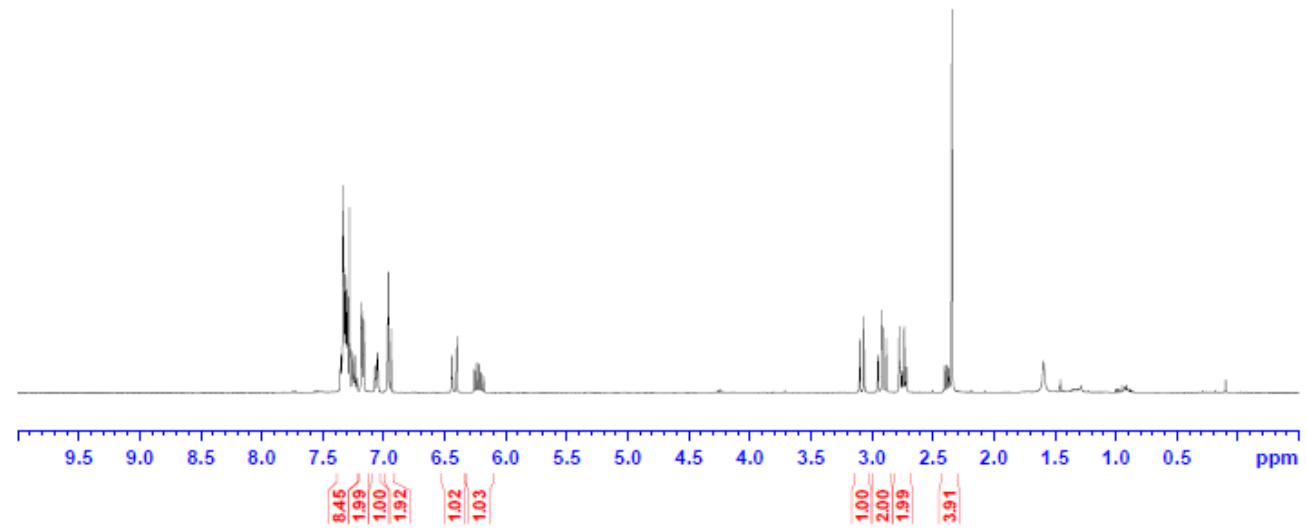

TB012089-3-C13
BBF1

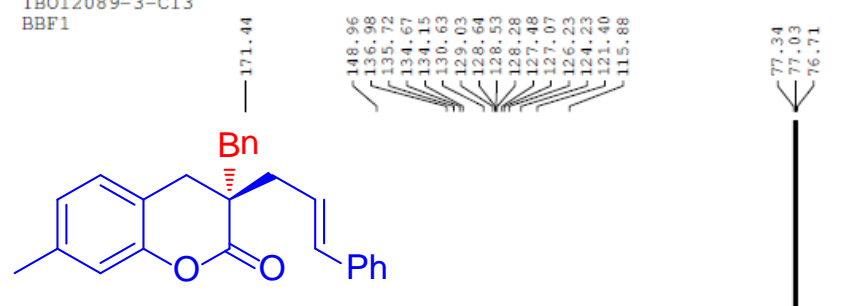

|

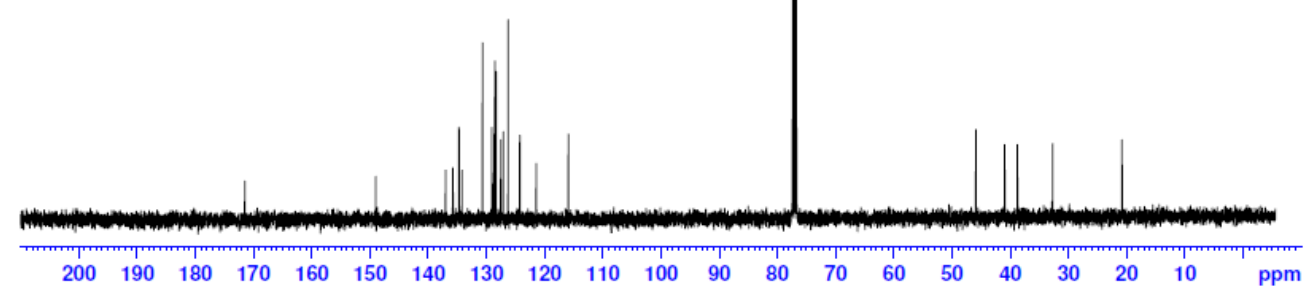


TB013073-12-H1

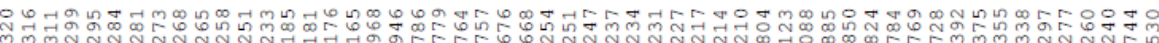

-<smiles>COc1ccc2c(c1)CC(CC=C(C)C)(CC=C(C)C)C(=O)O2</smiles>

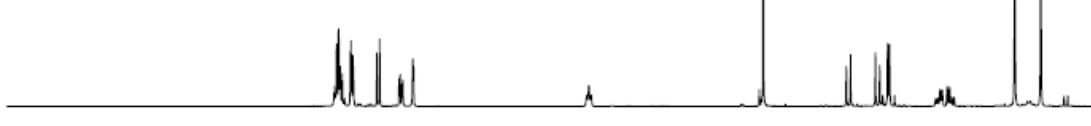

$\begin{array}{lllllllllllllllllllllll}9.5 & 9.0 & 8.5 & 8.0 & 7.5 & 7.0 & 6.5 & 6.0 & 5.5 & 5.0 & 4.5 & 4.0 & 3.5 & 3.0 & 2.5 & 2.0 & 1.5 & 1.0 & 0.5 & \mathrm{ppm}\end{array}$

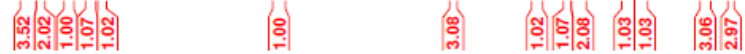

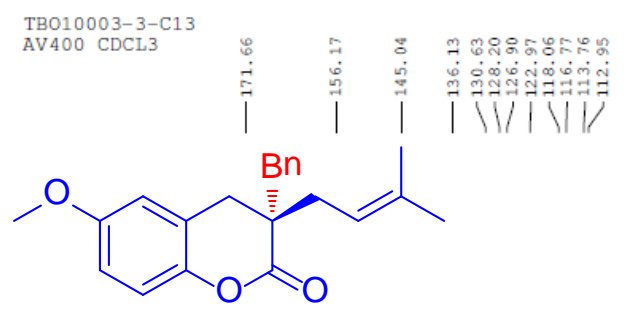

为

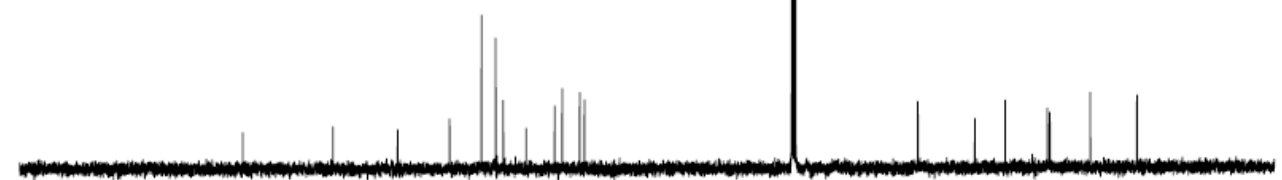

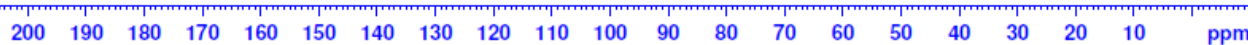




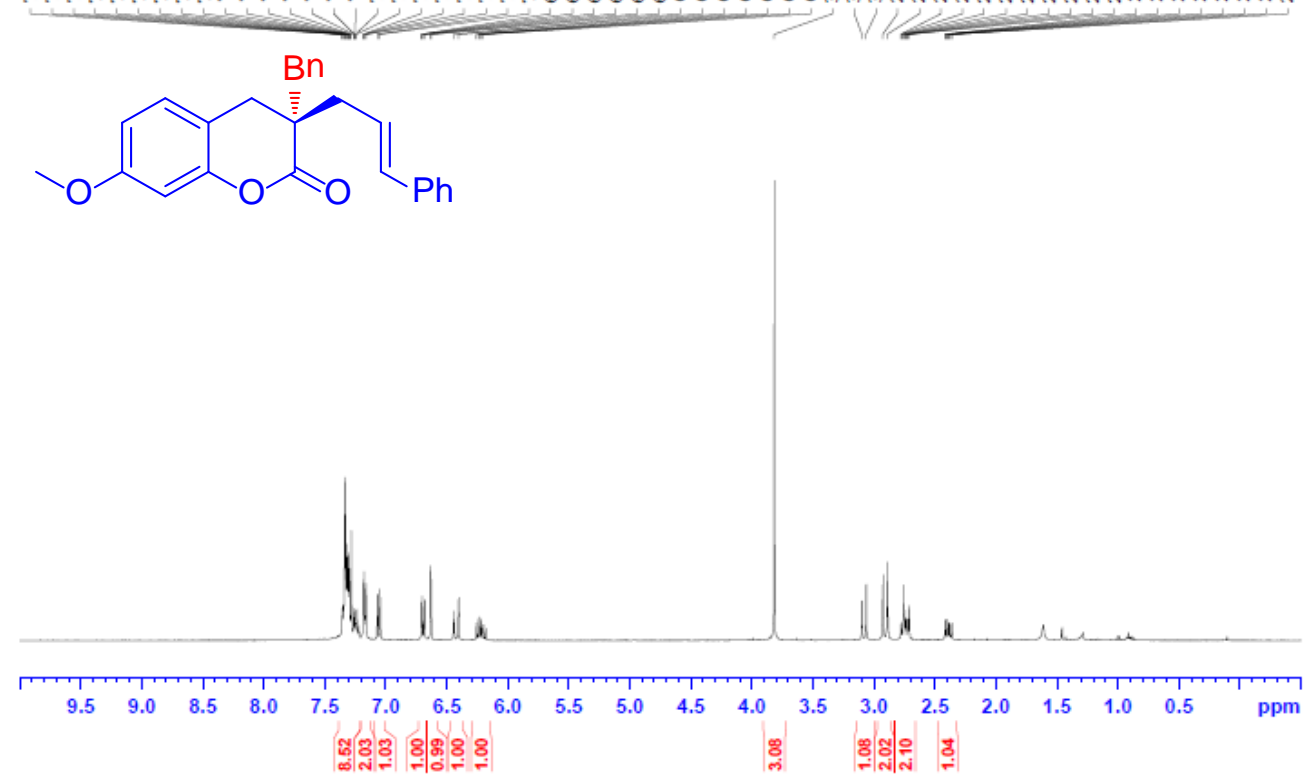<smiles>COc1ccc2c(c1)OC(=O)[C@](C/C=C/c1ccccc1)(Cc1ccccc1)C2</smiles>

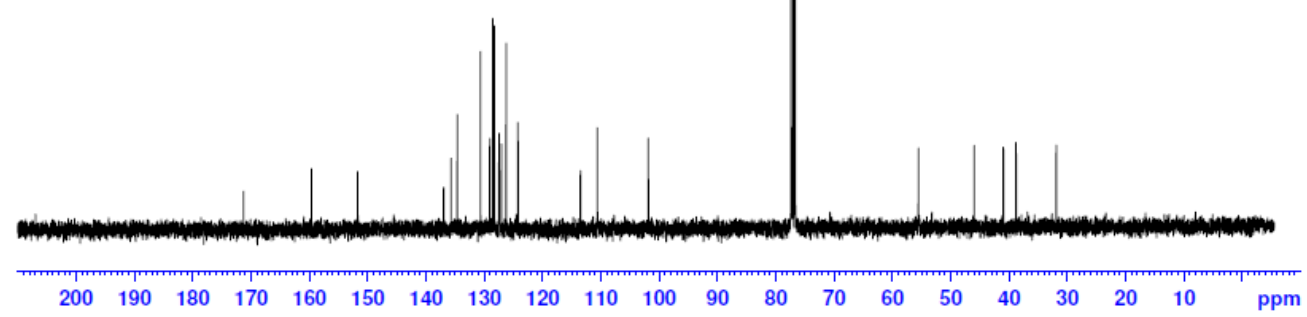



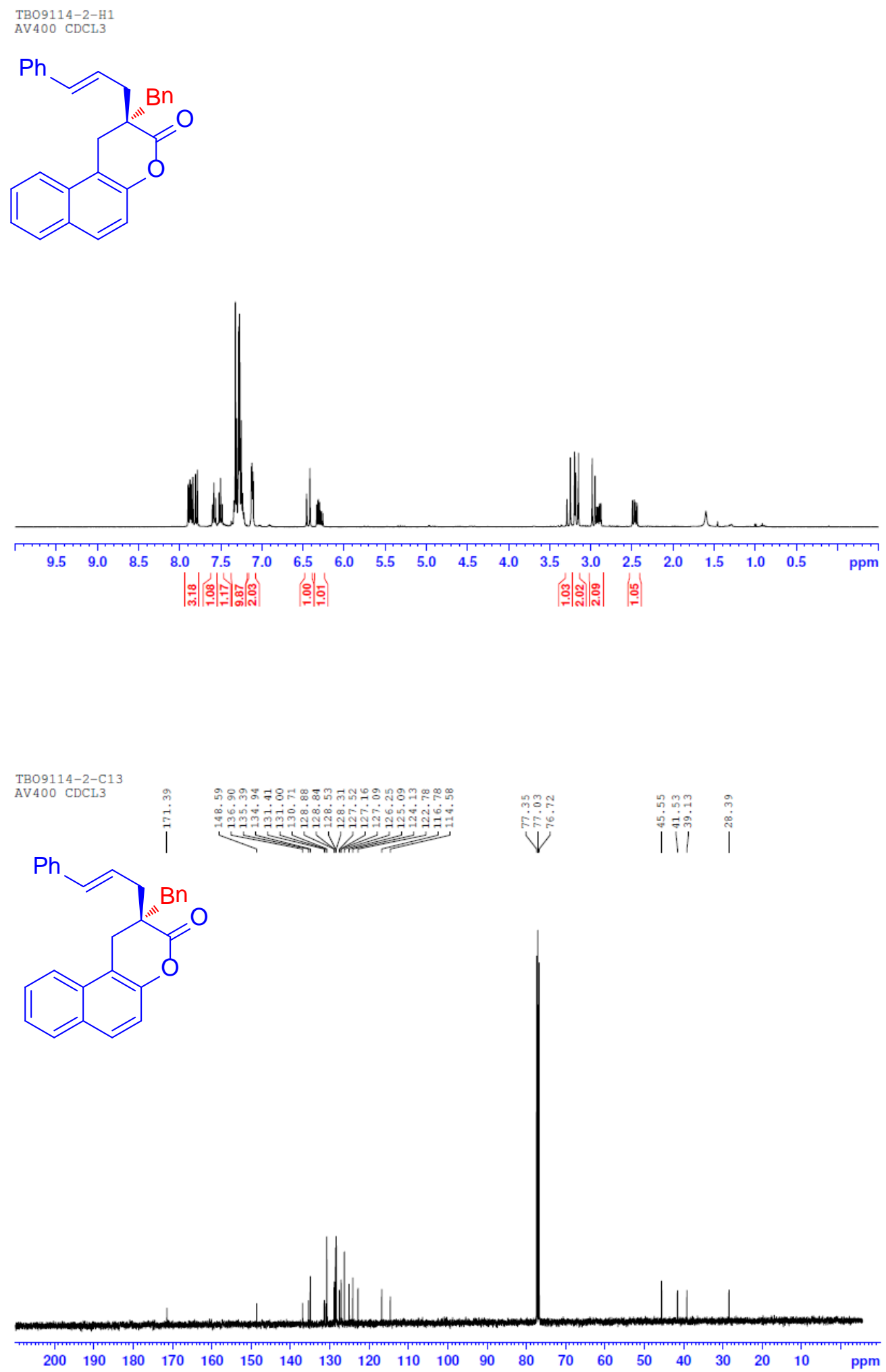

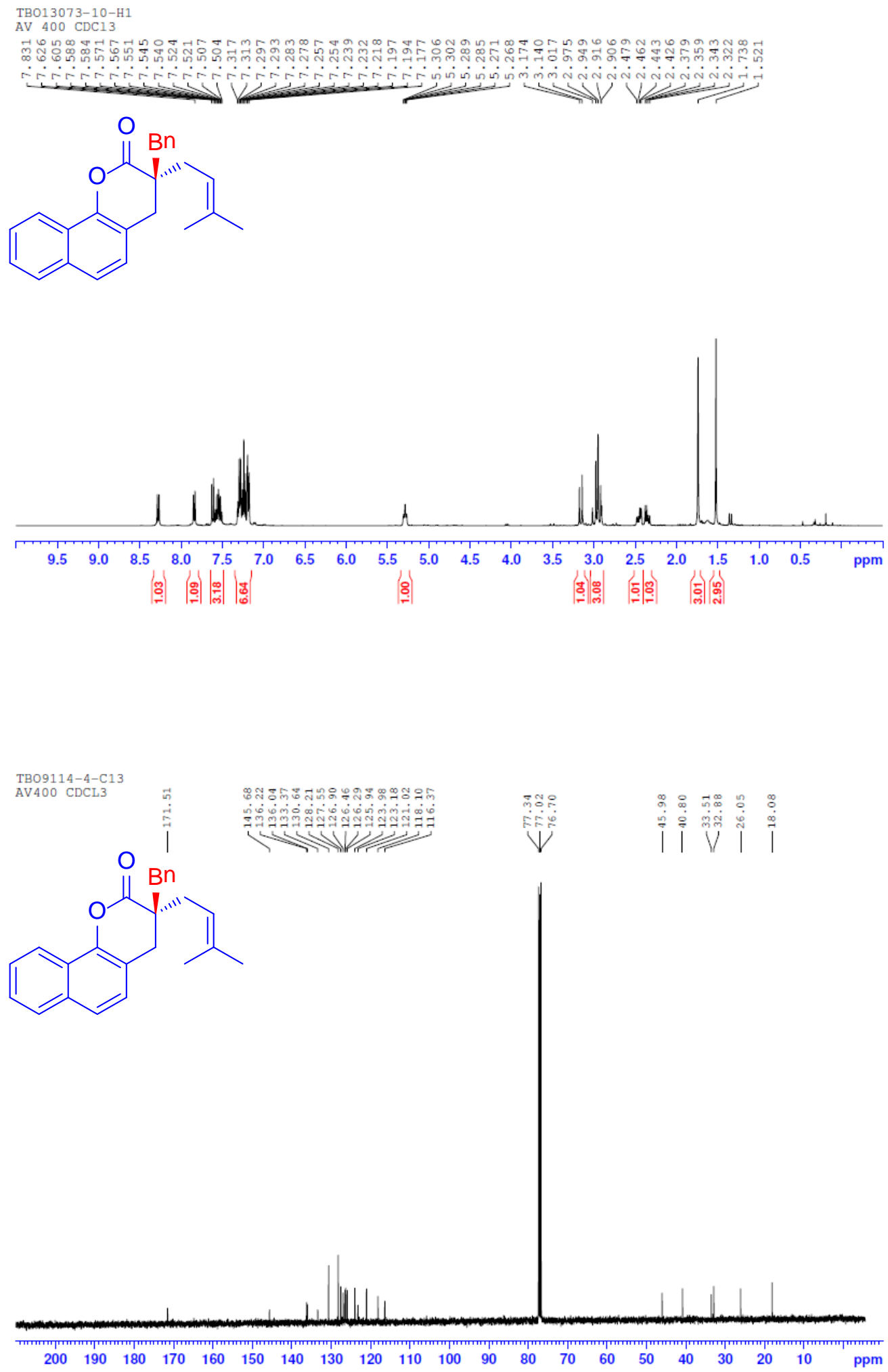

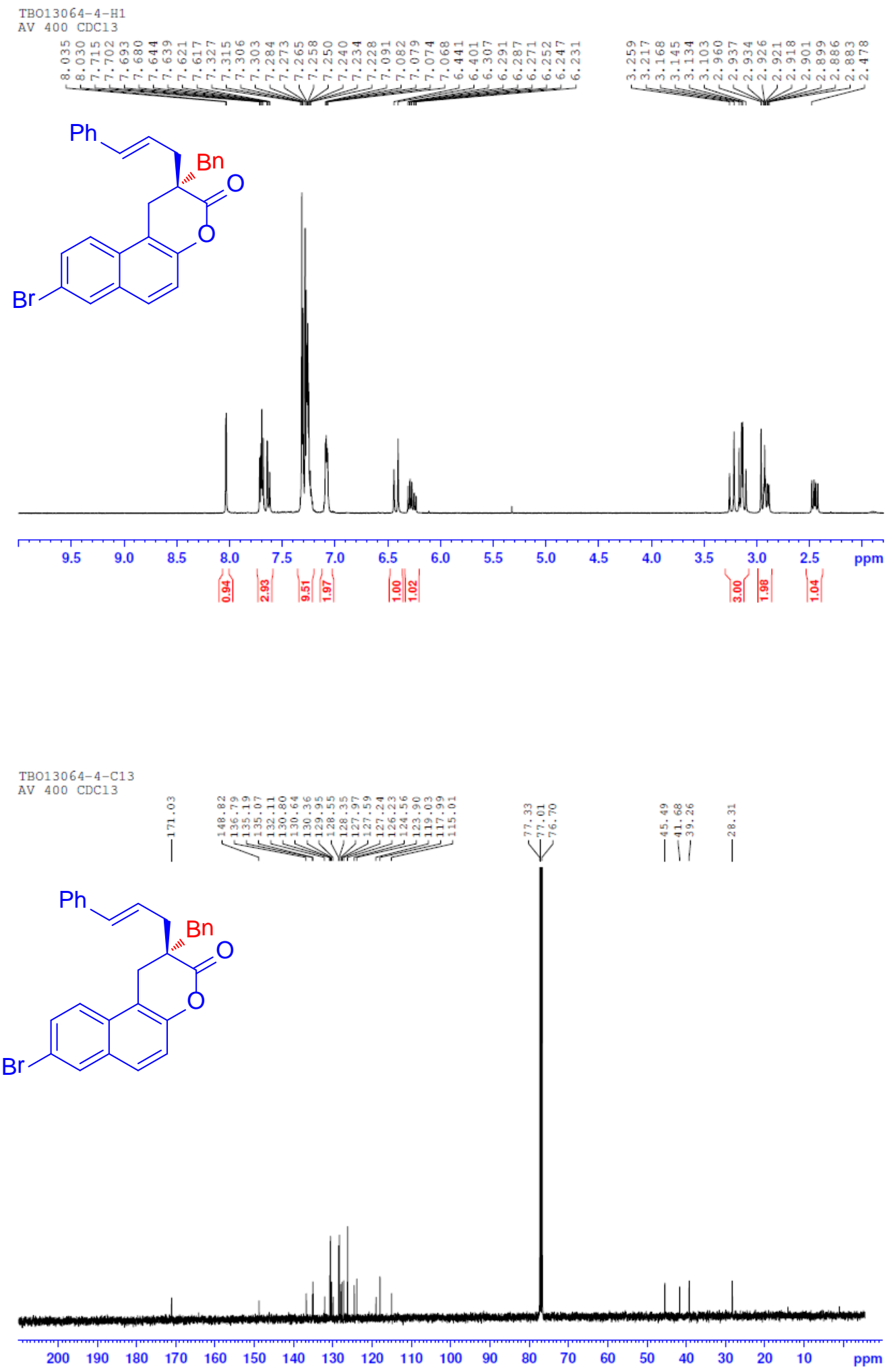


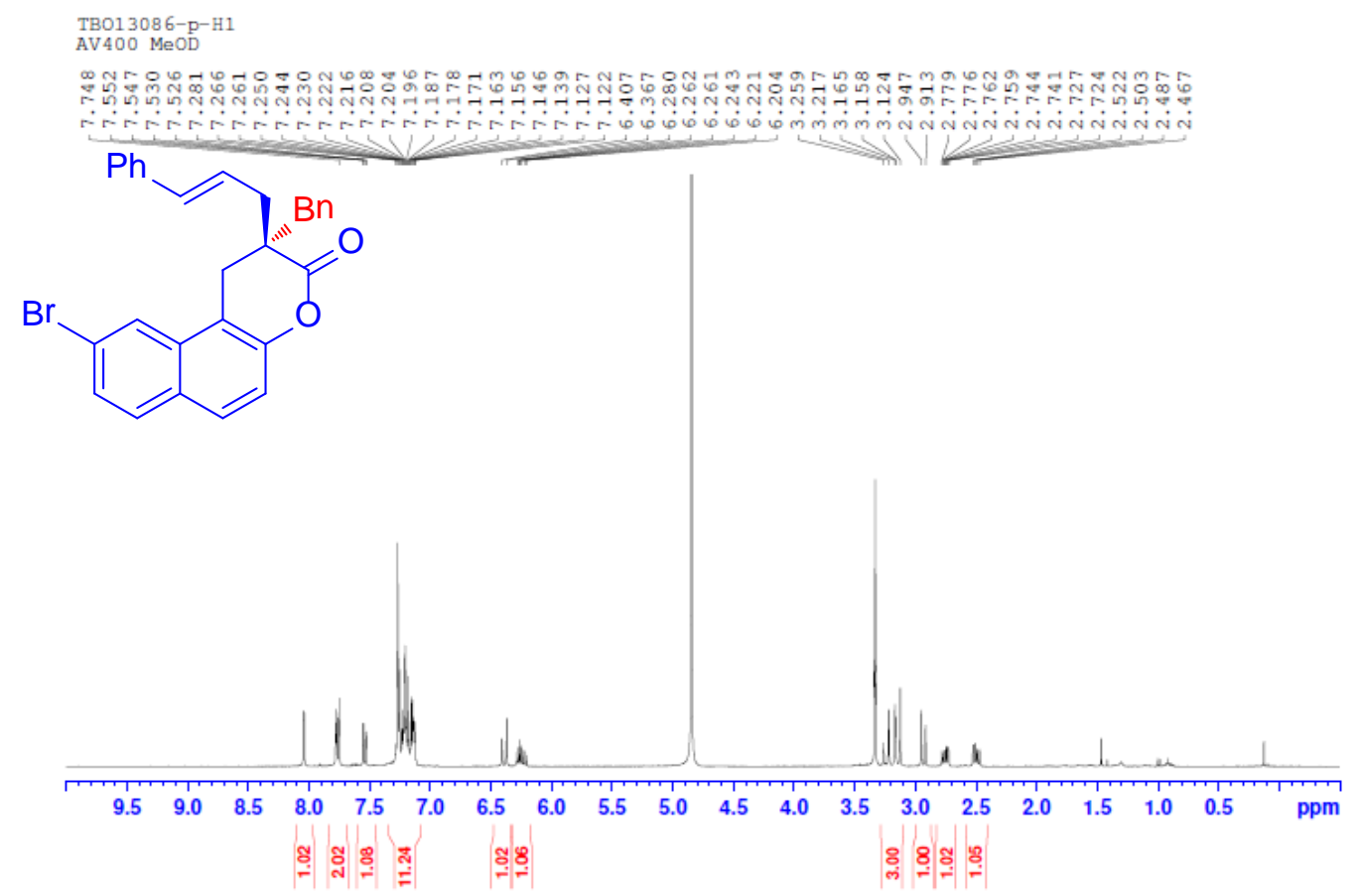

TB013086-p-C1

AV4 $00 \mathrm{NeOD}$

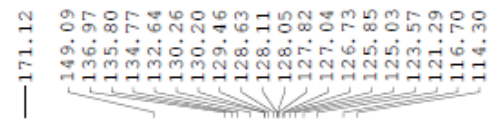

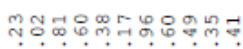

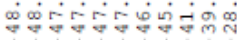<smiles>O=C1Oc2ccc3ccc(Br)cc3c2C[C@]1(C/C=C/c1ccccc1)Cc1ccccc1</smiles>

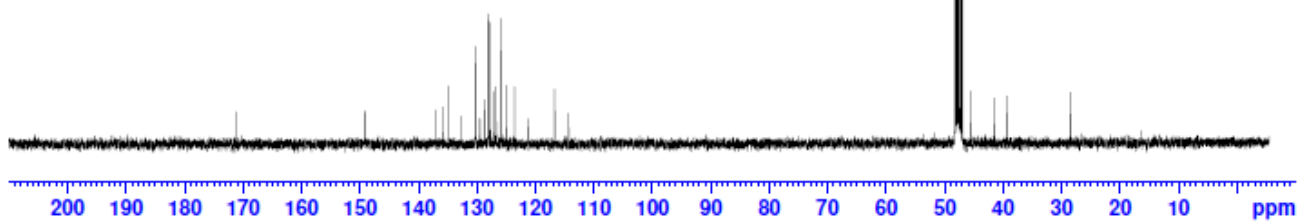


TB013090-1-H1
AV400 CDCL3 3

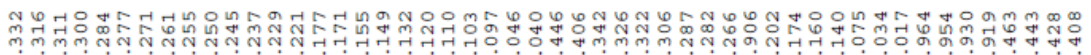

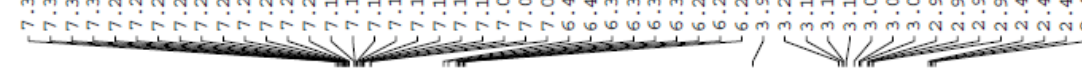<smiles>COc1ccc2ccc3c(c2c1)C[C@](Br)(C/C=C/c1ccccc1)C(=O)O3</smiles>

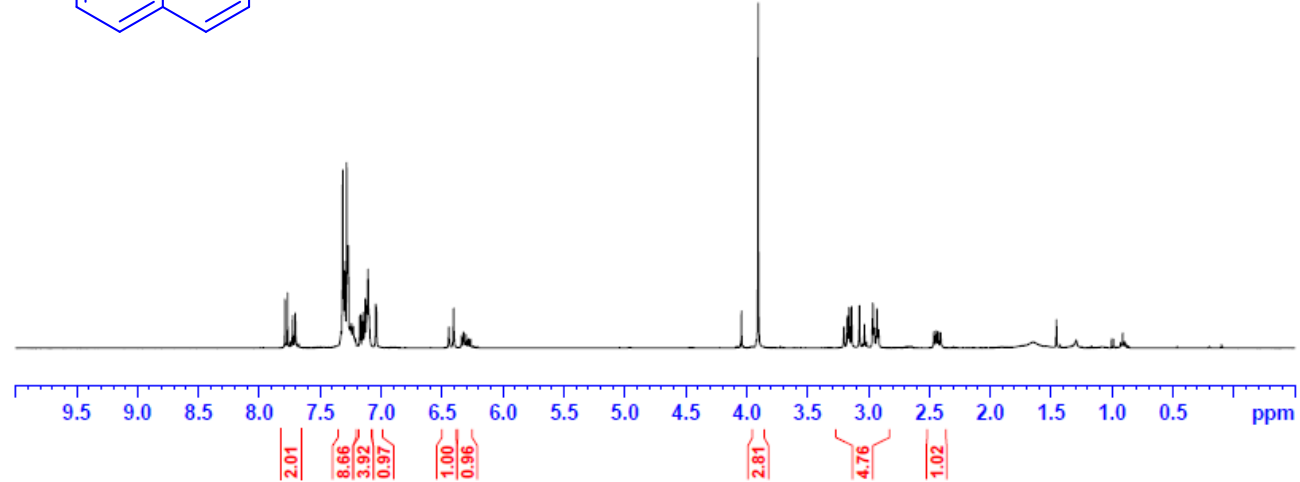

TB013090-1-C1
AV400 CDCL3

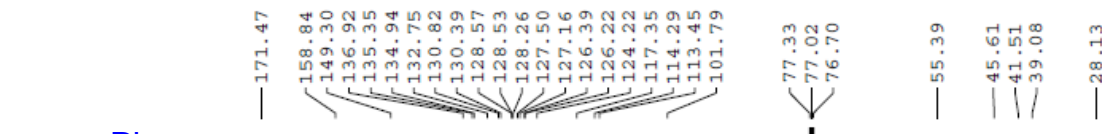<smiles>COc1ccc2ccc3c(c2c1)C[C@](Br)(C/C=C/[Pb])C(=O)O3</smiles>

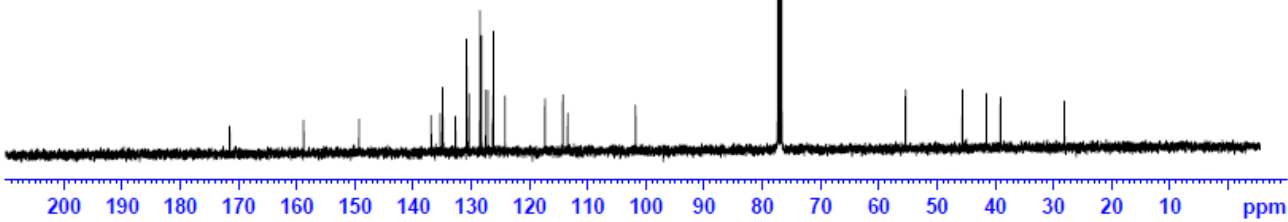


TBO13142-1-H1

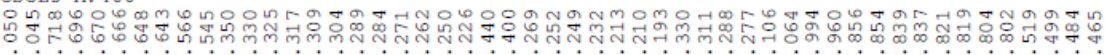

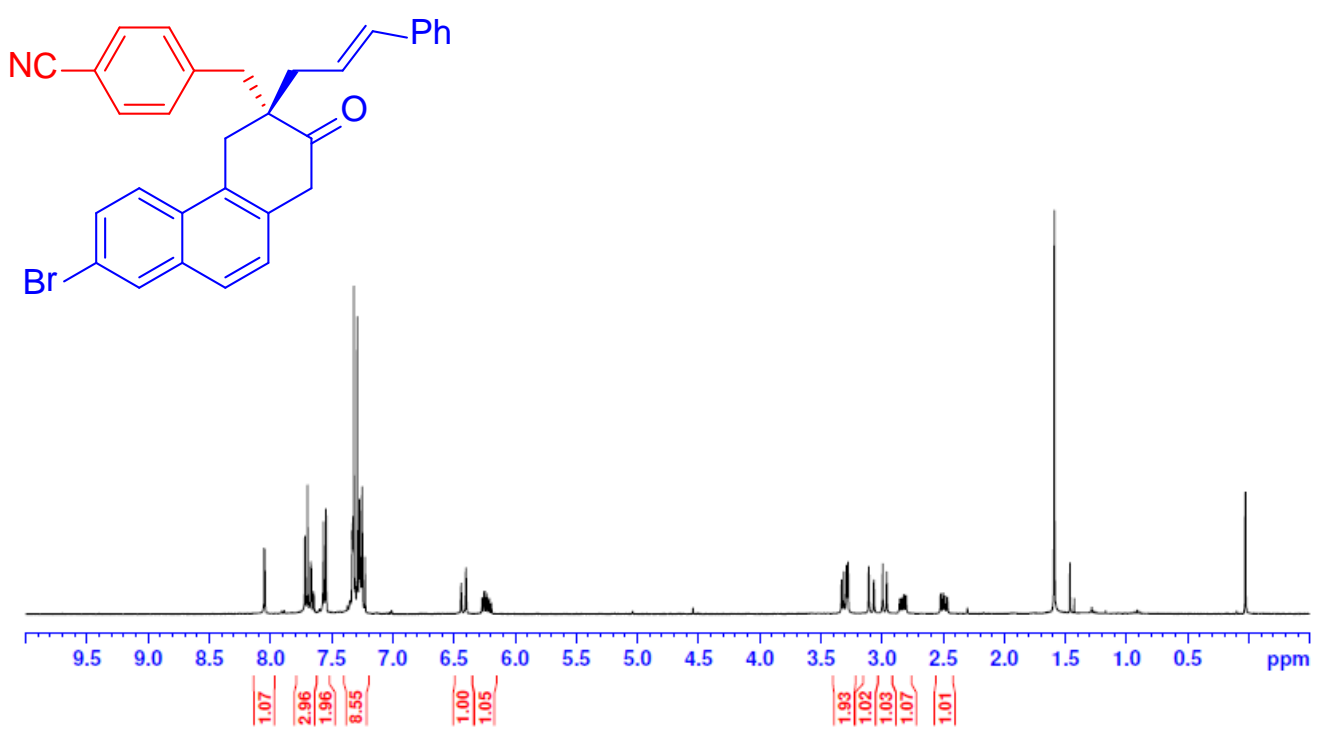

TB013142-1-C13

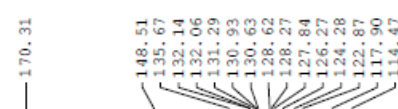

Vू<smiles>N#Cc1ccc(C[C@]2(C/C=C/c3ccccc3)Cc3c(ccc4cc(Br)ccc34)CC2=O)cc1</smiles>

$\begin{array}{lllllllllllllllllllll}200 & 190 & 180 & 170 & 160 & 150 & 140 & 130 & 120 & 110 & 100 & 90 & 80 & 70 & 60 & 50 & 40 & 30 & 20 & 10 & \text { ppm }\end{array}$ 
TBO13074-7-H1
AV $400 \mathrm{CDC13}$

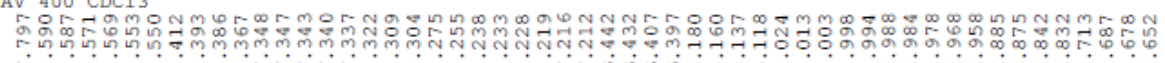

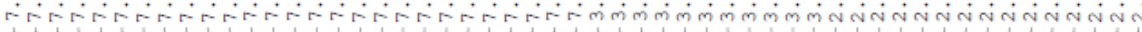<smiles>O=C1c2ccccc2CC1Cc1ccccc1</smiles>

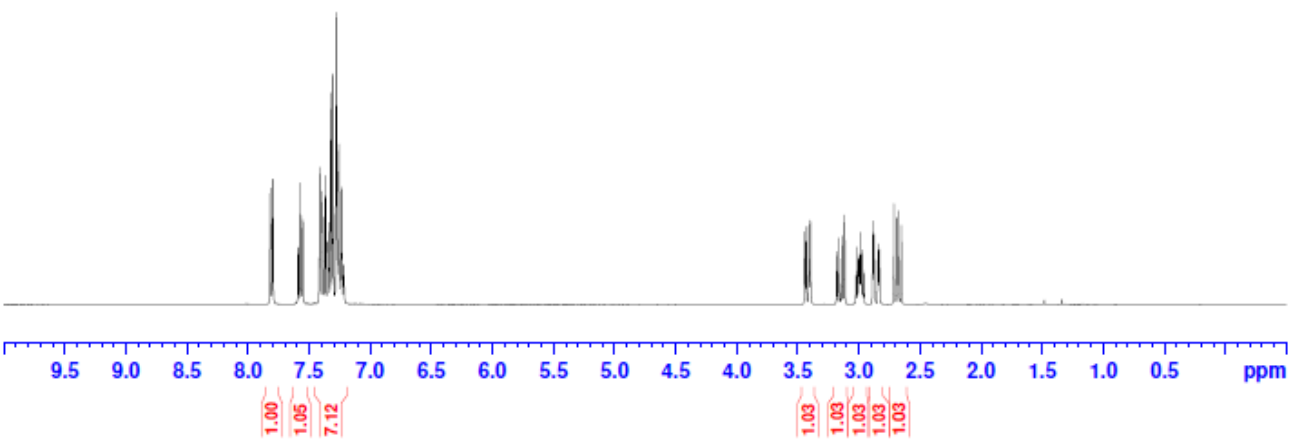

TBO13074-7-C1

AV $400 \quad \mathrm{CDCl}^{3}$

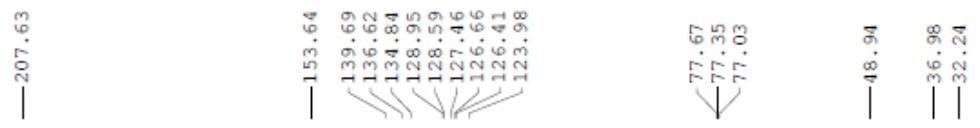<smiles>O=C1c2ccccc2CC1Cc1ccccc1</smiles>

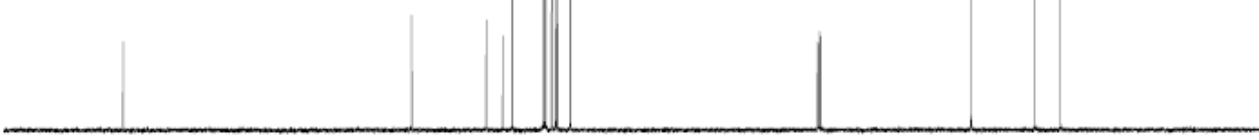

$\begin{array}{lllllllllllllllllllllll}220 & 210 & 200 & 190 & 180 & 170 & 160 & 150 & 140 & 130 & 120 & 110 & 100 & 90 & 80 & 70 & 60 & 50 & 40 & 30 & 20 & 10 & \text { ppm }\end{array}$ 
TBO13074-2-H1

CDCL 3

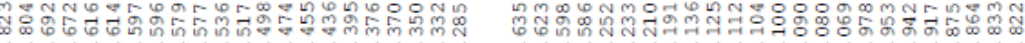

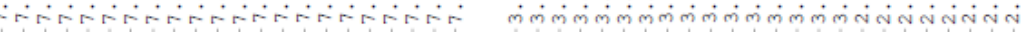<smiles>O=C1c2ccccc2CC1Cc1ccccc1C(F)(F)F</smiles>

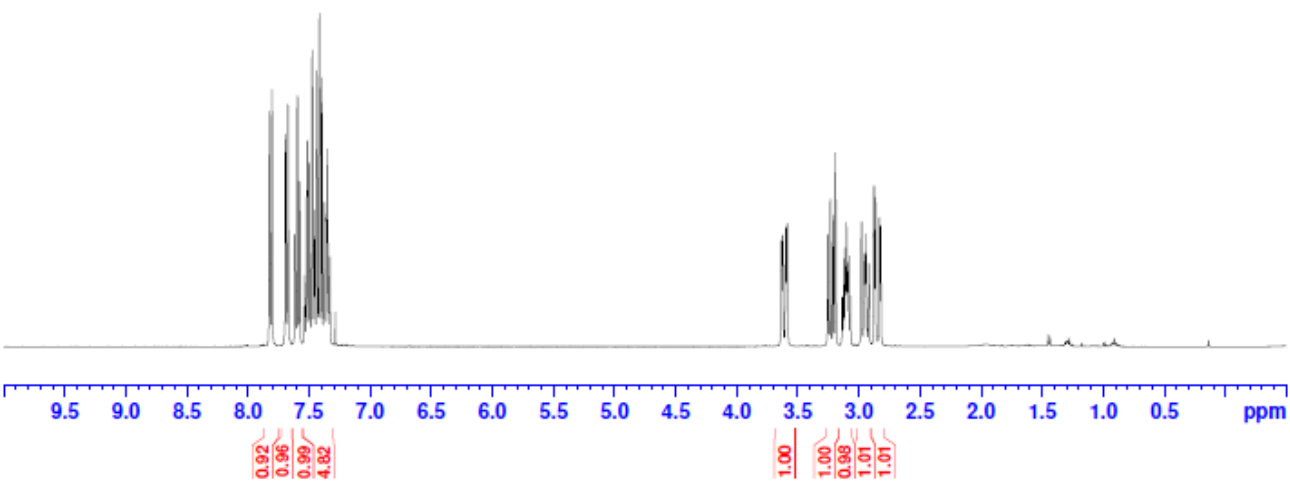

TBO13074-2-C13

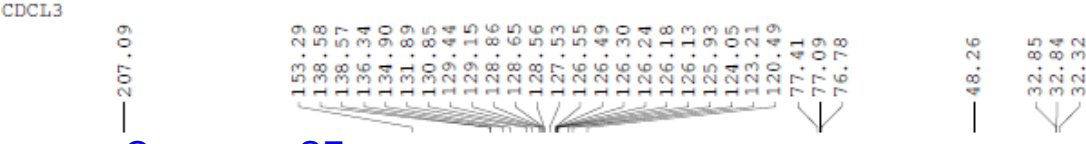<smiles>O=C1c2ccccc2CC1Cc1ccccc1C(F)(F)F</smiles>

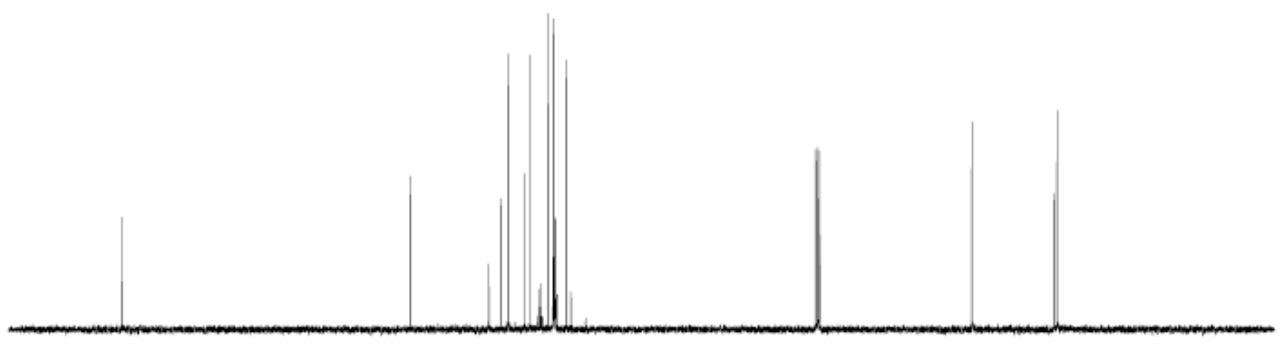

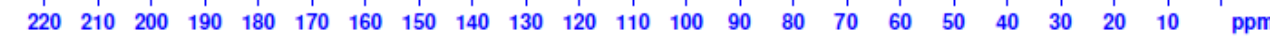




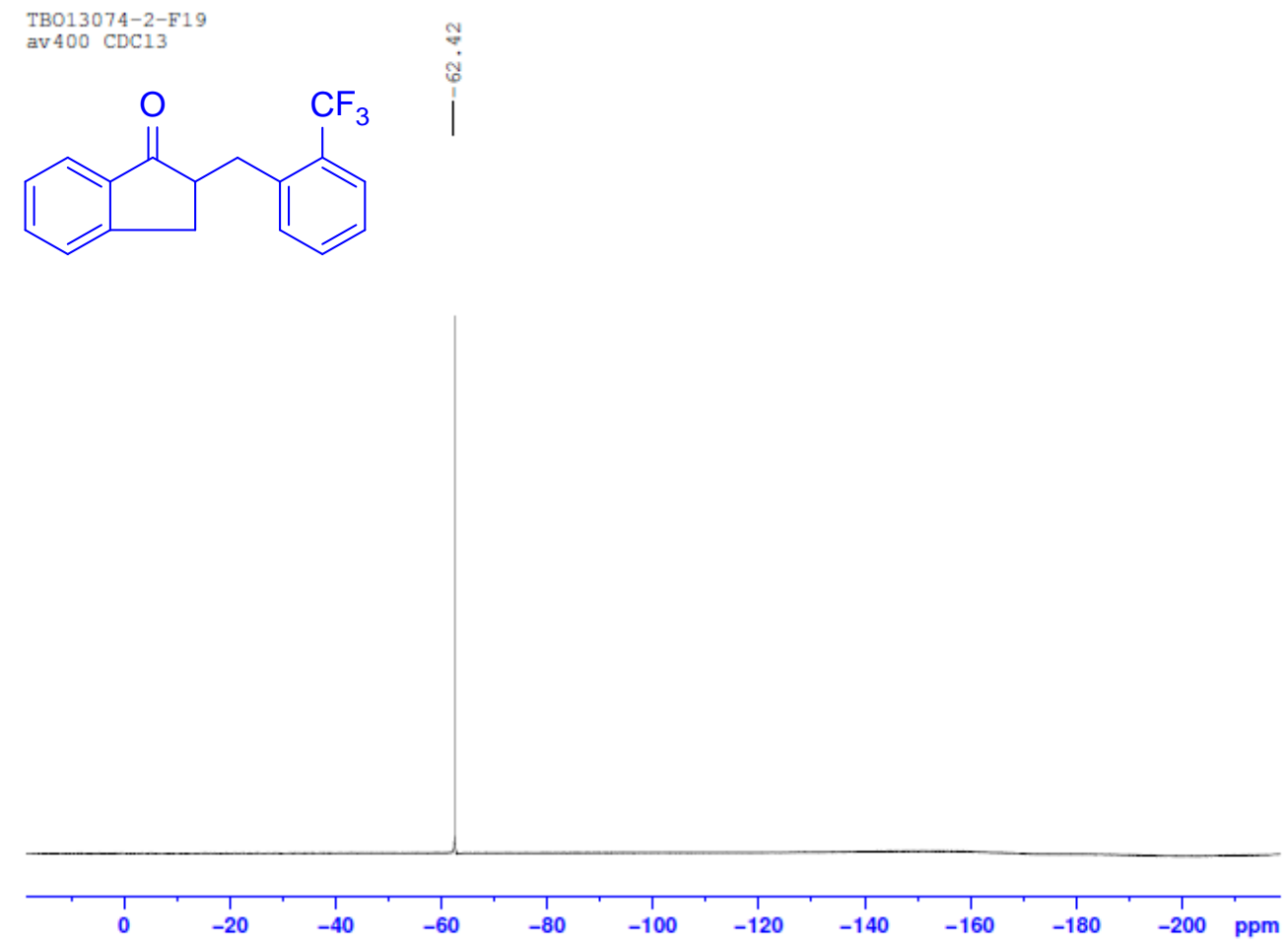

TBO $13074-4-\mathrm{H} 1$
AV 400 CDC13

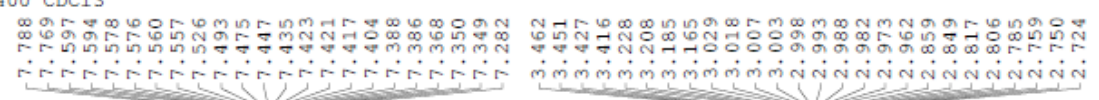

(N)

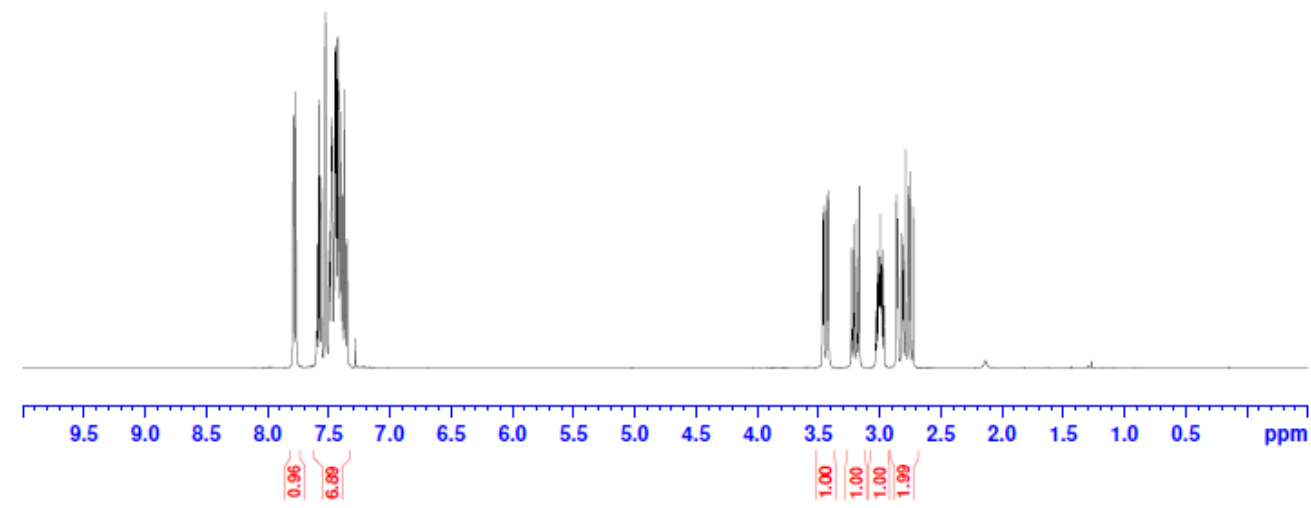


TB0 $13074-4-\mathrm{C} 13$

AV $400 \mathrm{CDCl}$

|

${ }_{1}^{C F_{3}}$

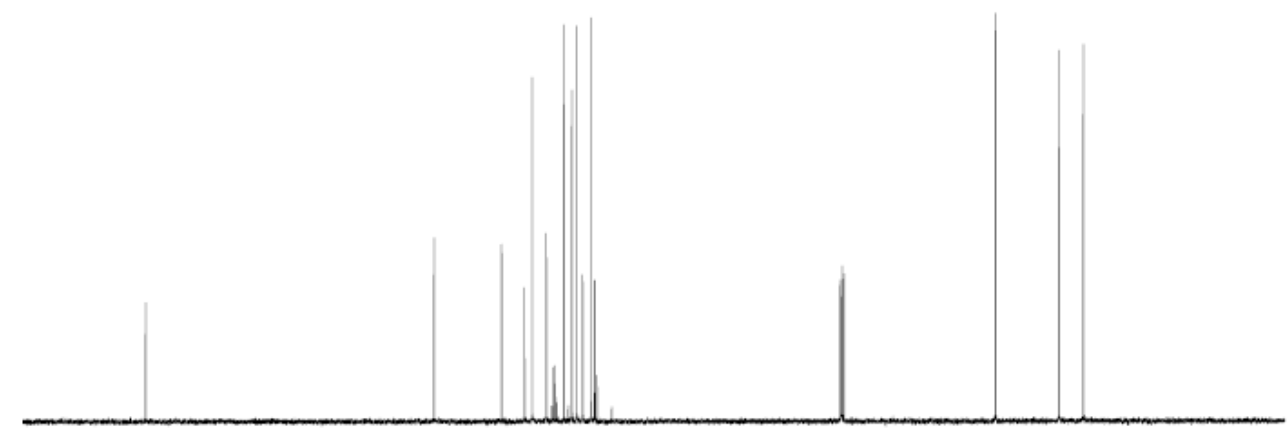

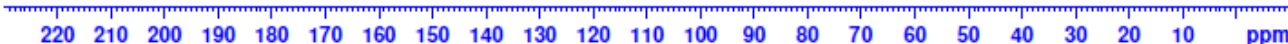

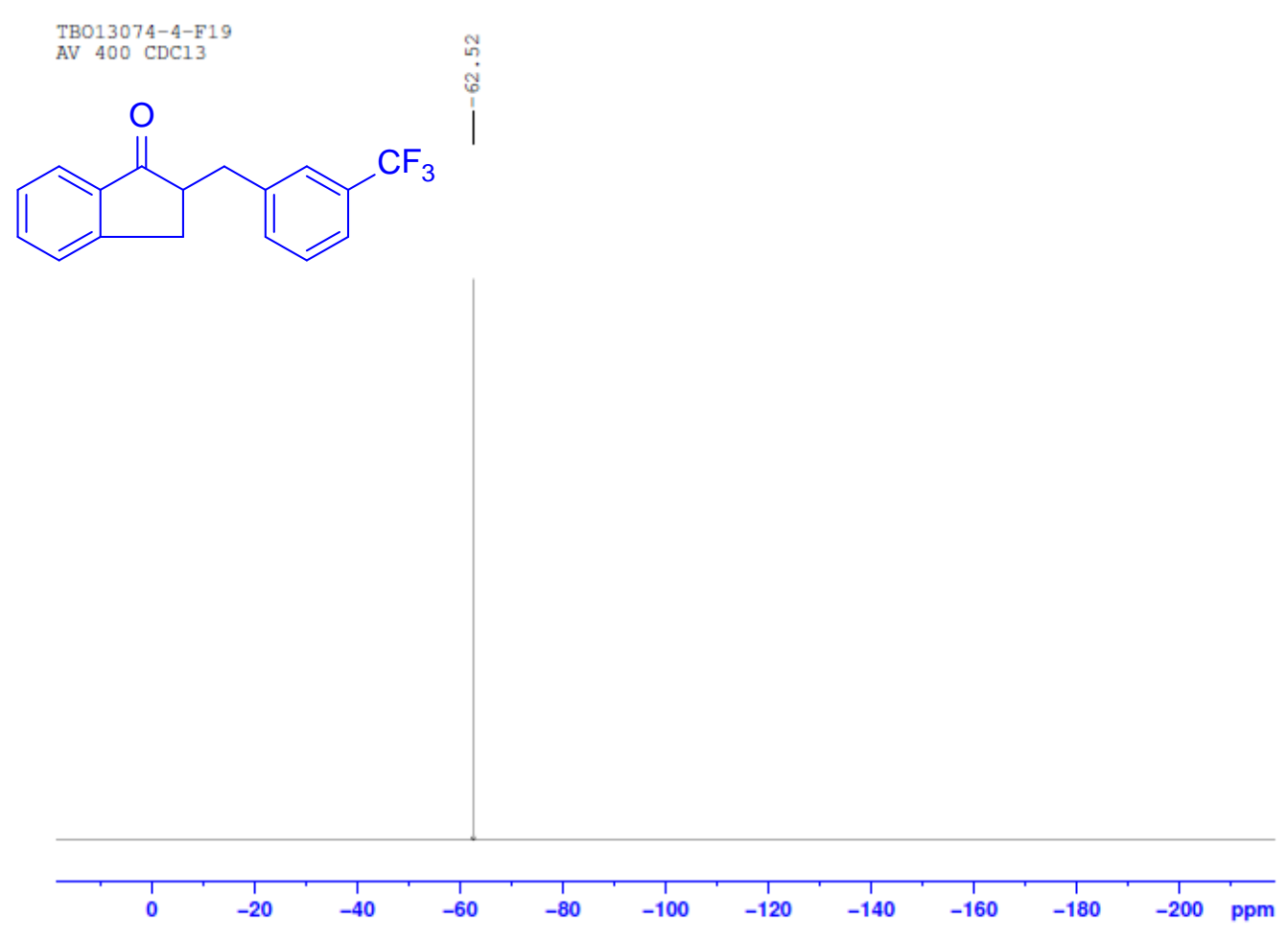




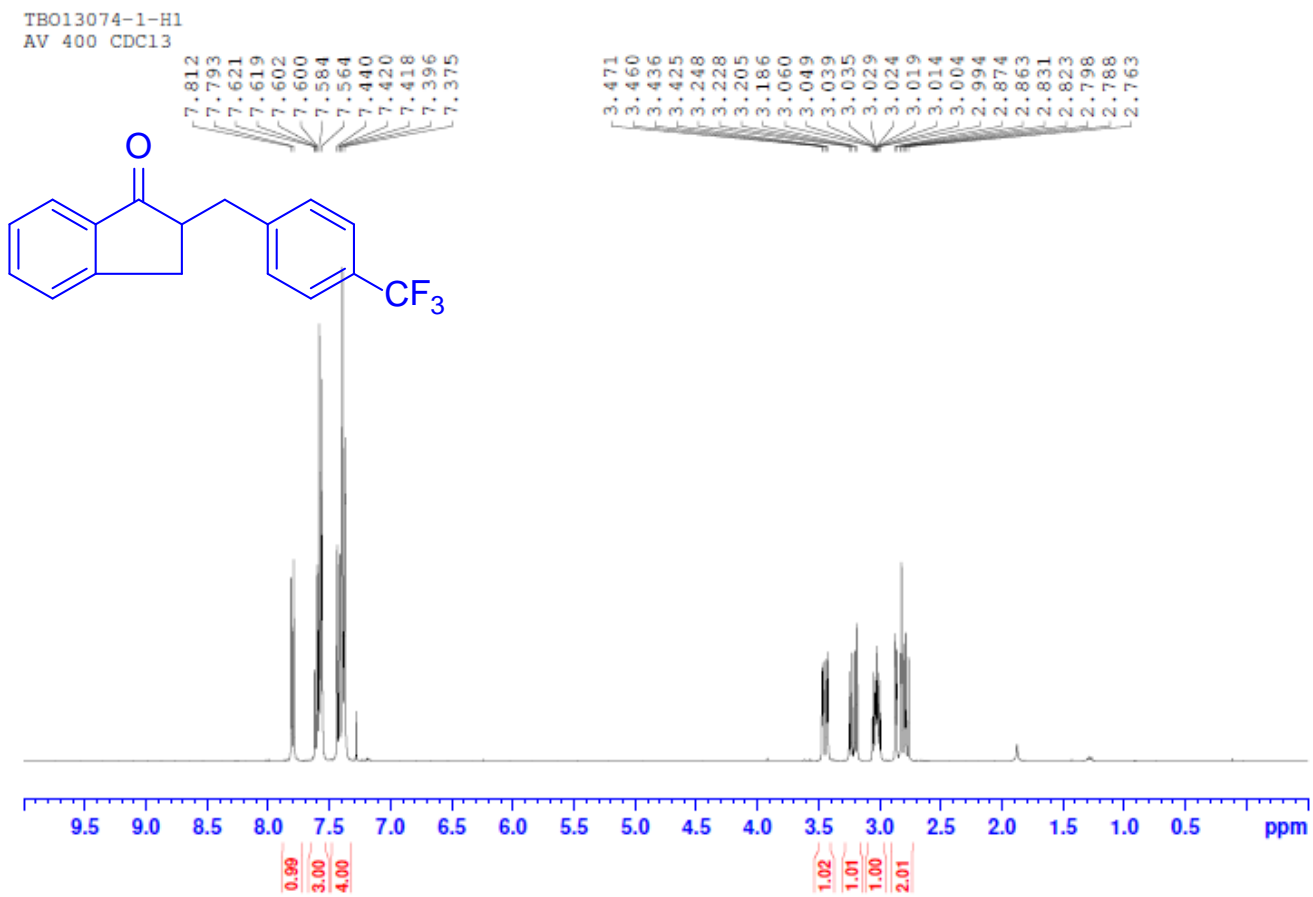

TB013074-1-C13

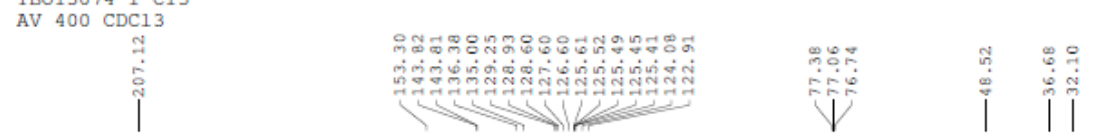<smiles>O=C1c2ccccc2CC1Cc1ccc(C(F)(F)F)cc1</smiles>

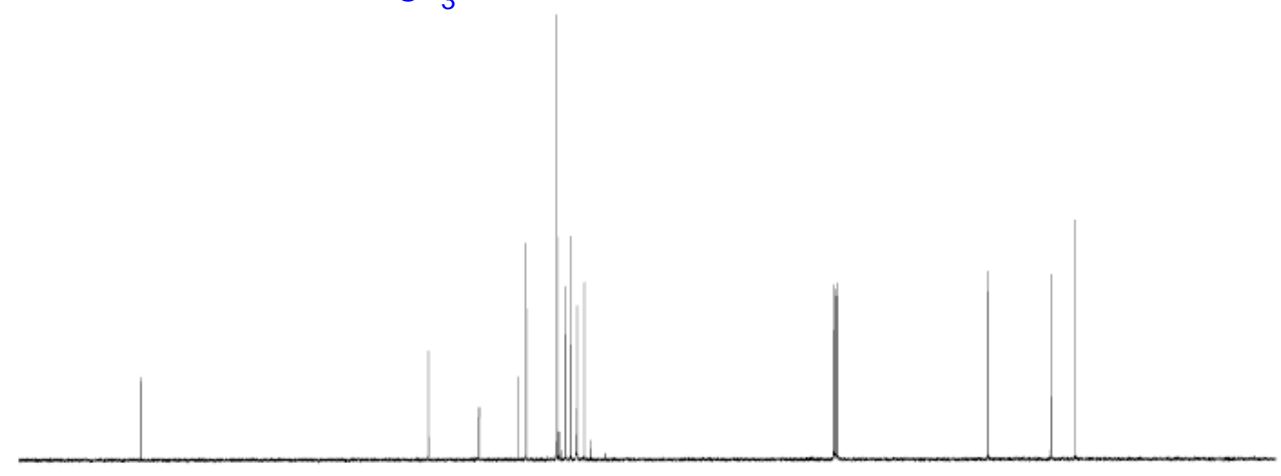

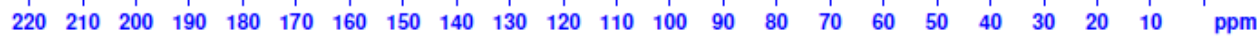




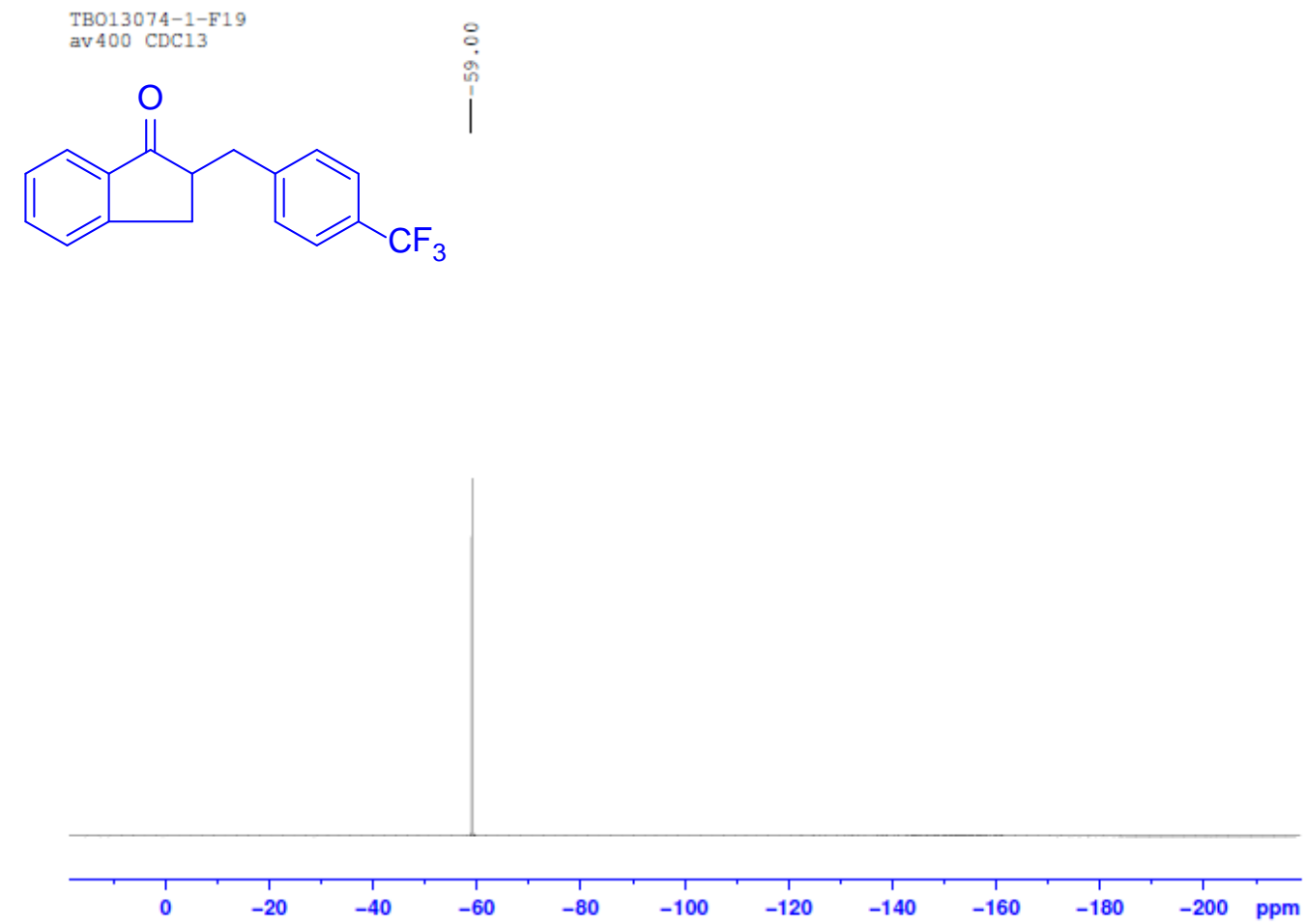

TBO13074-3-H1

AV $400 \quad \mathrm{CDCl} 3$

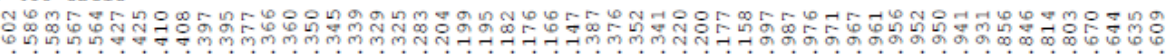

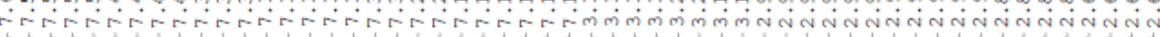

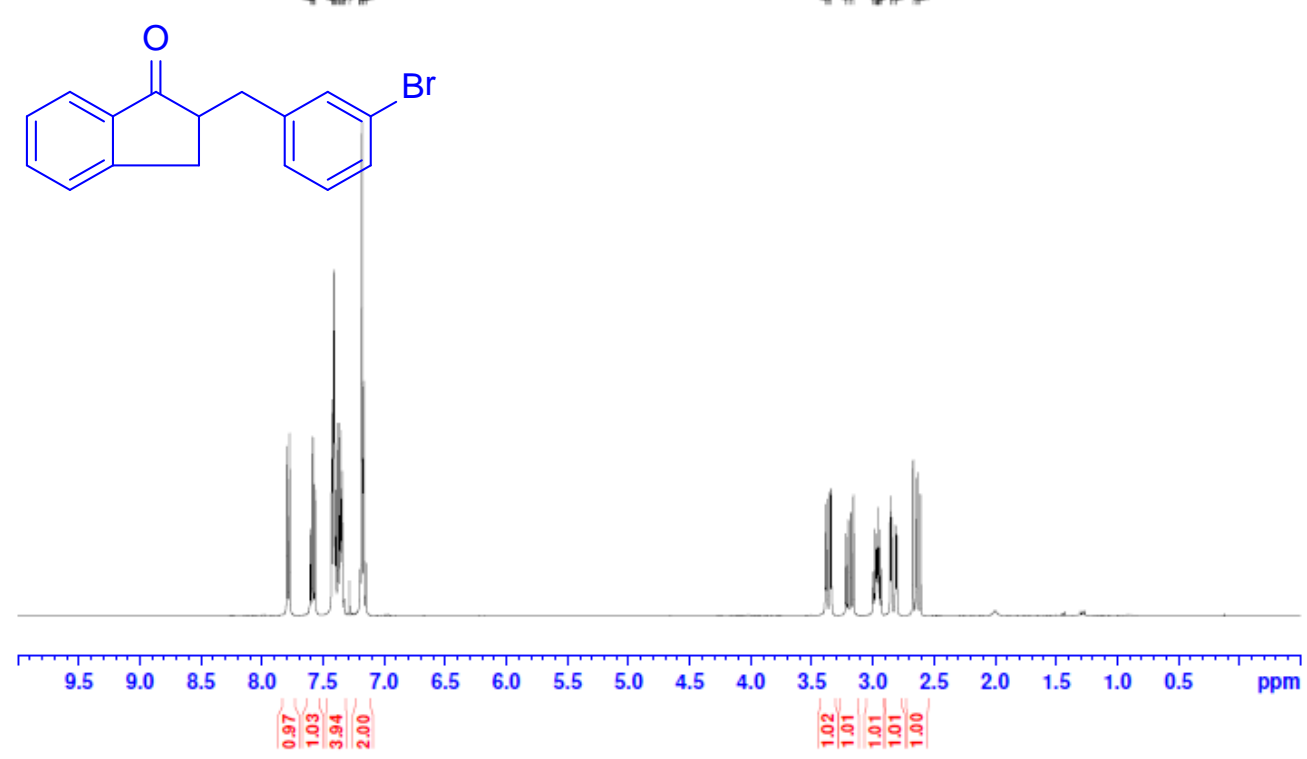




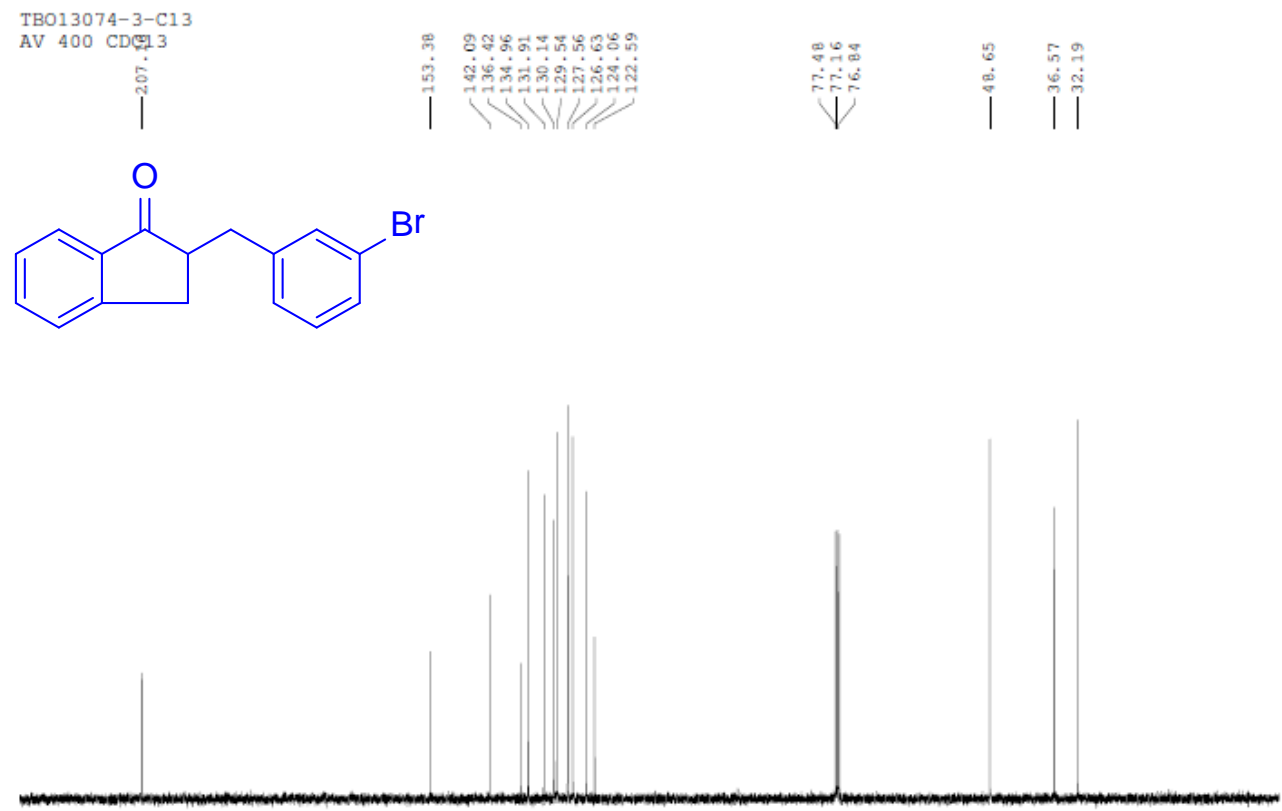

$\begin{array}{lllllllllllllllllllllllllllllll}220 & 210 & 200 & 190 & 180 & 170 & 160 & 150 & 140 & 130 & 120 & 110 & 100 & 90 & 80 & 70 & 60 & 50 & 40 & 30 & 20 & 10 & p p m\end{array}$

TBO13074-6-H1
AV $400 \mathrm{CDC13}$

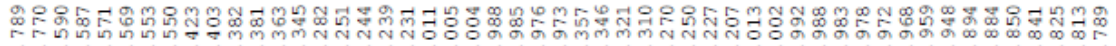<smiles>O=C1c2ccccc2CC1Cc1ccsc1</smiles>

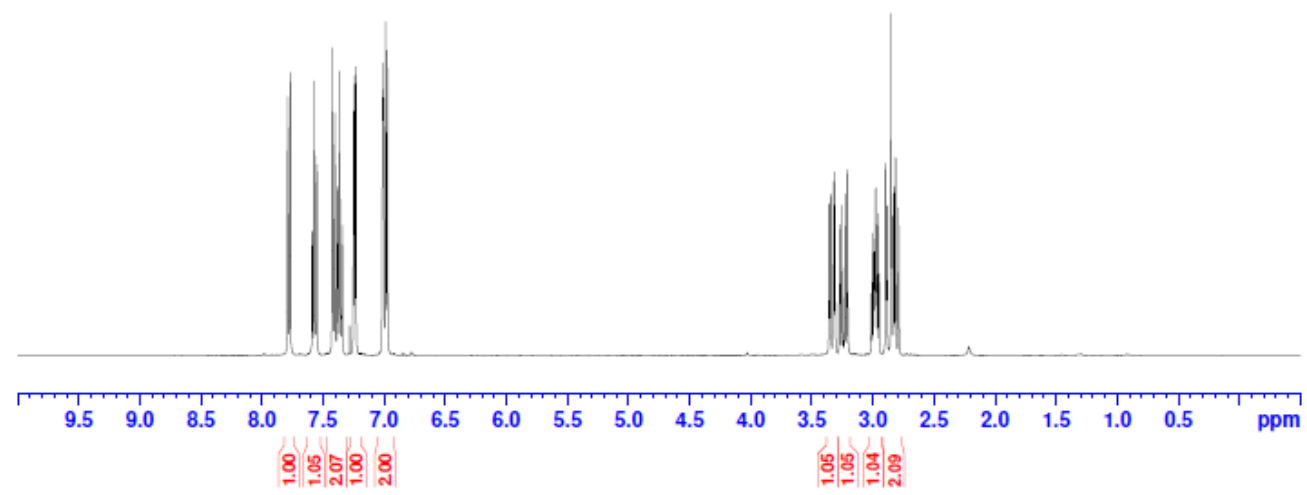




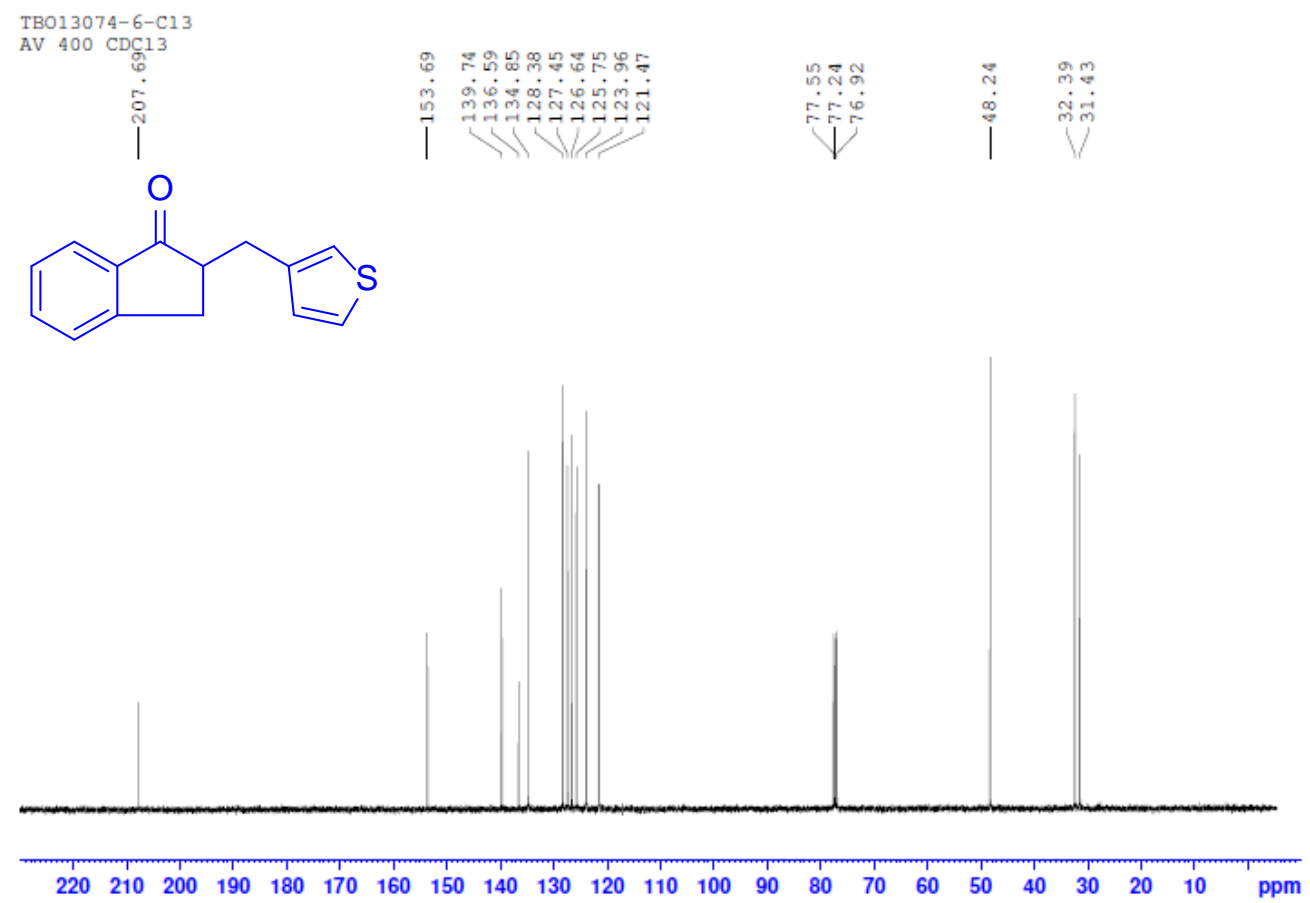

TBO $13074-5-\mathrm{H}_{1}$
AV 400 CDC13

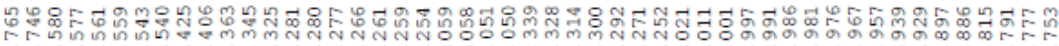
सr.

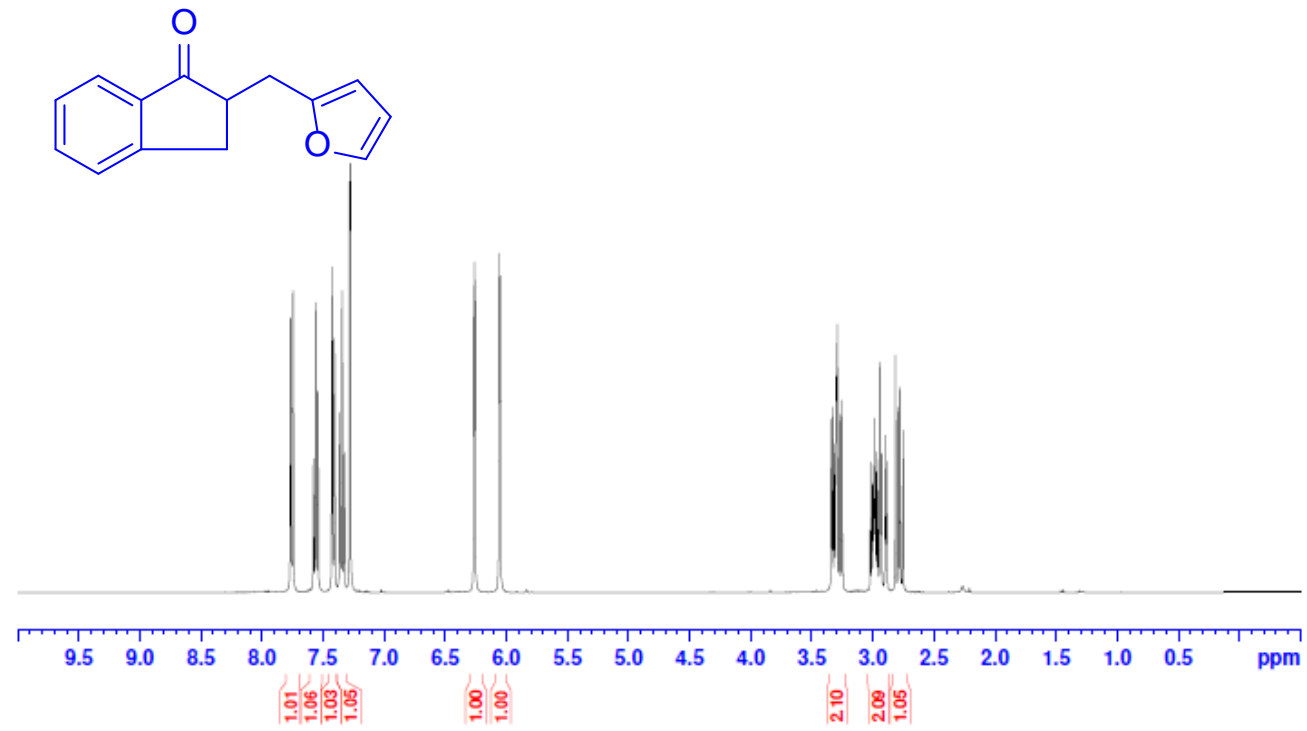




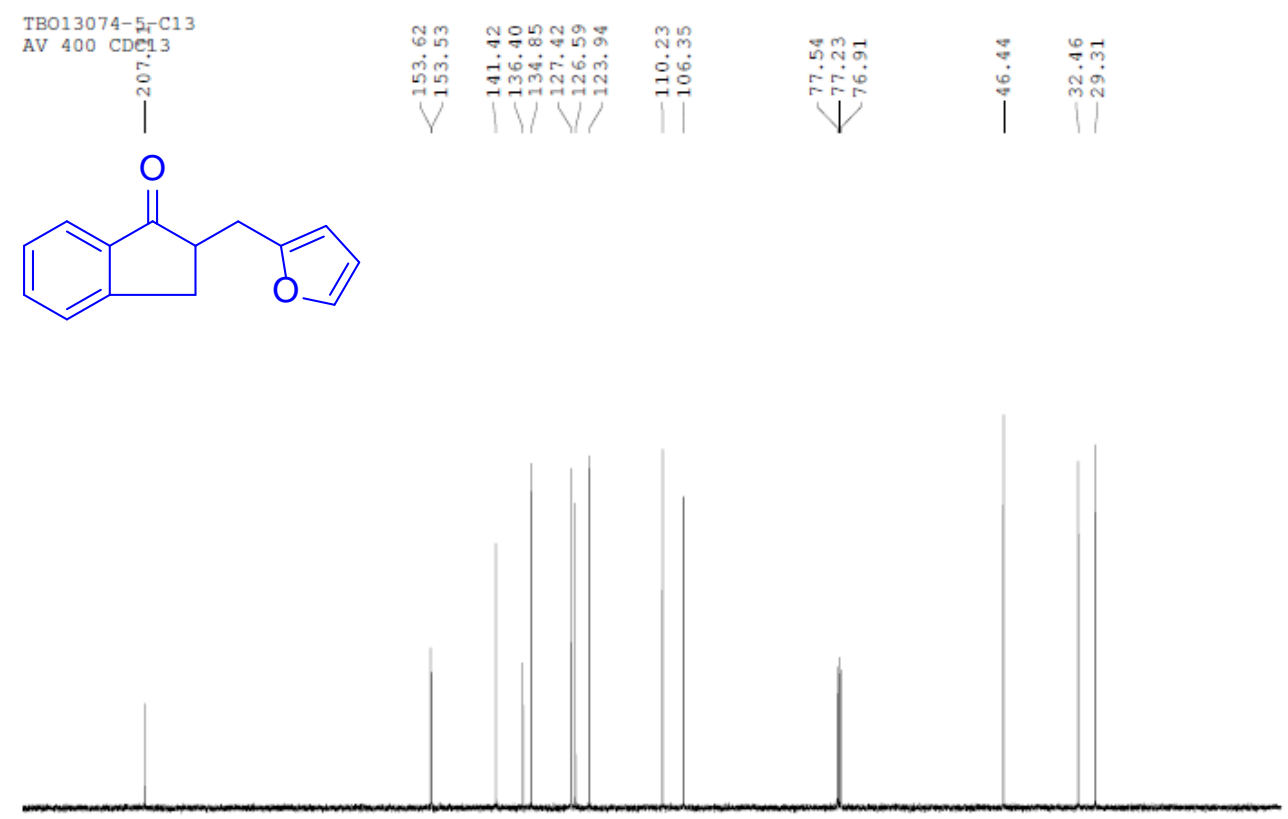

$\begin{array}{llllllllllllllllllllllll}220 & 210 & 200 & 190 & 180 & 170 & 160 & 150 & 140 & 130 & 120 & 110 & 100 & 90 & 80 & 70 & 60 & 50 & 40 & 30 & 20 & 10 & \mathrm{ppm}\end{array}$

TBO13074-14-H1
AV400 CDCL3 等<smiles>Cc1ccc2c(c1)C(=O)C(Cc1ccccc1C(F)(F)F)C2</smiles>

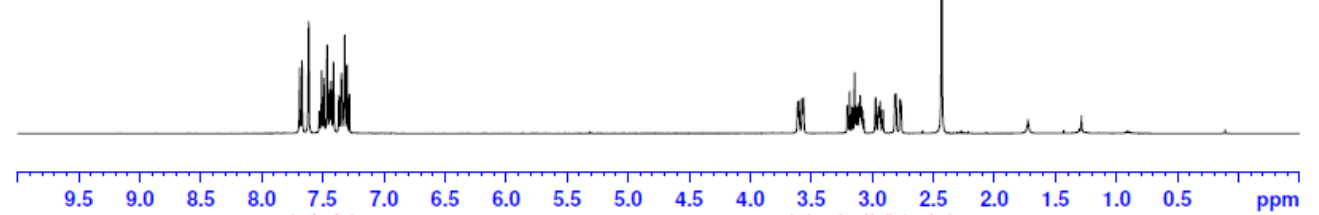
:

(:) 

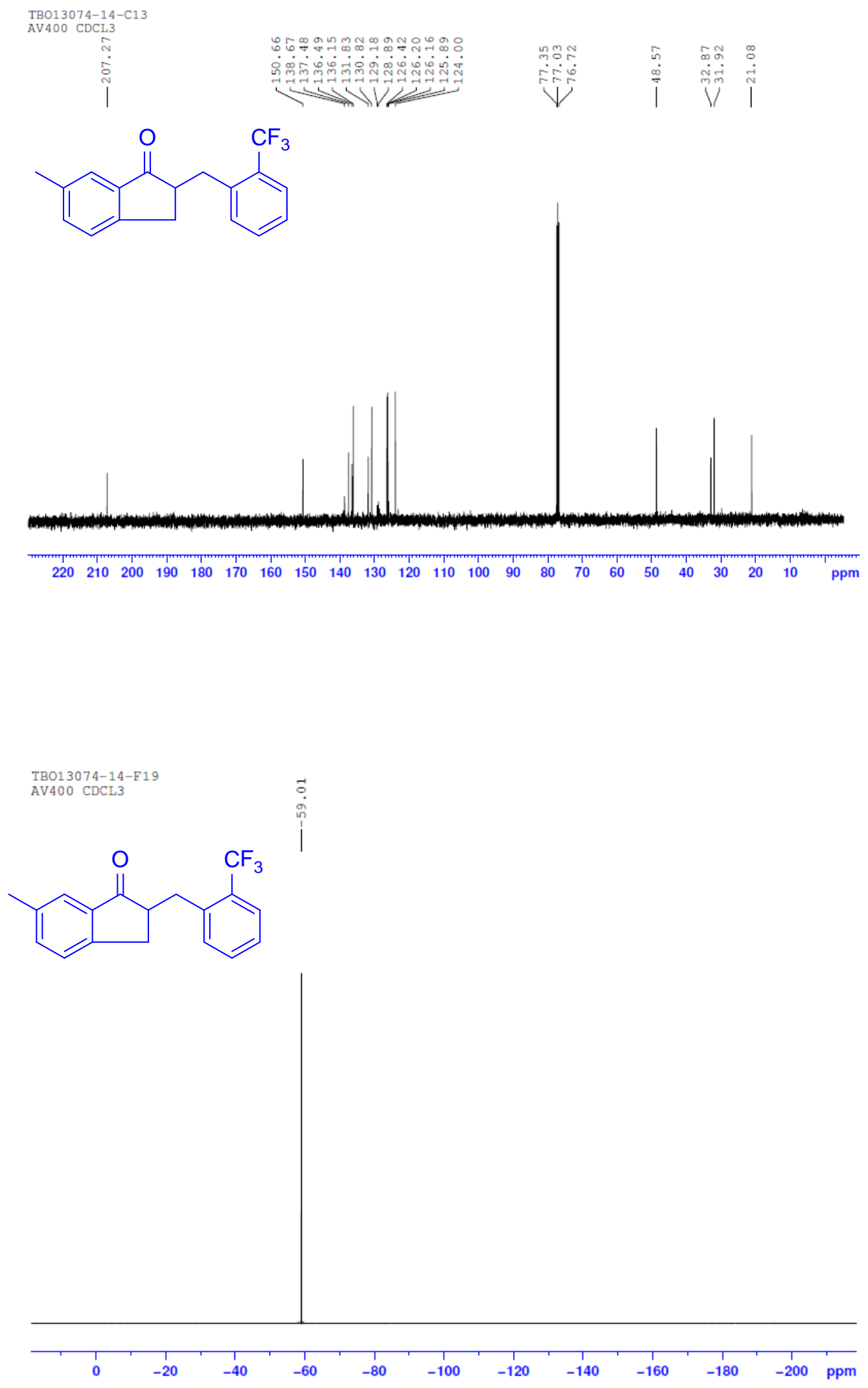

S135 


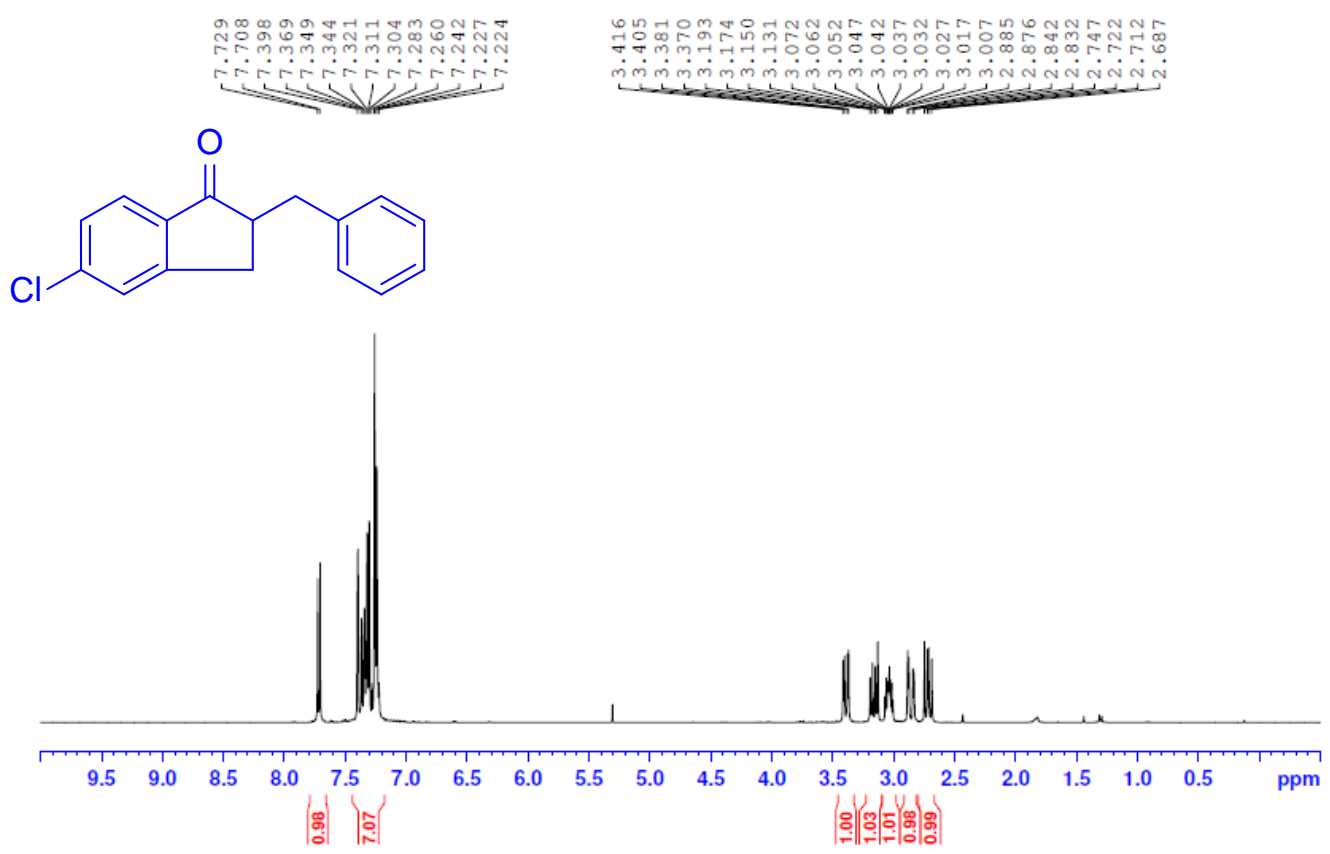

TB013074-15-C13

${ }_{\text {AV } 400}$ CDCL3 3
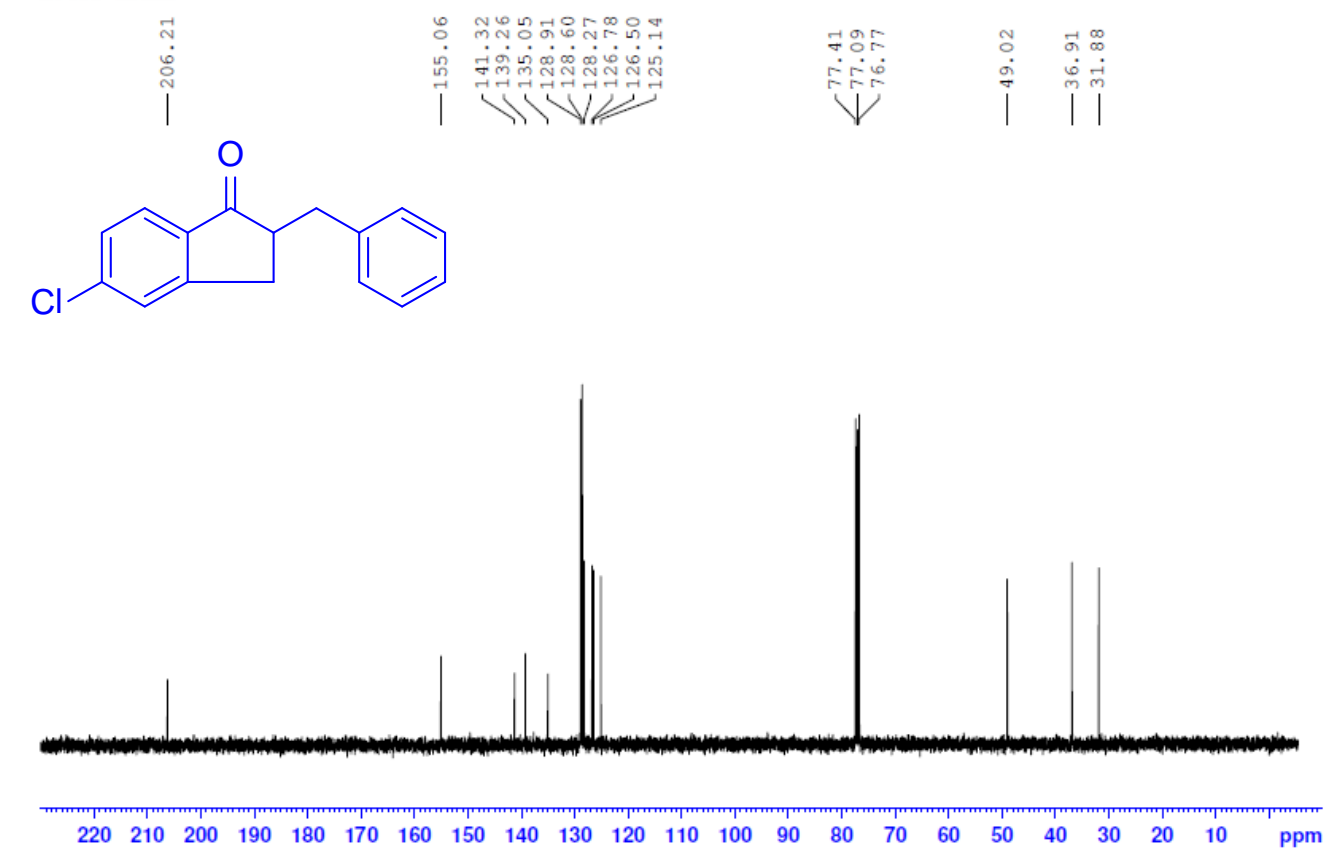


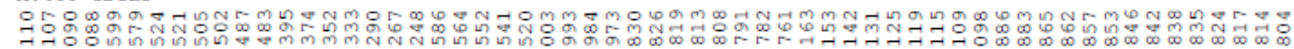

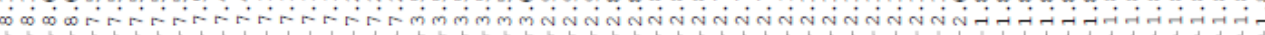<smiles>O=C1c2ccccc2CCC1Cc1ccc(C(F)(F)F)cc1</smiles>

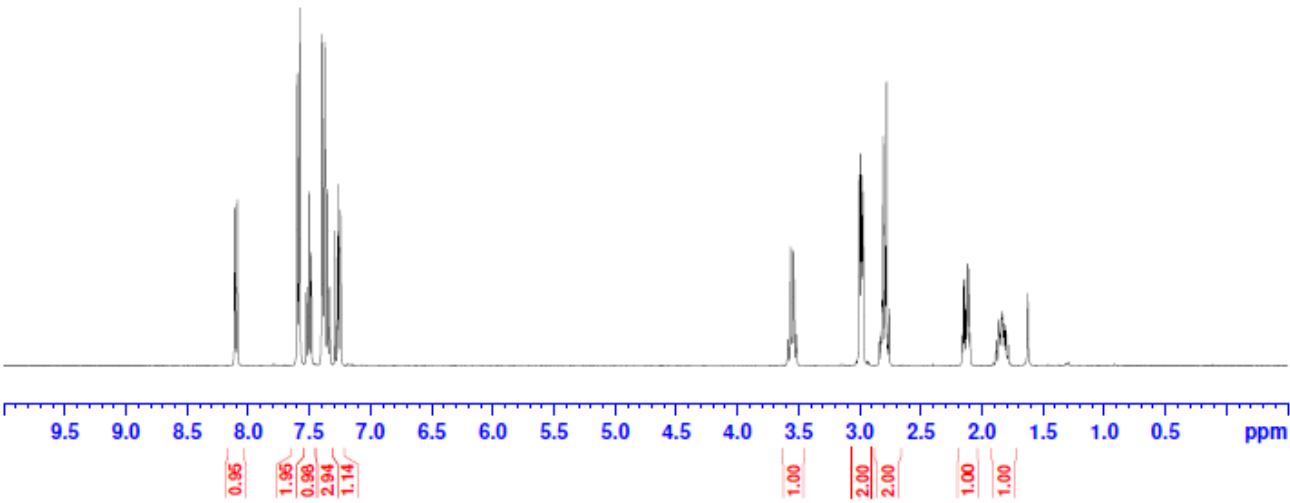

TB013074-8-C13

AV $400 \mathrm{CDC13}$<smiles>O=C1c2ccccc2CCC1Cc1ccc(C(F)(F)F)cc1</smiles>

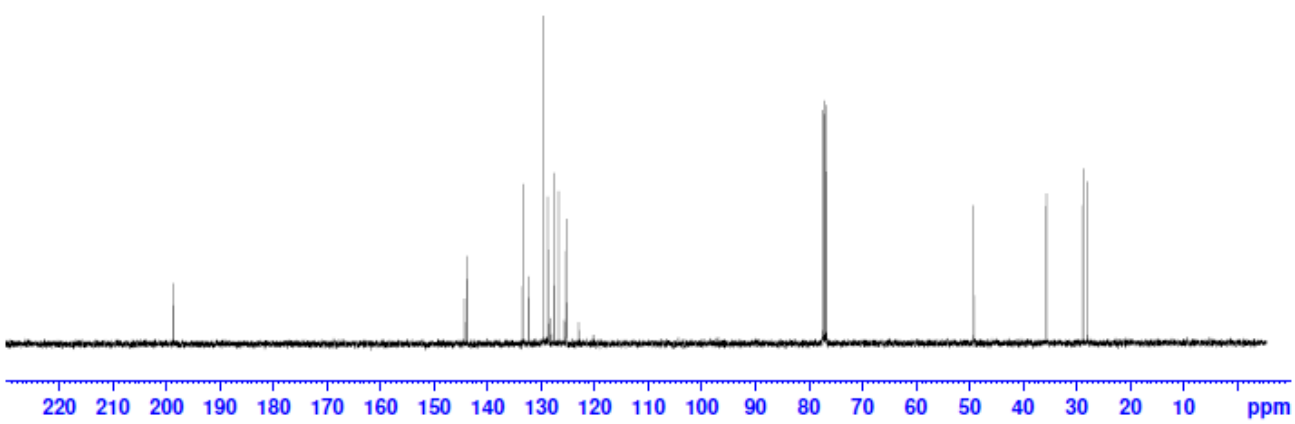




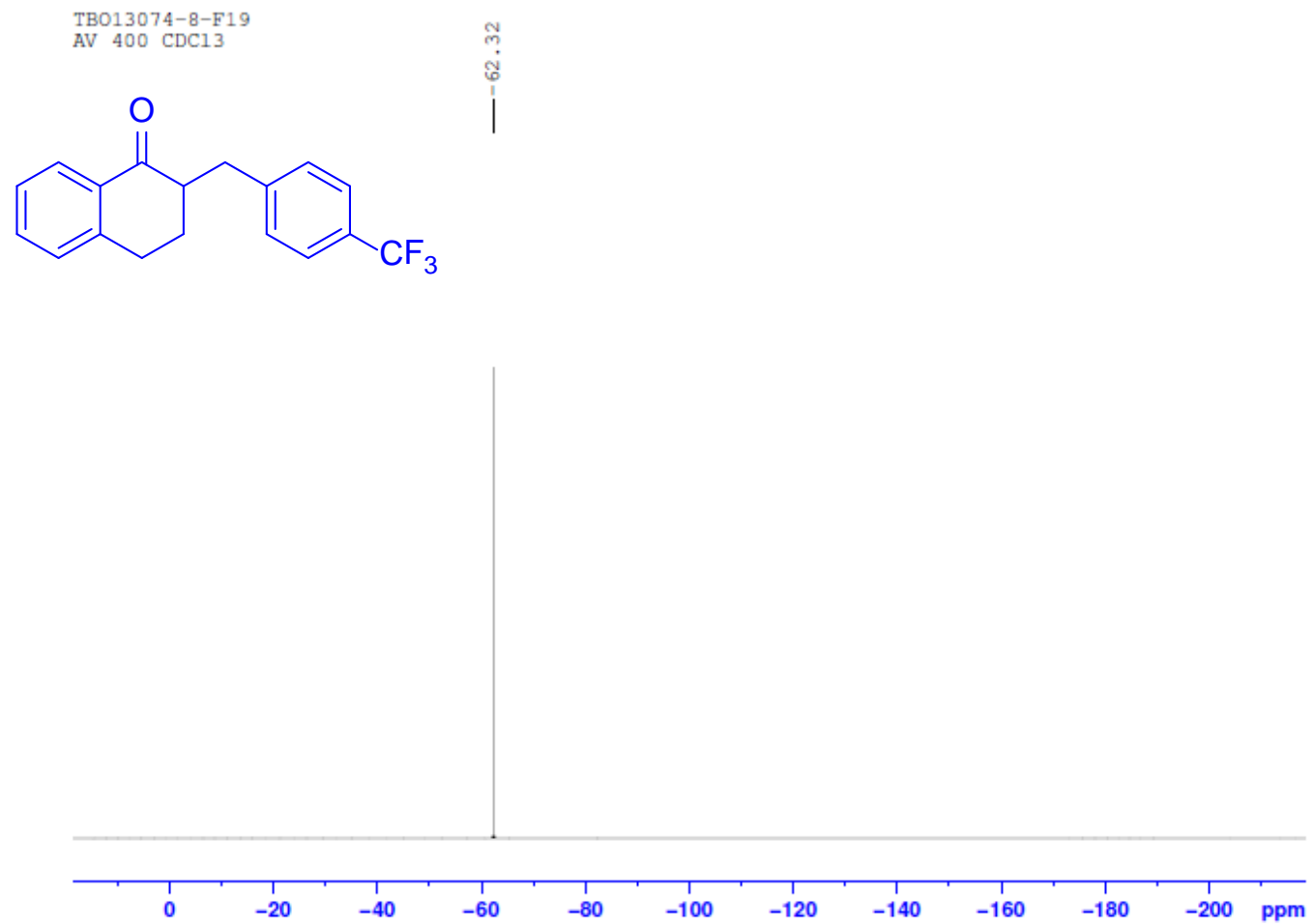

TBO $13074-12-\mathrm{H} 1$
AV $400 \mathrm{CDC13}$

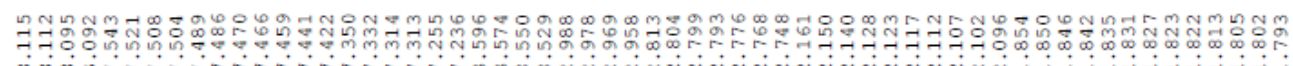

क w m<smiles>O=C1c2ccccc2CCC1Cc1cccc(C(F)(F)F)c1</smiles>

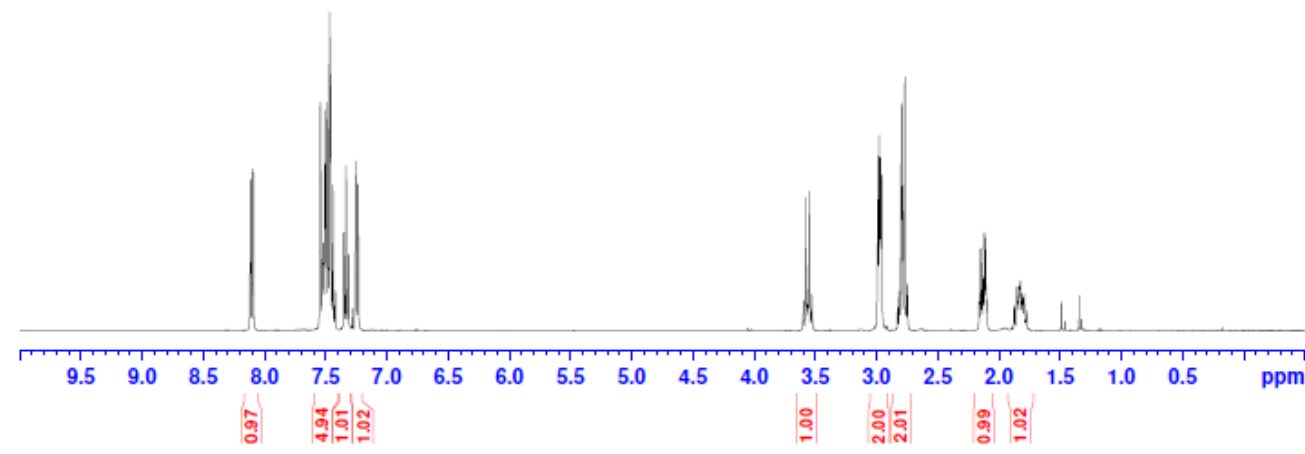


TB013074-12-C13

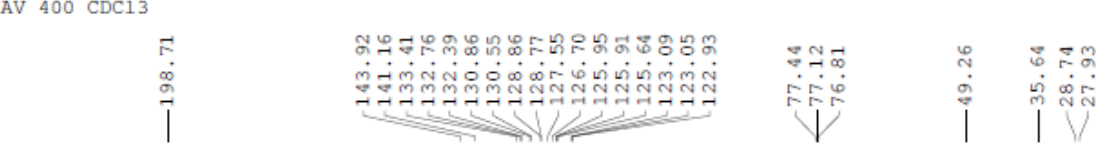<smiles>O=C1c2ccccc2CCC1Cc1cccc(C(F)(F)F)c1</smiles>

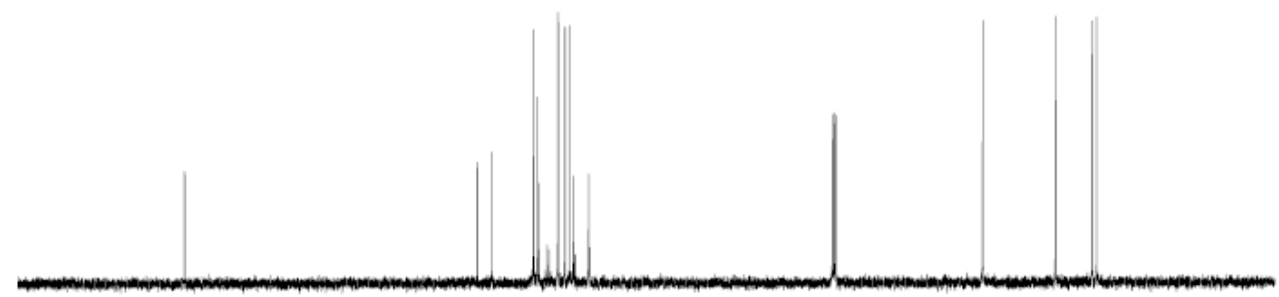

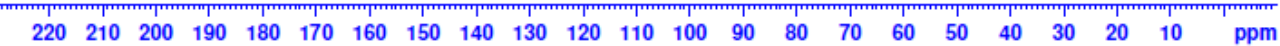<smiles>O=C1c2ccccc2CCC1Cc1cccc(C(F)(F)F)c1</smiles>

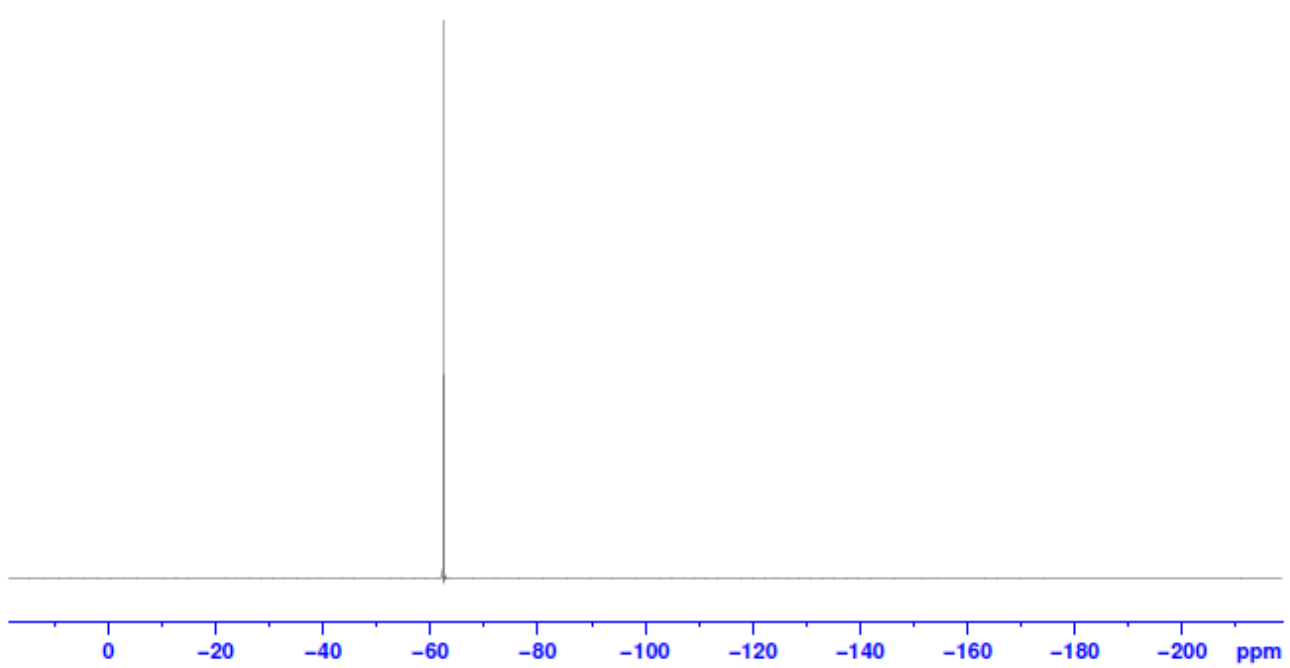



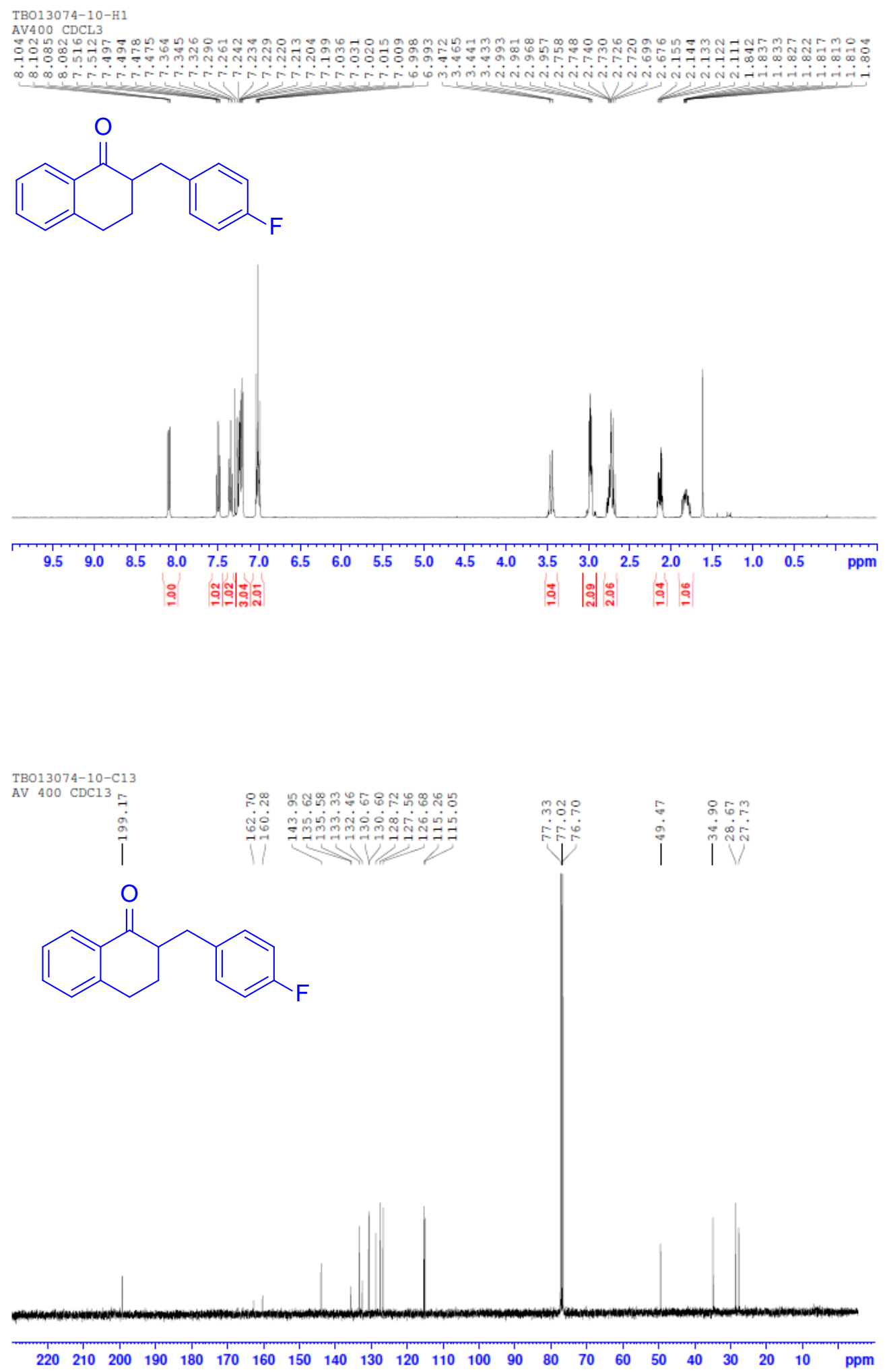

S140 


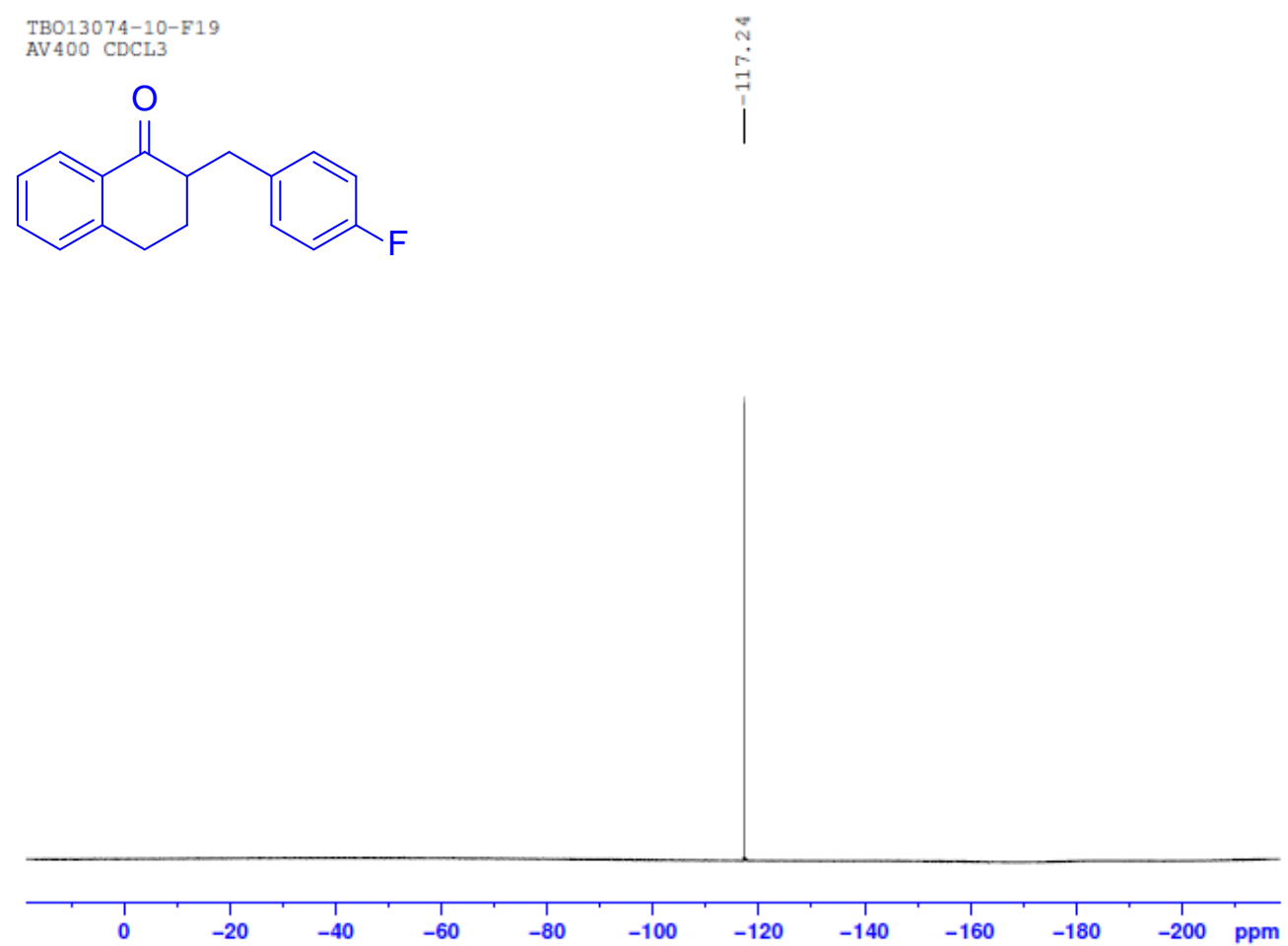

TBO13074-13-H1

av $400 \mathrm{CDC} 13$

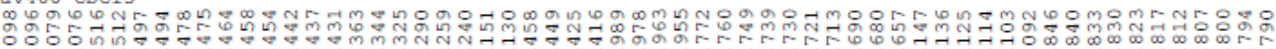

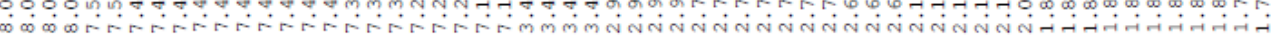

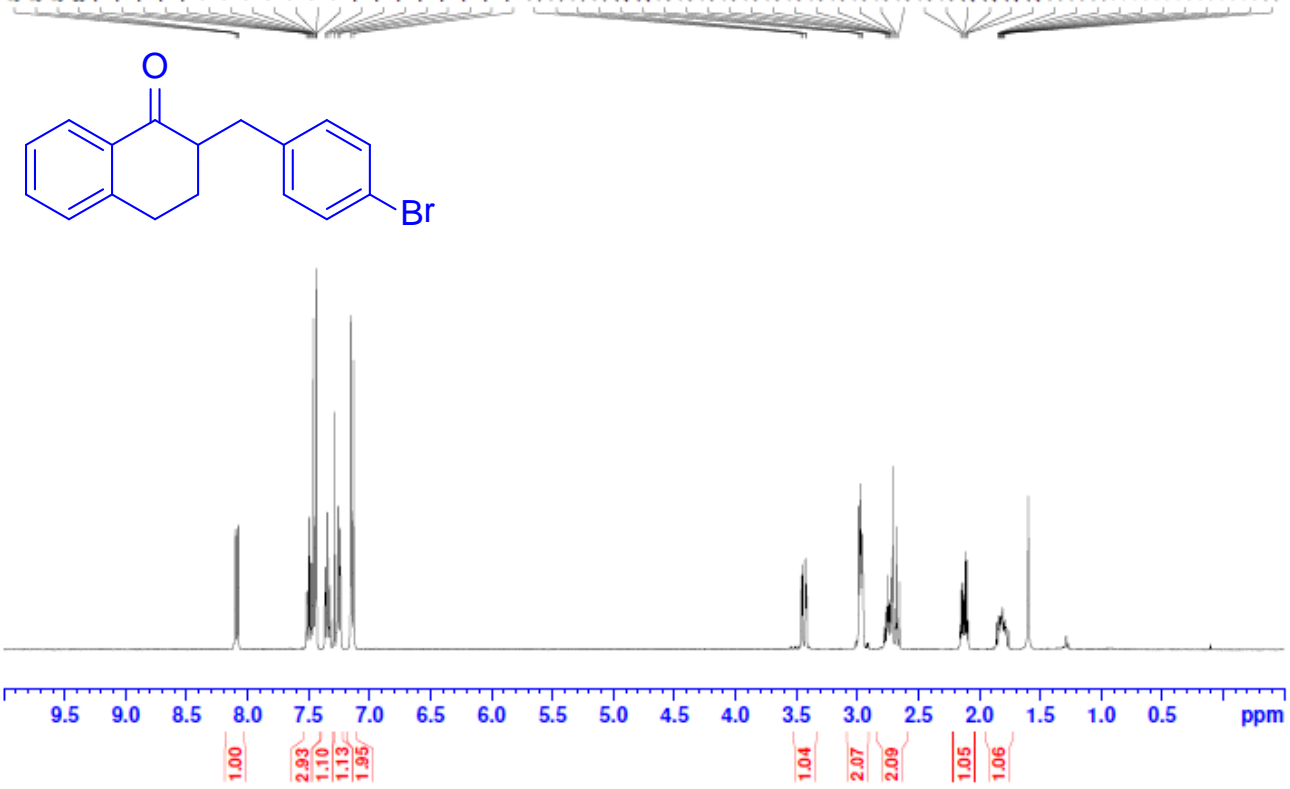




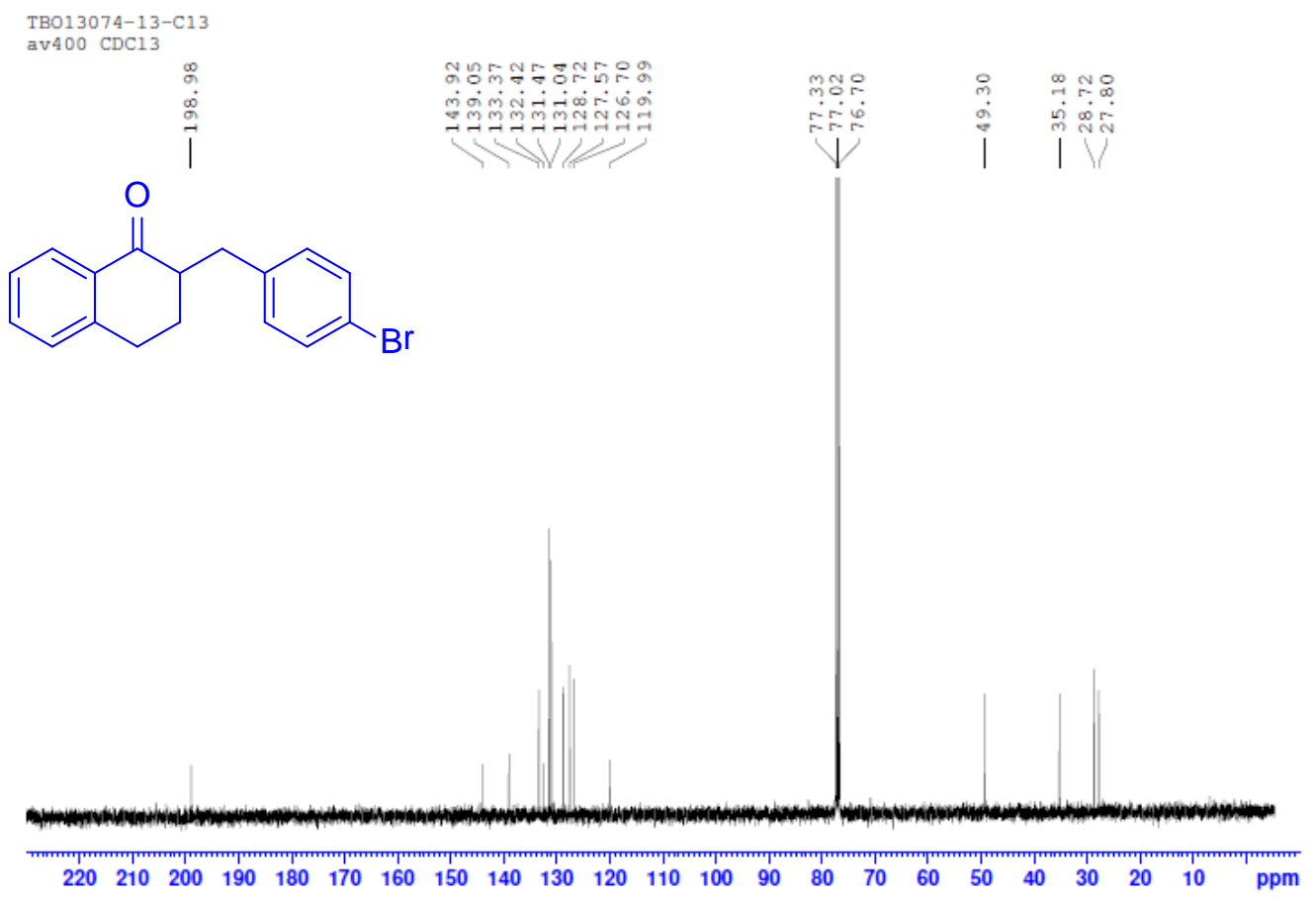

TBO $13074-11-\mathrm{H} 1$

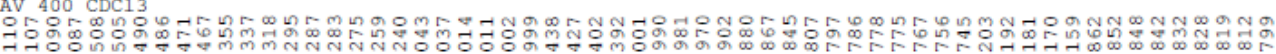

os o<smiles>O=C1c2ccccc2CCC1Cc1ccsc1</smiles>

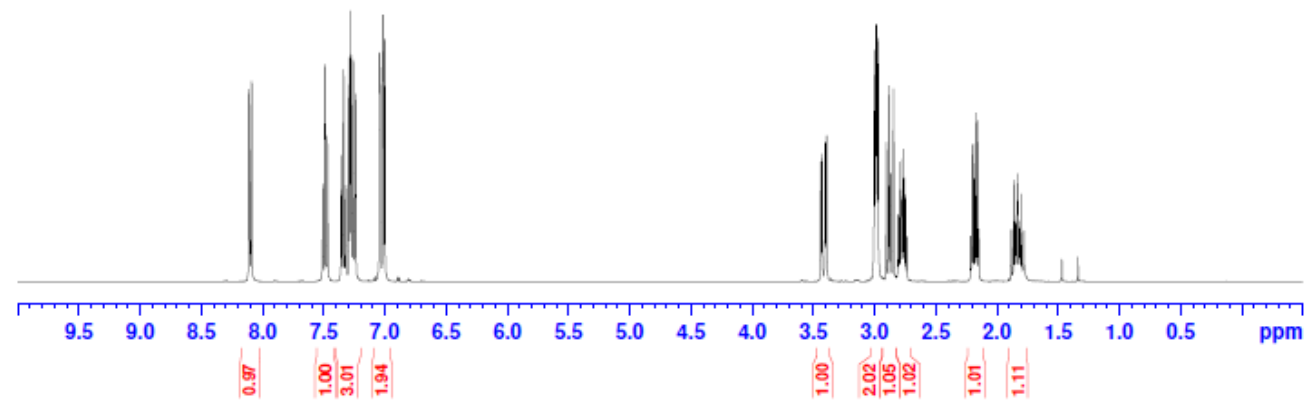


TB013074-11-C13
AV $400 \mathrm{CDC13}$
$\stackrel{2}{\circ}$

(C)

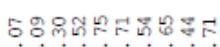

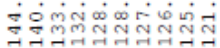

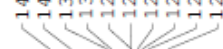

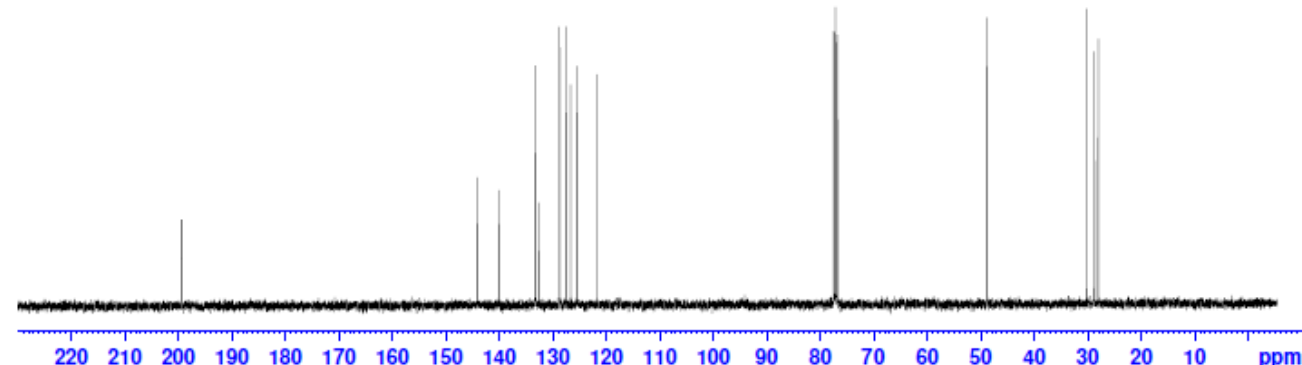

TBO13075-15-H1

av400 NeOD

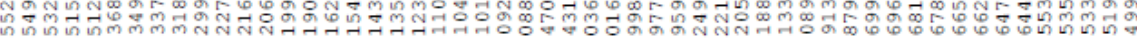

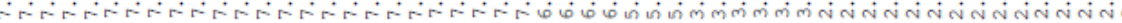<smiles>O=C1c2ccccc2CC1(C/C=C/c1ccccc1)Cc1ccccc1</smiles>
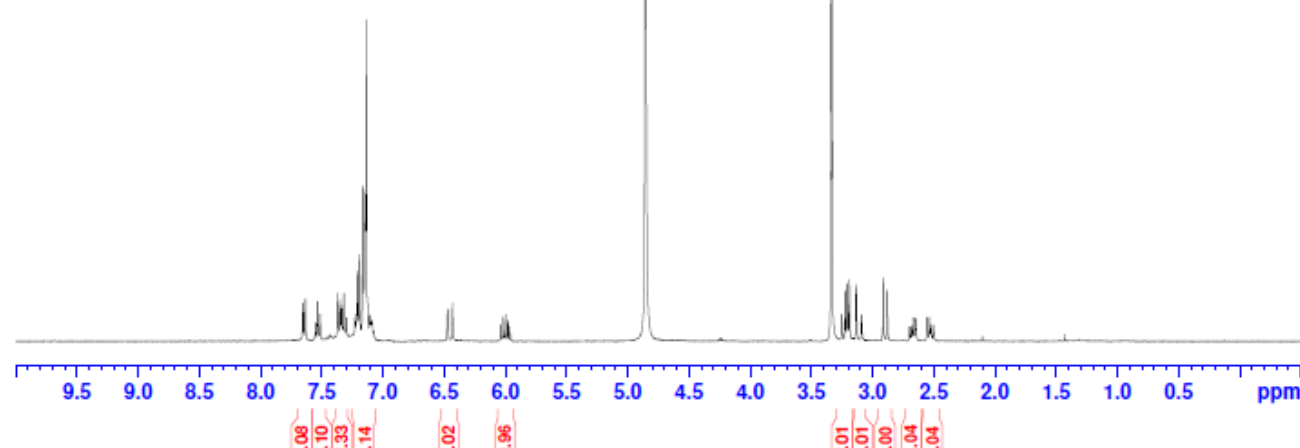

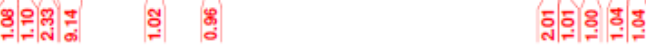




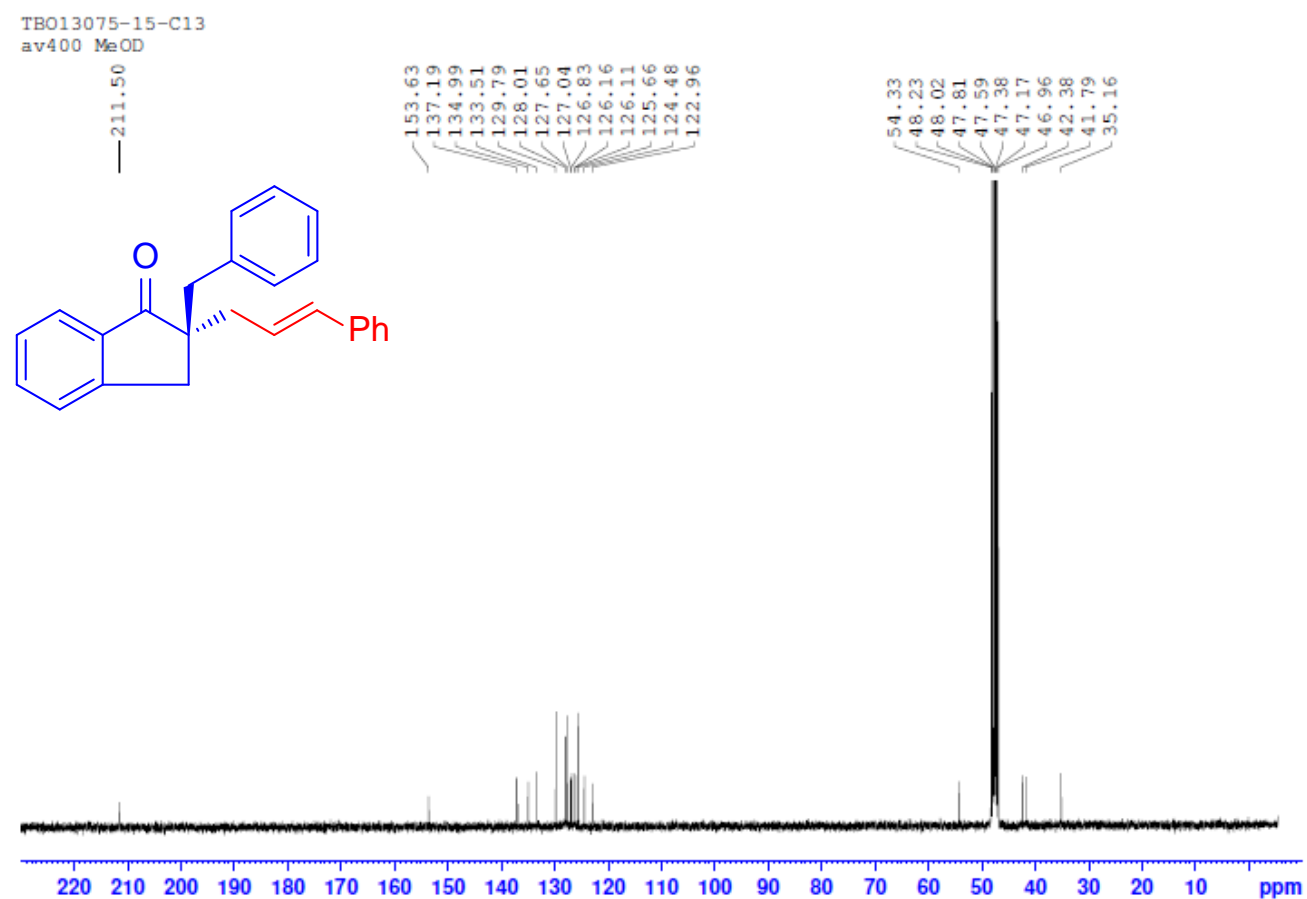<smiles>O=C1c2ccccc2CC1(C/C=C\P)C/C=C/P</smiles>

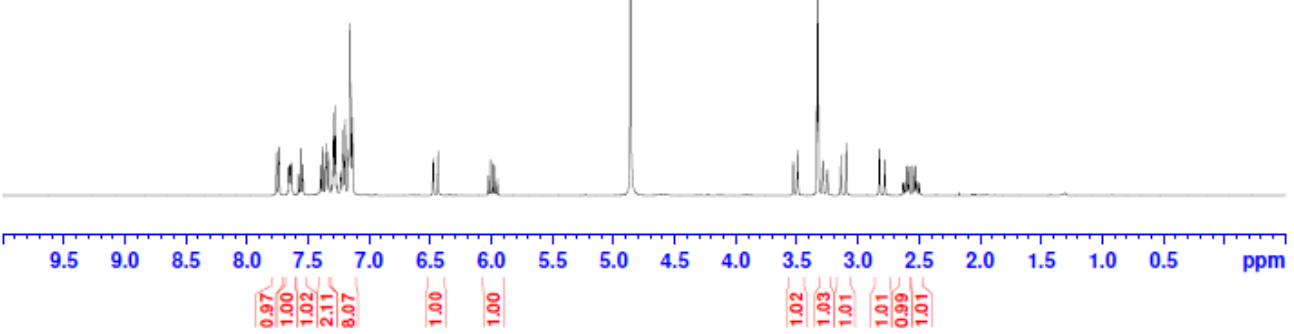




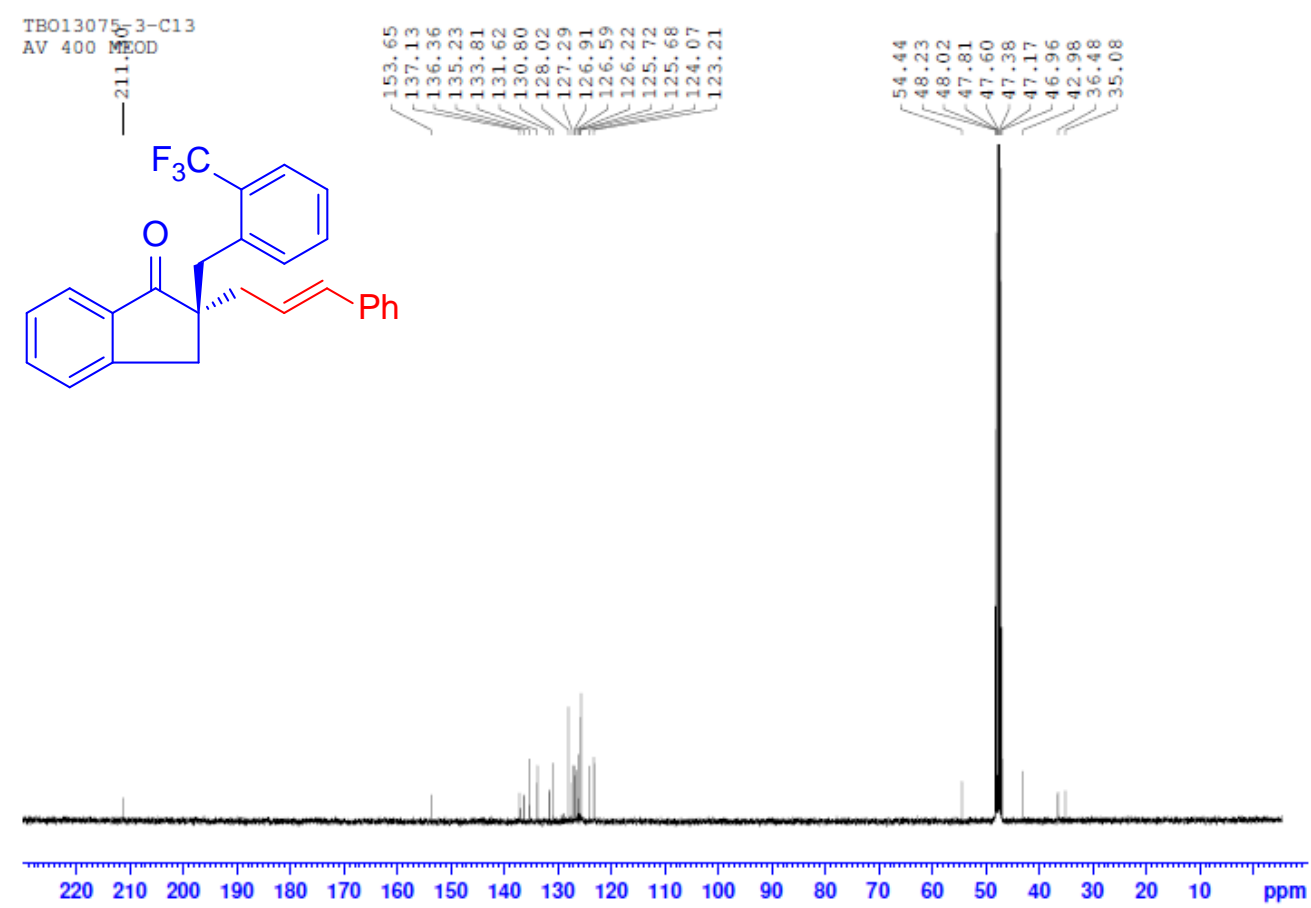<smiles>Cc1ccccc1C[C@@]1(C/C=C/c2ccccc2)Cc2ccccc2C1=O</smiles>

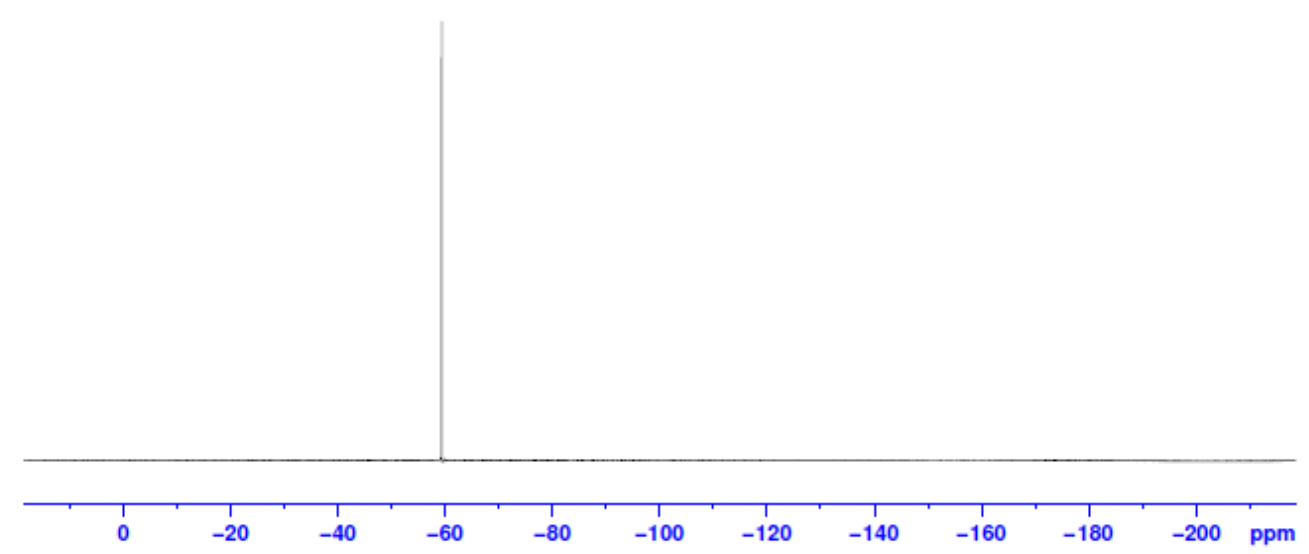



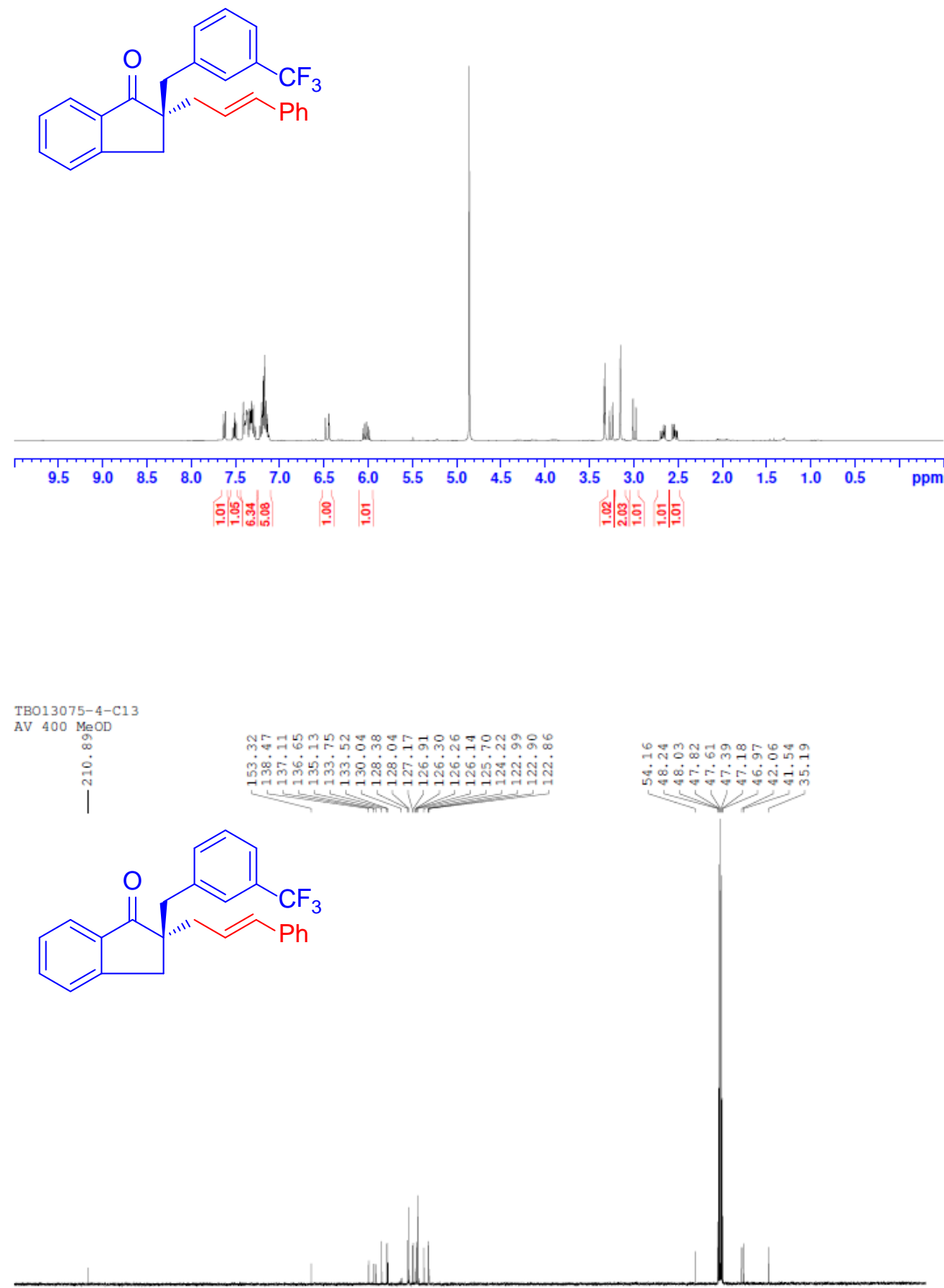

$\begin{array}{lllllllllllllllllllllll}220 & 210 & 200 & 190 & 180 & 170 & 160 & 150 & 140 & 130 & 120 & 110 & 100 & 90 & 80 & 70 & 60 & 50 & 40 & 30 & 20 & 10 & \text { ppm }\end{array}$ 


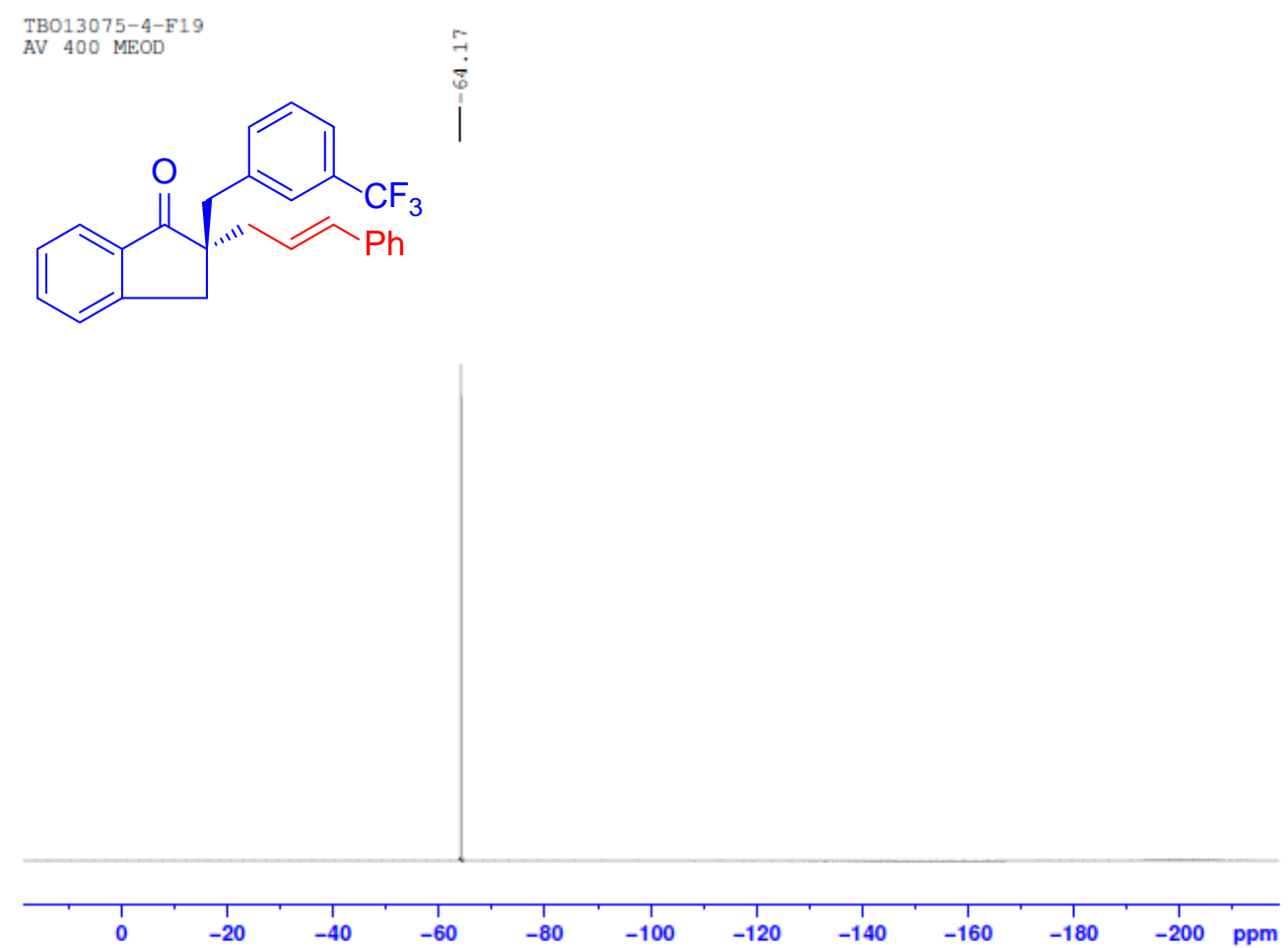

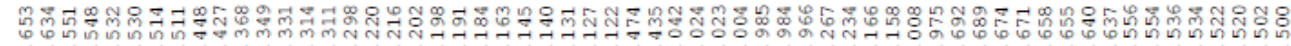

rivir<smiles>O=C1c2ccccc2CC1(C/C=C/c1ccccc1)Cc1ccc(C(F)(F)F)cc1</smiles>

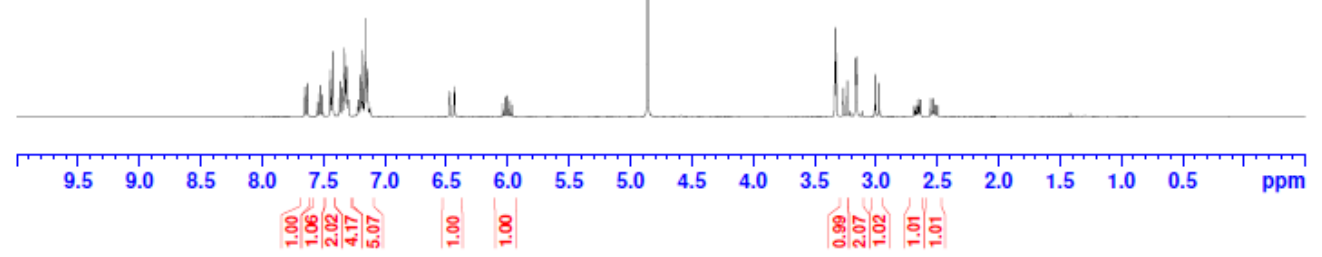



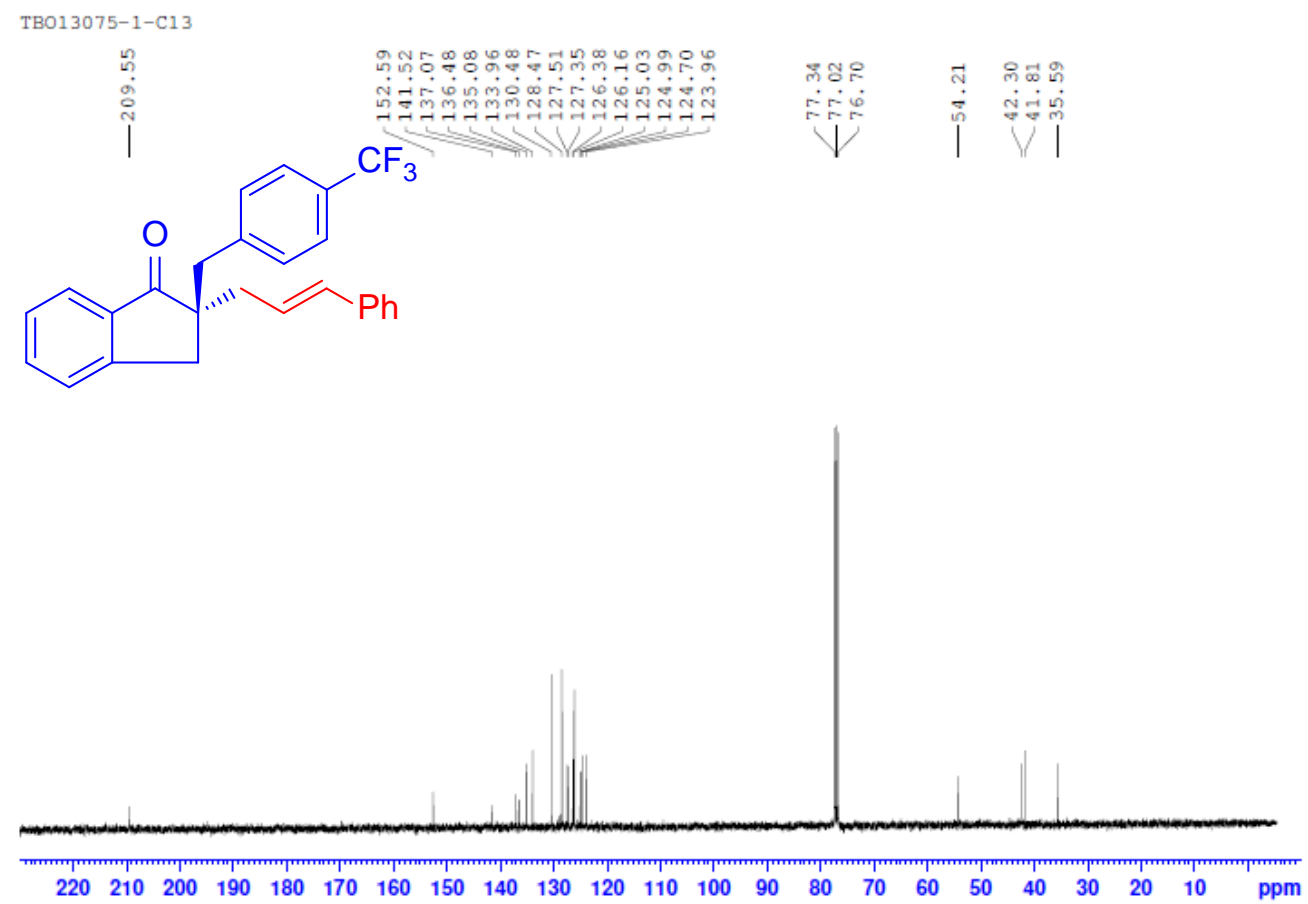

(B)

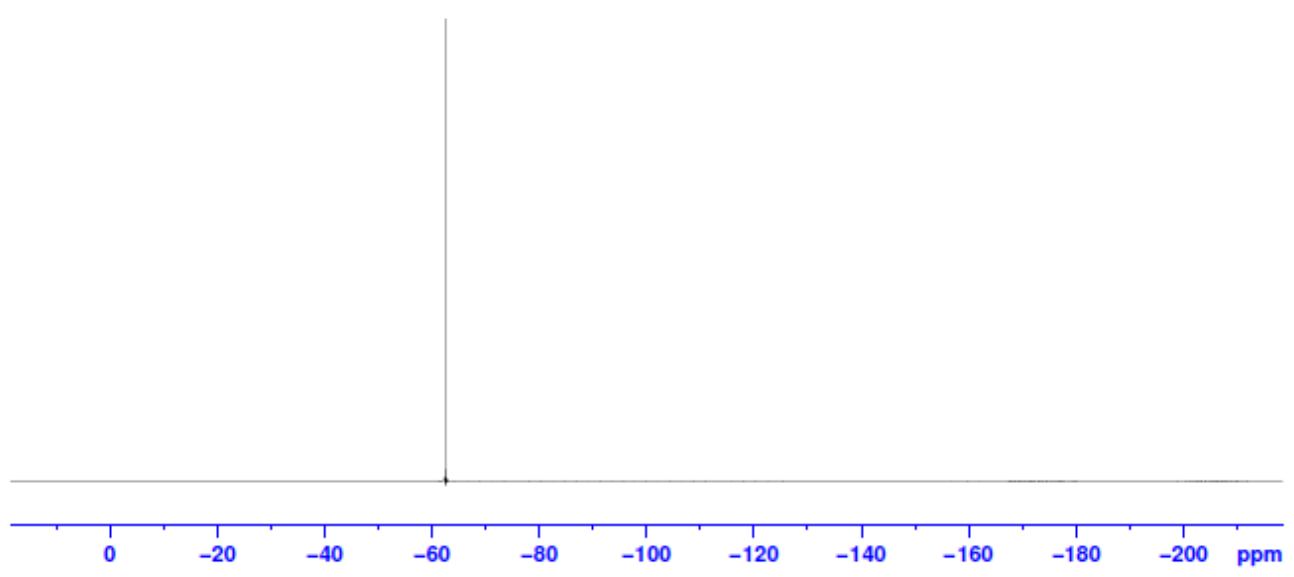




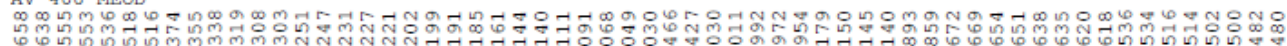
r.r.<smiles>O=C1c2ccccc2CC1(C/C=C/c1ccccc1)Cc1ccccc1</smiles>

Whe

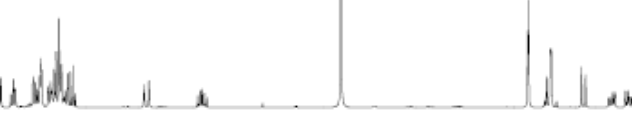
$\begin{array}{lllllllllllllllllllll}9.5 & 9.0 & 8.5 & 8.0 & 7.5 & 7.0 & 6.5 & 6.0 & 5.5 & 5.0 & 4.5 & 4.0 & 3.5 & 3.0 & 2.5 & 2.0 & 1.5 & 1.0 & 0.5 & \mathrm{ppm}\end{array}$

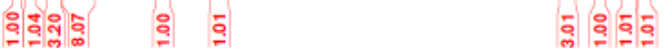

TB013075-5-C13<smiles>CNC(=O)OCCCCCCCC[C@@]1(Cc2cccc(Br)c2)Cc2ccccc2C1=O</smiles>

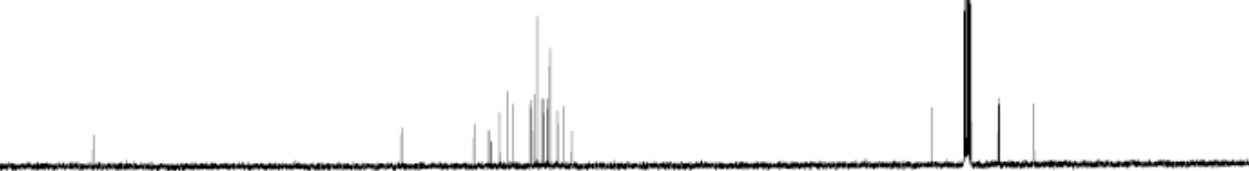

$\begin{array}{lllllllllllllllllllllll}220 & 210 & 200 & 190 & 180 & 170 & 160 & 150 & 140 & 130 & 120 & 110 & 100 & 90 & 80 & 70 & 60 & 50 & 40 & 30 & 20 & 10 & \text { ppm }\end{array}$ 


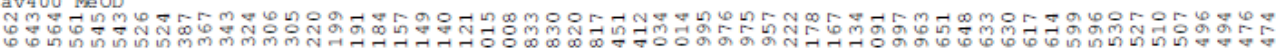

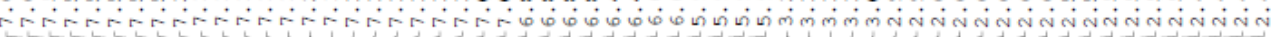<smiles>O=C1c2ccccc2CC1(C/C=C/c1ccccc1)Cc1ccccc1</smiles>
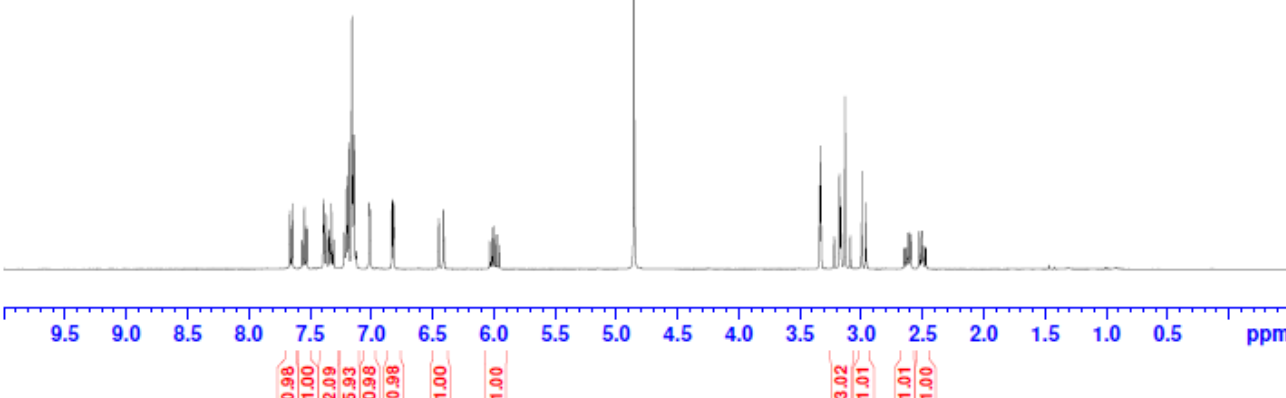

TB013075-16-C13

av4 400 NeOD

|<smiles>O=C1c2ccccc2C[C@]1(C/C=C/c1ccccc1)Cc1ccsc1</smiles>

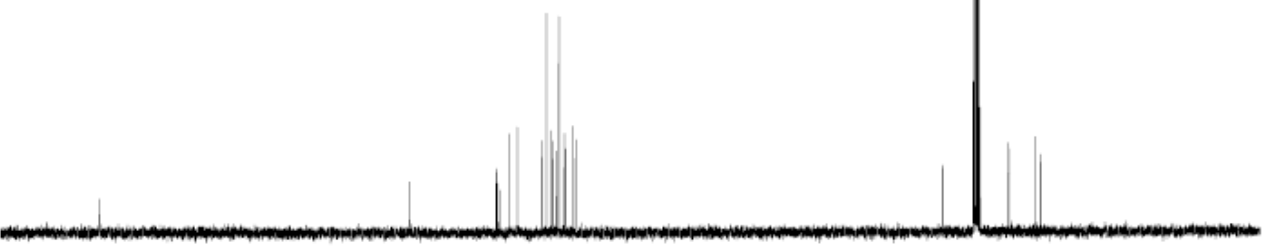

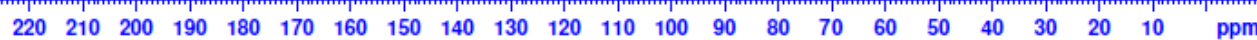



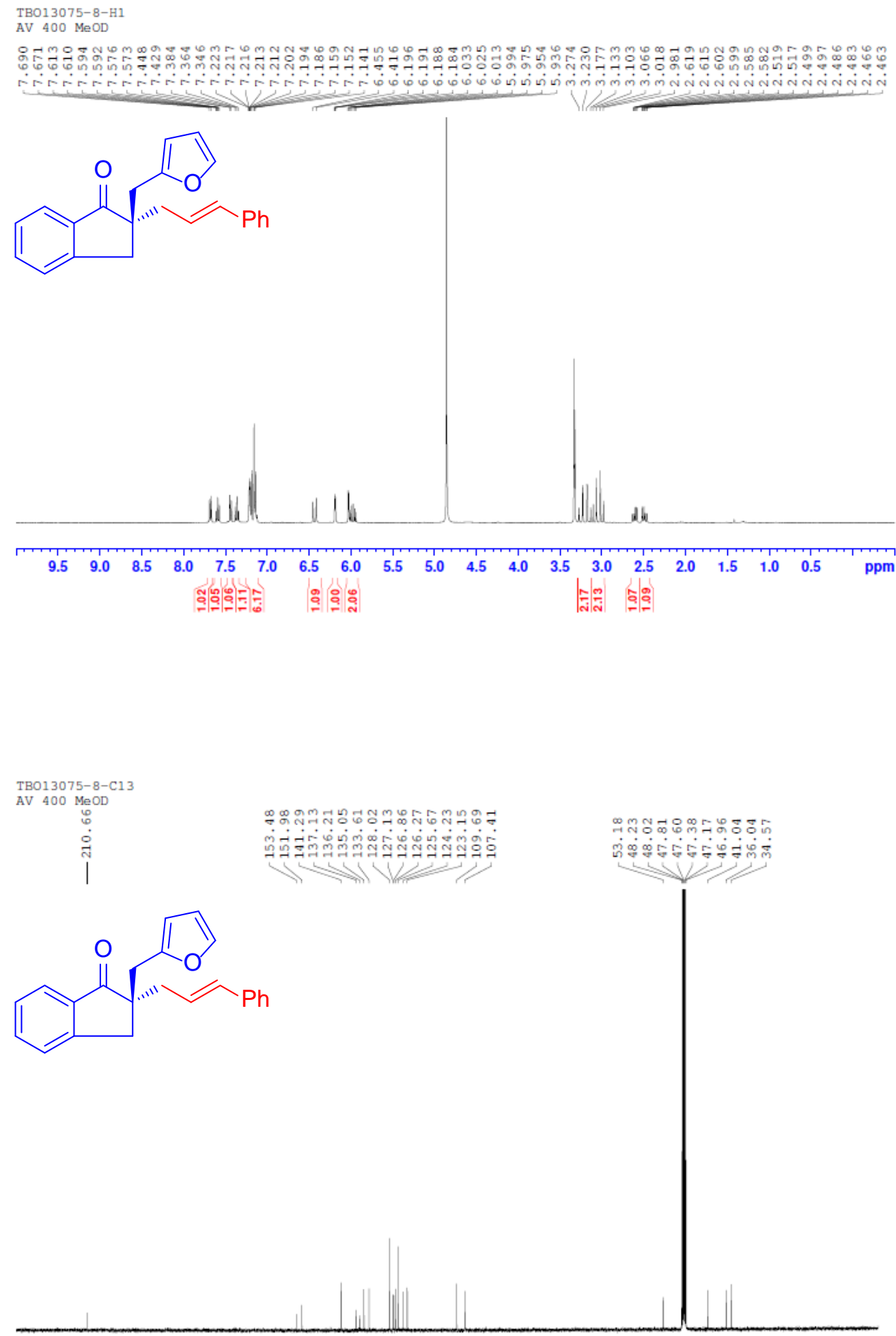

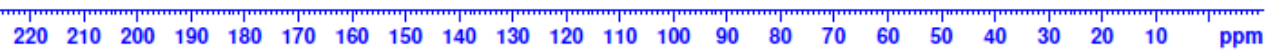



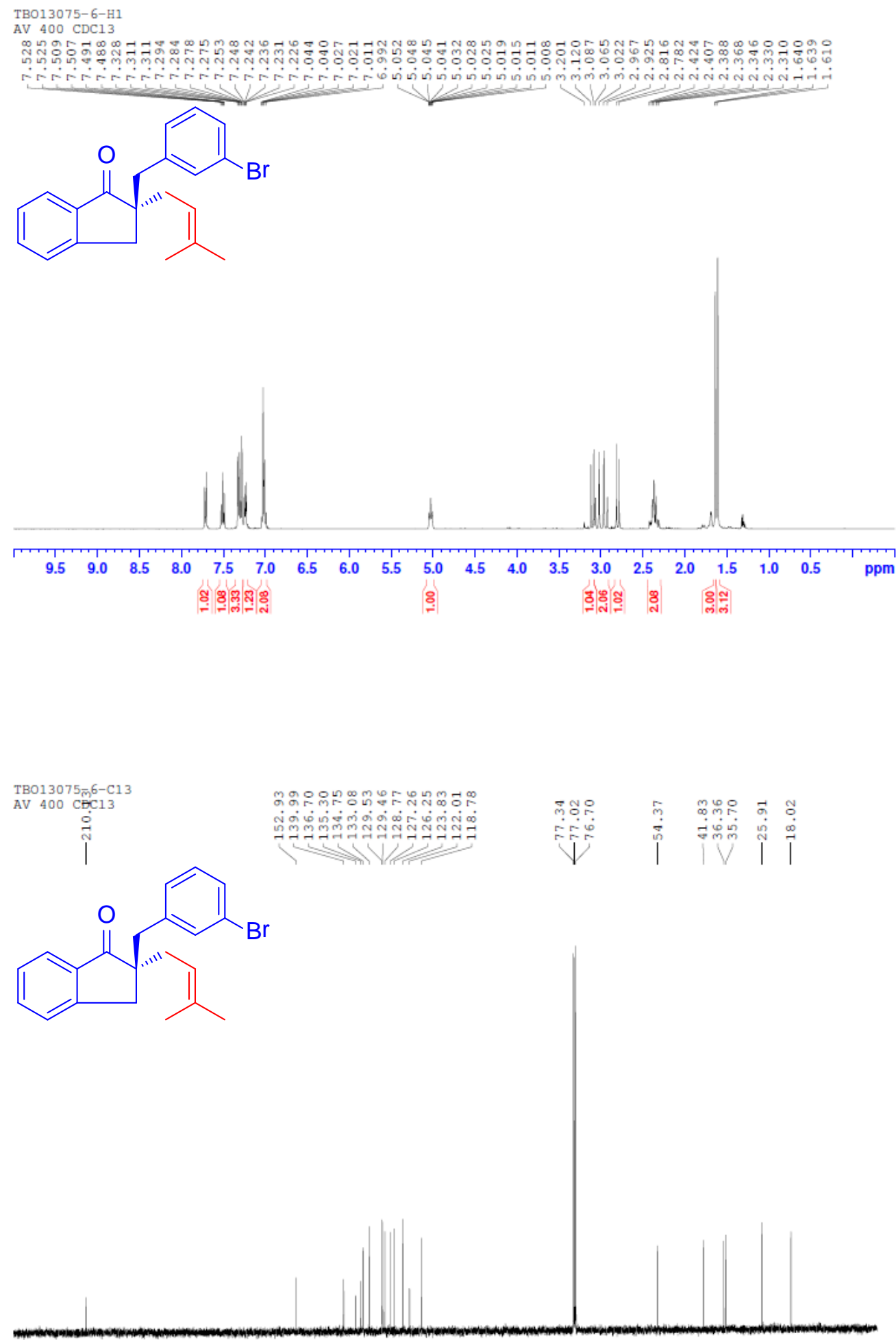

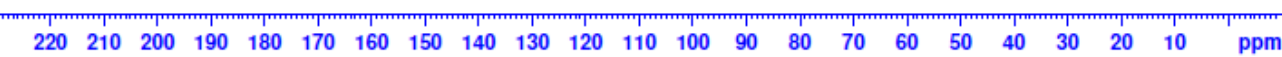




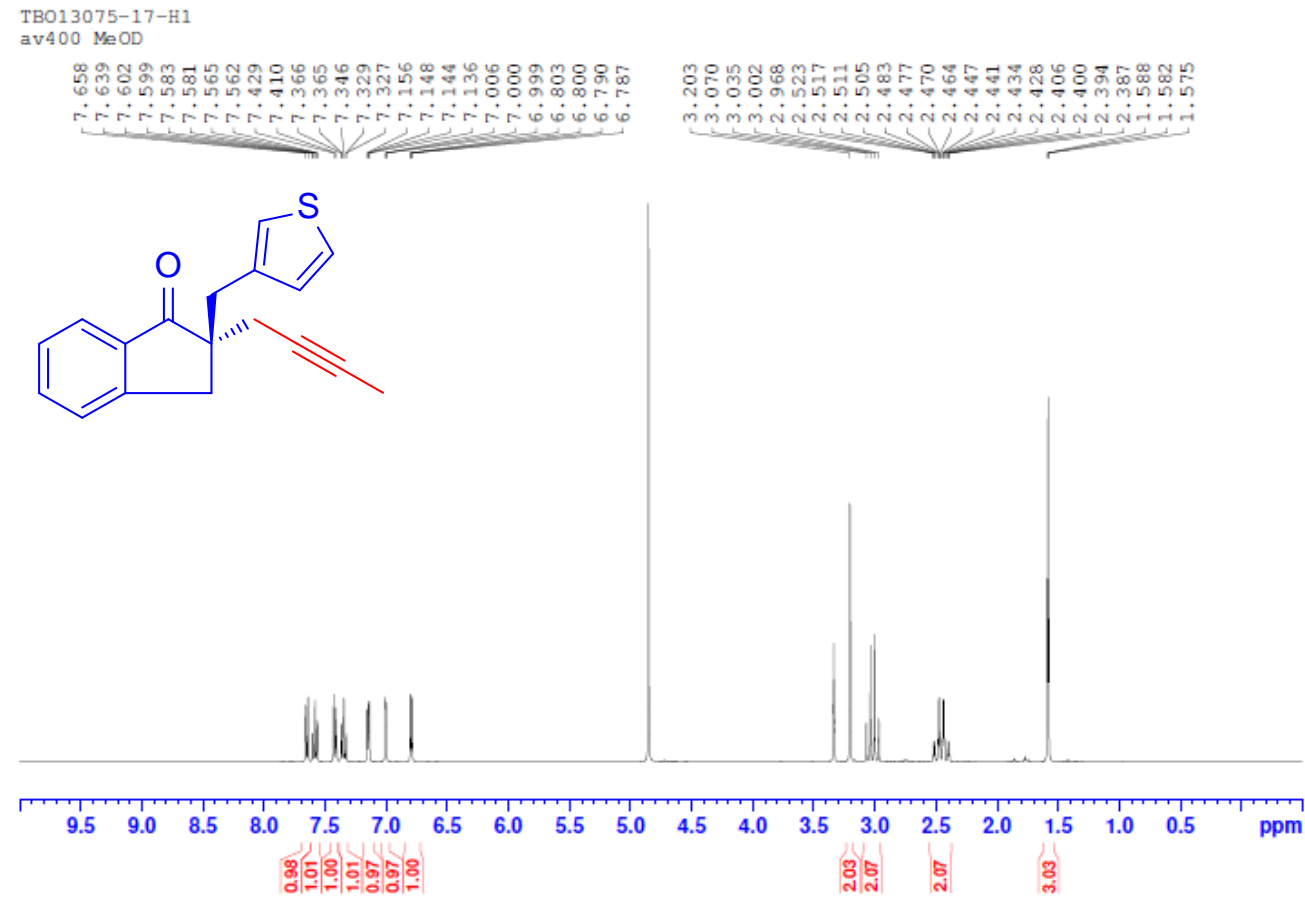

TB013075-17-C13

|

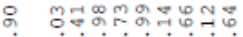

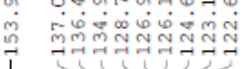

(N)

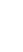



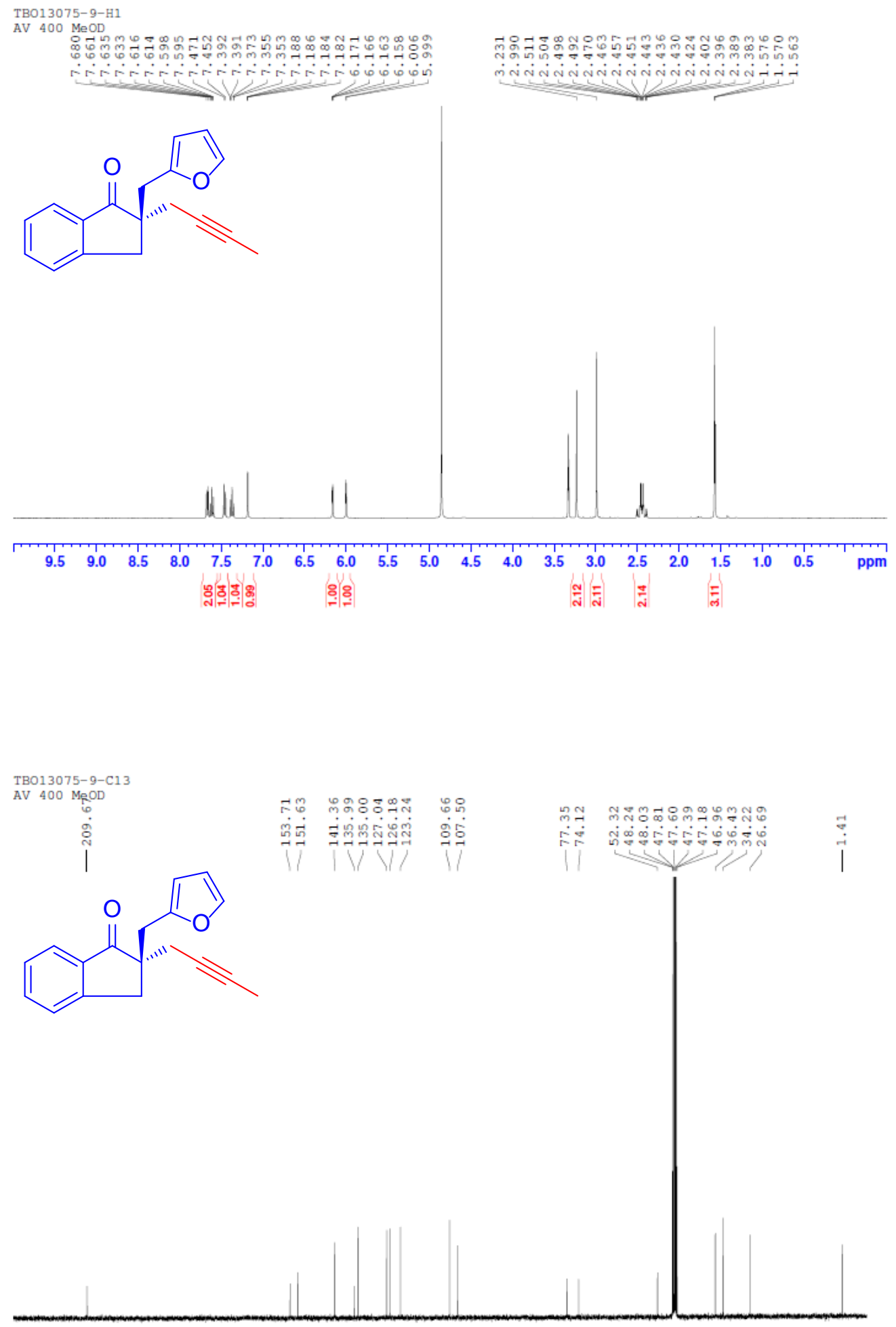

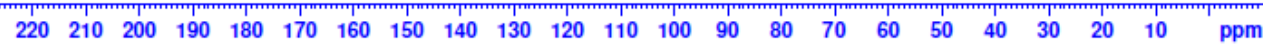



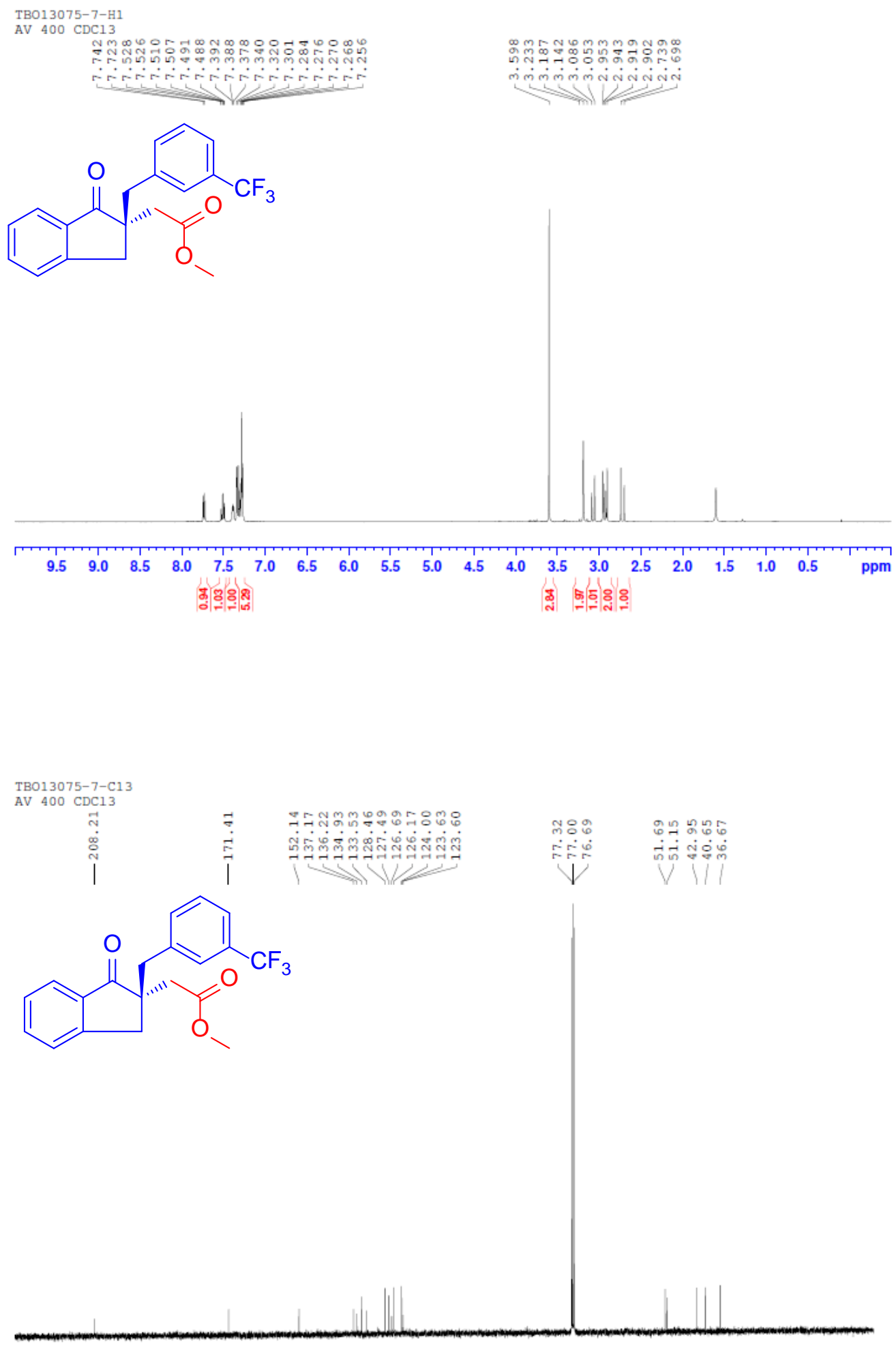

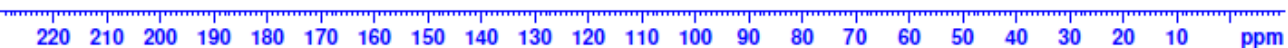




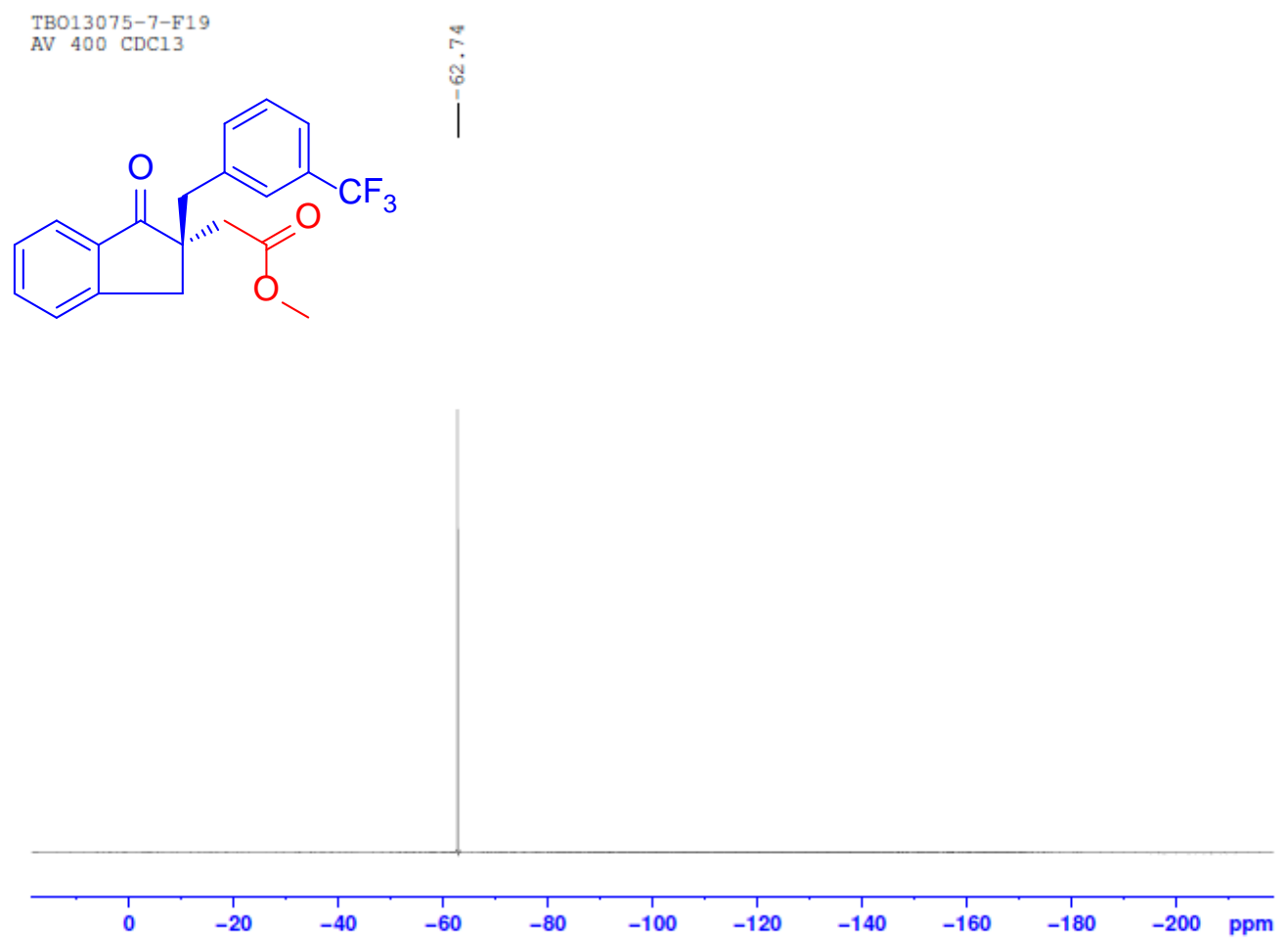

TBO $13075-2-\mathrm{H} 1$
AV 400 CDC13

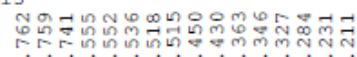

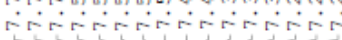

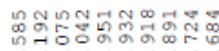

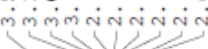

(c)

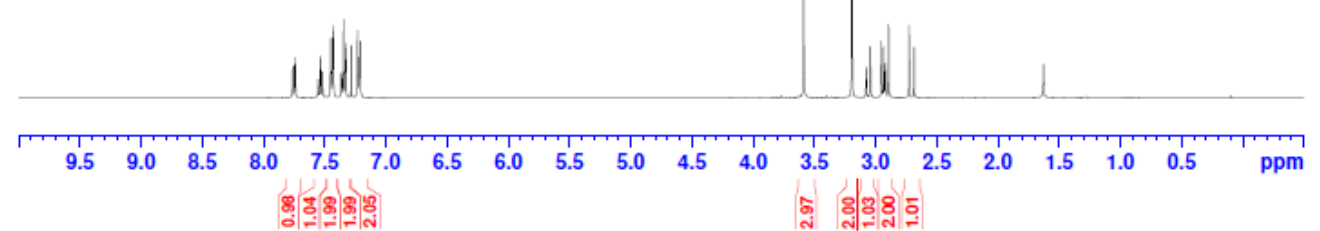

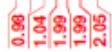

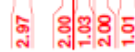




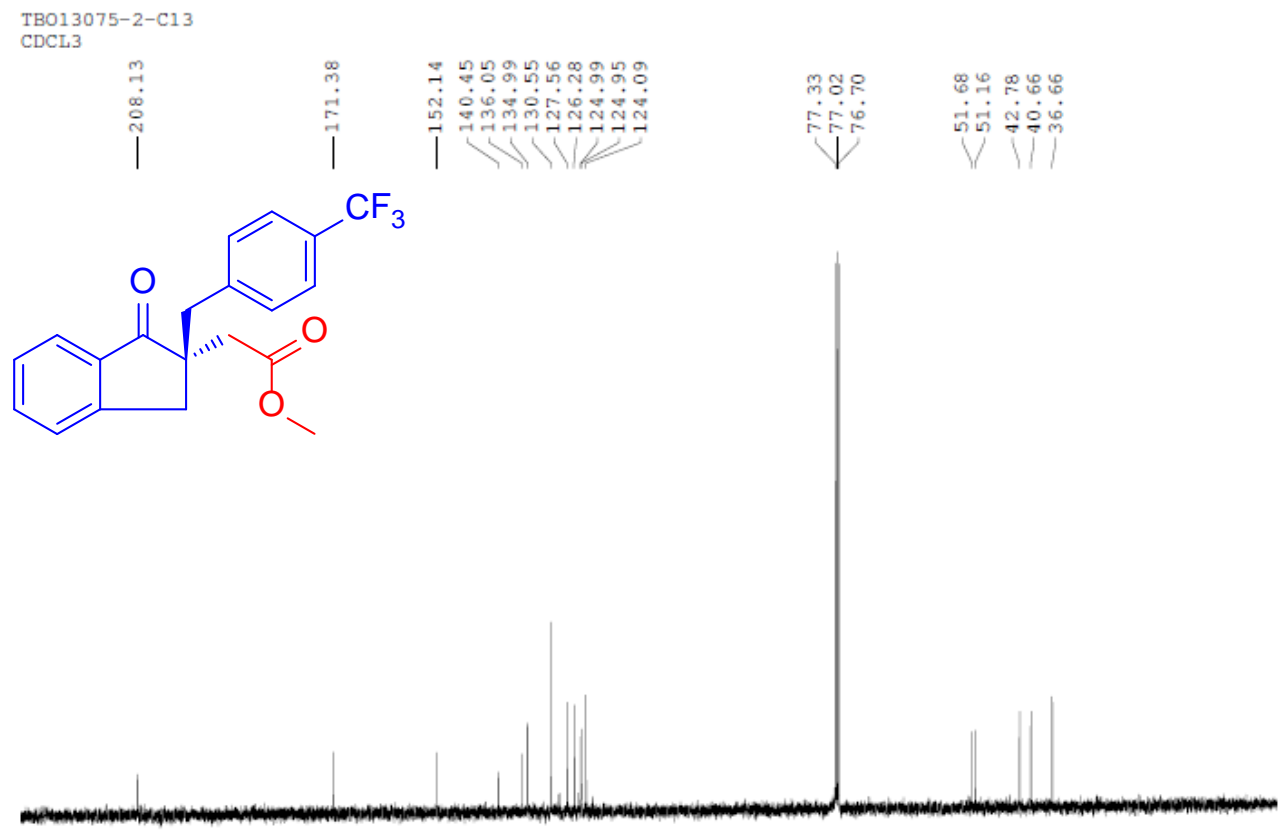

$\begin{array}{llllllllllllllllllllllll}220 & 210 & 200 & 190 & 180 & 170 & 160 & 150 & 140 & 130 & 120 & 110 & 100 & 90 & 80 & 70 & 60 & 50 & 40 & 30 & 20 & 10 & \mathrm{ppm}\end{array}$<smiles>COC(=O)C[C@@]1(Cc2ccc(C(F)(F)F)cc2)Cc2ccccc2C1=O</smiles>

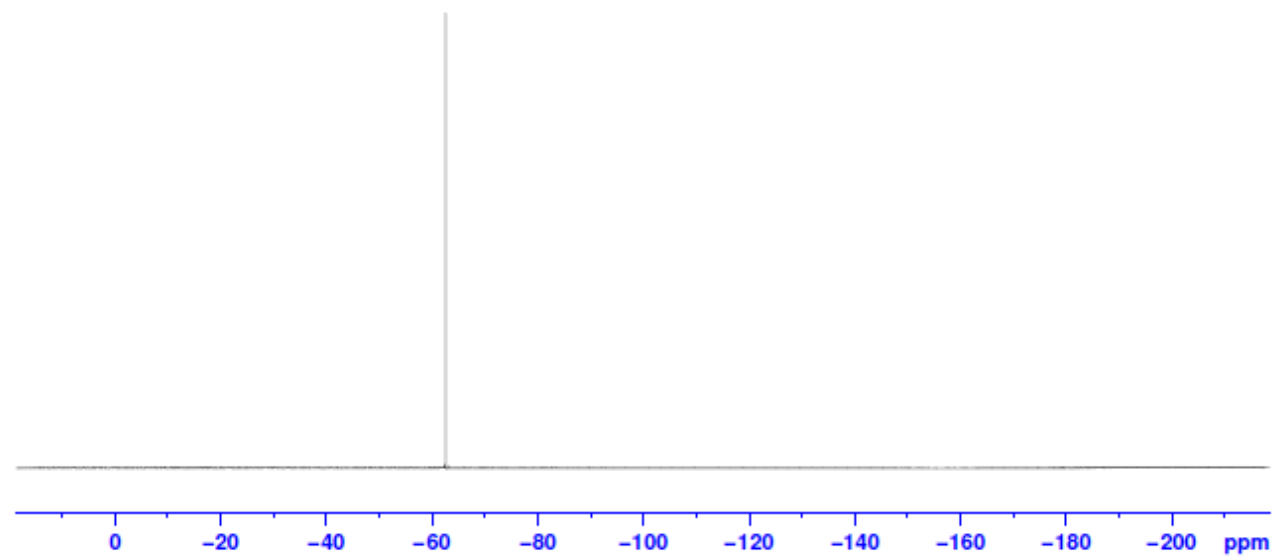




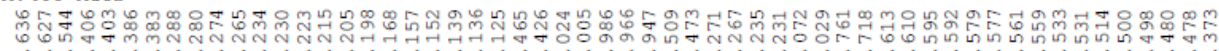

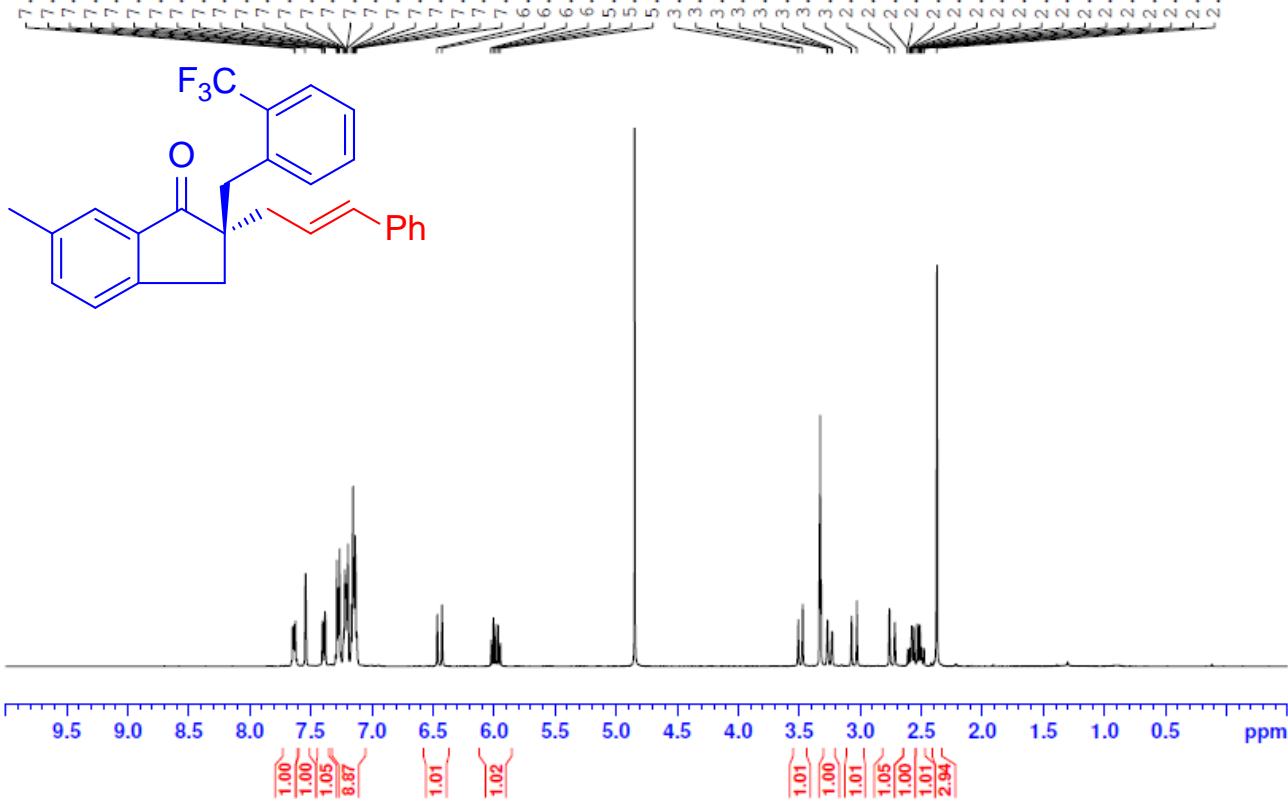

TB013075-20-C13

AV 400 MeOD
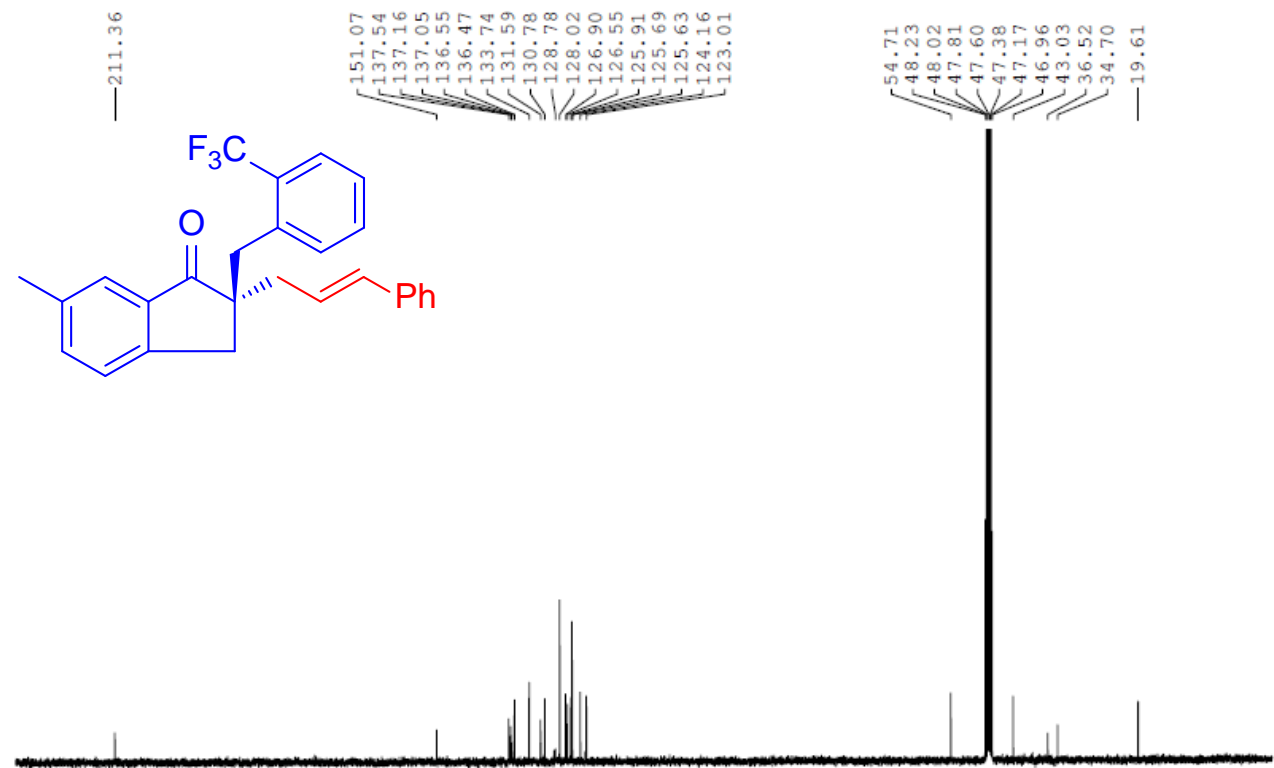

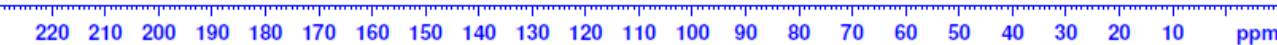



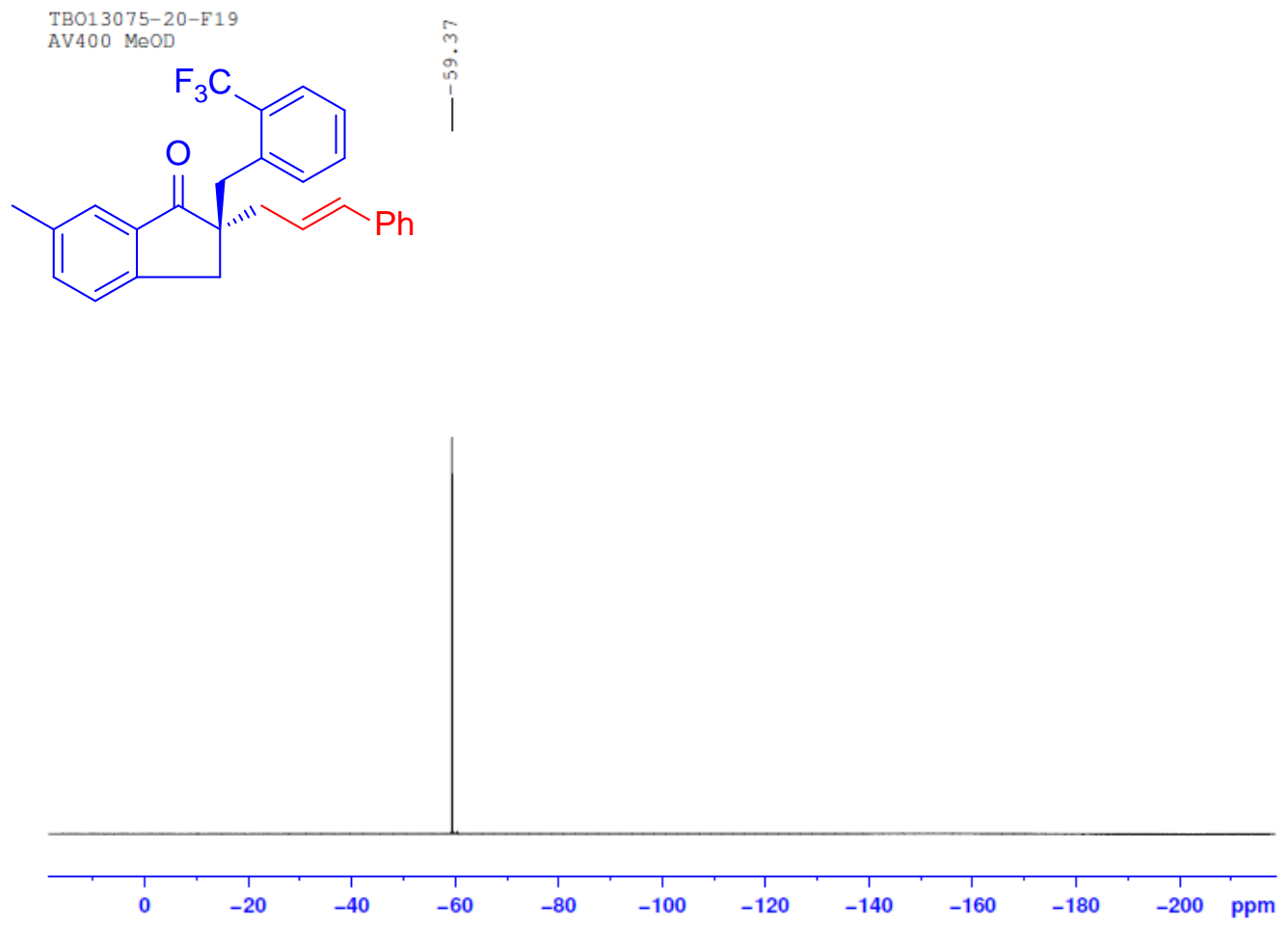<smiles>O=C1c2ccc(Cl)cc2CC1(C/C=C/c1ccccc1)Cc1ccccc1</smiles>

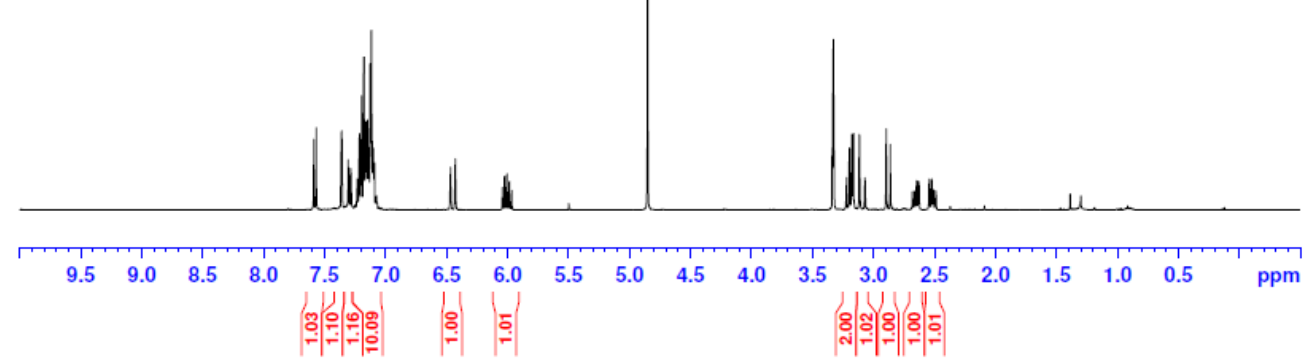




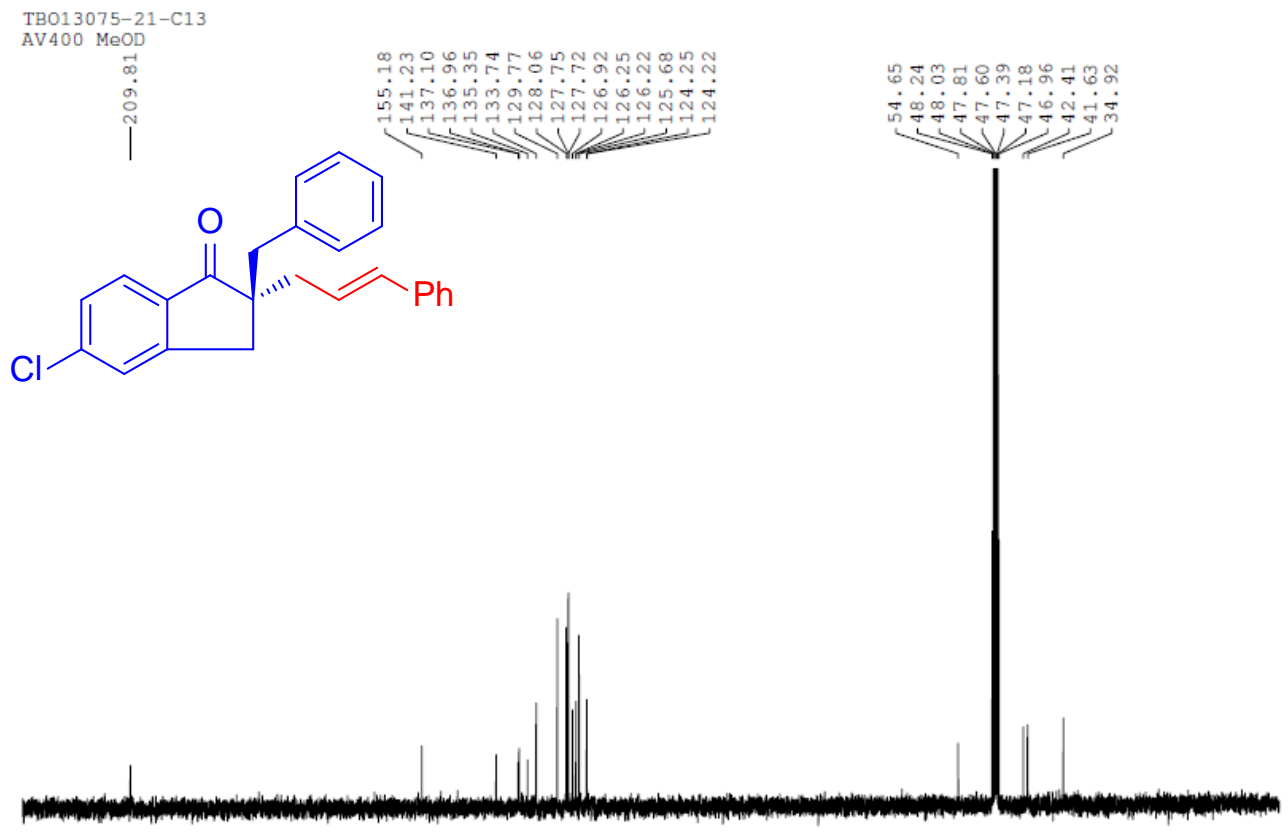

$\begin{array}{lllllllllllllllllllllll}220 & 210 & 200 & 190 & 180 & 170 & 160 & 150 & 140 & 130 & 120 & 110 & 100 & 90 & 80 & 70 & 60 & 50 & 40 & 30 & 20 & 10 & \mathrm{ppm}\end{array}$ TBO $13075-10-\mathrm{H} 1$
AV 400 MeOD

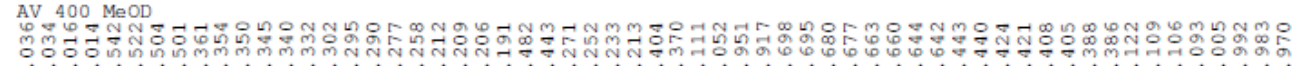

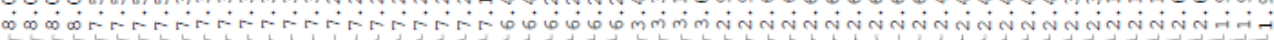<smiles>O=C1c2ccccc2CC[C@]1(C/C=C/c1ccccc1)Cc1ccc(C(F)(F)F)cc1</smiles>

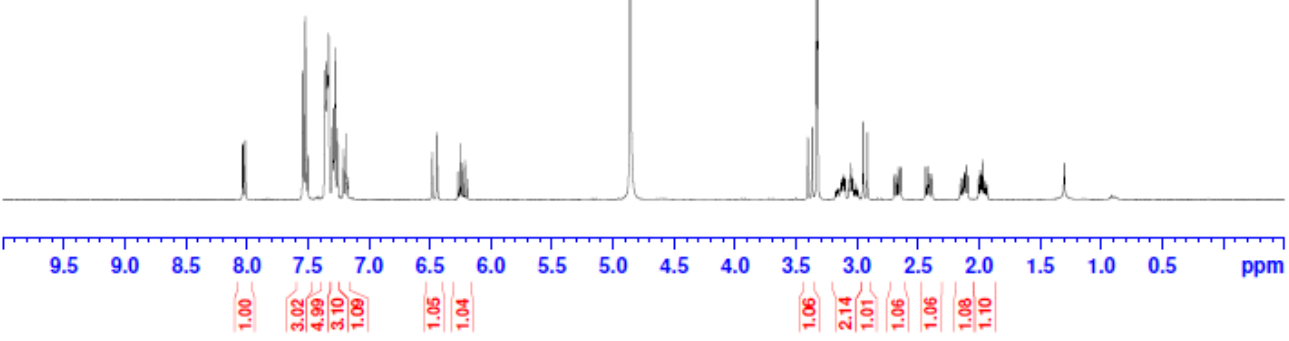



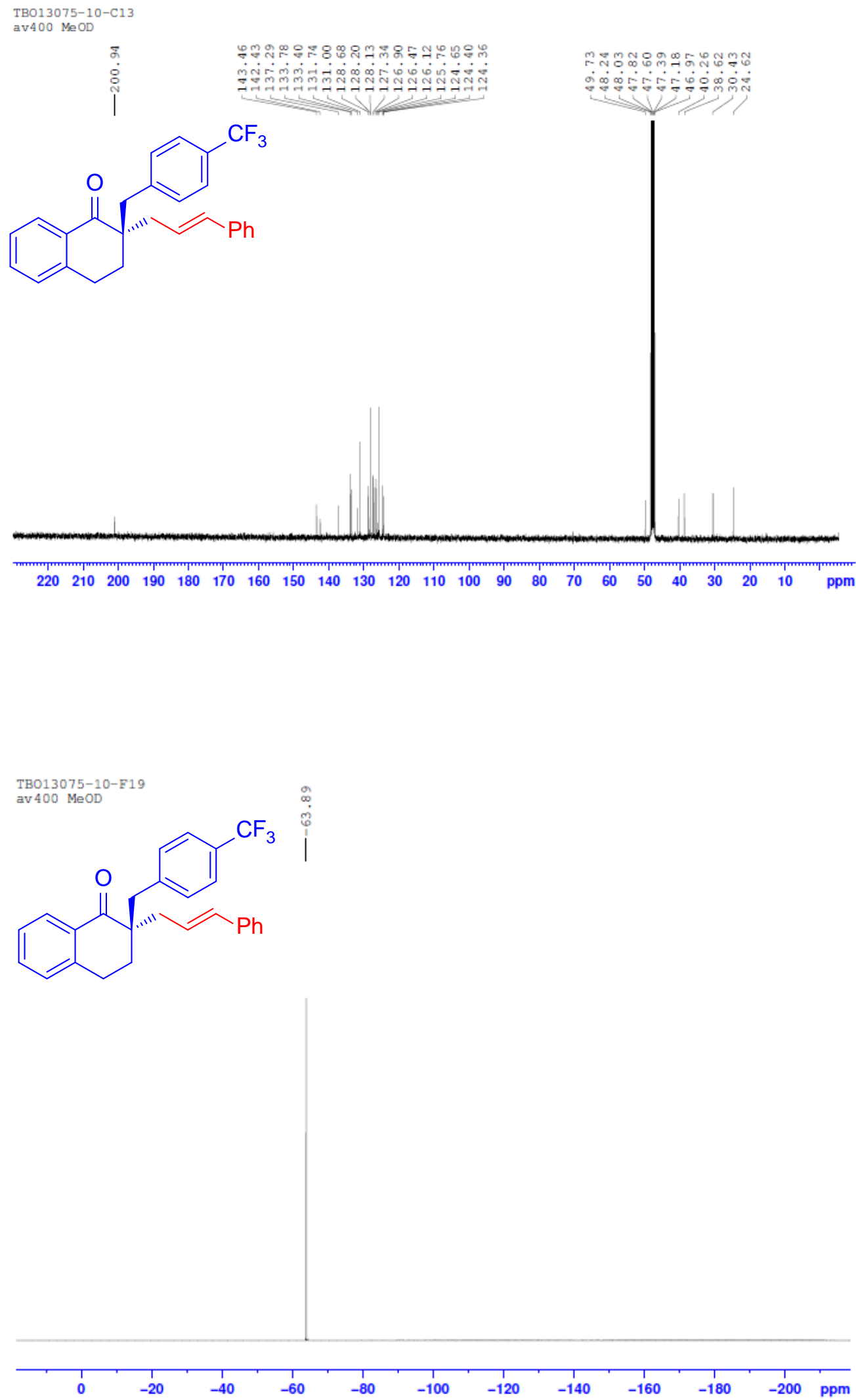

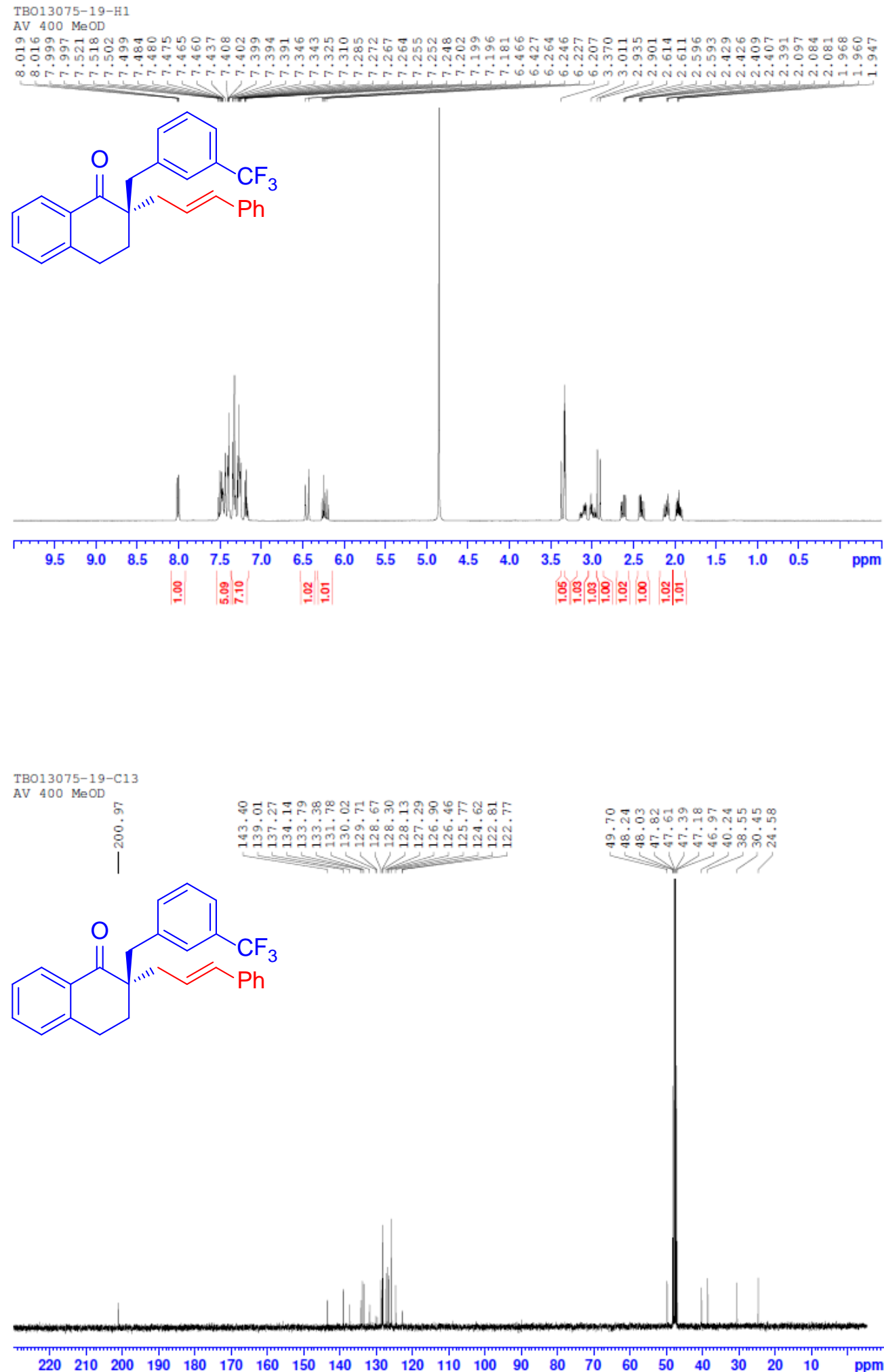

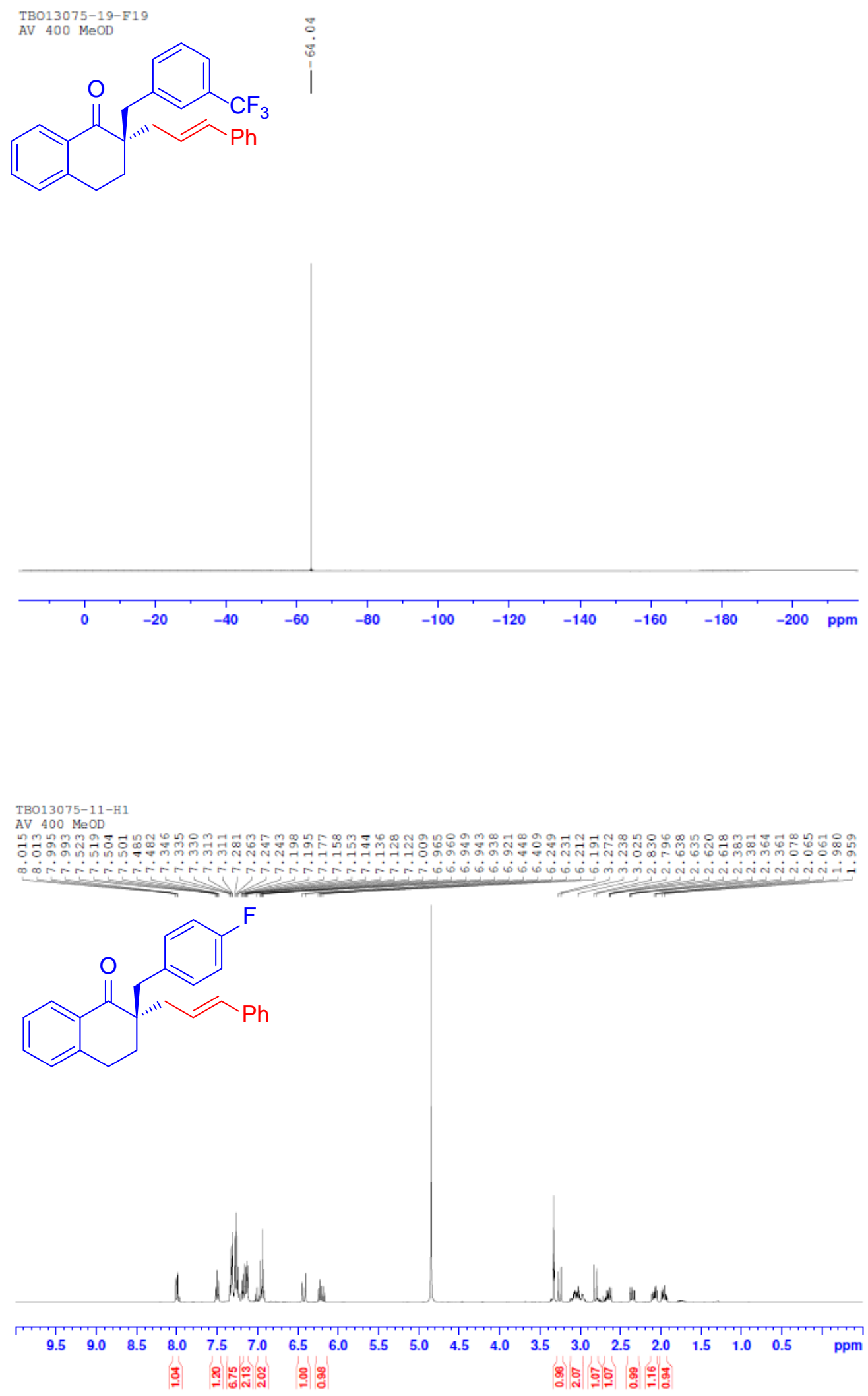


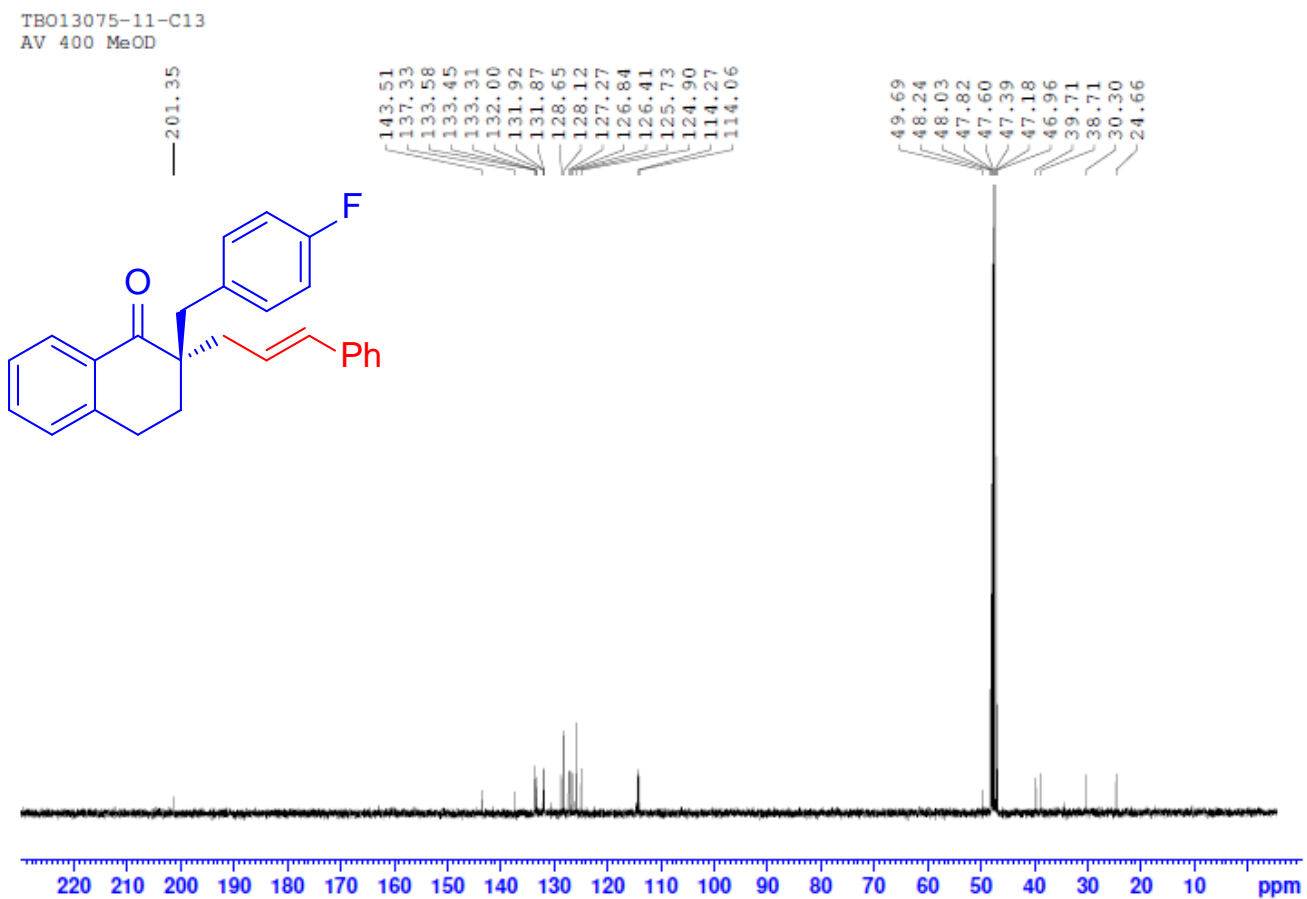<smiles>O=C1c2ccccc2CC[C@]1(C/C=C/c1ccc(F)cc1)Cc1ccccc1</smiles>

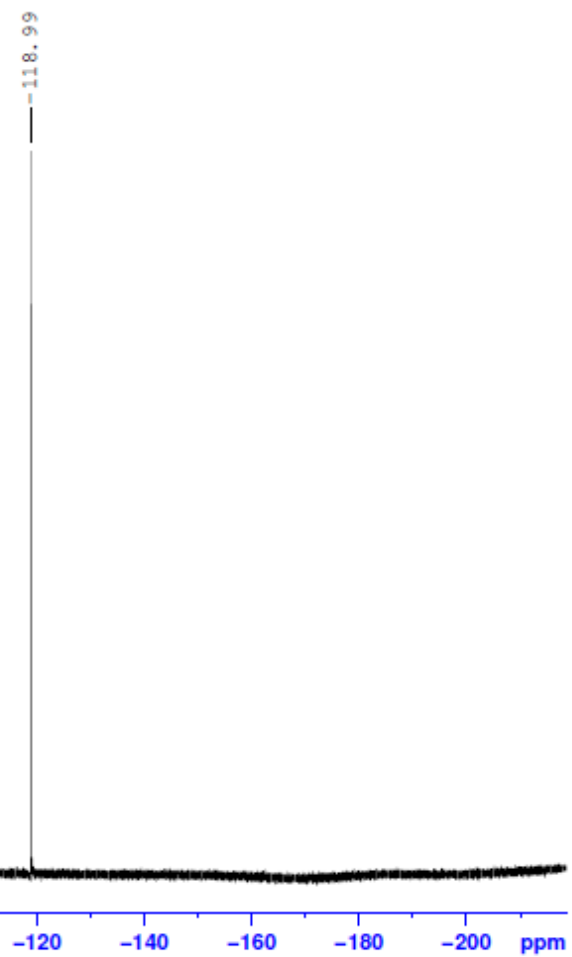




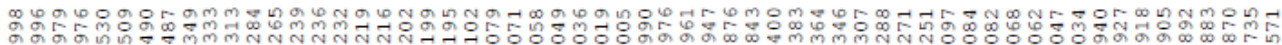

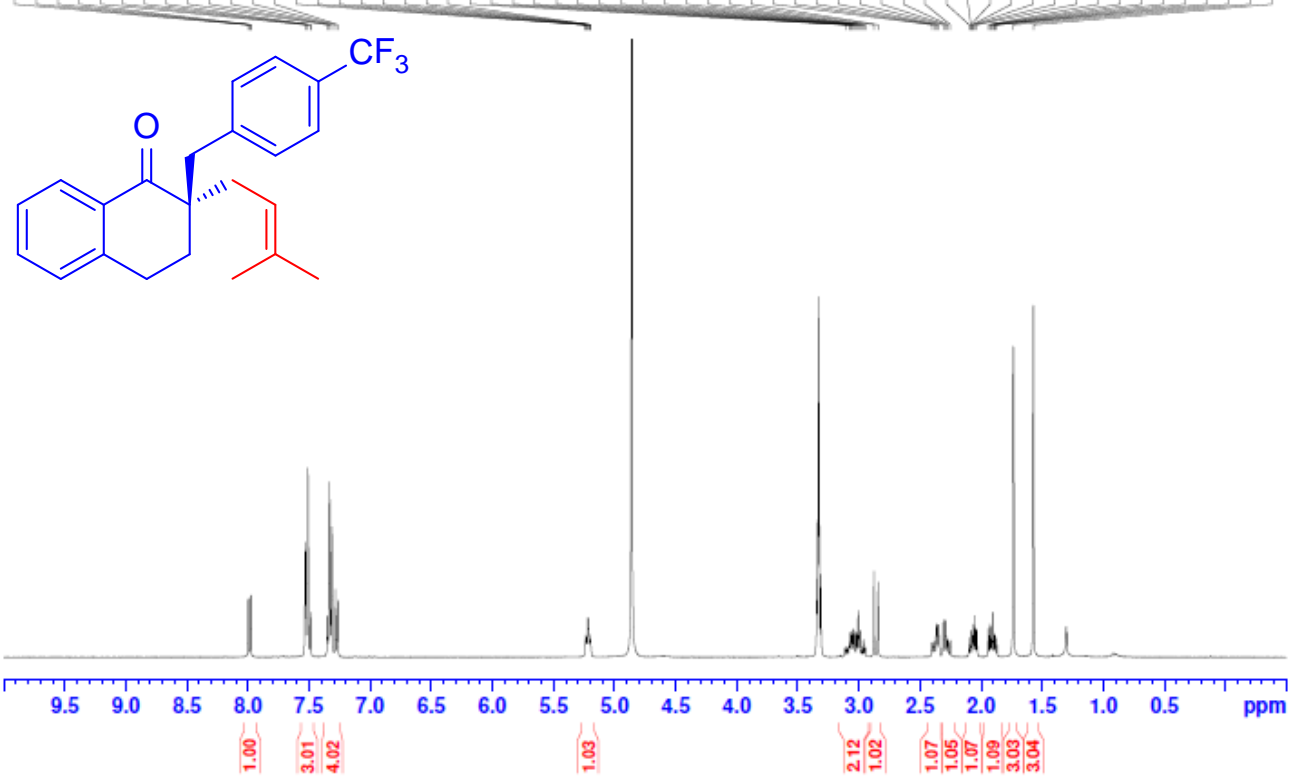

TBO13075-12-C13

av400 MeOD<smiles>CN=[W]O[Mg]</smiles>

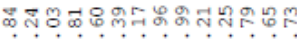

$\underset{\nabla}{\infty} \underset{\sim}{\infty}$

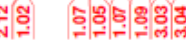

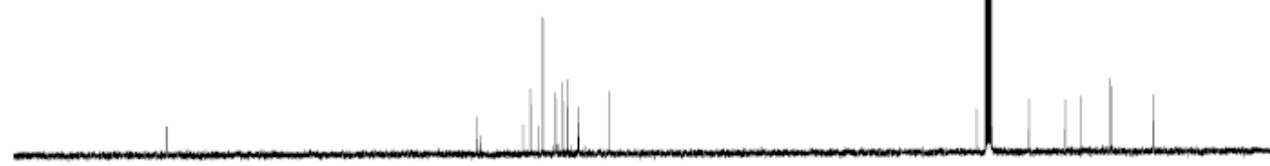

$\begin{array}{lllllllllllllllllllllll}220 & 210 & 200 & 190 & 180 & 170 & 160 & 150 & 140 & 130 & 120 & 110 & 100 & 90 & 80 & 70 & 60 & 50 & 40 & 30 & 20 & 10 & \mathrm{ppm}\end{array}$ 

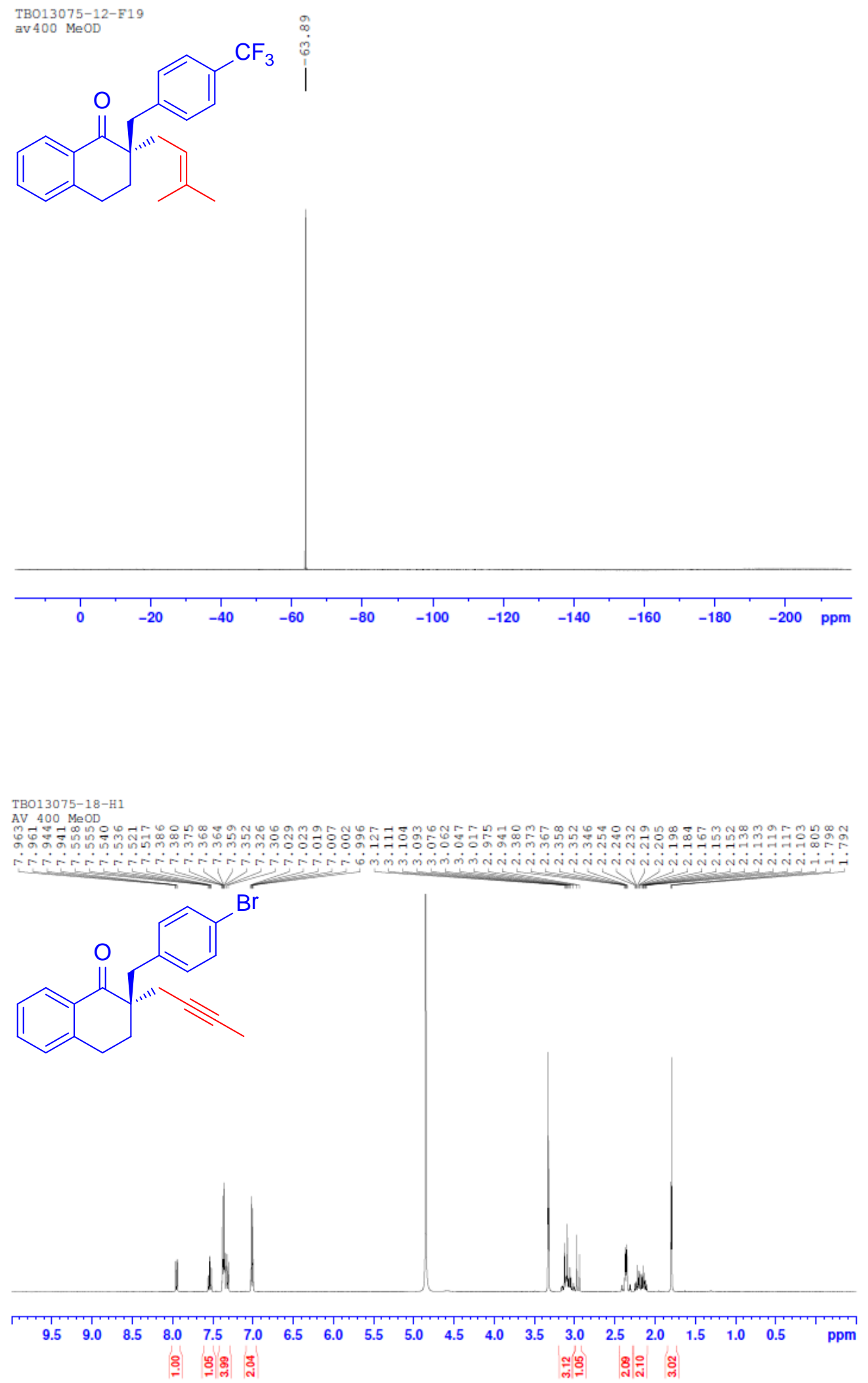


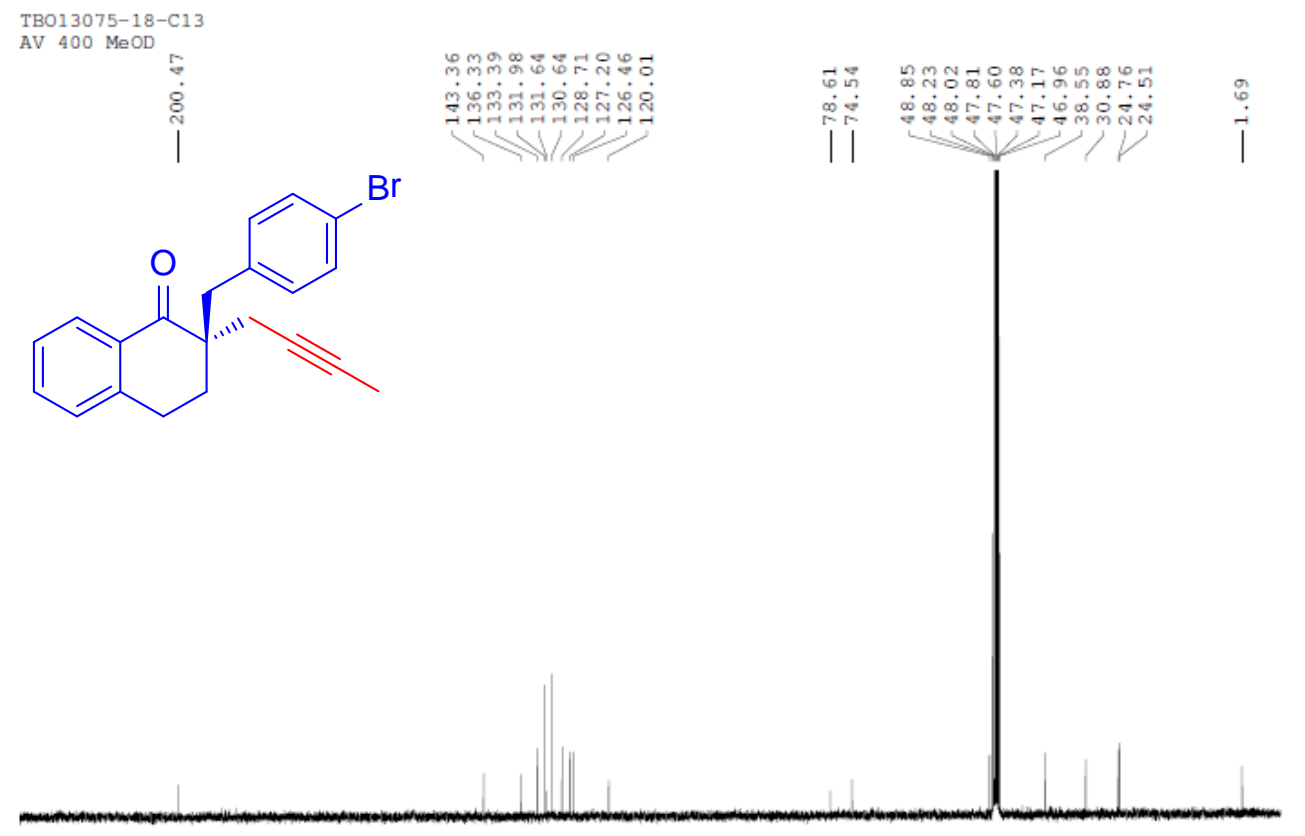

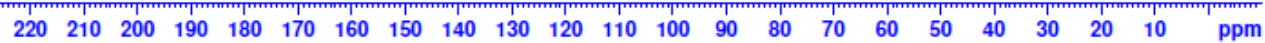

TB013075-13-H

av $400 \mathrm{MeOD}$

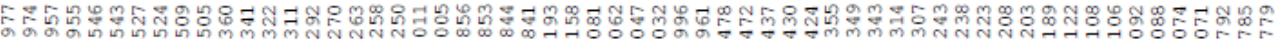

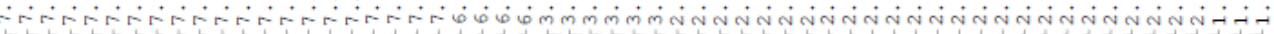

(1)
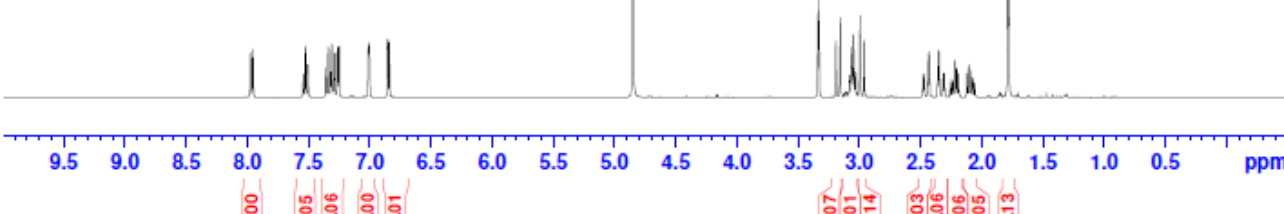

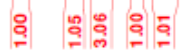

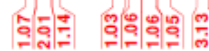




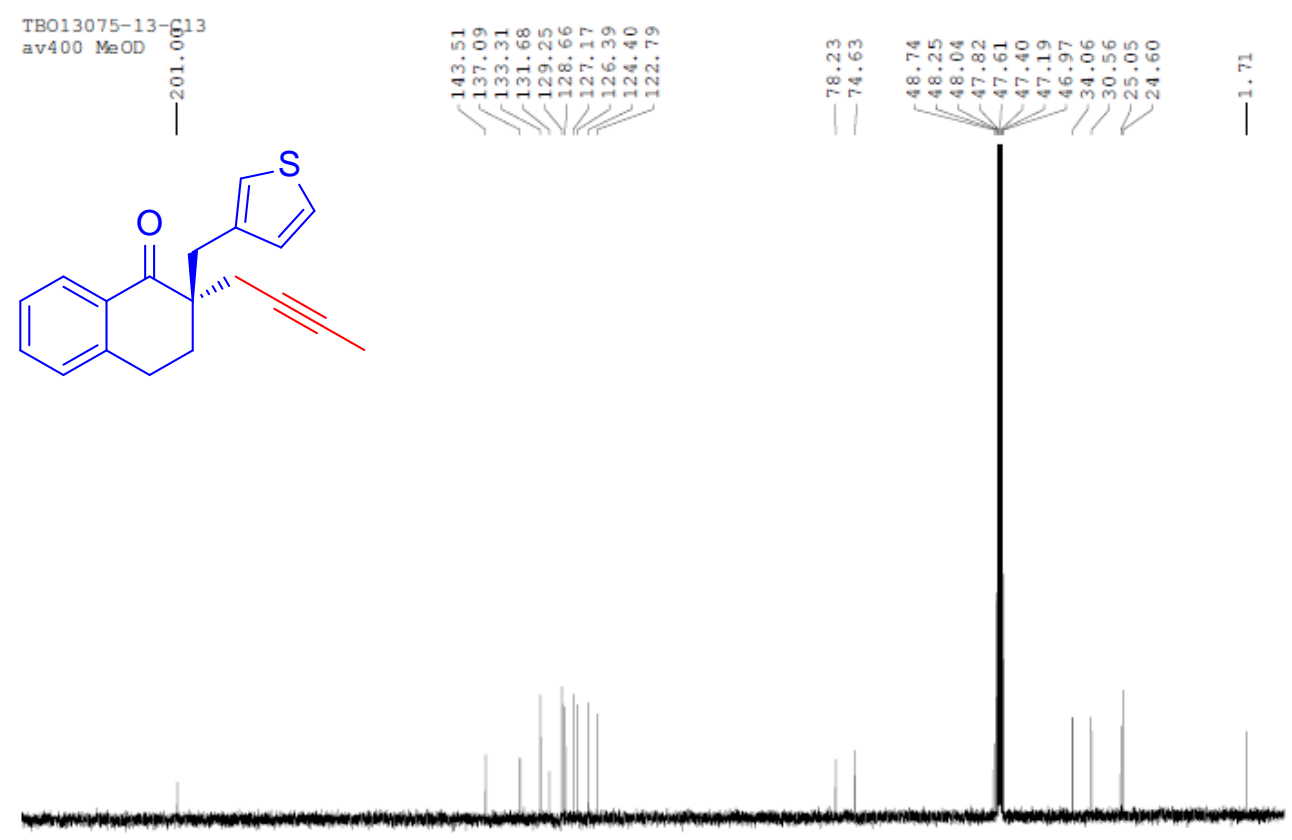

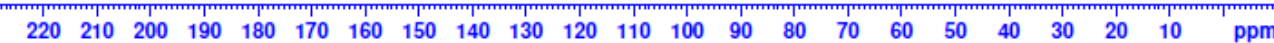

TBO13075-14-H

av4 00 NeOD

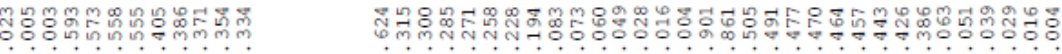

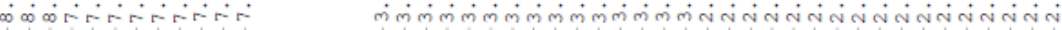

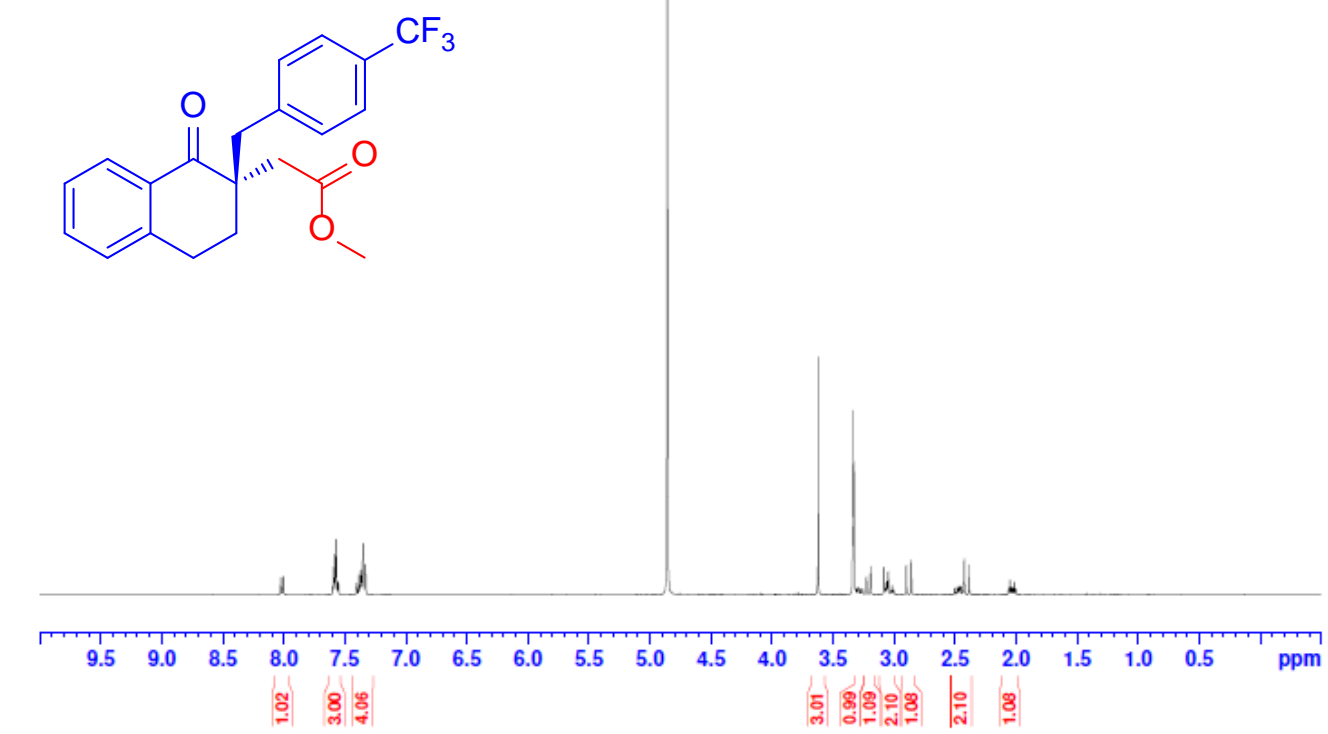




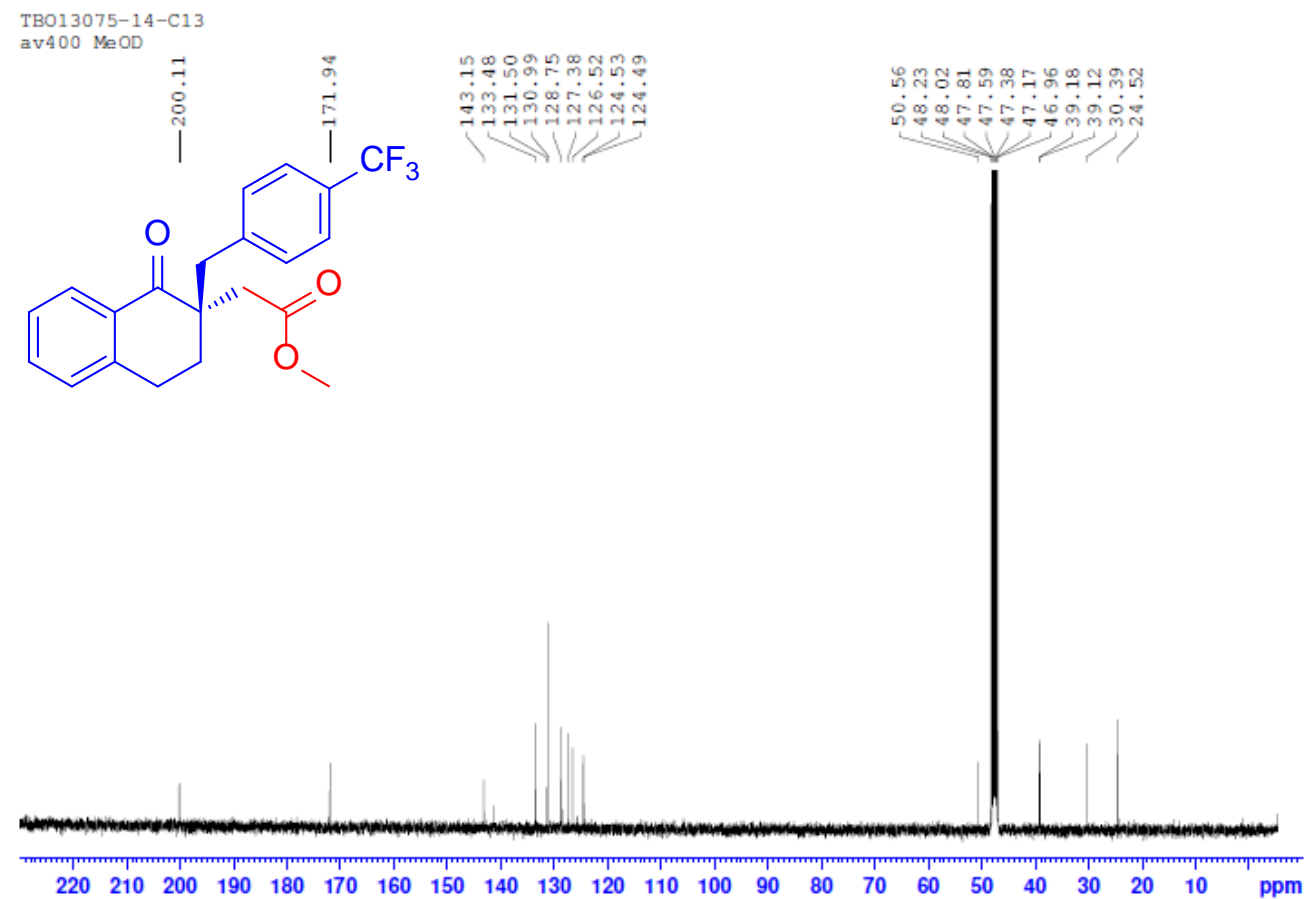<smiles>COC(=O)C[C@@]1(Cc2ccc(C(F)(F)F)cc2)CCc2ccccc2C1=O</smiles>

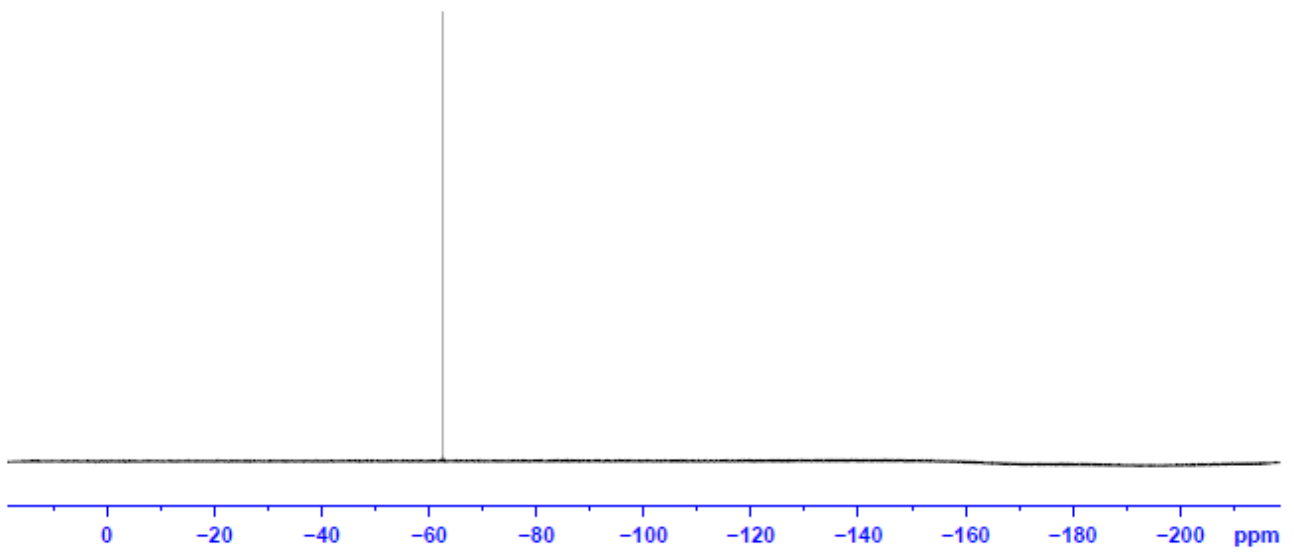


TB013081-1-H1

av300 CDCL3

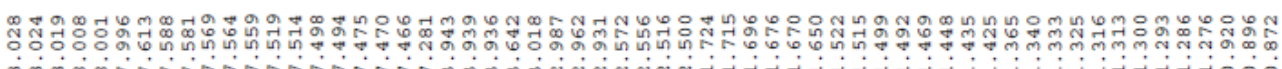

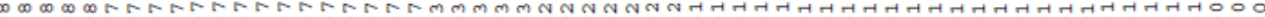
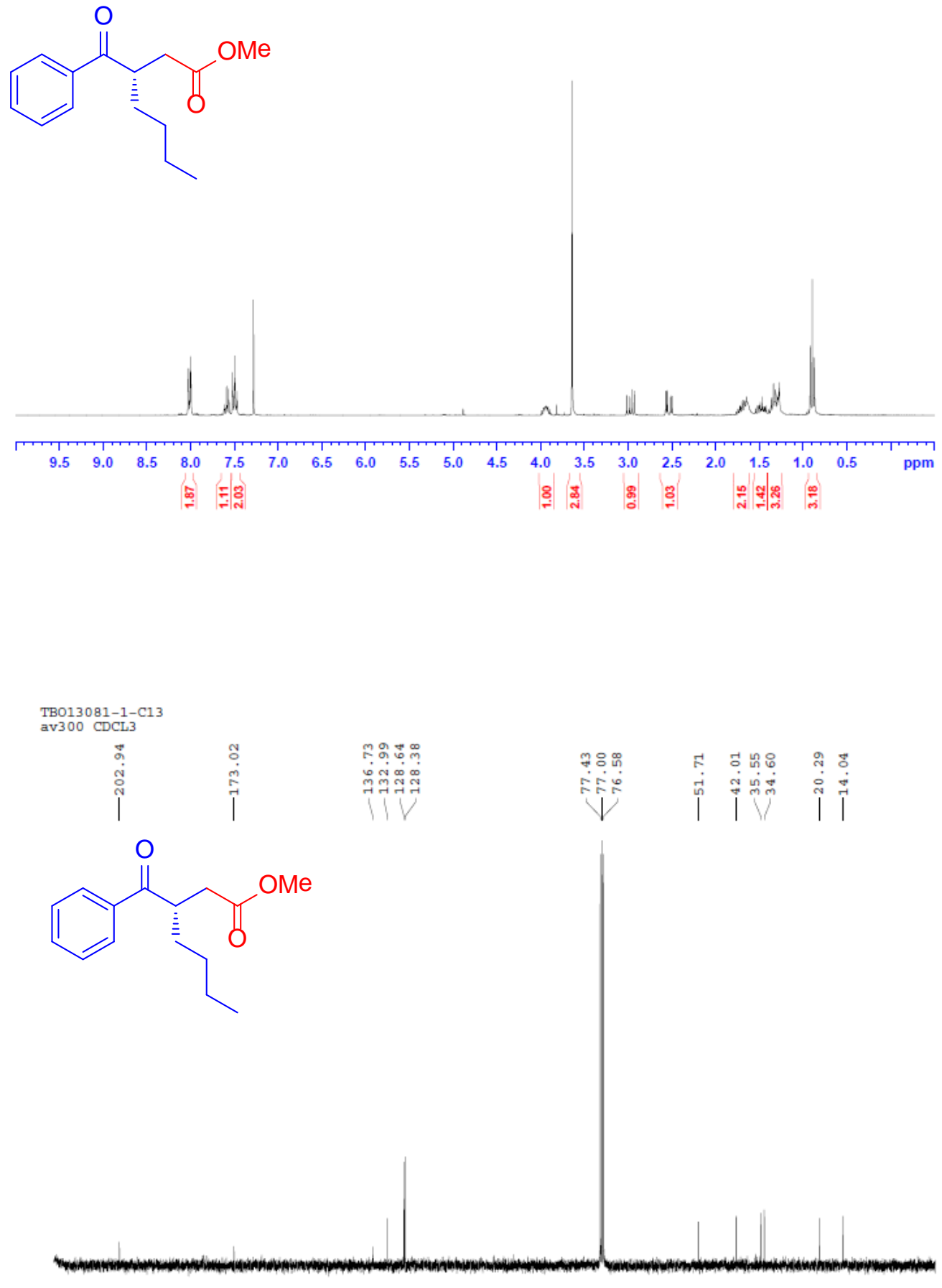

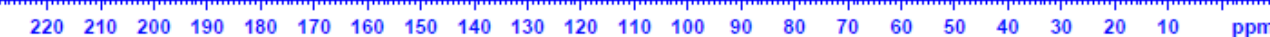


TBO13081-6-H1
AV $400 \mathrm{CDC13}$

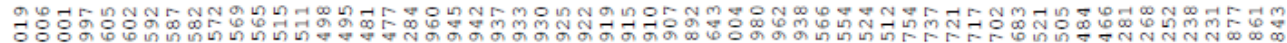

$\infty \omega_{0}$

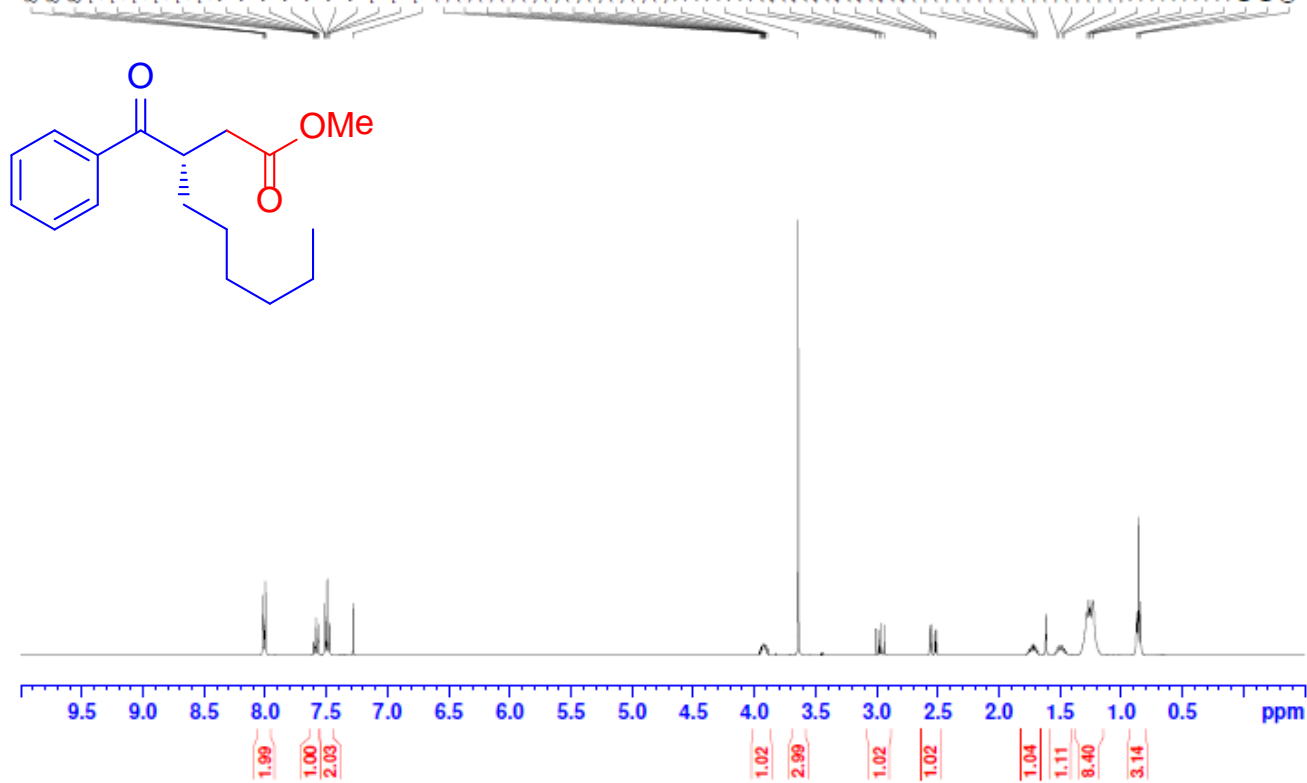

TB013081-6-C1

AV $400 \mathrm{CDC1} 3$

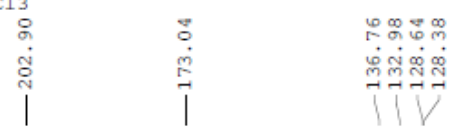

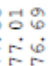

응 -

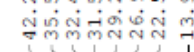

$\overbrace{(1)}^{O M e}$

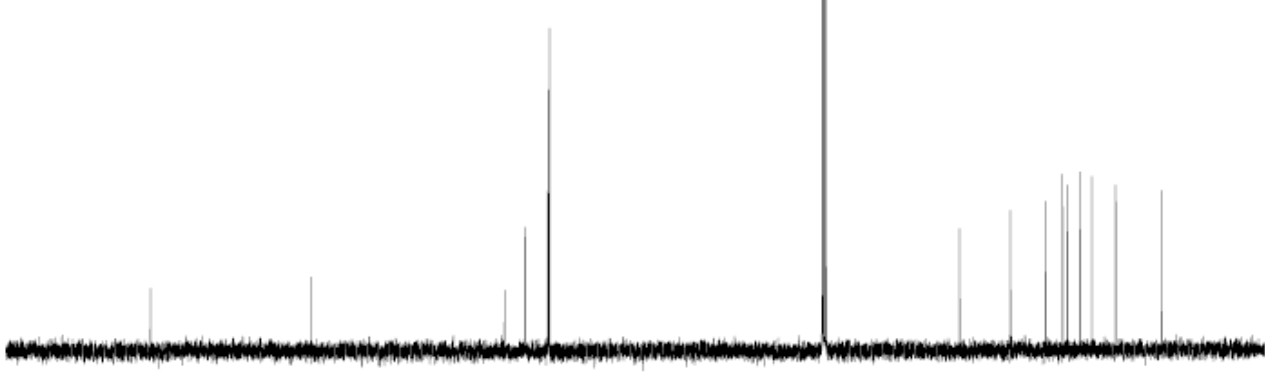

$\begin{array}{lllllllllllllllllllllll}220 & 210 & 200 & 190 & 180 & 170 & 160 & 150 & 140 & 130 & 120 & 110 & 100 & 90 & 80 & 70 & 60 & 50 & 40 & 30 & 20 & 10 & \mathrm{ppm}\end{array}$ 
TBO13081-5-H1

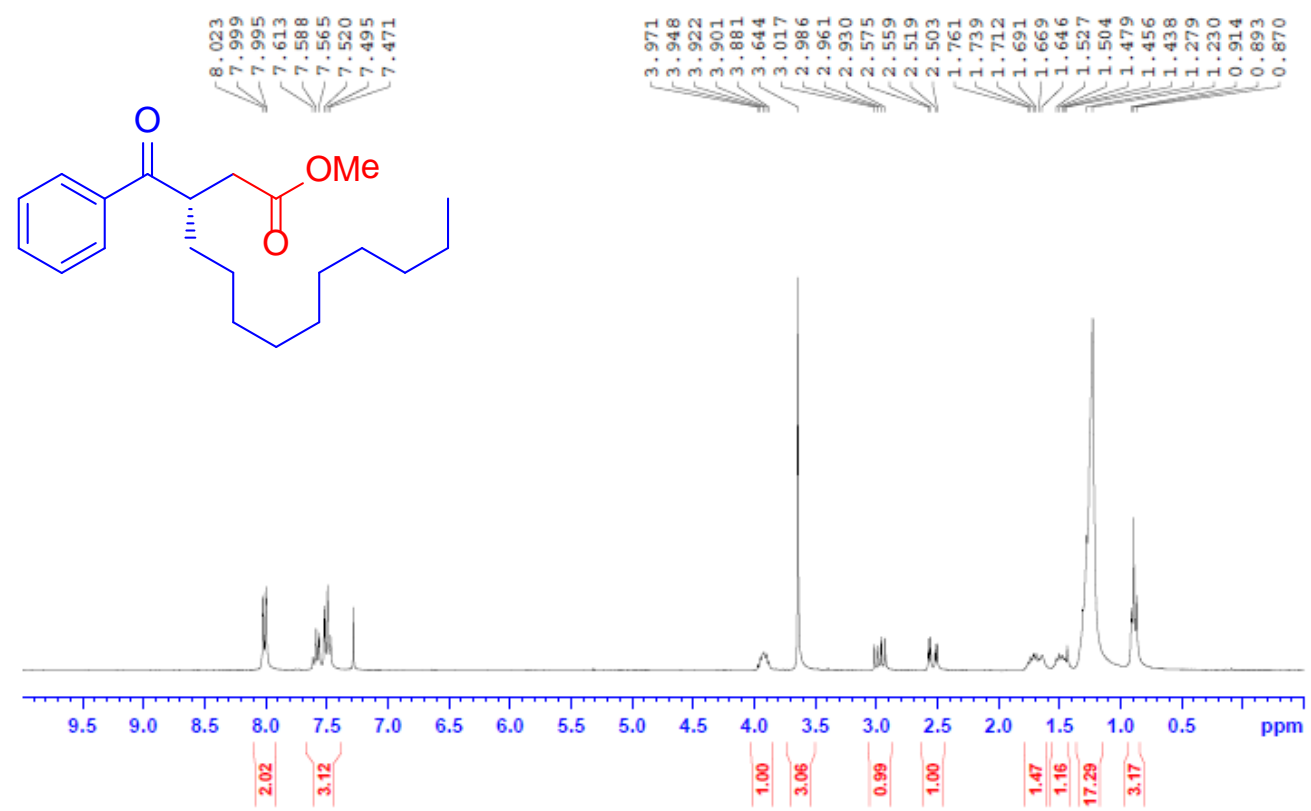

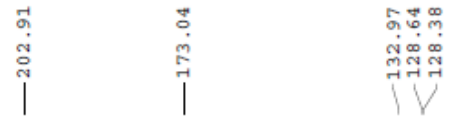<smiles>C=C(C[C@H](CCCCCCCCCC)C(=O)c1ccccc1)C(=O)OC</smiles> 


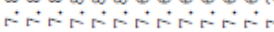

$\overbrace{(1)}^{O M M e}$

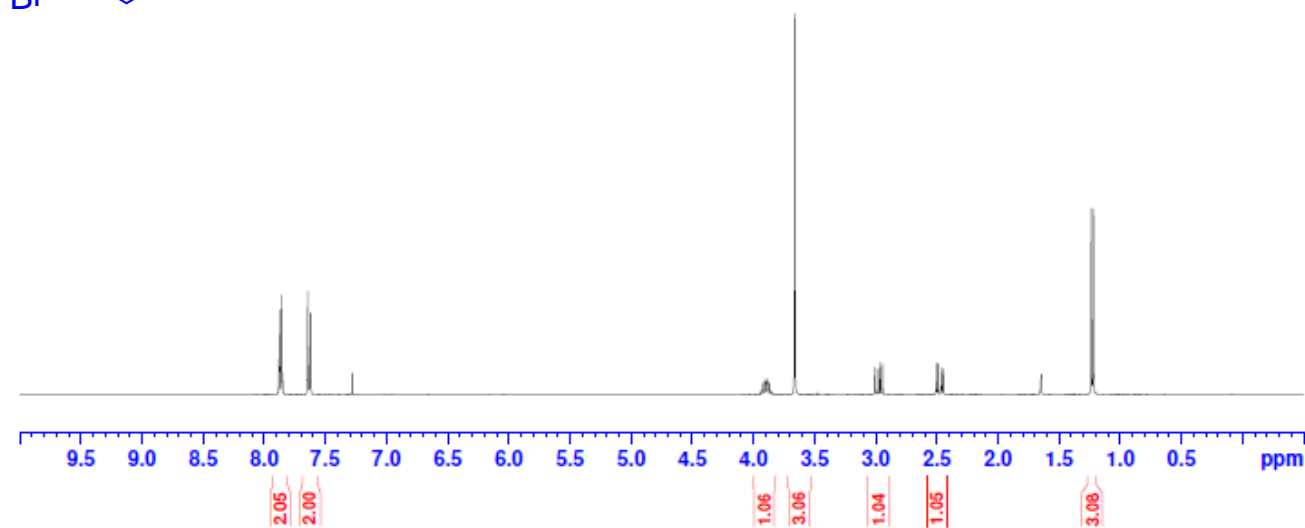

TB013081-7-C1

AV $400 \mathrm{CDC} 13$

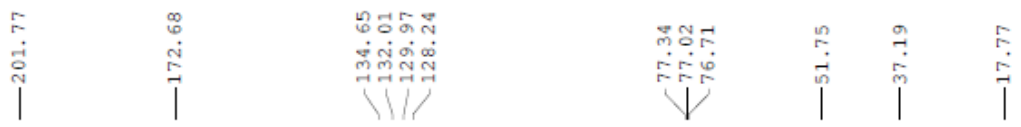<smiles>COC(=O)C[C@H](C)C(=O)c1ccc(Br)cc1</smiles>

$\mathrm{Br}$ 
TBO13081-3-H

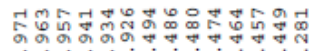

riscrivisis

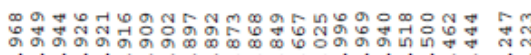

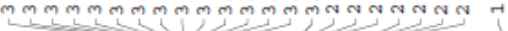<smiles>COC(=O)C[C@H](C)C(=O)c1ccc(Cl)cc1</smiles>

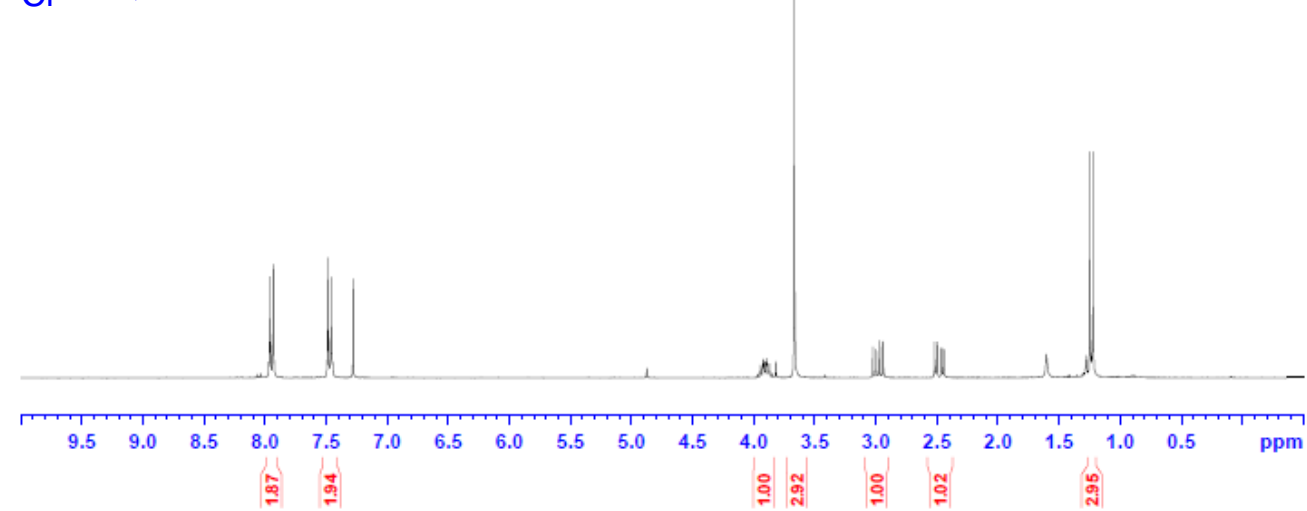

TB013081-3-C13
av300 CDCL 3

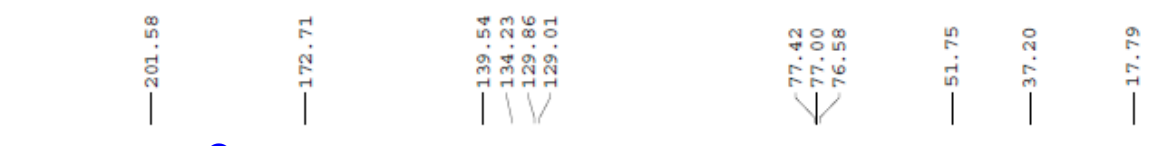<smiles>COC(=O)C[C@H](C)C(=O)c1ccc(Cl)cc1</smiles>

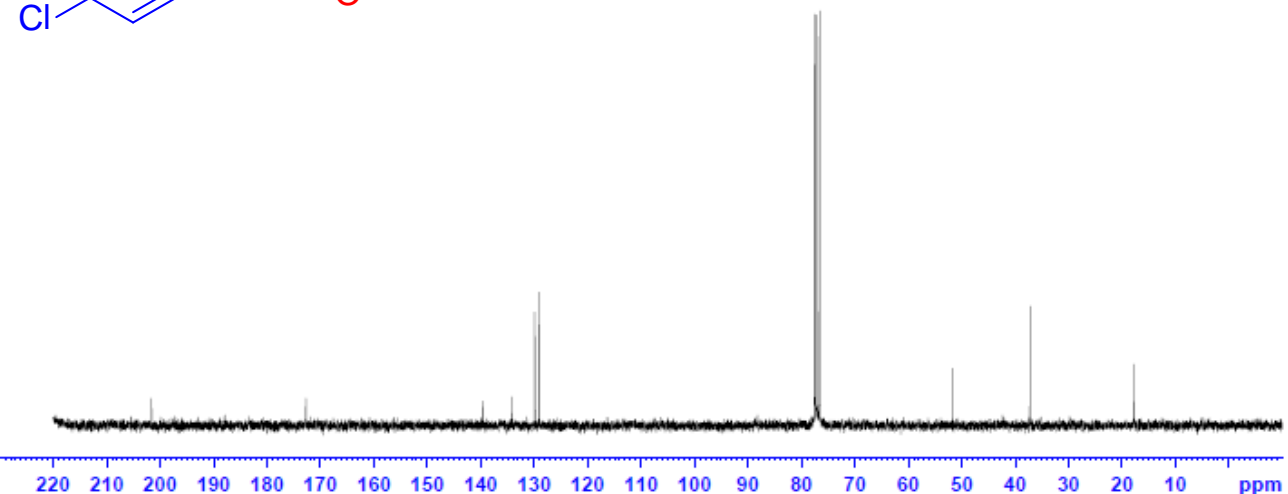


TBO13081-2-H1

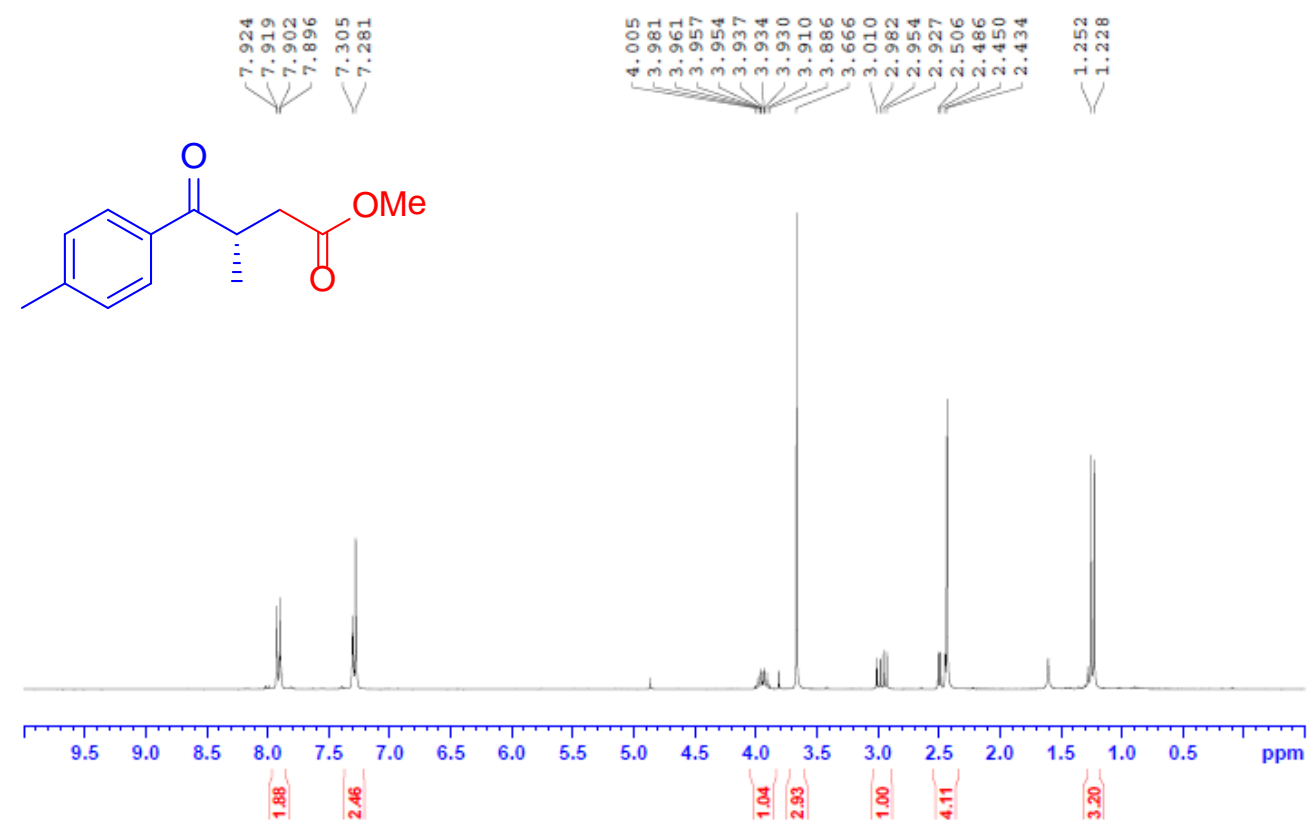

TBO $13081-2-\mathrm{C} 13$
av300 CDCL 3

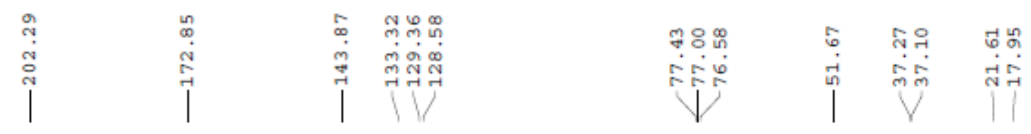<smiles>COC(=O)C[C@H](C)C(=O)c1ccc(C)cc1</smiles> 


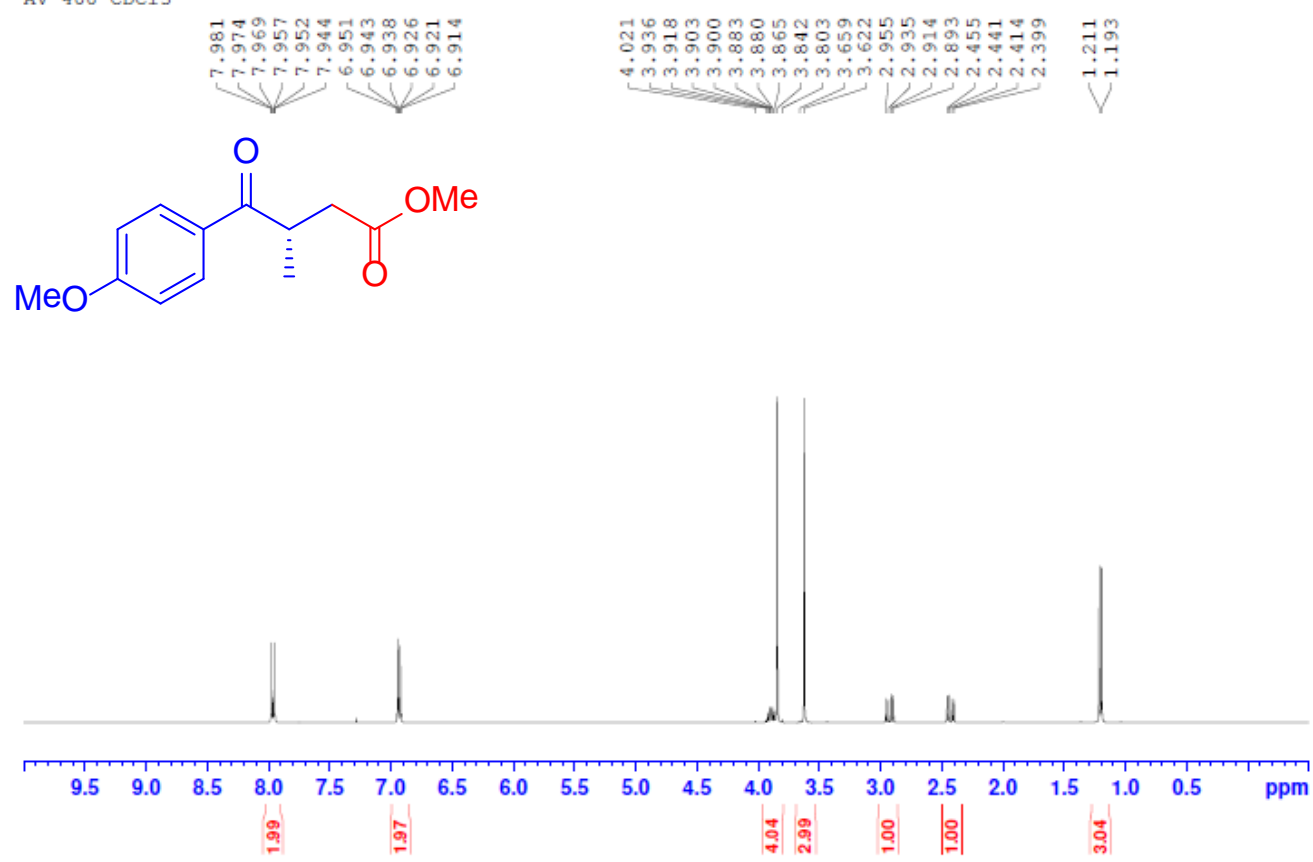

TB013081-4-C1

AV $400 \mathrm{CDC13}$

|<smiles>COC(=O)C[C@H](C)C(=O)c1ccc(OC)cc1</smiles>

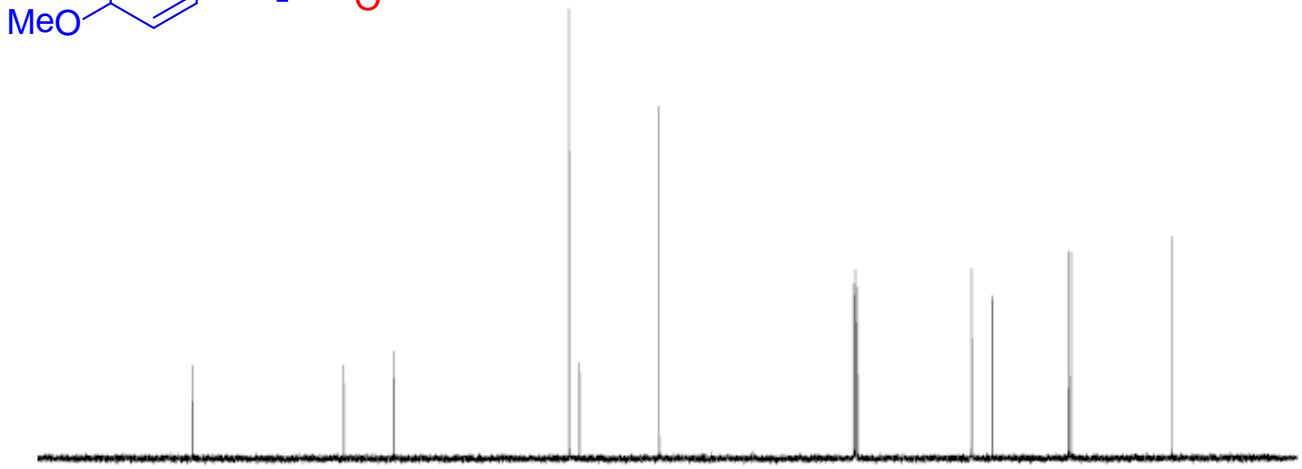

$\begin{array}{lllllllllllllllllllllll}220 & 210 & 200 & 190 & 180 & 170 & 160 & 150 & 140 & 130 & 120 & 110 & 100 & 90 & 80 & 70 & 60 & 50 & 40 & 30 & 20 & 10 & \mathrm{ppm}\end{array}$ 
TB013088-1-H1

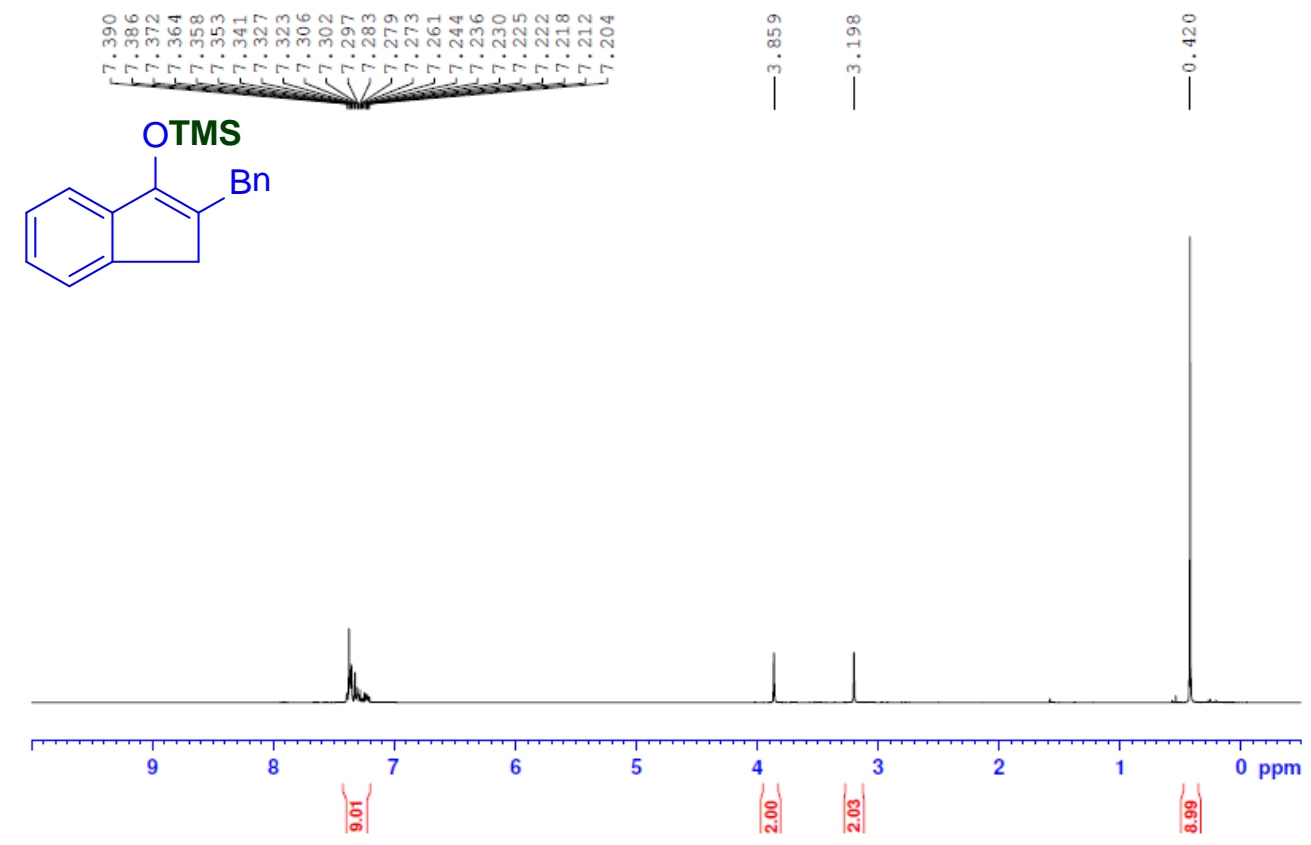

TB013088-1-C13
AV 400 CDC13
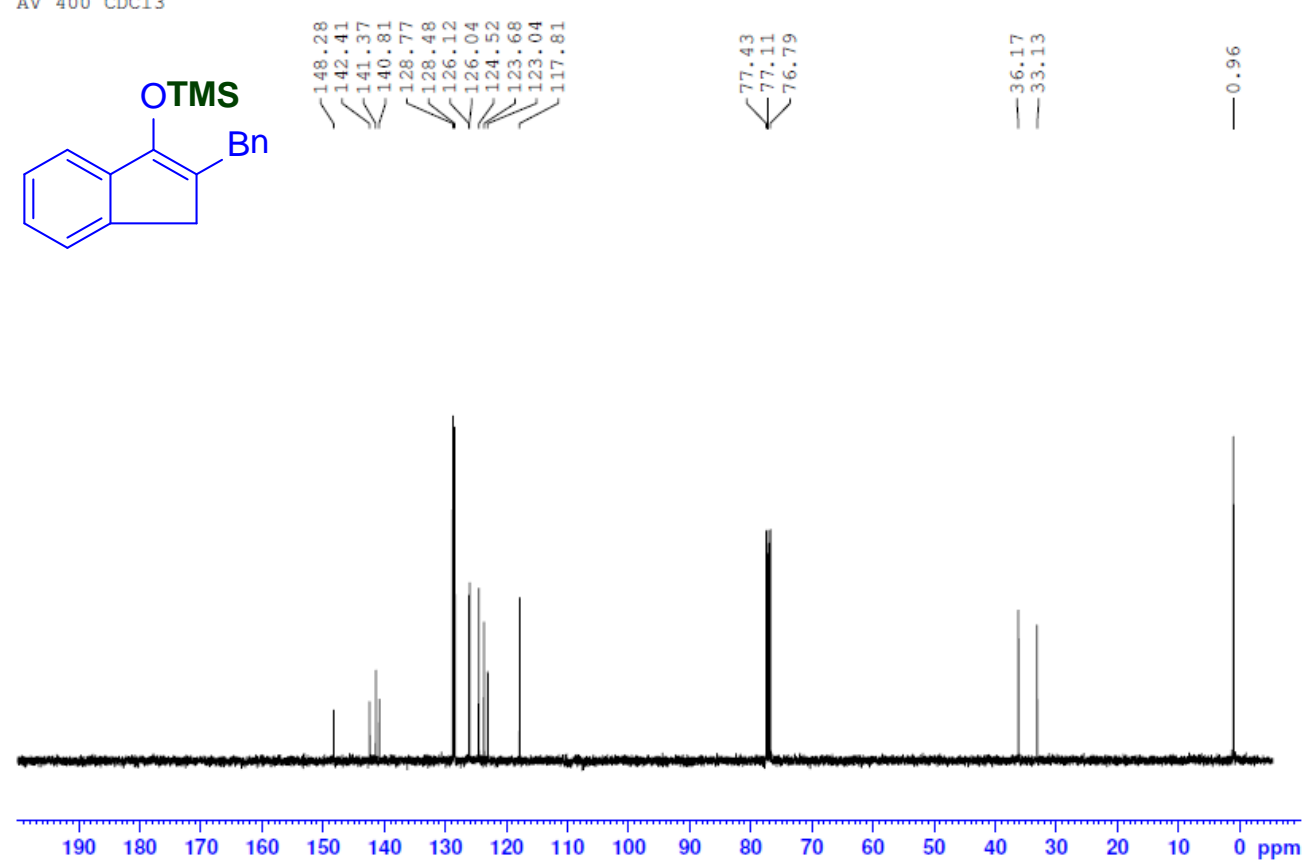
TB013088-2-H1

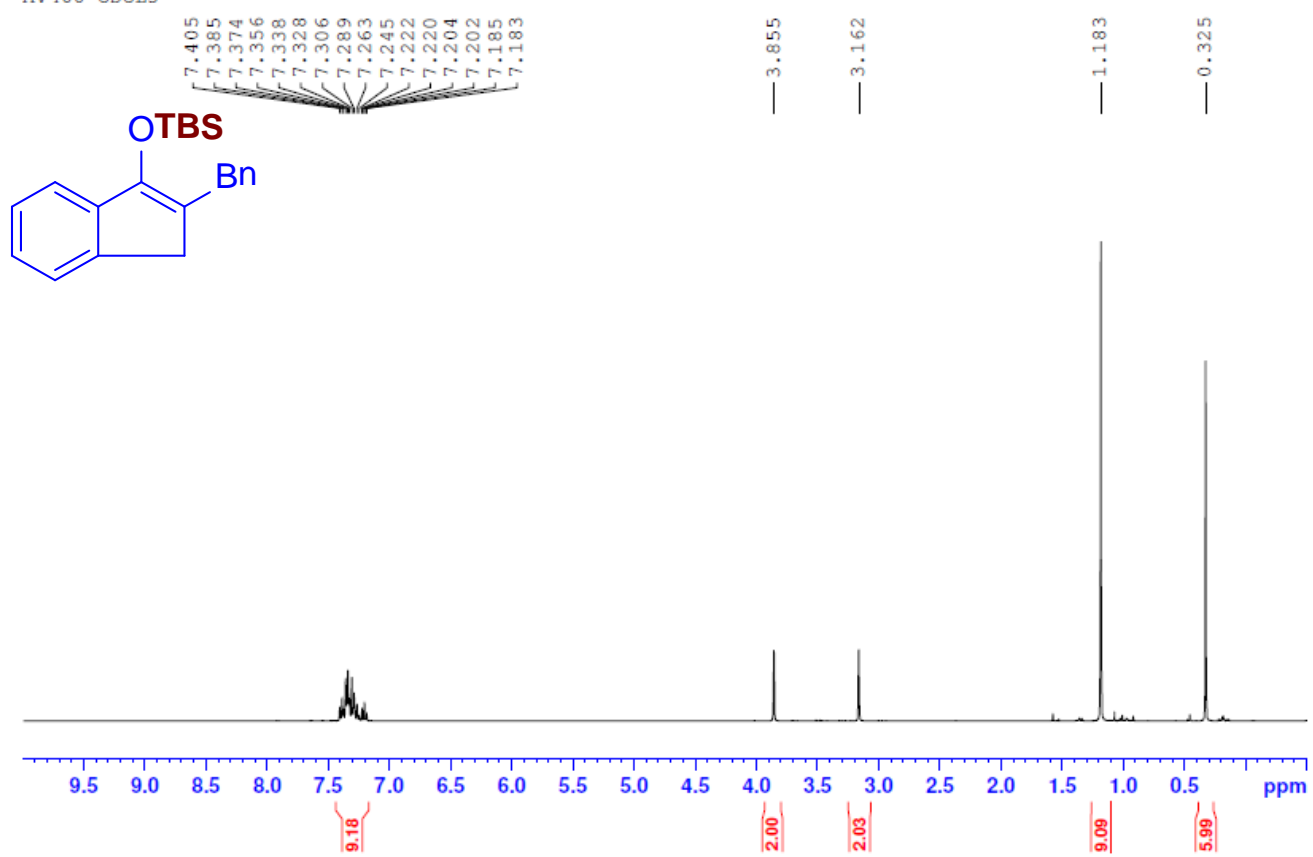

TB013088-2-C13

AV 400 CDCL 3
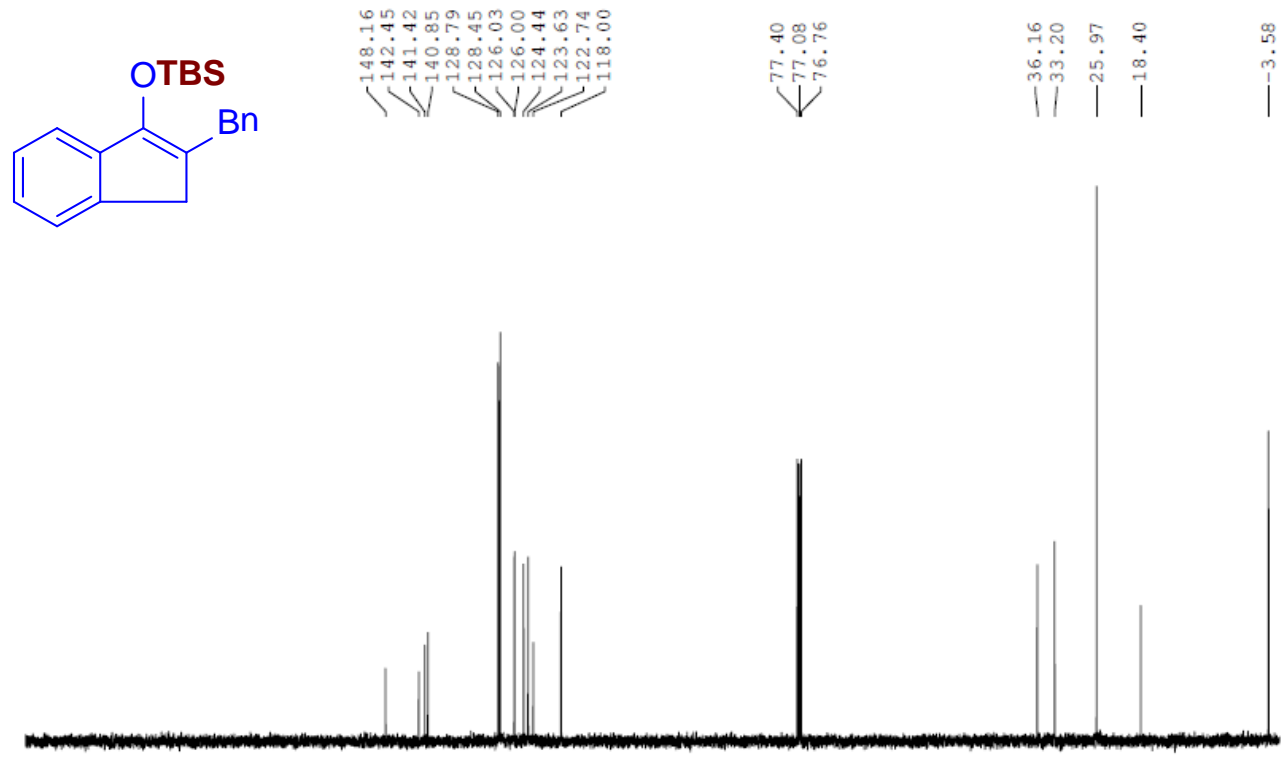

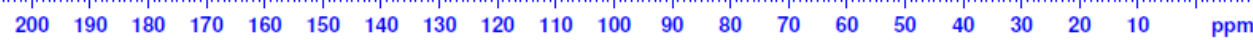




\section{X: HPLC Spectra of Products}<smiles>O=C1Oc2ccccc2CC1(Br)C/C=C/c1ccccc1</smiles>

96\% ee (OD-H, 90/10 hexanes/i-PrOH, 1.0 ml/min)

mAU
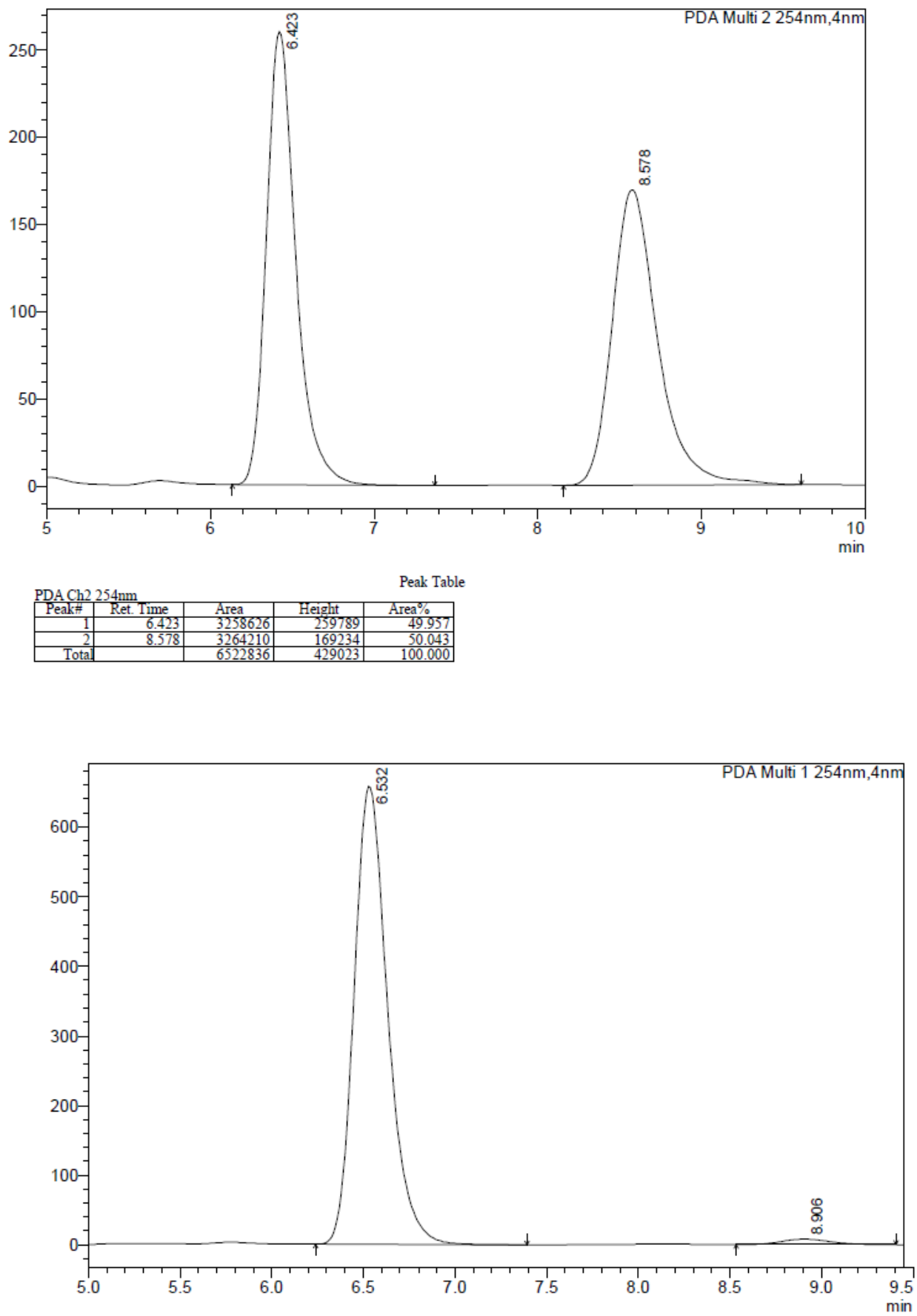

Peak Table

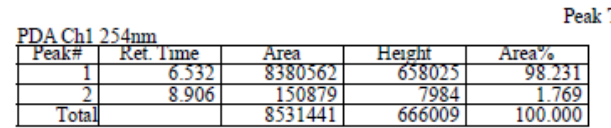


<smiles>C=CC[C@]1(Br)Cc2ccccc2OC1=O</smiles>

85\% ee (OJ-H, 95/5 hexanes/i-PrOH, 1.0 ml/min)

$\mathrm{mAU}$

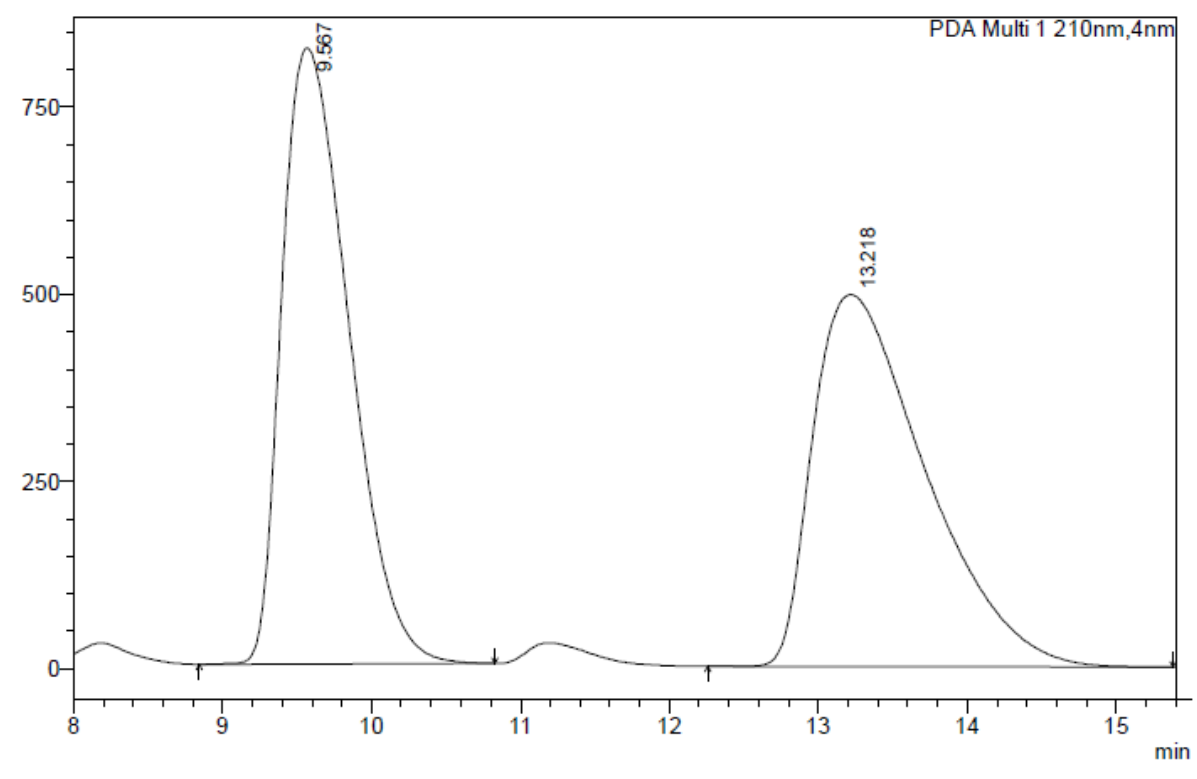

Peak Table

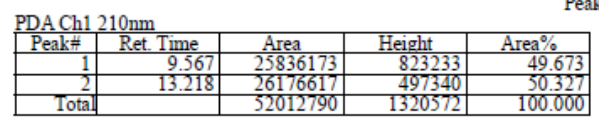

mAU

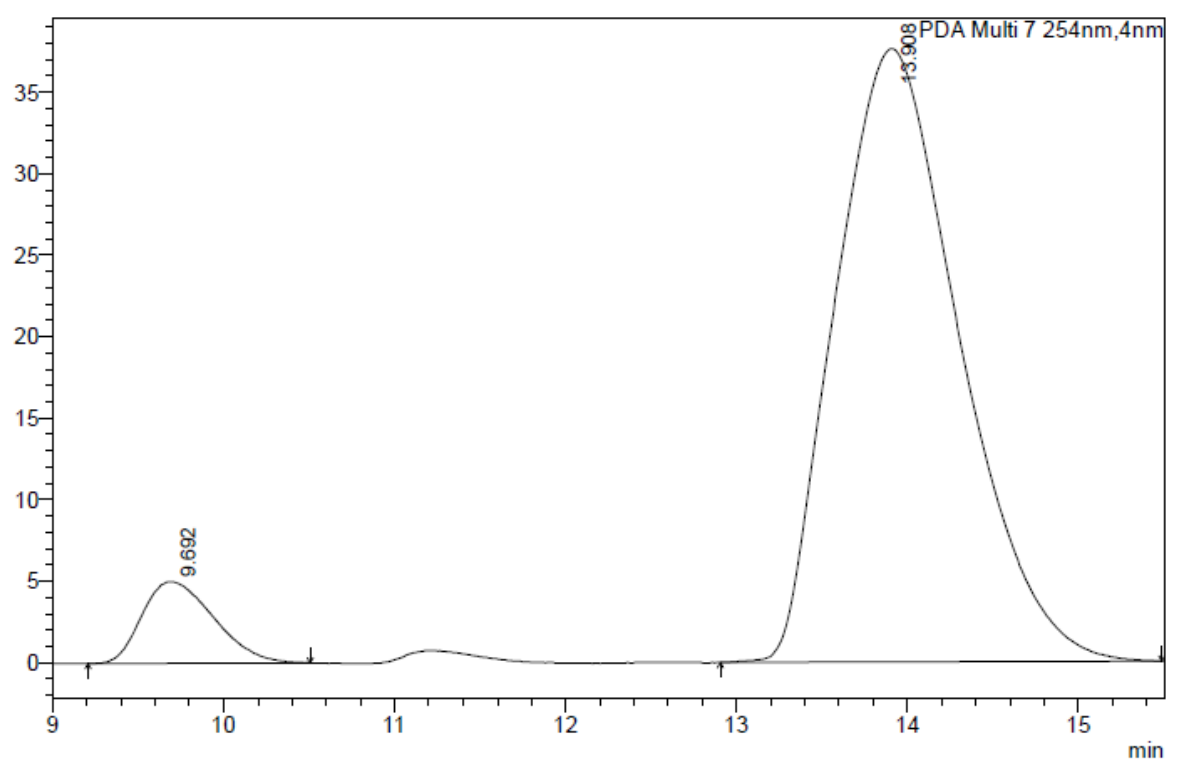

Peak Table

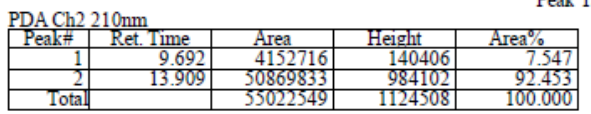


<smiles>CC(C)=CC[C@]1(Br)Cc2ccccc2OC1=O</smiles>

93\% ee (AD-H, 99/1 hexanes/i-PrOH, 1.0 ml/min)

mAU

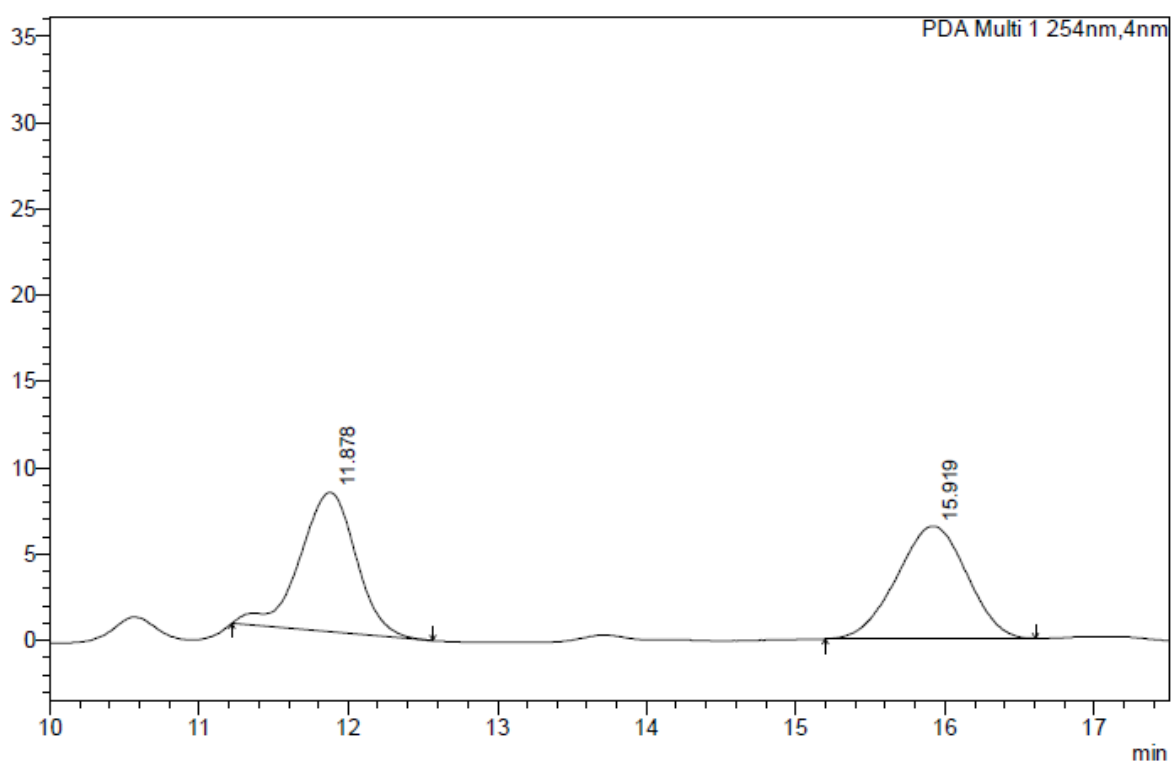

Peak Table

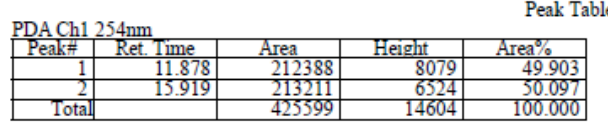

mAU
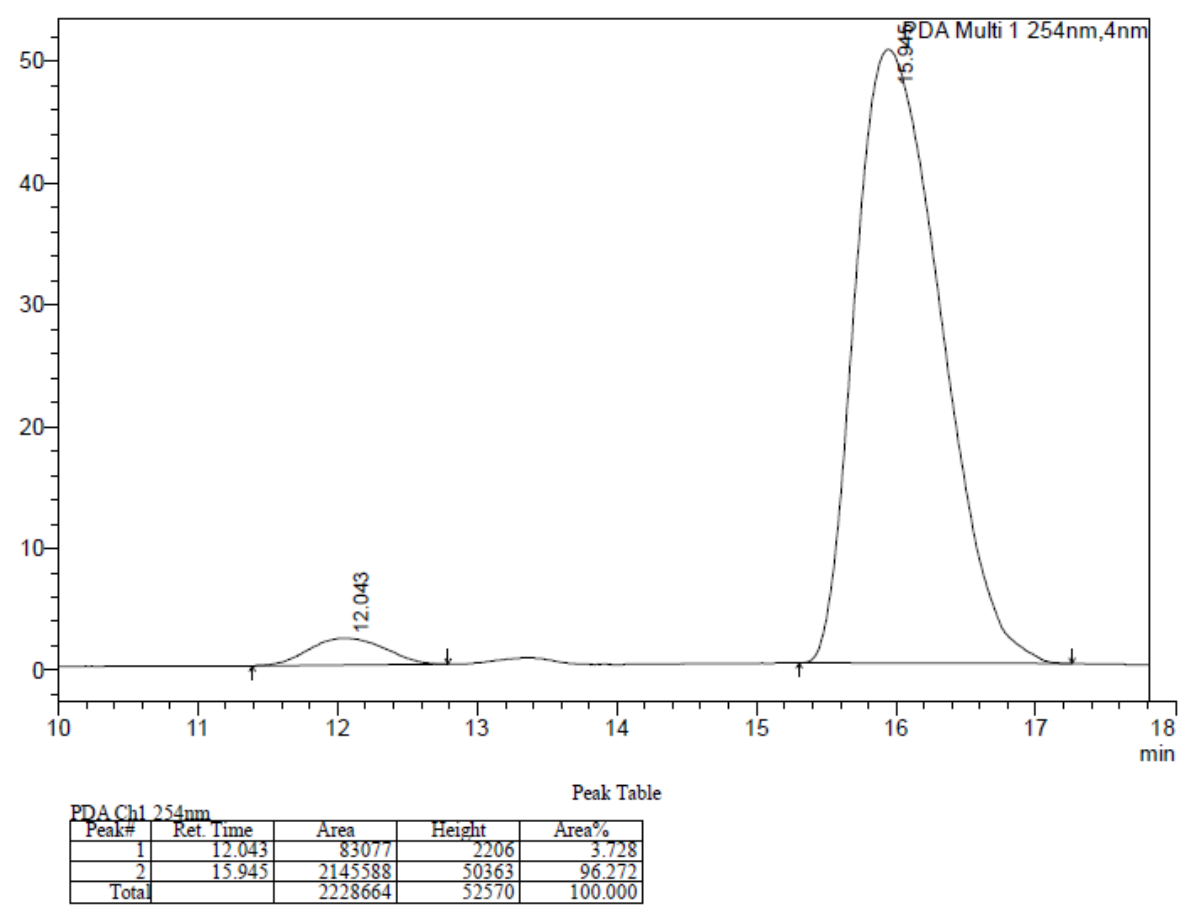


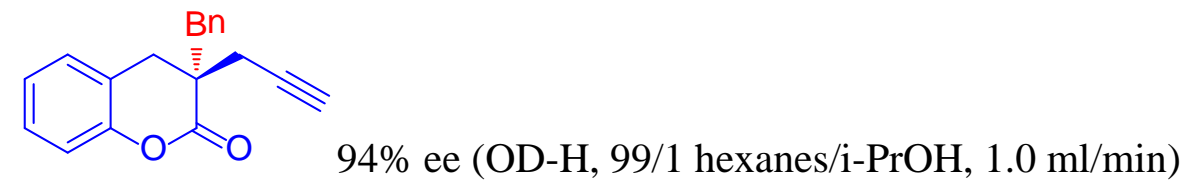

mAU
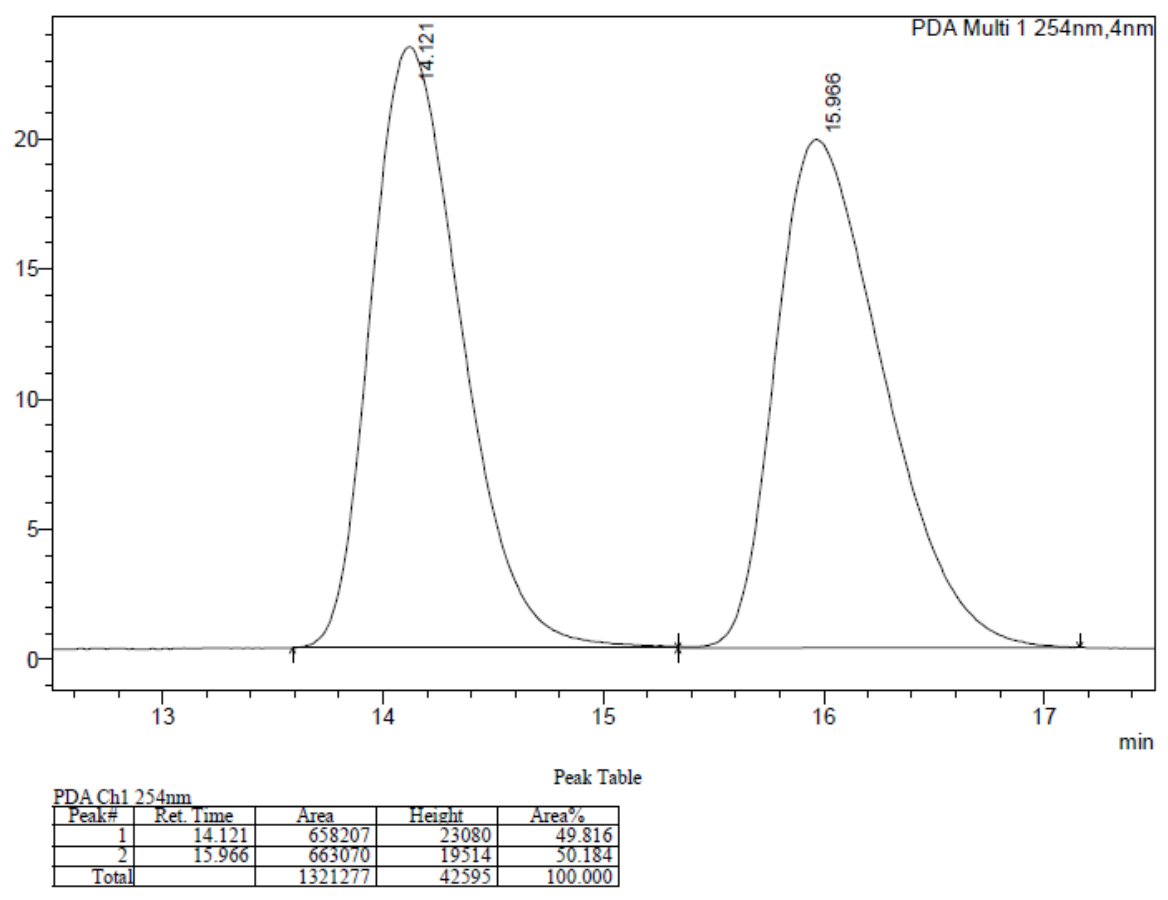

mAU
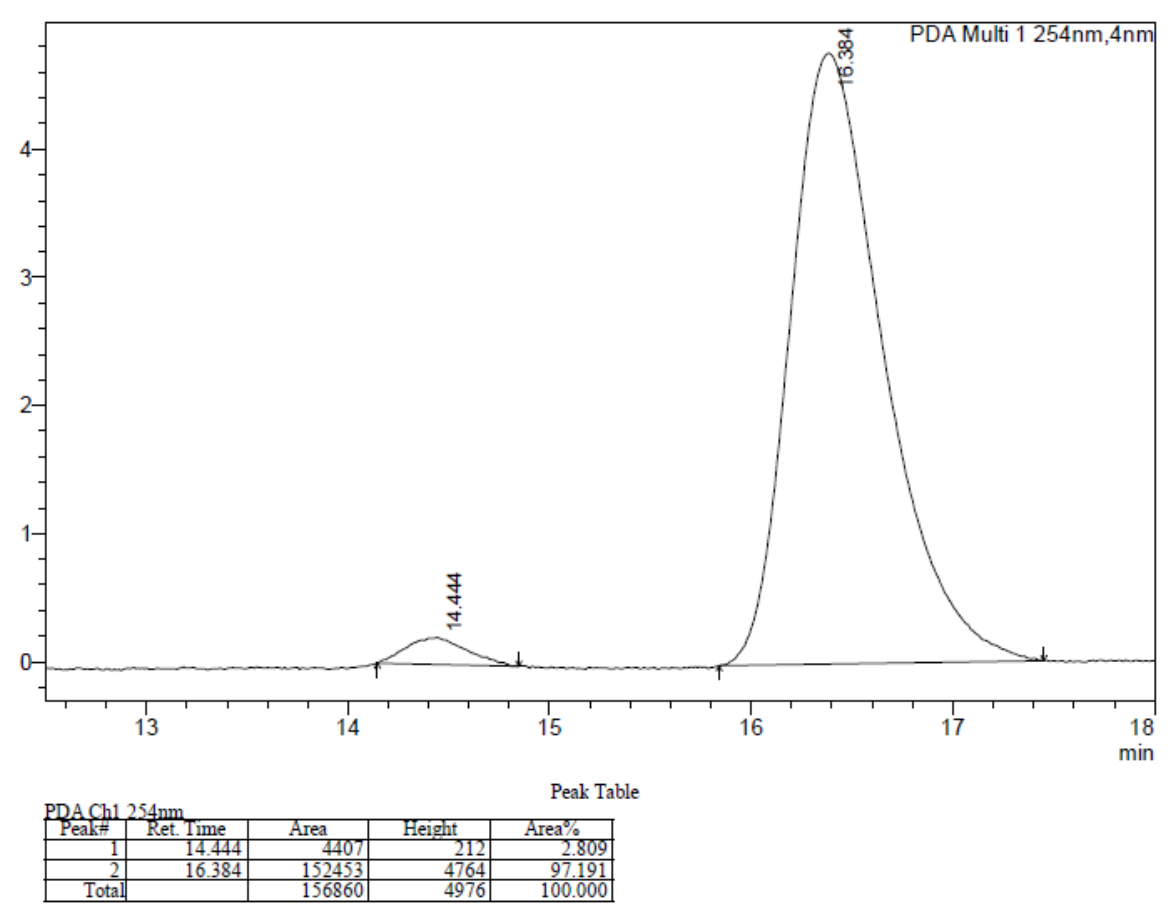


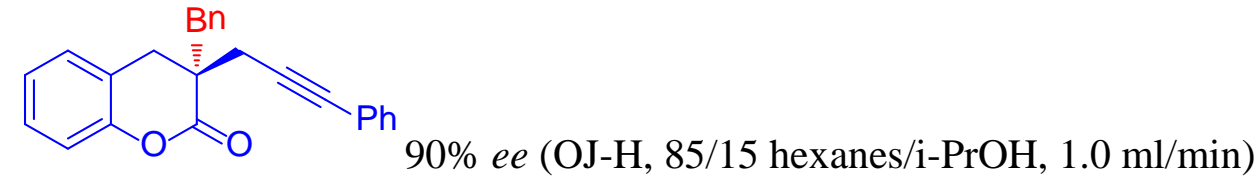

mAU
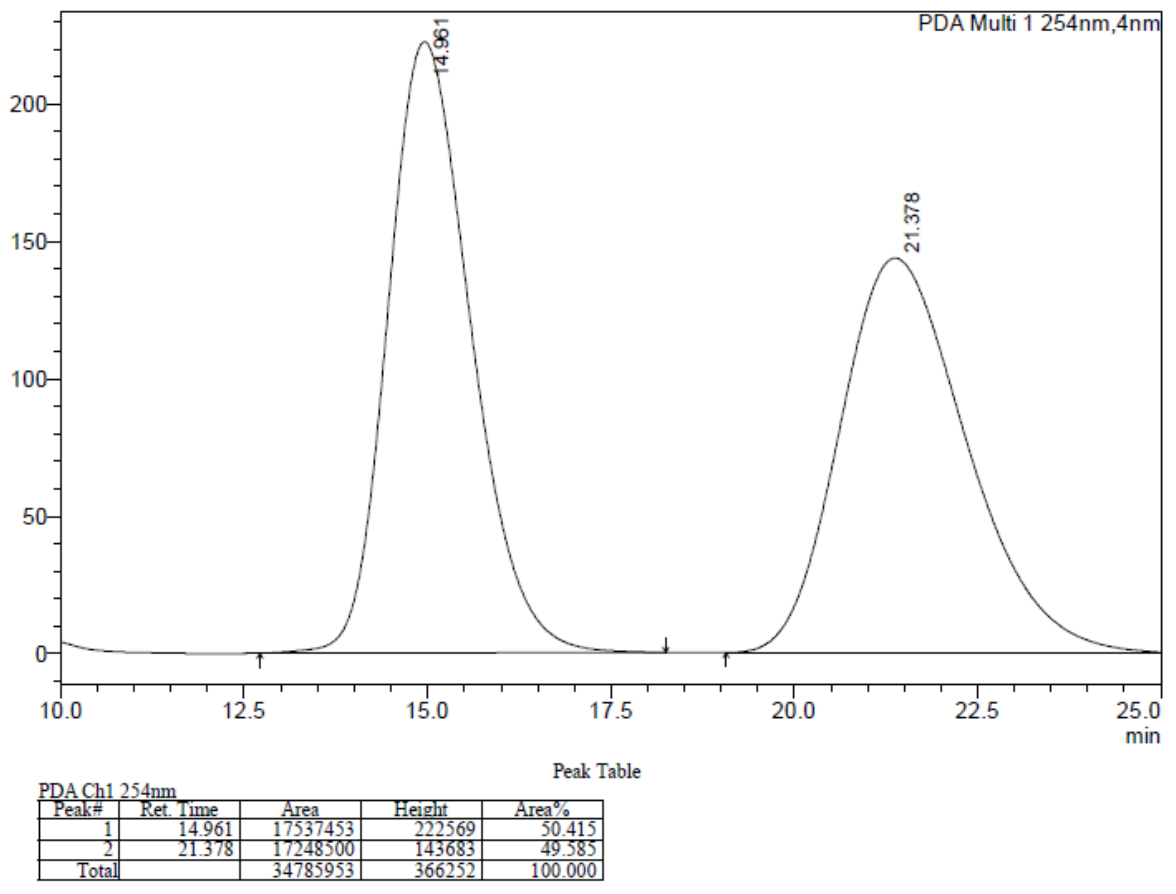

mAU
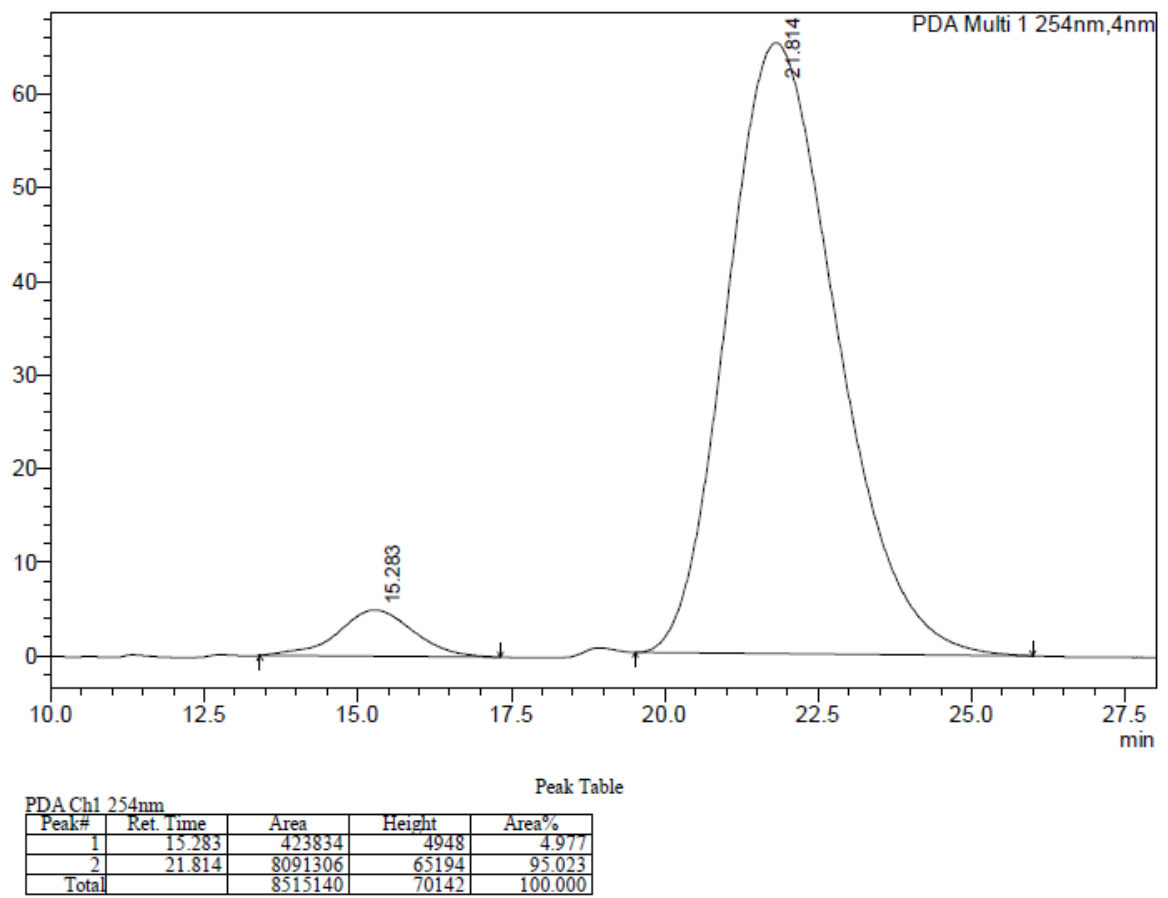

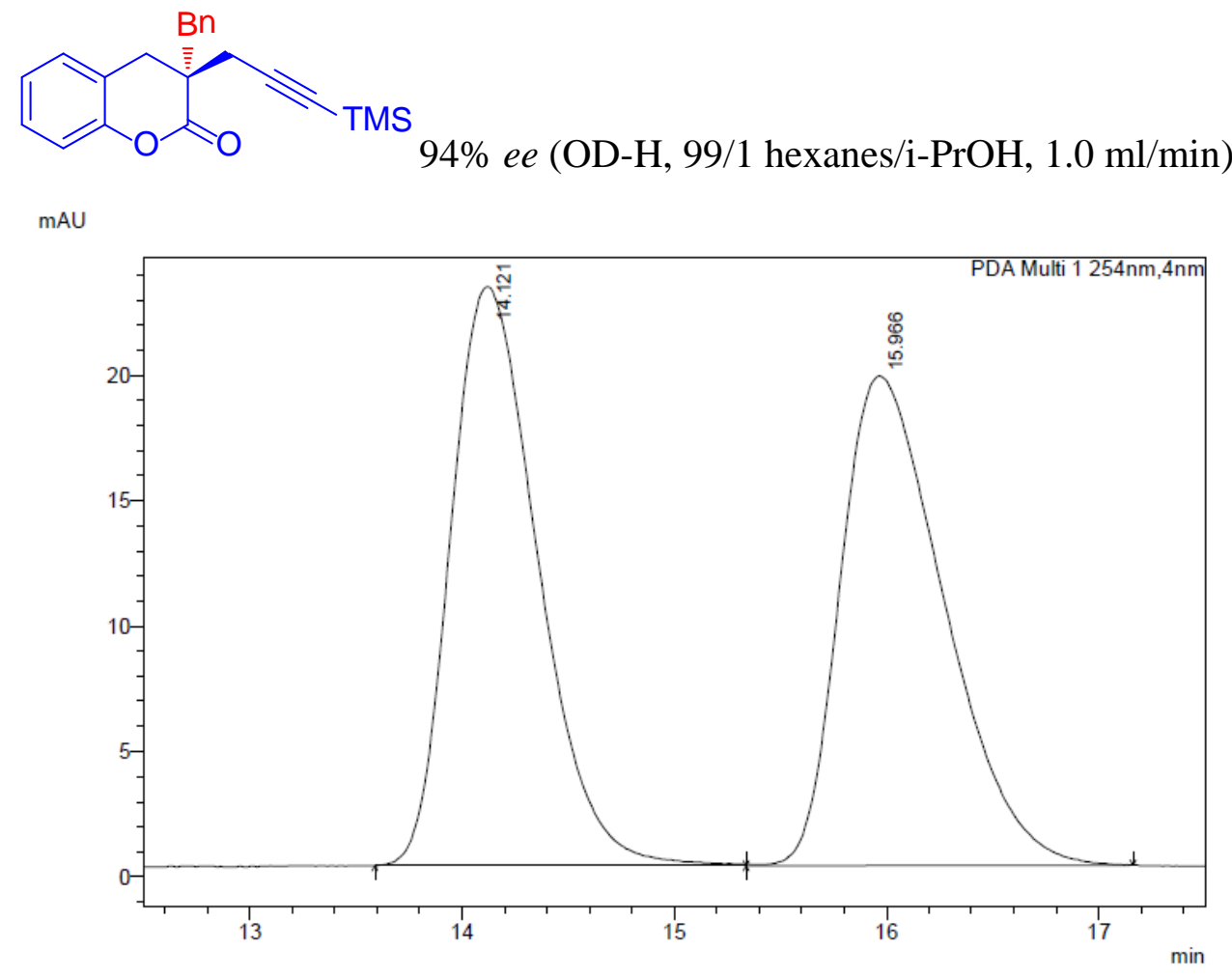

Peak Table

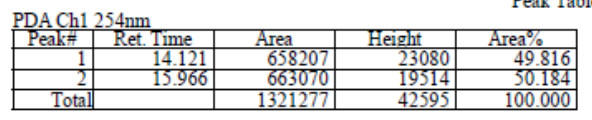

mAU
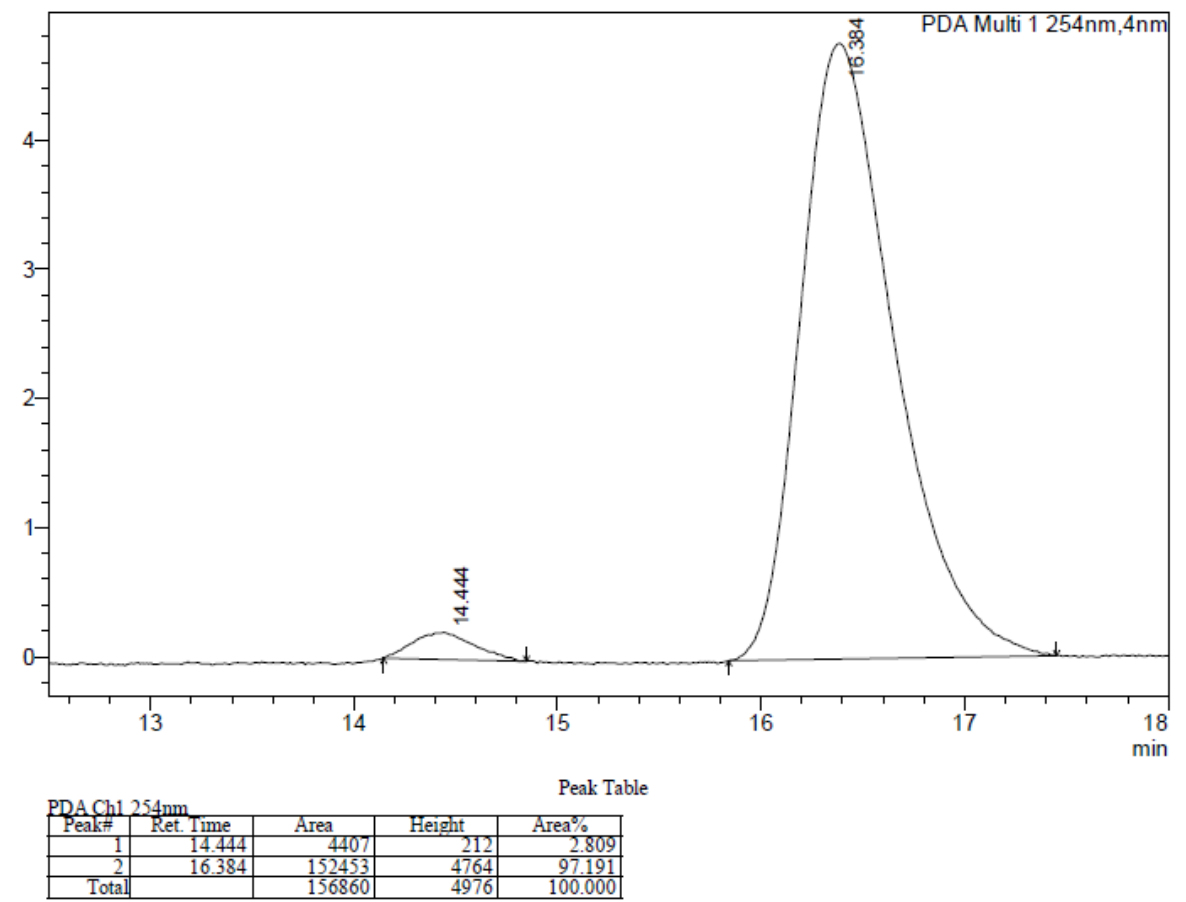
<smiles>CC(C)=CCC/C(C)=C/C[C@]1(Cc2ccccc2)Cc2ccccc2OC1=O</smiles>

93\% eе (AD-H, 99/1 hexanes/i-PrOH, 1.0 ml/min)

$\mathrm{mAU}$

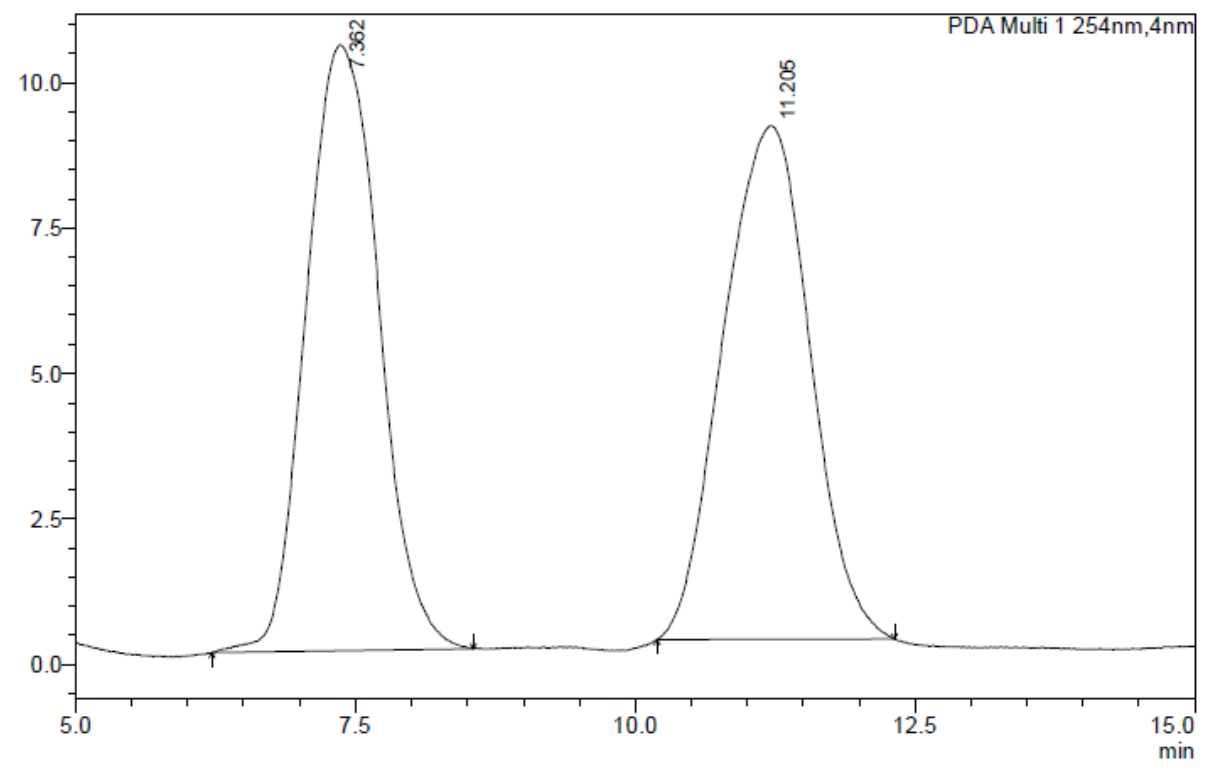

Peak Table

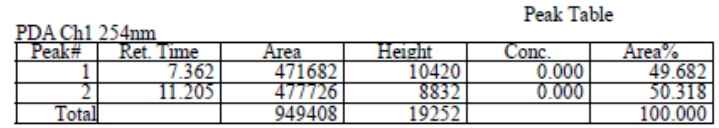

mAU

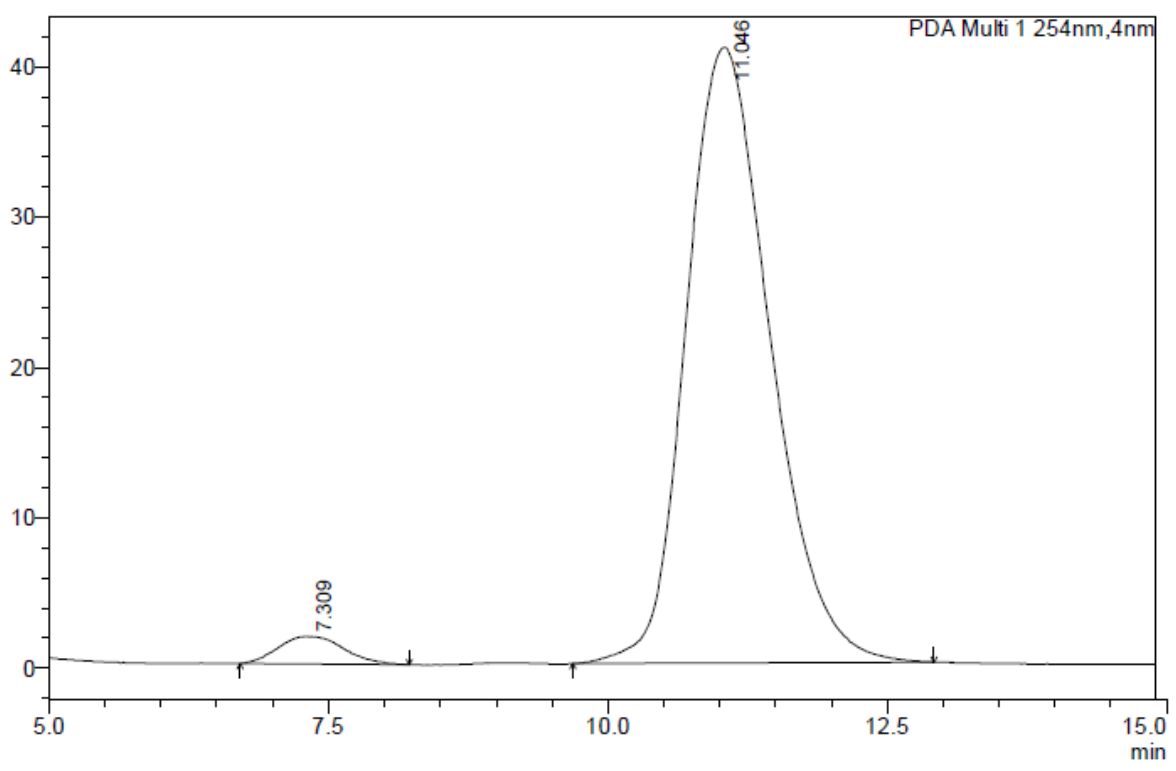

Peak Table

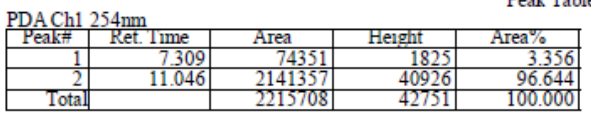




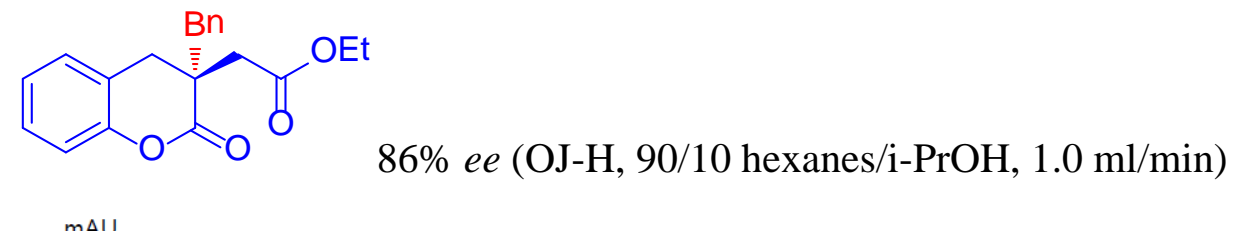

$\mathrm{mAU}$

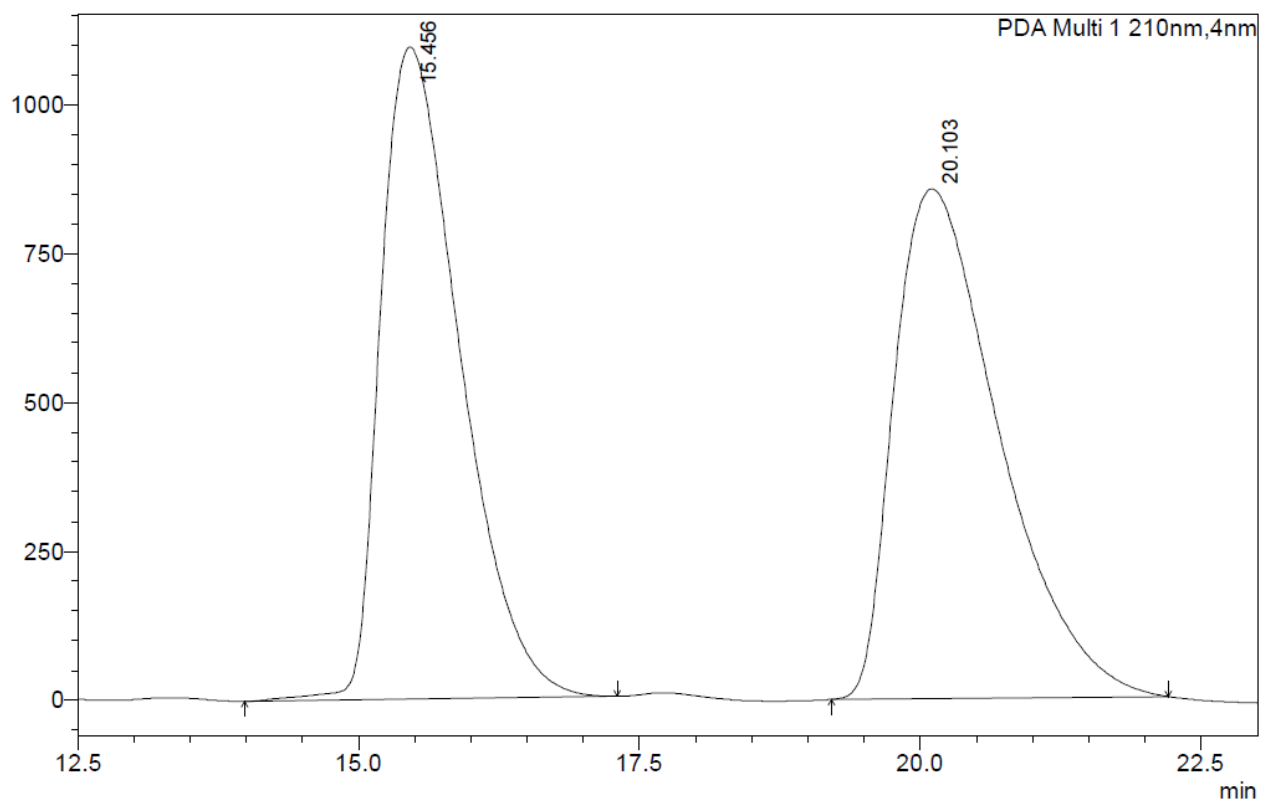

Peak Table

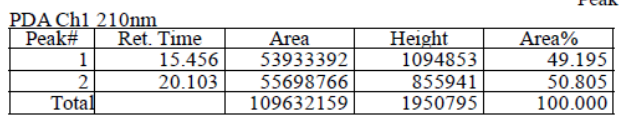

mAU

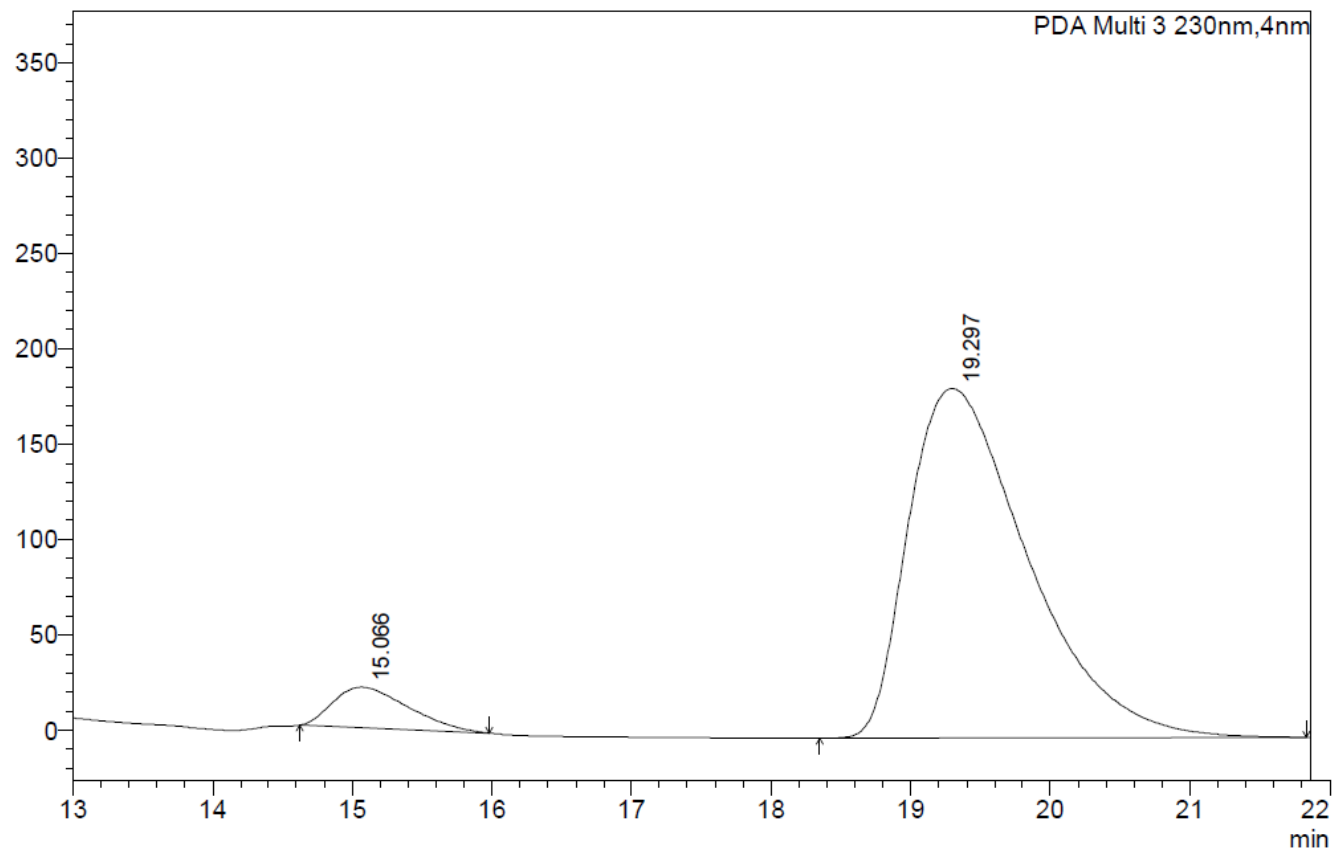

Peak Table

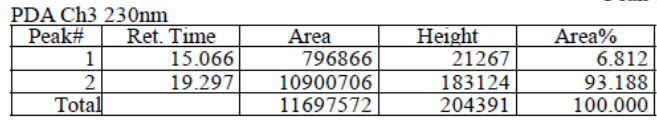




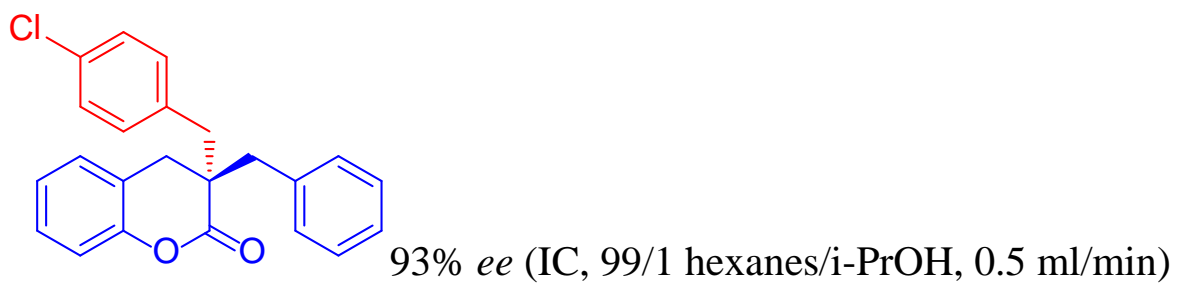

mAU
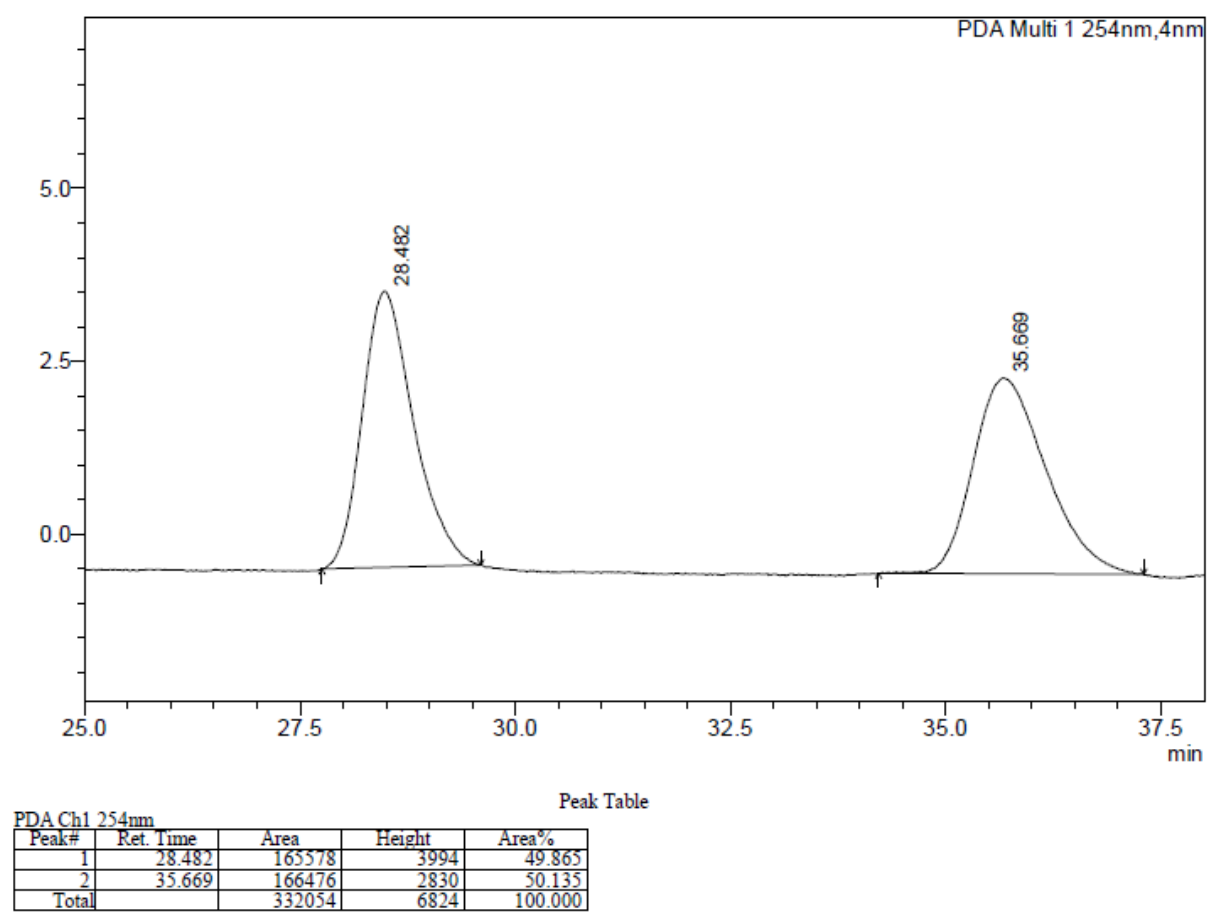

$\mathrm{mAU}$
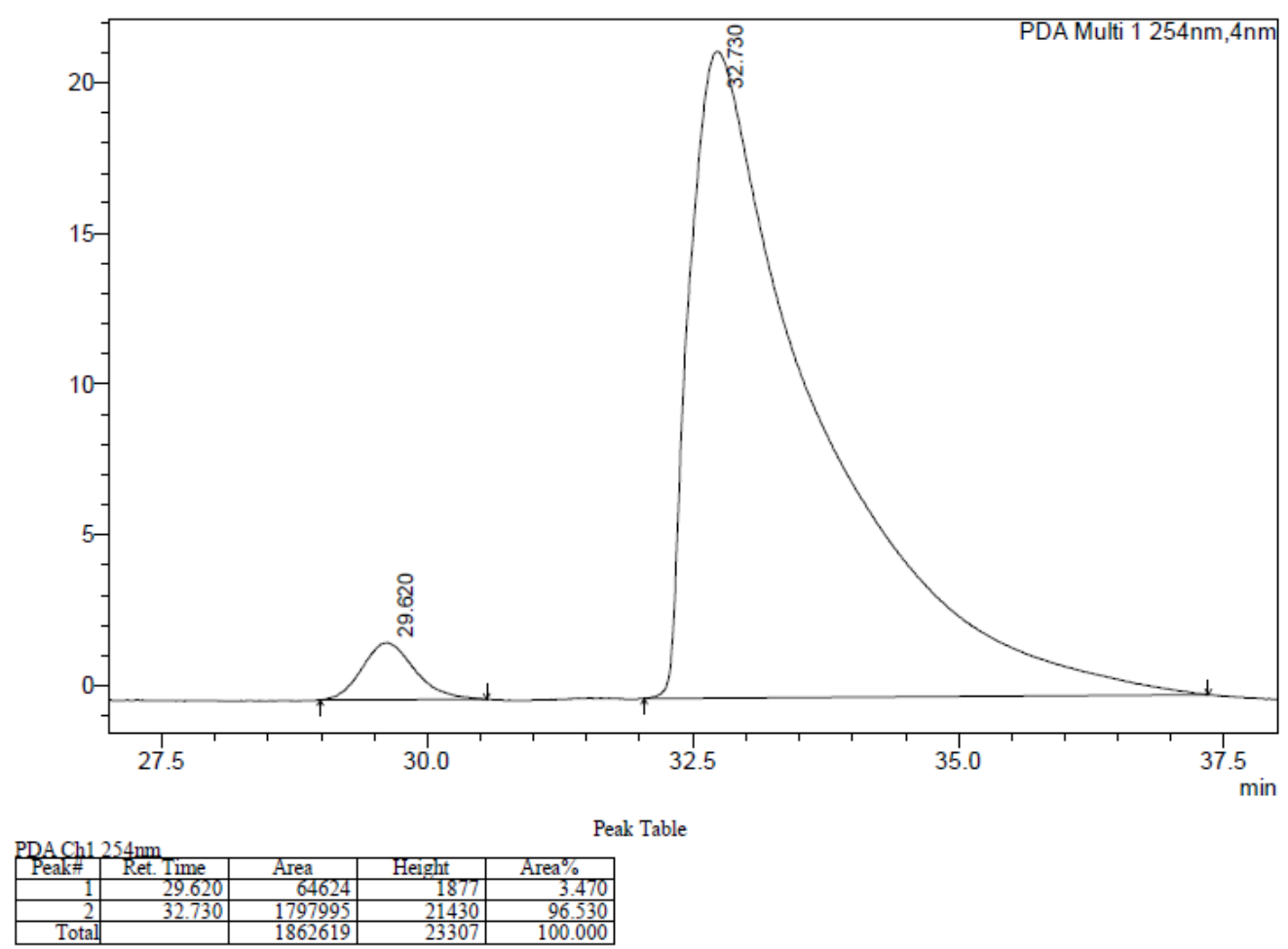


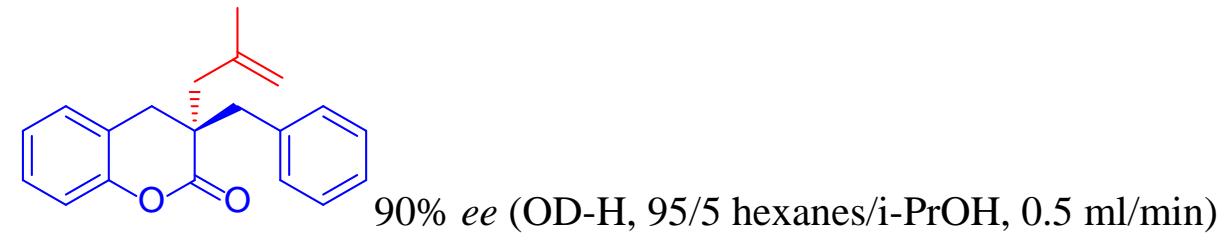

mAU

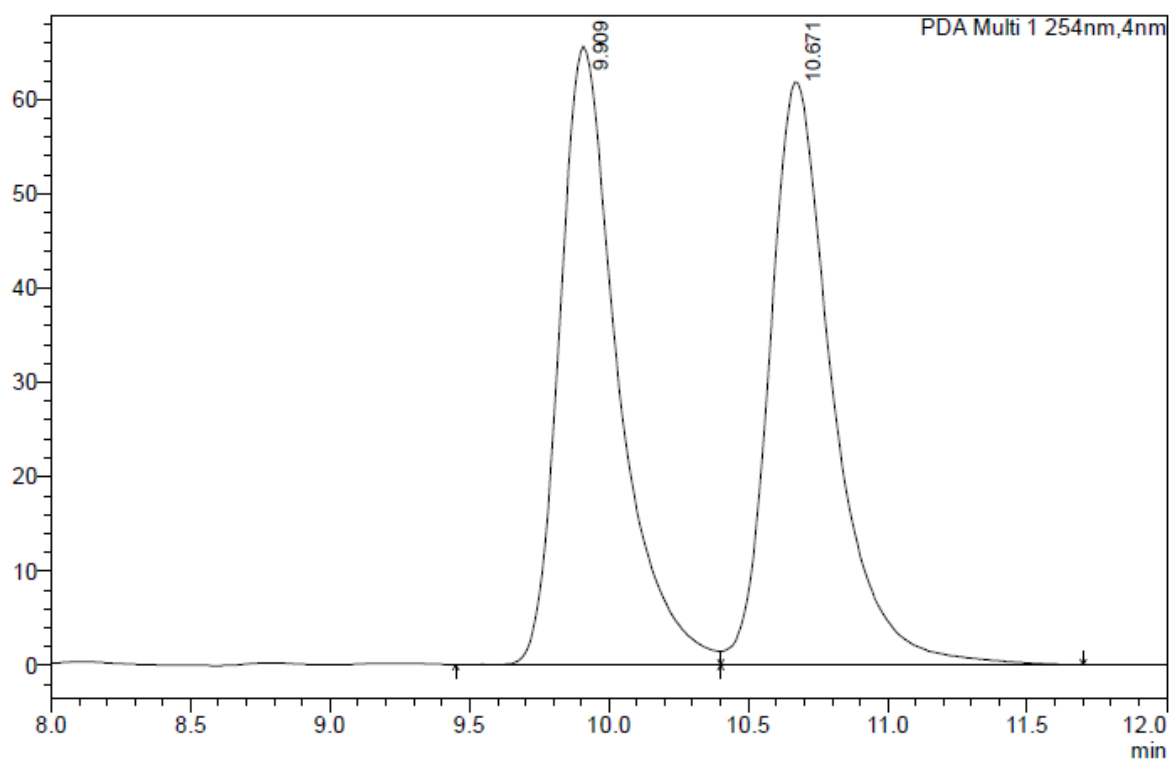

Peak Table

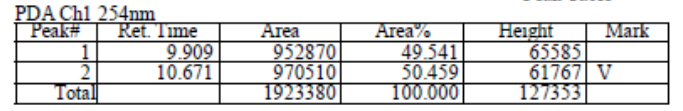

mAU

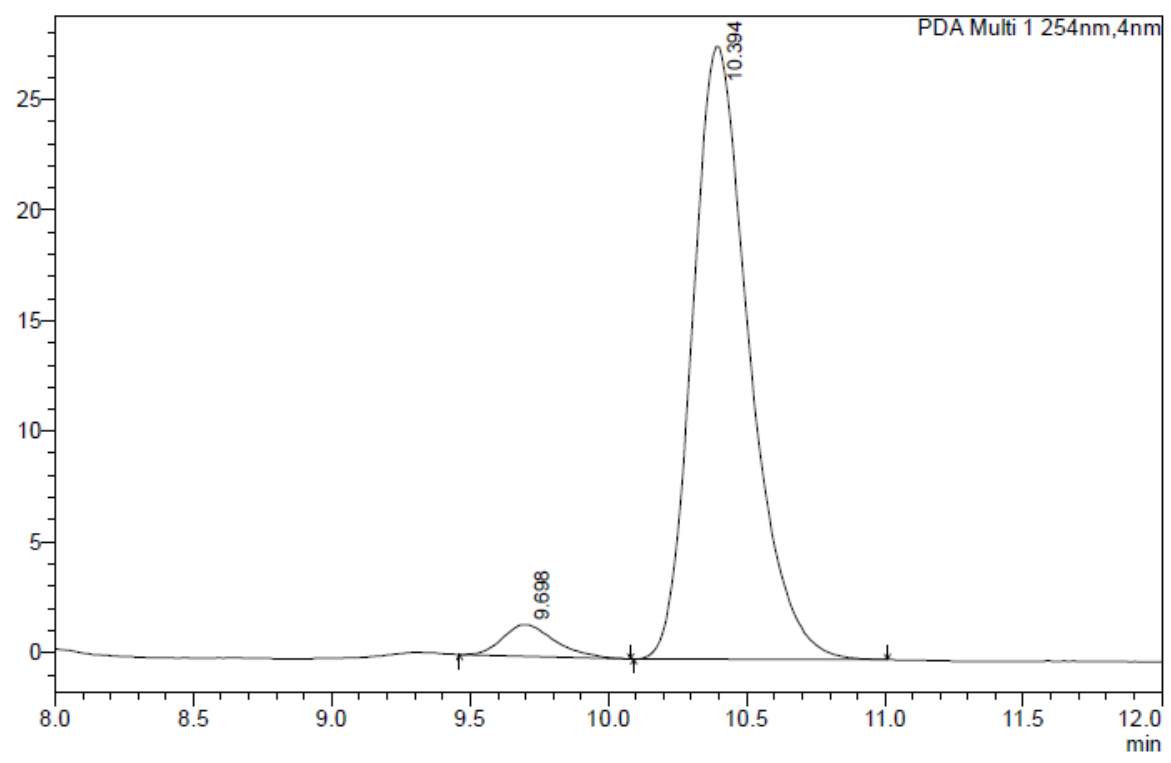

Peak Table

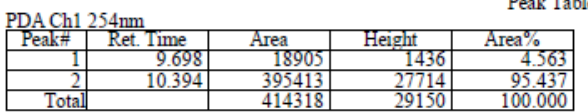




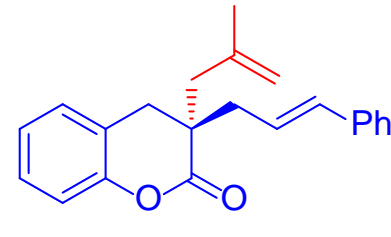

89\% ee (OD-H, 90/10 hexanes/i-PrOH, 0.5 ml/min)

mAU

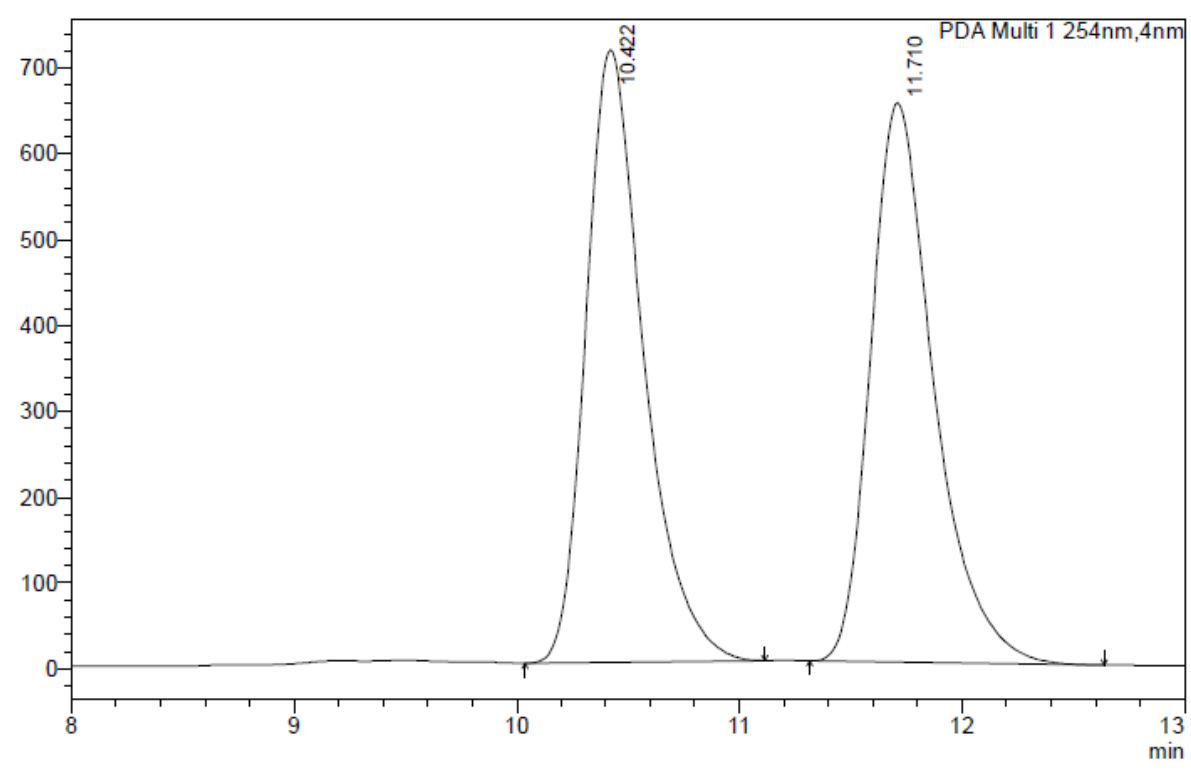

Peak Table

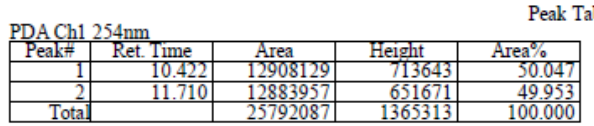

mAU

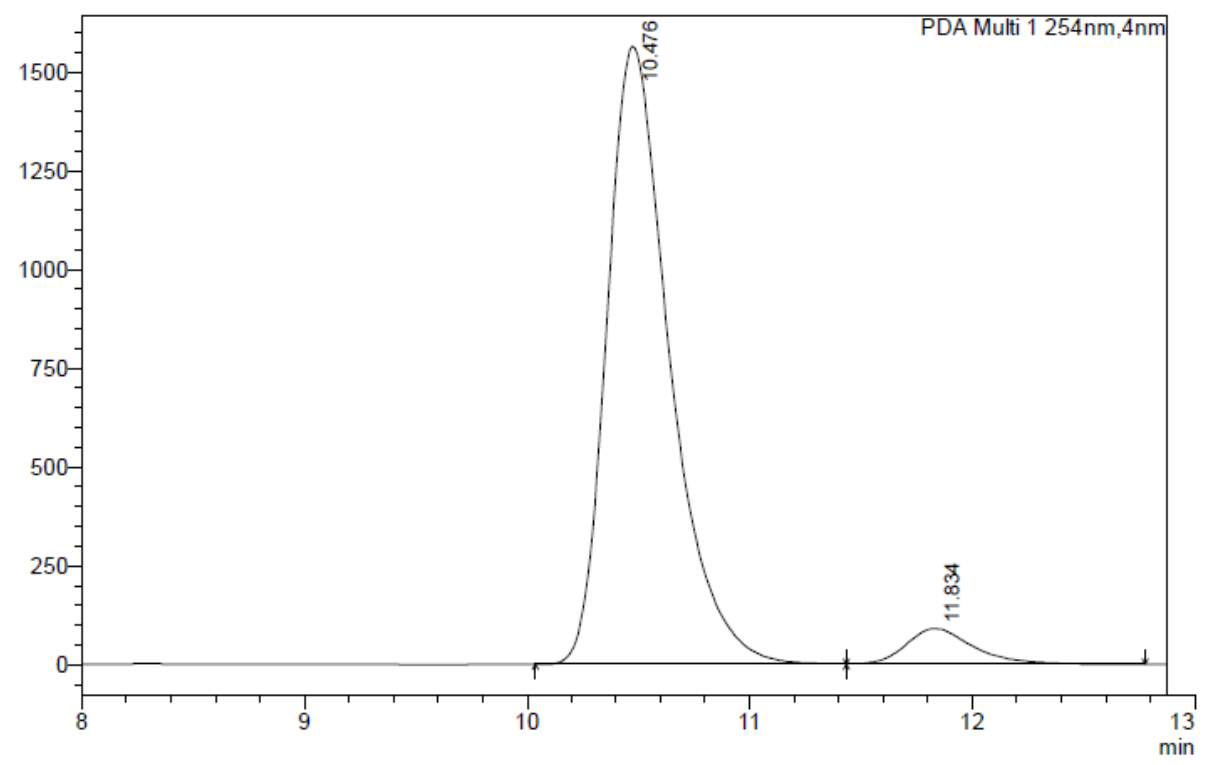

Peak Table

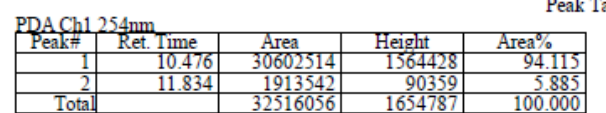


<smiles>C/C(=C\c1ccccc1)C[C@]1(/C=C/P)Cc2ccccc2OC1=O</smiles>

91\% ee (OD-H, 95/5 hexanes/i-PrOH, 0.5 ml/min)

mAL

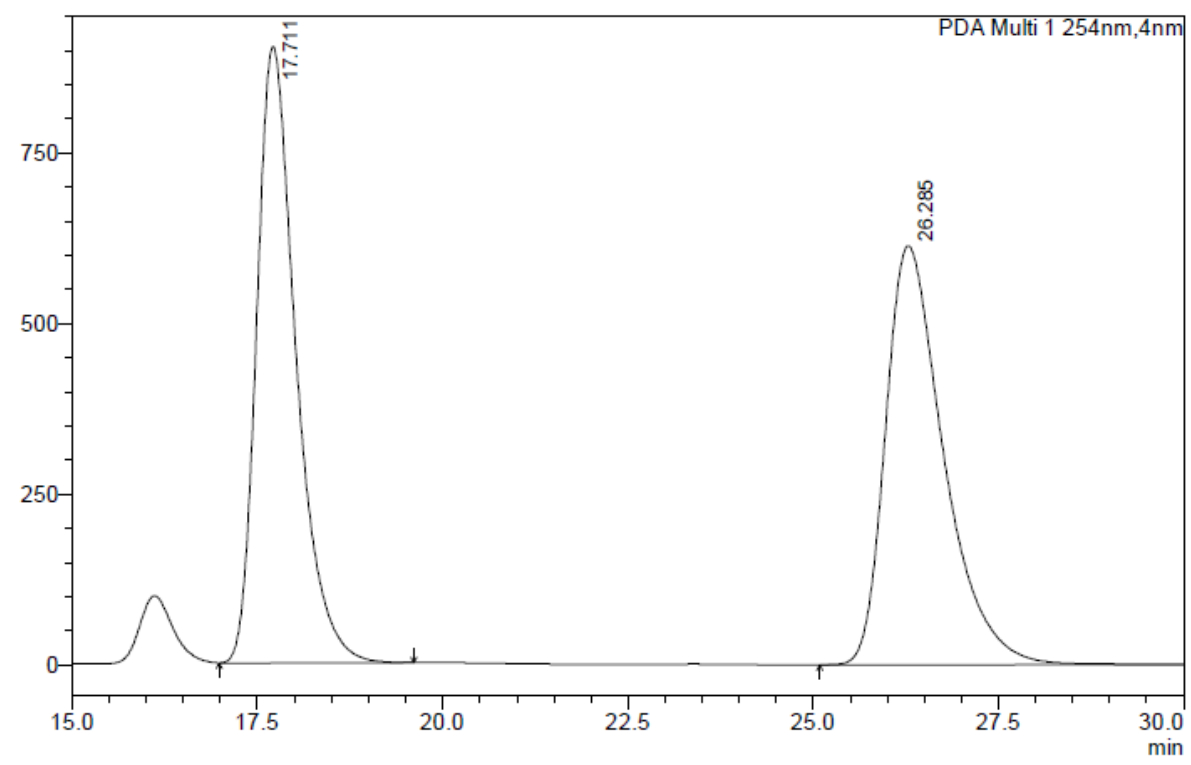

Peak Table

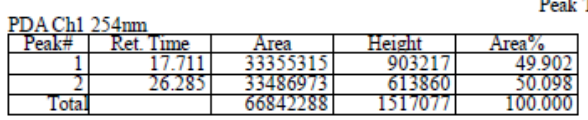

$\mathrm{mAU}$

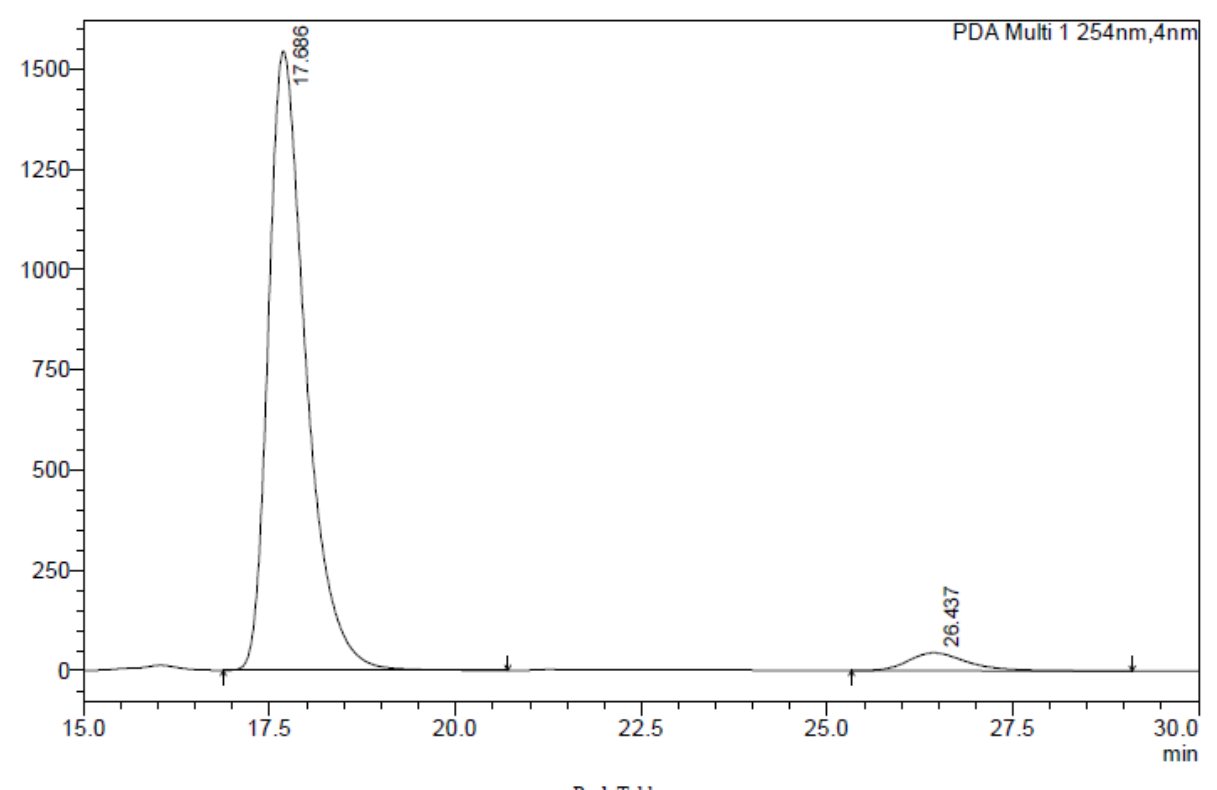

PDA Ch1 $254 \mathrm{~nm}$
\begin{tabular}{r|r|r|r|r|}
\hline Peak\# & Ret. Tme & \multicolumn{1}{c}{ Area } & \multicolumn{1}{c}{ Helght } & \multicolumn{1}{c|}{ Area $\%$} \\
\hline 1 & 17.686 & 54428632 & 1544137 & 95.668 \\
\hline 2 & 26.437 & 2464769 & 44675 & 4.332 \\
\hline Total & & 56893401 & 1588812 & 100.000 \\
\hline
\end{tabular} 


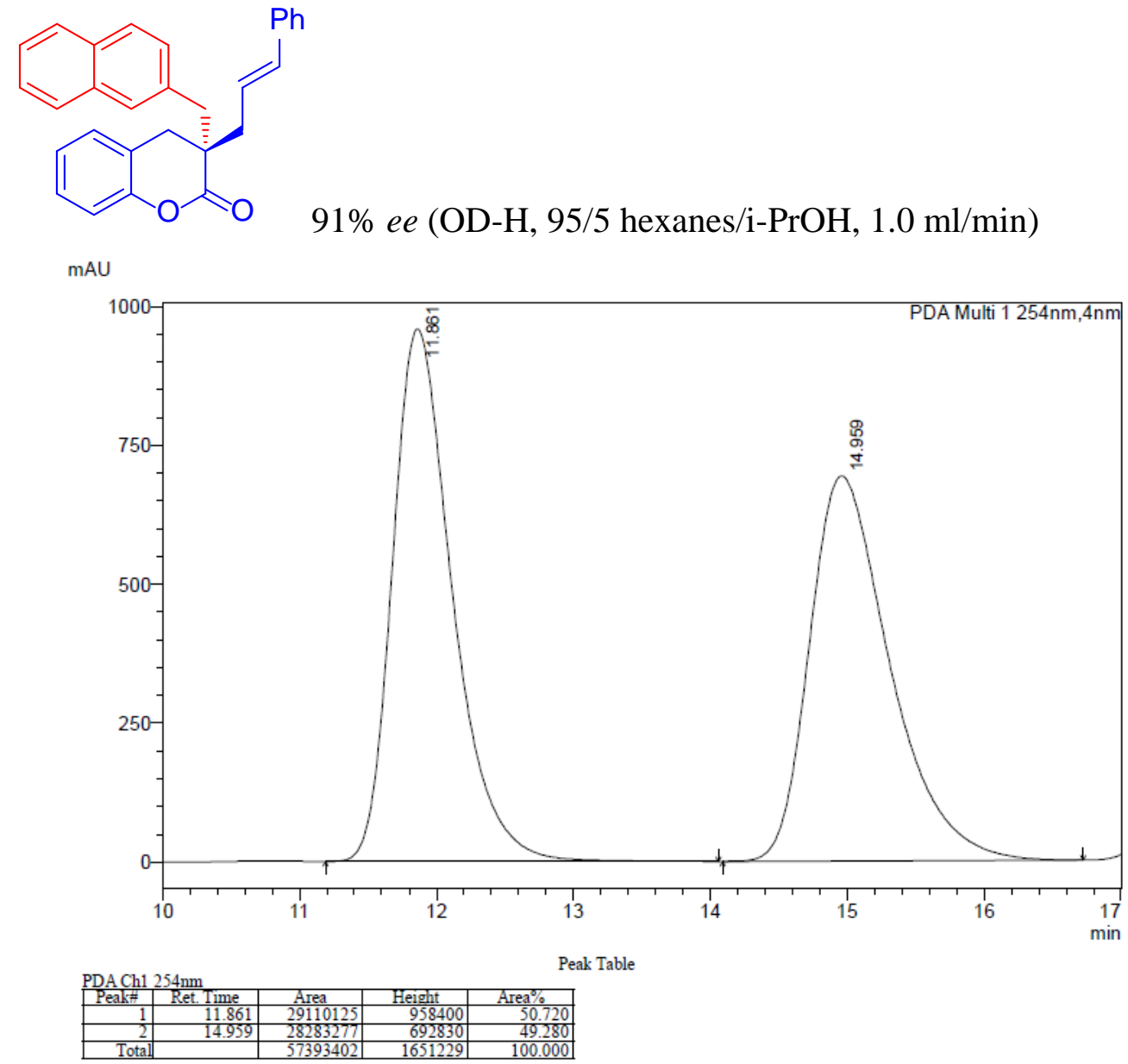

mAU
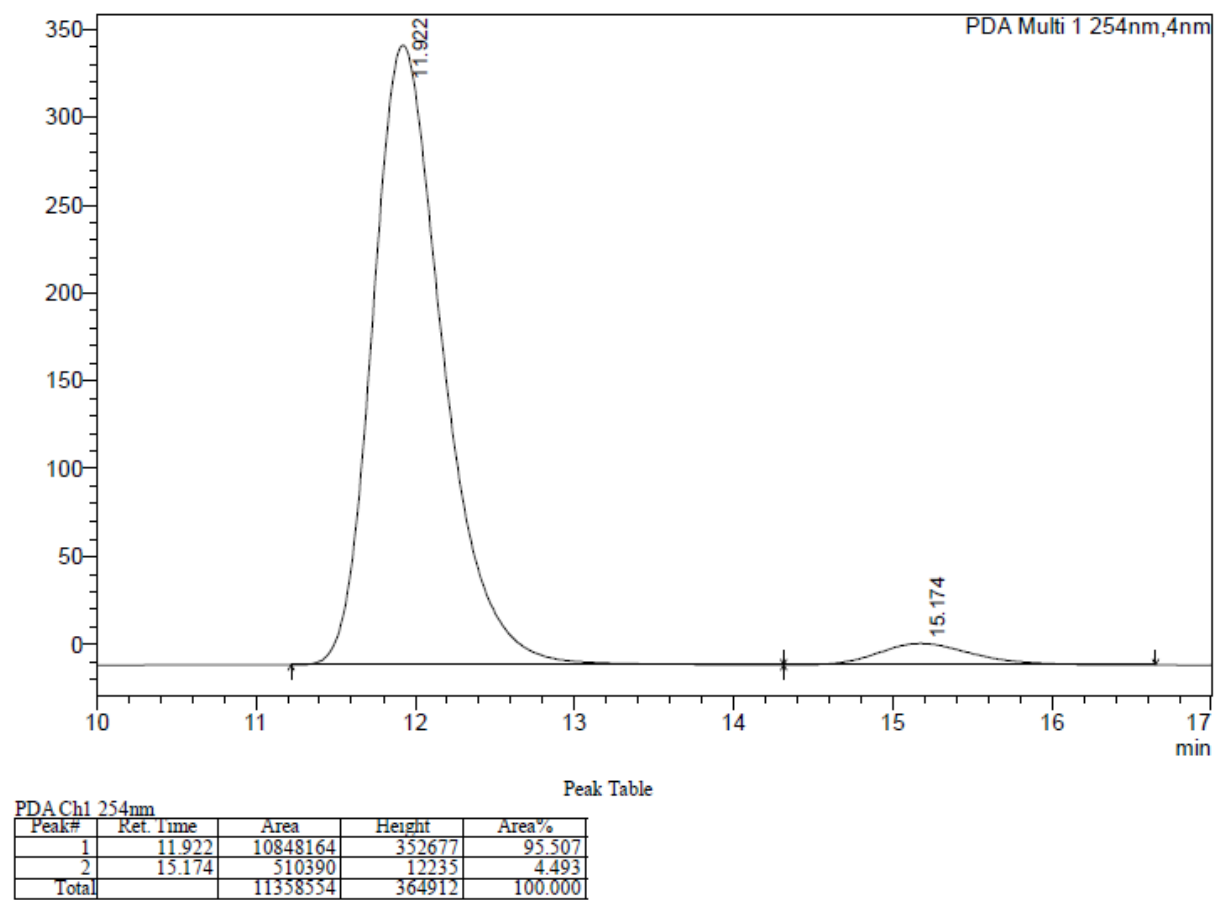
<smiles>COc1cccc(C/C=C/C[C@H]2Cc3ccccc3OC2=O)c1</smiles>

95\% ee (OD-H, 90/10 hexanes/i-PrOH, $1.0 \mathrm{ml} / \mathrm{min}$ )

mAU

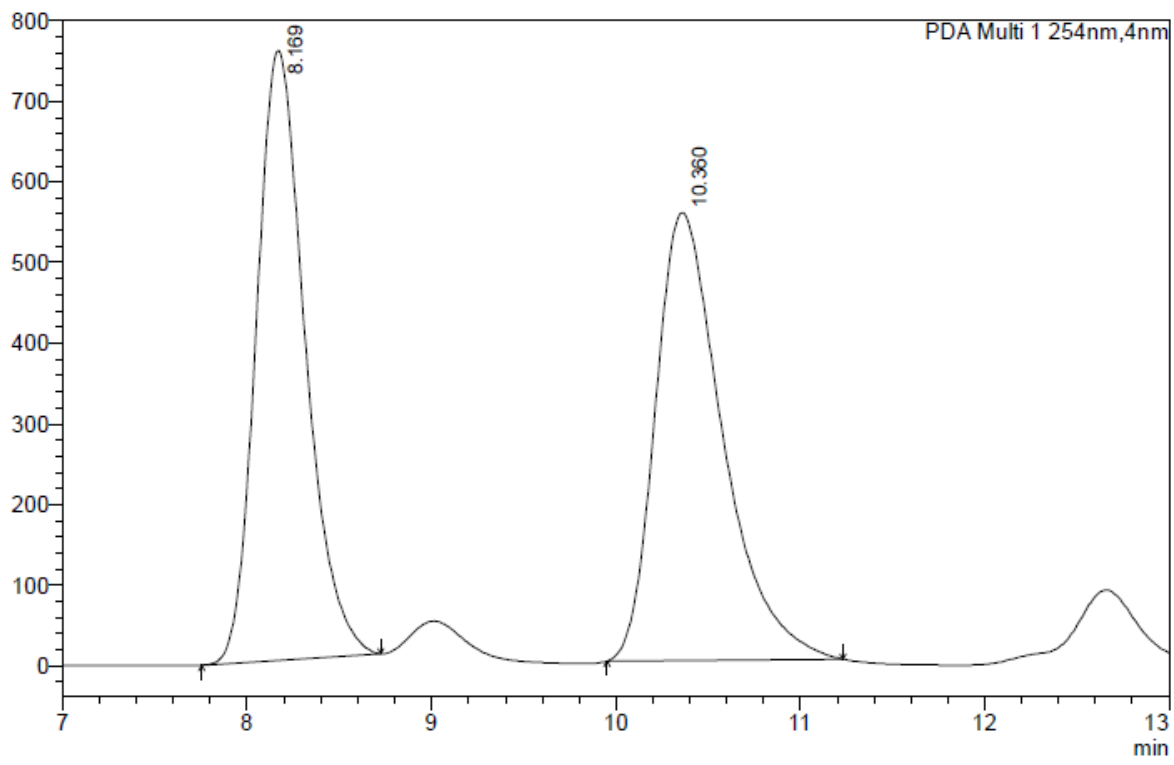

Peak Table

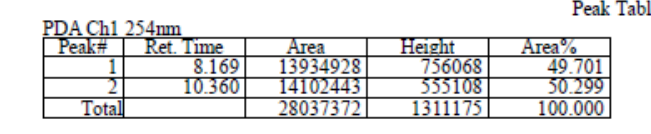

mAU
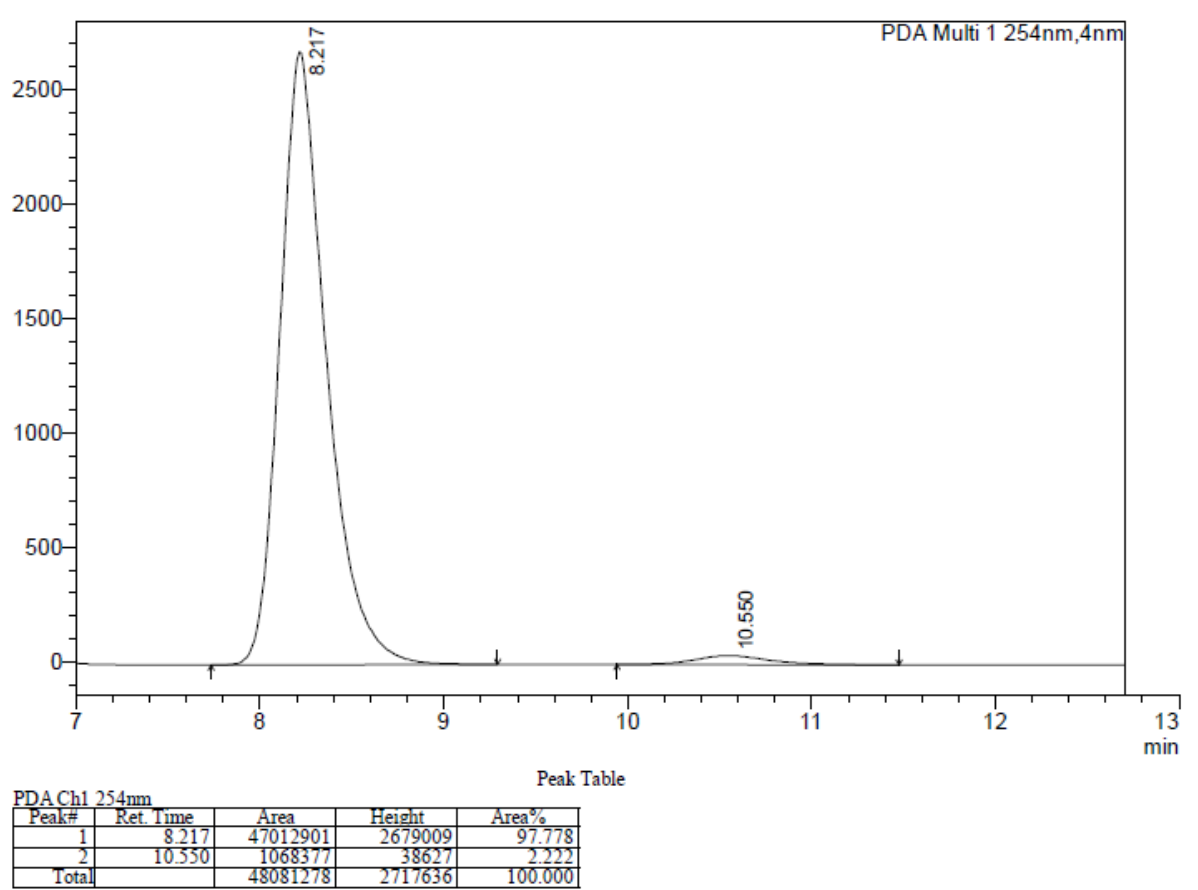
<smiles>O=C1Oc2ccccc2CC1(C/C=C/c1ccccc1)Cc1ccccc1Br</smiles>

89\% ee (OD-H, 95/5 hexanes/i-PrOH, 0.5 ml/min)
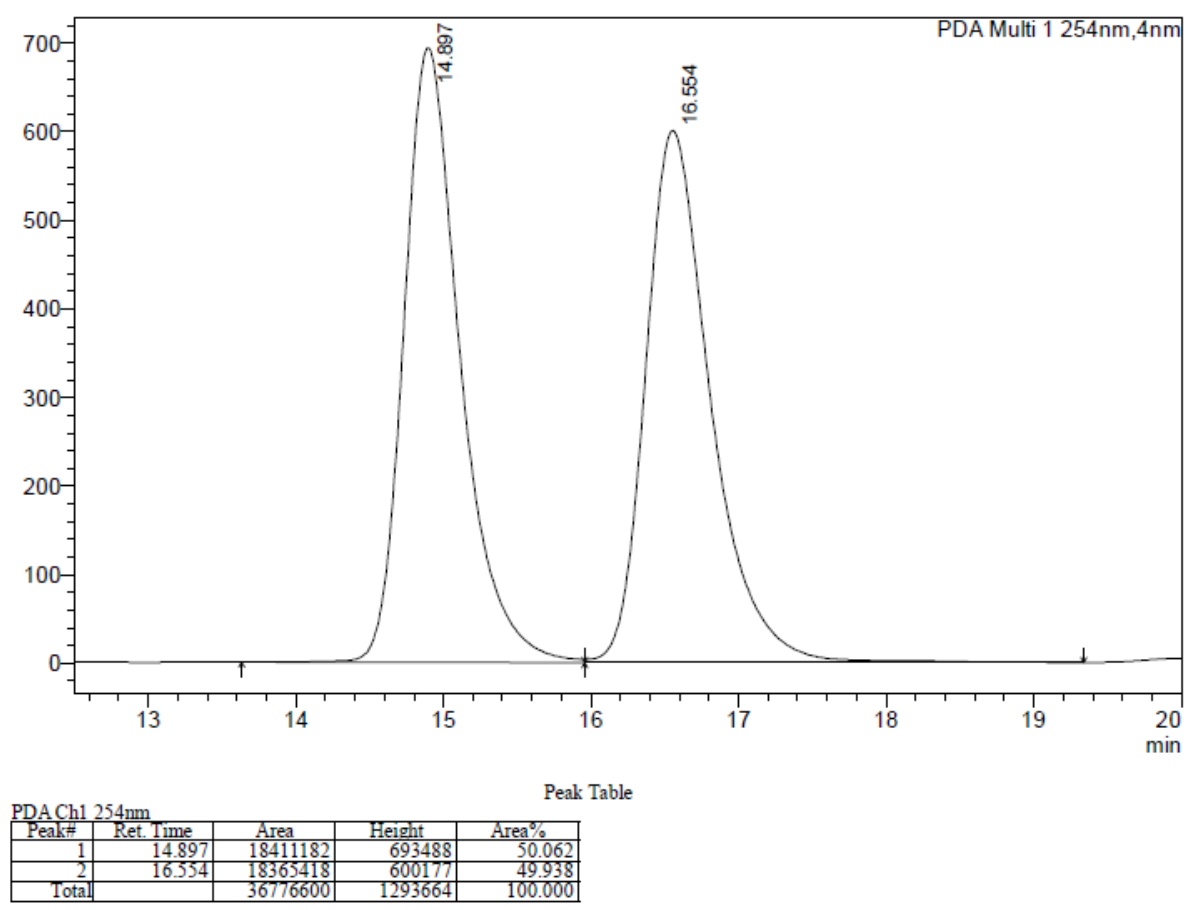

$\mathrm{mAU}$

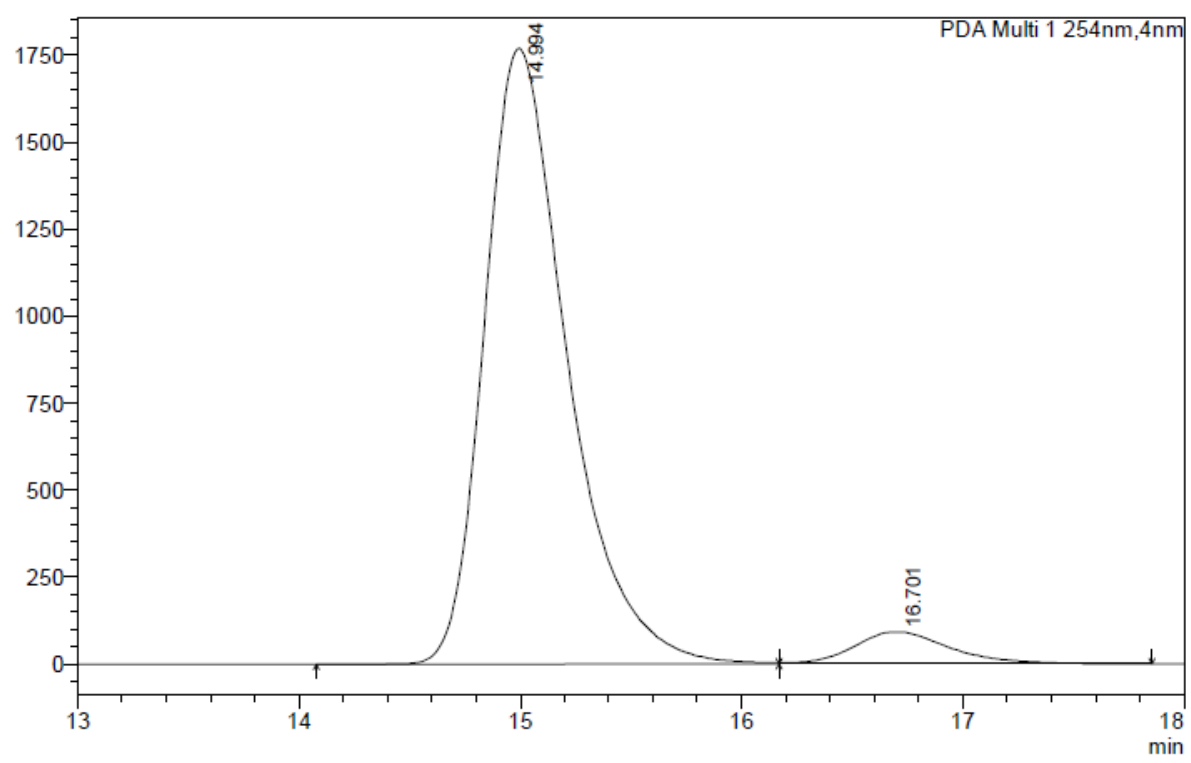

Peak Table

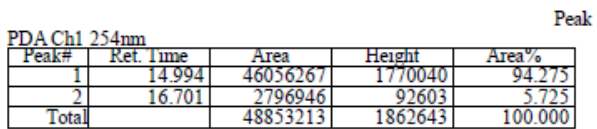


<smiles>Cc1ccc(C/C=C/C[C@H]2Cc3ccccc3OC2=O)cc1</smiles>

91\% ee (OD-H, 9O/10 hexanes/i-PrOH, 1.0 ml/min)

$\mathrm{mAU}$

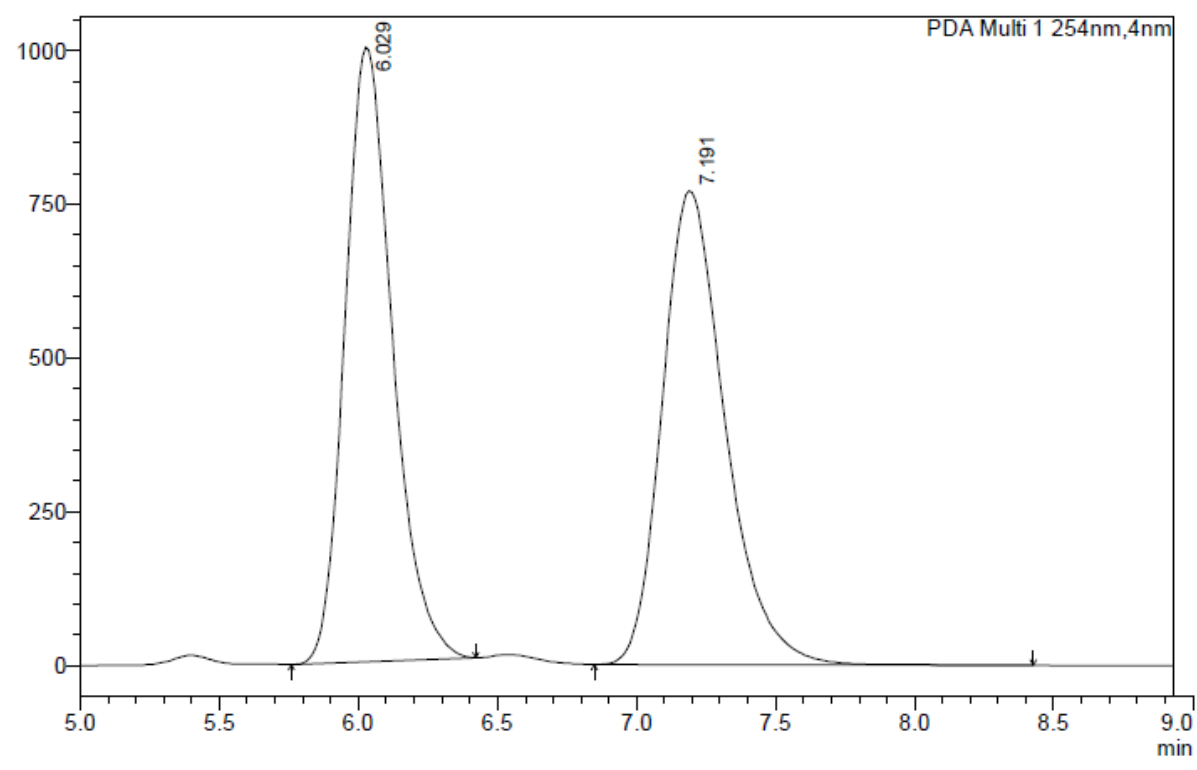

Peak Table

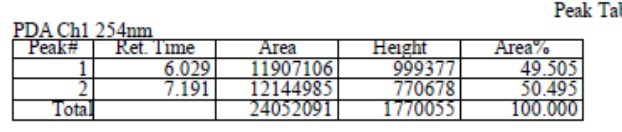

mAU
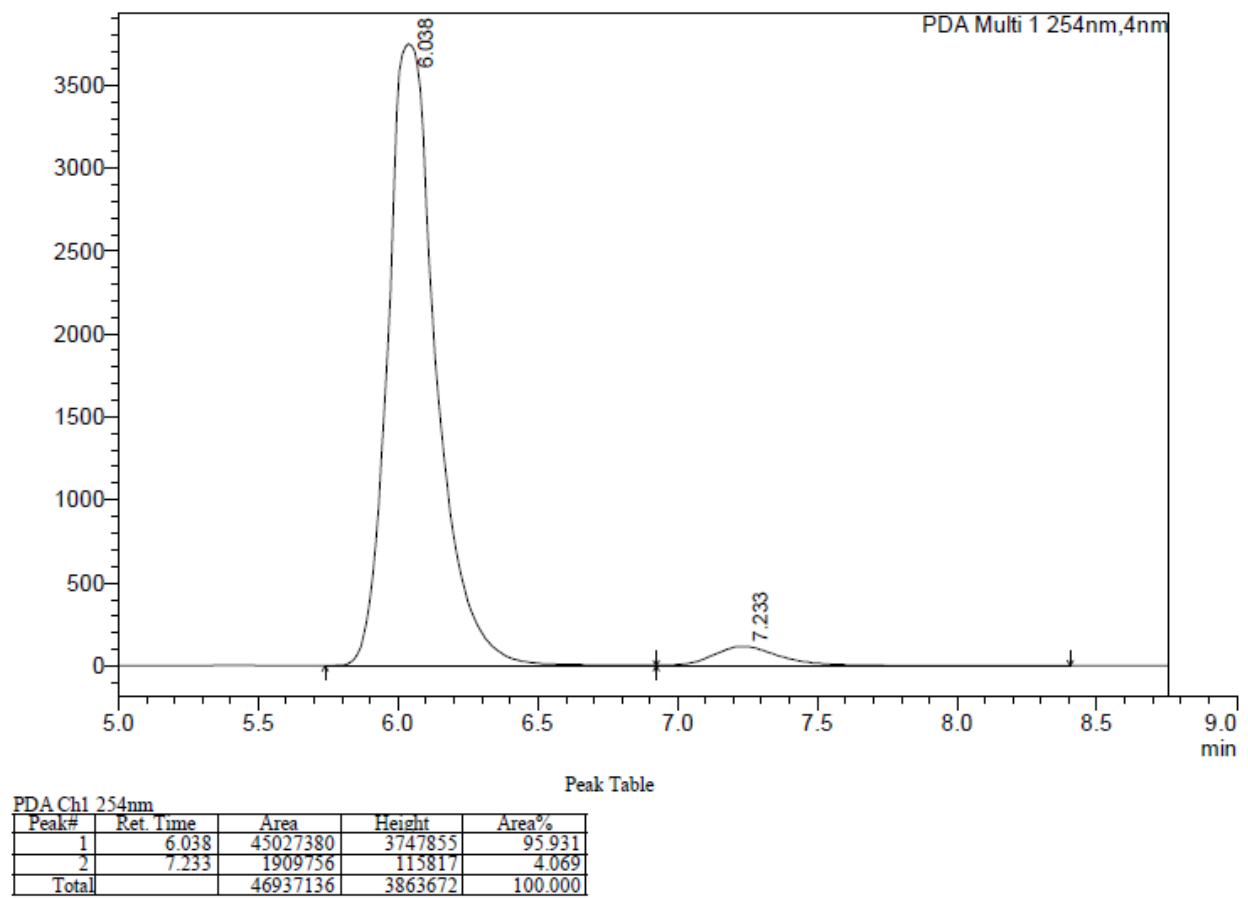
<smiles>O=C1Oc2ccccc2C[C@H]1C/C=C/c1ccc(F)cc1</smiles>

93\% ee (OD-H, 95/5 hexanes/i-PrOH, 1.0 ml/min)

mAU

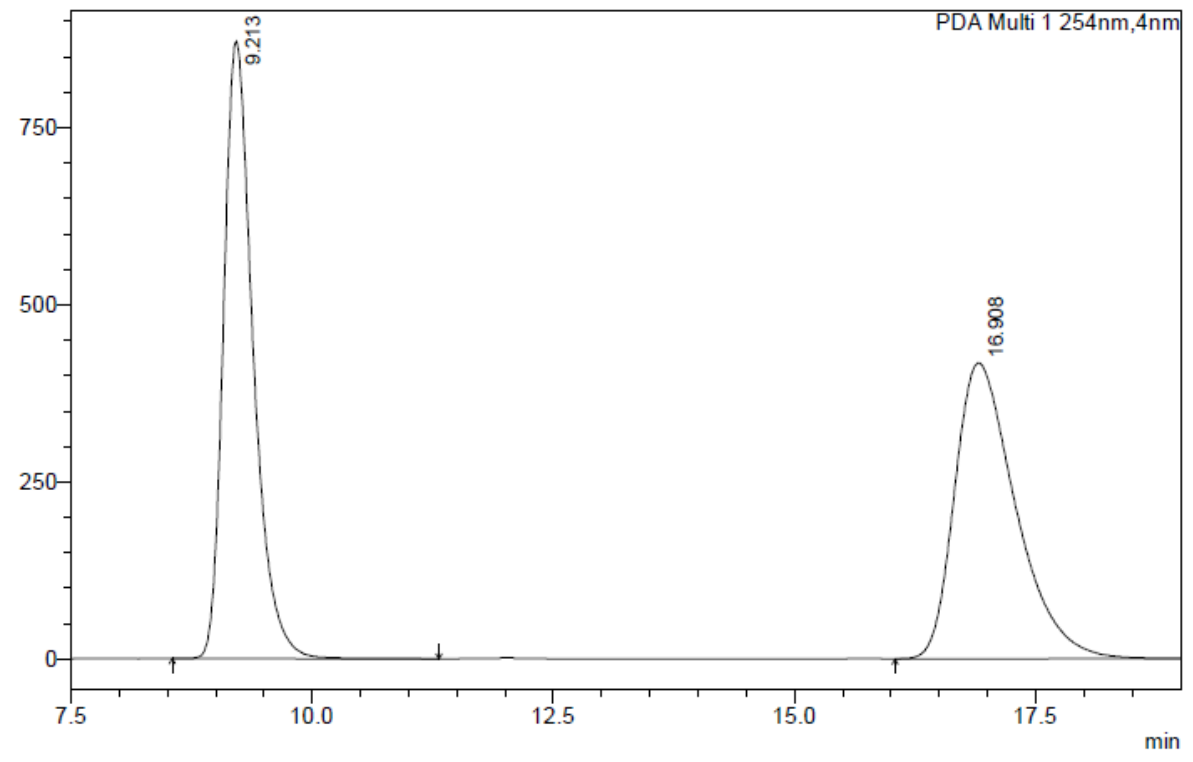

Peak Table

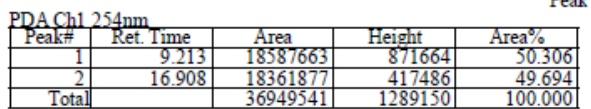

mAU

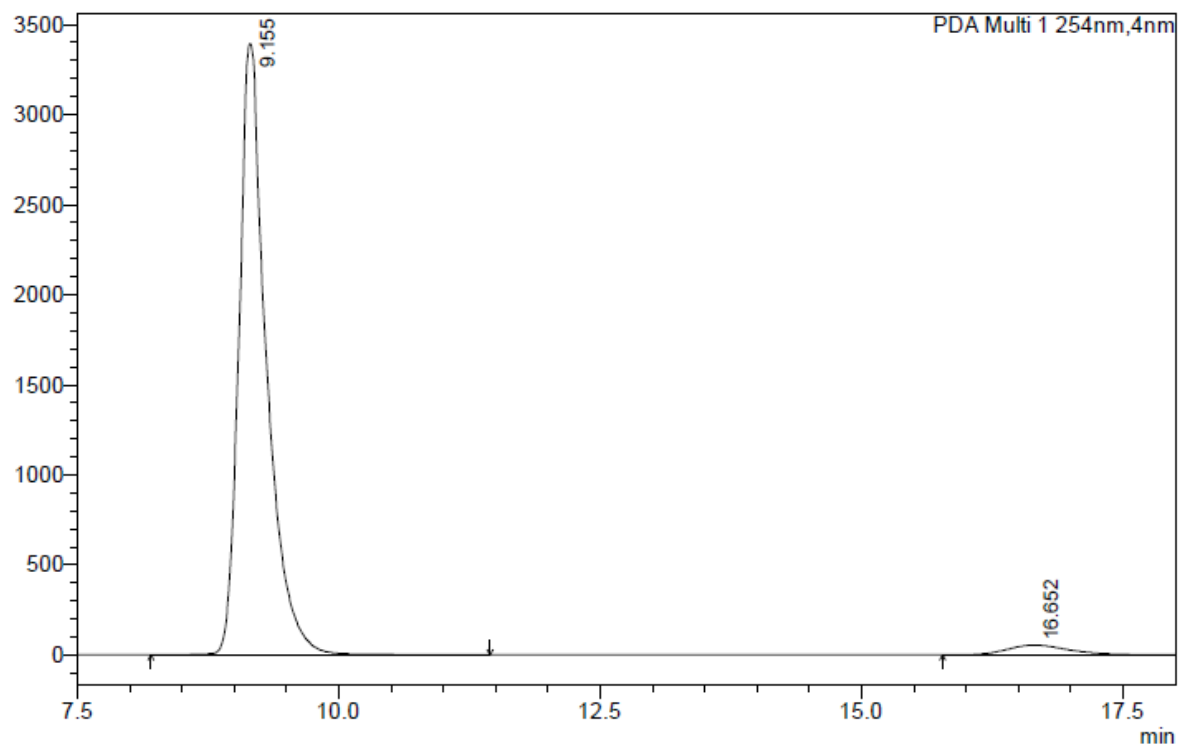

Peak Table

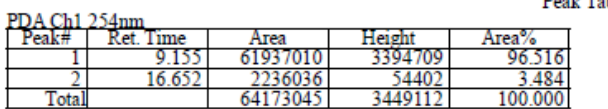


<smiles>Cc1ccc(C(F)(F)F)cc1</smiles>

91\% eе (OD-H, 90/10 hexanes/i-PrOH, $1.0 \mathrm{ml} / \mathrm{min}$ )

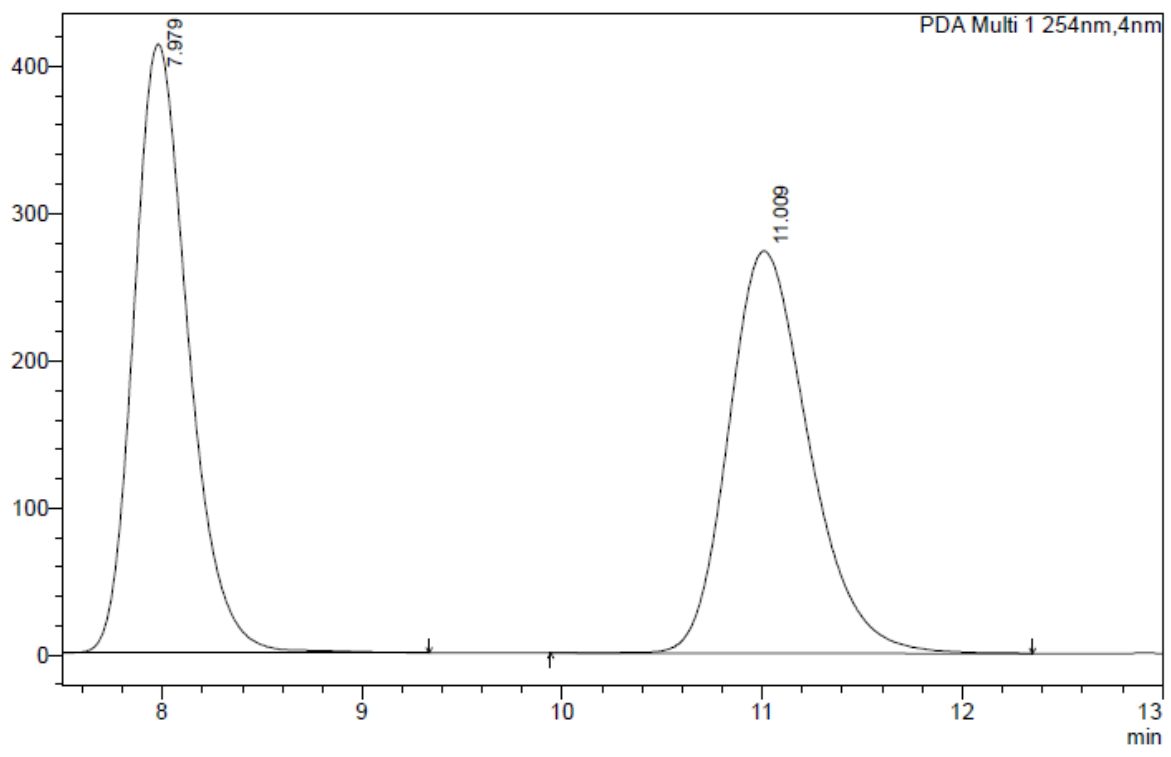

Peak Table
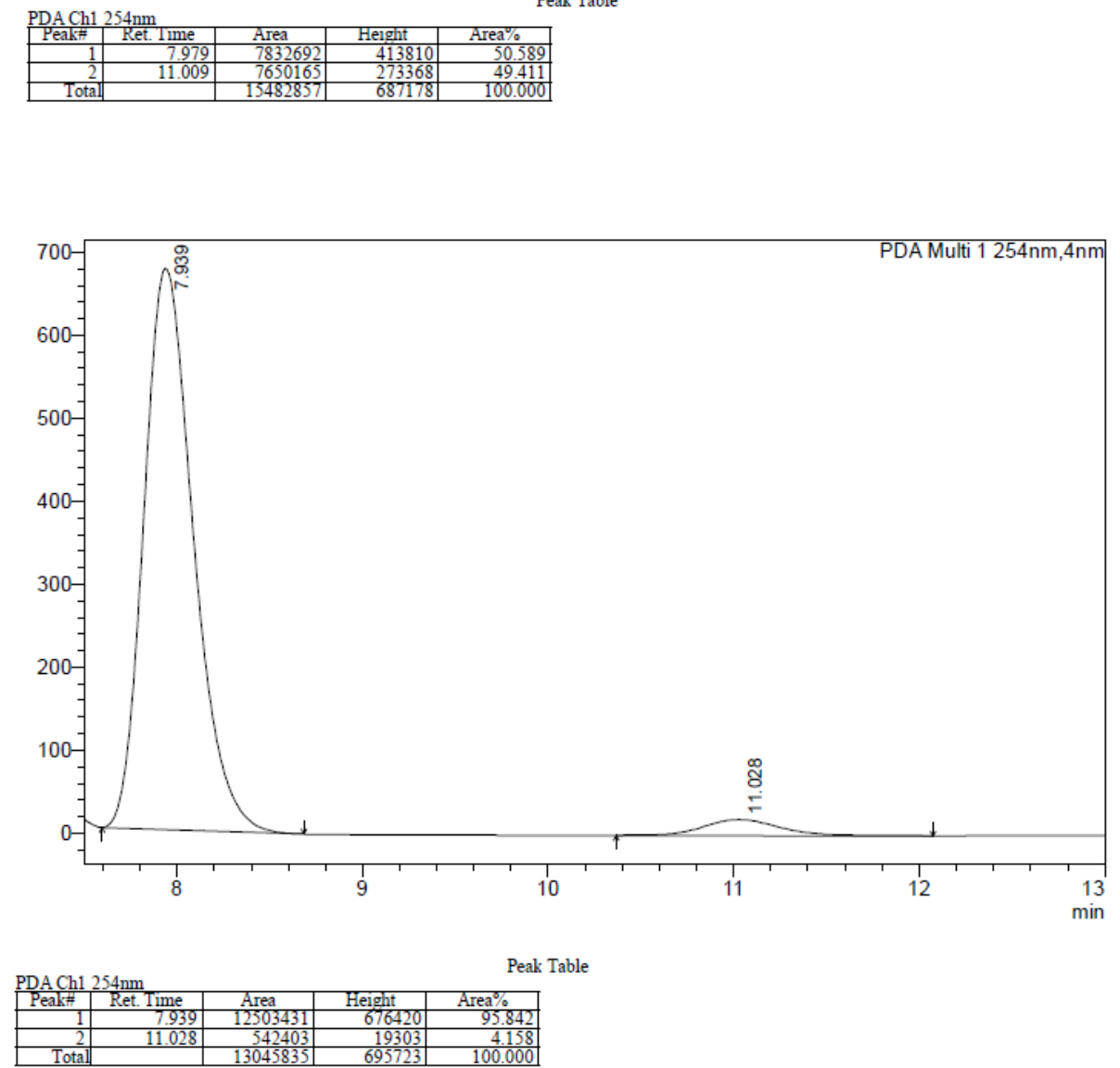
<smiles>CC(C)=CC[C@]1(Cc2ccccc2)Cc2cc(Br)cc(Br)c2OC1=O</smiles>

90\% eе (IC, 99/1 hexanes/i-PrOH, 0.5 ml/min)

mAU
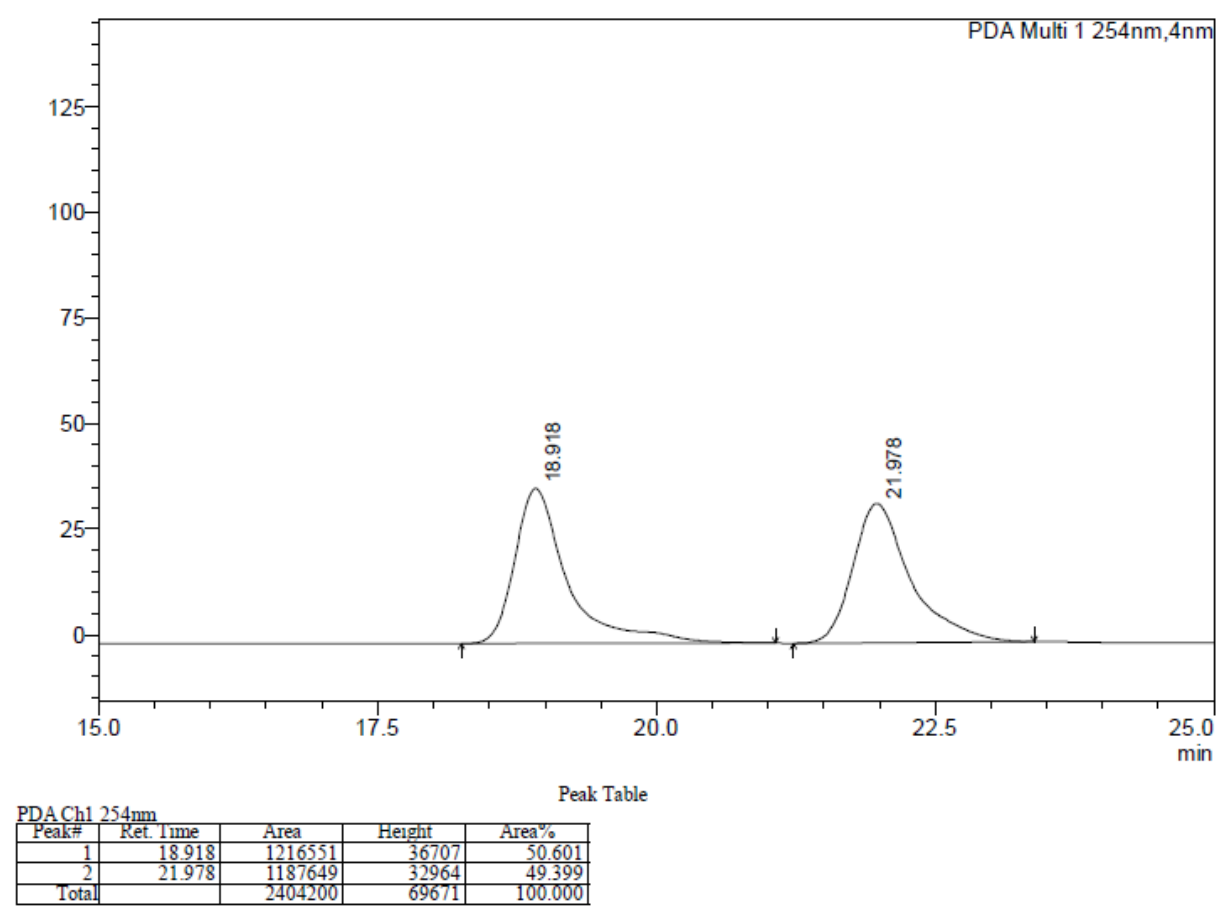

mAU
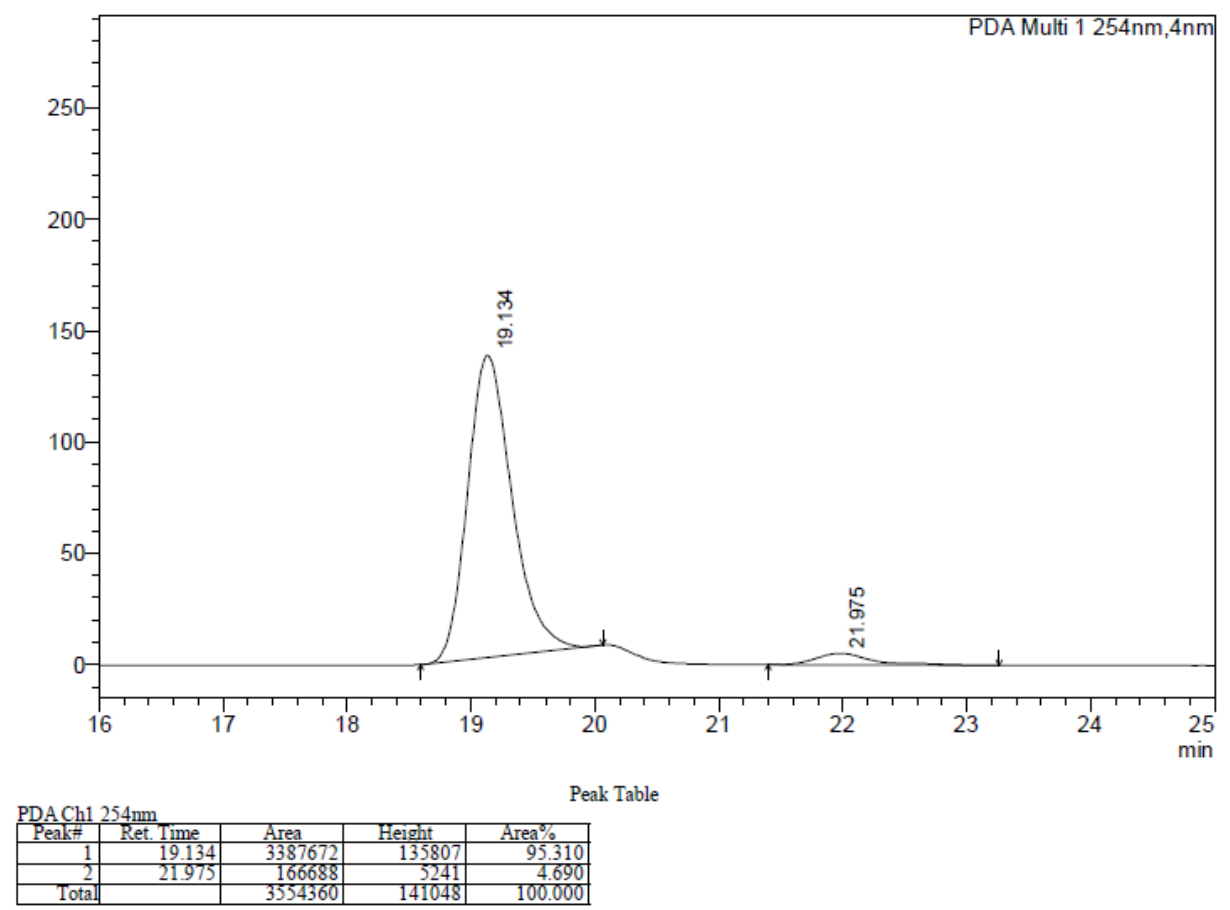
<smiles>CC(C)=CC[C@]1(Br)Cc2cccc(C)c2OC1=O</smiles>

95\% ee (OJ-H, 99/1 hexanes/i-PrOH, 0.5 ml/min)

mAU
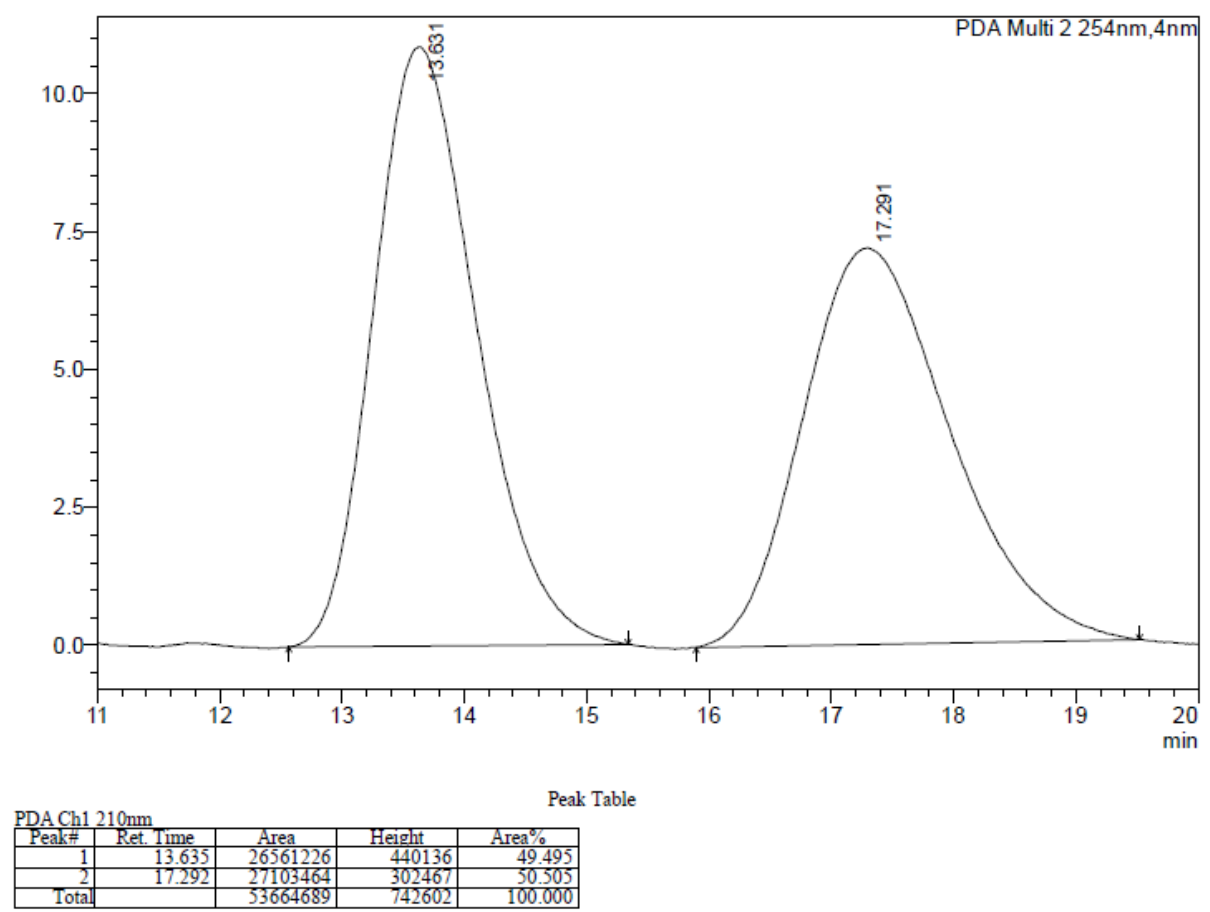

mAU

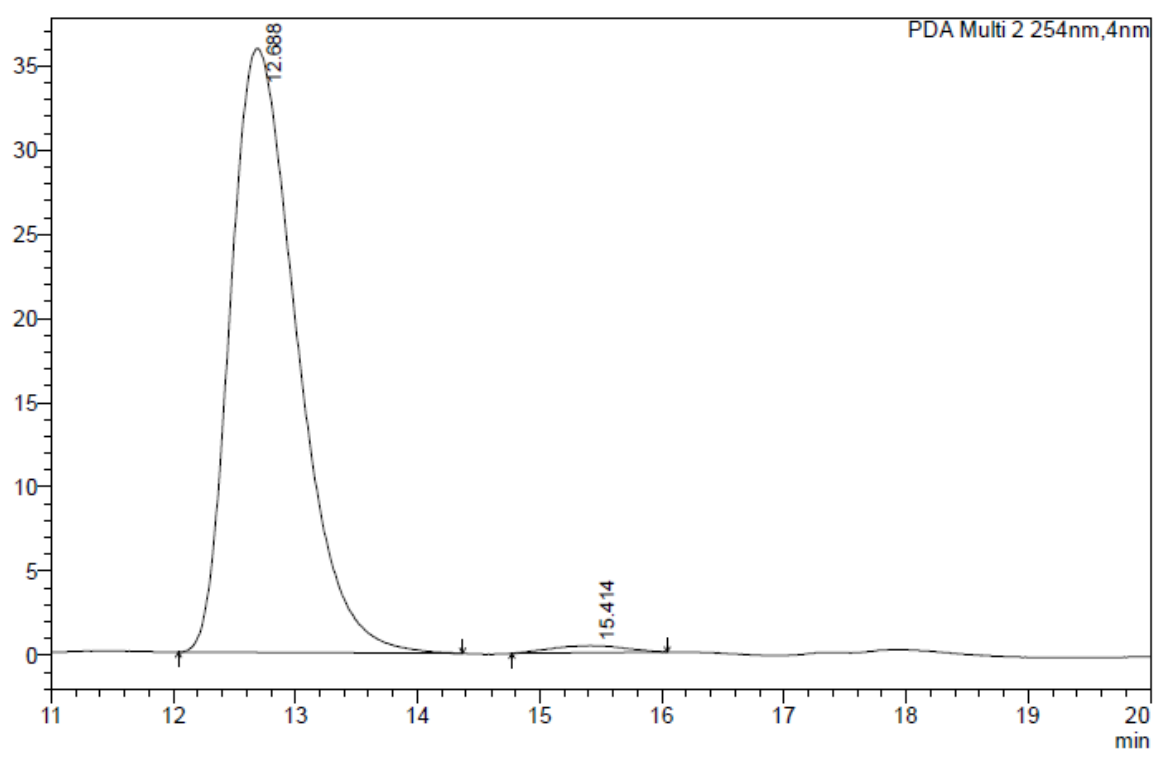

Peak Table

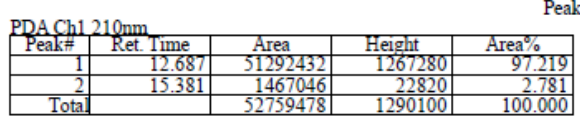



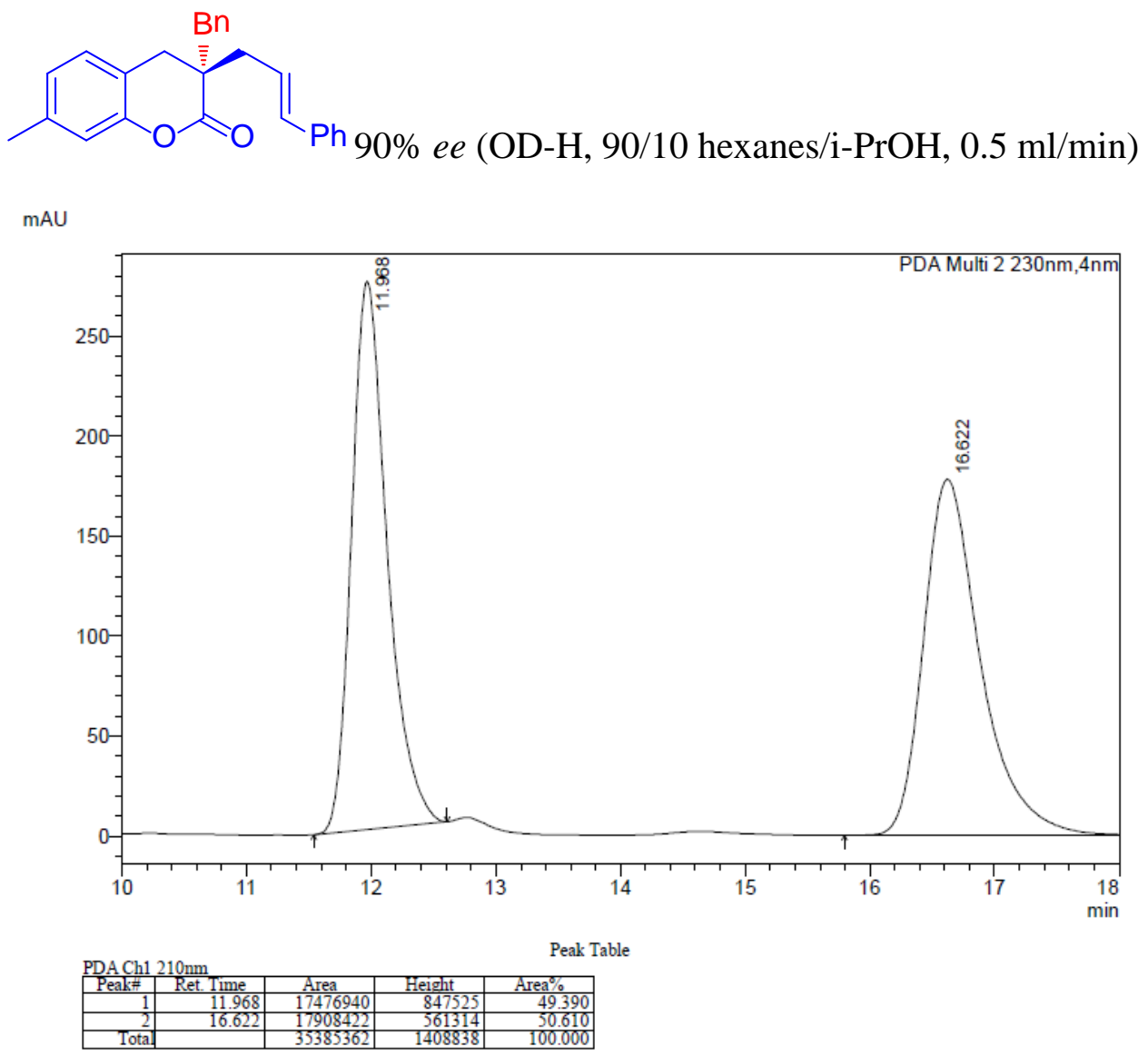

mAU
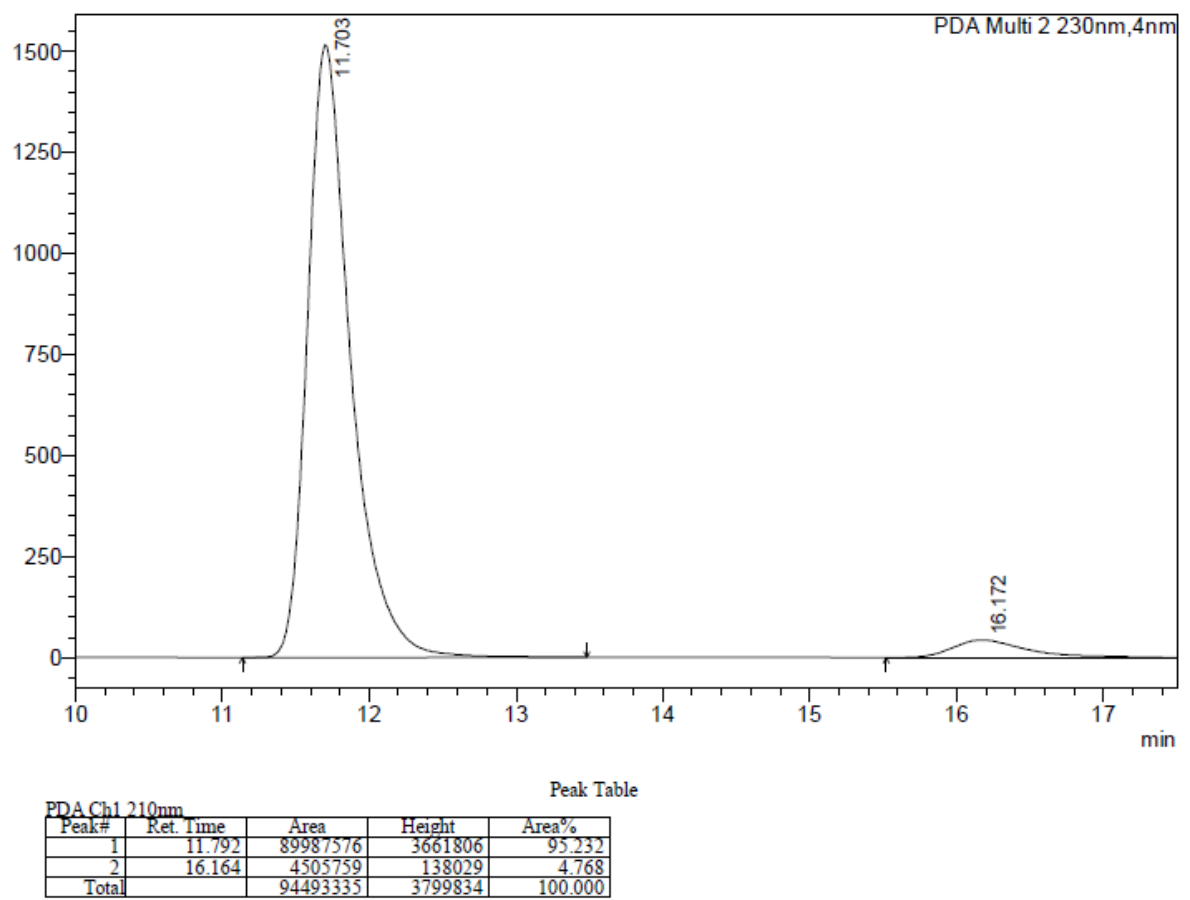


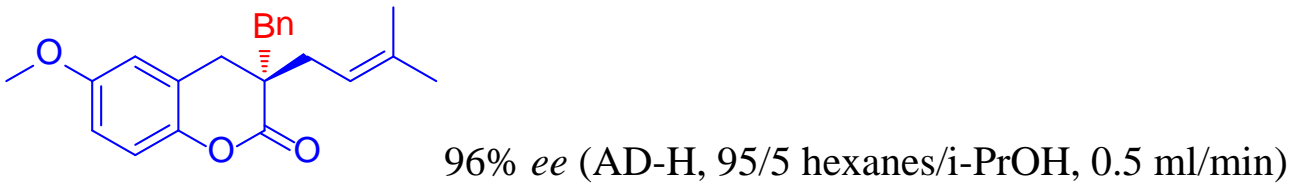

mAU
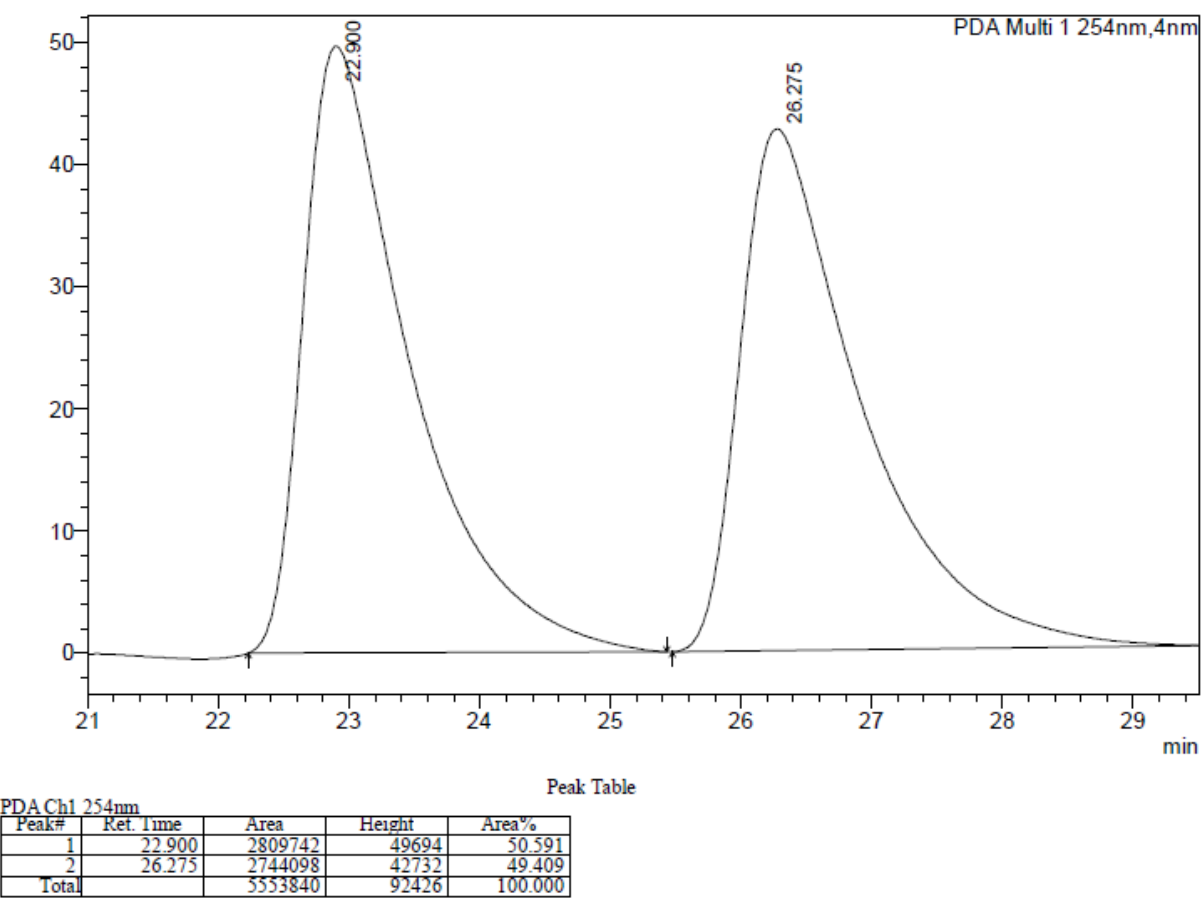

mAU

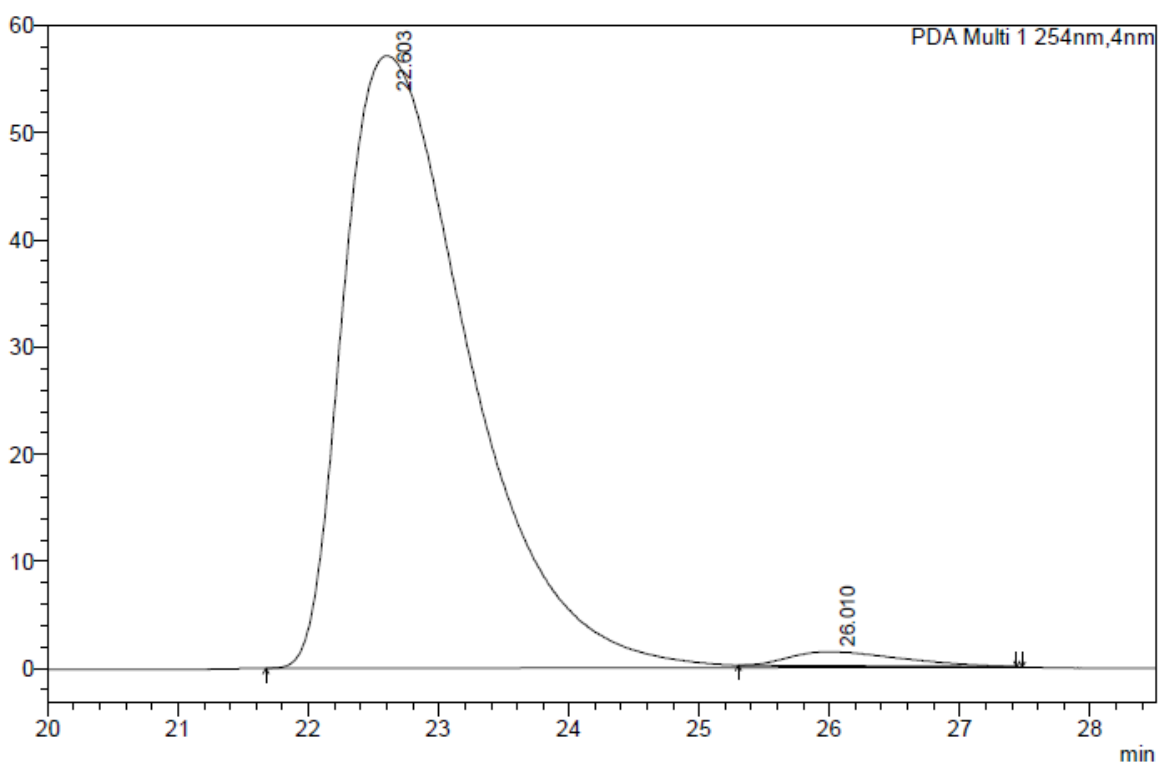

Peak Table

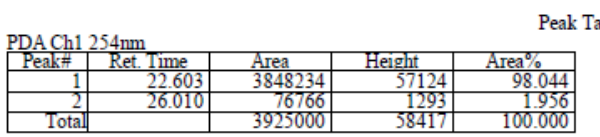



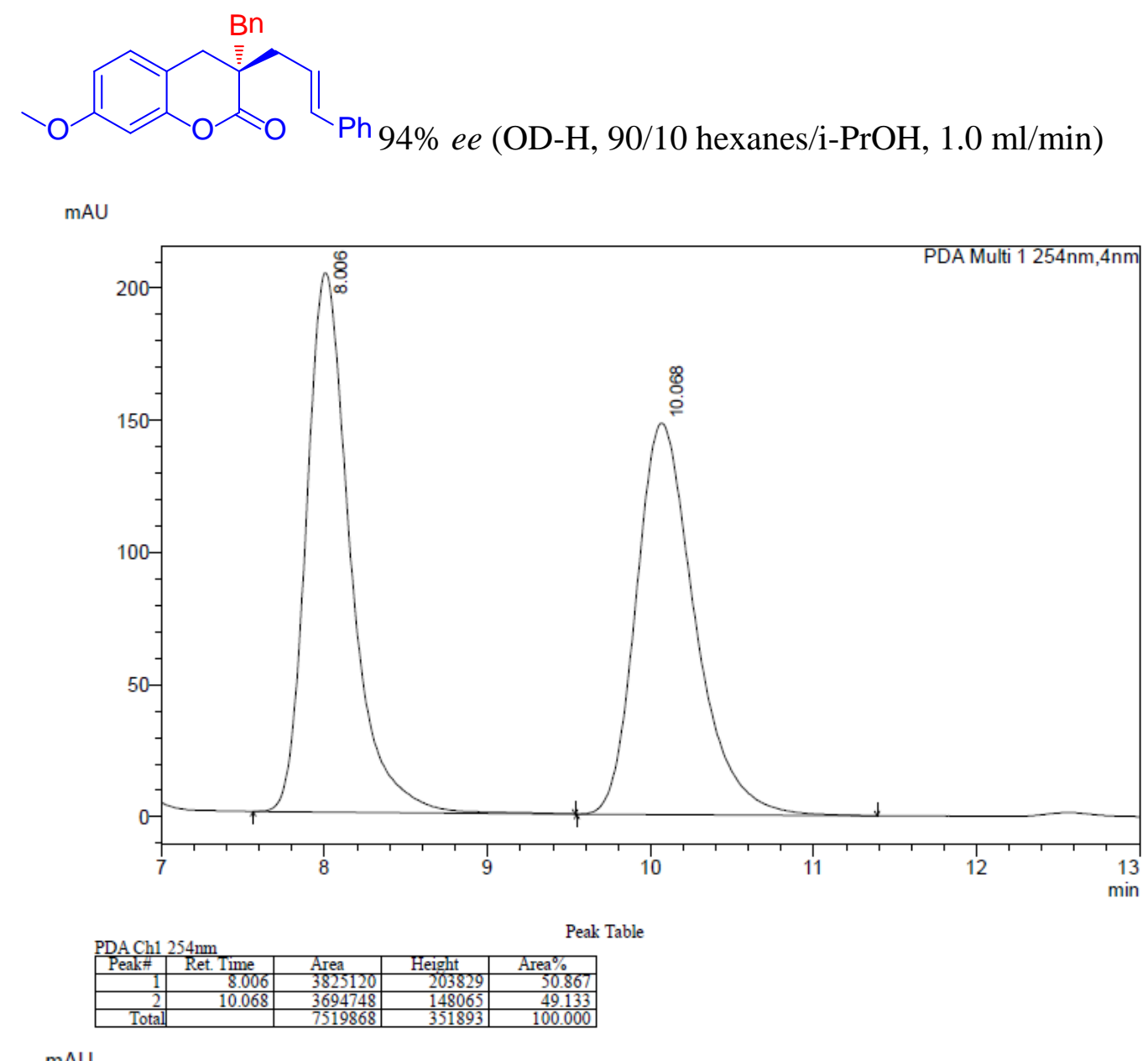

mAU

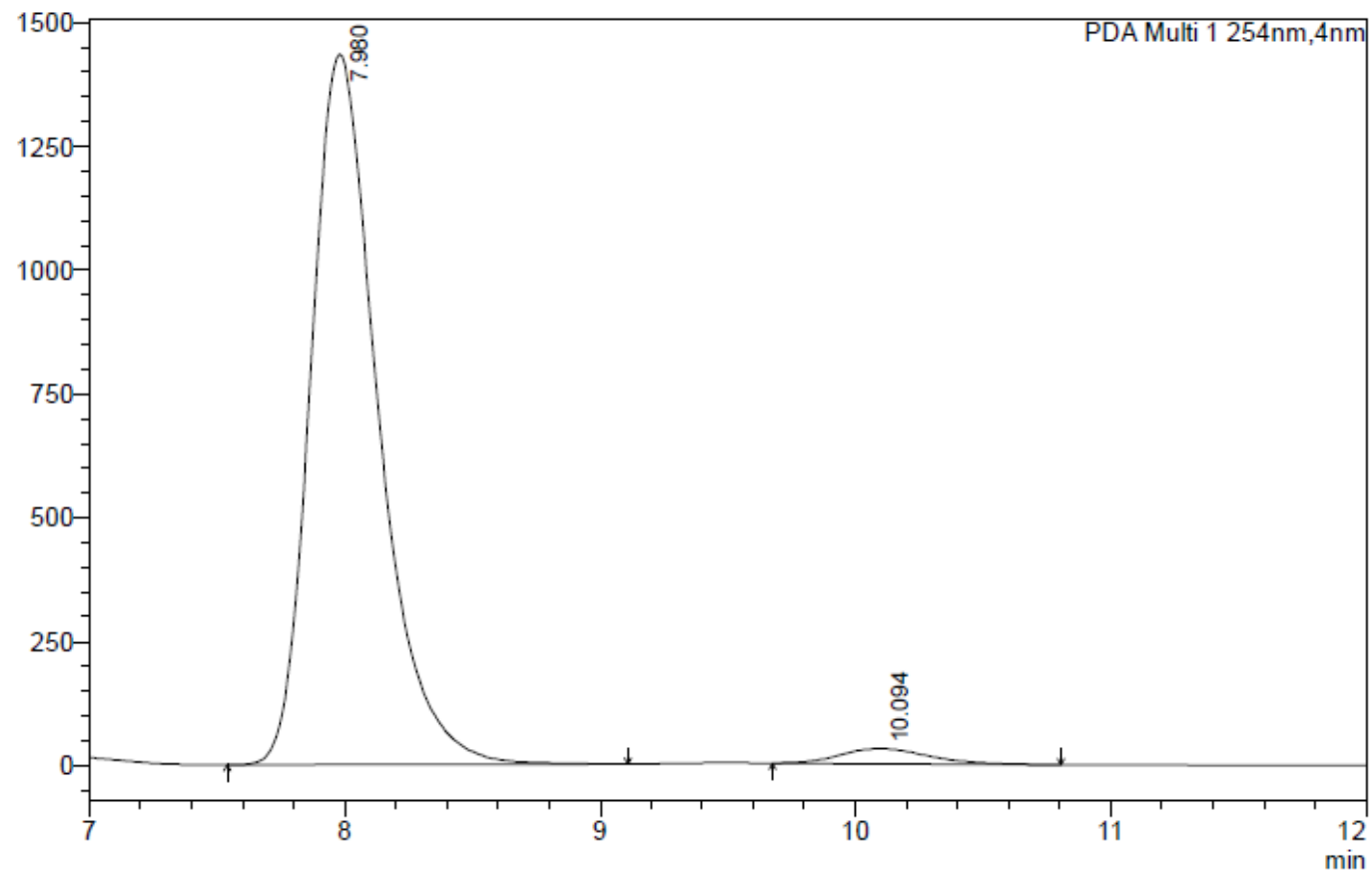

Peak Table

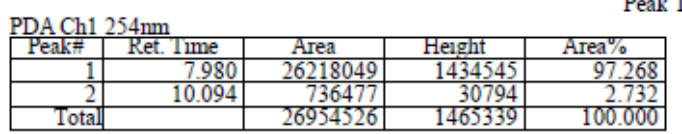


<smiles>O=C1Oc2ccc3ccccc3c2CC1(C/C=C/c1ccccc1)Cc1ccccc1</smiles>

90\% ee (OD-H, 90/10 hexanes/i-PrOH, $1.0 \mathrm{ml} / \mathrm{min}$ )

mAU

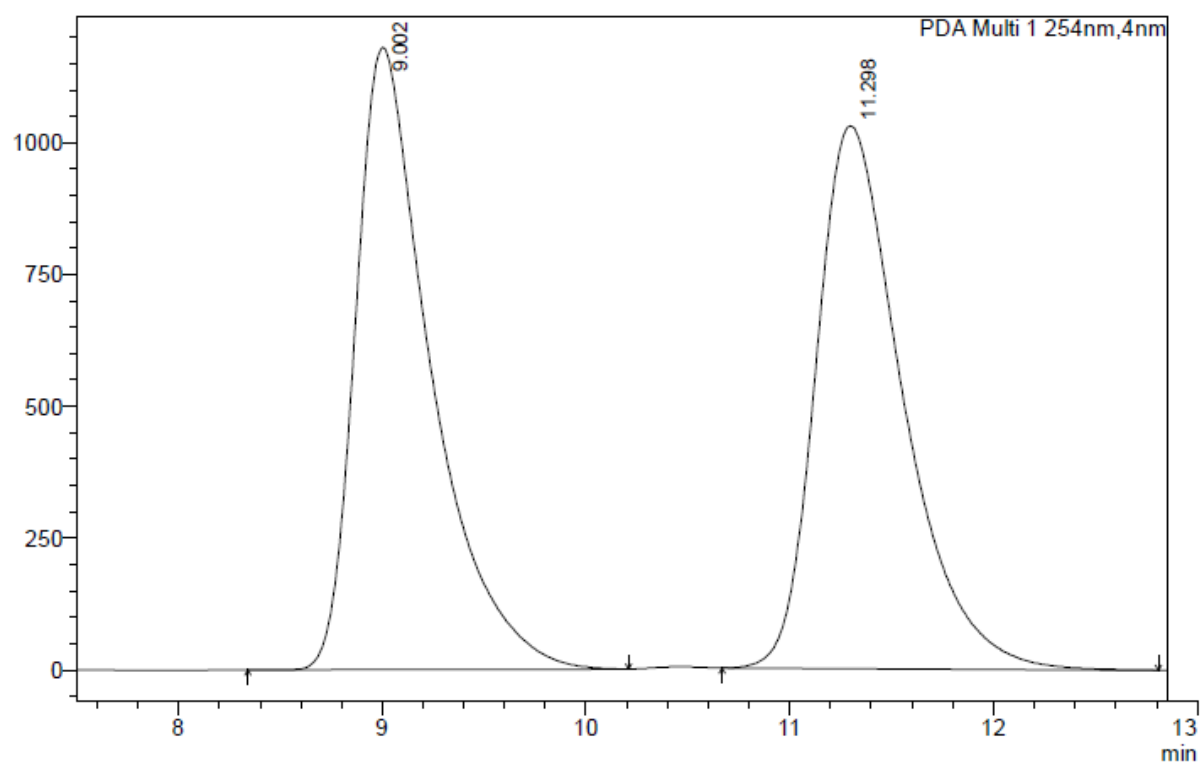

Peak Table

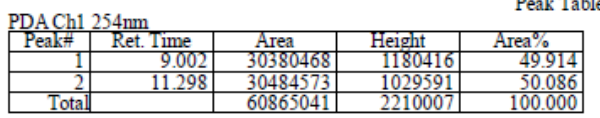

mAU

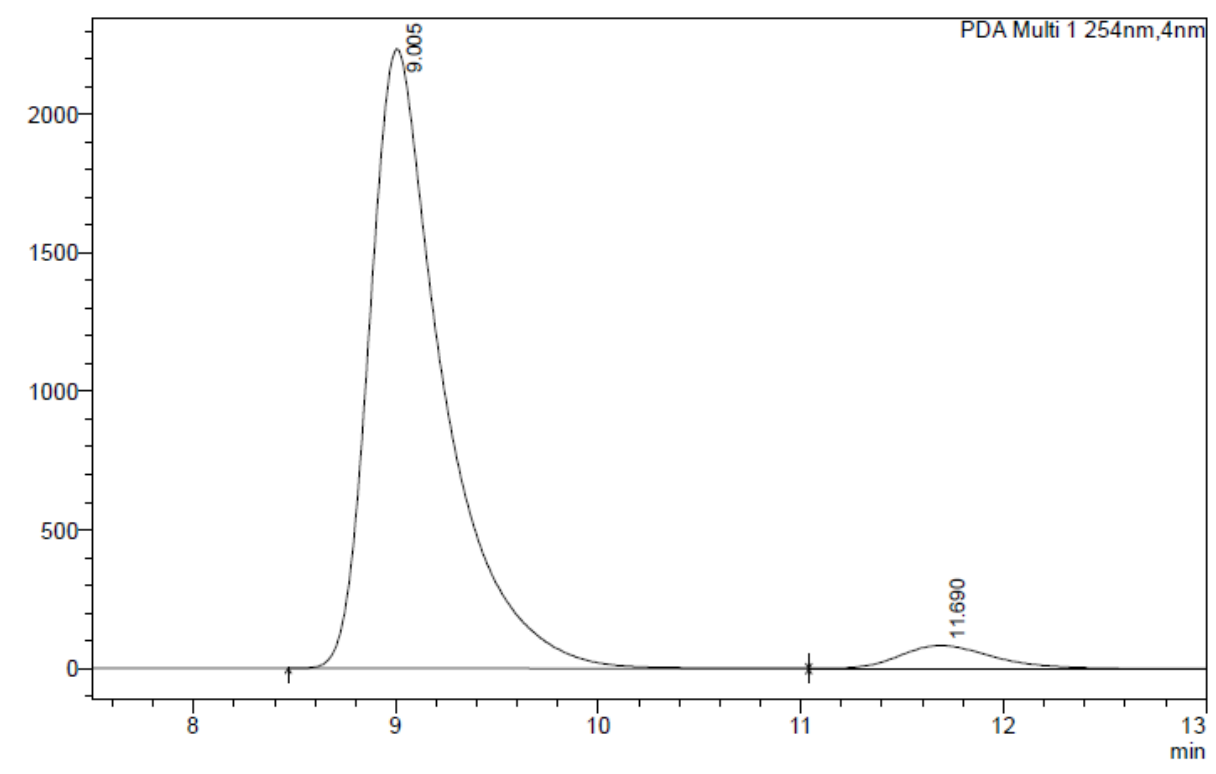

Peak Table

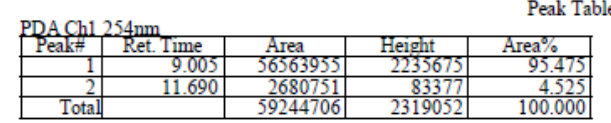




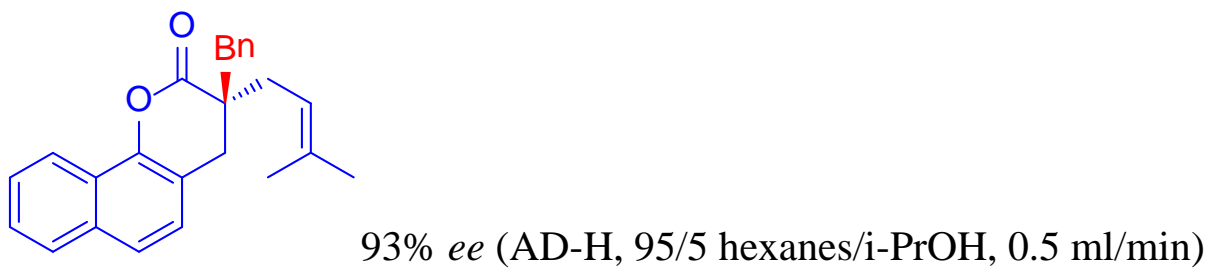

mAU
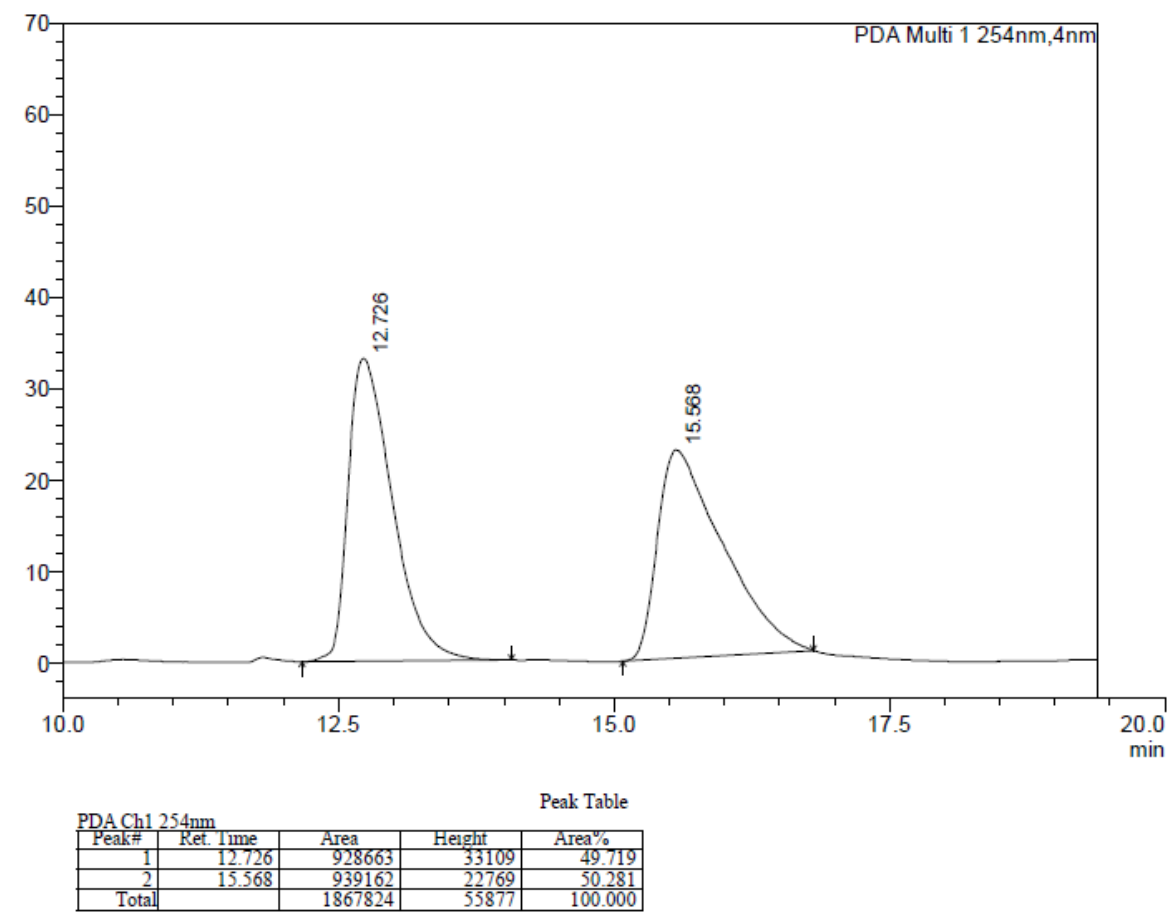

mAU

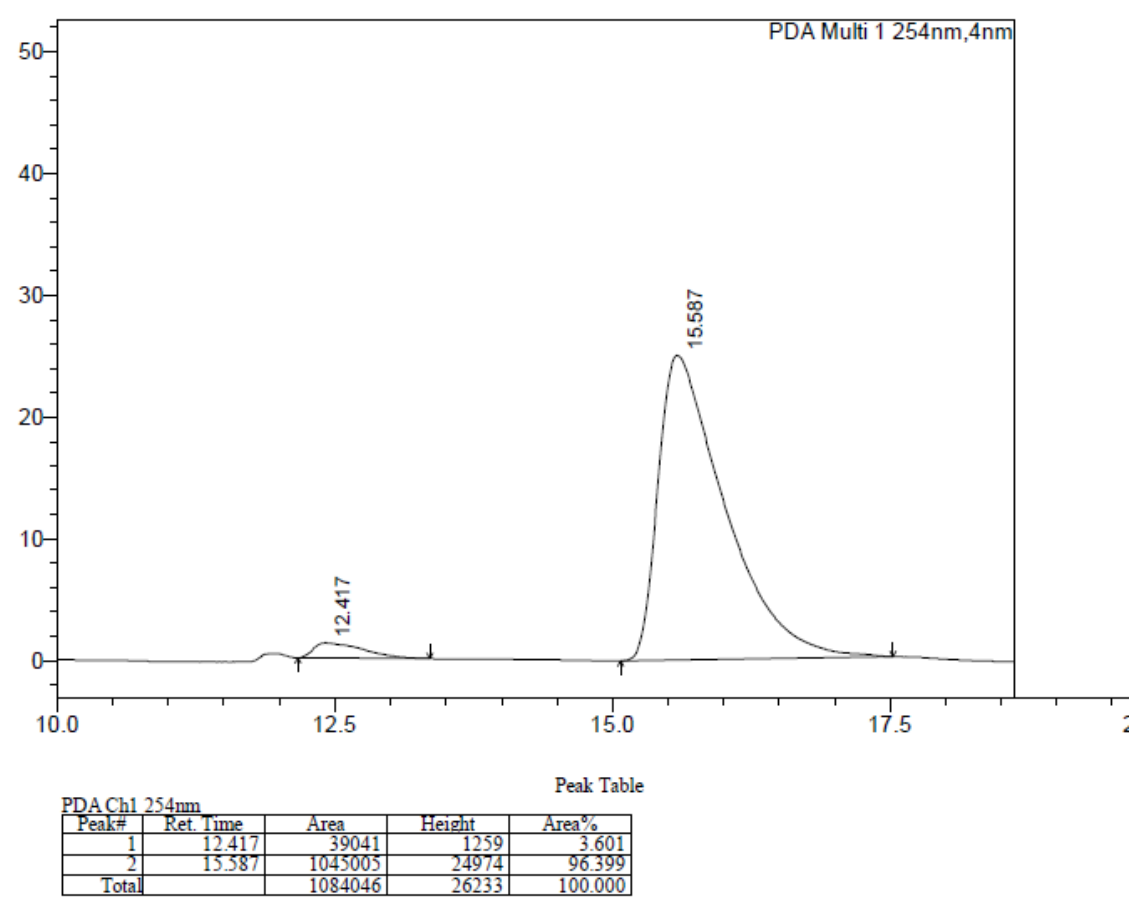


<smiles>O=C1Oc2ccc3cc(Br)ccc3c2C[C@]1(C/C=C/c1ccccc1)Cc1ccccc1</smiles>

95\% ee (OD-H, 90/10 hexanes/i-PrOH, 1.0 ml/min)

mAU

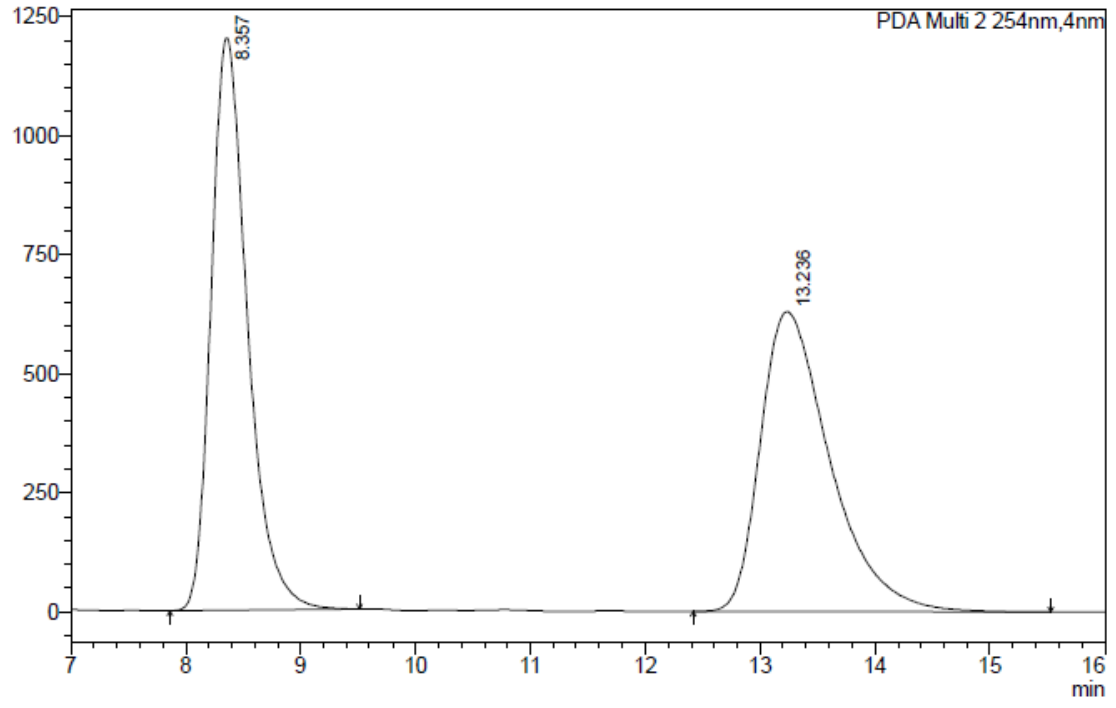

Peak Table

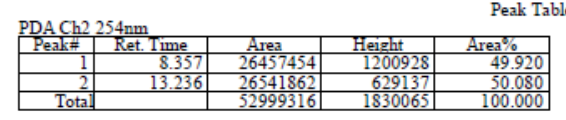

mAU

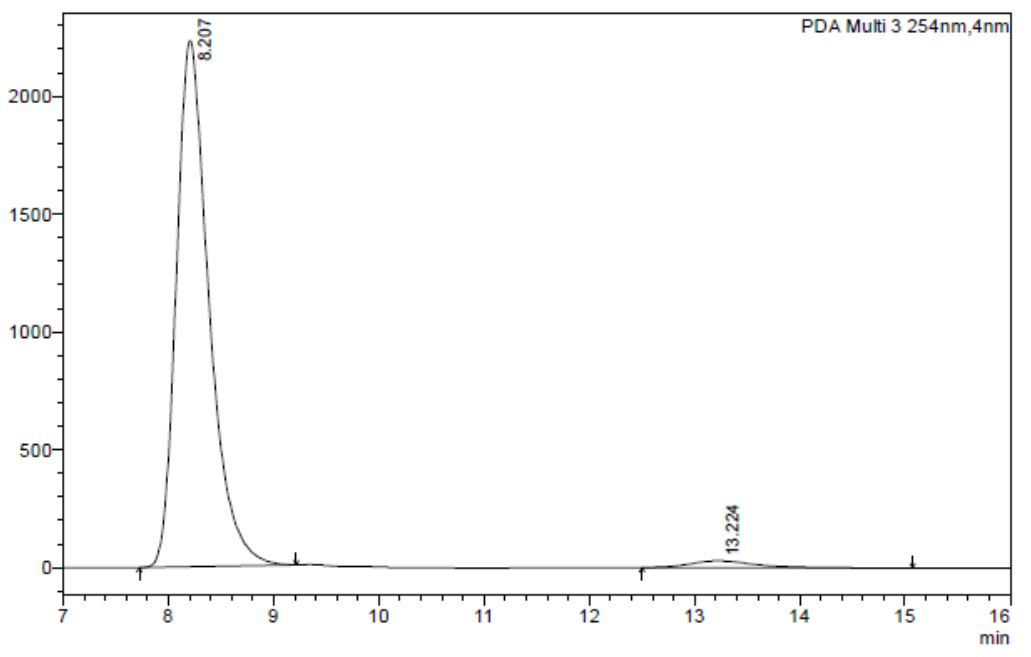

Peak Table

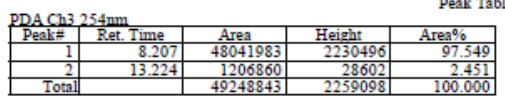


<smiles>O=C1Oc2ccc3ccc(Br)cc3c2C[C@]1(C/C=C/c1ccccc1)Cc1ccccc1</smiles>

94\% ee (OD-H, 95/5 hexanes/i-PrOH, 1.0 ml/min)

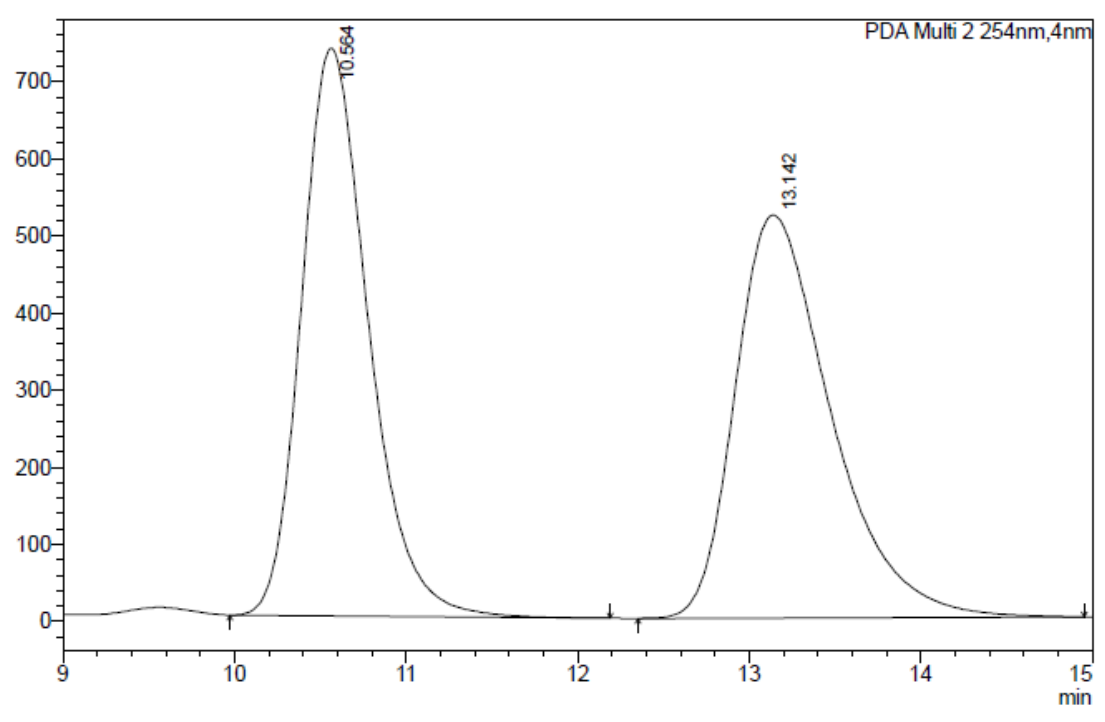

Peak Table
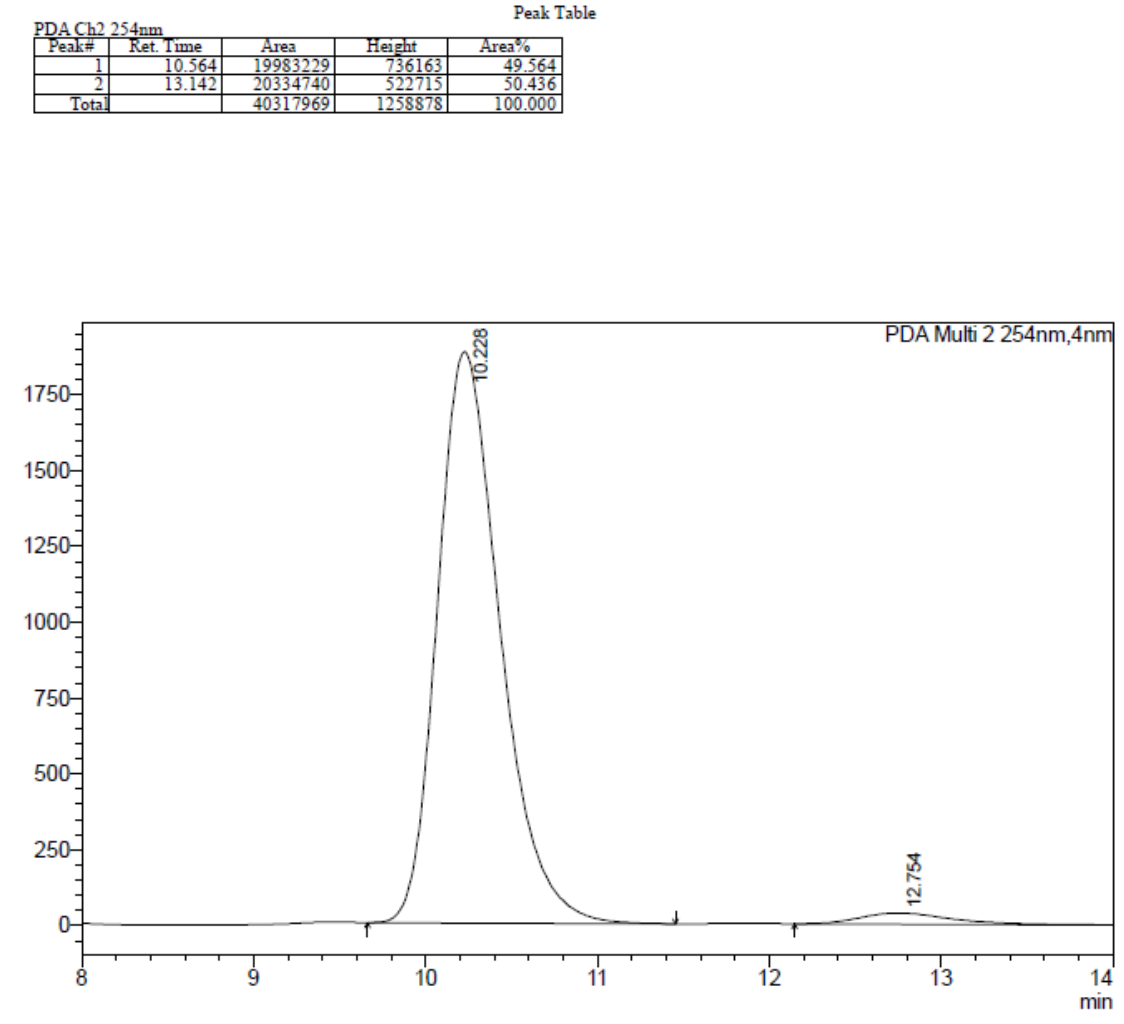

Peak Table

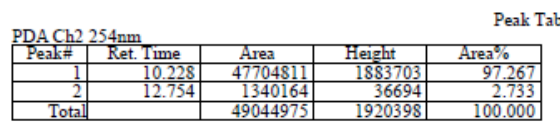


<smiles>COc1ccc2ccc3c(c2c1)CC(C/C=C/c1ccccc1)(Cc1ccccc1)C(=O)O3</smiles>

96\% eе (OD-H, 90/10 hexanes/i-PrOH, 1.0 ml/min)

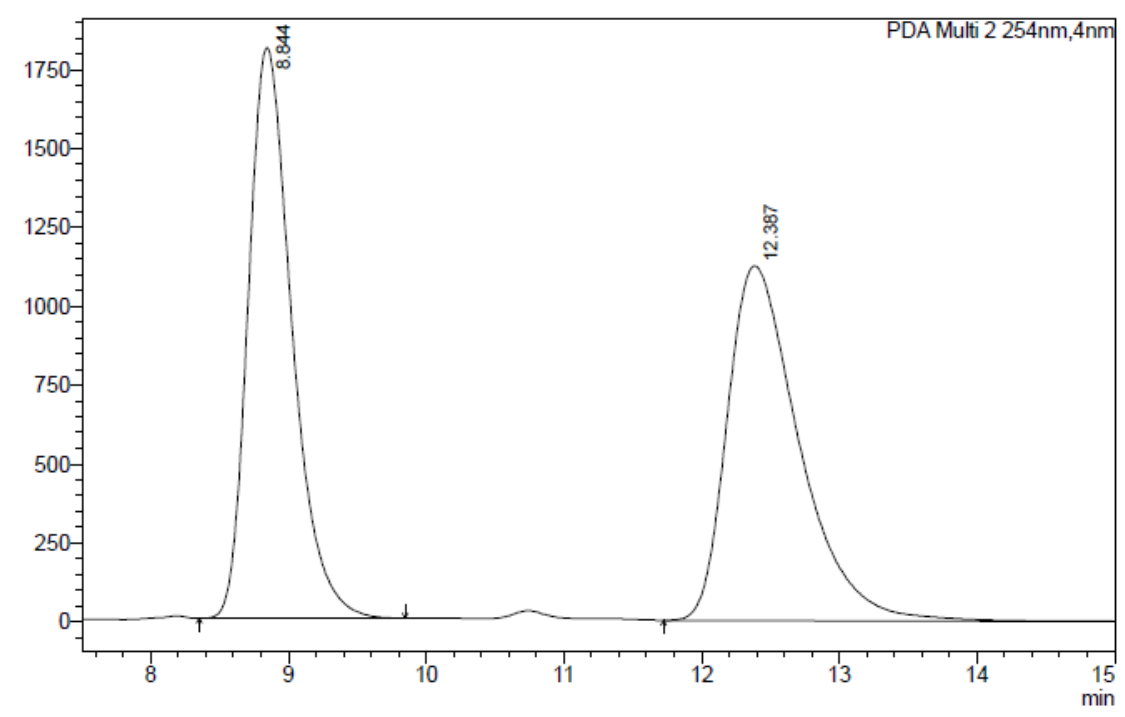

Peak Table
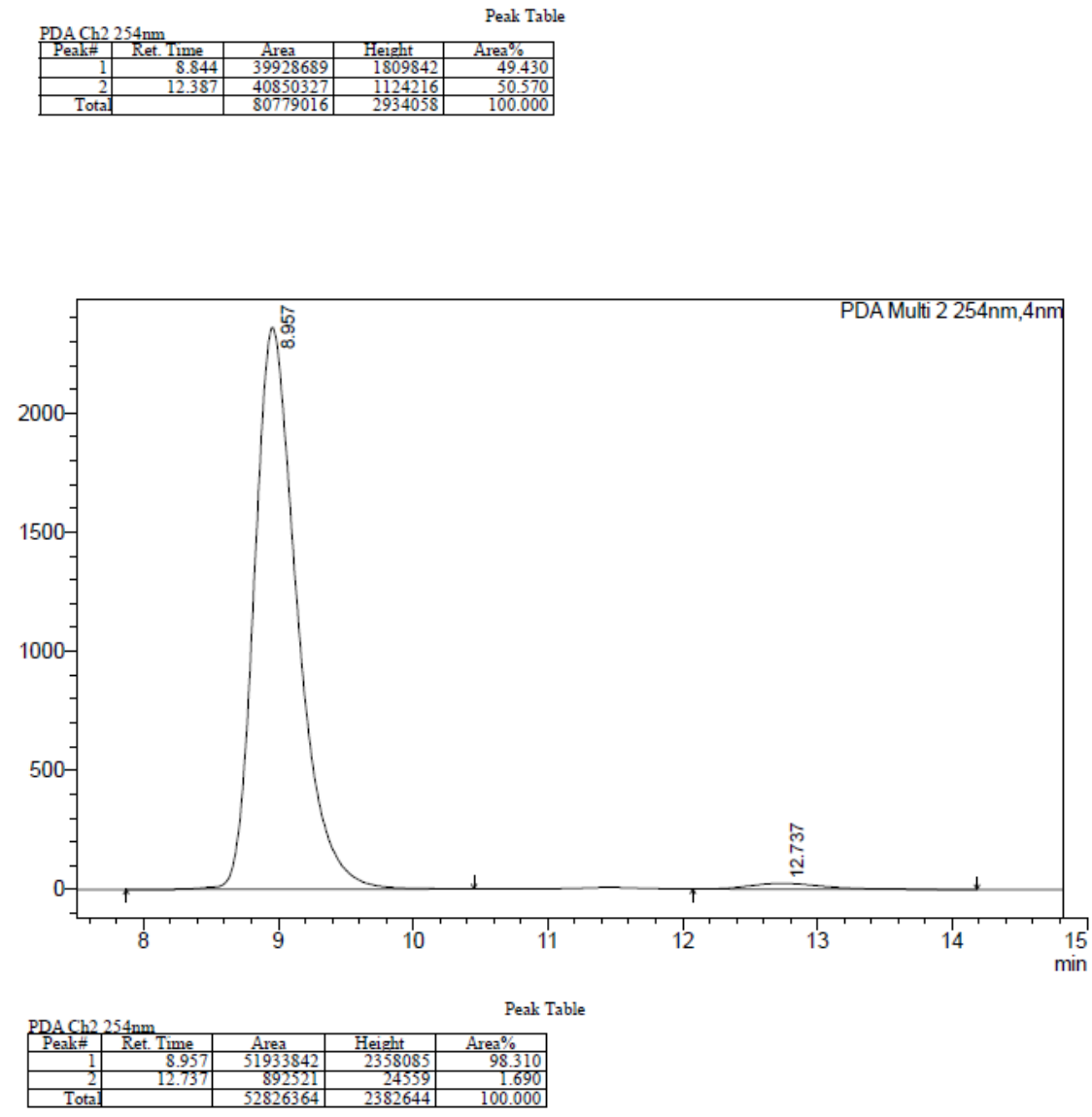


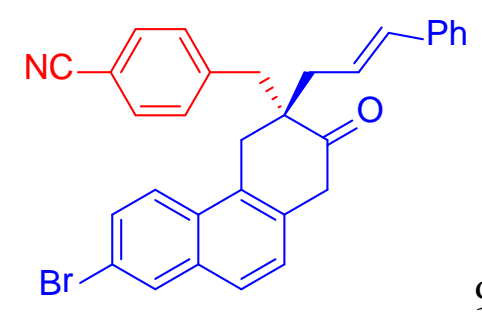

90\% ee (OD-H, 70/30 hexanes/i-PrOH, 1.0 ml/min)

MAU
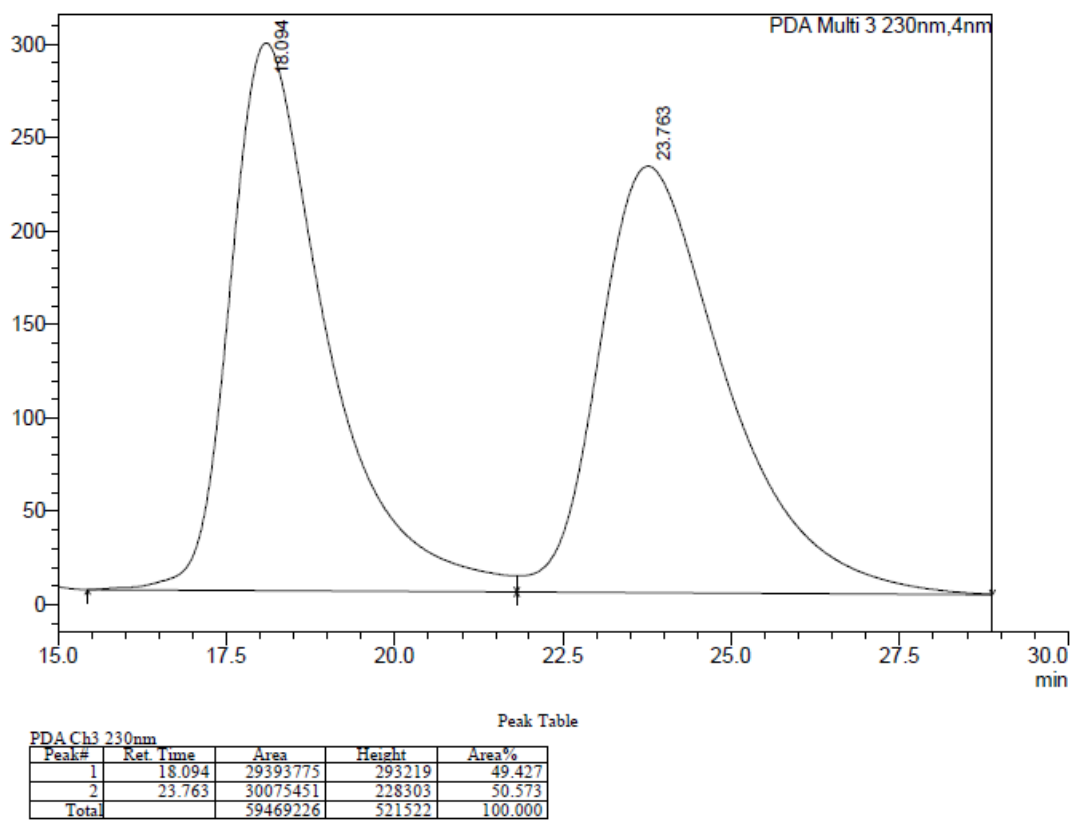

mAU

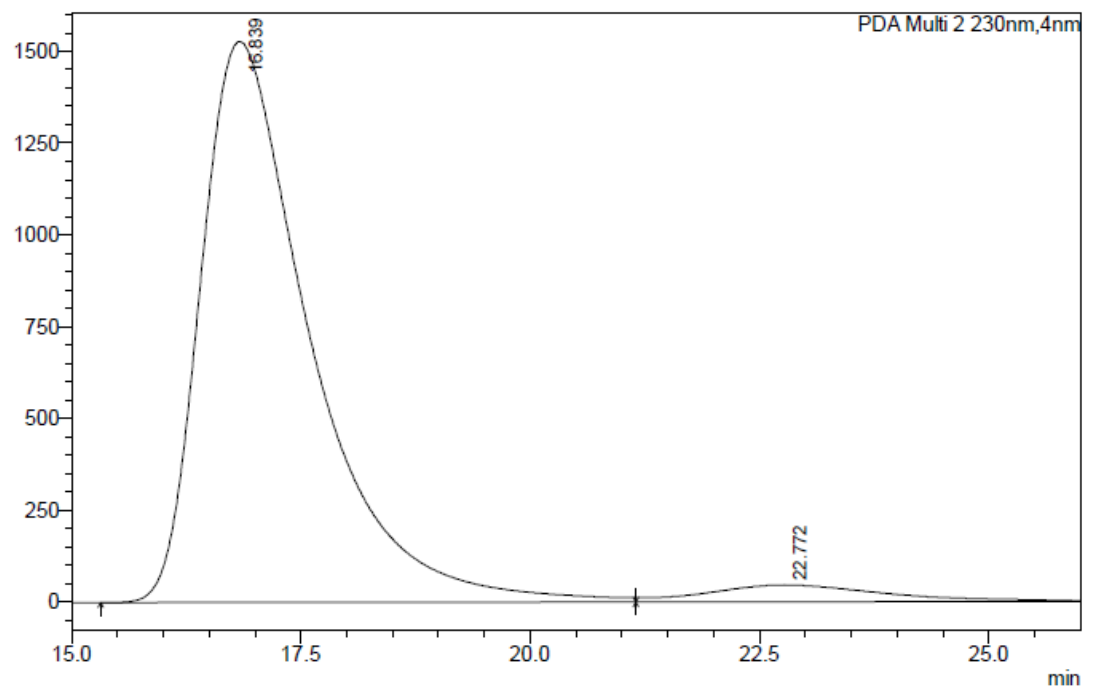

Peak Table

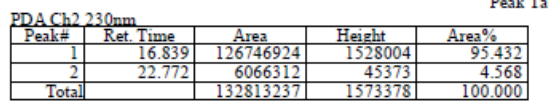


<smiles>O=C1c2ccccc2CC1(C/C=C/c1ccccc1)Cc1ccccc1</smiles>

95\% ee (OJ-H, 70/30 hexanes/i-PrOH, 1.0 ml/min)
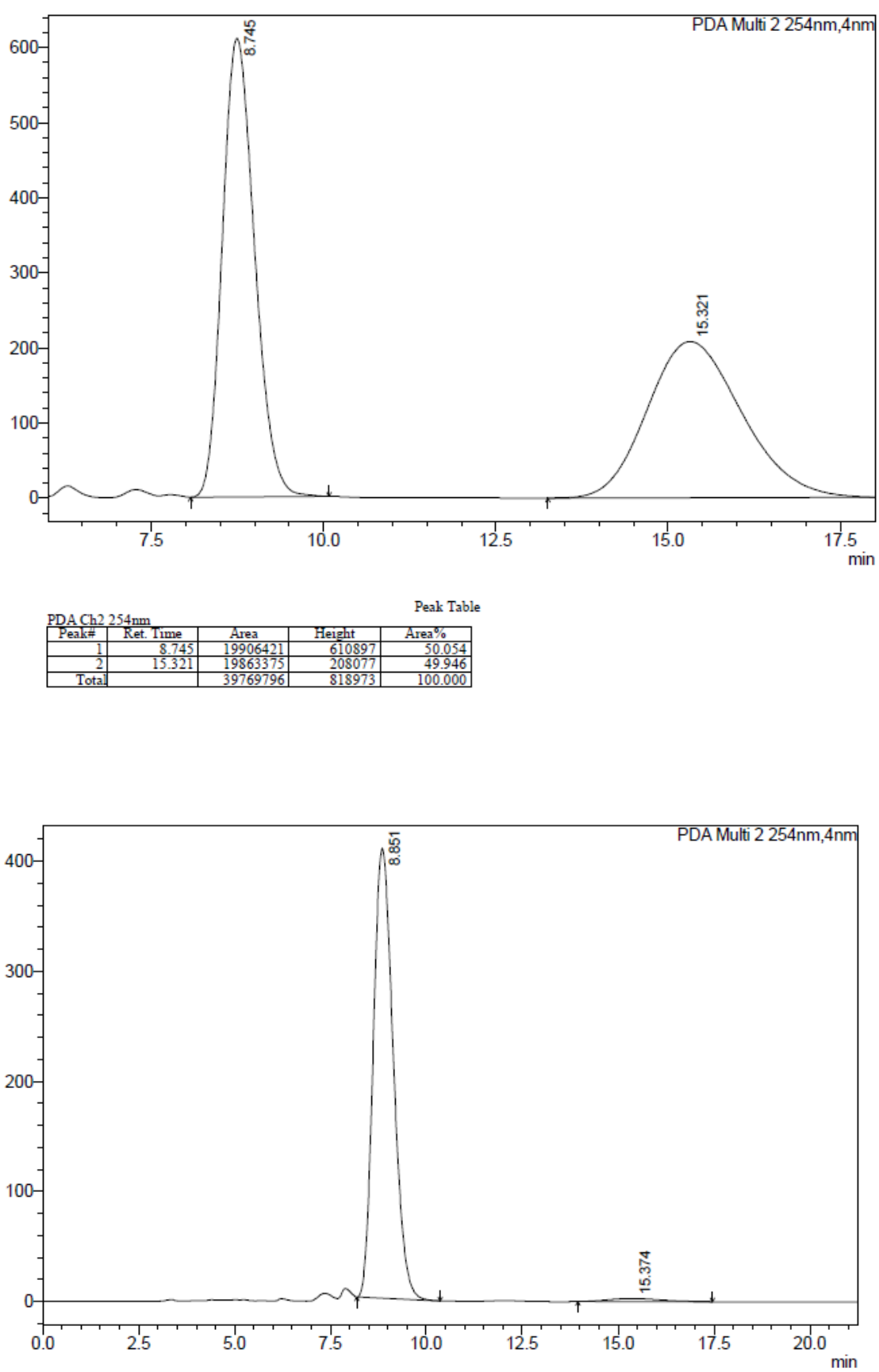

Peak Table

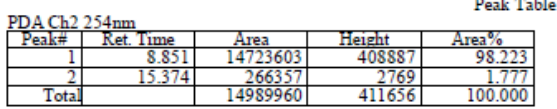



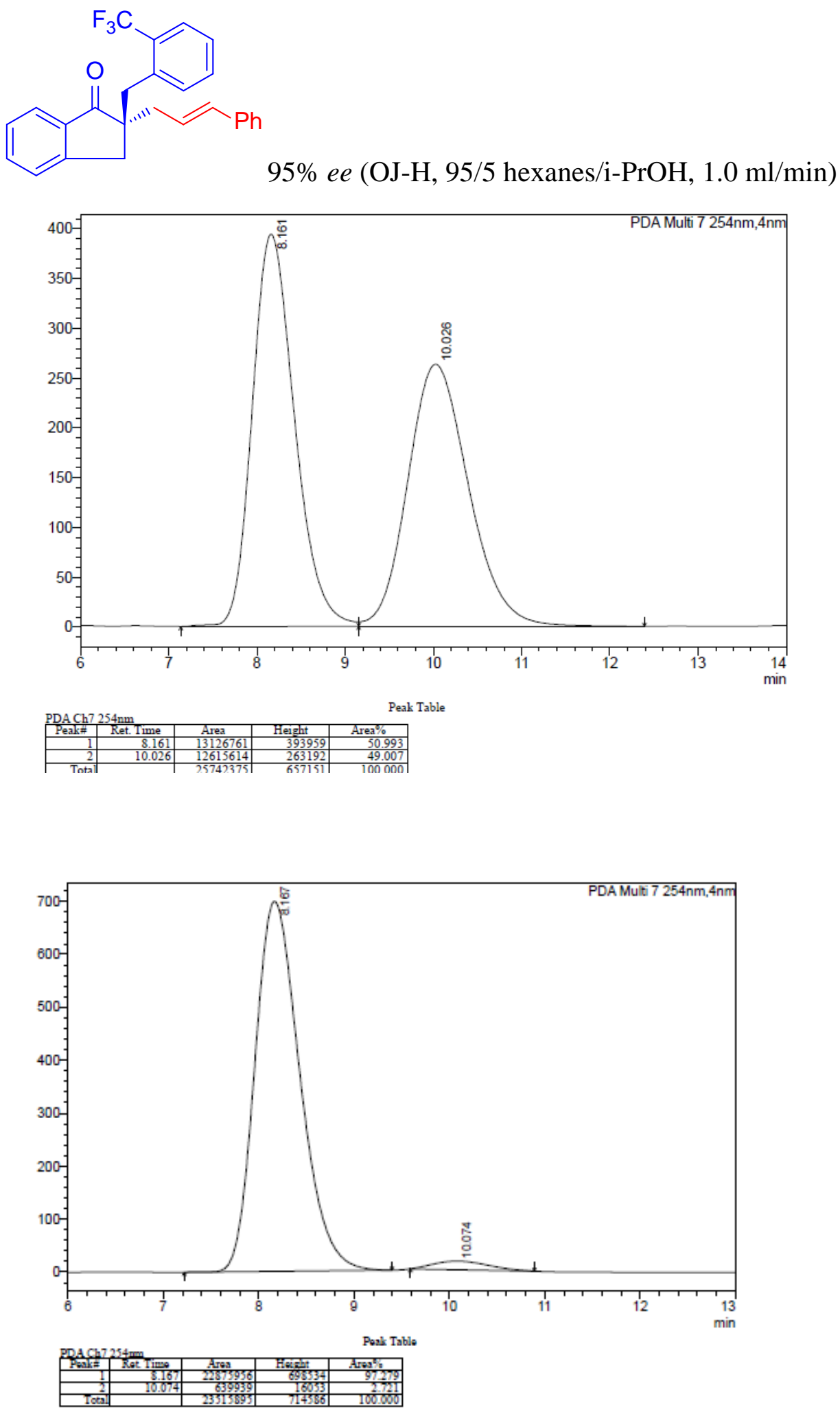

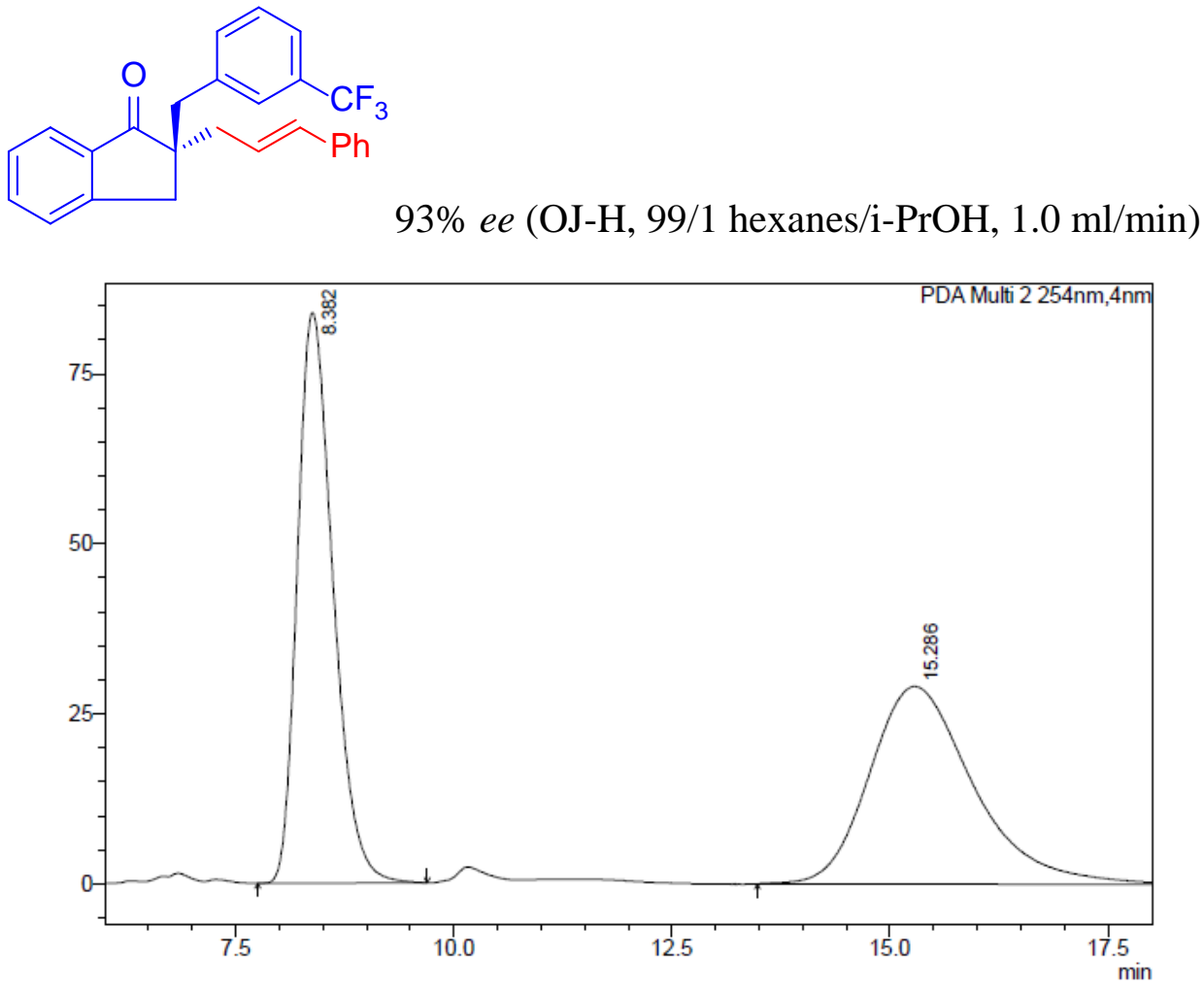

Peak Table
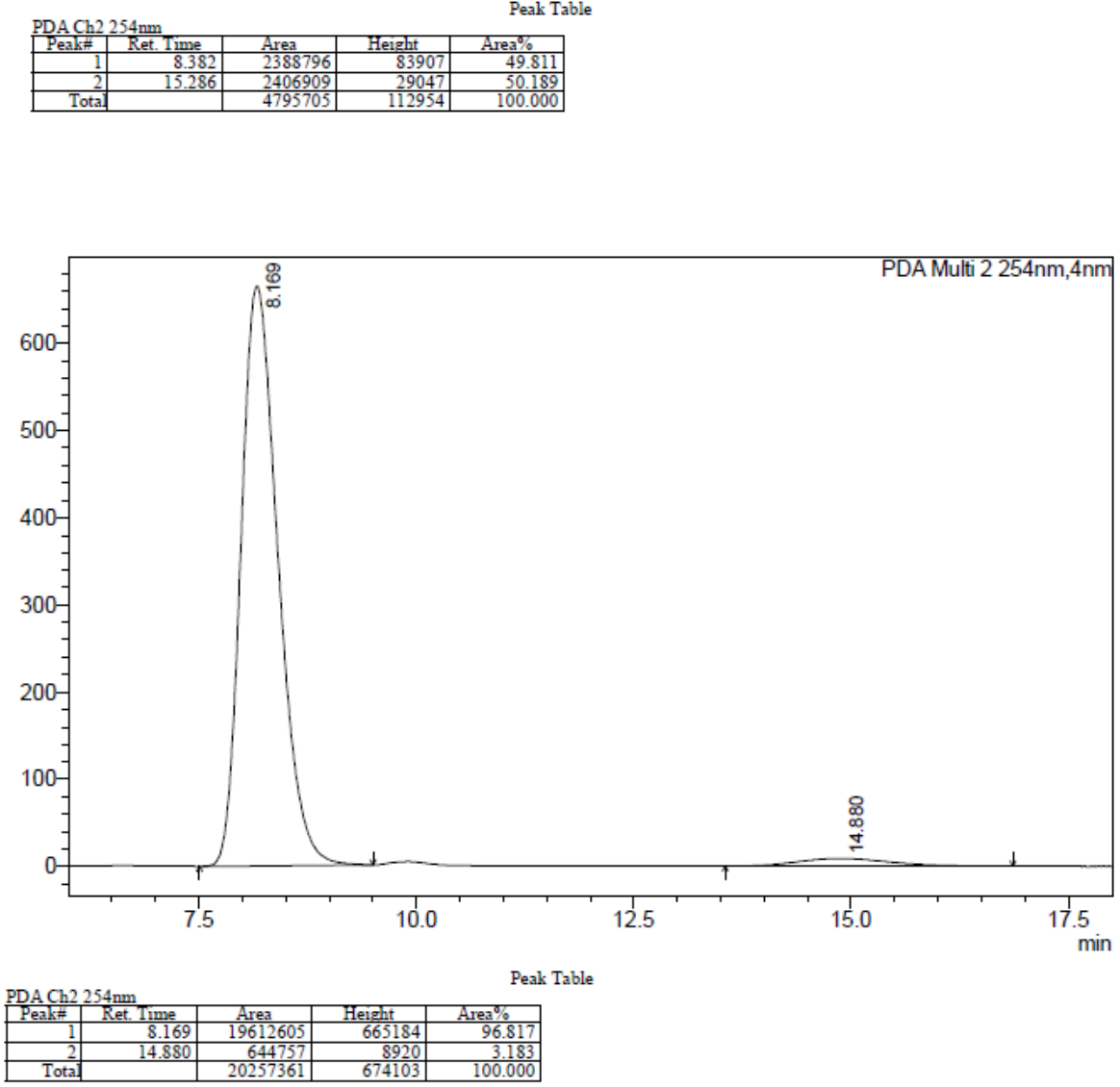

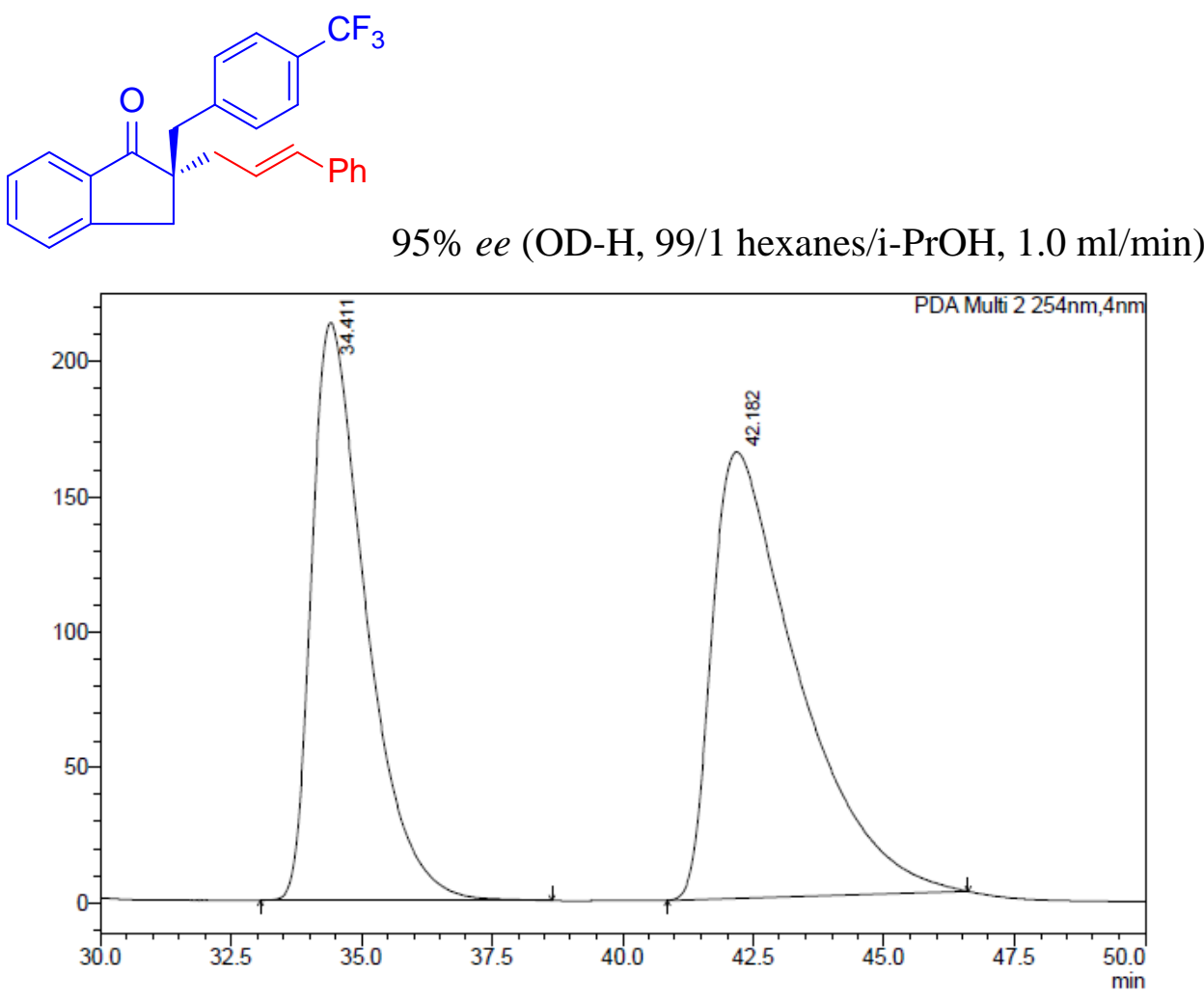

Peak Table
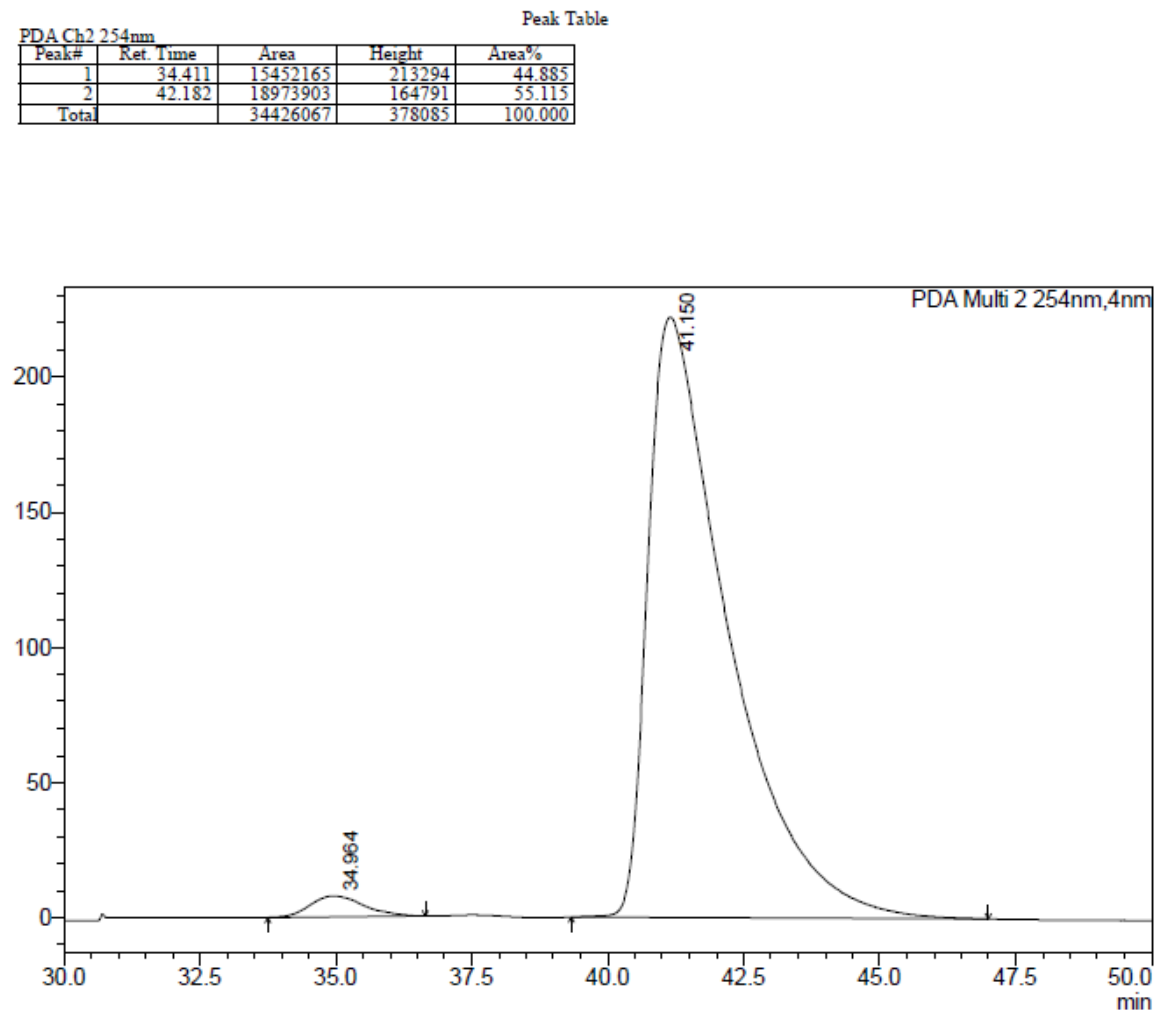

Peak Table

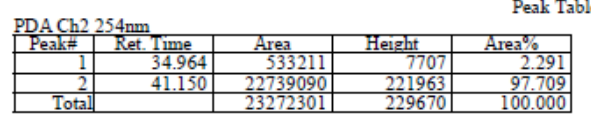


<smiles>O=C1c2ccccc2CC1(C/C=C/c1ccccc1)Cc1cccc(Br)c1</smiles>

99\% eе (IA, 98/2 hexanes/i-PrOH, 1.0 ml/min)

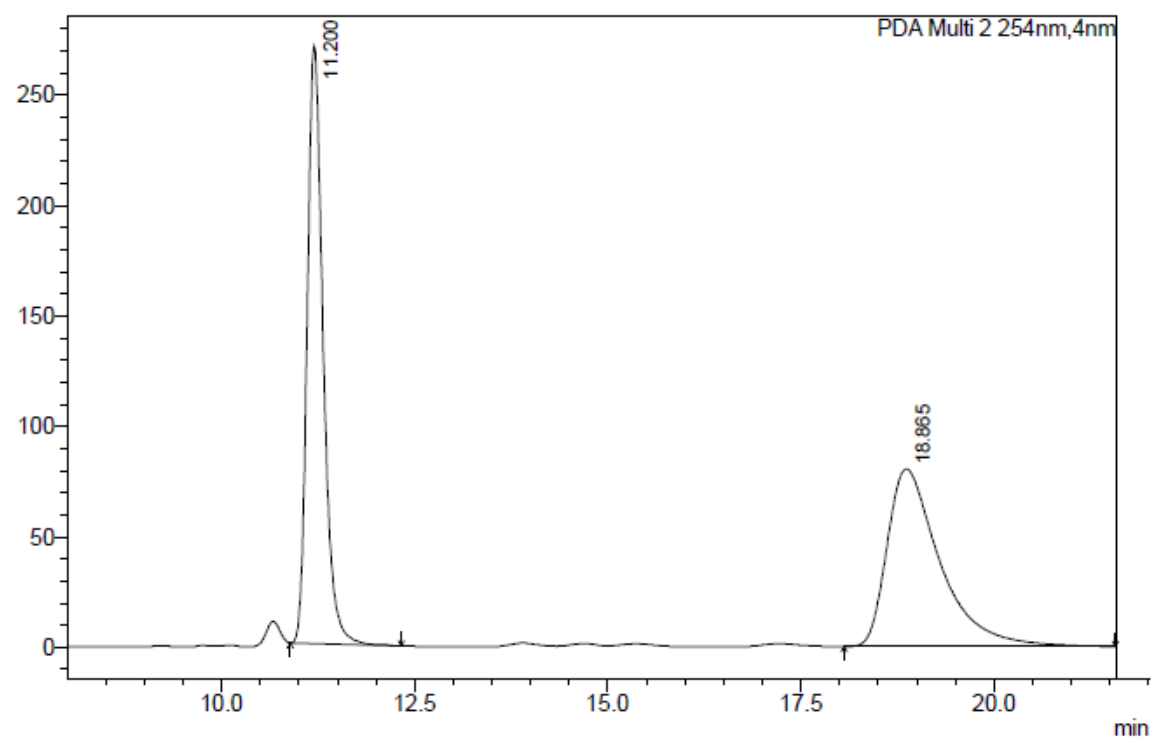

Peak Table
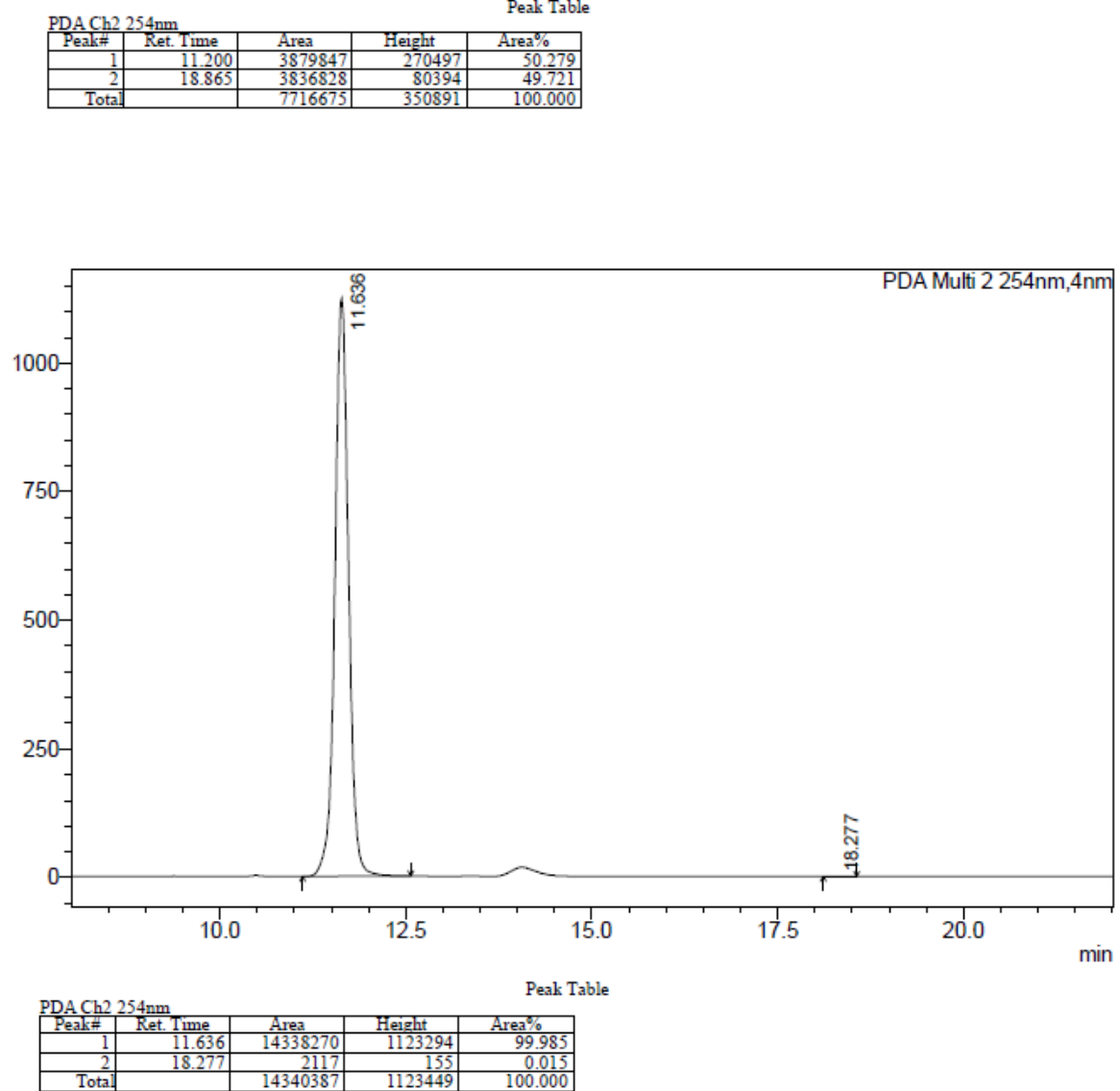
<smiles>O=C1c2ccccc2C[C@]1(C/C=C/c1ccccc1)Cc1ccsc1</smiles>

89\% eе (OJ-H, 80/20 hexanes/i-PrOH, 1.0 ml/min)
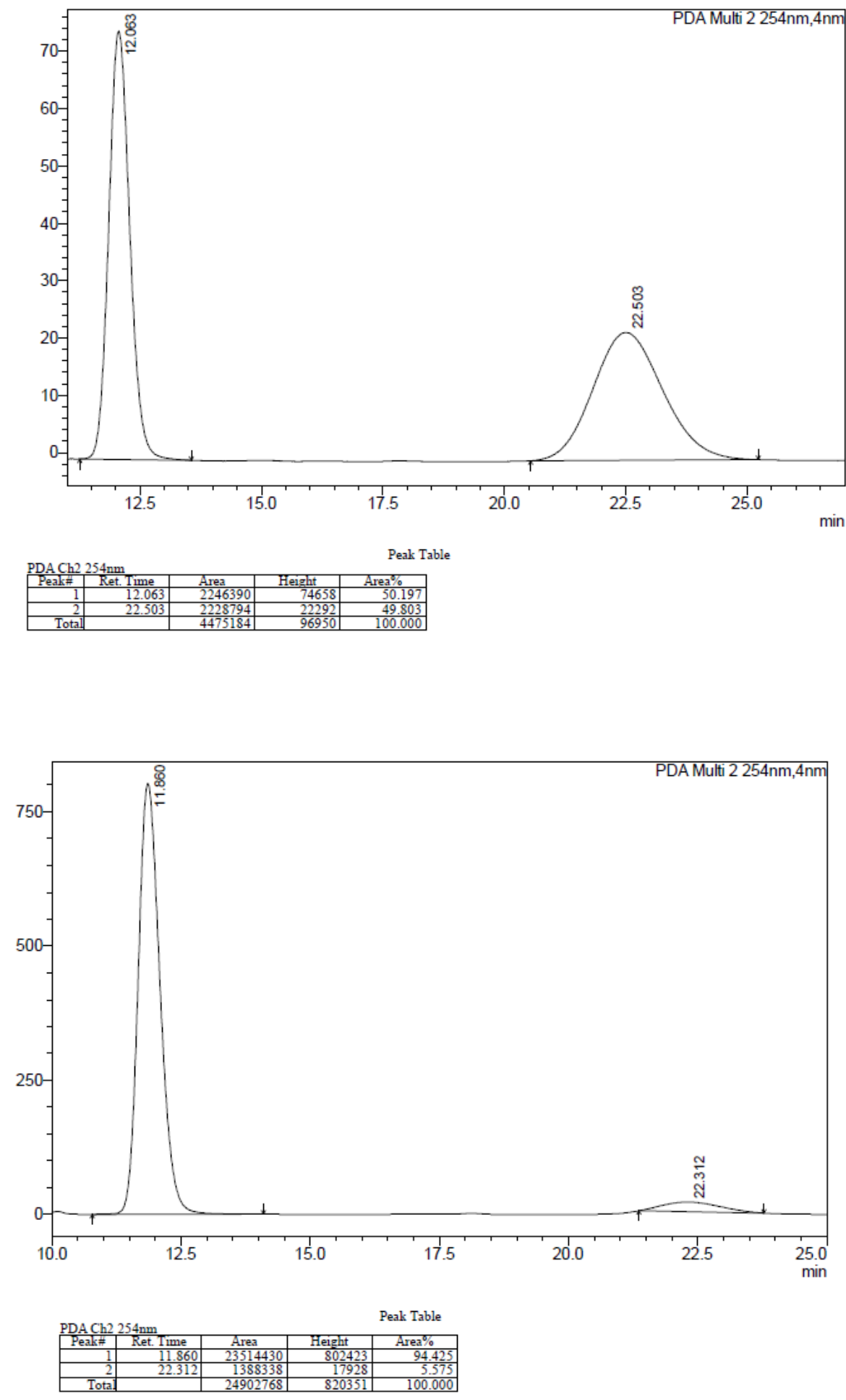
<smiles>O=C1c2ccccc2C[C@]1(C/C=C/c1ccccc1)Cc1ccco1</smiles>

85\% ee (OD-H, 98/2 hexanes/i-PrOH, 1.0 ml/min)

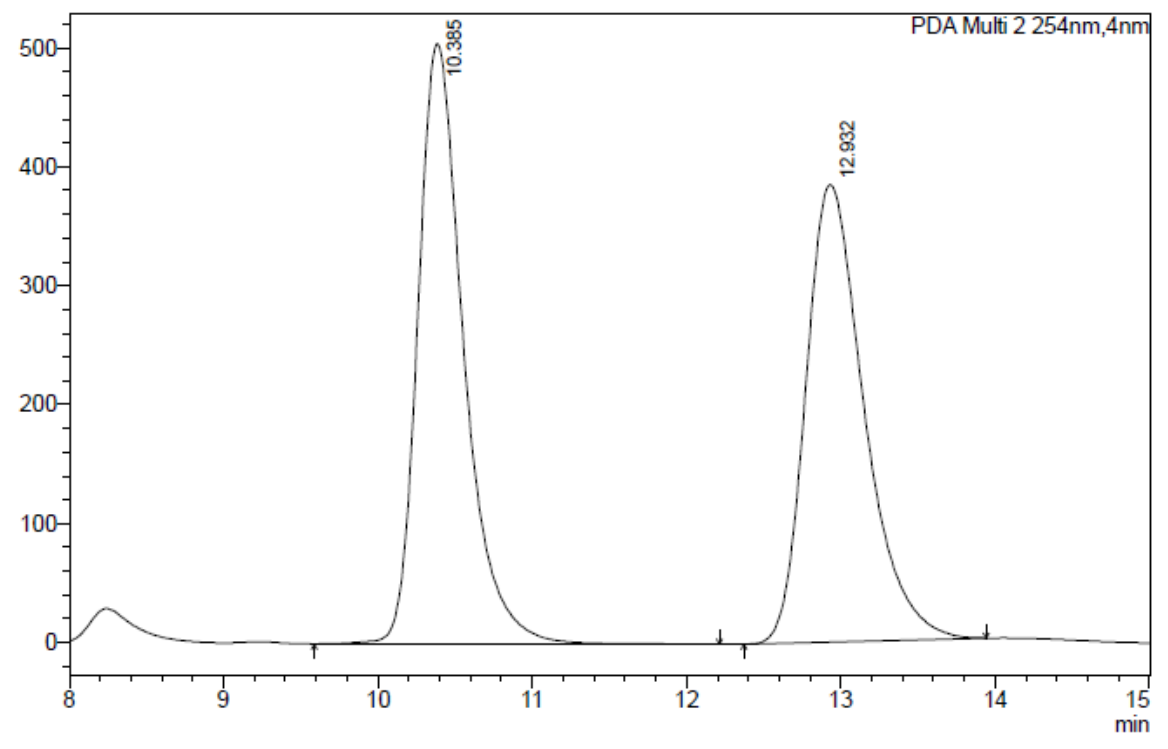

Peak Table
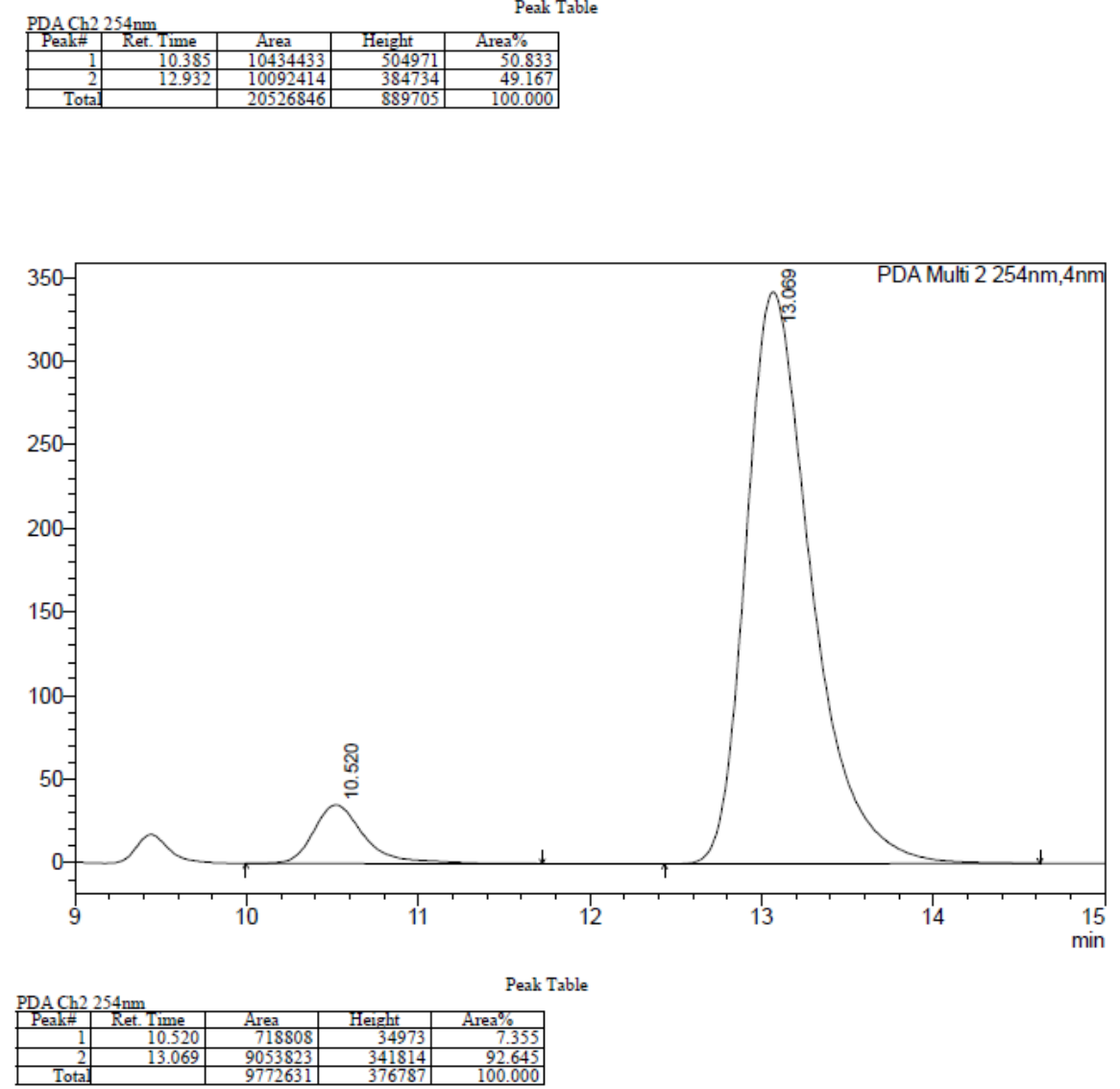
<smiles>CC(C)=CC[C@]1(Cc2cccc(Br)c2)Cc2ccccc2C1=O</smiles>

91\% ee (OJ-H, 99/1 hexanes/i-PrOH, 1.0 ml/min)

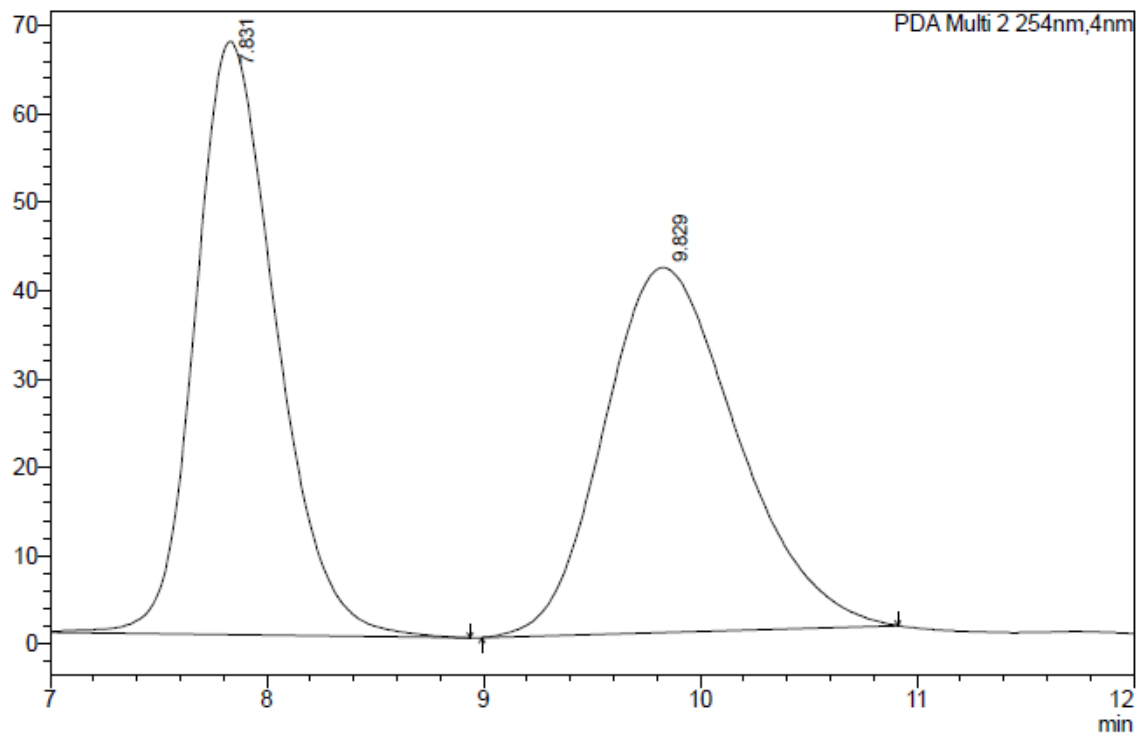

Peak Table
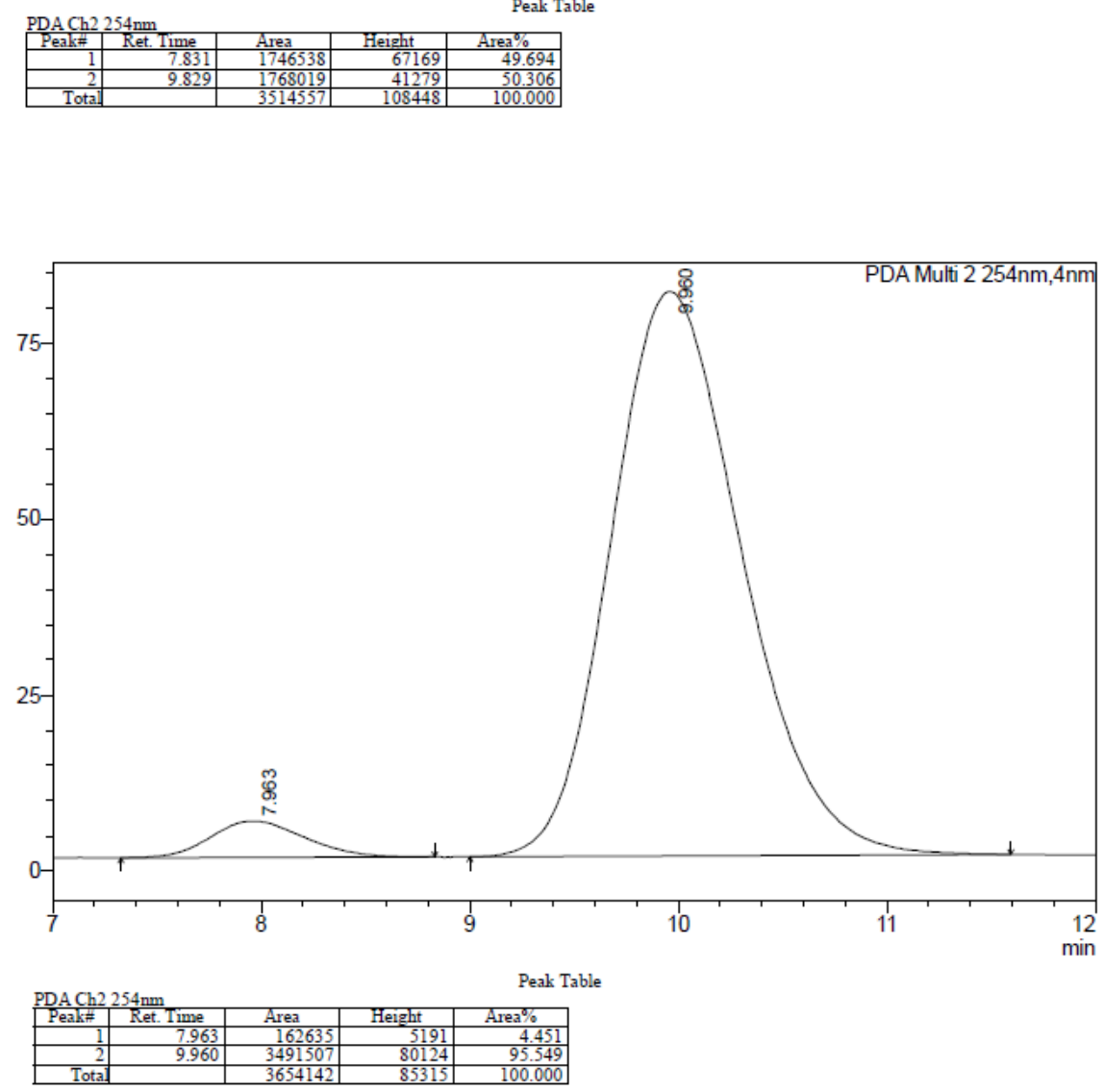

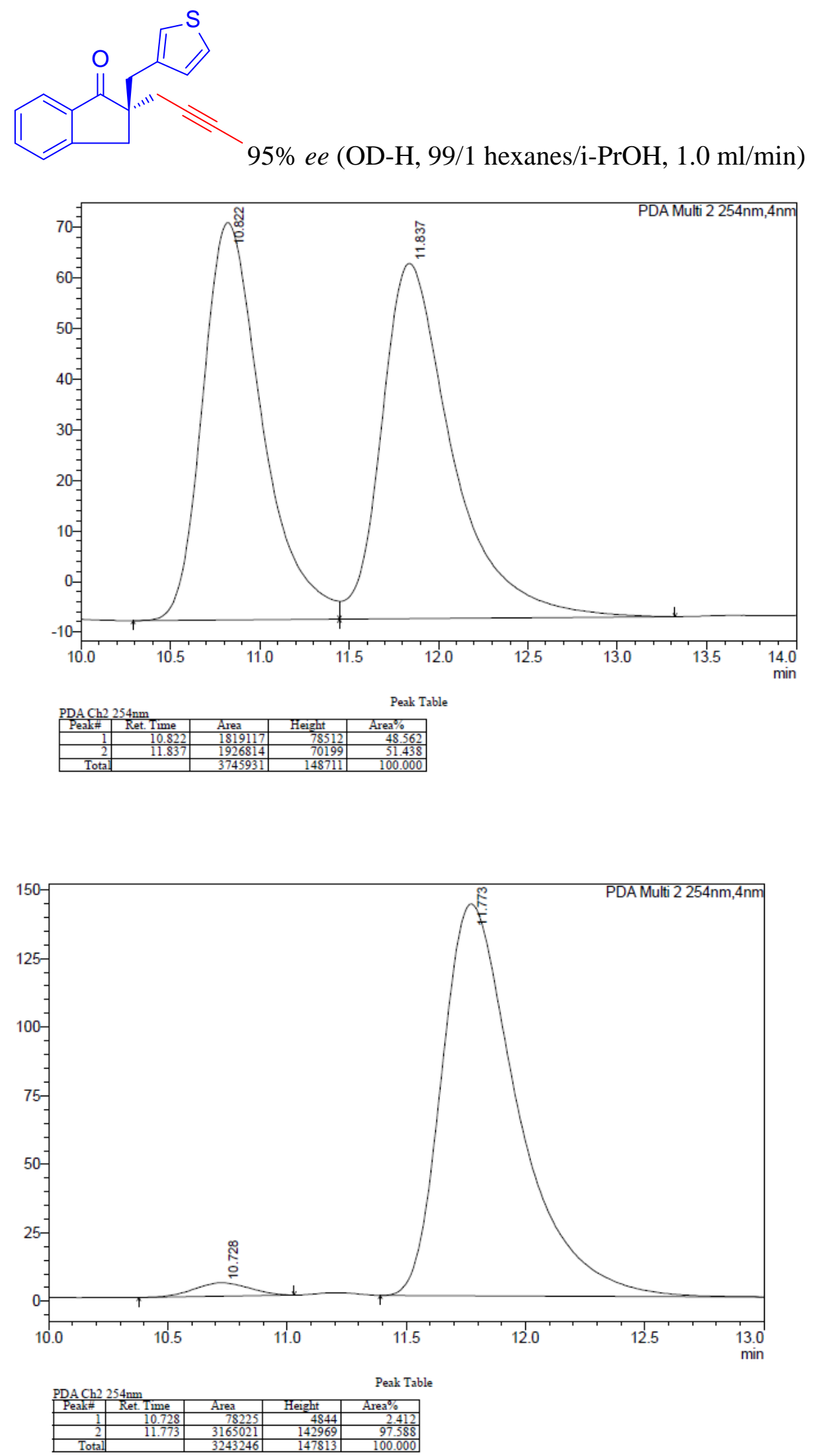

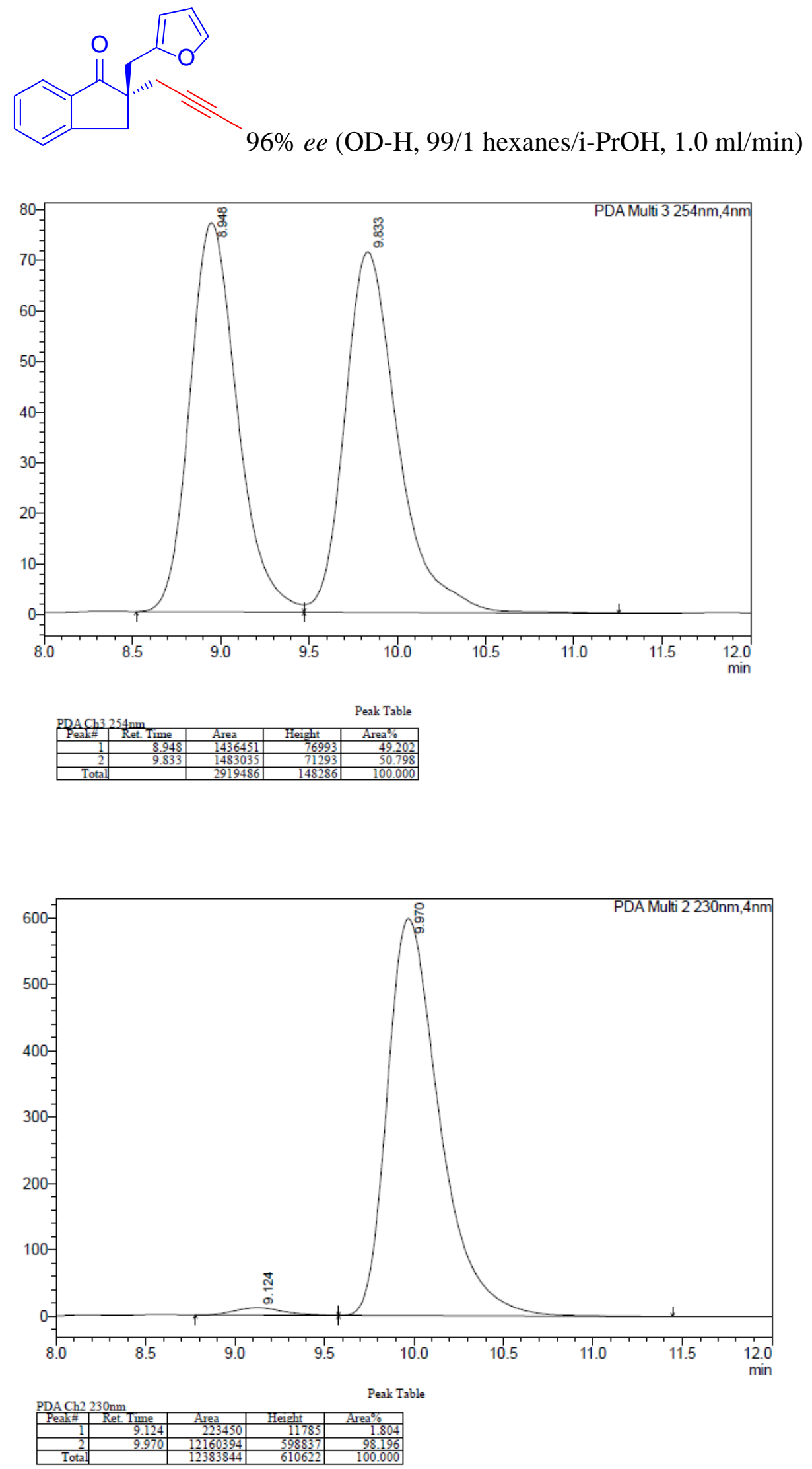
<smiles>COC(=O)C[C@]1(Cc2cccc(C(F)(F)F)c2)Cc2ccccc2C1=O</smiles>

90\% ee (OD-H, 90/10 hexanes/i-PrOH, 1.0 ml/min)
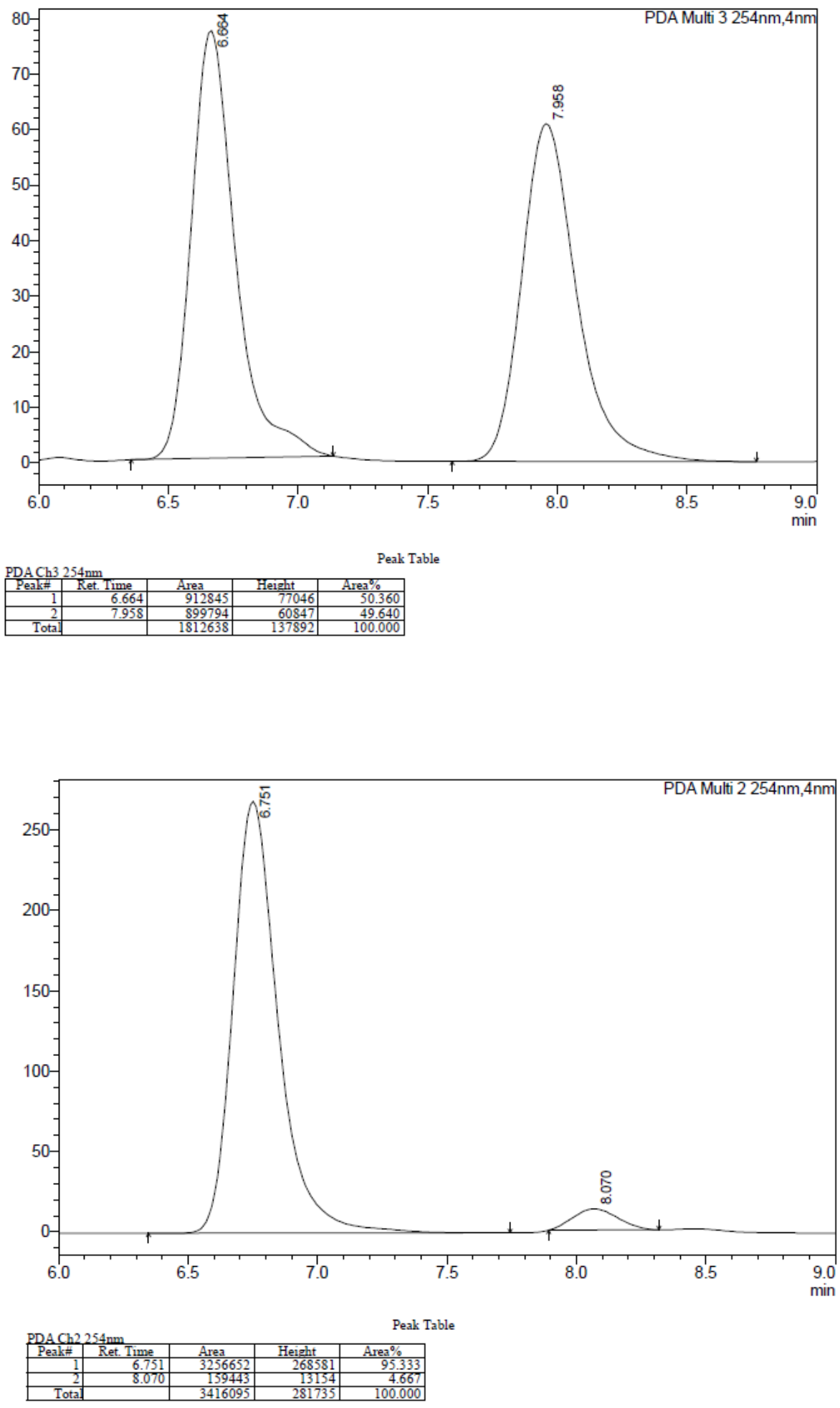
<smiles>COC(=O)C[C@]1(Cc2ccc(C(F)(F)F)cc2)Cc2ccccc2C1=O</smiles>

87\% ee (OD-H, 90/10 hexanes/i-PrOH, 1.0 ml/min)
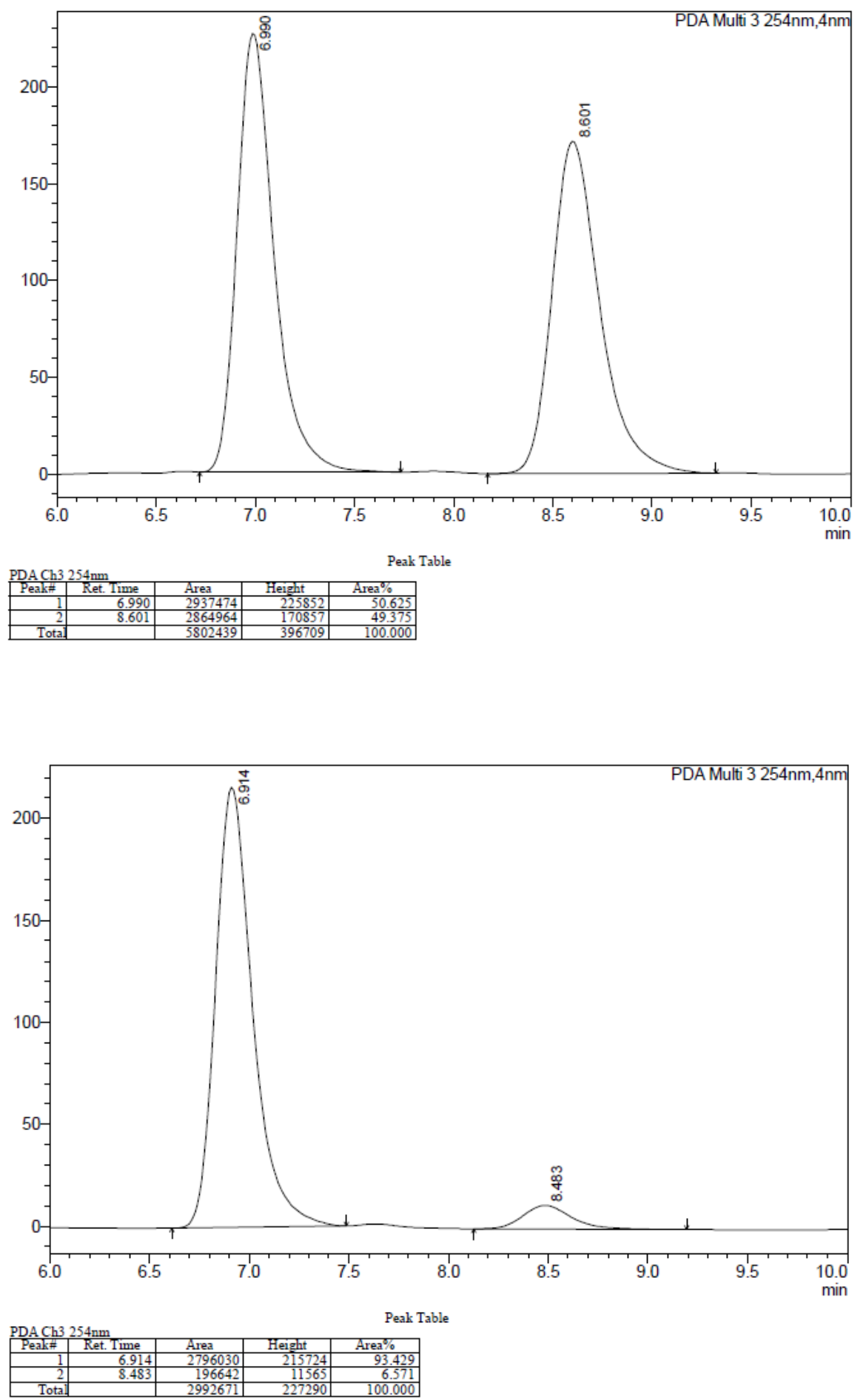

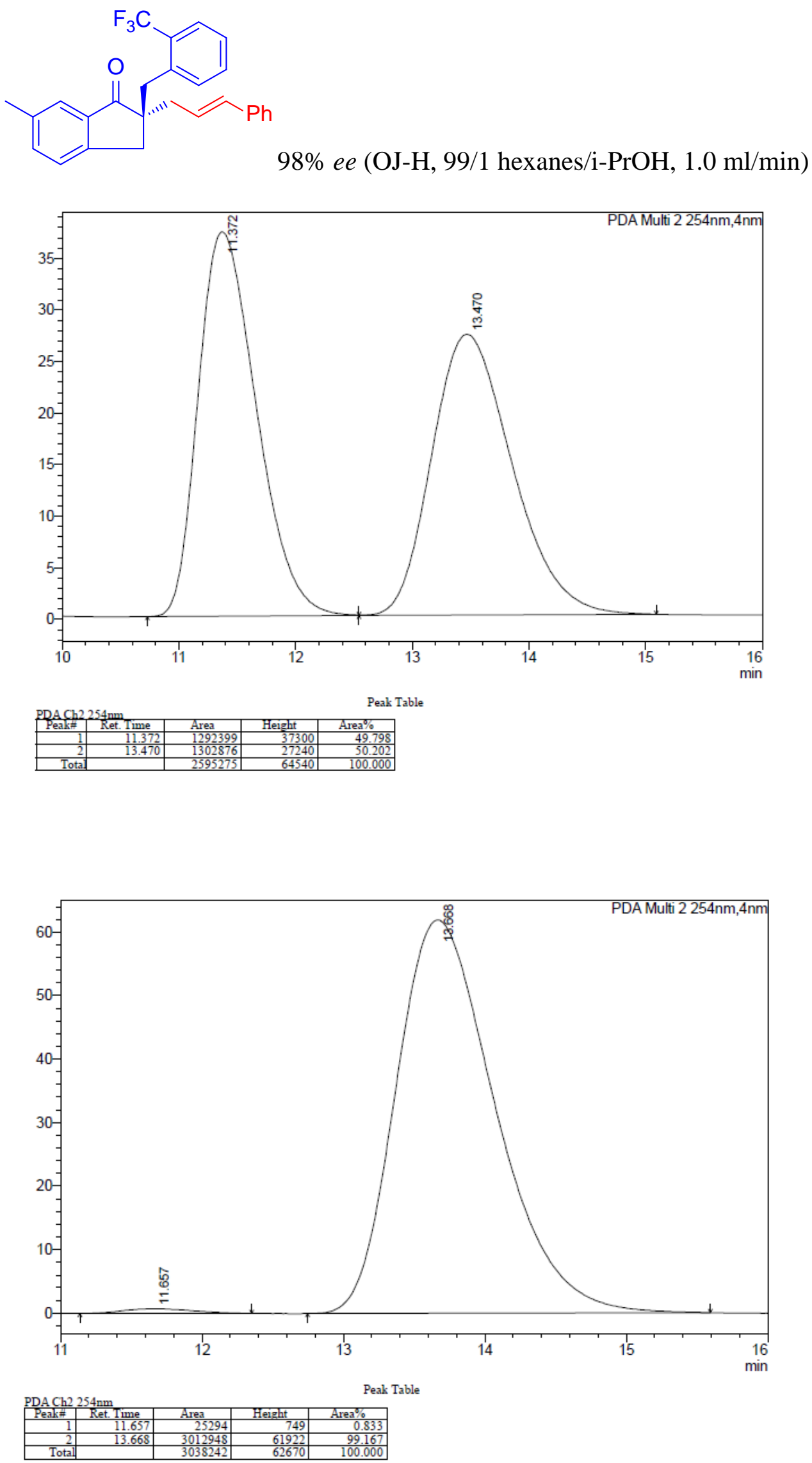
<smiles>O=C1c2ccc(Cl)cc2CC1(C/C=C/c1ccccc1)Cc1ccccc1</smiles>

92\% ee (OJ-H, 90/10 hexanes/i-PrOH, 1.0 ml/min)
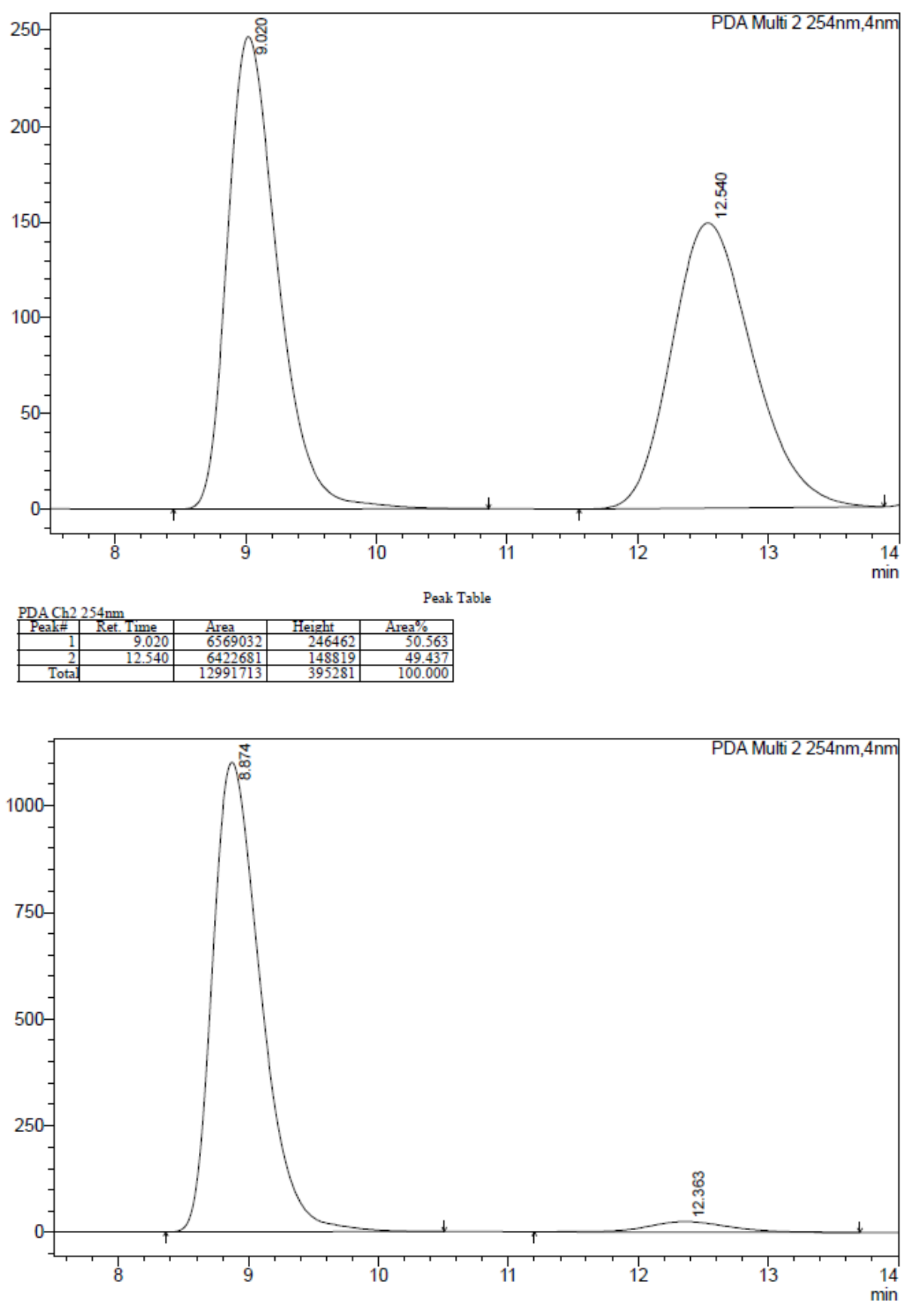

Peak Table

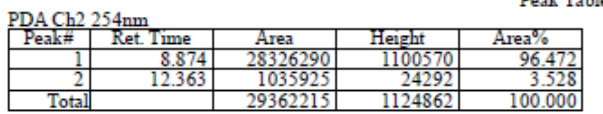



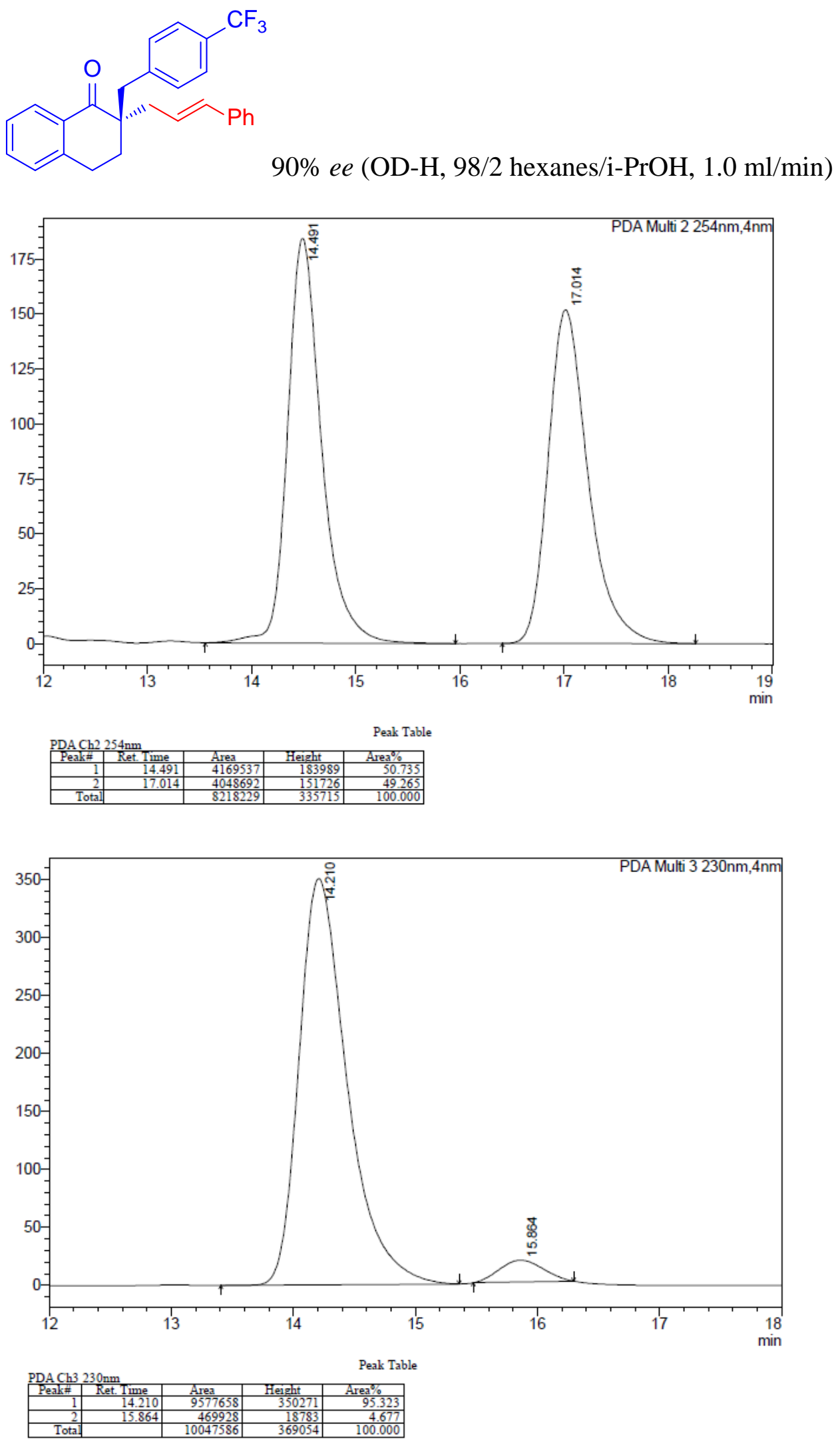
<smiles>O=C1c2ccccc2CC[C@]1(C/C=C/c1ccccc1)Cc1cccc(C(F)(F)F)c1</smiles>

91\% ee (OJ-H, 90/10 hexanes/i-PrOH, 1.0 ml/min)
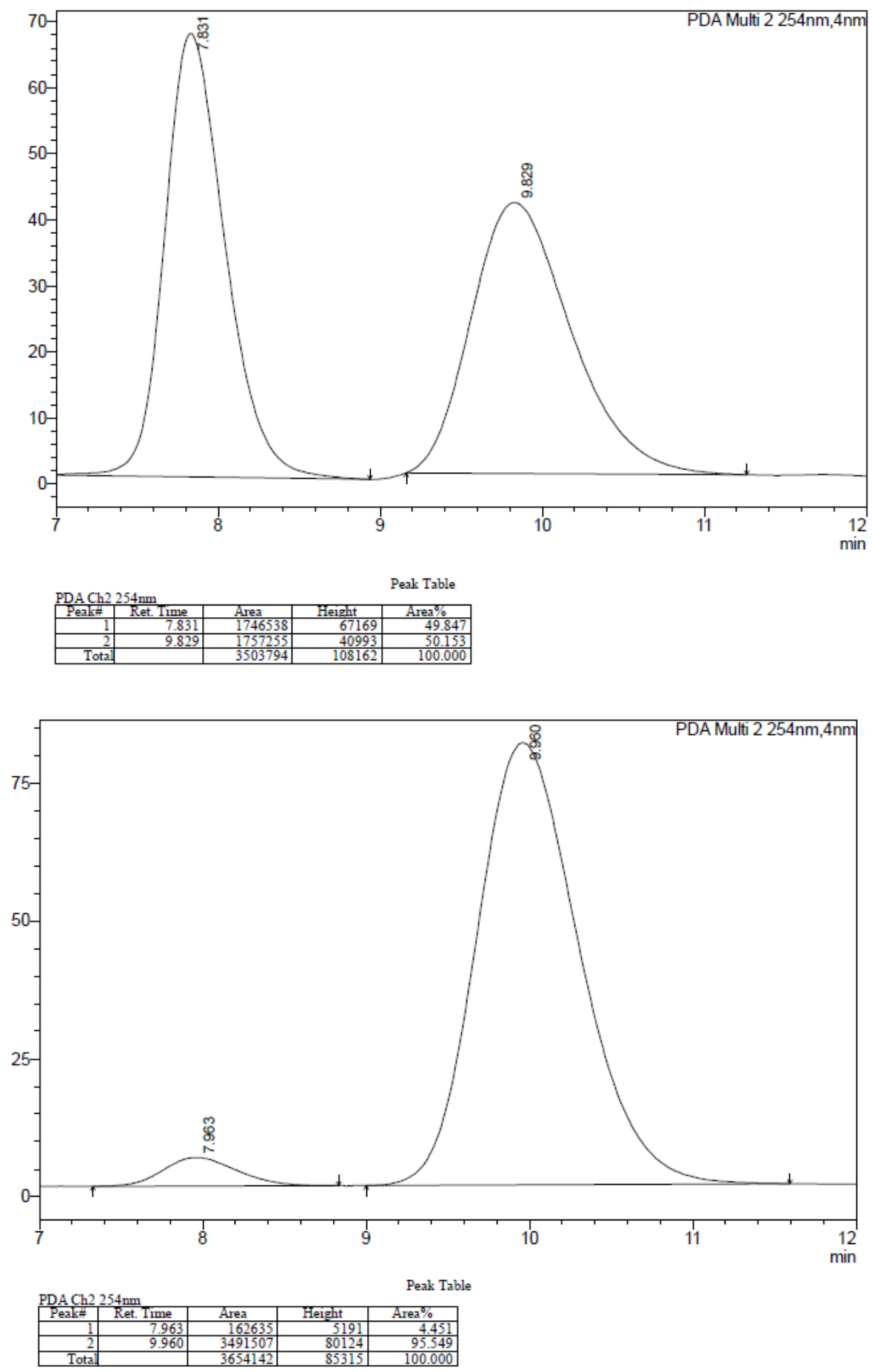

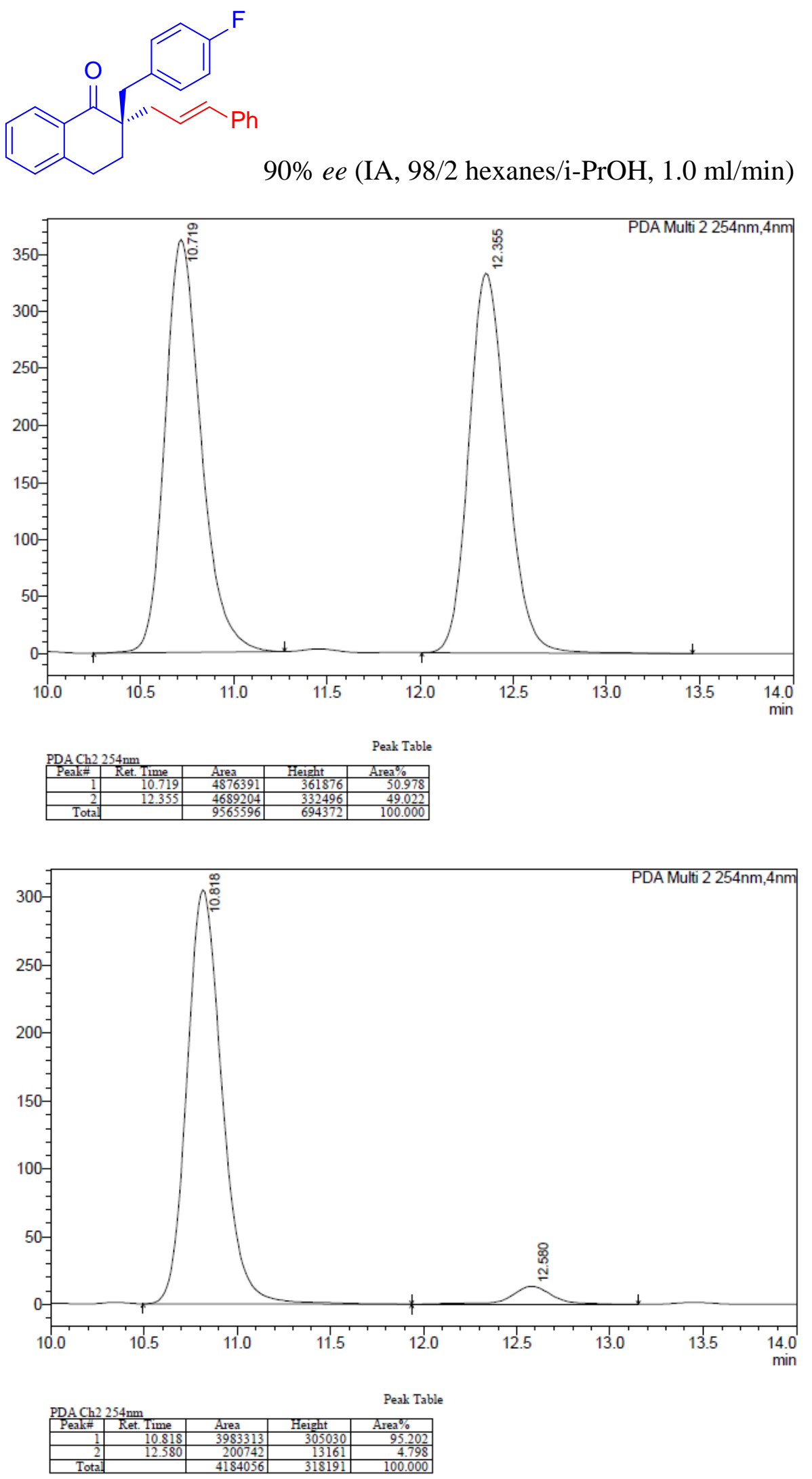
<smiles>CC(C)=CC[C@]1(Cc2ccc(C(F)(F)F)cc2)CCc2ccccc2C1=O</smiles>

92\% ee (OJ-H, 85/15 hexanes/i-PrOH, 1.0 ml/min)

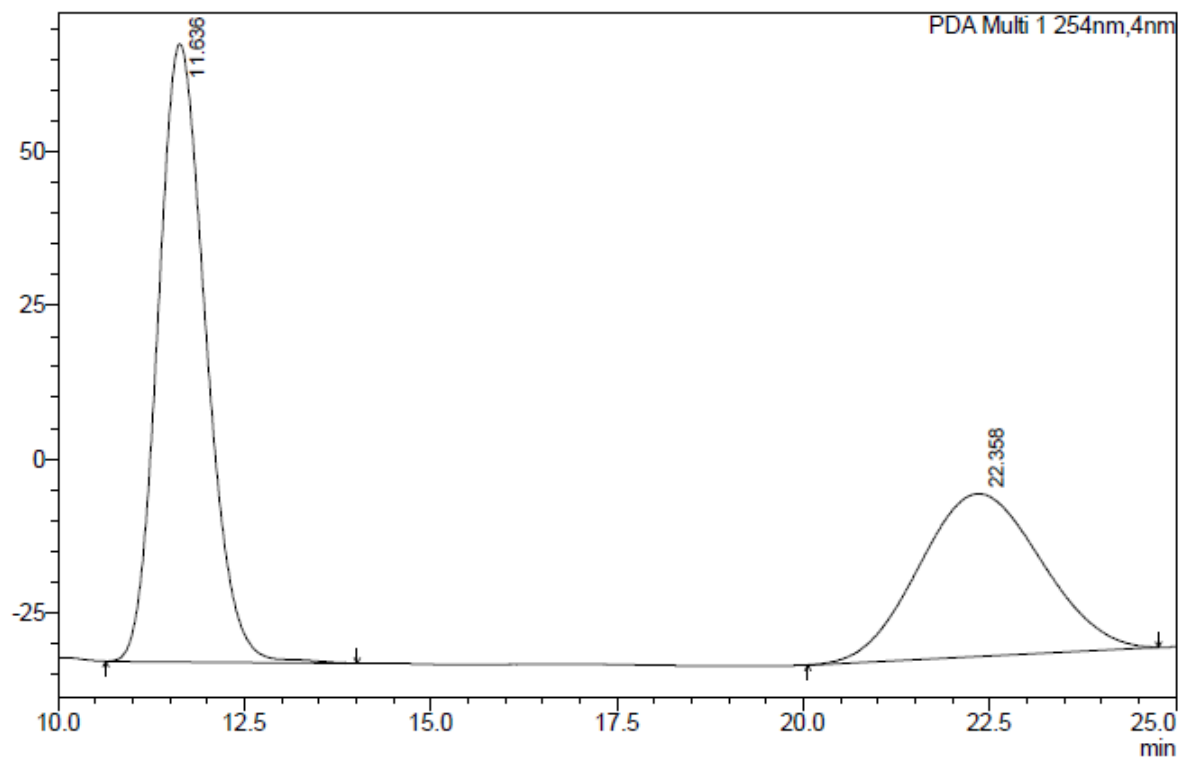

Peak Table
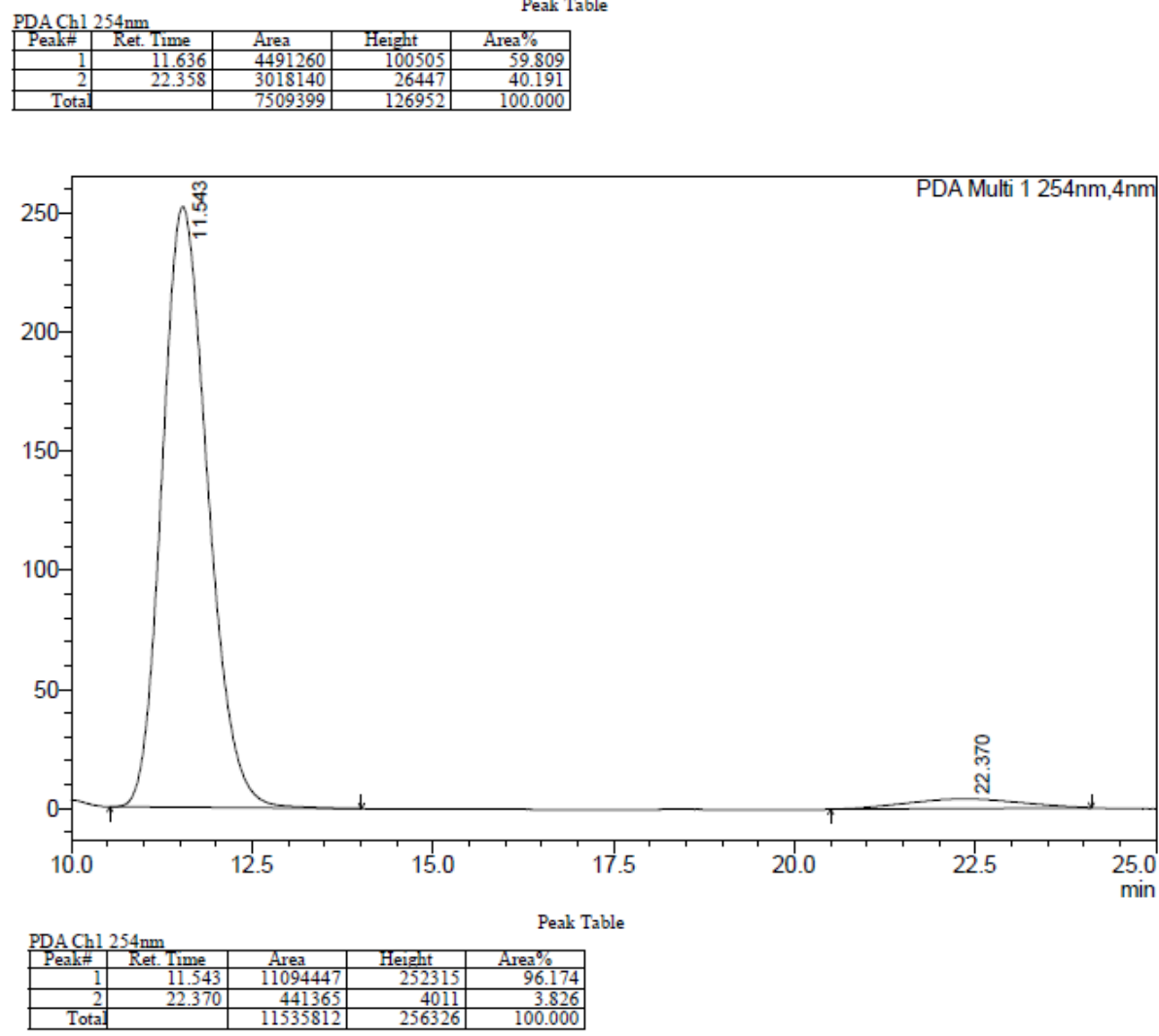
<smiles>CC#CC[C@]1(Cc2ccc(Br)cc2)CCc2ccccc2C1=O</smiles>

87\% ee (OD-H, 98/2 hexanes/i-PrOH, 1.0 ml/min)

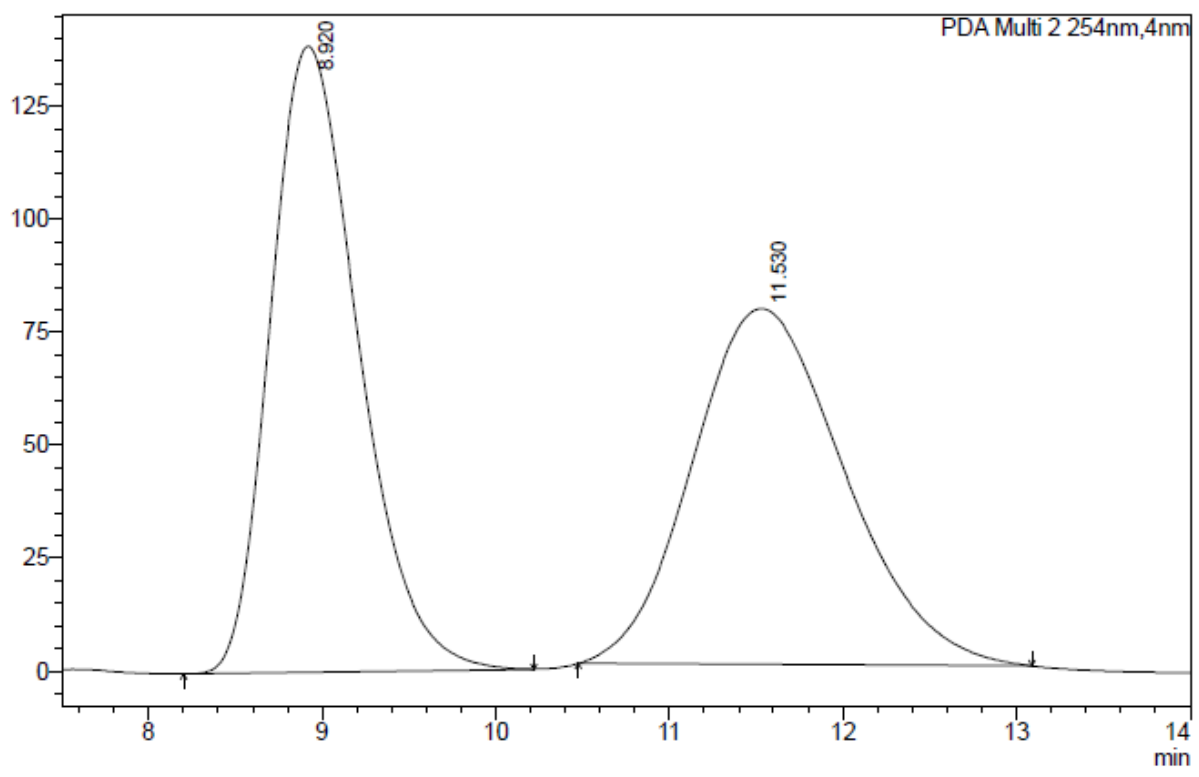

Peak Table
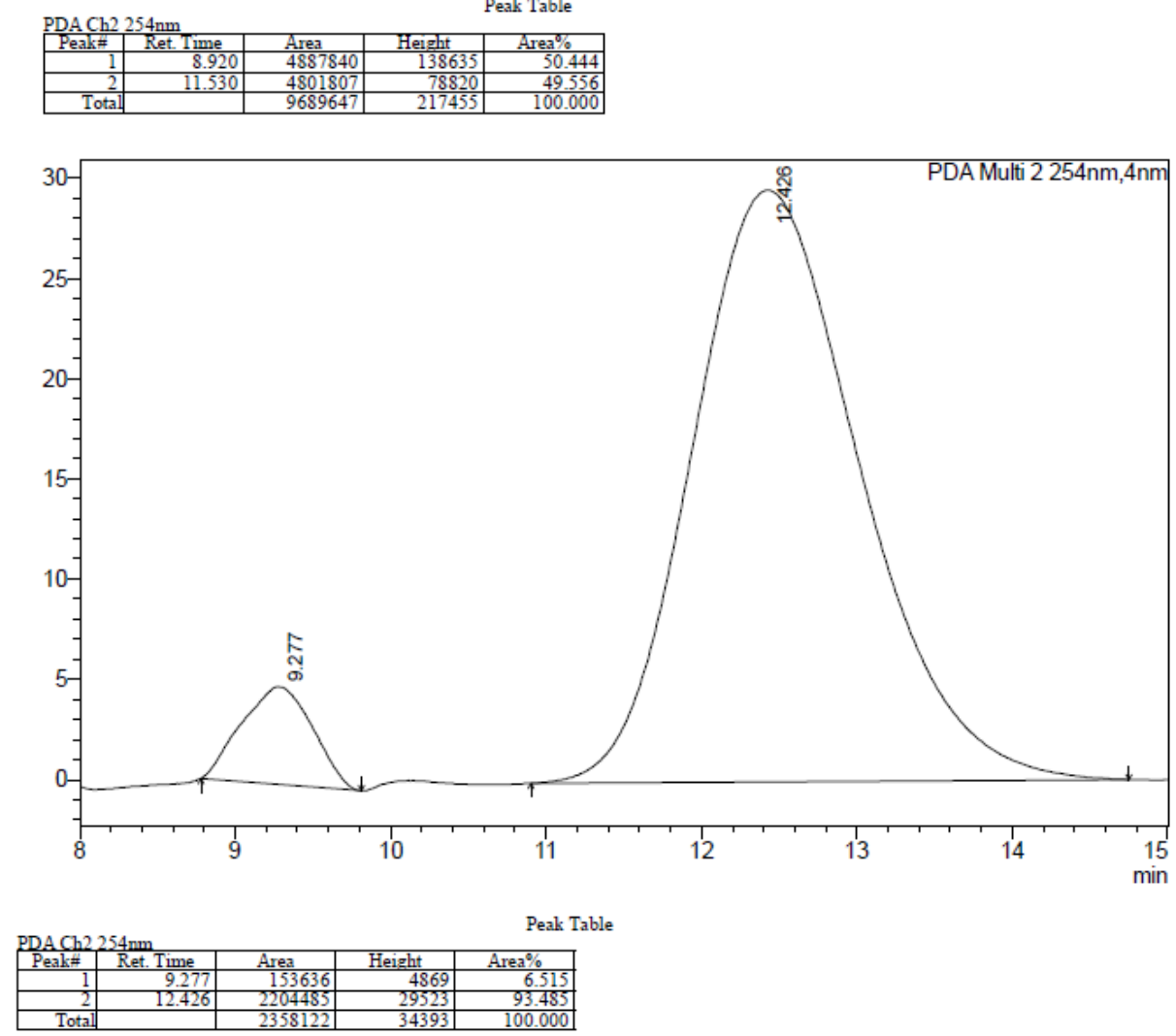

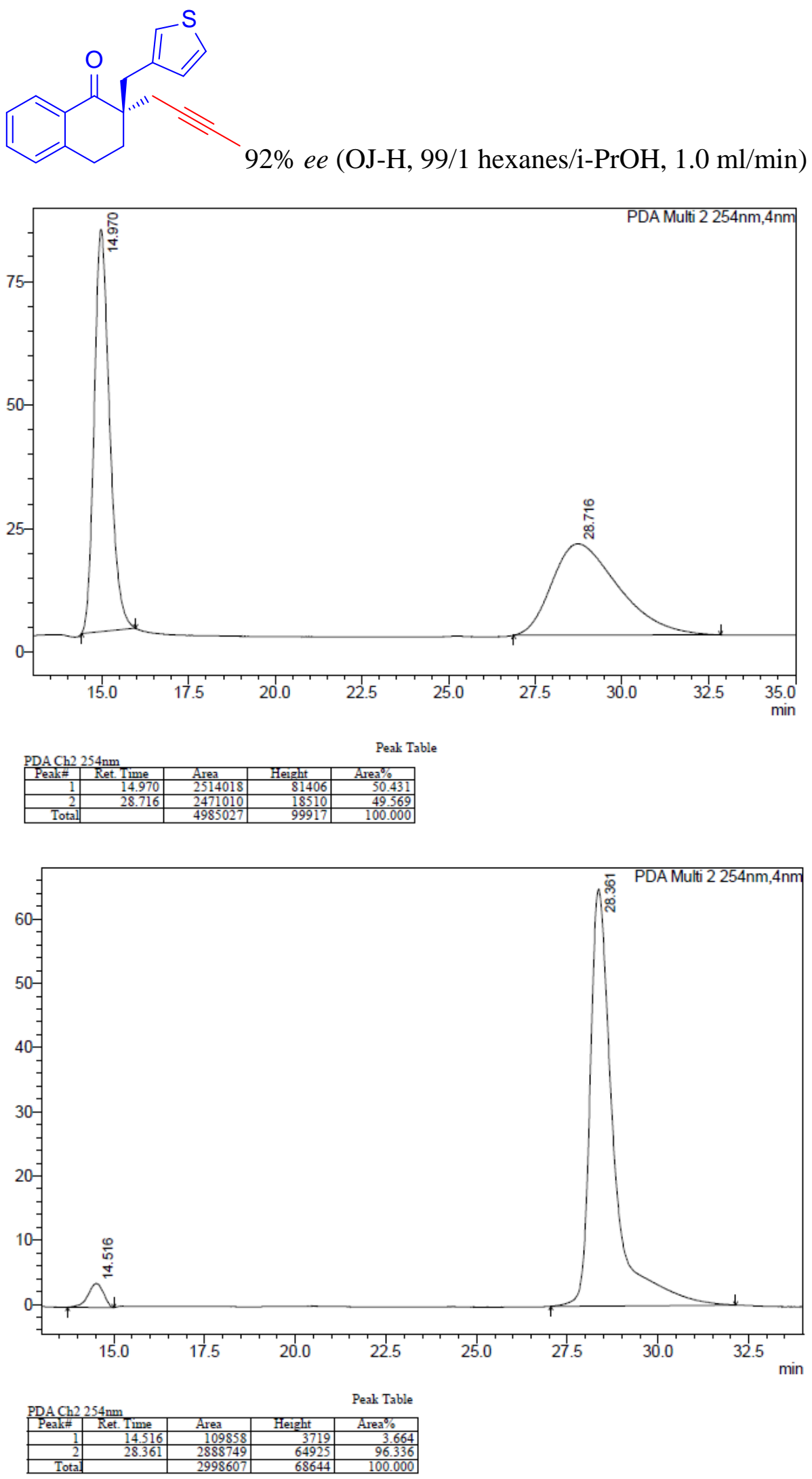
<smiles>COC(=O)C[C@]1(Cc2ccc(C(F)(F)F)cc2)CCc2ccccc2C1=O</smiles>

77\% ee (OD-H, 90/10 hexanes/i-PrOH, 1.0 ml/min)
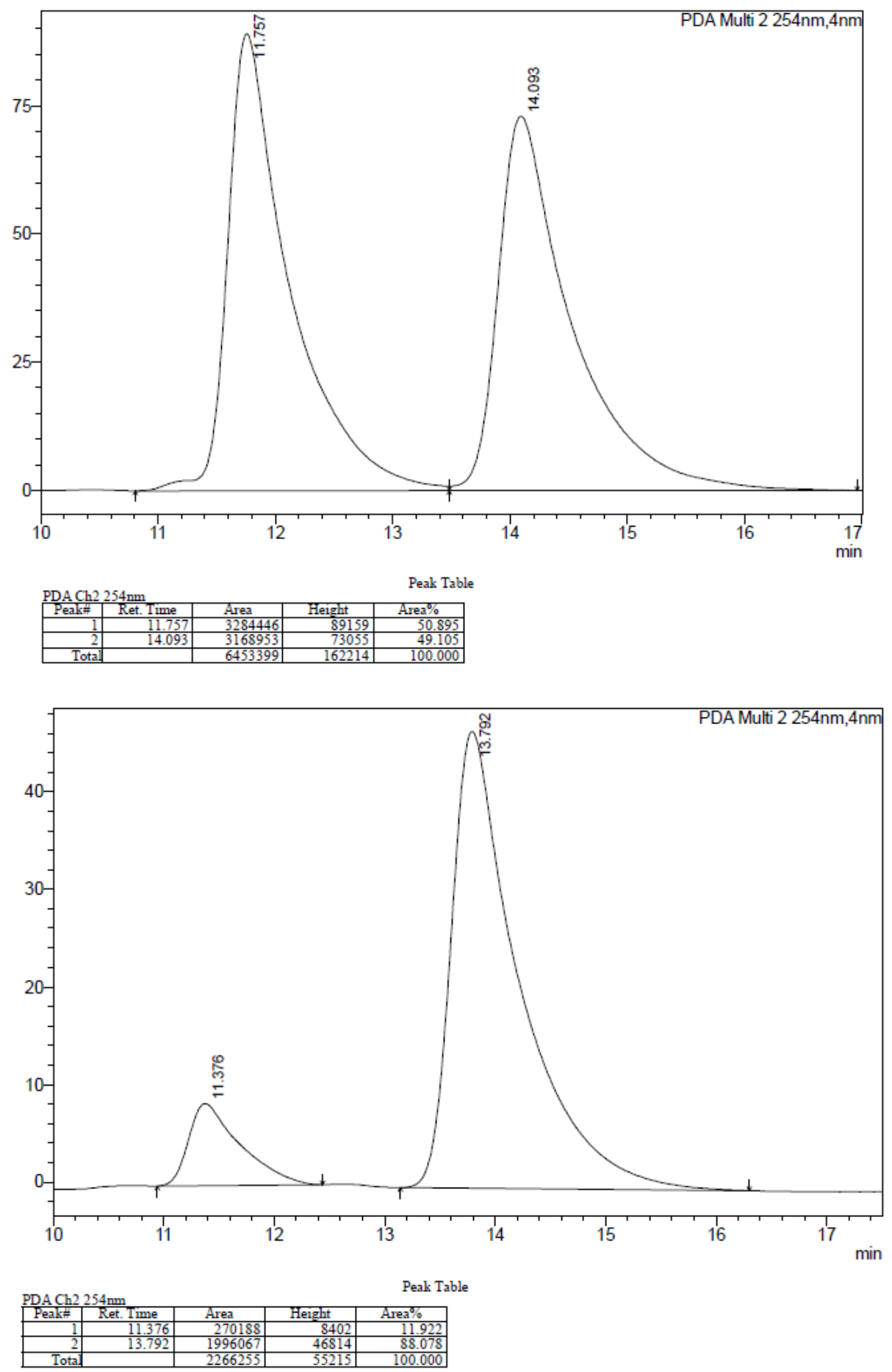
$\overbrace{i}^{O M e}$

85\% ee (OD-H, 90/10 hexanes/i-PrOH, 1.0 ml/min)

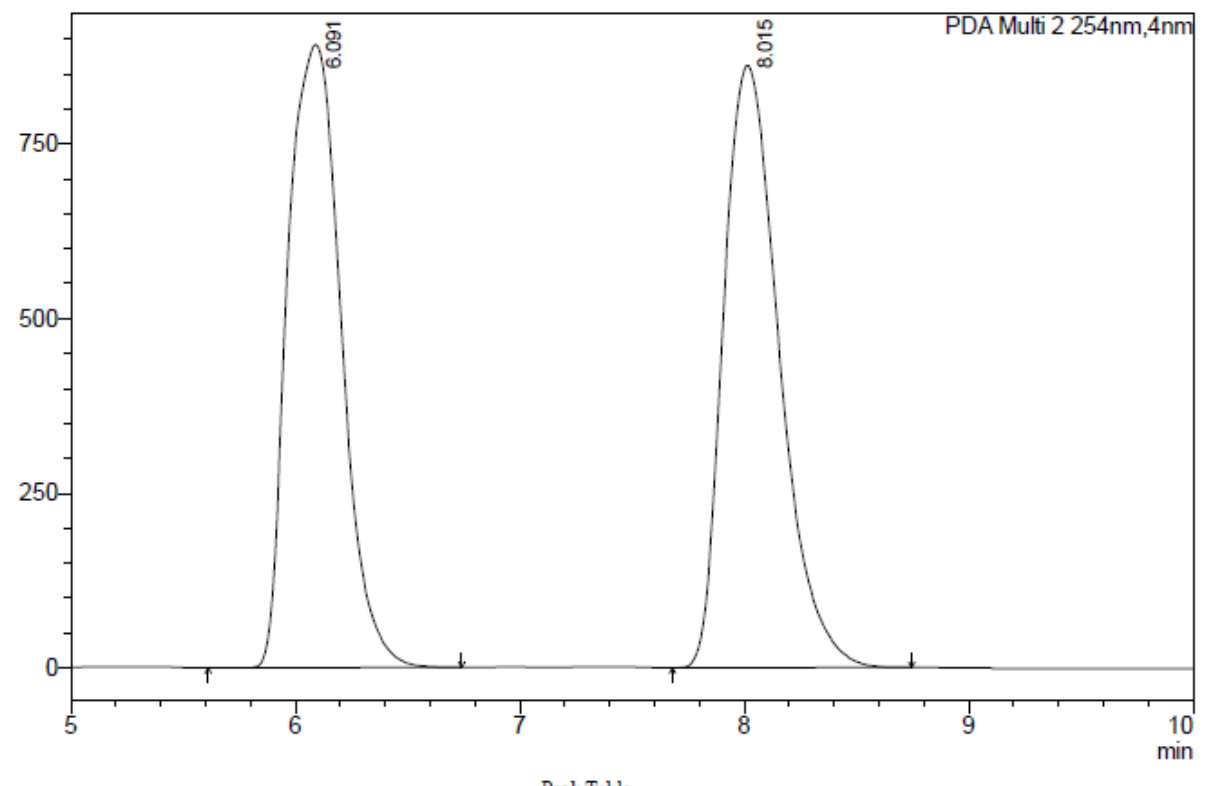

Peak Table
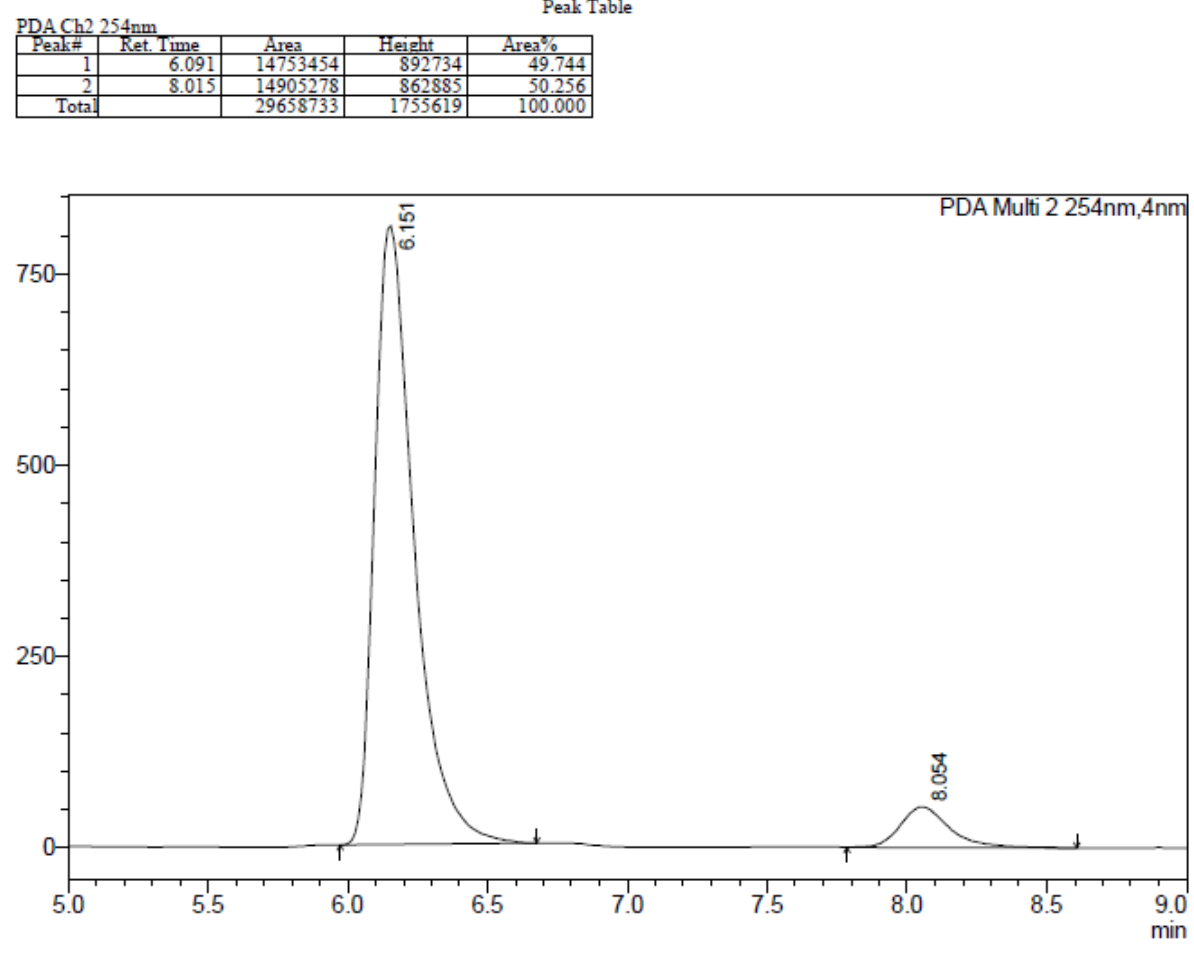

Peak Table

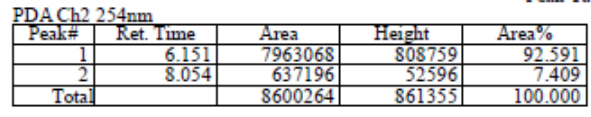


<smiles>CCCC[C@H](CC(=O)OC)C(=O)c1ccccc1</smiles>

89\% ee (OD-H, 90/10 hexanes/i-PrOH, $1.0 \mathrm{ml} / \mathrm{min}$ )
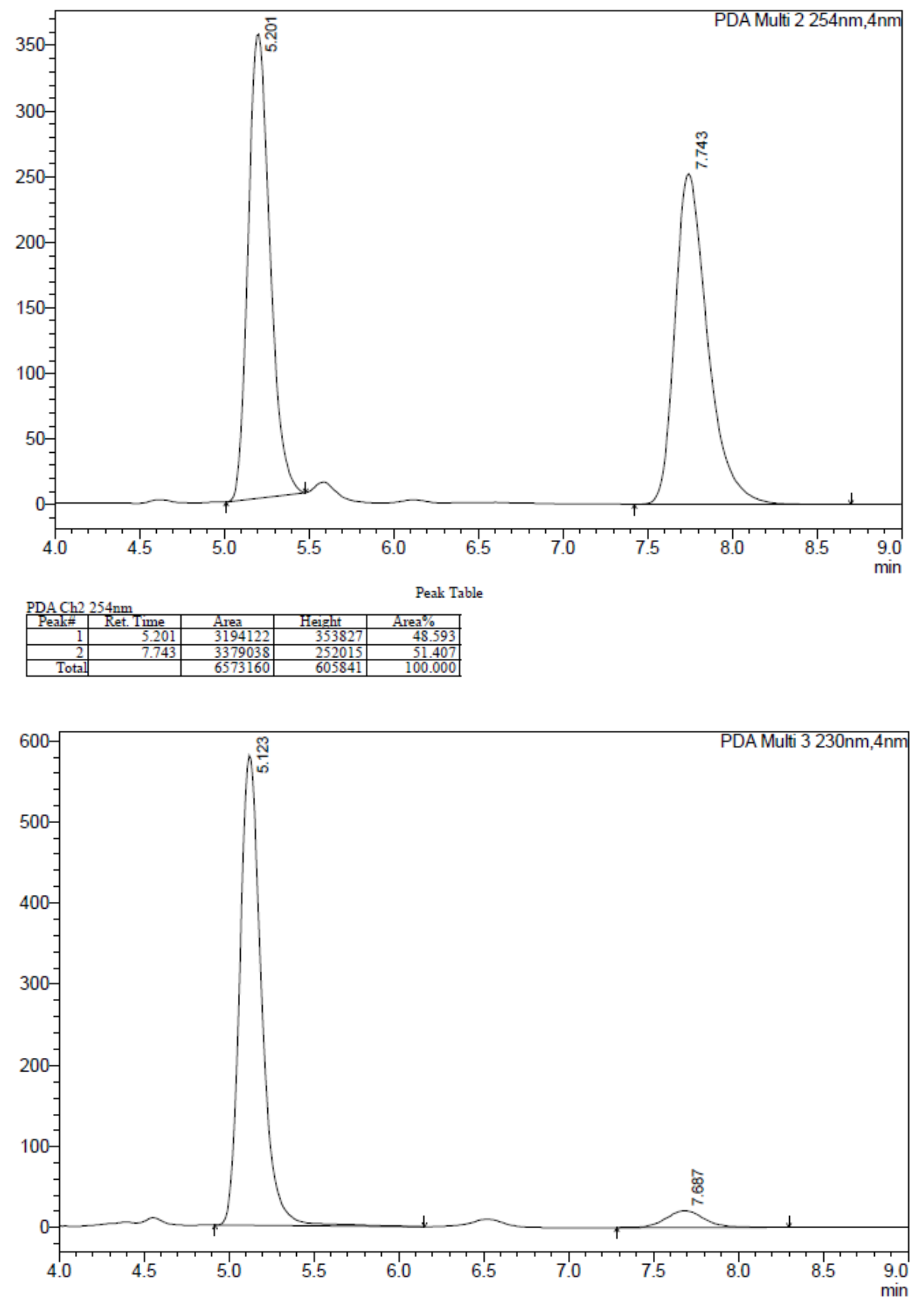

Peak Table

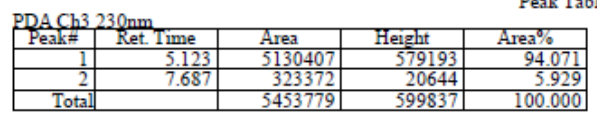


<smiles>CCCCCC[C@H](CC(=O)OC)C(=O)c1ccccc1</smiles>

89\% eе (OD-H, 90/10 hexanes/i-PrOH, 1.0 ml/min)
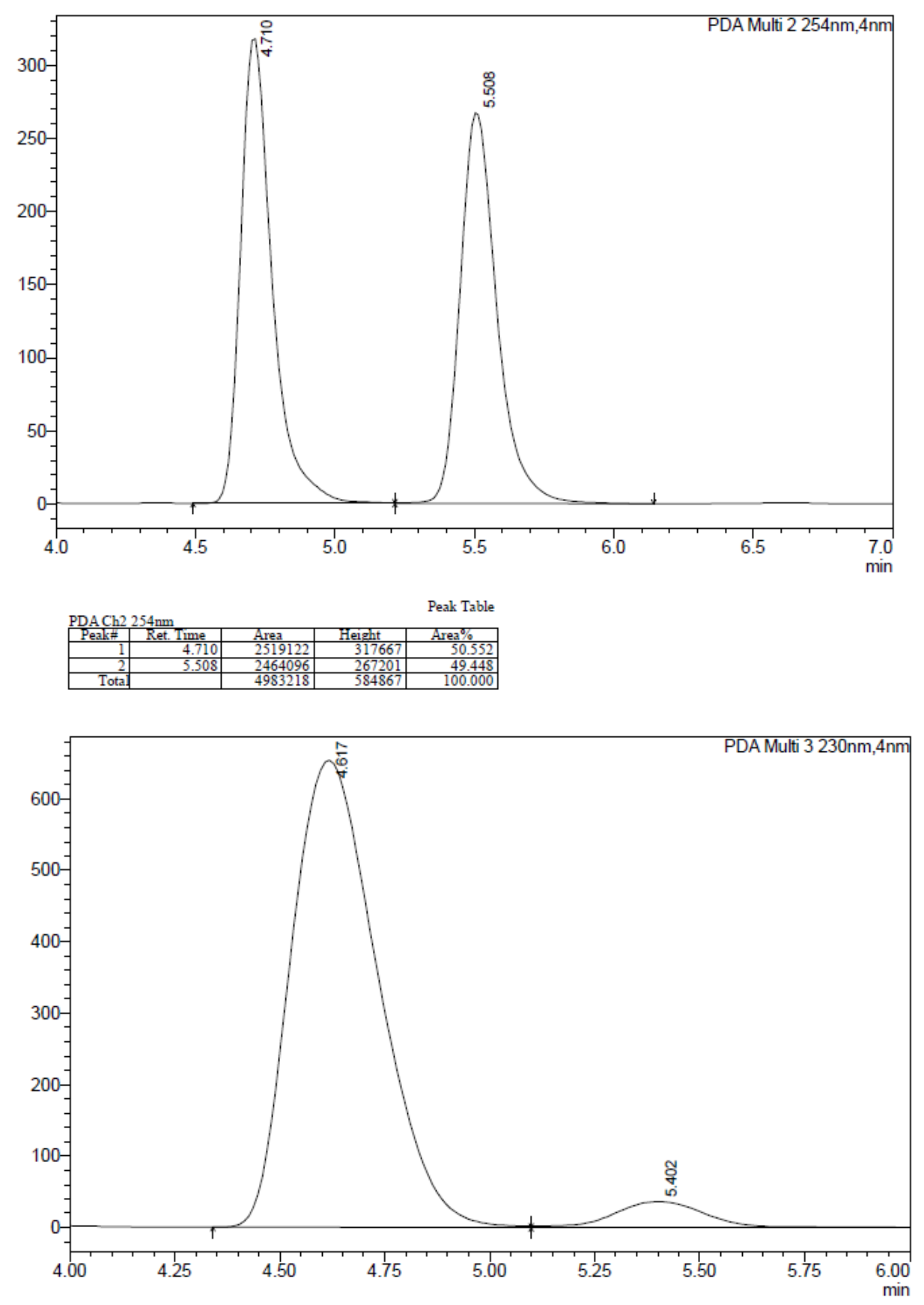

Peak Table

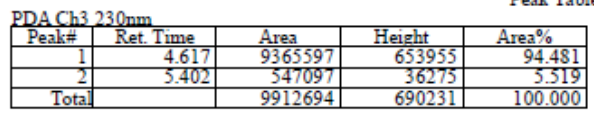


<smiles>CCCCCCCCC[C@H](CC(=O)OC)C(=O)c1ccccc1</smiles>

91\% ee (IB, 98/2 hexanes/i-PrOH, 1.0 ml/min)

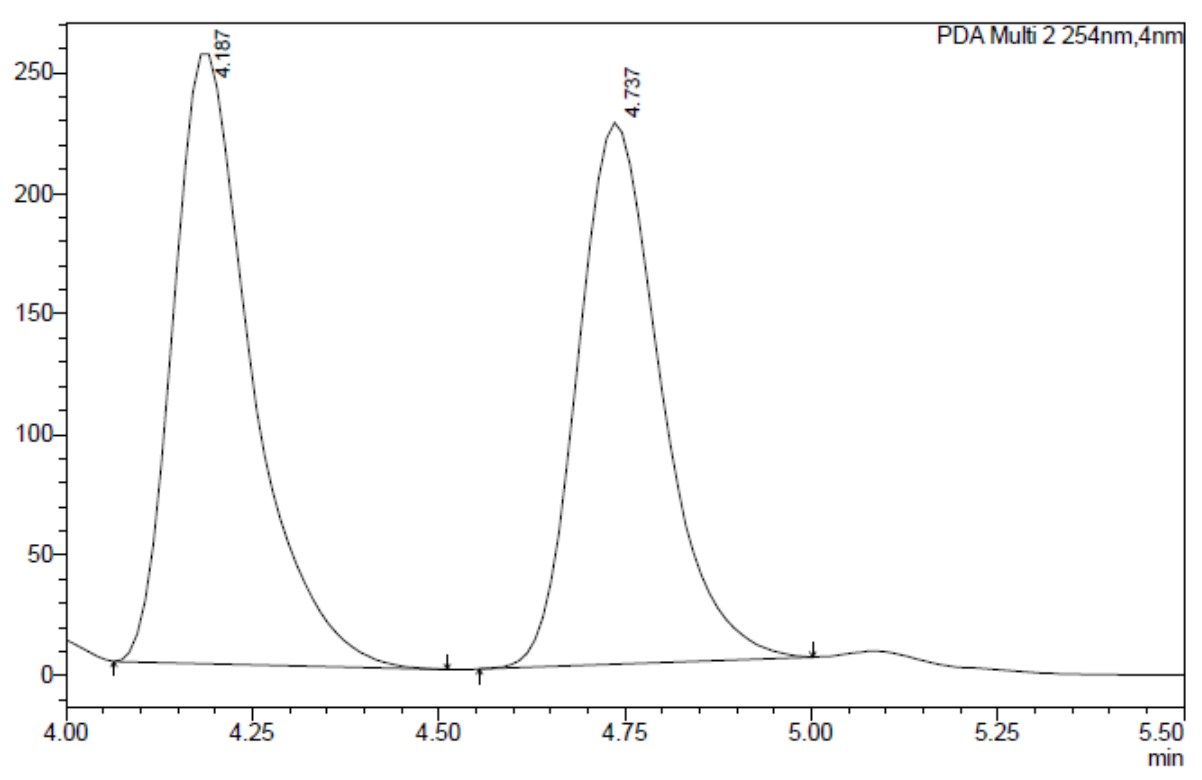

Peak Table
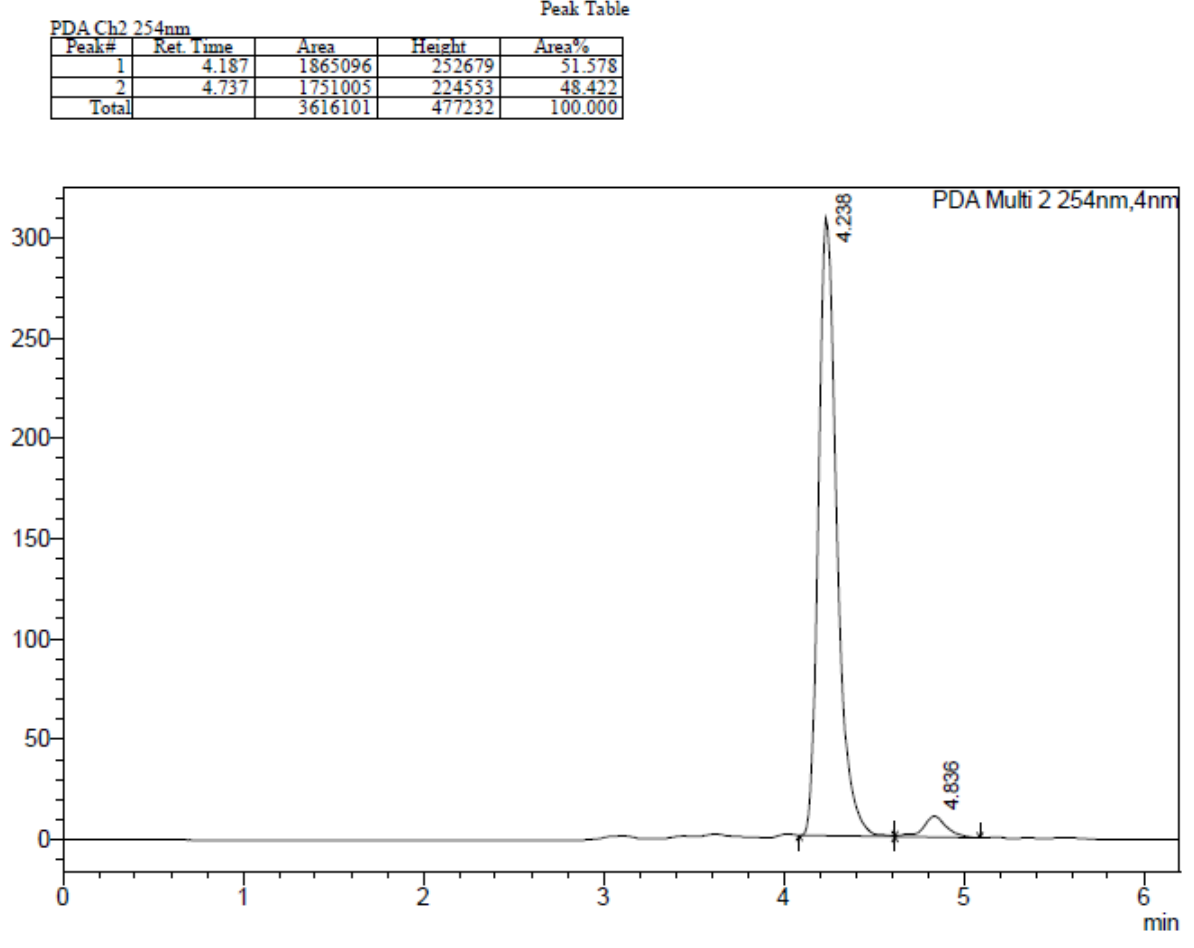

Peak Table

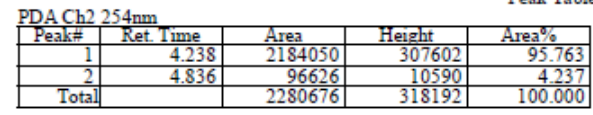


<smiles>CCOC(=O)C[C@H](C)C(=O)c1ccccc1</smiles>

85\% ee (OD-H, 90/10 hexanes/i-PrOH, 1.0 ml/min)

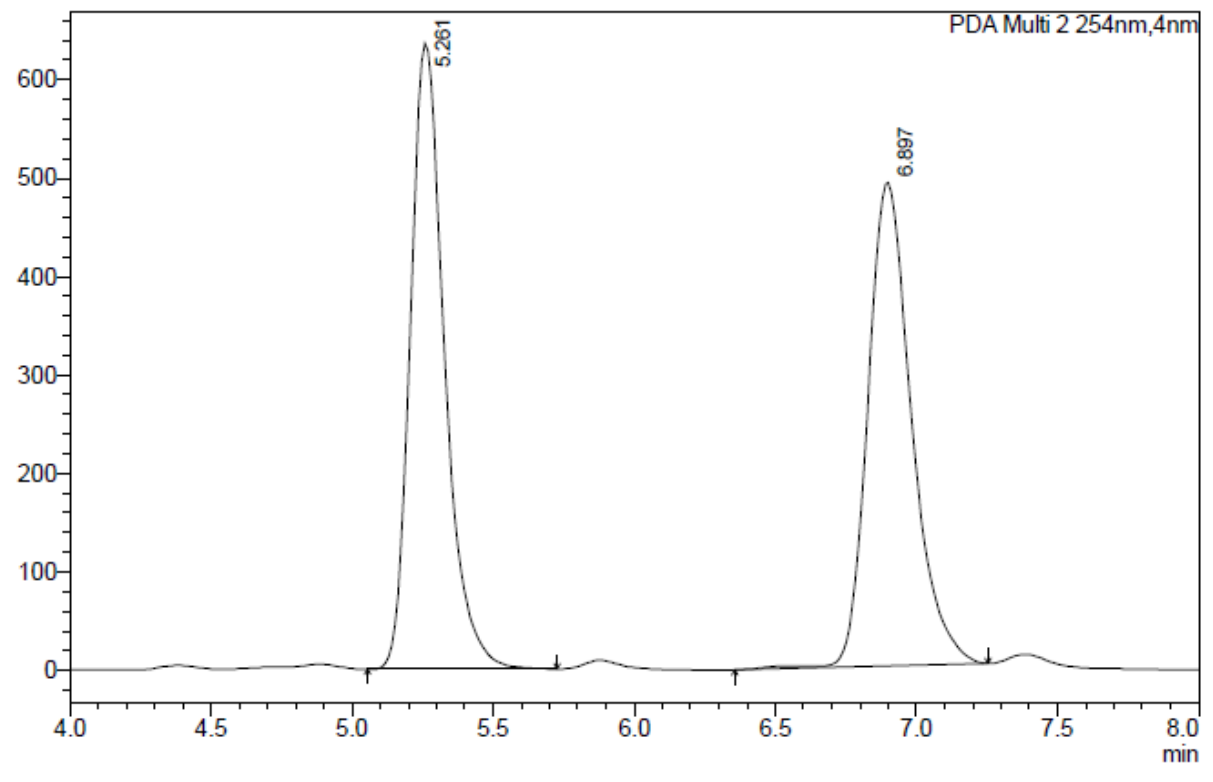

Peak Table
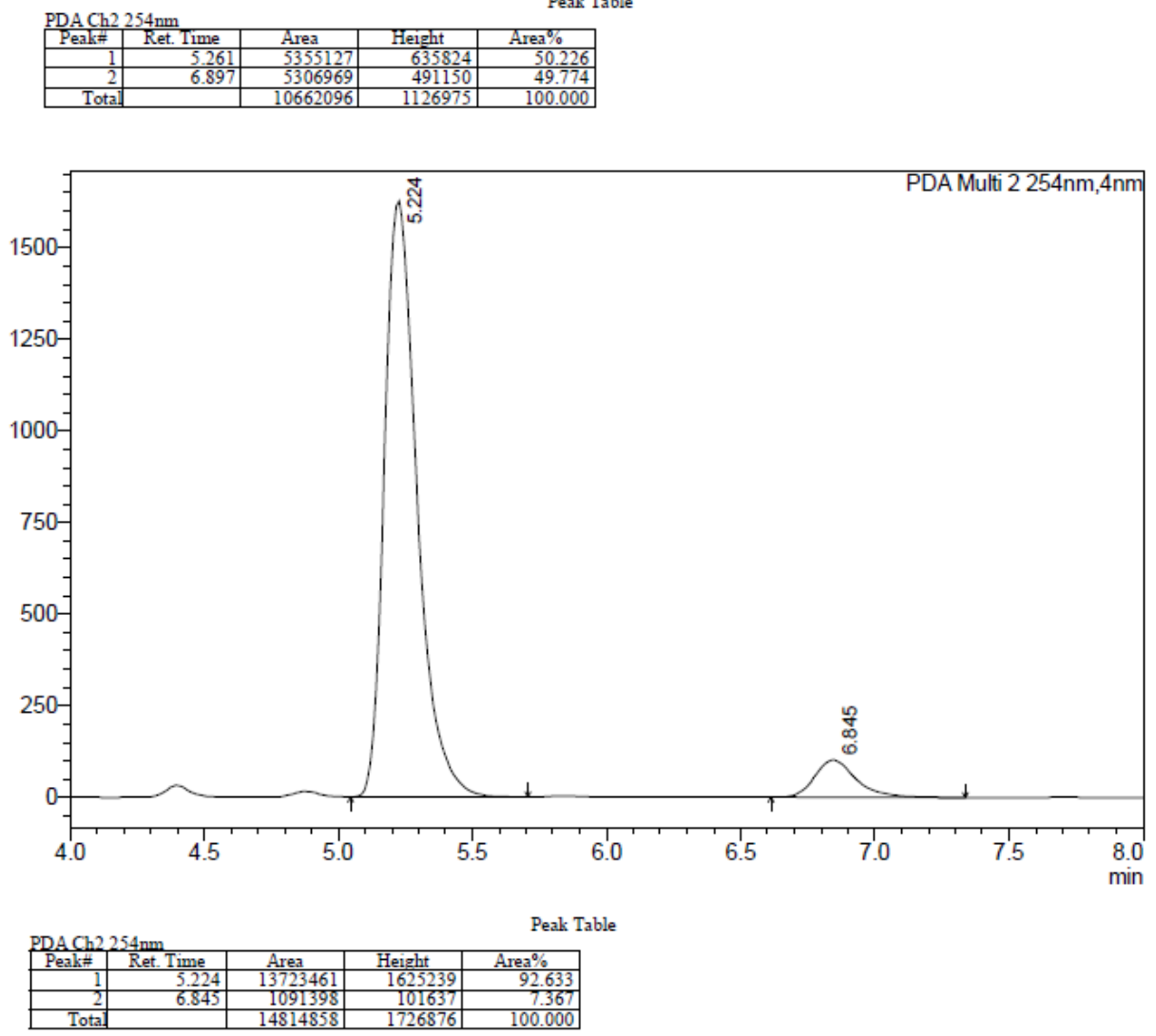
$\overbrace{O=}^{O M M e}$

85\% eе (OD-H, 90/10 hexanes/i-PrOH, 1.0 ml/min)

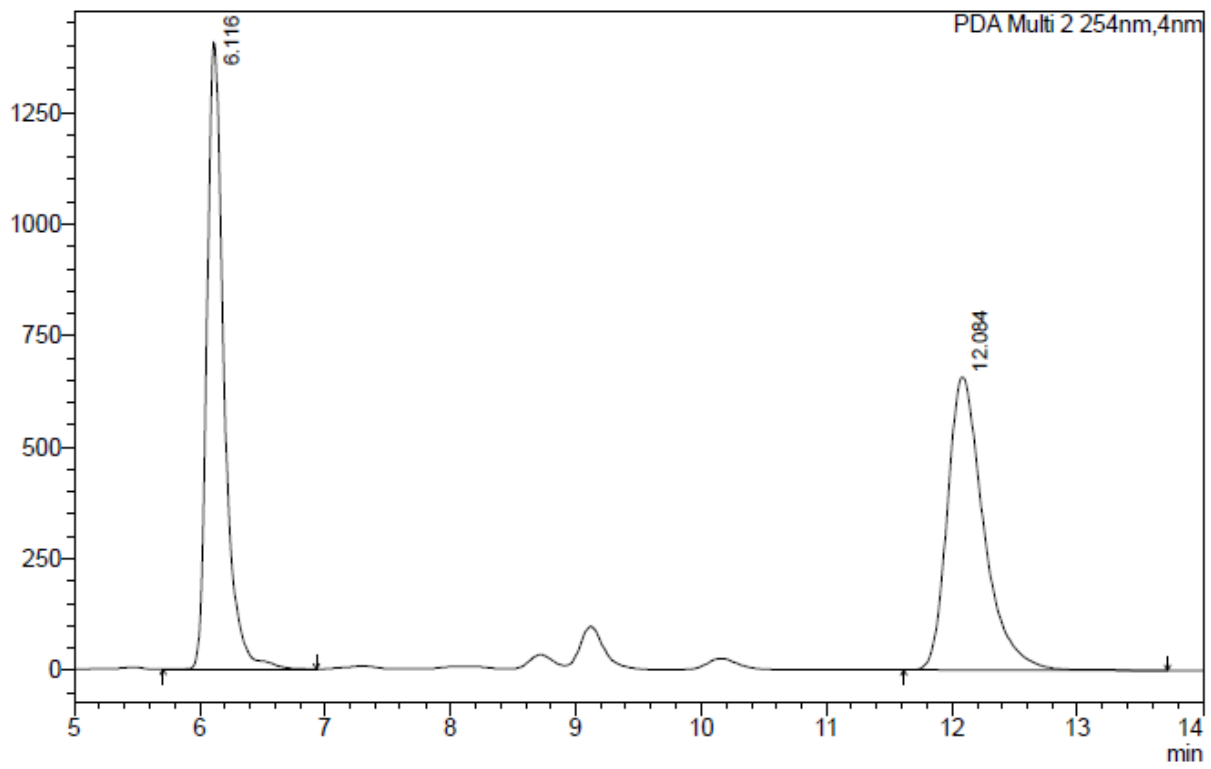

Peak Table
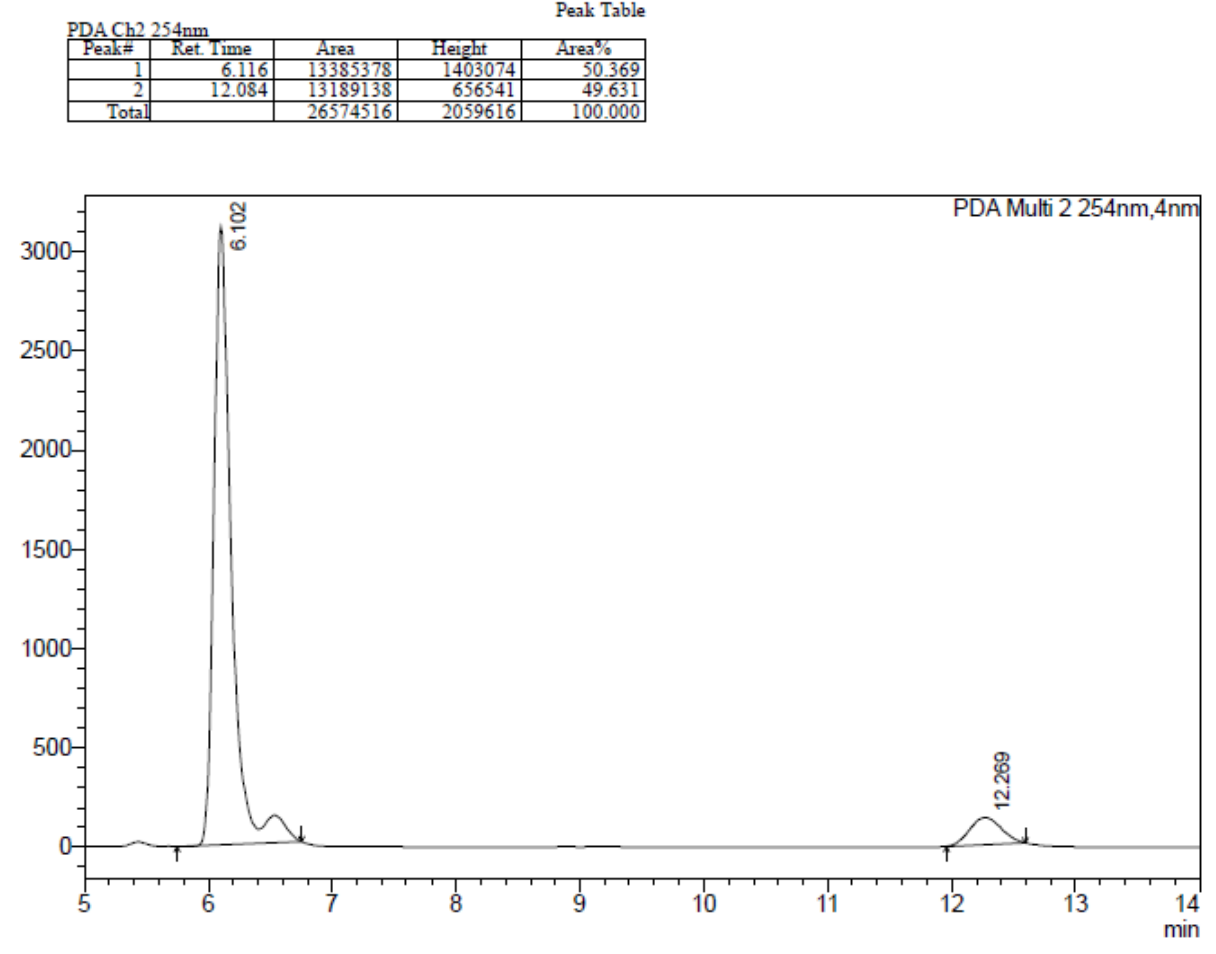

Peak Table

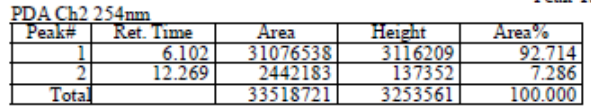


<smiles>COC(=O)C[C@H](C)C(=O)c1ccc(Cl)cc1</smiles>

86\% ee (OD-H, 90/10 hexanes/i-PrOH, $1.0 \mathrm{ml} / \mathrm{min}$ )
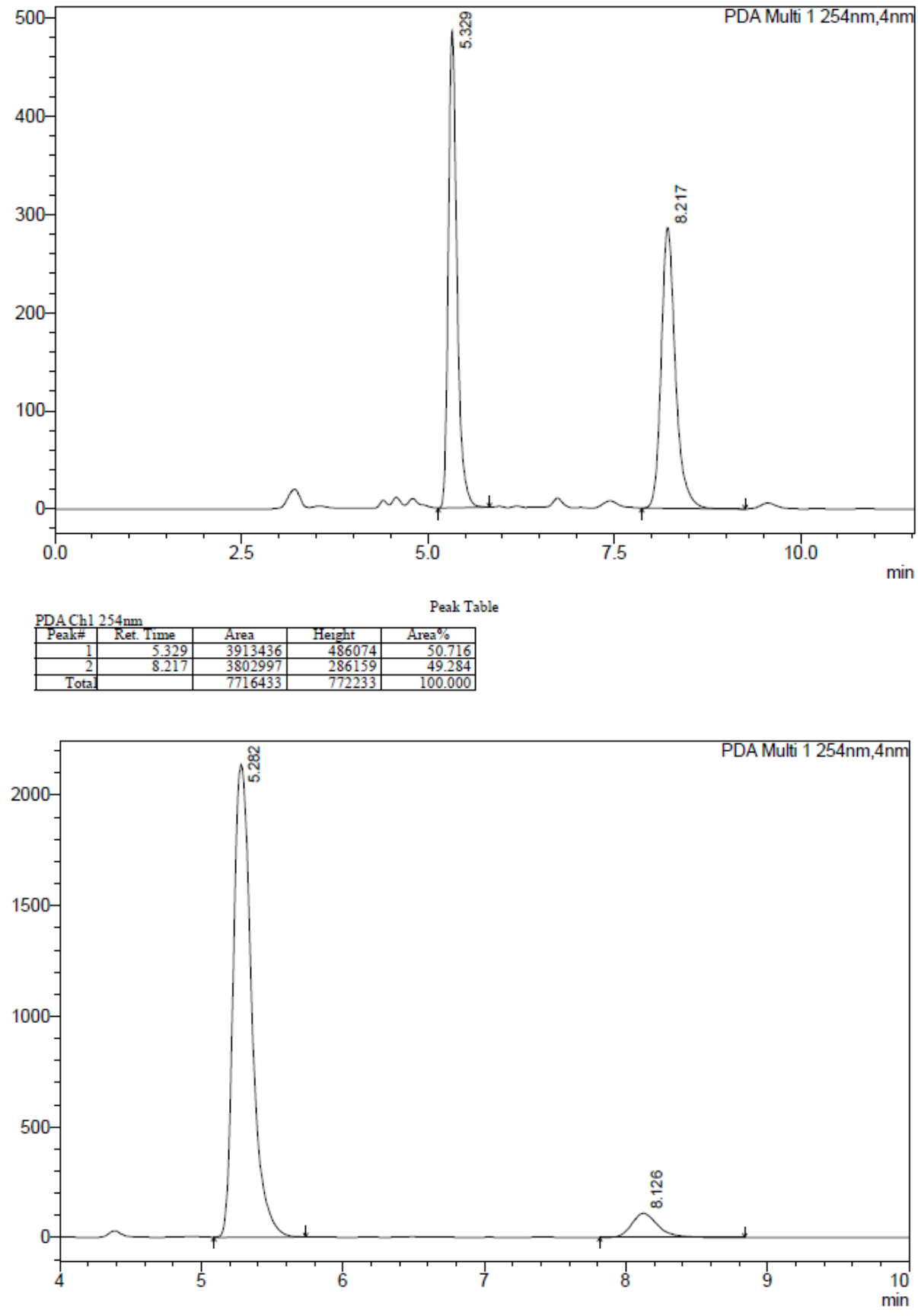

Peak Table

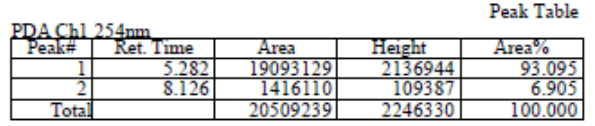


<smiles>COC(=O)C[C@H](C)C(=O)c1ccc(C)cc1</smiles>

90\% ee (OD-H, 90/10 hexanes/i-PrOH, 1.0 ml/min)

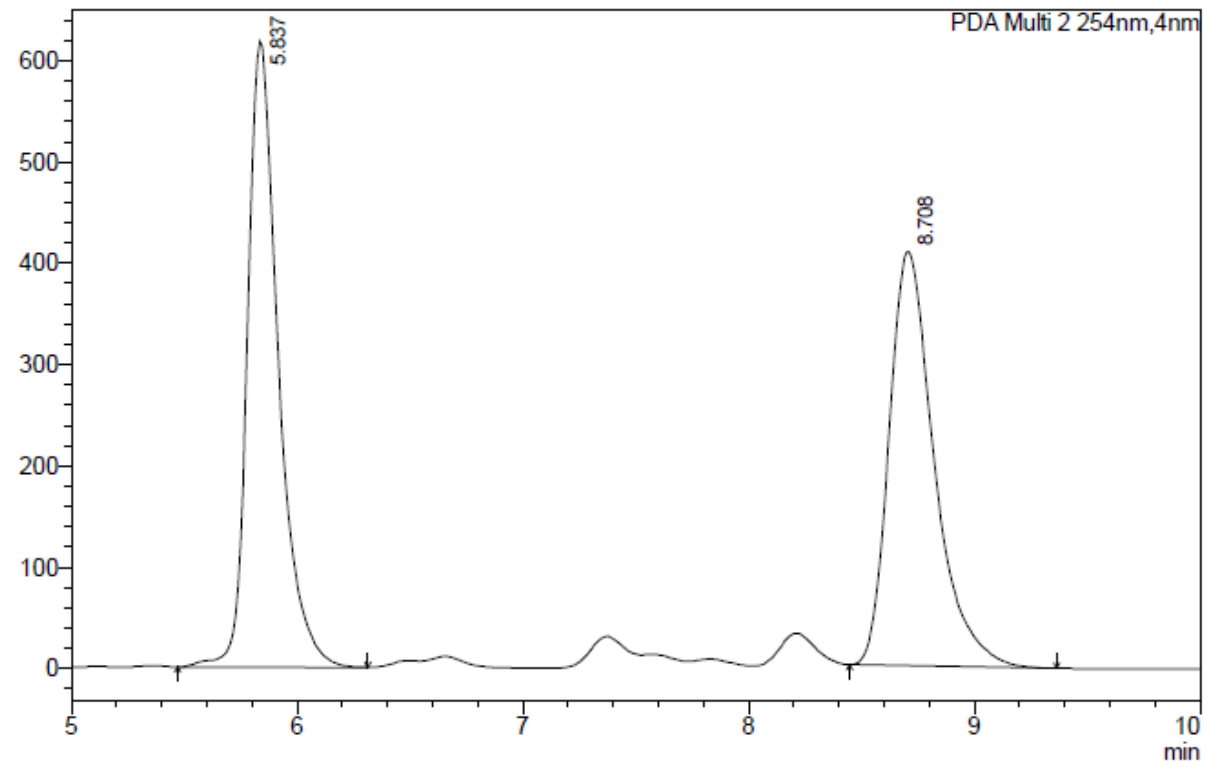

Peak Table
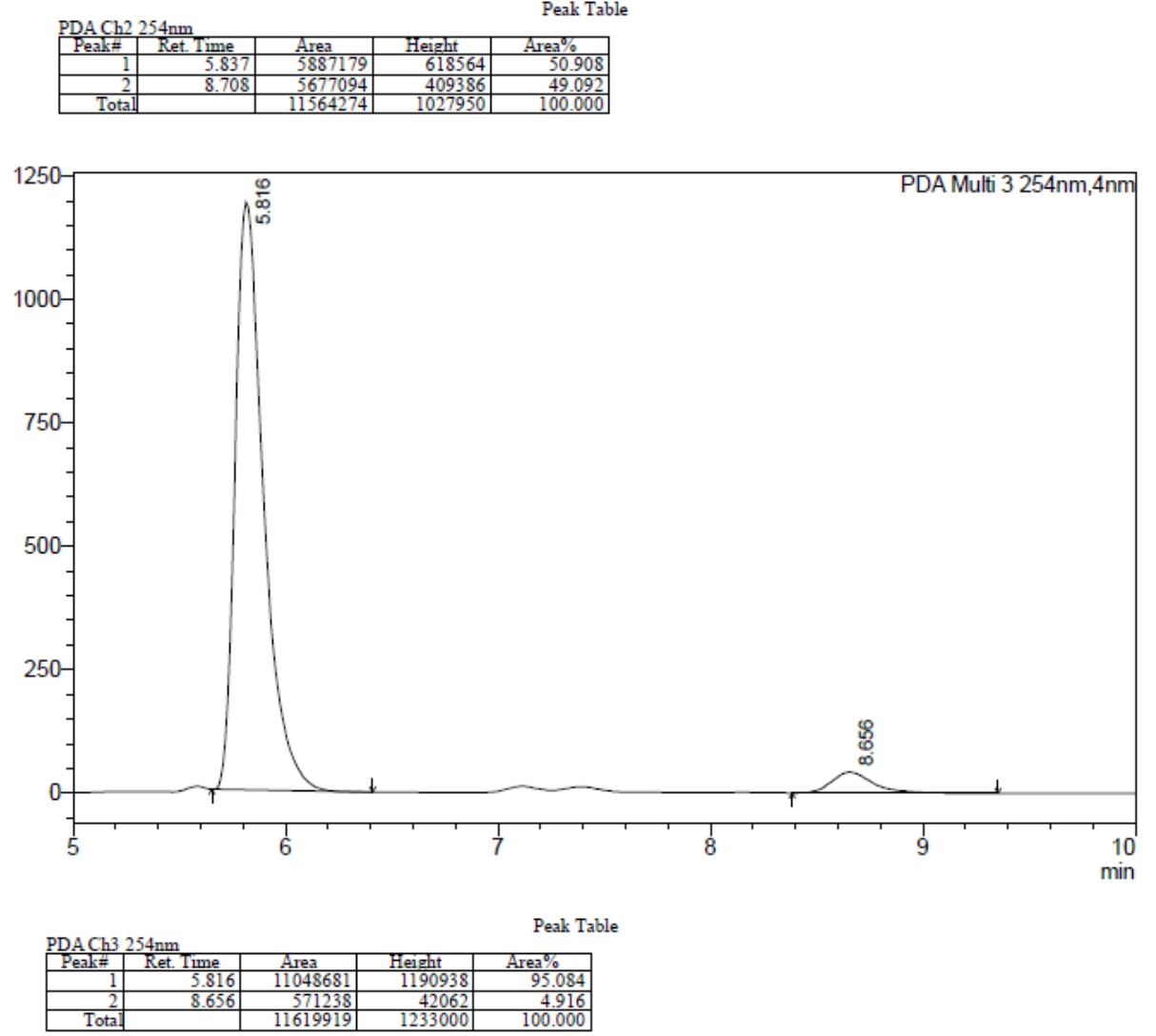
<smiles>COC(=O)C[C@H](C)C(=O)c1ccc(OC)cc1</smiles>

83\% ee (OD-H, 90/10 hexanes/i-PrOH, $1.0 \mathrm{ml} / \mathrm{min}$ )
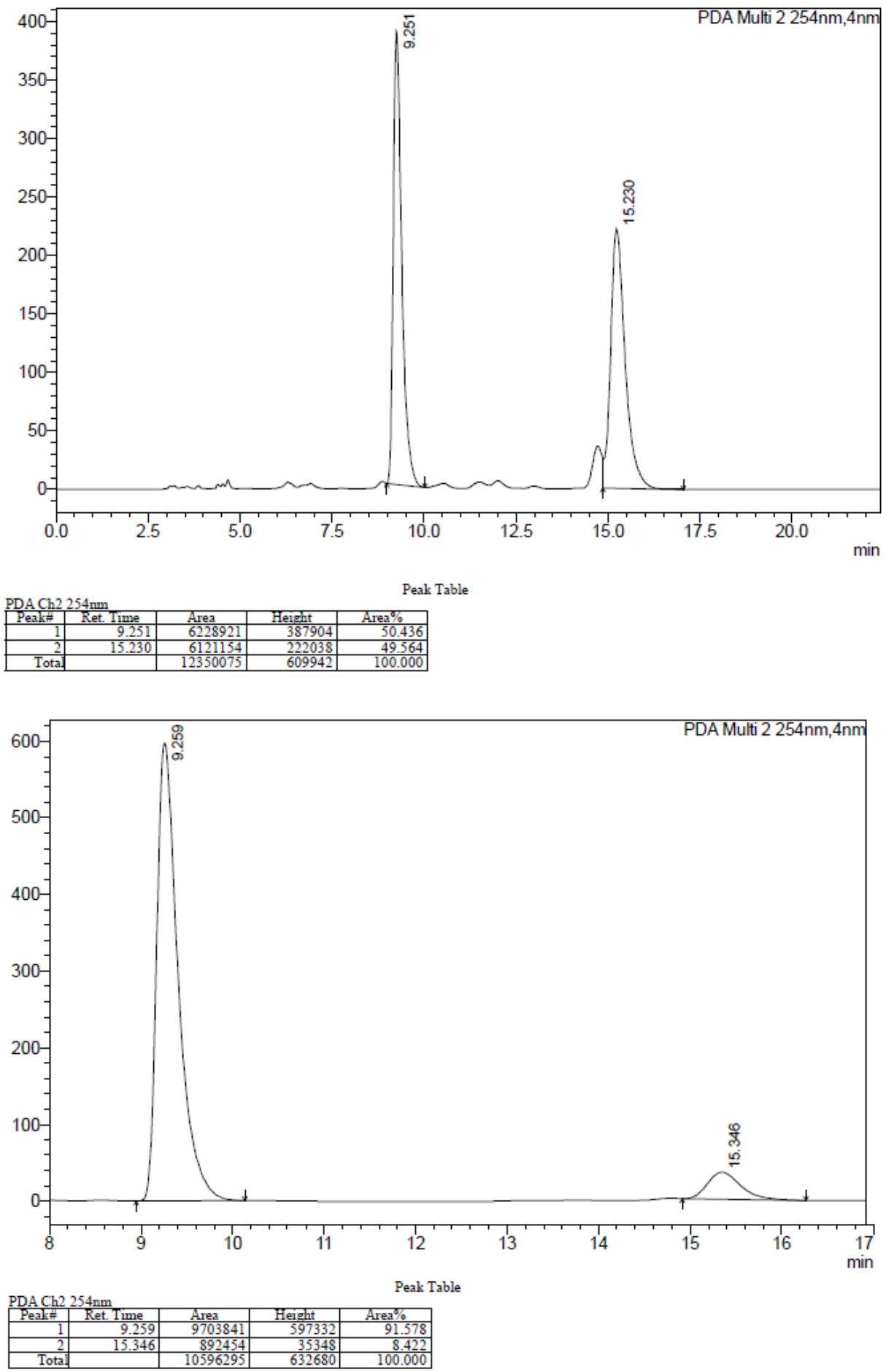